\title{
ZOOTAXA
}

2373

\section{Nomenclatural Studies Toward a World List of Diptera Genus-Group Names. Part I: André-Jean-Baptiste Robineau-Desvoidy}

\author{
Neal L. Evenhuis ${ }^{1}$, James E. O’Hara ${ }^{2}$, ThOmas PaPE ${ }^{3} \&$ Adrian C. Pont $^{4}$
}

1. J. Linsley Gressitt Center for Research in Entomology, Bishop Museum, 1525 Bernice Street, Honolulu, Hawaii 96817-2704, USA; email: NealE@bishopmuseum.org

2. Agriculture and Agri-Food Canada, 960 Carling Avenue, K.W. Neatby Building, Ottawa, Ontario, K1A 0C6, Canada; email: james.ohara@agr.gc.ca

3. Natural History Museum of Denmark, Universitetsparken 15, 2100 Copenhagen, Denmark; email:TPape@snm.ku.dk

4. Oxford University Museum of Natural History, Parks Road, Oxford OX1 3PW, UK;

email: pont.muscidae@btinternet.com

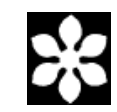

Magnolia Press

Auckland, New Zealand 
NEAL L. EVENHUIS, JAMES E. O'HARA, THOMAS PAPE \& ADRIAN C. PONT

Nomenclatural Studies Toward a World List of Diptera Genus-Group Names.

Part I: André-Jean-Baptiste Robineau-Desvoidy

(Zootaxa 2373)

265 pp.; $30 \mathrm{~cm}$.

26 February 2010

ISBN 978-1-86977-459-2 (paperback)

ISBN 978-1-86977-460-8 (Online edition)

FIRST PUBLISHED IN 2010 BY

Magnolia Press

P.O. Box 41-383

Auckland 1346

New Zealand

e-mail: zootaxa@mapress.com

http://www.mapress.com/zootaxa/

(C) 2010 Magnolia Press

All rights reserved.

No part of this publication may be reproduced, stored, transmitted or disseminated, in any form, or by any means, without prior written permission from the publisher, to whom all requests to reproduce copyright material should be directed in writing.

This authorization does not extend to any other kind of copying, by any means, in any form, and for any purpose other than private research use.

ISSN 1175-5326 (Print edition)

ISSN 1175-5334 (Online edition) 


\section{Table of contents}

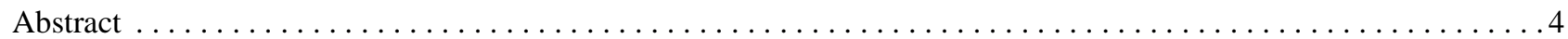

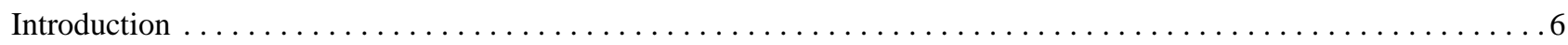

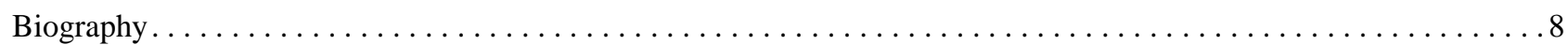

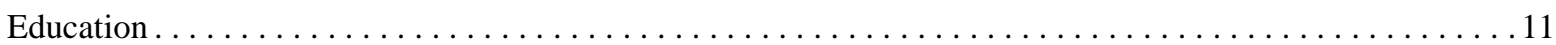

Robineau-Desvoidy's Character and Personality $\ldots \ldots \ldots \ldots \ldots \ldots \ldots \ldots \ldots \ldots \ldots \ldots \ldots \ldots \ldots$

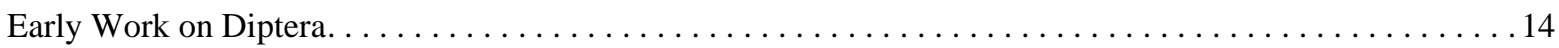

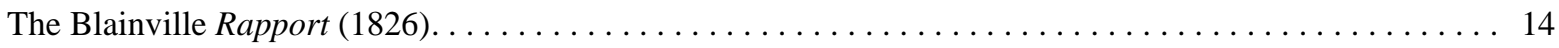

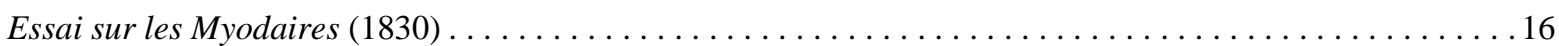

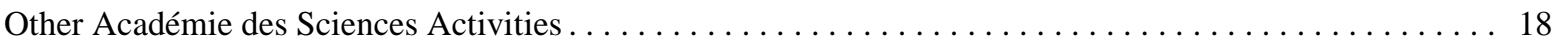

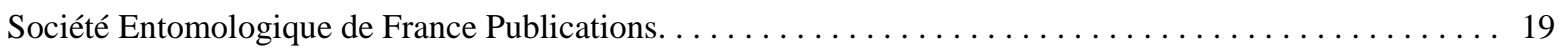

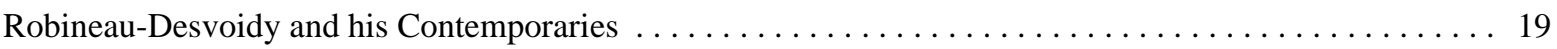

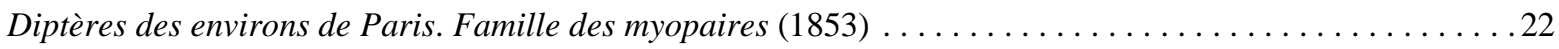

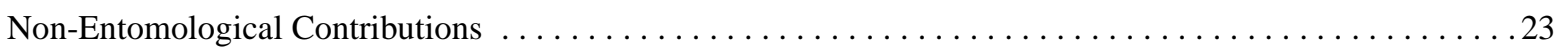

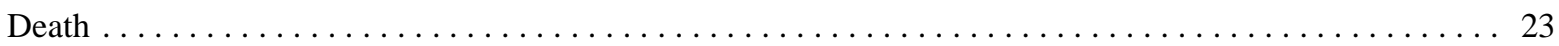

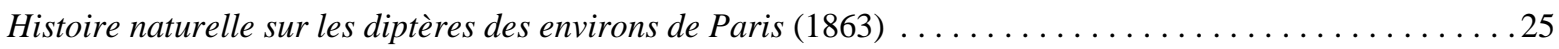

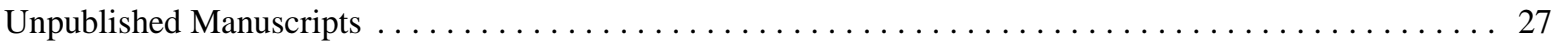

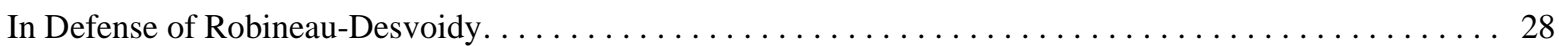

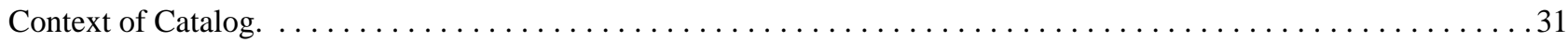

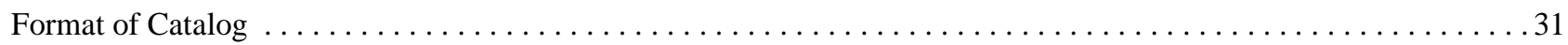

Catalog of Diptera Genus-Group Names of André-Jean-Baptiste Robineau-Desvoidy . . . . . . . . . . . . 33

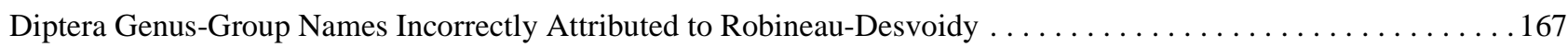

List of Diptera Genus-Group Names of Robineau-Desvoidy by Family. . . . . . . . . . . . . . . . . . . . 169

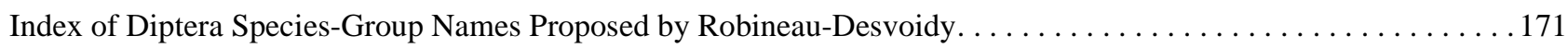

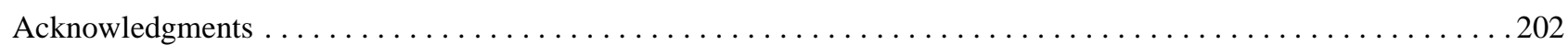

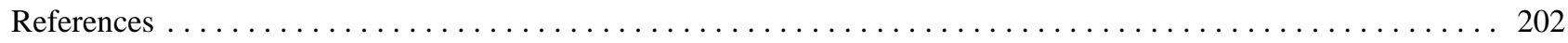

Appendix I. Complete Bibliography of André-Jean-Baptiste Robineau-Desvoidy. . . . . . . . . . . . . . . . . 224

Appendix II. Robineau-Desvoidy's Collections, Collectors, and Localities $\ldots \ldots \ldots \ldots \ldots \ldots \ldots$

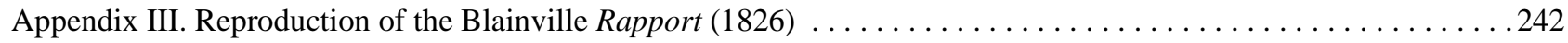




\section{Abstract}

This is the first in a series of rigorous nomenclatural studies of selected Diptera workers, undertaken as part of the Biosystematic Database of World Diptera (BDWD). A total of 566 genus-group names of Diptera proposed by A.-J.-B. Robineau-Desvoidy are listed, each with all originally included nominal species, method of typification, current taxonomic status, and emendations. A detailed biography of Robineau-Desvoidy is given with discussion of his works and his relationships with contemporaries. In addition, an index to all the species-group names of Diptera proposed by RobineauDesvoidy $(3,204)$ is given with bibliographic reference to each original citation. Appended to this study are a full bibliography of Robineau-Desvoidy's works, a list of collectors on which Robineau-Desvoidy based his Diptera studies, a list of collecting localities mentioned in his Diptera works, and a reproduction of the little-known but valuable 1826 Blainville Rapport of Robineau-Desvoidy's 1830 Essai sur les Myodaires.

Type species are designated for the following genus-group names: Dasyphora Robineau-Desvoidy, 1830 [Muscidae]; Dyctia Robineau-Desvoidy, 1830 [Sciomyzidae]; Fimetia Robineau-Desvoidy, 1830 [Sphaeroceridae]; and Haematobia Robineau-Desvoidy, 1830 [Muscidae].

Acting as First Reviser of multiple original spellings, the following are selected as correct original spellings for genus-group names: Arisbaea Robineau-Desvoidy, 1863 [Tachinidae]; Carbonia Robineau-Desvoidy, 1863 [Tachinidae]; Elbaea Robineau-Desvoidy, 1863 [Tachinidae]; Eversmania Robineau-Desvoidy, 1863 [Tachinidae]; Faedoria Robineau-Desvoidy, 1863 [Tachinidae]; Fairmairia Robineau-Desvoidy, 1853 [Conopidae]; Gymnodia Robineau-Desvoidy, 1863 [Muscidae]; Lylibaea Robineau-Desvoidy, 1863 [Tachinidae]; Phaenicia Robineau-Desvoidy, 1863 [Calliphoridae]; Solieria Robineau-Desvoidy, 1849 [Tachinidae]; and Stephensia Robineau-Desvoidy, 1863 [Tachinidae]; and for species-group names: Cyzenis haemisphaerica Robineau-Desvoidy, 1863 [Tachinidae].

Earlier or corrected type-species designations are given for the following genus-group names: Elpigia RobineauDesvoidy, 1863 [Sarcophagidae]; Erigone Robineau-Desvoidy, 1830 [Tachinidae]; Kirbya Robineau-Desvoidy, 1830 [Tachinidae]; Lilaea Robineau-Desvoidy, 1863 [Tachinidae]; Meckelia Robineau-Desvoidy, 1830 [Ulidiidae]; Melinda Robineau-Desvoidy, 1830 [Calliphoridae]; Misellia Robineau-Desvoidy, 1863 [Sarcophagidae]; Myophora RobineauDesvoidy, 1830 [Sarcophagidae]; Myoris Robineau-Desvoidy, 1830 [Ulidiidae]; Odinia Robineau-Desvoidy, 1830 [Odiniidae]; Phoraea Robineau-Desvoidy, 1830 [Anthomyiidae]; Rhinia Robineau-Desvoidy, 1830 [Rhiniidae]; Rhinophora Robineau-Desvoidy, 1830 [Rhinophoridae]; Sabethes Robineau-Desvoidy, 1827 [Culicidae]; Salticella RobineauDesvoidy, 1830 [Sciomyzidae]; and Urellia Robineau-Desvoidy, 1830 [Tephritidae].

The following new synonymies are proposed as junior synonyms under their respective valid genus-group names: under Acemya Robineau-Desvoidy, 1830 [Tachinidae] is Acemyia Schiner, 1861, n. syn.; under Aplomya Robineau-Desvoidy, 1830 [Tachinidae] is Aplomyia Agassiz, 1846, n. syn.; under Billaea Robineau-Desvoidy, 1830 [Tachinidae] is Nicea Marschall, 1873, n. syn.; under Bohemania Robineau-Desvoidy, 1863 [Tachinidae] is Bohemannia Bezzi \& Stein, 1907, n. syn.; under Botanophila Lioy, 1864 [Anthomyiidae] is Aegeria Agassiz, 1846, n. syn.; under Campylocheta Rondani, 1859 [Tachinidae] is Goedartia Bezzi \& Stein, 1907, n. syn.; under Cephenemyia Latreille, 1818 [Oestridae] is Cephenemya Robineau-Desvoidy, 1830, n. syn.; under Chrysomya Robineau-Desvoidy, 1830 [Calliphoridae] is Chrysomyia Macquart, 1835, n. syn.; under Chyromya Robineau-Desvoidy, 1830 [Chyromyidae] is Chyromyia Schiner, 1864, n. syn.; under Cistogaster Latreille, 1829 [Tachinidae] is Palassa Lioy, 1864, n. syn.; under Clairvillia Robineau-Desvoidy, 1830 [Tachinidae] is Phaniomyia Brauer \& Bergenstamm, 1889, n. syn.; under Conops irritans Linnaeus, 1758 [Muscidae] is Haematobia ferox Robineau-Desvoidy, 1830, n. syn.; under Cylidria Robineau-Desvoidy, 1830 [Sciomyzidae] is Cylindria Hendel, 1900, n. syn.; under Dinera Robineau-Desvoidy, 1830 [Tachinidae] is Myiocera Brauer \& Bergenstamm, 1889, n. syn.; under Dufouria Robineau-Desvoidy, 1830 [Tachinidae] is Silbermannia Marschall, 1873, n. syn.; under Dumerillia Robineau-Desvoidy, 1830 [Tachinidae] are Dumerilia Duponchel in d'Orbigny, 1844, n. syn., Dumerilia Agassiz, 1846, n. syn.; under Elodia Robineau-Desvoidy, 1830 [Tachinidae] is Westwoodia Herting, $1974, \mathbf{n}$. syn.; under Elomya Robineau-Desvoidy, 1830 [Tachinidae] is Helomyia Agassiz, 1846, n. syn.; under Elophoria Robineau-Desvoidy, 1830 [Tachinidae] is Helophoria Bezzi \& Stein, 1907, n. syn.; under Eriothrix Meigen, 1803 [Tachinidae] is Oliviera Lioy, 1864, n. syn.; under Estheria Robineau-Desvoidy, 1830 [Tachinidae] is Myiostoma Brauer \& Bergenstamm, 1889, n. syn.; under Eurithia Robineau-Desvoidy, 1844 [Tachinidae] is Eurythia Brauer \& Bergenstamm, 1889, n. syn.; under Eurysthaea Robineau-Desvoidy, 1863 [Tachinidae] is Euristhaea Marschall, 1873, n. syn.; under Fannia Robineau-Desvoidy, 1830 [Fanniidae] is Philintha Agassiz, 1846, n. syn.; under Freraea Robineau-Desvoidy, 1830 [Tachinidae] is Frerea Agassiz, 1846, n. syn.; under Gimmenthalia Robineau-Desvoidy, 1863 [Tachinidae] is Gimmerthalia Bezzi \& Stein, 1907, n. syn.; under Gonia Meigen, 1803 [Tachinidae] are Pissemyia Schiner, 1861, n. syn., Redia Bezzi \& Stein, 1907, n. syn.; under Haematobia Le Peletier \& Serville, 1828 [Muscidae] are Haematobia Robineau-Desvoidy, 1830, n. syn., Hoematobia Bigot, 1892, n. syn.; under Hermya Robineau-Desvoidy, 1830 [Tachinidae] is Hermyia 
Verrall in Scudder, 1882, n. syn.; under Hydrotaea Robineau-Desvoidy, 1830 [Muscidae] is Ophira Bigot, 1892, n. syn.; under Hydromya Robineau-Desvoidy, 1830 [Sciomyzidae] is Hydromyia Curtis, 1837, n. syn.; under Hylemya Robineau-Desvoidy, 1830 [Anthomyiidae] is Hylemia Marié, 1930, n. syn.; under Leiophora Robineau-Desvoidy, 1863 [Tachinidae] is Liophora Bezzi \& Stein, 1907, n. syn.; under Linnaemya Robineau-Desvoidy, 1830 [Tachinidae] is Linnaemyia Aldrich, 1905, n. syn.; under Loevia Robineau-Desvoidy, 1863 [Tachinidae] is Loewia Herting, 1974, n. syn.; under Macromya Robineau-Desvoidy, 1830 [Tachinidae] is Macromyia Agassiz, 1846, n. syn.; under Megarhinus Robineau-Desvoidy, 1827 [Culicidae] is Megarhina Osten Sacken, 1881, n. syn.; under Minettia Robineau-Desvoidy, 1830 [Lauxaniidae] is Terennia Schiner, 1868, n. syn.; under Musca Linnaeus, 1758 [Muscidae] are Plaxemyia Schiner, 1861, n. syn., Byomyia Williston, 1908, n. syn.; under Oestrus Linnaeus, 1758 [Oestridae] is Cephalemya Robineau-Desvoidy, 1830, n. syn.; under Oswaldia Robineau-Desvoidy, 1863 [Tachinidae] is Edomyia Bezzi \& Stein, 1907, n. syn.; under Otites Latreille, 1804 [Otitidae] is Meckelia Robineau-Desvoidy, 1830, n. syn.; under Palpomya Robineau-Desvoidy, 1830 [Platystomatidae] is Palpomyia Macquart, 1835, n. syn.; under Pegomya Robineau-Desvoidy, 1830 [Anthomyiidae] is Pegomyia Curtis, 1837, n. syn.; under Phaonia Robineau-Desvoidy, 1830 [Muscidae] are Fellea Agassiz, 1846, n. syn., Euphemyia Lioy, 1864, n. syn.; under Phasia Latreille, 1804 [Tachinidae] is Allophora Bezzi \& Stein, 1907, n. syn.; under Phryxe Robineau-Desvoidy, 1830 [Tachinidae] is Erinnia Bezzi \& Stein, 1907, n. syn.; under Phyllomya Robineau-Desvoidy, 1830 [Tachinidae] is Phyllomyia Agassiz, 1846, n. syn.; under Polystodes Robineau-Desvoidy, 1830 is Polistodes Speiser, 1915, n. syn.; under Pyrellia Robineau-Desvoidy, 1830 [Muscidae] is Pyrelia Am Stein, 1857, n. syn.; under Rhinomya Robineau-Desvoidy, 1830 [Tachinidae] are Rhinomyia Agassiz, 1846, n. syn., Rhynomyia Schiner, 1861, n. syn.; under Rhyncomya Robineau-Desvoidy, 1830 [Rhiniidae] is Rhynchomyia Agassiz, 1846, n. syn.; under Rivellia Robineau-Desvoidy, 1830 [Platystomatidae] is Revellia Agassiz, 1846, n. syn.; under Sarcophaga Meigen, 1826 [Sarcophagidae] is Myiophora Bezzi \& Stein, 1907, n. syn.; under Solieria Robineau-Desvoidy, 1849 [Tachinidae] is Myiobia Mik, 1890, n. syn.; under Strauzia Robineau-Desvoidy, 1830 [Tephritidae] is Straxissia Verrall in Scudder, 1882, n. syn.; under Tachina Meigen, 1803 [Tachinidae] is Servilia Am Stein, 1857, n. syn.; under Taxigramma Perris, 1852 [Sarcophagidae] is Misella Brauer \& Bergenstamm, 1893, n. syn.; under Thecophora Rondani, 1845 [Conopidae] is Occemyia Schiner, 1861, n. syn.; under Uramya Robineau-Desvoidy, 1830 [Tachinidae] are Uramyia Agassiz, 1846, n. syn., Uromyia Brauer \& Bergenstamm, 1893, n. syn.; under Xyphosia Robineau-Desvoidy, 1830 [Tephritidae] is Xiphosia Agassiz, 1846, n. syn.

New combinations resulting from research conducted here include: Paracalobata ephippium (Fabricius, 1794), n. comb.; Paracalobata octoannulata (Strobl, 1899), n. comb. [Micropezidae].

Additionally, Gymnodia Robineau-Desvoidy, 1863 is treated as a valid senior synonym of Brontaea Kowarz, 1873, n. status [Muscidae]; Lyciella Collin, 1948, n. status, is treated as an invalid junior synonym of the valid name Meiosimyza Hendel, 1925, n. status [Lauxaniidae].

Reversal of precedence is invoked for four cases of subjective synonymy to promote stability in nomenclature: Thricops Rondani, 1856, nomen protectum and Phyllis Robineau-Desvoidy, 1830, nomen oblitum [in Muscidae]; Musca halterata Panzer, 1798, nomen protectum and Musca nigrita Scopoli, 1763, nomen oblitum [in Sarcophagidae]; Tachina moerens Meigen, 1830, nomen protectum and Kirbya vernalis Robineau-Desvoidy, 1830, nomen oblitum [in Tachinidae]; Trypeta longipennis Wiedemann, 1830, nomen protectum and Struzia inermis Robinea-Desvoidy 1830, nomen oblitum [Tephritidae]. 
"L'histoire des mouches est immense; leur étude est difficile; de plus, la vie de l'homme est courte et ses moyens d'investigation sont bornés ... je reviens à vous, Mouches, qui avez toujours fait mes plus chères délices. Je vous ai suivies dans presque toutes les conditions de vos existences si diverses; vous pouvez me considérer comme votre homme-lige." [The science of flies is immense; their study is difficult; moreover, a man's life is short and his means for investigation are limited ... I am returning to you, Flies, you who have always given me my most cherished delights. I have followed you into the most diverse conditions of your existence; you can consider me to be your liegeman.]

— Robineau-Desvoidy, 1863

\section{Introduction}

The short quotation above summarizes the enthusiasm, dedication and almost spiritual devotion to his study of the Diptera by André-Jean-Baptiste Robineau-Desvoidy (1799-1857), one of the most unfortunate and maligned characters in all of dipterology. A life-long bachelor living alone in his house "Ermitage" in the village of Saint-Sauveur-en-Puisaye, in a marshy, foggy, and unhealthy area of Burgundy in central France, Robineau-Desvoidy was an extremely industrious taxonomist who was ahead of his time in certain of his observations and theories but was ignored or vilified for others. For example, his conclusions about the function and morphology of the dipteran halter was published in 1827 but went largely unnoticed, while a M. Jousset of Bellesme published a paper many years later on the same subject and received all the accolades [see Giard (1878) for details, in which the award of a prize must have played a big part in Jousset's omission of all reference to Robineau-Desvoidy's work]. However, Robineau-Desvoidy is probably better known for the negative views of dipterists towards his work. For example, his new classification of higher Diptera with many new tribes and families was based on the associations of the adults and immatures with plants and insect hosts, while he was alleged somewhat unfairly to have ignored the work of his predecessors and contemporaries. However, probably the most criticized of his actions was his habit of describing many species based on small differences in coloration, only to have them synonymized later, even by he himself. An extreme example of this is given by Wainwright (1928) in pointing to the Palaearctic Diptera catalog (Bezzi \& Stein, 1907) where no fewer than 248 Robineau-Desvoidy names were put into synonymy under one single species, the tachinid Phryxe vulgaris (Fallén), a feat that has possibly never been surpassed in systematic zoology ${ }^{1}$. We can add to

1. On this very subject, Robineau-Desvoidy (1863a: 325) wrote: “On est violemment tenté de n'admettre qu'une seule espèce de Phryxe, tant il est difficile d'établir des différences et des distinctions dans ce genre ... Les éclosions nous prouvent qu'à chaque pas nous faisons rencontre d'espèces différentes. J'en connais déjà plus de deux cent espèces aux environs de Paris. Un fort volume ne contiendra pas celles qui vivent sur le Globe. Il devient impossible à notre langage de les bien établir: on a sans cesse le retour des mêmes phrases et des mêmes mots. Leur discernement complet sera la patience humaine poussée à son apogée." [One is seriously tempted to recognize no more than a single species of Phryxe, as it is so difficult to define differences and distinctions in this genus ... Reared specimens prove to us that at every step we are meeting with different species. I already know more than two hundred species from the Paris district. A large volume would not be enough to contain all the species that inhabit the world. It is becoming impossible to define them clearly by means of words: one sees the endless repetition of the same phrases and the same words. Their complete definition will push human patience to its absolute limits.] We provide two photographs of a portion of the collection of Robineau-Desvoidy's Phryxe that exists today in the Paris Museum (see Figs. 12-13). 
this a little-known fact and one that exemplifies the difficulties that he (and indeed most of his contemporaries) found in their attempts to classify small organisms using just a hand lens ${ }^{2}$ : his description of a new beetle (Xenillus clypeator) from mushrooms in a cherry tree near his home in 1839. It was shown later to be a soil-dwelling oribatid mite (cf. Jacot, 1929). Peyerimhoff (1932), in a history of entomology in France, could find little more to say about Robineau-Desvoidy than the following unnecessarily derogatory personal comment:

\footnotetext{
"Mort à Paris en 1857, il s'est fait enterrer, avec son cheval et son chien, dans sa propriété Renards à SaintSauveur-en-Puisaye. Son visage était d'une laideur singulière." [Dying in Paris in 1857, he was buried with his horse and his dog on his property, Renards, at Saint-Sauveur-en-Puisaye. His face was singularly ugly.] (Peyerimhoff, 1932: 52).
}

No doubt because of limits of working only with a hand lens, he felt more comfortable describing the larger muscoid and acalypterate flies and avoided the tinier of the acalypterates and also the nematocerans-apart from his first paper on Culicidae. Throughout his life, Robineau-Desvoidy's reputation had its ups and downs, but even now, despite his shortcomings, he is still considered one of the foremost of French dipterists, one of the first French entomologists to specialize in Diptera (together with his contemporary Justin Pierre Marie Macquart, 1778-1855), and one of the world's first tachinid specialists.

His proclivity to name new genera $(566)$ and species $(3,204)$ necessitates a diligent examination of his taxa, and as part of the Biosystematic Database of World Diptera (BDWD) project [http://www.diptera.org], a review is undertaken here of all the Diptera genus-group names proposed by Robineau-Desvoidy. The genusgroup names were proposed from 1827 through to his posthumous work in 1863 . They span a number families but, apart from his first dipterological work on Culicidae in 1827, they are primarily restricted to the higher Brachycera, focusing especially on the calypterate families. In this study, we review all 566 nomenclaturally available genus-group names as well as nomenclaturally unavailable genus-group names that either he proposed or that have been attributed to him. Genus-group entries are presented alphabetically and list all originally included species, type species, current status, and the emendations that we have been able to locate. A list of all species-group names of Diptera proposed by Robineau-Desvoidy is also given with date and page combinations that link to original publication of these nominal species in his bibliography. Appended to the study are the following: a full bibliography of all of Robineau-Desvoidy's known published works, including his non-entomological publications; lists of collectors and their collections and localities found in the works of Robineau-Desvoidy; and a reproduction of Blainville et al.'s 1826 Rapport of Robineau-Desvoidy's 1830 Essai sur les Myodaires.

2. An impression of the optical equipment available in the early part of the 19th century can be gained from a portrait of the French coleopterist Dejean, which shows him in his early 40s at ca. 1820-1825 (Boisduval, 1846: plate). In his left hand Dejean is holding a group of three hand lenses, attached together at their bases, which can be used singly or with two or all three overlapping to give added magnification. This hand lens is barely visible in the full resolution of the online version of this portrait: (http://upload.wikimedia.org/wikipedia/commons/2/20/ Auguste_Dejean_1780-1845.jpg). 


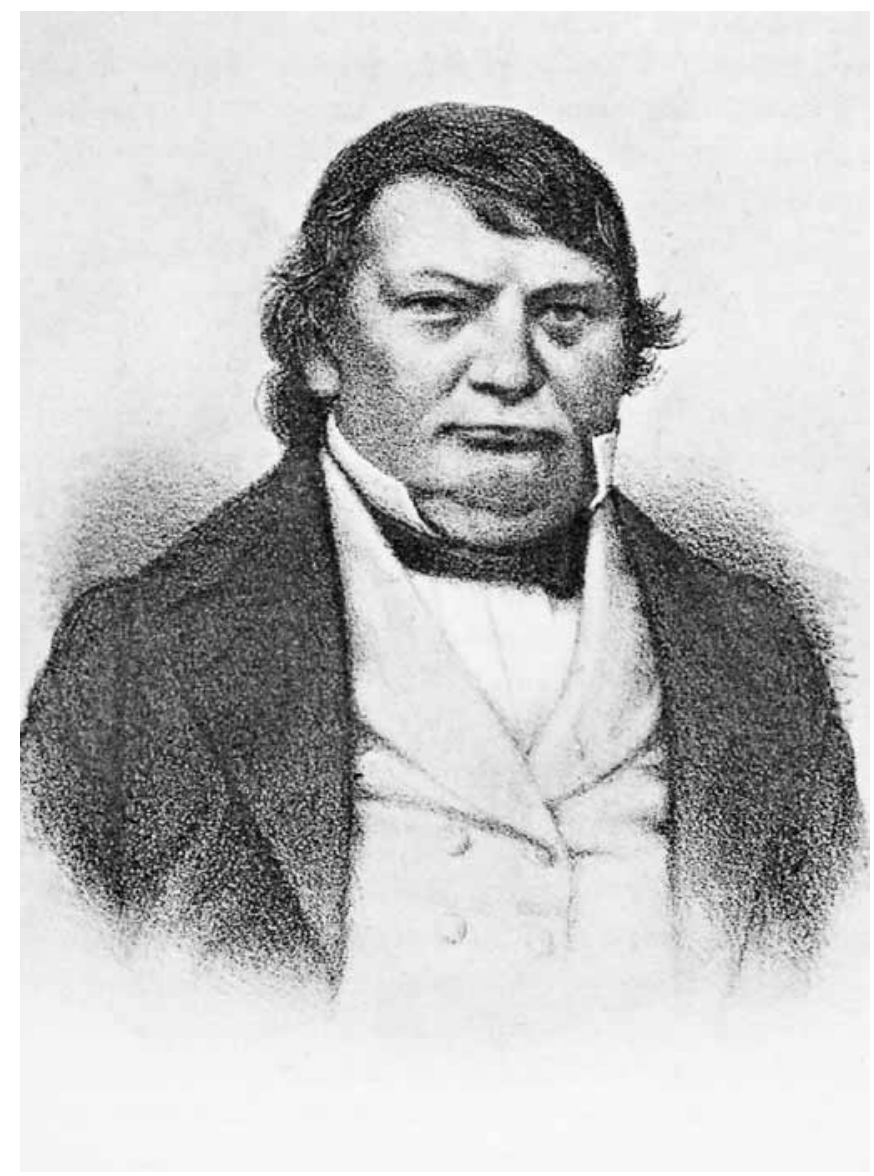

FIGURE 1. Portrait of André-Jean-Baptiste Robineau-Desvoidy.

\section{Biography}

André-Jean-Baptiste Robineau-Desvoidy was born on 1 January 1799 in Saint-Sauveur-en-Puisaye, a small Burgundy village in the department of Yonne, France, about 160 kilometers south of Paris. His father was Jean-Baptiste Joseph Marie Robineau-Desvoidy (1771-1838) and his mother was Angélique Adelaïde Bourgoin (1780-1846). He had one sister, Marie Anne Victorine, who married Dieudonné Prudent Gandrille and whose only son Victor (1827-1879) died without issue. Robineau-Desvoidy himself remained a bachelor.

The descendants of our Robineau-Desvoidy's great-grandfather, Jean-Charles Alexandre Robineau, include the surnames Robineau-Desvoidy, Robineau-Bourgneuf, Robineau-Duclos, and plain Robineau according to the genealogical table of the Robineau family given by Rocher (2003: 163). These secondary patronyms were apparently added to distinguish the various branches of the family one from another although their use seems to have been somewhat arbitrary: Robineau-Desvoidy's father had this multiple surname, but his grandfather and uncle were plain "Robineau" while his cousin was "Robineau-Bourgneuf". The first known publication of A.-J.-B. Robineau-Desvoidy, published in 1818 while he was still a medical student, was printed under the name "J.-B. Robineau-Duvoidy" [the first indication that he preferred dropping his first given name "André", which was absent from most of his subsequent papers], whereas all subsequent publications have his surname given as "Robineau-Desvoidy". His second surname has appeared in various orthographies (e.g., "Desvoidy", "Duvoidy", "Des Voidy", "des Voidy" and "Devoidy"), and the first name even as "Robinot", making searches for all of his published works (and for reviews of them) sometimes difficult. The most common spelling of his surname and the one seen in his signatures is "Robineau-Desvoidy" [see Figs. 23; and see sample in Séguy (1925: 131)]. On his death certificate, he is recorded as "Jean Baptiste Robineau Desvoidy", without hyphens (Royer, 1931). 


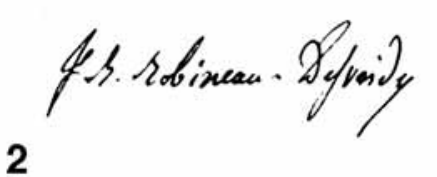

2

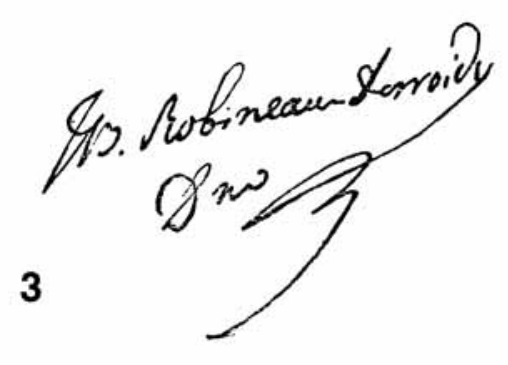

FIGURES 2-3. Robineau-Desvoidy signature samples.

Apparently the Robineau family was an ancient one in the Puisaye region, dating back to Celtic times as Robineau-Desvoidy himself believed, but was certainly represented by the Actes Latins du Martyre de Saint Prix written down in the reign of the West Frankish king Charles the Bald (823-877) and countersigned in the 10 th century by the priest of Saint-Sauveur-en-Puisaye who bore the Latin name Robinaldus. His genealogical association with what he called "une race éminemment Poyaudine" [residents of the Puisaye region] was apparently lost through the centuries (Duché, 1859), but he himself listed a number of his bourgeois antecedents in his Essai statistique sur le canton de Saint-Sauveur-en-Puisaye (1838) and considered his family to have been one of the most eminent in the region: Vincent Robineau, procurator fiscal (1627); Simon Robineau, judge (1640); Edmonde Robineau, apothecary merchant (1644); Etienne Robineau, curate (ca. 1645); and in the period 1650-1700, another Simon Robineau (judge and lieutenant of the bailiwick) and Vincent Robineau (lawyer). He considered his family line to have survived because the members lived quietly and unobtrusively and never left the area. The only one to have left was yet another Vincent Robineau, and he was killed at age 21 at the Battle of Leipzig (1813). [The dates indicate when the names were recorded in historical documents (presumably found by Robineau-Desvoidy, and then listed in his Essai statistique); they are cited here merely to show the antiquity and diversity of the Robineau tribe.]

In the early 19th century, the village of Saint-Sauveur was an isolated and unhealthy place. There were few tracks connecting it with neighboring villages, and it was surrounded by dense forests and extensive marshes. In his 1838 Essai statistique sur le canton de Saint-Sauveur-en-Puisaye, Robineau-Desvoidy referred many times to the unhealthy climate of the area. He wrote of the valleys that were enveloped in a thick blanket of fog every evening, which lasted throughout the night and had a detrimental effect on people already exhausted by their daytime labors. The vast forests prevented the free circulation of air. Added to this was the poor quality of the water. There were no freshwater springs, and all the available water was filtered through the marshes and contained many unhealthy substances. To his descriptions of the area we should add that such conditions were ideal for the development of numerous biting Diptera such as Culicidae, Ceratopogonidae, and Tabanidae. Life expectancy was low, and there was a high incidence of rickets among the population. When newcomers arrived and settled in the village, their robust and vigorous good health was sapped within a short time and few people lived into middle or old age. Robineau-Desvoidy's own grandfather died at the age of 45 and his grandmother at 49 . Over the centuries, the entire canton had failed to produce anyone of note. Robineau-Desvoidy believed that the survival of his family down the centuries was due to the fact that the Robineau men only married women from outside the area.

The Saint-Sauveur-en-Puisaye of Robineau-Desvoidy's description can no longer be recognized. The village has enlarged, there is a good network of country roads leading in various directions and marshes have been drained, but the huge forests remain and give the area a rural and unspoiled feeling. The house "Ermitage" that Robineau-Desvoidy built for himself (Figs. 4-5) still exists on the edge of the town and is now called "Les Renards". 

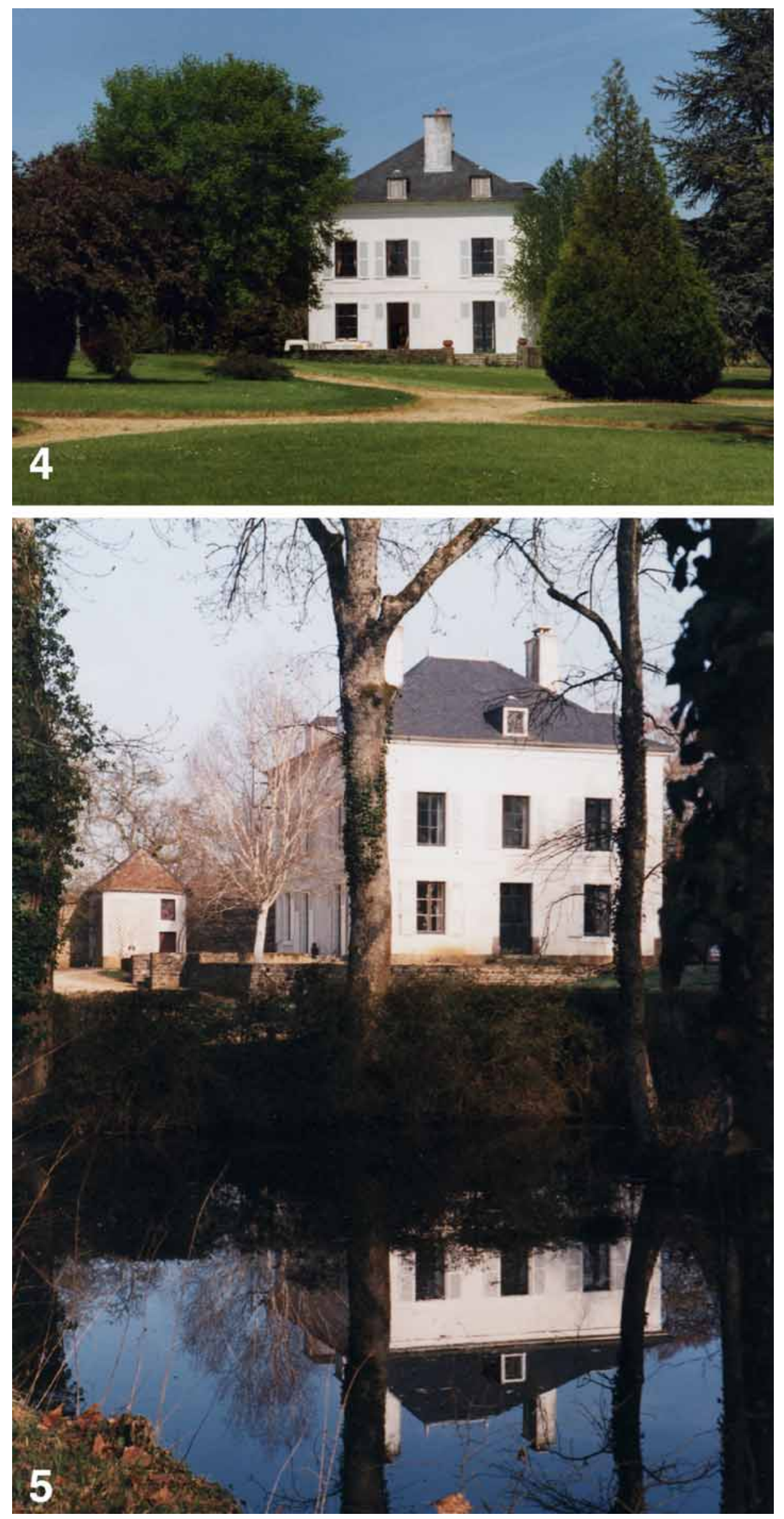

FIGURES 4-5. Robineau-Desvoidy's home in Saint-Sauveur, "L'Ermitage". Photos: B. Ceusters. 


\section{Education}

Robineau-Desvoidy initially studied classical Greek and Latin languages and literature at the Collège d'Auxerre, where he showed great aptitude for work and a vivid imagination. This classical education is clearly reflected in the euphonious and well-formed names for genera and species that he coined, and was responsible for his brilliant and incisive literary style. In 1817, at the age of 18, he went to Paris to study medicine and attended the classes of such scientific notables of the time as Étienne-Geoffroy Saint-Hilaire (1772-1844), Henri Marie Ducrotray de Blainville (1777-1850), Pierre-André Latreille (1762-1833), and Jean-BaptistePierre-Antoine de Monet, Chevalier de Lamarck (1744-1829). They opened up the world of natural history to him and from then on he was enthralled with the study of nature and his natural surroundings. It was a combination of those initial natural history teachings and a remarkable golden yellow tachinid [Clytia gentilis Robineau-Desvoidy, 1830, now known as Heliozeta helluo (Fabricius, 1805)] that he collected in May 1821 that led him to focus his life's work on the higher flies of the Saint-Sauveur region, as he wrote:

“Ce fut sa capture, en 1821, dans la vallée de Montmorency, qui nous inspira l'idée d'étudier les Mouches. Si ce fatal insecte ne fût jamais tombé sous notre main, de combien de peines et d'études n'eussions-nous pas été exempt!" [It was the capture of this [Clytia gentilis], in 1821, in the Montmorency valley, that gave us the idea of studying Flies. If this fatal insect had never fallen into our hands, how many troubles and how many studies we would have been spared!] (Robineau-Desvoidy, 1863b: 290).

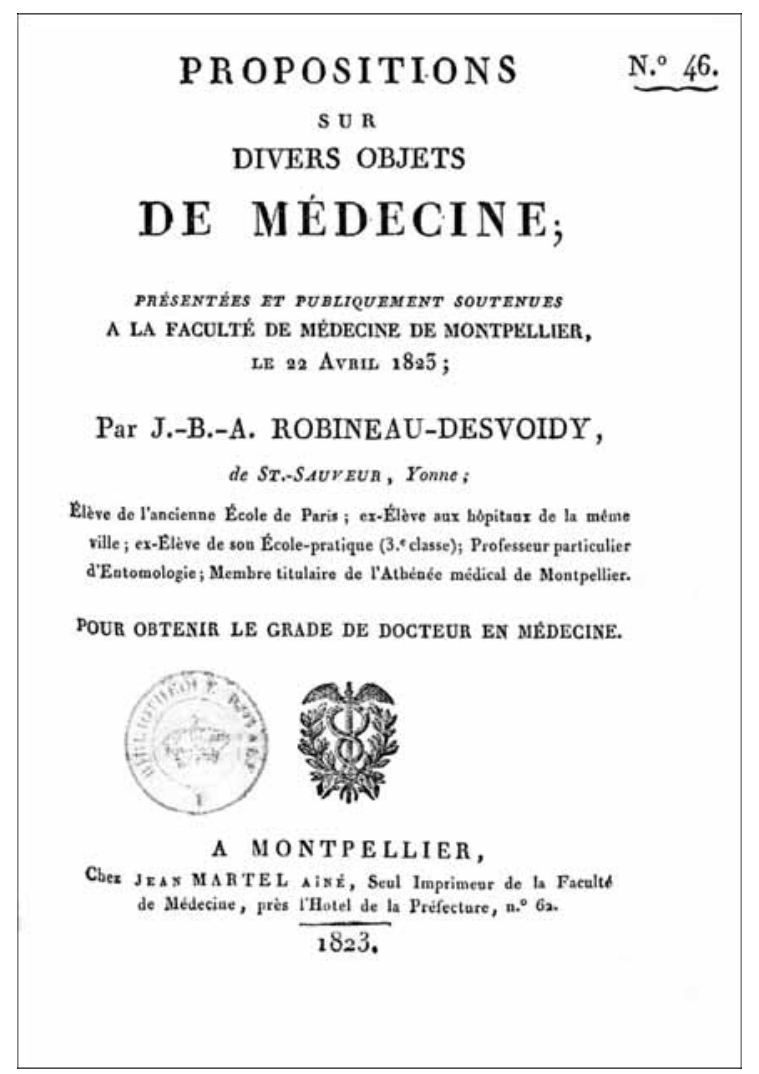

FIGURE 6. Title page of Robineau-Desvoidy's thesis.

The School of Medicine in Paris was closed toward the end of 1822, so Robineau-Desvoidy went to Montpellier where, under the tutelage of anatomy and physiology professor Jacques Lordat (1773-1870), he took his exams and submitted his thesis, which was subsidized by the university. His thesis was on the chemical composition of the human body, which he admitted was hastily copied from works by the chemists Baron LouisJacques Thénard (1777-1857) and Thomas Thomson (1773-1852). According to Duché (1859: 91), midway through the ceremony when he was on the point of receiving his diploma, he was summoned before a committee of professors who had discovered his plagiarism and judged that the thesis was therefore not legally his. Miraculously, rather than expelling him or prosecuting him, he was given a second chance and soon fulfilled 
the requirements of his degree by completing a second thesis (see Fig. 6), which he undertook at his own expense and without reimbursement from the college.

After obtaining his qualifications to practice as a physician at Montpellier, Robineau-Desvoidy returned to Saint-Sauveur and, apart from visits to Paris for scientific meetings and study, remained there for the rest of his life. He worked out of his home as a physician to the poor, devoting himself selflessly to the care of the local people and often not accepting payment for his services. He was fortunate enough to have private means, and was able to live comfortably and without the economic imperative of having to earn his living. A solitary and fundamentally unsociable individual, he bought a marshy plot of land well away from the village, drained it, and built himself a retreat called "Ermitage", where he could spend his time studying his many collections and writing articles. His love of nature and his environs never ceased. He was consumed with learning about many aspects of the Puisaye region and published papers on paleontology, archaeology, geology, entomology, and general history. He designed the garden of his "Ermitage" with flowers, trees, and water features, and kept his numerous collections arranged systematically in his study. Oddly, although professionally a physician, only one publication by him could be found pertaining to medicine-a short pamphlet pertaining to his thesis (Robineau-Desvoidy, 1823).

\section{Robineau-Desvoidy's Character and Personality}

Robineau-Desvoidy was a true child of the Enlightenment and of the French Revolution, inheriting the 18th century belief in progress through reason, science, order and equality. His life illustrates his belief in equality and the duty of every citizen not only to care for his fellow-man but also to contribute to the progress of society and of science in whatever way he can. Early 19th century France had largely freed itself from the shackles of revealed religion, and Robineau-Desvoidy was outspoken in his comments on the remaining Roman Catholic clergy, who sought to suppress parts of his publications, and on those who still clung to ancient privilege or abused newly acquired privilege. He was a deist rather than an atheist, and had little time for the superannuated clergy who still had influence in post-Napoleonic France. His free-thinking and independent views are best summed up by these statements:

“... le mérite individuel a pour le moins autant de valeur que la possession héritée de vastes domaines." [... individual merit is at least of equal value to the hereditary possession of huge estates.] (Robineau-Desvoidy, 1838: 77).

“Espérons que Saint-Sauveur n'aura plus ni seigneurs, ni marquis." [Let us hope that Saint-Sauveur will never again have lords or marquises.] (Robineau-Desvoidy, 1838: 90).

“Tous mes compatriotes reconnaissent aujourd'hui que le travail et l'ordre sont les seuls Dieux dignes de la nouvelle société!" [All my compatriots now recognize that work and order are the only gods worthy of the new society.] (Robineau-Desvoidy, 1838: 100).

So far as historical research is concerned, his attitude was surprisingly modern:

"Nous savons combien de serfs coûtait le coursier chargé de porter un de nos seigneurs; nous avons le détail exact des agneaux, des chèvres, des poules, des oeufs, de l'orge, de l'avoine, des liards et deniers que chacune de nos paroisses payait à l'évêque d'Auxerre. Mais nous ne possédons aucune donnée, ni sur la vie des habitants, ni sur leur nombre, ni sur leurs industries, ni sur les efforts qu'ils ont dû tenter à diverses reprises pour secouer le joug." [We know how many serfs were needed for the charger that bore one of our lords; we have exact details about the lambs, the goats, the hens, the eggs, the barley, the oats, the farthings and the cents that each of our parishes paid to the bishop of Auxerre. But we have no documentation about the life of the inhabitants, their numbers, their businesses, or even of the efforts that they repeatedly had to make to shake off the yoke.] (Robineau-Desvoidy, 1838: 12). 
His sarcasm and wit could be excoriating and was certainly not calculated to ingratiate him with those who had power or influence. For example, when attempts were made to introduce smallpox vaccination for the people:

"Chaque fois que l'autorité supérieure du département a voulu se mêler de la vaccination dans notre canton, elle n'a fait que montrer son ineptie. Lorsque le préfet actuel, le sieur Taillepied, débuta dans nos contrées, il s'annonça comme le premier philanthrope de France; nos cantons ne pouvaient former trop de comités pour la vaccine. Que résultat-il d'un si beau zèle [?]. Du vent. M. le préfet ferait mieux de continuer l'élégant hygiène de ses ongles; qu'il nous laisse au moins les devoirs de la lancette." [Each time that the higher authority of the Department has wished to involve itself in the vaccination for our canton, it has done nothing except show its own ineptitude. When the current prefect, the Master Taillepied, took office in our region, he looked as though he would be France's premier philanthropist; our cantons could not form enough committees for the vaccine. And what resulted from all this fine zeal? Pure wind. The prefect would do better to continue with the elegant manicure of his nails; he should leave to us at least the duties of the lancet.] (Robineau-Desvoidy, 1838: 49).

We ask ourselves: is anything different nowadays?

In his privately published Recherches sur l'organisation vertébral des crustacés, des arachnides et des insectes (1828), he devoted a very large part of the introductory letter addressed to his friend François-Vincent Raspail $^{3}$ (1794-1878) to attacks on the members of the French Académie des Sciences who had rejected this manuscript for publication. For a young man writing about respected academicians, his tone was bitter and wounding as he attacked the cliques in the Académie and what he saw as old-fashioned research, blinkered attitudes, and erroneous scientific results. This seriously upset the Académie, and the publication of this paper was further seen as a mark of ingratitude towards those members of the Académie who had earlier given their imprimatur to his Essai sur les Myodaires (Blainville et al., 1826).

The committee of clerics who would not approve his 1838 Essai statistique for publication were the recipients of his sharpest sarcasm:

"Révérends Pères, ô rayons d'intelligence émanés de l'esprit saint, je m'abaisse devant la sagesse de vos décisions, je m'anéantis devant la profondeur de vos jugements; je suis consumé par le feu qui vous anime. Vous m'avez condamné! J'avais manifesté quelque orgeuil, vous m'avez ordonné de me taire: j'essayais de sortir de la poussière, vous m'y enfoncez davantage. Que votre volonté soit faite! puissiez-vous seulement agréer la sincérité de mon repentir! ... Avec vos honneurs, vos titres, vos rubans, vos crachats, vos cierges, vos dignités, vos majorats, l'impertinence de vos prétensions, la nullité de vos opinions politiques, et le renouvellement toujours facile de vos serments, vous savez trop bien que vous n'êtes que des marionettes devant la sévère et majestueuse raison de mes principes." [Reverend Fathers, oh rays of intelligence emanating from the Holy Spirit, I humble myself before the wisdom of your decisions, I am prostrate before the profundity of your opinions; I am consumed by the fire that drives you. You have condemned me! I have shown pride, and you have ordered me to be silent: I have tried to raise myself from the dust, you thrust me back down again. May your will be done! if you will only accept the sincerity of my repentance! ... With your honors, your titles, your ribbons, your decorations, your candles, your dignities, your inalienable rights, the impertinence of your pretensions, the nullity of your political opinions, and the always easy renewal of your oaths, you know only too well that you are no more than puppets in the face of the severe and majestic reason of my principles.] (Robineau-Desvoidy, 1838: ii-iii, vi).

3. Raspail was one of the founders of the cell theory in biology, and was an early proponent of the use of the microscope in the study of plants. He was also an early advocate of the use of antisepsis and better sanitation and diet. After the revolution of 1830, Raspail became involved in politics. He was President of the Human Rights Society, and was imprisoned for that role. While in prison, he tended sick inmates, and studied their diseases. He became convinced of the value of camphor, which he believed worked by killing extremely small parasites-a version of the germ theory of disease. 
Much of this criticism of the canton's clerics is now a matter of minor or local historical interest, but the disputes in print with his contemporary dipterist Macquart had a considerable bearing on the development of 19th century dipterology and also had a negative effect on Robineau-Desvoidy's scientific reputation, which has continued to this day.

While some of his writings and exchanges with fellow-dipterists show him to have been a sensitive and irascible character and combative in defense of his own views, he was not always so and seems rather to have become more impassioned with the passing years as he saw his work ignored or adversely criticized and condemned. In his Essai sur les Myodaires (1830), he was frequently modest in describing his own efforts to reach an acceptable classification and was unafraid to admit that the solution to certain problems was eluding him. It is also clear that he had no problems accepting the recommendations of the three-man committee that was charged with appraising the manuscript of this work (Blainville et al., 1826). In fact, the manuscript clearly benefited from this advice and Robineau-Desvoidy proceeded to examine collections of Diptera more widely (see under "Collections" in Appendix II) and in many cases the types of the new species resulting from this enlarged study have survived. So, while he was ready to admit to what he did not know and was willing to accept justified criticism, what infuriated him most was what he regarded as unjustified criticism by those he judged incompetent to have opinions or those he considered to be in scientific error. The passage of time saw him becoming more stubborn in his views, but he was occasionally willing to admit to his mistakes: "Ici, $d u$ moins, il ne fait que marcher sur nos traces, car nous fûmes le premier coupable." [Here, at the least, we can only retrace our steps because we were the primary culprit.] (Robineau-Desvoidy, 1846b: 28).

\section{Early Work on Diptera}

Soon after his encounter with the golden yellow tachinid Heliozeta helluo (Fabricius), Robineau-Desvoidy began his first study of the flies of the region, a huge monograph entitled Essai sur les Myodaires, which contained a completely new classification and descriptions of hundreds of new genera and almost 1,500 new species. Latreille was aware of the work in progress as he mentioned it in his Familles naturelles $d u$ règne animal (Latreille, 1825: 499). On 28 August 1826 Robineau-Desvoidy finished his manuscript [initially entitled Sur les insectes qui composaient le genre des mouches (Musca) de Linné] and presented it to the Académie des Sciences in Paris, which immediately formed a committee consisting of Blainville, Latreille, and André Marie Constant Duméril (1774-1860), who were given the task of examining the work and submitting a detailed report back to the Académie on whether it was of publishable quality. With this massive tome, the young 27year old Robineau-Desvoidy was essentially proposing the new name of myodaires for the Linnaean concept of Musca.

\section{The Blainville Rapport (1826)}

After a little over one month spent examining the large manuscript, on 2 October 1826 Blainville, on behalf of the committee, presented the report, which was published as a 24-page pamphlet and handed out to members of the Académie (we provide a reproduction of this important but little-known pamphlet in Appendix III). The Rapport began with a brief history of dipterology in order to put Robineau-Desvoidy's study in context with previous works, then summarized the higher groups of Robineau-Desvoidy, characterizing them and enumerating the genera contained in each (and naming some of them), and concluded with a list of things that needed to be attended to. Osten Sacken (1903) gave an excellent overview of the contents of the Rapport and acknowledged the huge contribution that Robineau-Desvoidy had made, while implying that some of the criticisms the committee made might have been a bit unfair. Among these criticisms was Robineau-Desvoidy's failure to acknowledge the work of his contemporaries such as Carl Frederik Fallén (1764-1830) and Johann Wilhelm Meigen (1764-1845); and his failure to examine all the collections in Paris, instead primarily focusing his work on his own collections in the area of his home town. Another criticism concerned the many genus-group names he proposed as patronyms to honor his friends and colleagues. The committee understood 
the sentiment but thought that some of the names were not very easy to pronounce. Among these, some of the teachers during his studies in Paris and Montpellier impressed him enough to be rewarded with patronyms. These included his major professor at Montpellier, Lordat (Lordatia), Essai examining committee members Blainville (Blainvillia-twice!) and Latreille (Latreillia), and anatomy professor at Paris, Hercule StraussDürkheim (Strauzia). Nor did he overlook his fellow-dipterists Meigen (Meigenia) and Macquart (Macquartia). The other items needing attention included a better definition of the scope of the study, a glossary of his new terminology at the beginning, better synonymies of species based on the works of Fallén and Meigen, and, when finalizing the manuscript, to give a strict analysis of the facts rather than pretentious generalizations. In the end, the committee recommended publication in their Mémoires Présentes par Divers Savants à l'Académie Royale des Sciences de l'Institut de France as soon as the criticisms were attended to.

Four years elapsed from Blainville et al.'s (1826) Rapport of Robineau-Desvoidy's Essai to its publication in the Mémoires (Robineau-Desvoidy, 1830), a delay that upset at least one of his contemporaries, JeanVictor Audouin (1797-1841), who was scheduled to have his major work on the morphology and terminology of the insect thorax appear in the Mémoire after Robineau-Desvoidy's. The four-year delay was much longer than anyone anticipated and Audouin's work (which was never published) was delayed to the point that it was eventually "scooped" by a similar work by William Sharp Macleay (1831) [who at least credited some terminology he used to the unpublished manuscript of Audouin's] (Audouin in Macleay 1831) 4 . During these four years, Robineau-Desvoidy made a number of corrections and additions to his manuscript, found many more new taxa in new collections that he examined (see under "Collections" in Appendix III), and also continued to publish other works. These actions no doubt contributed to the delay but were not the only reasons. The minutes of the séance of 13 July 1829 of the Académie des Sciences show that the manuscript of Robineau-Desvoidy was then already at the printer and the Académie was encouraging the printer to finish the job since they had already paid the funds for it to be printed.

During those intervening four years, Robineau-Desvoidy authored a number of other natural history works. His first dipterological contribution was a short work on Culicidae (Robineau-Desvoidy, 1827) in which the new genera Megarhinus (= Toxorhynchites Theobald), Sabethes, and Psorophora were described, and new species were described in the genera Anopheles Meigen, Culex Linnaeus, Psorophora, and Sabethes. It is also the only work on Diptera in which he provided illustrations, which were very well executed and provided essential details for identification. That same year he published papers on an olfactory organ in crustaceans and on the function of the halteres of the Diptera. The following year he published a 228-page summary classificatory work on crustaceans, insects, and arachnids (Robineau-Desvoidy, 1828), which was dedicated to his mentor Étienne-Geoffroy Saint-Hilaire. During this four-year span he attended meetings of the Académie des Sciences, where he presented no fewer than nine notes or papers on insects, bryozoans, reptiles, and morphological observations (see Appendix I for a full list). By now it was becoming apparent that RobineauDesvoidy was a hard worker and prolific writer, and this was recognized in his home town if not immediately in Paris, so much so that French writer Sidonie-Gabrielle Colette, who was also of the Puisaye area, is said to have patterned the father of the character Claudine in her novel Claudine à Paris (Colette, 1901) after Robineau-Desvoidy. The father, one "Robineau-Declus", spent countless hours studying the snails of the "Montigny" region:

"It has been suggested that the notion of a tome on an obscure subject—and to most people a study of snails in one area of France might be described as obscure-was suggested to Colette by the achievements of a hardworking but unremembered relative of her mother's through her first marriage, Jean-Baptiste RobineauDesvoidy, who died in 1857. His writings covered subjects of localized interest in addition to more general works published by the Académie des Sciences. He even studied the flies of the canton of Saint-Sauveur, using for "fly" the obscure word myodaire which according to his biographer, monsieur Pierre Piétresson de SaintAubin (son of Sido's friend Madame de Saint-Aubin), is not in any dictionary." (Crosland, 1973: 81).

4. Audouin suffered a double humiliation in that, being editor of this volume of the Annales des Sciences, he not only had to endure Macleay beating him to publishing on the subject, but was forced to resort to producing small footnotes in explanation and defense throughout the article. 
The delay in publication also caused some nomenclatural problems of priority. The Rapport by Blainville et al. (1826) was apparently read by a number of entomological colleagues in France because some of RobineauDesvoidy's proposals of terminology and classification showed up in reviews of the Rapport and entries in encyclopédies and dictionnaires published before or in manuscripts of works submitted before Robineau-Desvoidy (1830) (e.g., Férussac, 1827; Guérin, 1827; Le Peletier \& Serville, 1828; Latreille, 1829, 1830; Huot, 1830). In one case (Haematobia Le Peletier \& Serville, 1828), despite the authors of the entry giving Robineau-Desvoidy credit for the name, expecting that his Essai would be published before their treatment of the genus, the descriptive information given under this genus-group name prior to Robineau-Desvoidy (1830) was enough to make the name available but with a different authorship. For many years after the 1830s, the name Haematobia was attributed to Robineau-Desvoidy by authors who either did not know of Le Peletier \& Serville's (1828) Encyclopédie entry or did not strictly adhere to a Principle of Priority.

Because some of the nomenclatural acts in the Blainville Rapport predated their intended debut in Robineau-Desvoidy, thereby potentially causing problems of stability of nomenclature and taxonomy in certain cases, Sabrosky (1988) applied to the ICZN Commission to suppress the work, which was ratified by action of I.C.Z.N. (1990 [Opinion 1601]). This obviated potential problems with names made available in Blainville et al. (1826), but there are still names in other pre-1830 works listed above that may need action by the ICZN Commission to conserve the names in Robineau-Desvoidy (1830).

\section{Essai sur les Myodaires (1830)}

Robineau-Desvoidy's Essai sur les Myodaires appeared in the Mémoires of the Académie on 6 June 1830. Its main purpose was to describe all the new species of myodaires, which was essentially a new name for the Linnaean concept of Musca and embraced the entire Schizophora (Calyptratae and Acalyptratae) at hand from his own collection as well as from the collections of many of his colleagues (see Appendix II), and to put them into a new classification based on their life histories. The 813-page work contains 323 new genus-group names and 1,531 new species-group names in addition to 61 mostly new family-group names, plus 31 genusgroup names and 236 species-group names for previously-described taxa. In terms of its geographic coverage, it was firmly based on the fauna of France and on specimens collected by a number of entomologists but most especially by Robineau-Desvoidy himself. A small number of species were described from other parts of Europe, and even fewer from other zoogeographic regions, particularly from North and South America.

The work opens with an account of the morphology of the myodaires (pages 3-24), which includes particularly detailed information on characters in the head and wings. Robineau-Desvoidy states that he had also studied the configuration of the abdomen, the sexual organs and the legs, but he gives no details of this. He first divides the myodaires into nine natural subdivisions based mainly on five character states found in the arista, the lower calypter, wing vein $\mathrm{M}$, body conformation, and larval habitats; for two groups, a character in the female ovipositor is added. Then each subdivision is described, discussed, and further subdivided into sections and then into genera. No type species or typical species are cited for the genera, and many of them are extremely small in size or even monotypic. Many are named after Robineau-Desvoidy's own teachers and colleagues, fellow French entomologists, and also entomologists in other European countries. Species are described with a Latin diagnosis and a French description, and locality and collector are usually cited; as a general rule neither the sex nor the number of specimens is given, but sometimes the location of the specimens, whether in the Muséum National d'Histoire Naturelle, Paris, or in a private collection, is given.

Given the vast number of genera and species described, this must have seemed a profoundly puzzling work when it first appeared. Yet it has its own logic and, beginning with the group we now know as the Tachinidae, it moves through the Sarcophagidae, Calliphoridae, Muscidae, Anthomyiidae, and then many of the families of the Acalyptratae. The sequence of descriptions, taken together with the taxonomic characters mentioned and the habits and life histories that are described, has made it possible to interpret a very large proportion of the names, with the result that many of our most familiar genus-group names stem from this work. 


\section{ESSA I}

\author{
SU R
}

\section{LES MYODAIRES,}

Par le Docteur J. B. ROBINEAU - DESVOIDY, de Saint-Sauveur (Yonne).

\section{CHAPITRE PREMIER}

L'ACADÉmie royale des Sciences, dans la séance du 2 octobre 1826 , vota limpression de mon Essai sur les Myodaires du canton de Saimt-Sauveur, département de l'Younc. Pour répondre dignement à cet honneur, jai dû revoir mon travail en totalité, l'assurer sur de plus larges bases, et profiter des critiques de ia Commission du Rapport. Javais prévu, ce qui est arrivé, que je travaillais sur un sujet sans fin. Les naturalistes n'exigeront donc pas de moi une perfection dont lidée s'éloigne à mesure qu'on étudie davantage. Pour s'expliquer la quantité d'individus que je décris, on saura que jai eu à ma disposition les principales collections de Paris. Je dois à MM. Am. Le Peletier de Saint-Fargeau, AudinetServille et Blondel, la connaissance d'un grand nombre d'espèces de Paris avec des détails sur les habitudes de quelquesunes. M. Carcel m'a communiqué le résultat de ses chasses

\section{7}

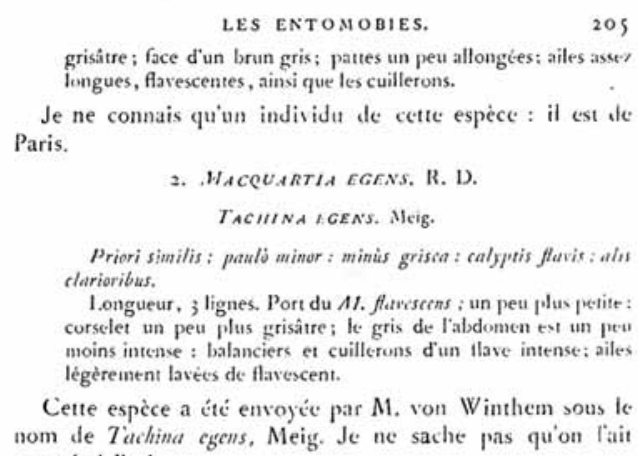

LES ENTOMOBIES.

205

grisatre; face d'un brun gris; pattes un peu allongies; ailes asse? longues, flarescentes, ainsi que les cuillerons. Paris.

Je ne connais quin individu de cette espece : il est de

2. WACQUARTIA EGENS. R. D.

TACIIINA IGEN'S. NeIg.

Priori similis : pauls minur : minis grisia : calygtis flavit: atis clarioribus.

1.ongueur, 3 lignes. Port du At. flatriserns ; un peu plus petite : curselet un peu plus grisâtre; le gris de labdomen tel un peet moins intenve : balanciers et cuillerons d'un flave intense: piles légèrement lavees de flavescent.

Cette espcce a été envoyéc par $M$. von Winthem sous ic nom de Tathina egens. Meig. Je ne sache pras qu'on lait trouvéc it Paris.

3. ALACQUARTIA RUREIPES, R. D.

1igra: gristo-tomintosa : primis antruna articulis pedibusyut rabris ; calyptis alarumque hasi flatis.

Longueur, 4 lignes $1 / 2$. O Corps noir, saupoudre $\mathrm{de}$ griv: frontaux noirs: face d'un brun-gris sovenx: premiers articles antennaires, pattes, rouges: tarses bruns: pralpes fauves; cuillesfon. et base des ailes jaunes.

J'ai trouvé cette espece à saint-Sauveur parmi des plantes littorales.

$$
\text { 4. MAACQUATTA GERHANIC.A. R. D. }
$$

Vigrat ; riv cincrascons : calyptis flavis ; ald sat clarte.

Longueur, $\{$ lignes $1 / 2$. Face d'un brun blanchátre: antenne. fromt, pattes, noirs; corselet nuir un peu luisam, très-ligèremen glacé de cendré: aldomen noir-luisant, conme un peu verdovam. avec quelques reflets cendrés: cuillerons jaunes; ailes claires. quoique légèrement lavies de nưrâtre

Cette espice a été envoyce didllemanne par M. von

8

FIGURES 7-8. Sample pages of Robineau-Desvoidy's 1830 “Essai sur les myodaires”.

Osten Sacken (1903) made comparisons between the manuscript of 1826 (based on the notes in the Blainville Rapport) and the 1830 publication and noted that not all of Robineau-Desvoidy's initially proposed families were treated in the 1830 work. In retrospect, Robineau-Desvoidy (1844a) wrote:

"Vers le fin de l'année 1826, l'Académie des sciences vota l'impression de notre Essai sur les Myodaires: les deux premiers tiers de cet ouvrage se trouvèrent imprimés en 1830, le dernier tiers reste encore inédit." [Toward the end of the year 1826, the Academy of Sciences approved our Essai sur les Myodaires for publication: the first two-thirds of this work were published in 1830, while the final third still remains unpublished.] (Robineau-Desvoidy, 1844a: 5).

There is indeed an apparent caesura in the 1830 publication, between pages 279 and 280, which may represent the dividing line between parts 1 and 2. But what happened to part 3? Did it contain the families initially proposed in the Blainville Rapport that did not appear in the 1830 work? It may be represented by one or more of the papers listed in the section "Unpublished Manuscripts" below (q.v.) or have been incorporated into his final, posthumous work (Robineau-Desvoidy, 1863a,b).

There is some evidence of carelessness resulting from the revisions that he made to his original 1826 manuscript. For example, he occasionally refers to a species (from France) that resembles the preceding species, but the preceding one or two species are actually from outside Europe and from the Dejean collection, and have clearly been inserted after 1826.

Reviews of the work were initially favorable and dipterists such as Macquart and Meigen courteously attempted to incorporate the genera of Robineau-Desvoidy into their studies of European Diptera (e.g., Mac- 
quart, 1834a,b, 1835; Meigen, 1838). However, after further examination, problems were found and resulting reviews leaned more toward the unfavorable. Those who were initially favorable or non-committal were primarily those unfamiliar with Diptera or the taxonomy and classification of the higher flies (e.g., Férussac, 1827; Germar, 1828), but not all were gentle:

\begin{abstract}
"[Robineau-Desvoidy is] a French physician, who has chiefly written various papers on the Diptera. In these he proposes upwards of 300 new generic names for the single family of Muscidae. The spirit in which this is done, may be judged by the fact of his having made no allusion to the admirable work of Meigen! and scarcely to any modern author. It is hardly necessary to say that the characters of these proposed groups are generally too trivial to deserve notice, and the whole work is undeserving of authority." (Swainson, 1840: 309-310).
\end{abstract}

However, those who were specialists on these groups were far less kind. An example of this was Loew (1844) who attempted to review the European taxa of the tephritid genus complex Trypeta Meigen and was forced to decipher the placement of the taxa described by Robineau-Desvoidy:

\begin{abstract}
"Das entomologische Publikum scheint über die monströse Publikation des Herrn Rob. Desvoidy so erstaunt gewesen zu sein, oder sich durch die großartigen Redensarten desselben so über die Haltlosigkeit seines Werkes haben täuschen zu lassen, dass es ihm die rechte Antwort: „erst Schüler zu werden, ehe er der Meister spielen will," bis jetzt leider schuldig geblieben ist." [The entomological public seems to have been so astounded by the monstrous publication of Mr Robineau Desvoidy, or to have been so deceived by his grandiloquent mode of writing as to the basic weakness of the work, that it has unfortunately been unable to give the right response: "first become a pupil before playing the master".] (Loew, 1844: 314-315).
\end{abstract}

This article by Loew (1844) contains a blistering attack on Robineau-Desvoidy's methodology in describing hundreds of new genera, for describing species without reference to previous work or to possible homonymies, and for setting himself up as an author while ignoring other work and yet criticizing the work of Macquart whose achievements were actually far greater than his.

\title{
Other Académie des Sciences Activities
}

Robineau-Desvoidy published two of his larger studies with the Académie des Sciences $(1828,1830)$ and these were early in his "entomological" career. The encouragement he received from reviews of his works by committees charged with examining them no doubt gave him a great deal of confidence. But these votes of encouragement were not necessarily because his works were thought of highly but more because of his relatively young age (for instance, presenting his first large work to the Académie at the age of 27), exuberance, and initiative; and that any mistakes on his part in these manuscripts could be forgiven due to his youth. Robineau-Desvoidy possibly saw things differently and attended meetings of the Académie from 1826 through 1833, presented papers for publication, and gave small notes on interesting observations. Following the death of Latreille on 6 February 1833, Robineau-Desvoidy put himself up for candidacy for Latreille's vacant seat of zoology of the Académie at the séance of 11 March 1833 and provided a list of his publications as his qualifications. At the same meeting, one of his former professors, Hercule Strauss-Dürkheim, also announced himself as a candidate for the seat and also gave a list of his publications as a testament to his qualifications. It is noteworthy that the published minutes of that meeting do not list Robineau-Desvoidy's publications but do list those of Strauss-Dürkheim. In the end, Robineau-Desvoidy did not make the final list of candidates, which included such luminaries of the time as Isidore Geoffroy Saint-Hilaire (6 years younger than Robineau-Desvoidy and the one who was eventually elected to the seat), Achille Valenciennes, Anselme Gaetan Desmarest, André d'Audebard baron de Férussac (who died just 3 years later), Hercule Strauss-Dürkheim, and Henri Milne-Edwards. Although a younger and possibly less experienced man got elected (being the son of Étienne Geoffroy Saint-Hilaire no doubt helped Isidore get votes), the votes for Isidore were three times as numerous as those for the next candidate. For Robineau-Desvoidy to think that he, as a country doctor, would be consid- 
ered by Parisian scientists of the Académie as being on the same social and academic level as them gives a glimpse into his mindset. Living alone in a small village 160 kilometers south of Paris gave him little opportunity for day-to-day interaction with colleagues and as such he probably did not have a good perspective on his social and collegial position within Parisian science. A short time after this he decided that he would not continue his involvement with the Académie.

\section{Société Entomologique de France Publications}

Robineau-Desvoidy was a member of a number of scientific societies (among them, the Société d'Histoire Naturelle de Paris, the Société Géologique de France, and a founding member of the Société des Sciences Historiques et Naturelles de l'Yonne), but it was his association with the Société Entomologique de France that served as the venue for most of his publications. Robineau-Desvoidy joined the Société Entomologique de France in 1833, one year after its founding and attended many of its meetings in Paris with only a few years' hiatus in membership (1835-1838). He remained a member thereafter until his death. He was a member for almost 10 years before he started publishing in the society's journals. After his 1830 Essai, he continued the study of his myodaires and, while accumulating information for his second tome on these flies, he published shorter papers on the subject in order to get into print the findings of new genera and species that he was discovering. Unfortunately, his ideas on classification and taxonomic concepts were not supported by many of his contemporaries and his continued ignoring of Macquart's generic concepts and higher classification led to some adversarial exchanges that were published in the Annales (Macquart, 1842; Robineau-Desvoidy, 1846c, 1847; Macquart, 1847).

In a prescient note in his local journal, Robineau-Desvoidy (1853c: 531) complained about the lack of a central medium where short observations could be published that would otherwise have remained unknown and confined to specimens in cabinet drawers. Such a possibility is now provided by, for example, the Entomologist's Monthly Magazine, which includes scientific articles together with short notes of one or two paragraphs recording interesting observations; plus a number of websites such as http://www.diptera.info.

\section{Robineau-Desvoidy and his Contemporaries}

The publication of the Essai sur les Myodaires in 1830 must have exploded like a thunderbolt on the French entomological community and particularly on those with some expertise in the Diptera. They were suddenly confronted with the new term myodaires in which the old Linnaean Musca was split into 61 family-group taxa, 354 genus-group taxa (of which 323 were new), and 1,767 species-group taxa (of which 1,531 were new).

The first criticism leveled at Robineau-Desvoidy was that he had ignored the work of his contemporaries, especially Fallén, Macquart, and Meigen. An early example is a review by Bengt Fries in 1834:

\footnotetext{
"Robineau Desvoidy Essai sur les Myodaires, utgör hela 2:dra Vol. af Mem. des Savans etr. de Paris, (813 sid.). Ett besynnerligt arbete, hvaruti mycken möda är nedlagd, och som för 30 år sedan skulle hafva gällt för ett mästerstycke. Nu torde det föga blifva begagnadt, då Författaren så litet tagit notice af alla de arbeten, som redan långt före hans eget utkommit öfver samma ämne, att man bland ett par 1000:nde Europeiska och exotiska arter, som deri beskrifvas, knappt skall finna öfver 30 eller 40, vid hvilka Fabricius eller Meigen äro citerade. Falléns, Wiedemanns m. fl. arbeten hafva varit honom alldeles obekante. Med namnet Myodaires betecknar han detsamma som Meigens Muscides, eller Falléns Haematomyzides, Muscides och alla följande familjer. Dessa indelas uti 9 stora afdelningar, som hufvudsakligen karakteriseras genom kroppsfärgen och larvernes vistelseort, ehuru de fleste larverne tyckas vara lika obekante för Förf. som för andra Entomologer. De 2 sista stora sectionerne äro blott nämde, men afhandlas ej vidare. De öfriga 7 indelas uti 354 genera, af hvilka 202 höra till första sectionen, som innefattar Oestri (hvilkas beskrifning äfven utelemnas), Falléns Rhizomyzides, Tachinae och de egentliga Muscides, eller alla, som hafva en tvärnerv bakom midten af vingen. Troligtvis skulle arbetet befinnas äga värde, för den, som hade tid och mod att derefter börja ett alldeles nytt studium af dessa insekter." [Robineau Desvoidy Essai sur les Myodaires takes up all of the second volume of
} 


\begin{abstract}
Mem. des Savans etr. de Paris (813 pp.). A peculiar work, which has taken much effort, and which 30 years ago would have been a masterpiece. Now it will find little use, as the author has taken so little notice of all those works that were published on the same subject long before his own, that among a couple of thousand European and exotic species that are described therein, are found hardly more than 30 or 40 for which Fabricius or Meigen are cited. The works of Fallén, Wiedemann and others have been entirely unknown to him. With the name Myodaires he is addressing the same as Meigen's Muscides, or Fallén's Haematomyzides, Muscides and all the following families. These are divided into 9 large sections, which mainly are characterized by the color of their body and the habitat of the larvae, even though most of the larvae seem to be as unknown to the author as they are to other entomologists. The 2 last sections are just mentioned, but do not receive further treatment. The other 7 are divided into 354 genera, of which 202 belong to the first section that contains Oestri (the description of which is even omitted), Fallén's Rhizomyzides, Tachinae and the true Muscides, or all of those with a crossvein in the distal half of the wing. The work would probably be valuable to a person with time and courage to start an entirely new study of these insects." (Fries, 1834: 120-121).
\end{abstract}

However, it must be pointed out in all fairness that in mid-August 1826, when Robineau-Desvoidy submitted his manuscript Essai for publication, Meigen's volumes 4 and $5(1824,1826)$ containing the calyptrate Diptera (within which the majority of Robineau-Desvoidy's taxa of study were found) had only just appeared ${ }^{5}$ and Macquart was still at the outset of his dipterological career, his publications in 1826 not having been published until autumn. However, in preparing the revised version, Robineau-Desvoidy seems to have taken these criticisms to heart since his new genera Macquartia and Meigenia certainly show respect for these two great dipterists and he indeed made specific reference to Meigen's excellent work. He wrote in the introduction to the Essai that Meigen excelled in the recognition and description of species, but that he (Robineau-Desvoidy) was only describing species that he had seen and studied, so that many of Meigen's species had to be omitted as they were unknown to him.

Regarding the omission of references to Fallén's work, Robineau-Desvoidy admitted openly that he knew of Fallén's work on Musca and the pre-1826 papers in the series Diptera Sveciae but had been unable to obtain them. For a young man living in the country, to locate and review the entire literature up to 1825 was probably too great a task, and evidently he did not have access to all the classic taxonomic works of the late 18th and early 19th centuries.

Macquart (1834a,b, 1835) was the first to come to grips with the Essai, and he synonymized numbers of its new species with species previously described by Fabricius, Fallén, and Meigen. He listed Fabricius, Latreille, Meigen, Wiedemann, Fallén, and Robineau-Desvoidy as having founded families, tribes, genera, and species, but gave the greatest credit to Meigen:

“En un mot, il a fait pour les Diptères ce qu'a fait pour l'Entomologie entière notre immortel Latreille, qui a élevé cette aimable science au rang des objets les plus dignes de nos veilles ..." [In a word, he has done for the Diptera what was done for the whole of Entomology by our immortal Latreille, who raised this delightful science to the level of those subjects that are most worthy of our waking hours.] (Macquart, 1834b: 23).

The self-opinionated and sensitive Robineau-Desvoidy could hardly have been pleased to see himself publicly downgraded in this way.

Meigen too attempted to deal with the plethora of names in the Essai, but could only do so in the final, supplementary volume [= seventh] of his Systematische Beschreibung der europäischen zweiflügeligen Insekten (1838), because his previous volumes had appeared or were sent to the printer before Robineau-Desvoidy's 1830 Essai. Meigen (1838) acknowledged the help of Macquart in supplying him with the most current names for species in the Essai. The genus-group names previously authored by Macquart were all listed with Macquart's surname after each name. But for every genus-group name authored by Robineau-Desvoidy there was a conspicuous absence of any attribution, though Meigen did attempt to incorporate a number of them into his own classificatory system (e.g., Aricia, Hylemya, Hydrotaea, Lucilia, Pyrellia). As regards the species-group names in Meigen (1838), if they were Macquart species, they were so attributed and usually

5. Meigen (1826) no doubt appeared a few months after Robineau-Desvoidy submitted his Essai in August 1826 as the preface to Meigen (1826) is dated 1 August 1826, so Robineau-Desvoidy would not have had any knowledge of its contents at that time. 
with a short description. However, if they were Robineau-Desvoidy species, they again lacked Robineau-Desvoidy's authorship and were usually only listed by name without any descriptive characters to aid in identification. It seems that the genera and species that could be recognized usually were, while the rest were ignored, and by-and-large this has been the fate of Robineau-Desvoidy's entire oeuvre.

At the end of the following decade and despite the publication of monumental works on the cyclorrhaphous Diptera by Macquart (1834a,b, 1835) and Meigen (1838), Robineau-Desvoidy made an embittered submission to the Société Entomologique de France at their meeting of 6 February 1839:

\begin{abstract}
"M. Robineau-Desvoidy lit une courte notice où il se plaint amèrement de la nullité des progrès de la science dans l'étude des Mouches, depuis la publication de son essai sur les Myodaires. Il pense qu'il faut s'en prendre surtout au défaut d'études réelles dans cette matière, et déclare qu'en France aucun entomologiste ne s'est encore sérieusement occupé de ce sujet." [Mr Robineau-Desvoidy made a brief announcement in which he complained bitterly about the lack of progress in the scientific study of Flies since the publication of his essai sur les Myodaires. He considered that it was necessary to take it up, especially in view of the lack of real studies of this topic, and stated that no French entomologist had yet engaged seriously with this subject.] (RobineauDesvoidy, 1839: vi).
\end{abstract}

This was a particularly pointed barb directed at Macquart, and led to a series of exchanges in the pages of the Annales from which it is clear that these two had fundamental differences at all levels of their dipterological activity, both in systematic theory and in taxonomic practice. However, their public differences were confined to the professional level and avoided all personal attacks. The most derogatory comment by Robineau-Desvoidy (1850a: 173) was mild: "Il faut admettre que M. Macquart n'a examiné cet individu qu'à un faux jour" [It has to be admitted that Mr Macquart can only have examined this specimen on the wrong day] compared to what regularly appears in some self-published journals today. Yet he continued to respect Macquart, as his Occemya macquarti of 1853 shows.

Robineau-Desvoidy (1842a) accused Macquart of misidentifying species and of proposing unnecessary generic names. Referring to one of Macquart's papers, he wrote (page 260): "Là, il n'y a que désordre, confusion, absence de toute espèce d'observation et de toute idée d'organisation" [There (in that work), there is nothing but disorder, confusion, an absence of all type of observation and of all idea of organization]. In a second attack on Macquart that immediately followed the previous one (Robineau-Desvoidy, 1842b), he professed admiration for the "Insectes diptères du nord de la France" (Macquart, 1834b), but considered the two volumes on the Histoire Naturelle des Insectes. Diptères (Macquart, 1834a, 1835) to be "une simple et indigeste compilation" [a straightforward and undigested compilation] (page 264). And he accused Macquart of having slavishly followed German authors whose work, in Robineau-Desvoidy's opinion, showed serious shortcomings in their treatment of the cyclorrhaphous Diptera.

Macquart (1842) responded in a calm and lucid manner, dealing first with Robineau-Desvoidy's general points and then turning to the more specific matters. He wrote:

"M. Robineau-Desvoidy me critique de baser, ainsi que les diptérologistes allemands, la classification sur l'organisation, au lieu de prendre, comme lui, pour base, les moeurs des diptères." [Mr Robineau-Desvoidy criticizes me for basing my classification on structure, as do the German dipterists, instead of taking the habits of the Diptera as the basis, as he does.] (Macquart, 1842: 165).

This is the nub of the matter, and is the reason for the subsequent rejection of Robineau-Desvoidy's classification: he based his groups on habits, hosts, flower visits, etc., giving morphology a secondary role. Macquart then followed with what has proved to be the experience of every subsequent dipterist trying to use the works of Robineau-Desvoidy:

“...j’avoue que, malgré l'étude approfondie que je fis de cet ouvrage, j’en trouvai l'emploi très-difficile pour la détermination des genres et des espèces." [I must confess that in spite of the thorough study that I made of this work I have found it very difficult to use for the identification of the genera and species.] (Macquart, 1842: 167168). 
This dispute crackled on for several years. Robineau-Desvoidy (1846c) returned to a critique of Macquart's treatment of his Essai and other papers. He claimed priority for his name myodaires and again gave a justification of it. He discussed Macquart's "créophiles" as being the same as his "entomobies", and accused Macquart of creating generic homonyms. He took issue with various points of systematics in Macquart's work, and explained why he placed the Myopinae away from the other Conopidae and in his "entomobies". Macquart (1847) replied by noting that there were plenty of family-group names proposed by Fallén and Meigen without the need for the newly proposed "myodaires", and he pointed out, with less justification, that genus-group names should be based on taxonomic characters rather than on personal names. He quoted extensively from the Blainville Rapport, and echoed the verdict that too much weight was given by Robineau-Desvoidy to a classification based on habits, behavior and biology rather than one based on structure, and he again criticized the excessive number of genera, some of which he accepted but many of which he considered to be artificial or based on trivial characters.

After receiving Macquart's 1847 response, the Société Entomologique de France decided to print it but said that enough was enough, and refused to publish any more ripostes in this dispute:

"Sur le demande de l'auteur, la Société décide que ce mémoire sera inséré dans le plus prochain numéro de ses Annales, et elle exprime le désir que la discussion qui s'est élevée entre MM. Robineau-Desvoidy et Macquart n'ait pas d'autres suites." [At the request of the author, the Society decided that this note should be included in the next issue of the Annales, and also expressed the wish that there should be no further continuation of the discussion that has arisen between Messrs Robineau-Desvoidy and Macquart.] (Desmarest, 1847: xiv).

But all through the 1850s the Société continued to publish papers on Tachinidae by both Robineau-Desvoidy and Macquart, despite their obviously opposing views on classification.

\section{Diptères des environs de Paris. Famille des myopaires (1853)}

In this work, Robineau-Desvoidy separated the myopaires from the remaining Conopidae and treated them as a distinct family, being "misled by his idiosyncrasy of founding the classification on the habits of the larvae" (Osten Sacken, 1894: 381). Robineau-Desvoidy wrote:

"Nous n'hésitons pas de proposer un mode de classification qui ne repose sur des modifications organiques: ce mode est tout-à-fait artificiel. Mais après de mûres réflexions, nous l'avons jugé préférable à d'autres modes qui ne présentent pas la même facilité d'application." [We do not hesitate to propose a method of classification that is not based on structural modifications: this method is completely artificial. But after mature reflection, we have considered it to be preferable to other methods which are not so easy to apply.] (Robineau-Desvoidy, 1853a: 90).

This work also illustrates how much Robineau-Desvoidy had accepted criticisms of his Essai and how far his presentational technique had advanced: it contains an excellent and comprehensive review of previous work on the myopaires including his own, type species are cited for all the genera, there are lists with appropriate discussion of the synonyms and misidentifications in the works of other authors including himself, and it also includes a detailed account of Meigen's work. This methodology was carried over into his final, posthumous work on the myodaires (1863a,b).

This work too had a cool reception; a century later, J.E. Collin wrote about it as follows:

“... he proposed no less than ten generic names, and published descriptions (apparently most of them quite unrecognizable) of twenty-eight new species. This work is particularly full (even for one by Desvoidy) of mistakes and contradictory statements, and no subsequent student has been able to understand, or make sense of, what the author had published." (Collin, 1960: 145). 
Robineau-Desvoidy was more than just a dipterist or entomologist. He was insatiable in his quest for knowledge about the Puisaye area and published a number of non-entomological articles in addition to short notes on non-Diptera. As a founding member of the Société des Sciences Historiques et Naturelles de l'Yonne, he supplied its members with many articles on history, archaeology, geology, and paleontology of the region. He is probably best known outside of entomology for his discovery of the Venus Anadymene statue in some slag heaps in the town of Mezilles (Robineau-Desvoidy, 1849h) and his book on the demography and history of Saint-Sauveur, Essai statistique sur le canton de Saint-Sauveur-en-Puisaye (1838). The latter was planned to be published by the Société des Sciences Historiques et Naturelles de l'Yonne but was withdrawn as the members of the publications committee thought it contained too many errors as well as numerous preposterous Celtic etymologies. Funds not being an object for him, Robineau-Desvoidy had it printed privately. It remains a fundamental but little-known reference work for the region as it contains a prodigious amount of statistical research. A facsimile edition of 300 copies was published in 2002 in the series Monographies des villes et villages de France edited by M.-G. Micberth (Le Livre d'Histoire-Lorisse, Paris).

It is known that Robineau-Desvoidy attended two of the Congrès Archéologiques de France, in 1850 and 1851, at which he was an active participant (see his Bibliography in Appendix I). But even here it appears that he ruffled a number of feathers:

"M. Robineau-Desvoidis [sic] se défend d'avoir voulu porter atteinte à la vénération que l'on a pour les trois saints du pays Auxerrois. L'incident est terminé." [M. Robineau-Desvoidy denied that he had wished to make an attack on the veneration in which the three saints of the Auxerre area are held. The incident was closed.] (Quantin, 1851: 40).

He also attended the 1851 Congrès Scientifique de France, making a number of contributions relating to geology. He was co-opted on to a committee appointed to examine the diseases of grapes, and his opinions and researches are quoted throughout the report (Des Moulins, 1852).

\section{Death}

No doubt the unhealthy conditions of his home and surroundings led to his early death. He continually suffered from asthma and respiratory problems, but continued to attend to his impoverished and sickly patients in the unhealthy, cold and humid environment of his home town despite the encouragement of his friends to move away. Not long before the end, he was apparently resigned to his fate and wrote of his failed health to his friend, the librarian Lemercier:

\footnotetext{
"Au milieu de cette misère, continuation d'amour pour le travail. Plus je sens la vie qui me quitte, plus mon ardeur pour l'étude semble prendre des forces nouvelles. Expliquez cela. Je crois que je mourrai en loupant un diptère!" [In the midst of this misery, love of the work continues. The more I feel the life that is leaving me, the more my ardor for study seems to take on fresh strength. Explain that. I think that I shall die while holding a dipteran under the magnifying glass!] (Duché, 1859: 25).
}

When his health seemed to be at its worst and with no signs of improvement, he was sent to a private hospital on the Avenue Montaigne in Paris where it was believed he could receive more assiduous care than at his home in Saint-Sauveur, but he died at that hospital in Paris on 25 June 1857 (Royer, 1931).

Obituaries of Robineau-Desvoidy are few and their rarity is no doubt a reflection of the impression people had of him towards the end. However, one French dipterist, Jacques Marie Frangile Bigot (1818-1893), gave an eloquent eulogy of him in the Annales de la Société Entomologique de France, including this memorable line: 


\begin{abstract}
"Malheur bien grand, car avec lui vient de s'éteindre une des lumières de la science entomologique, avec lui nous perdons le dernier des Diptéristes français!" [What a great misfortune, because with his death one of the lights of entomological science has been extinguished, with him we have lost the last of the French Dipterists!] (Bigot, 1858: cxxxii).
\end{abstract}

This is ironic, since it derives from another French dipterist whose work was not well perceived by his colleagues.

Robineau-Desvoidy was interred in the grounds of his home and, according to the wishes of his will, was buried between his horse and his dog (Duché, 1859). His grave was subsequently moved and he was interred in the grounds of the château of Saint-Sauveur. A picture of the tomb as it appeared in 1928 was published by Royer (1929) and is reproduced here (Fig. 9). This property is now a retirement home for the elderly, and a visit there by one of us (ACP) in 1997 could find no trace of the burial or any marker and it may have been moved to another part of the grounds after the 1928 photo was taken. The area that was most probably the site of the grave is now an exercise area for horses. Officials at the home did not know of any grave in the grounds.

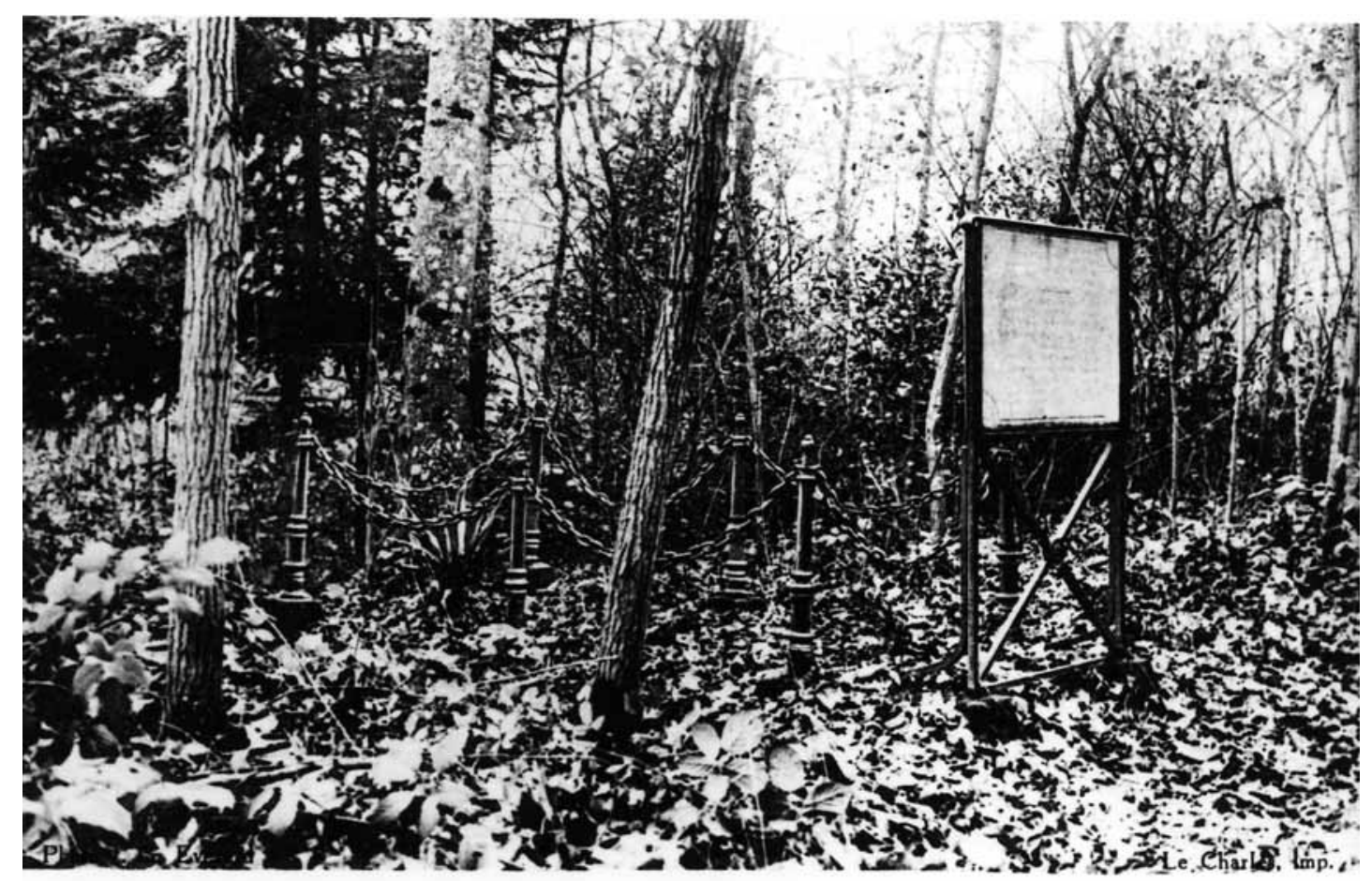

La tombe de Robineau-Desvoidy dans le Parc du Château de Saint-Sauveur-en-Puisaye (Yonne)

FIGURE 9. Tomb of Robineau-Desvoidy in 1928 (from Royer, 1929).

Robineau-Desvoidy never married. He had one sister who married a Gandrille, and it was his nephew, Victor Gandrille, who was the executor of his estate and saw to it that Robineau-Desvoidy's library and collections were donated to the Société des Sciences Historiques et Naturelles de l'Yonne in Auxerre (Quantin, 1858). Victor was the last lord of the manor at Saint-Sauveur, and he bequeathed the Château, which he had inherited from his father, to the municipality of Saint-Sauveur and, on moving into Robineau-Desvoidy's "Ermitage", renamed it as "Les Renards", the name that it still bears. Gandrille was a noted drinker and lecher who walked around the village in a white smock over which a large white beard cascaded. He died in 1879, aged 58. "Les Renards" was sold to pay Gandrille's debts, and Robineau-Desvoidy's remains were subsequently moved in 1888 to the Château park and re-buried. 
Robineau-Desvoidy's long-time friend, Henri Monceaux (1830-1900), secretary of the Société des Sciences Historiques et Naturelle de l'Yonne, decided to fulfill the last wishes of the deceased. With the support of Robineau-Desvoidy's family, he gathered together the voluminous notes of Robineau-Desvoidy's last major tome, one that he had been working on since his 1830 paper, in order to get it to a point where it could be published. A year after Robineau-Desvoidy's death, Monceaux presented the situation to the Société Entomologique de France (Monceaux, 1858) and received their recommendation to publish the work (Fairmaire $e t$ al., 1858). Despite the Société's recommendation, funds to cover publication were a problem and only after they were guaranteed by Robineau-Desvoidy's nephew, Victor Gandrille, was the manuscript finalized and, after being printed in Auxerre (limited to just 250 copies), was published by Victor Masson in Paris, in cooperation with Franz Wagner in Leipzig and Williams \& Norgate in London (see sample pages, Figs. 10-11).

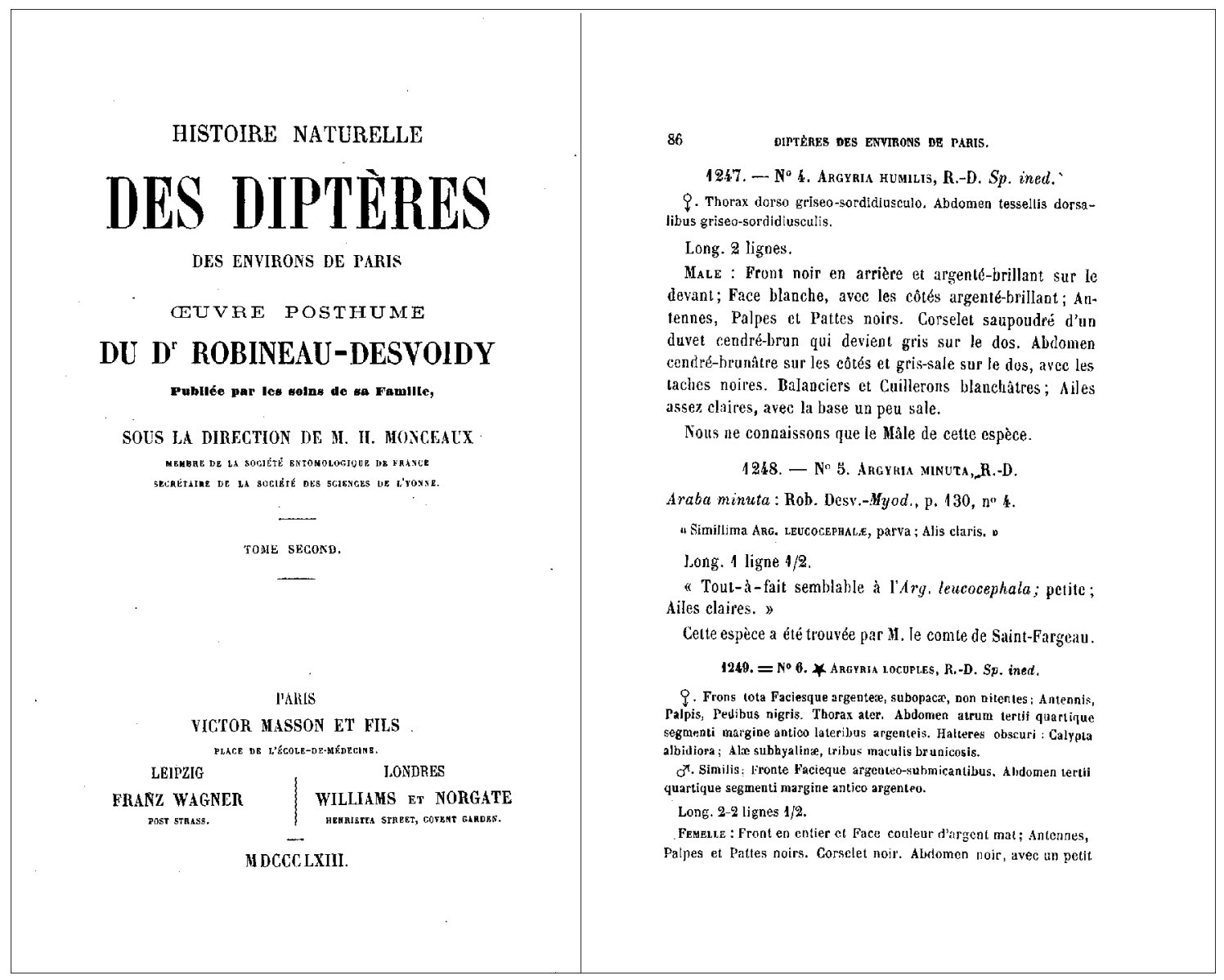

FIGURES 10-11. Sample pages of Robineau-Desvoidy's 1863 "Histoire naturelle des diptères des environs de Paris".

The four-year effort was an indication that despite the manuscript being "fit for printing" when RobineauDesvoidy penned his avant-propos four months before his death (Robineau-Desvoidy, 1863a: 44), a lot of work was obviously still required to get the manuscript into a publishable state. Osten Sacken (1903: 191) explained some of the work Monceaux was required to do. Apparently the unfinished portion of the work was between pages 86 and 91, which was left blank and for Monceaux to fill in as best he could. It seems he simply filled the missing pages word-for-word with what appeared on pages 10-11 in Robineau-Desvoidy (1830) and gave a caveat about synonymies in a footnote on page 91 . As some have quipped, it might have been better for all of dipterology if some of these "lacunae" had never been filled or even that the 1863 work had never been published as, despite the enormous amount of work that Robineau-Desvoidy put into it for 26 years, the 
resulting troubles with nomenclature and taxonomy have more than offset any gains that might have been made.

Gerstaecker (1865) was one of the first to review the work and his words were not favorable. He put things in context by giving a short preface concerning Robineau-Desvoidy's 1830 work:

"Es ist wohl kaum von den Dipterologen ein Werk so allgemein und mit so großem Recht angegriffen worden, als der Essai sur les Myodaires des ebengenannten Verf.'s, welcher ohne die Kenntniss des darin behandelten Gegenstandes irgendwie zu fördern, ausschließlich darauf berechnet schien, die Synonymie bis in's Aeusserste zu verwirren." [There is hardly any work that has been so generally and so justifiably attacked by dipterologists as the above-mentioned author's Essai sur les Myodaires, which, without in any way advancing knowledge of the topic that it deals with, seems exclusively calculated to push synonymy into the utmost confusion.] (Gerstaecker, 1865: 399).

Gerstaecker then summarized the 1863 work with:

"Sich durch die Beschreibungen von 2,240 Arten und 370 Gattungen, wie sie der Verf. hier vorlegt, durchzuarbeiten, um schließlich eine immense Zeit und Mühe vollkommen nutzlos verschwendet zu haben, dazu kann sich ein ernster Forscher wohl in der That nicht gut hergeben!" [To work through the descriptions of the 2,240 species and 370 genera that are here presented by the author and in the end to have wasted the time and effort in complete futility is something to which a serious researcher cannot actually devote himself!] (Gerstaecker, 1865: 399).

\section{And went on to say:}

"Sollte aber auch Jemand eine solche Artenzahl für möglich halten, so wird er sich bei näherer Einsicht des Robineau'schen Buches wenigstens leicht davon überzeugen können, dass sie sich höchstens auf dem Papier, nicht aber in natura vorfindet; die Beschreibungen des Verf.'s liefern hiervon in den verschiedensten Gattungen den besten Beweis, gleichzeitig freilich auch den, dass sie mit einiger Sicherheit zu deuten, ein vergebliches Bemühen sein würde. Gewisse Gattungen bieten in der That das Unglaublichste dar, was man in der Aufstellung neuer Arten verlangen kann." [But if someone were to consider such a number of species to be possible, closer study of Robineau's book would easily be able to convince him that this number exists at most on paper and not in nature; the author's descriptions in the most diverse genera offer the best proof that this is so, and at the same time that the attempt to interpret them with any certainty would be a futile endeavor. Certain genera do indeed offer the most unbelievable facts that one could demand for the erection of new species.] (Gerstaecker, 1865: 400).

However, the work should not be condemned out of hand. Despite the plethora of new taxa and the concomitant problems with their interpretation, it has to be admitted that the technical side of the work and its presentation show a vast improvement on his 1830 book and indeed on most of his other published papers except for his revision of the myopaires discussed above (Robineau-Desvoidy, 1853a). It shows clearly his decades of experience in the field and in his study, poring over the thousands of his myodaires with a hand lens. In this 1863 work, as in the 1853 paper, we see each family-group taxon named, diagnosed (often by means of a rudimentary key), and described. Each genus-group taxon includes a list of synonyms and earlier erroneous interpretations, a description in both French and Latin, often a statement of a type species and, when relevant, a discussion of other pertinent matters. Each species-group taxon includes bibliographic references, synonymies, misidentifications in the case of previously described species; a diagnosis in Latin; a statement of the size; a description of each sex in French; a statement of his material, its provenance, the collector (when not himself), and in some cases a statement of the exact number of specimens and where the specimens are located when not in his personal collection; where available, information is included on biology, reproduction, hosts, flight period, habitats, flower visits, and relationships. The work concludes with a list of Lepidoptera, Coleoptera, Hymenoptera and Hemiptera hosts with their tachinoid parasitoids. 
The work is marred by Robineau-Desvoidy's continued eccentric views on what is a genus and what is a species:

"Y a-t-il des Genres ou n'y a-t-il pas? Oui, si vous réunissez plusieurs Espèces offrant presque les mêmes caractères, ayant les moeurs analogues ou ne se laissant distinguer entre elles que par des variations de taille ou de teinte ... Naguère, on m'avait fait le reproche d'avoir exagéré le nombre des Espèces." [Are there such things as Genera or are there not? Yes, if you unite together several Species that display almost the same characters, that have analogous habits, or that can only be distinguished from each other by variations in size or color ... Formerly I was reproached for having exaggerated the number of Species.] (Robineau-Desvoidy, 1863a: x).

The majority of the species described in this work were from Robineau-Desvoidy's own collecting. However, he was able to include species from Bigot's collection (from Piedmont, Corsica, and France), a large number of species reared from their hosts by the French lepidopterist Bellier de la Chavignerie, and reared material sent or given to him by other lepidopterists such as Bercé, Bagriot, Duponchel, Goureau, and Guérin-Méneville (see under "Collectors" in Appendix II below).

He spent some time, at least in 1855, working in the Paris Museum, where he studied Macquart's types and also redescribed most of Meigen's species from their original material (Robineau-Desvoidy, 1863a: e.g., $157,167,177,836$, etc.). As an aside, it is worth noting that he knew of the Meigen plates of Diptera, which were packed away some time after that and not re-discovered until over 100 years later by Loïc Matile (1974) and published in full color by Günter Morge shortly thereafter (see Morge, 1975, 1976a,b): "D'après la communication qui nous a été faite du volume des figures de Meigen ..." [According to the communication that was made to us of the volume of Meigen's illustrations ...] (Robineau-Desvoidy, 1863a: 735). Through his acquaintance with the young Bigot, he also saw the types of some Macquart species that were located in Bigot's private collection. He paid considerable attention to the works of Meigen, Macquart, and Zetterstedt, correcting what he perceived to be erroneous identifications and noting those that he considered correct. He was unafraid to synonymize his own 1830 names where appropriate (e.g., see under Roeselia antiqua Meigen in 1863a: 608) and he also pointed out that in a number of cases his earlier material had been destroyed:

“Le Phorocère apicans ( $\left.n^{\circ} 4\right)$ n'est pas un Phorocère; nous l'avons perdu ...” [Phorocera apicans $\left(\mathrm{n}^{\circ} 4\right)$ is not

a Phorocera; we have lost it ...] (Robineau-Desvoidy, 1863a: 508).

"Comme nous n'en possédons plus que le débris ..." [As we now have nothing more than the debris ...]

(Robineau-Desvoidy, 1863b: 384, under Ptilocera palpalis Robineau-Desvoidy, 1830).

"Par malheur les Dermestes ont pénétré dans leur boîte ..." [Unfortunately Dermestes got into their box ...] (Robineau-Desvoidy, 1863a: 753).

“Comme j'ai perdu l'échantillon typique ..." [As I have lost the typical specimen ...] (Robineau-Desvoidy, 1863a: 689, under Ceranthia microcera Robineau-Desvoidy, 1830).

Despite its faults, which are many, this work represents an almost unparalleled degree of industry and is a testimonial to Robineau-Desvoidy's acuity in the field, collecting, observing, and studying.

\section{Unpublished Manuscripts}

Monceaux (1863), in his introduction to the two-volumed posthumous work by Robineau-Desvoidy, mentioned some of the unfinished manuscripts on Diptera that did not make it to print. These included one on "Syrphiaires", one on acalyptrates, the Diptera of the Paris Museum, the entomophagous myodaires sent to him from Prussia by Hartig, the myodaires of the Bigot collection, and the flies of the county of Nice in southern France. Other of his manuscripts were listed by Duché (1859), focusing mainly on the history of the Pui- 
saye region, and include such titles as Études sur la Puisaye, Essai sur l'origine du culte du diocèse d'Auxerre, and Dissertation sur le nom d'Auxerre. Robineau-Desvoidy's manuscripts remain in the archives of the Société des Sciences Historiques et Naturelles de l'Yonne (Rocher, 2003: 162).

\section{In Defense of Robineau-Desvoidy}

Robineau-Desvoidy certainly incurred the wrath of some of his dipterological colleagues and endured being ignored by others. But he persisted in his beliefs, and frequently complained that no one had followed his lead and taken up the study of the myodaires.

Osten Sacken was prudent in his review of the two major works of Robineau-Desvoidy and wrote:

"My detailed study of Rob.-Desvoidy's Prefaces has convinced me that this author does not deserve the reproach often urged against him of having neglected the work of his predecessors, Fallén and Meigen. He had done what he could to study and assimilate their publications, especially Meigen's, but he is not to be blamed if he failed to achieve the impossible." (Osten Sacken, 1903: 192).

Sabrosky (1974) also came to Robineau-Desvoidy's defense:

"The sudden proliferation of genera in the Essai was the basis for the aspersions cast by Swainson and Coquillett, among others. Yet a high proportion of the genera are recognized today, and many of RobineauDesvoidy's generic names are familiar currency to those who deal with muscoid Diptera. ... It is true that his efforts in his posthumous work of 1863 were not as successful, although some important generic names such as Phaenicia, Ravinia, Bessa, and Drino do date from that work. Nevertheless, one must conclude from the 1830 Essai that he has been treated unfairly by too much of history. Certainly in the generic classification of Tachinidae he was in reality far ahead of his time, and deserving of respect rather than obloquy." (Sabrosky, 1974: 220, 221).

The defense by Sabrosky was primarily of the 1830 work and he admitted that the 1863 work was "not as successful". Whether or not there is a high proportion of genera recognized today is contestable. Based on the genus-group study in this paper, the overall percentage of genus-group names proposed by Robineau-Desvoidy that are currently valid taxonomically is $51 \%$ (see Table 1). Within the Tachinidae, where by far the most genus-group names were proposed (310), only $49 \%$ are currently considered valid taxonomically (these include nomina dubia). This percentage is even lower if the large number of those nomina dubia are treated as not taxonomically valid. Among the calyptrate flies, two of the lowest percentages are in Sarcophagidae (37\%) and Muscidae (36\%) while he seems to have fared much better in Calliphoridae (75\%). However, these figures need to be viewed against the general trend of generic "lumping" in Sarcophagidae and Muscidae, and of generic "splitting" in Calliphoridae (still continuing ad absurdum in the so-called "Bengaliidae"), while most of the best-known generic names in Muscidae still in use today were coined by Robineau-Desvoidy (e.g., Phaonia, Helina, Hydrotaea, Limnophora, Dasyphora, Morellia, Azelia, Graphomya, Muscina, Pyrellia, Mydaea). Of those families for which he proposed more than 10 genus-group names, he did not do well at all with the Conopidae (17\% validity).

Osten Sacken (1894) gave a thoughtful and sympathetic account of Robineau-Desvoidy and came to the following conclusion:

\footnotetext{
"Robineau makes on me the impression of having been one of those men whose remarkable talents are interfered with by a defect in the character, by the want of that self-control which is indispensable in the production of useful and enduring work. Still, it would be worth while, for some competent dipterologist, to undertake a critical review of the whole work of Robineau on the Myodaires. Gifted as he was, a sincere lover and diligent observer of nature, he must have left some grains of gold in his, apparently confused, mass of publications." (Osten Sacken, 1894: 386).
} 
TABLE 1. Percentage of taxonomically valid Robineau-Desvoidy genus-group names by family.

\begin{tabular}{|c|c|c|c|}
\hline Family & total & valid & $\%$ valid \\
\hline Anthomyiidae & 19 & 12 & 63 \\
\hline Calliphoridae & 20 & 15 & 75 \\
\hline Chamaemyiidae & 1 & 0 & 0 \\
\hline Chyromyidae & 3 & 1 & 33 \\
\hline Conopidae & 12 & 2 & 17 \\
\hline Culicidae & 3 & 2 & 67 \\
\hline Drosophilidae & 1 & 1 & 100 \\
\hline Dryomyzidae & 1 & 0 & 0 \\
\hline Ephydridae & 8 & 5 & 63 \\
\hline Fanniidae & 3 & 1 & 33 \\
\hline Glossinidae & 1 & 1 & 100 \\
\hline Heleomyzidae & 8 & 5 & 63 \\
\hline Lauxaniidae & 4 & 1 & 25 \\
\hline Micropezidae & 1 & 0 & 0 \\
\hline Muscidae & 39 & 14 & 36 \\
\hline Neriidae & 1 & 0 & 00 \\
\hline Odiniidae & 1 & 1 & 100 \\
\hline Oestridae & 2 & 0 & 0 \\
\hline Platystomatidae & 5 & 4 & 80 \\
\hline Psilidae & 2 & 1 & 50 \\
\hline Rhiniidae & 4 & 3 & 75 \\
\hline Rhinophoridae & 8 & 6 & 75 \\
\hline Richardiidae & 3 & 3 & 100 \\
\hline Sarcophagidae & 43 & 16 & 37 \\
\hline Scathophagidae & 9 & 5 & 56 \\
\hline Sciomyzidae & 12 & 9 & 67 \\
\hline Sepsidae & 3 & 3 & 0 \\
\hline Sphaeroceridae & 6 & 6 & 100 \\
\hline Tachinidae & 310 & 150 & 49 \\
\hline Tanypezidae & 1 & 0 & 0 \\
\hline Tephritidae & 18 & 15 & 83 \\
\hline Ulidiidae & 11 & 5 & 45 \\
\hline Unplaced Acalyptratae & 3 & 1 & 33 \\
\hline
\end{tabular}


How should we now judge Robineau-Desvoidy and his achievements? As a man he was both a philanthrope and a misanthrope, caring for the poor and needy in the unhealthy region of Saint-Sauveur-en-Puisaye while preferring to live alone and well away from other people. He was irascible and combative, yet was remembered with affection by his friends long after his death. As a scientist, his work was brilliant but flawed and it was recognized early on that he had too much imagination, extracting unwarranted conclusions from too little evidence. He was not even 30 years old when he submitted his monumental Essai sur les Myodaires for publication and there has rarely been a person who devoted himself so wholeheartedly and with such passion to the study of Diptera, yet his basic concepts of what constitutes a genus and species were far removed from what anyone except the tachinid specialist Charles Henry Tyler Townsend (1863-1944) has considered to be the case. Townsend was of the opinion that a genus should consist of individuals capable of interbreeding and that the species should be the color and other variations within those populations. This fits very well with Robineau-Desvoidy's philosophy, which no doubt is what led him to create hundreds of "paper species" that had no biological reality. Consequently, his categories above species-group became over inflated: what we would now consider to be genera he often ranked as tribes or families. His contemporaries were understandably perplexed by so many names and categories and also found unacceptable his underlying philosophy that a classification should be based on habits and biology rather than on morphological structure. Unsurprisingly, his work was largely ignored by contemporaries and subsequent workers as it has always been so difficult to interpret. Confounding these problems, his collection was mostly destroyed, so interpretation of almost all his genera and species has had to be based on close and repeated reading of his work combined with intuition and guesswork. 


\section{Context of Catalog}

We depend on names for proper and efficient communication about the evolutionary units-species and higher taxa-that make up our biota. The scientific community is currently creating the infrastructure for dealing efficiently and unambiguously with biological names: Global Names Architecture, Catalogue of Life, Index Fungorum, International Plant Names Index, ZooBank, etc. An acutely critical issue, which tends to be neglected or at least left alarmingly under-funded, is dealing with the "quality" of the names themselves; i.e., taking every measure to ensure that every biological name and its inherent properties (e.g., spelling, type fixation, authorship, accurate date of publication) are fully consistent and correct according to current nomenclatural legislature. Since 1984, the Diptera community has been working towards a unified, shared, authoritative resource for names of Diptera, the Biosystematic Database of World Diptera, which is now close to having completed the harvest of all names from the major primary and secondary sources (for a brief overview and history, see Evenhuis et al., 2010). The family-group names of Diptera have been completed by Sabrosky (1999). The next step is publication of a fully peer reviewed World List of Diptera Genus-Group Names. As a means of fulfilling this goal, we are here introducing the first installment in a series of planned "Nomenclatural Studies Toward a World List of Diptera Genus-Group Names". While the ultimate goal is a complete and separately published World List of Diptera Genus-Group Names, the presently planned series of articles is targeted at a subset of these names, namely those proposed by some of the most productive early authors. The following are those for whom work has already begun: A.-J.-B. Robineau-Desvoidy, P.-J.-M. Macquart, J.W. Meigen, C. Rondani, G. Enderlein, and H. Loew. Together, the genus-group names proposed by these authors make up slightly over $10 \%$ of the total number of genus-group names in Diptera, but we expect that these names contain a particularly high frequency of complications and intricacies, and a peer review of these names will provide an explicit protocol and a solid template for the ultimate peer review of every genus-group name ever proposed within Diptera.

\section{Format of Catalog}

The list of genus-group names below presents all known names proposed by or attributed to Robineau-Desvoidy.

HEADING: All nomenclaturally available genus-group names are numbered. Of those, names that are taxonomically valid are placed in boldface. All taxonomically invalid names (junior synonyms, junior homonyms, unjustified emendations) are presented in italics. Nomenclaturally unavailable names (incorrect spellings, nomina nuda) are placed in square brackets [ ] and are unnumbered. The date and page for the first appearance of the published name is given for all names and its full citation can be found in the references. When more than one publication appeared in the same year, a letter suffix is given that corresponds to the chronological order of publication.

ORIGINALLY INCLUDED SPECIES: A full list of originally included species is given with original combination, author and date, which is essential in determining valid typifications of genus-group names. In some cases, Robineau-Desvoidy did not give the actual (or original) author of a name and determining the difference between a new proposal of a taxon and the listing of a previously described taxon proved difficult. In some of these cases, he listed the name of the person that appeared on a museum label for a particular specimen (Robineau-Desvoidy annotates these instances under the respective species). However, research in some cases showed that no name was ever proposed by that person. In some of these cases, the name could be attributed to another author, in other cases, when no name was ever proposed by that person, Robineau-Desvoidy is treated as the author of that name.

TYPE SPECIES: The type species is listed in its original combination and with its form of typification. If it is currently considered a junior synonym (or more rarely, an unavailable senior synonym) of another nominal species, then the name of the latter species is given in square brackets. 
CURRENT STATUS: Current status follows the most recent world or regional catalogs for various families as well as the latest revisionary work(s) for that particular genus-group name if superseding a previously published catalog treatment. For cases of unavailable names (that by definition do not enter into synonymy) we use the phrase "treated under" to indicate current placement of the name.

FAMILY: Family assignment follows the family standards of the Biosystematic Database of World Diptera (Evenhuis et al., 2009).

REMARKS: Genus-group names or typifications needing further clarification or presenting nomenclatural or taxonomic problems are annotated. For all cases of multiple original spellings of a genus-group name, the First Reviser to have selected one of them to be the correct original spelling is indicated.

EMENDATIONS: All known emendations of each genus-group name are listed with an indication of their justification in parenthesis. This list is probably not exhaustive, but presents those emendations that have been previously recorded or have been found during this study. The ICZN Code Article 33.2 states that emendations are "Any demonstrably intentional change in the original spelling of a name other than a mandatory change" and three criteria are given in Article 33.2.1 that can each be used independent of the others in determining what is considered "demonstrably intentional": 1. "when in the work itself or in an author's (or publisher's) corrigenda, there is an explicit statement of intention"; or 2. "when both the original and the changed spelling are cited and the latter is adopted in place of the former", or 3. "when two or more names in the same work are treated in a similar way". Few workers have realized the significance of criterion 3, since this can include names that may previously have been recognized as incorrect subsequent spellings. However, if there are two or more names in the same work that are "treated in a similar way" they become emendations. As a result, there are no doubt numerous uncataloged emendations in published papers of what were previously thought to be merely incorrect subsequent spellings that have escaped notice. Also, it may not be possible to distinguish between a newly proposed emendation and an acceptance of an earlier emendation. We have chosen to consider the earliest cases of such emendations through similar treatment as separate emendations; later homonymous changed spellings that fit criterion 3, whether by the same author or by others, are here considered subsequent usages as they essentially fit Article 33.5. Those earliest discovered emendations indicated here as "new synonymies" are junior synonyms of the current valid genus-group name given above it in the CURRENT STATUS line. Emendations by other authors in our nomenclatural studies series (e.g., Rondani, Meigen, Macquart, Enderlein, and Loew) not previously published as such are not listed here, but will be presented later in those studies.

A summary list at the end of the genus-group name catalog gives a breakdown of the genus-group names proposed by Robineau-Desvoidy by family and maintains the same formatting of boldface, italics, etc. to indicate nomenclatural and taxonomic status.

ABBREVIATIONS: For brevity, we abbreviate the International Commission on Zoological Nomenclature as "I.C.Z.N." for literature references in the catalog. To further differentiate, we use "ICZN Code" to refer to the "International Code of Zoological Nomenclature (1999)" and "ICZN Commission" to refer to the actual Commission.

6. We understand that emendations are synonyms at the time of their proposal, so they cannot be "new" synonyms in this work. We prefer instead to list each newly discovered emendation here as a "new synonymy" to indicate a new action of synonymization, which also allows abstracting services to catch all these names that otherwise have not previously been listed as available names. 


\section{Catalog of Diptera Genus-Group Names of Robineau-Desvoidy}

\section{Acemya Robineau-Desvoidy, 1830: 202.}

ORIGINALly INCLUDED SPECIES: Acemya oblonga Robineau-Desvoidy, 1830; Acemya subrotunda RobineauDesvoidy, 1830; Acemya myoidea Robineau-Desvoidy, 1830 (as “Acemya myoïdea. R. D.”).

TYPE SPECIES: Acemya oblonga Robineau-Desvoidy, 1830 [= Tachina acuticornis Meigen, 1824], by subsequent designation (Desmarest in d'Orbigny, 1849a: 318).

CURRENT STATUS: Valid genus [teste Herting \& Dely-Draskovits (1993: 172)].

FAMILY: TACHINIDAE.

REMARKS: Rondani (1856: 75) and Brauer (1893: 499) designated Acemya subrotunda Robineau-Desvoidy, 1830 as type species but these were later.

Emendations: Acomyia Agassiz, 1846b: 3, 5 (unjustified); Acemyia Schiner, 1861b: 472 (unjustified), n. syn.

2. Acidia Robineau-Desvoidy, 1830: 720.

ORIGINALly INCLUDED SPECIES: Acidia guerini Robineau-Desvoidy, 1830; Tephritis cognata Wiedemann, 1817 (as "Tephrytis cognata. Wintheim").

TYPE SPECIES: Tephritis cognata Wiedemann, 1817, by subsequent designation [Rondani, 1870: 10 (as "Tripeta cognata. Mgn.")].

CURRENT StATUS: Valid genus [teste Norrbom et al. (1999: 67)].

FAMILY: TEPHRITIDAE.

REMARKS: White (1986: 146) assumed that the type species designated by Rondani (1870: 10) of "Tripeta cognata. Mgn." was a misidentification; and therefore considered the earliest designation not based on a misidentification to be that of Tephritis cognata Wiedemann by Hendel (1914a: 85). White's (1986) assumption was incorrect and Rondani's designation, although getting the authorship incorrect, is valid under the ICZN Code.

\section{Acinia Robineau-Desvoidy, 1830: 775.}

ORIGINALLY INCLUDED SPECIES: Acinia jaceae Robineau-Desvoidy, 1830; Acinia pediculariarum RobineauDesvoidy, 1830; Acinia arctii Robineau-Desvoidy, 1830; Acinia annulata Robineau-Desvoidy, 1830; Acinia millefolii Robineau-Desvoidy, 1830; Acinia plantaris Robineau-Desvoidy, 1830; Acinia claripennis Robineau-Desvoidy, 1830.

TYPE SPECIES: Acinia jaceae Robineau-Desvoidy, 1830 [= Tephritis corniculata Zetterstedt, 1819], by subsequent designation [Duponchel in d'Orbigny, 1839: 85 (as "Acinia corniculata Fabricius ou l'A. jaceae Rob. Desv.")].

CURRENT STATUS: Valid genus [teste Norrbom et al. (1999: 69)].

FAMILY: TEPHRITIDAE.

REMARKS: By placing an originally included species in synonymy with Acinia corniculata, the designation by Duponchel in d'Orbigny (1839) is valid. Rondani (1871: 4) designated the same nominal species but this was later.

\section{Aciura Robineau-Desvoidy, 1830: 773.}

ORIGINALly INCLUDED SPECIES: Aciura femoralis Robineau-Desvoidy, 1830; Aciura tibialis RobineauDesvoidy, 1830.

TYPE SPECIES: Aciura femoralis Robineau-Desvoidy, 1830 [= Musca coryli Rossi, 1794], by subsequent designation (Rondani, 1856: 113).

CURRENT STATUS: Valid genus [teste Norrbom et al. (1999: 70)]. 
FAMILY: TEPHRITIDAE.

EMENDATIONS: Acidura Agassiz, 1846b: 4 (unjustified).

\section{Actia Robineau-Desvoidy, 1830: 85.}

ORIGinally InCluded SPECIES: Actia cingulata Robineau-Desvoidy, 1830; Actia pilipennis RobineauDesvoidy, 1830.

TYPE SPECIES: Roeselia lamia Meigen, 1838, by subsequent designation (I.C.Z.N., 1987: 71 [Opinion 1432]). CURRENT StATUS: Valid genus [teste Herting \& Dely-Draskovits (1993: 328)].

FAMILY: TACHINIDAE.

REMARKS: The history leading to the action by the I.C.Z.N. (1987: 71 [Opinion 1432]) to fix the type designation of Actia is complex and was laid out clearly by O'Hara (1985) in his application to the ICZN Commission. In summary, the Commission was requested to designate Roeselia lamia Meigen, 1838 as the type species for Actia Robineau-Desvoidy, 1830 and Actia cingulata Robineau-Desvoidy, 1830 as type species for Elfia Robineau-Desvoidy, 1850. By doing this, Elfia was essentially removed from objective synonymy with Actia since the earliest respective subsequent type designations for Actia (Coquillett, 1910: 503) and Elfia (Robineau-Desvoidy, 1863a: 672) were both of Actia cingulata Robineau-Desvoidy, 1830.

\section{Adenia Robineau-Desvoidy, 1863a: 1041.}

ORIGINALly INCLUDED SPECIES: Adenia irrorata Robineau-Desvoidy, 1863; Adenia spreta RobineauDesvoidy, 1863; Adenia funebris Robineau-Desvoidy, 1863; Adenia propinqua Robineau-Desvoidy, 1863 (with "Tachina atrata : Rob. Desv." in synonymy); Adenia intacta Robineau-Desvoidy, 1863; Tachina grisea Robineau-Desvoidy, 1830; Adenia vigil Robineau-Desvoidy, 1863; Tachina nigra Robineau-Desvoidy, 1830; Adenia potatoria Robineau-Desvoidy, 1863; Adenia profuga RobineauDesvoidy, 1863.

TYPE SPECIES: Tachina grisea Robineau-Desvoidy, 1830 [preoccupied by Tachina grisea Fallén, 1810; =

Tachina rustica Fallén, 1810], by original designation.

CuRrent Status: Valid subgenus of Exorista Meigen, 1803 [teste Herting \& Dely-Draskovits (1993: 126)].

FAMILY: TACHINIDAE.

[Adenia] Robineau-Desvoidy, 1863b: 403.

CURRENT STATUS: Incorrect original spelling of Arenia Robineau-Desvoidy, 1863 [teste Robineau-Desvoidy (1863b: 920)].

FAMILY: TACHINIDAE.

REMARKS: The spelling of Adenia (page 403) was corrected to Arenia (page 920) in the errata of RobineauDesvoidy (1863b).

7. Adia Robineau-Desvoidy, 1830: 558.

ORIGINALLY INCLUDED SPECIES: Adia oralis Robineau-Desvoidy, 1830.

TYPE SPECIES: Adia oralis Robineau-Desvoidy, 1830 [= Musca cinerella Fallén, 1817], by monotypy. CURRENT STATUS: Valid genus [teste Dely-Draskovits (1993: 13)].

FAMILY: ANTHOMYIIDAE.

[Aebalia] Robineau-Desvoidy, 1863b: 347.

CURRENT STATUS: Incorrect original spelling of Oebalia Robineau-Desvoidy, 1863 [teste Pape (1996: 119)]. FAMILY: SARCOPHAGIDAE. 
8. Aetylia Robineau-Desvoidy, 1863a: 270.

ORIGINAlly InCLUDED SPECIES: Aetylia laeta Robineau-Desvoidy, 1863; Aetylia tranquilla RobineauDesvoidy, 1863; Aetylia demens Robineau-Desvoidy, 1863; Tachina hortulana Meigen, 1826.

TYPE SPECIES: Aetylia laeta Robineau-Desvoidy, 1863 [= Exorista glirina Rondani, 1859], by original designation.

CURRENT StATUs: Junior synonym of Phebellia Robineau-Desvoidy, 1846 [teste Herting \& Dely-Draskovits (1993: 184)].

FAMILY: TACHINIDAE.

[Afrella] Robineau-Desvoidy MS name (Blainville et al., 1826: 12).

CURRENT STATUS: Unavailable name; suppressed by action of I.C.Z.N. (1990: 162 [Opinion 1601]); treated as a nomen dubium in Tachinidae [teste this work].

FAMILY: TACHINIDAE.

9. Afrellia Robineau-Desvoidy, 1863a: 933.

ORIGINALLY INCLUDED SPECIES: Afrellia nigrita Robineau-Desvoidy, 1863.

TYPE SPECIES: Afrellia nigrita Robineau-Desvoidy, 1863, by original designation.

CURRENT StATUS: Placed in "Doubtful taxa in Tachinidae" by Herting \& Dely-Draskovits (1993: 435).

FAMILY: TACHINIDAE.

10. Afzelia Robineau-Desvoidy, 1863a: 582.

ORIGINALLY INCLUDED SPECIES: Afzelia exigua Robineau-Desvoidy, 1863.

TYPE SPECIES: Afzelia exigua Robineau-Desvoidy, 1863, by monotypy.

CuRRENT STATUS: Placed in "Doubtful taxa in Tachinidae" by Herting \& Dely-Draskovits (1993: 435).

FAMILY: TACHINIDAE.

11. Agria Robineau-Desvoidy, 1830: 376.

ORIGINALly INCLUDED SPECIES: Agria punctata Robineau-Desvoidy, 1830; Agria grisea RobineauDesvoidy, 1830; Agria grisescens Robineau-Desvoidy, 1830; Agria punctulata Robineau-Desvoidy, 1830; Agria bipunctata Robineau-Desvoidy, 1830; Agria gesnerioidea Robineau-Desvoidy, 1830 (as “Agria gesnerioïdea. R. D.”).

TYPE SPECIES: Agria punctata Robineau-Desvoidy, 1830 [= Musca affinis Fallén, 1817], by subsequent designation (Townsend, 1916: 6).

CURRENT StATUS: Valid genus [teste Pape (1996: 158)].

FAMILY: SARCOPHAGIDAE.

REMARKS: An earlier type-species designation by Coquillett (1910: 504) of Musca affinis Fallén, 1817 is invalid as it was not an originally included species.

12. Ahrensia Robineau-Desvoidy, 1863b: 14.

ORIGINAlly INCLUded SPECIES: Phania flavipalpis Macquart, 1835; Ahrensia femoralis RobineauDesvoidy, 1863; Ahrensia nitida Robineau-Desvoidy, 1863.

TYPE SPECIES: Phania flavipalpis Macquart, 1835 [= Tachina exigua Meigen, 1824], by original designation.

CURREnT STAtus: Junior synonym of Microsoma Macquart, 1855 [teste Herting \& Dely-Draskovits (1993: 397)].

FAMILY: TACHINIDAE. 
13. Albinia Robineau-Desvoidy, 1830: 209.

ORIGINALLY INCLUDED SPECIES: Albinia buccalis Robineau-Desvoidy, 1830.

TYPE SPECIES: Albinia buccalis Robineau-Desvoidy, 1830, by monotypy.

CURRENT STATUS: Placed in "Doubtful taxa in Tachinidae" and considered possibly Rhinophoridae by Herting \& Dely-Draskovits (1993: 435).

FAMILY: TACHINIDAE.

14. Alina Robineau-Desvoidy, 1863b: 639.

ORIGINALly INCLUDED SPECIES: Morellia agilis Robineau-Desvoidy, 1830; Alina pratensis RobineauDesvoidy, 1863; Morellia concolor Robineau-Desvoidy, 1830; Alina pilipes Robineau-Desvoidy, 1863.

TYPE SPECIES: Alina pratensis Robineau-Desvoidy, 1863 [= Cyrtoneura simplex Loew, 1857], by subsequent designation (Coquillett, 1910: 504).

CurRent Status: Preoccupied by Risso, 1826; Récluz, 1842; junior synonym of Morellia RobineauDesvoidy, 1830 [teste Pont (1986b: 94)].

FAMILY: MUSCIDAE.

15. Alophora Robineau-Desvoidy, 1830: 293.

ORIGINAlly InCluded SPECIES: Syrphus subcoleoptratus Fabricius, 1775; Syrphus hemipterus Fabricius, 1794 (as "Phasia hemiptera. Meig."); Alophora pilosa Robineau-Desvoidy, 1830; Alophora ferruginea Robineau-Desvoidy, 1830.

TYPE SPECIES: Syrphus hemipterus Fabricius, 1794, by subsequent designation [Robineau-Desvoidy, 1863b: 226 (as "Thereva hemiptera de Fabricius")].

CURRENT STATUS: Junior synonym of Phasia Latreille, 1804 [teste Herting \& Dely-Draskovits (1993: 409)].

FAMILY: TACHINIDAE.

REMARKS: Brauer (1893: 497), Coquillett (1910: 505), and Townsend (1916: 6) designated the same type species but were later.

EMENDATIONS: Halophora Agassiz, 1846b: 171 (unjustified); Allophora Mik, 1894: 49 (unjustified).

16. Amedea Robineau-Desvoidy, 1830: 207.

ORIGINALLY INCLUDED SPECIES: Amedea scutellaris Robineau-Desvoidy, 1830.

TYPE SPECIES: Amedea scutellaris Robineau-Desvoidy, 1830, by monotypy.

CURRENT STATUS: Placed in "Doubtful taxa in Tachinidae" by Herting \& Dely-Draskovits (1993: 435).

FAMILY: TACHINIDAE.

17. Amenia Robineau-Desvoidy, 1830: 443.

ORIGINALly INCLUDED SPECIES: Amenia imperialis Robineau-Desvoidy, 1830; Musca leonina Fabricius, 1775 (as “Amenia leonina. R. D.”).

TYPE SPECIES: Musca leonina Fabricius, 1775, by subsequent designation (Macquart, 1844: 116[273]).

CURRENT STATUS: Valid genus [teste Kurahashi (1989: 716)].

FAMILY: CALLIPHORIDAE.

REMARKS: Although the second included species is listed as "Amenia leonina. R. D.", it is clear that Robineau-Desvoidy was not describing a new species but was treating Musca leonina Fabricius since he stated after the diagnosis of the genus-group name "Je pense que la réunion de tous ces caractères empêchera aisément de confondre ce genre avec les Chrysomyes. Il renferme les plus belles Muscides connues. Il faut sans doute lui rapporter le Musca leonina de Fabricius." 
18. Amesia Robineau-Desvoidy, 1863b: 363.

ORIGINALLY INCLUDED SPECIES: Amesia variabilis Robineau-Desvoidy, 1863.

TYPE SPECIES: Amesia variabilis Robineau-Desvoidy, 1863 [= Microphthalma europaea Egger, 1860], by monotypy.

CURRENT STATUS: Preoccupied by Duncan, 1841; junior synonym of Microphthalma Macquart, 1843 [teste Herting \& Dely-Draskovits (1993: 351)].

FAMILY: TACHINIDAE.

19. Amina Robineau-Desvoidy, 1830: 629.

ORIGINALLY INCLUDED SPECIES: Amina parisiensis Robineau-Desvoidy, 1830.

TYPE SPECIES: Amina parisiensis Robineau-Desvoidy, 1830, by monotypy.

CURRENT STATUS: Junior synonym of Scathophaga Meigen, 1803 [teste Gorodkov (1986: 29)].

FAMILY: SCATHOPHAGIDAE.

REMARKS: Gorodkov (1986: 29) synonymized the genus-group name with Scathophaga but left its only species, Amina parisiensis, in "Doubtful genera and species" of Scathophagidae (Gorodkov, 1986: 40).

20. Aminta Robineau-Desvoidy, 1830: 569.

Originally InCluded SPECIES: Aminta ludibunda Robineau-Desvoidy, 1830; Aminta grisea RobineauDesvoidy, 1830; Aminta brunipennis Robineau-Desvoidy, 1830; Aminta rivularis Robineau-Desvoidy, 1830; Aminta floralis Robineau-Desvoidy, 1830.

TYPE SPECIES: Aminta ludibunda Robineau-Desvoidy, 1830 [= Anthomyia lepida Wiedemann, 1817], by subsequent designation (Coquillett, 1910: 505).

CURREnT StATUs: Junior synonym of Fannia Meigen, 1803 [teste Pont (1986a: 43)].

FAMILY: FANNIIDAE.

21. Amobia Robineau-Desvoidy, 1830: 96.

ORIGINALly INCLUDED SPECIES: Amobia conica Robineau-Desvoidy, 1830.

TYPE SPECIES: Amobia conica Robineau-Desvoidy, 1830 [= Tachina signata Meigen, 1824], by monotypy.

CURRENT STATUS: Valid genus [teste Pape (1996: 71)].

FAMILY: SARCOPHAGIDAE.

EMENDATIONS: Ammobia Bezzi \& Stein, 1907: 519 (unjustified).

22. Amphisa Robineau-Desvoidy, 1863a: 129.

ORIGINALLY INCLUDED SPECIES: Amphisa laticornis Robineau-Desvoidy, 1863.

TYPE SPECIES: Amphisa laticornis Robineau-Desvoidy, 1863 [= Micropalpus lithosiophagus Rondani, 1859], by monotypy.

CURRENT STATUS: Preoccupied by Curtis, 1828; senior (but invalid) synonym of Homoeonychia Brauer \& Bergenstamm, 1889 (subgenus of Linnaemya Robineau-Desvoidy, 1830) [teste Herting (1984: 99)].

FAMILY: TACHINIDAE.

23. Amyclaea Robineau-Desvoidy, 1863b: 404.

ORIGINALLY INCLUDED SPECIES: Amyclaea serva Robineau-Desvoidy, 1863.

TYPE SPECIES: Amyclaea serva Robineau-Desvoidy, 1863 [= Musca carinifrons Fallén, 1817], by monotypy.

CURRENT StATUS: Junior synonym of Dinera Robineau-Desvoidy, 1830 [teste Herting \& Dely-Draskovits (1993: 361)].

FAMILY: TACHINIDAE. 
24. Andrina Robineau-Desvoidy, 1863a: 835.

ORIGINALLY INCLUDED SPECIES: Tachina lentis Meigen, 1824 (as “Masicera senilis : Meig.").

TYPE SPECIES: Tachina lentis Meigen, 1824 (as "Masicera senilis : Meig.") [= Tachina spathulata Fallén, 1820], by monotypy.

CURRENT Status: Junior synonym of Ramonda Robineau-Desvoidy, 1863 (subgenus of Periscepsia Gistel, 1848) [teste this work].

FAMILY: TACHINIDAE.

REMARKS: Robineau-Desvoidy (1863a: 836) wrote the following concerning the type species: "D'après l'étiquette du Muséum, cette espèce est originaire d'ALLEMAGNE. Meigen en avait d'abord fait une MASICERA; depuis il la rapporta parmi les CLISTES, ainsi que le prouve l'étiquette actuelle du Muséum, vérifiée en 1853". It appears Robineau-Desvoidy misread "lentis" as "senilis" on the handwritten label of the specimen upon which he based the description [see Herting (1984: 191, note 112)]. Herting \& DelyDraskovits (1993: 377) treated Andrina as a junior synonym of Ramonda Robineau-Desvoidy, 1863 and O'Hara \& Wood (2004: 65) treated Ramonda as a subgenus of Periscepsia Gistel, 1848.

\section{Anemya Robineau-Desvoidy, 1863a: 850.}

ORIGINALLY INCLUDED SPECIES: Anemya clausa Robineau-Desvoidy, 1863.

TYPE SPECIES: Anemya clausa Robineau-Desvoidy, 1863, by original designation.

CURRENT StATUS: Placed in "Doubtful taxa in Tachinidae" by Herting \& Dely-Draskovits (1993: 435).

FAMILY: TACHINIDAE.

EMENDATIONS: Anemyia Verrall in Scudder, 1882: 22 (unjustified).

\section{Anetia Robineau-Desvoidy, 1863a: 868.}

ORIGINALLY INCLUDED SPECIES: Anetia occlusa Robineau-Desvoidy, 1863.

TYPE SPECIES: Anetia occlusa Robineau-Desvoidy, 1863, by original designation.

CURRENT STATUS: Preoccupied by Hübner, 1823; no new replacement name proposed; placed in "Doubtful taxa in Tachinidae" by Herting \& Dely-Draskovits (1993: 435).

FAMILY: TACHINIDAE.

REMARKS: Sabrosky \& Arnaud (1965: 1103) placed Anetia in synonymy with Lydella Robineau-Desvoidy, 1830 and treated its type species (Anetia occlusa Robineau-Desvoidy, 1863) as a junior synonym of Lydella grisescens Robineau-Desvoidy, 1830, the type species of Lydella. Herting $(1974,1984)$ did not treat it; but Herting \& Dely-Draskovits (1993), no doubt because of the absence of a type specimen of the type species, reasoned that no conclusive placement could be made and relegated it to "Doubtful taxa in Tachinidae".

\section{Anicia Robineau-Desvoidy, 1863b: 99.}

ORIGINALly InCLUDED SPECIES: Anicia sabulosa Robineau-Desvoidy, 1863; Anicia insidiosa RobineauDesvoidy, 1863; Anicia demissa Robineau-Desvoidy, 1863; Anicia muricola Robineau-Desvoidy, 1863.

TYPE SPECIES: Anicia sabulosa Robineau-Desvoidy, 1863 [= Tachina campestris Fallén, 1810], by subsequent designation (Coquillett, 1910: 506).

CurRent Status: Junior synonym of Metopia Meigen, 1803 [teste Pape (1996: 97)].

FAMILY: SARCOPHAGIDAE.

28. Anthomya Robineau-Desvoidy, 1830: 581.

CURRENT STATUS: Unjustified emendation of Anthomyia Meigen, 1803; junior synonym of Anthomyia Meigen, 1803 [teste Sabrosky (1999: 47)]. 
FAMILY: ANTHOMYIIDAE.

REMARKS: Robineau-Desvoidy (1830: 581) listed his spelling Anthomya and Meigen's spelling Anthomyia together and consistently treated his spelling as valid. Thus we follow Sabrosky (1999: 47) in considering this to be an intentional act by Robineau-Desvoidy and as such an (unjustified) emendation for Anthomyia Meigen, 1803.

\section{Aphria Robineau-Desvoidy, 1830: 89.}

ORIGINALly InCludED SPECIES: Aphria abdominalis Robineau-Desvoidy, 1830; Aphria servillii RobineauDesvoidy, 1830.

TYPE SPECIES: Aphria abdominalis Robineau-Desvoidy, 1830 [= Tachina longirostris Meigen, 1824], by subsequent designation (Robineau-Desvoidy, 1863a: 767).

CurRent Status: Valid genus [teste Herting \& Dely-Draskovits (1993: 336)].

FAMILY: TACHINIDAE.

REMARKS: Robineau-Desvoidy (1863a: 767) designated as type species Tachina longirostris Meigen, 1824, which was not originally included by Robineau-Desvoidy (1830) in Aphria. However, because Tachina longirostris was placed in synonymy in Robineau-Desvoidy (1863a: 767) with the originally included species Aphria abdominalis Robineau-Desvoidy, 1830, the subsequent designation by RobineauDesvoidy (1863a: 767) is valid by ICZN Code Article 69.2.2.

\section{Aplomya Robineau-Desvoidy, 1830: 184.}

ORIGINALly INCLUDED SPECIES: Aplomya nitens Robineau-Desvoidy, 1830; Aplomya zonata RobineauDesvoidy, 1830.

TYPE SPECIES: Aplomya zonata Robineau-Desvoidy, 1830 [= Tachina confinis Fallén, 1820], by subsequent designation (Robineau-Desvoidy, 1863a: 459).

CURRENT STATUS: Valid genus [teste Herting \& Dely-Draskovits (1993: 183)].

FAMILY: TACHINIDAE.

REMARKS: Robineau-Desvoidy (1863a: 459) designated as type species Tachina confinis Fallén, 1820, which was not originally included by Robineau-Desvoidy (1830) in Aplomya. Tachina confinis was placed in synonymy in that work as "Masicera zonata: Macq.-Buff. II, p. 120, no. 1", which refers to Phryxe zonata Robineau-Desvoidy (1830: 159) and not Aplomya zonata Robineau-Desvoidy (1830: 185). However, as Sabrosky (1999: 51) indicated, the discussion by Robineau-Desvoidy (1863a: 459) explains that he considered Phryxe zonata and Aplomya zonata to be synonymous: "Sons [sic] le nom Phryxe zonata et d'Aplomya zonata, j'avais donné un double emploi au même Insecte ...", thus the type designation is valid under ICZN Code Article 69.2.2. Townsend (1916: 6) designated Aplomya nitens RobineauDesvoidy, 1830 as type species, but this was later.

Emendations: Aplomyia Agassiz, 1846a: 3 (unjustified), n. syn.; Haplomyia Agassiz, 1846b: 172 (unjustified).

31. Araba Robineau-Desvoidy, 1830: 127.

ORIGINALLY INCLUDED SPECIES: Tachina fastuosa Meigen, 1824; Araba philanthi Robineau-Desvoidy, 1830; Tachina argyrocephala Meigen, 1824; Araba minuta Robineau-Desvoidy, 1830; Musca leucocephala Rossi, 1790 (as "Tachina leucocephala. Meig."); Araba obscura Robineau-Desvoidy, 1830; Araba assimilis Robineau-Desvoidy, 1830; Araba squamipallens Robineau-Desvoidy, 1830; Araba grisea Robineau-Desvoidy, 1830; Araba fulva Robineau-Desvoidy, 1830.

TYPE SPECIES: Tachina argyrocephala Meigen, 1824, automatic [by designation of the same species (by original designation of Robineau-Desvoidy, 1863b: 88) for Arabella Robineau-Desvoidy, 1863].

CuRrent Status: Junior synonym of Metopia Meigen, 1803 [teste Pape (1996: 97)]. 
FAMILY: SARCOPHAGIDAE.

REMARKS: To make the genus-group name orthography conform in a more euphonious manner with his French vernacular for the genus ("Arabette"), Robineau-Desvoidy (1863b: 88) changed the spelling of his 1830 Araba to Arabella (an unjustified emendation for Araba) and designated a type species for Arabella (see also No. 32, Arabella below).

EMENDATIONS: Arabella Robineau-Desvoidy, 1863b: 88 (unjustified).

\section{Arabella Robineau-Desvoidy, 1863b: 88.}

CURRENT STATUS: Preoccupied by Grube, 1850; unjustified emendation of Araba Robineau-Desvoidy, 1830; junior synonym of Metopia Meigen, 1803 [teste Pape (1996: 97)].

FAMILY: SARCOPHAGIDAE.

REMARKS: Arabella was proposed by Robineau-Desvoidy (1863b: 88) as a new spelling (= unjustified emendation) for his Araba of 1830 when he, for reasons of euphony, changed the vernacular name from "Arabette" to "Arabelle".

\section{Arenia Robineau-Desvoidy, 1863b: 403 (920).}

ORIGINALLY INCLUDED SPECIES: Arenia volucris Robineau-Desvoidy, 1863 (as "Adenia volucris").

TYPE SPECIES: Arenia volucris Robineau-Desvoidy, 1863 (as "Adenia volucris"), by monotypy.

CURRENT STATUS: Junior synonym of Billaea Robineau-Desvoidy, 1830 [teste Herting \& Dely-Draskovits (1993: 357)].

FAMILY: TACHINIDAE.

REMARKS: This is the correct spelling of Adenia Robineau-Desvoidy (1863b: 403) [not Adenia RobineauDesvoidy (1863b: 1041)], the correction of which is in the errata on page 920. The description and included species for the nominal genus are found on page 403 under the incorrect original spelling "Adenia".

\section{Arge Robineau-Desvoidy, 1863a: 182.}

ORIGINALLY INCLUDED SPECIES: Arge terminalis Robineau-Desvoidy, 1863.

TYPE SPECIES: Arge terminalis Robineau-Desvoidy, 1863 [= Musca quadripustulata Fabricius, 1794], by original designation.

CuRReNT STATUS: Preoccupied by Schrank, 1802; Hübner, 1819; junior synonym of Winthemia RobineauDesvoidy, 1830 [teste Herting \& Dely-Draskovits (1993: 180)].

FAMILY: TACHINIDAE.

REMARKS: The preoccupation of the name Arge was noted by Monceaux in the errata to Robineau-Desvoidy (1863b: 917), but he left the matter to someone more experienced to take nomenclatural action. No replacement name was ever subsequently published.

35. Argyrella Robineau-Desvoidy, 1863b: 87.

ORIGINALly INCLUDED SPECIES: Argyrella dissimilis Robineau-Desvoidy, 1863; Argyrella festinans Robineau-Desvoidy, 1863.

TYPE SPECIES: Argyrella dissimilis Robineau-Desvoidy, 1863 [= Tachina argyrocephala Meigen, 1824], by subsequent designation (Townsend, 1916: 6).

CuRRent Status: Junior synonym of Metopia Meigen, 1803 [teste Pape (1996: 97)].

FAMILY: SARCOPHAGIDAE.

REMARKS: An earlier type-species designation by Coquillett (1910: 509) of Musca leucocephala Rossi, 1790 is invalid as it was not an originally included species. Coquillett may have been misled that it was an 
originally included species since Robineau-Desvoidy (1863b: 87) mentioned in the note under Argyrella dissimilis "semblable à l'Arg. leucocephala", but this is in reference to "Argyria leucocephala" and not a species in Argyrella.

36. Argyria Robineau-Desvoidy, 1863b: 82.

ORIGINAlly InCluded SPECIES: Musca leucocephala Rossi, 1790 (as “Musca leucocephala : Panz."; Araba philanthi Robineau-Desvoidy, 1830 (as “Araba Philanti : Rob. Desv.”); Argyria hilaris RobineauDesvoidy, 1863; Argyria humilis Robineau-Desvoidy, 1863; Araba minuta Robineau-Desvoidy, 1830; Argyria locuples Robineau-Desvoidy, 1863.

TYPE SPECIES: Musca leucocephala Rossi, 1790 [preoccupied by Musca leucocephala Villers, 1789; = Tachina argyrocephala Meigen, 1824], by original designation (as “Musca leucocephala, Panz.").

CuRRENT Status: Preoccupied by Hübner, 1818; junior synonym of Metopia Meigen, 1803 [teste Pape (1996: 97)].

FAMILY: SARCOPHAGIDAE.

37. Aria Robineau-Desvoidy, 1830: 309.

ORIGINALLY INCLUDED SPECIES: Aria fulvicrus Robineau-Desvoidy, 1830.

TYPE SPECIES: Aria fulvicrus Robineau-Desvoidy, 1830, by monotypy.

CURRENT STATUS: Preoccupied by Pallas, 1811; no new replacement name proposed; placed in "Doubtful taxa in Tachinidae" by Herting \& Dely-Draskovits (1993: 435).

FAMILY: TACHINIDAE.

38. Aricia Robineau-Desvoidy, 1830: 486.

ORIGINALly INCLUDED SPECIES: Aricia impunctata Robineau-Desvoidy, 1830; Aricia rustica RobineauDesvoidy, 1830; Aricia claripennis Robineau-Desvoidy, 1830; Aricia luteipes Robineau-Desvoidy, 1830; Aricia biguttata Robineau-Desvoidy, 1830; Aricia nigricans Robineau-Desvoidy, 1830; Aricia bisbinotata Robineau-Desvoidy, 1830 (as “Aricia bis-binotata. R. D.”); Aricia quatuorpunctata Robineau-Desvoidy, 1830 (as “Aricia quatuor-punctata. R. D.”); Aricia vittata Robineau-Desvoidy, 1830.

TYPE SPECIES: Aricia impunctata Robineau-Desvoidy, 1830 [= Musca impuncta Fallén, 1825], by subsequent designation (Coquillett, 1901: 144).

CURRENT StATUS: Preoccupied by Reichenbach, 1817; Savigny, 1822; junior synonym of Helina RobineauDesvoidy, 1830 [teste Pont (1986b: 136)].

FAMILY: MUSCIDAE.

REMARKS: Earlier type-species designations by Westwood (1840: 141) of Musca lardaria Fabricius, 1781 and by Rondani (1856: 95) of Musca erratica Fallén, 1825 are not valid as these were not originally included species.

39. Arina Robineau-Desvoidy, 1830: 696.

ORIGINALLY INCLUDED SPECIES: Arina obscura Robineau-Desvoidy, 1830.

TYPE SPECIES: Arina obscura Robineau-Desvoidy, 1830, by monotypy.

CURRENT STATUS: Nomen dubium [teste this work].

FAMILY: SCIOMYZIDAE.

REMARKS: Rozkošný \& Elberg (1984: 193) listed the only included species (Arina obscura) among their

"Doubtful species" but omitted listing of the genus-group name; we treat Arina Robineau-Desvoidy, 
1830 as a nomen dubium in Sciomyzidae until such time as its proper taxonomic status can be ascertained.

EMENDATIONS: Arhina Agassiz, 1846b: 34 (unjustified).

40. Arisbaea Robineau-Desvoidy, 1863b: 290.

ORIGINAlly InCluded SPECIES: Arisbaea lateralis Robineau-Desvoidy, 1863 (as "Zophomya lateralis : Macq.").

TYPE SPECIES: Arisbaea lateralis Robineau-Desvoidy, 1863 (as "Zophomya lateralis : Macq.") [= Musca tachinoides Fallén, 1817], by monotypy.

CURREnt Status: Junior synonym of Stomina Robineau-Desvoidy, 1830 [teste Herting \& Dely-Draskovits (1993: 391)].

FAMILY: TACHINIDAE.

REMARKS: There are two original spellings of this genus-group name in Robineau-Desvoidy (1863b): Arisbaea (page 290) and Arisbea (page 851). Acting as First Reviser, we select Arisbaea as the correct original spelling.

[Arisbea] Robineau-Desvoidy, 1863b: 851.

CURRENT STATUS: Incorrect original spelling of Arisbaea Robineau-Desvoidy, 1863a [teste this work].

FAMILY: TACHINIDAE.

41. Arraltia Robineau-Desvoidy, 1863b: 72.

ORIGINALLY INCLUDED SPECIES: Arraltia atra Robineau-Desvoidy, 1863.

TYPE SPECIES: Arraltia atra Robineau-Desvoidy, 1863 [= Tachina praefica Meigen, 1824], by monotypy.

CURRENT Status: Junior synonym of Macquartia Robineau-Desvoidy, 1830 [teste Herting \& DelyDraskovits (1993: 312)].

FAMILY: TACHINIDAE.

42. Asbella Robineau-Desvoidy, 1863b: 398.

ORIGINALLY INCLUDED SPECIES: Asbella ruficornis Robineau-Desvoidy, 1863.

TYPE SPECIES: Asbella ruficornis Robineau-Desvoidy, 1863 [= Dexia triangulifera Zetterstedt, 1844], by monotypy.

CURRENT STAtus: Junior synonym of Billaea Robineau-Desvoidy, 1830 [teste Herting \& Dely-Draskovits (1993: 357)].

FAMILY: TACHINIDAE.

43. Ateria Robineau-Desvoidy, 1863a: 809.

ORIGINALLY INCLUDED SPECIES: Ateria nitida Robineau-Desvoidy, 1863.

TyPE SPECIES: Ateria nitida Robineau-Desvoidy, 1863 [= Phorichaeta prunaria Rondani, 1861], by monotypy.

CURRENT StAtus: Junior synonym of Ramonda Robineau-Desvoidy, 1863 (subgenus of Periscepsia Gistel, 1848) [teste this work].

FAMILY: TACHINIDAE.

REMARKS: Herting \& Dely-Draskovits (1993: 377) treated Ateria as a junior synonym of Ramonda RobineauDesvoidy, 1863 and O'Hara \& Wood (2004: 65) treated Ramonda as a subgenus of Periscepsia Gistel, 1848. 


\section{Athrycia Robineau-Desvoidy, 1830: 111.}

ORIGINALly INCLUDED SPECIES: Athrycia erythrocera Robineau-Desvoidy, 1830; Athrycia flavescens Robineau-Desvoidy, 1830.

TYPE SPECIES: Athrycia erythrocera Robineau-Desvoidy, 1830 [= Tachina trepida Meigen, 1824], by subsequent designation (Robineau-Desvoidy, 1863a: 830).

CurRent Status: Valid genus [teste Herting \& Dely-Draskovits (1993: 382)].

FAMILY: TACHINIDAE.

EMENDATIONS: Atrichia Verrall in Scudder, 1882: 38 (unjustified).

45. Atilia Robineau-Desvoidy, 1863a: 475.

ORIGINAlly InCluded SPECIES: Atilia occlusa Robineau-Desvoidy, 1863; Atilia flavisquamis RobineauDesvoidy, 1863; Atilia potatoria Robineau-Desvoidy, 1863; Atilia ambulans Robineau-Desvoidy, 1863; Atilia praeceps Robineau-Desvoidy, 1863.

TYPE SPECIES: Atilia occlusa Robineau-Desvoidy, 1863, by original designation.

CURRENT STATUS: Preoccupied by Adams, 1853; no new replacement name proposed; placed in "Doubtful taxa in Tachinidae" by Herting \& Dely-Draskovits (1993: 435).

FAMILY: TACHINIDAE.

46. Atrania Robineau-Desvoidy, 1863a: 814.

ORIGINALLY INCLUDED SPECIES: Atrania hyalinata Robineau-Desvoidy, 1863.

TYPE SPECIES: Atrania hyalinata Robineau-Desvoidy, 1863 [= Wagneria gagatea Robineau-Desvoidy, 1830], by monotypy.

CURRENT STATUS: Junior synonym of Wagneria Robineau-Desvoidy, 1830 [teste Herting \& Dely-Draskovits (1993: 380)].

FAMILY: TACHINIDAE.

47. Aubaea Robineau-Desvoidy, 1863a: 185.

ORIGINAlly InCluded SPECIES: Aubaea cita Robineau-Desvoidy, 1863; Aubaea campestris RobineauDesvoidy, 1863; Aubaea pyralidis Robineau-Desvoidy, 1863; Aubaea aurulenta Robineau-Desvoidy, 1863; Aubaea nigrita Robineau-Desvoidy, 1863; Aubaea minuta Robineau-Desvoidy, 1863.

TYPE SPECIES: Aubaea aurulenta Robineau-Desvoidy, 1863 [= Tachina floralis Fallén, 1810], by original designation.

CURRENT Status: Junior synonym of Nemorilla Rondani, 1856 [teste Herting \& Dely-Draskovits (1993: 182)].

FAMILY: TACHINIDAE.

REMARKS: Aubaea was proposed as a new genus in Robineau-Desvoidy (1863a: 185; with six originally included species, fixation by original designation) and also in Robineau-Desvoidy (1863b: 182; with one included species, fixation by monotypy). As odd as it may seem to propose two separate genera with the same name in the same family and in the same work, the two names are homonyms as they represent two separate generic concepts. Thus, both genus-group names are listed here. See also No. 48 Aubaea below.

48. Aubaea Robineau-Desvoidy, 1863b: 182.

ORIGINALLY INCLUDED SPECIES: Ocyptera interrupta Meigen, 1824 (with “Ocyptera cylindrica : Fall." in synonymy).

TYPE SPECIES: Ocyptera interrupta Meigen, 1824 sensu Robineau-Desvoidy, 1863b [misidentification; = Ocyptera pusilla Meigen, 1824], by monotypy. 
CURRENT STATUS: Preoccupied by Robineau-Desvoidy, 1863a; junior synonym of Ocypterula Rondani, 1856 (subgenus of Cylindromyia Meigen, 1803) [teste Herting (1984: 179)].

FAMILY: TACHINIDAE.

REMARKS: ICZN Code Article 70.3.2 is not invoked to fix the type species as Ocyptera pusilla Meigen, 1824 because Aubaea Robineau-Desvoidy, 1863b is preoccupied and there is no threat to nomenclatural stability. See also No. 47 Aubaea above.

\section{Azelia Robineau-Desvoidy, 1830: 592.}

ORIGINALly InCluded SPECIES: Azelia florea Robineau-Desvoidy, 1830; Azelia gentilis RobineauDesvoidy, 1830; Azelia nebulosa Robineau-Desvoidy, 1830; Azelia duodecimpunctata RobineauDesvoidy, 1830 (as “Azelia duodecim-punctata. R. D.”); Azelia obscura Robineau-Desvoidy, 1830; Azelia dorsalis Robineau-Desvoidy, 1830; Azelia agilis Robineau-Desvoidy, 1830; Azelia vernalis Robineau-Desvoidy, 1830; Azelia tibialis Robineau-Desvoidy, 1830.

TYPE SPECIES: Azelia florea Robineau-Desvoidy, 1830 [= Anthomyia triquetra Wiedemann, 1817], by subsequent designation (Rondani, 1866: 72).

CuRrent Status: Valid genus [teste Pont (1986b: 62)].

FAMILY: MUSCIDAE.

REMARKS: Coquillett (1901: 142) designated Azelia nebulosa Robineau-Desvoidy, 1830, but this was later.

\section{Bacchis Robineau-Desvoidy, 1830: 803.}

ORIGINALLY INCLUDED SPECIES: Bacchis cellarum Robineau-Desvoidy, 1830; Bacchis ludibunda RobineauDesvoidy, 1830; Bacchis leucoptera Robineau-Desvoidy, 1830; Bacchis putris Robineau-Desvoidy, 1830; Bacchis geniculata Robineau-Desvoidy, 1830.

TYPE SPECIES: Bacchis cellarum Robineau-Desvoidy, 1830, by subsequent designation (Duponchel in d'Orbigny, 1842a: 409).

CURREnT Status: Nomen dubium; transferred to Drosophilidae [teste Roháček in Roháček (2001: 38)]; treated as incertae sedis in Drosophilidae [teste this work].

FAMILY: DROSOPHILIDAE.

REMARKS: Roháček in Roháček (2001: 38) followed Duda (1938) in transferring this genus-group name from Sphaeroceridae to Drosophilidae, primarily because the type species (Bacchis cellarum) was said to occur "sur le vin corrompu et exposé à l'air" by Robineau-Desvoidy (1830: 804).

\section{Bebricia Robineau-Desvoidy, 1863a: 1112.}

ORIGINALLY INCLUDED SPECIES: Macquartia microcera Robineau-Desvoidy, 1830; Macquartia brachycera Robineau-Desvoidy, 1830.

TYPE SPECIES: Macquartia microcera Robineau-Desvoidy, 1830 [= Tachina praefica Meigen, 1824], by original designation.

CURRENT StATUS: Junior synonym of Macquartia Robineau-Desvoidy, 1830 [teste Herting \& DelyDraskovits (1993: 312)].

FAMILY: TACHINIDAE.

\section{Belida Robineau-Desvoidy, 1863b: 45.}

ORIGINALLY INCLUDED SPECIES: Belida flavipalpis Robineau-Desvoidy, 1863.

TYPE SPECIES: Belida flavipalpis Robineau-Desvoidy, 1863, by monotypy.

CURRENT STATUS: Valid genus [teste Herting \& Dely-Draskovits (1993: 144)].

FAMILY: TACHINIDAE. 


\section{Bellardia Robineau-Desvoidy, 1863b: 548.}

ORIGINALLY INCLUDED SPECIES: Bellardia vernalis Robineau-Desvoidy, 1863.

TYPE SPECIES: Bellardia vernalis Robineau-Desvoidy, 1863 [= Tachina obsoleta Meigen, 1824], by monotypy.

CURRENT STATUS: Valid genus [teste Rognes (1991: 34)].

FAMILY: CALLIPHORIDAE.

REMARKS: Rondani (1863: 81) also proposed the name Bellardia for a tabanid. Robineau-Desvoidy (1863a,b) was announced as published by Monceaux at the 11 January 1863 meeting of the Société des Sciences Historiques et Naturelles de l'Yonne; Rondani's 1863 work is assumed to have come out much later in the year, so Robineau-Desvoidy (1863b) has priority.

\section{Bellieria Robineau-Desvoidy, 1863b: 432.}

ORIGINAlly INCLUDED SPECIES: Bellieria rubricornis Robineau-Desvoidy, 1863; Myophora cinerea Robineau-Desvoidy, 1830.

TYPE SPECIES: Myophora cinerea Robineau-Desvoidy, 1830, by original designation.

CURRENT STATUS: Junior synonym of Sarcophaga Meigen, 1826 but subgenerically unplaced [teste Pape (1996: 419)].

FAMILY: SARCOPHAGIDAE.

\section{Bellina Robineau-Desvoidy, 1863b: 194.}

ORIGINALLY INCLUDED SPECIES: Bellina melanura Robineau-Desvoidy, 1863.

TYPE SPECIES: Bellina melanura Robineau-Desvoidy, 1863, by monotypy.

CURRENT STATUS: Valid genus [teste Crosskey (1977: 591)].

FAMILY: TACHINIDAE.

\section{Belvosia Robineau-Desvoidy, 1830: 103.}

ORIGINALly INCLUDED SPECIES: Belvosia bicincta Robineau-Desvoidy, 1830.

TYPE SPECIES: Belvosia bicincta Robineau-Desvoidy, 1830, by monotypy.

CURRENT STATUS: Valid genus [teste O'Hara \& Wood (2004: 160)].

FAMILY: TACHINIDAE.

REMARKS: Robineau-Desvoidy indicated that this genus-group name was dedicated to the memory of Palisot de Beauvois, thus the genus-group name is Robineau-Desvoidy's latinization of "Beauvois".

\section{Bengalia Robineau-Desvoidy, 1830: 425.}

ORIGINALly INCLUDED SPECIES: Bengalia testacea Robineau-Desvoidy, 1830; Bengalia labiata RobineauDesvoidy, 1830; Bengalia pallens Robineau-Desvoidy, 1830; Bengalia melanocera Robineau-Desvoidy, 1830.

TYPE SPECIES: Bengalia testacea Robineau-Desvoidy, 1830 [= Musca torosa Wiedemann, 1819], by subsequent designation (Duponchel in d'Orbigny, 1842a: 542).

CURRENT StATUS: Valid genus [teste Rognes (2006: 467)].

FAMILY: CALLIPHORIDAE.

58. Beraldia Robineau-Desvoidy, 1863a: 906.

ORIGINALLY INCLUDED SPECIES: Beraldia aurifacies Robineau-Desvoidy, 1863; Erycia vanessae RobineauDesvoidy, 1850; Beraldia concolor Robineau-Desvoidy, 1863. 
TYPE SPECIES: Erycia vanessae Robineau-Desvoidy, 1850, by original designation.

CURRENT STATUS: Placed in "Doubtful taxa in Tachinidae" by Herting \& Dely-Draskovits (1993: 435).

FAMILY: TACHINIDAE.

59. Bercaea Robineau-Desvoidy, 1863b: 549.

ORIGINALly INCLUDED SPECIES: Bercaea strenua Robineau-Desvoidy, 1863; Myophora florea RobineauDesvoidy, 1830; Bercaea floridula Robineau-Desvoidy, 1863; Bercaea haemathura Robineau-Desvoidy, 1863; Bercaea agilis Robineau-Desvoidy, 1863; Musca haemorrhoidalis Fallén, 1817 (as "Bercaea haemorrhoïdalis, Fall.”); Bercaea oralis Robineau-Desvoidy, 1863; Bercaea agraria RobineauDesvoidy, 1863; Bercaea morio Robineau-Desvoidy, 1863; Bercaea laeta Robineau-Desvoidy, 1863; Bercaea sponsa Robineau-Desvoidy, 1863; Bercaea apricata Robineau-Desvoidy, 1863; Bercaea anceps Robineau-Desvoidy, 1863.

TYPE SPECIES: Musca haemorrhoidalis Fallén, 1817 sensu Robineau-Desvoidy, 1863b (as "Bercaea haemorrhö̈dalis, R.-D.”) [misidentification; = Musca africa Wiedemann, 1824], by original designation. CURRENT STAtus: Valid subgenus of Sarcophaga Meigen, 1826 [teste Pape (1996: 302)].

FAMILY: SARCOPHAGIDAE.

60. Beria Robineau-Desvoidy, 1830: 418.

ORIGINALLY INCLUDED SPECIES: Beria inflata Robineau-Desvoidy, 1830.

TYPE SPECIES: Beria inflata Robineau-Desvoidy, 1830, by monotypy.

CURRENT STATUS: Junior synonym of Rhyncomya Robineau-Desvoidy, 1830 [teste Rognes (2002: 28)].

FAMILY: RHINIIDAE.

61. Bessa Robineau-Desvoidy, 1863b: 164.

ORiginally InCluded SPECIES: Bessa secutrix Robineau-Desvoidy, 1863; Bessa blanda RobineauDesvoidy, 1863; Bessa palpalis Robineau-Desvoidy, 1863.

TYPE SPECIES: Bessa secutrix Robineau-Desvoidy, 1863 [= Tachina selecta Meigen, 1824], by original designation.

CURRENT STATUS: Valid genus [teste Herting \& Dely-Draskovits (1993: 142)].

FAMILY: TACHINIDAE.

62. Besseria Robineau-Desvoidy, 1830: 232.

ORIGINALLY INCLUDED SPECIES: Besseria reflexa Robineau-Desvoidy, 1830.

TYPE SPECIES: Besseria reflexa Robineau-Desvoidy, 1830 [= Tachina melanura Meigen, 1824], by monotypy.

CurRent StATUS: Valid genus [teste Herting \& Dely-Draskovits (1993: 432)].

FAMILY: TACHINIDAE.

\section{Bigotia Robineau-Desvoidy, 1863a: 1048.}

ORIGINALLY INCLUDED SPECIES: Tachina brevicornis Macquart, 1854.

TYPE SPECIES: Tachina brevicornis Macquart, 1854 [preoccupied by Tachina brevicornis Macquart, 1839], by original designation.

CURRENT Status: Placed in "Doubtful taxa in Tachinidae" by Herting \& Dely-Draskovits (1993: 435).

FAMILY: TACHINIDAE.

REMARKS: No new replacement name is proposed for the type species because it is considered a nomen dubium. 
64. Billaea Robineau-Desvoidy, 1830: 328.

ORIGINALLY INCLUDED SPECIES: Billaea grisea Robineau-Desvoidy, 1830.

TYPE SPECIES: Billaea grisea Robineau-Desvoidy, 1830 [= Dexia pectinata Meigen, 1826], by monotypy.

CurRent Status: Valid genus [teste Herting \& Dely-Draskovits (1993: 356)].

FAMILY: TACHINIDAE.

[Biomye] Robineau-Desvoidy MS name (Blainville et al., 1826: 11).

CURRENT STATUS: Unavailable name; suppressed by action of I.C.Z.N. (1990: 162 [Opinion 1601]); treated under Musca Linnaeus, 1758 [teste this work].

REMARKS: This is likely a typesetter's error in misreading the handwriting of what was intended to be "Biomya". Because it was typeset in italics, we feel that this was probably the MS name that was published later as Byomya Robineau-Desvoidy (1830: 392) and not a vernacular usage.

FAMILY: MUSCIDAE.

65. Bithia Robineau-Desvoidy, 1863a: 770.

ORIGINALLY INCLUDED SPECIES: Tachina spreta Meigen, 1824.

TYPE SPECIES: Tachina spreta Meigen, 1824, by original designation.

CurRent StATUS: Valid genus [teste Herting \& Dely-Draskovits (1993: 338)].

FAMILY: TACHINIDAE.

\section{Blainvillia Robineau-Desvoidy, 1830: 514.}

ORIGINALLY INCLUDED SPECIES: Blainvillia palpata Robineau-Desvoidy, 1830.

TYPE SPECIES: Blainvillia palpata Robineau-Desvoidy, 1830, by monotypy.

CURRENT STATUS: Junior synonym of Hydrotaea Robineau-Desvoidy, 1830 [teste Pont (1986b: 74)].

FAMILY: MUSCIDAE.

REMARKS: Robineau-Desvoidy (1830) proposed this name twice for two different genera in this work. The Blainvillia on page 514 was for a single species collected in 1828, two years after he presented his original draft of the Myodaires to the Académie des Sciences, with an etymology "Je dédie ce genre à M. Ducrotay de Blainville, un de mes maîtres, et l'auteur du rapport qui m'a valu les honneurs de l'impression pour les Myodaires". The second Blainvillia (on page 713, see entry below) has no date of collection and only a short dedication to "M. de Blainville, auteur du Rapport sur mes Myodaires". It is possible that the second Blainvillia (on page 713) originally had another name and Robineau-Desvoidy changed it soon after Blainville gave his report to the Académie in 1826 in order to honor him. Two years later (in 1828), he collected a new genus in Saint-Sauveur and wanted to add it to his paper with a name honoring his "maitre" but forgot he had already changed the name of one of his genera to honor the same person. While it is possible to synonymize this Blainvillia (1830: 514) with Hydrotaea because of its position in the text between Hydrotaea and Ophyra Robineau-Desvoidy (also a junior synonym of Hydrotaea), the type species cannot be interpreted because Robineau-Desvoidy clearly described two different species (one with plumose arista, one with pubescent arista) as the male and female of his Blainvillia palpata.

67. Blainvillia Robineau-Desvoidy, 1830: 713.

ORIGINAlly INCLUded SPECIES: Musca formosa Panzer, 1798 (as “Otites formosa. Latr.”); Blainvillia jucunda Robineau-Desvoidy, 1830.

TYPE SPECIES: Musca formosa Panzer, 1798 [preoccupied by Musca formosa Scopoli, 1763; = Otites elegans Latreille, 1805], by subsequent designation (Hennig, 1939: 46). 
CuRRENT STATUS: Preoccupied by Robineau-Desvoidy, 1830: 514; junior synonym of Otites Latreille, 1804 [teste Soós (1984: 47)].

FAMILY: ULIDIIDAE.

REMARKS: See also No. 66 Blainvillia above.

68. Blissonia Robineau-Desvoidy, 1863b: 648.

ORIGINALLY INCLUDED SPECIES: Blissonia caesia Robineau-Desvoidy, 1863; Muscina fungivora RobineauDesvoidy, 1830 (as "Musca fungivora : Rob. Desv."; with "Curtonevra fungivora : Macq." in synonymy); Blissonia rustica Robineau-Desvoidy, 1863.

TYPE SPECIES: Blissonia caesia Robineau-Desvoidy, 1863 [= Musca levida Harris, 1780], by subsequent designation (Townsend, 1916: 6).

CURRENT Status: Junior synonym of Muscina Robineau-Desvoidy, 1830 [teste Pont (1986b: 59)].

FAMILY: MUSCIDAE.

REMARKS: Musca fungivora Robineau-Desvoidy, 1830 was designated as type species by Hennig (1962b: 759), but this was later.

69. Blondelia Robineau-Desvoidy, 1830: 122.

ORIGINALly INCLUDED SPECIES: Blondelia nitida Robineau-Desvoidy, 1830; Blondelia abdominalis Robineau-Desvoidy, 1830; Blondelia pallidipalpis Robineau-Desvoidy, 1830; Blondelia fasciata Robineau-Desvoidy, 1830.

TYPE SPECIES: Blondelia nitida Robineau-Desvoidy, 1830 [= Tachina nigripes Fallén, 1810], by subsequent designation (Duponchel in d'Orbigny, 1842a: 609).

CURRENT STATUS: Valid genus [teste Herting \& Dely-Draskovits (1993: 168)].

FAMILY: TACHINIDAE.

REMARKS: Townsend (1916: 6) designated the same type species but this was later.

70. Blumia Robineau-Desvoidy, 1863a: 468.

ORIGINALLY INCLUDED SPECIES: Blumia occlusa Robineau-Desvoidy, 1863.

TYPE SPECIES: Blumia occlusa Robineau-Desvoidy, 1863 [= Exorista caudata Rondani, 1859], by monotypy.

CuRRENT Status: Junior synonym of Phryxe Robineau-Desvoidy, 1830 [teste Herting \& Dely-Draskovits (1993: 192)].

FAMILY: TACHINIDAE.

71. Bohemania Robineau-Desvoidy, 1863b: 10.

TYPE SPECIES: Tachina curvicauda Fallén, 1820, automatic [by designation of the same species (by subsequent designation of Rondani, 1856: 75) for Uromyia Meigen, 1838].

CuRrent Status: Preoccupied by Stål, 1855; junior synonym of Phania Meigen, 1824 [teste Herting \& Dely-Draskovits (1993: 434)].

FAMILY: TACHINIDAE.

REMARKS: This genus-group name was proposed by Robineau-Desvoidy (1863b: 10) as a replacement name for Uromyia Meigen, 1838 [as "Uramyia"]; thinking it was preoccupied by Uramya Robineau-Desvoidy, 1830.

EMENDATIOns: Bohemannia Bezzi \& Stein, 1907: 749 (unjustified), n. syn. 
72. Boisduvalia Robineau-Desvoidy, 1830: 730.

ORIGINALLY INCLUDED SPECIES: Boisduvalia rutilans Robineau-Desvoidy, 1830; Boisduvalia testacea Robineau-Desvoidy, 1830; Boisduvalia nitida Robineau-Desvoidy, 1830; Boisduvalia cyanea RobineauDesvoidy, 1830; Boisduvalia tibialis Robineau-Desvoidy, 1830.

TYPE SPECIES: Boisduvalia rutilans Robineau-Desvoidy, 1830, by subsequent designation (Duponchel in d'Orbigny, 1842a: 637).

CURRENT STATUS: Valid genus [teste Evenhuis \& Thompson (1990: 233)].

FAMILY: PLATYSTOMATIDAE.

REMARKS: The family placement is provisional. Although Macquart (1835: 437) placed the type species rutilans in the genus Ortalis Fallén, 1810 [Ulidiidae], Loew (1873: 18) synonymized this genus-group name with Rivellia Robineau-Desvoidy, 1830. The genus has not been treated in the major regional Diptera catalogs and comprises five species: one Oriental, two Afrotropical, and two patria ignota. The genus-group name is not to be found in the platystomatid chapters of the Oriental catalog (Steyskal, 1977) or Afrotropical catalog (Steyskal, 1980). Hendel (1914b: 169) did treat the genus [in his "Platystominae"] and designated Boisduvalia rutilans Robineau-Desvoidy, 1830 as type species but this was later than the designation in d'Orbigny (1842a).

73. Bonellia Robineau-Desvoidy, 1830: 56.

ORIGINAlly InCludED SPECIES: Bonellia tessellans Robineau-Desvoidy, 1830; Bonellia lateralis RobineauDesvoidy, 1830; Bonellia rubiginosa Robineau-Desvoidy, 1830.

TYPE SPECIES: Bonellia tessellans Robineau-Desvoidy, 1830, by subsequent designation (Townsend, 1916: $6)$.

CURRENT STATUS: Preoccupied by Rolando, 1822; senior (but invalid) synonym of Bonellimyia Townsend, 1919 (subgenus of Linnaemya Robineau-Desvoidy, 1830) [teste Herting \& Dely-Draskovits (1993: 285)].

FAMILY: TACHINIDAE.

REMARKS: Robineau-Desvoidy (1863a: 134) designated "Tachina haemorrhoïdalis, Fall." as type species but this was not an originally included species.

74. Bonnetia Robineau-Desvoidy, 1830: 55.

ORIGINALly INCLUDED SPECIES: Bonnetia longipes Robineau-Desvoidy, 1830; Bonnetia oenanthis Robineau-Desvoidy, 1830.

TYPE SPECIES: Bonnetia oenanthis Robineau-Desvoidy, 1830 [= Tachina comta Fallén, 1810], by subsequent designation (Townsend, 1916: 6).

CURRENT STATUS: Junior synonym of Linnaemya Robineau-Desvoidy, 1830 sensu stricto [teste Herting \& Dely-Draskovits (1993: 284)].

FAMILY: TACHINIDAE.

75. Brachelia Robineau-Desvoidy, 1830: 61.

ORIGINALLY INCLUDED SPECIES: Brachelia westermanni Robineau-Desvoidy, 1830.

TYPE SPECIES: Brachelia westermanni Robineau-Desvoidy, 1830 [preoccupied by and = Tachina westermanni Wiedemann, 1819], by monotypy.

CURRENT StATUS: Valid genus [teste Crosskey (1980: 845)].

FAMILY: TACHINIDAE.

REMARKS: Robineau-Desvoidy (1830: 62) indicated that Latreille had labeled the specimen upon which the species was described as "Tachina Westermanni" (no doubt Wiedemann's 1819 name), but still described this as a new species. 
76. Bremia Robineau-Desvoidy, 1863a: 243.

ORIGINALLY INCLUDED SPECIES: Carcelia velox Robineau-Desvoidy, 1830.

TYPE SPECIES: Carcelia velox Robineau-Desvoidy, 1830, by original designation.

CURRENT STATUS: Preoccupied by Rondani, 1860; no replacement name proposed; placed in "Doubtful taxa in Tachinidae" by Herting \& Dely-Draskovits (1993: 435).

FAMILY: TACHINIDAE.

77. Brullaea Robineau-Desvoidy, 1863a: 773.

ORIGINALly INCLUDED SPECIES: Brullaea ocypteroidea Robineau-Desvoidy, 1863 (as "Brullaea ocypterö̈dea, R.-D.”).

TYPE SPECIES: Brullaea ocypteroidea Robineau-Desvoidy, 1863 (as “Brullaea ocypterö̈dea, R.-D.”), by monotypy.

CURRENT STATUS: Valid genus [teste Herting \& Dely-Draskovits (1993: 422)].

FAMILY: TACHINIDAE.

[Buquetia] Robineau-Desvoidy, 1846a: 107.

CURRENT STATUS: Unavailable name; proposed without description or included species; treated under Buquetia Robineau-Desvoidy, 1847 [teste this work].

FAMILY: TACHINIDAE.

78. Buquetia Robineau-Desvoidy, 1847: 286.

ORIGINALLY INCLUDED SPECIES: Buquetia musca Robineau-Desvoidy, 1847.

TYPE SPECIES: Buquetia musca Robineau-Desvoidy, 1847, by monotypy.

CURRENT STATUS: Valid genus [teste Herting \& Dely-Draskovits (1993: 191)].

FAMILY: TACHINIDAE.

79. Byomya Robineau-Desvoidy, 1830: 392.

ORIGINALLY INCLUDED SPECIES: Byomya carnifex Robineau-Desvoidy, 1830; Byomya violacea Robineau-

Desvoidy, 1830; Byomya stimulans Robineau-Desvoidy, 1830.

TYPE SPECIES: Byomya violacea Robineau-Desvoidy, 1830 [= Musca tempestiva Fallén, 1817], by subsequent designation (Townsend, 1915: 434).

CURRENT Status: Junior synonym of Musca Linnaeus, 1758 [teste Pont (1986b: 87)].

FAMILY: MUSCIDAE.

EMENDATIOns: Byomyia Agassiz, 1846b: 56 (unjustified); Biomyia Williston, 1908: 373 (unjustified), n. syn.

80. Caenis Robineau-Desvoidy, 1863a: 675.

ORIGINALLY INCLUDED SPECIES: Caenis prompta Robineau-Desvoidy, 1863; Tachina pullata Meigen, 1824.

TYPE SPECIES: Caenis prompta Robineau-Desvoidy, 1863 [= Erythrocera scutellaris Robineau-Desvoidy, 1848], by original designation.

CURRENT STATUS: Preoccupied by Stephens, 1833; junior synonym of Eurysthaea Robineau-Desvoidy, 1863

[teste Herting \& Dely-Draskovits (1993: 245)].

FAMILY: TACHINIDAE. 
[Calliphora] Robineau-Desvoidy MS name (Blainville et al., 1826: 11).

CURRENT STATUS: Unavailable name; suppressed by action of I.C.Z.N. (1990: 162 [Opinion 1601]); treated under Calliphora Robineau-Desvoidy, 1830 [teste this work].

FAMILY: CALLIPHORIDAE.

81. Calliphora Robineau-Desvoidy, 1830: 433.

ORIGINALLY INCLUDED SPECIES: Calliphora fulvibarbis Robineau-Desvoidy, 1830; Calliphora brunibarbis Robineau-Desvoidy, 1830; Musca vomitoria Linnaeus, 1758; Calliphora littoralis Robineau-Desvoidy, 1830; Calliphora vicina Robineau-Desvoidy, 1830; Calliphora spitzbergensis Robineau-Desvoidy, 1830; Calliphora musca Robineau-Desvoidy, 1830; Calliphora myoidea Robineau-Desvoidy, 1830 (as “Calliphora myoüdea. R. D.”); Calliphora monspeliaca Robineau-Desvoidy, 1830; Calliphora nana Robineau-Desvoidy, 1830; Calliphora aurulans Robineau-Desvoidy, 1830; Calliphora viridescens Robineau-Desvoidy, 1830; Calliphora villosa Robineau-Desvoidy, 1830; Calliphora peruviana Robineau-Desvoidy, 1830; Calliphora oceaniae Robineau-Desvoidy, 1830; Calliphora compressa Robineau-Desvoidy, 1830; Calliphora rostrata Robineau-Desvoidy, 1830.

TYPE SPECIES: Musca vomitoria Linnaeus, 1758, by original designation.

CURRENT STATUS: Valid genus [teste Rognes (1991: 59)].

FAMILY: CALLIPHORIDAE.

REMARKS: Type-species designation confirmed and Calliphora placed on the Official List of Generic Names in Zoology by action of I.C.Z.N. (1954: 75 [Opinion 274]).

[Calyptia] Robineau-Desvoidy, 1863b: 59.

CURRENT STATUS: Incorrect original spelling of Calyptidia Robineau-Desvoidy, 1863 [teste RobineauDesvoidy (1863b: 920)].

FAMILY: TACHINIDAE.

REMARKS: The spelling of Calyptia (page 59) was corrected to Calyptidia (page 920) in the errata of Robineau-Desvoidy (1863b).

82. Calyptia Robineau-Desvoidy, 1863b: 576.

ORIGINALly InCluded SPECIES: Calyptia carceli Robineau-Desvoidy, 1863; Calyptia fuliginosa RobineauDesvoidy, 1863.

TYPE SPECIES: Calyptia carceli Robineau-Desvoidy, 1863 [= Musca halterata Panzer, 1798], by subsequent designation (Townsend, 1916: 6).

CURRENT STATUS: Junior synonym of Nyctia Robineau-Desvoidy, 1830 [teste Pape (1996: 165)].

FAMILY: SARCOPHAGIDAE.

83. Calyptidia Robineau-Desvoidy, 1863b: 59 (920).

ORIGINALly InCluded SPECIES: Calyptidia occlusa Robineau-Desvoidy, 1863 (as "Calyptia occlusa, R.D.”).

TYPE SPECIES: Calyptidia occlusa Robineau-Desvoidy, 1863 (as “Calyptia occlusa, R.-D.”), by monotypy.

CURRENT StAtus: Junior synonym of Dufouria Robineau-Desvoidy, 1830 [teste Herting \& Dely-Draskovits (1993: 393)].

FAMILY: TACHINIDAE.

REMARKS: This is the correct spelling of Calyptia Robineau-Desvoidy (1863b: 59), the correction of which is in the errata on page 920. The description of the new genus and new species is on pages 59-60 under the incorrect original spelling "Calyptia". 
84. Camilla Robineau-Desvoidy, 1863b: 641.

ORIGINALLY INCLUDED SPECIES: Morellia aenescens Robineau-Desvoidy, 1830; Camilla fuscana Robineau-

Desvoidy, 1863; Camilla vivida Robineau-Desvoidy, 1863.

TYPE SPECIES: Morellia aenescens Robineau-Desvoidy, 1830, by subsequent designation (Coquillett, 1910: 517).

CuRRENT Status: Preoccupied by Haliday, 1837; junior synonym of Morellia Robineau-Desvoidy, 1830 [teste Pont (1986b: 94)].

FAMILY: MUSCIDAE.

[Carbonaria] Robineau-Desvoidy, 1863a: 808.

CURRENT STATUS: Incorrect original spelling of Carbonia Robineau-Desvoidy, 1863 [teste this work].

FAMILY: TACHINIDAE.

85. Carbonia Robineau-Desvoidy, 1863a: 808.

ORIGINALLY INCLUDED SPECIES: Carbonia impatiens Robineau-Desvoidy, 1863 (as "Carbonaria impatiens, R.-D.”).

TYPE SPECIES: Carbonia impatiens Robineau-Desvoidy, 1863 [= Ocyptera costata Fallén, 1815], by original designation (as “Carbonaria impatiens, R.-D.”).

CURRENT StATUs: Junior synonym of Wagneria Robineau-Desvoidy, 1830 [teste Herting \& Dely-Draskovits (1993: 380)].

FAMILY: TACHINIDAE.

REMARKS: Robineau-Desvoidy (1863a) used two different spellings when proposing this genus: Carbonia and Carbonaria (both on page 808). Acting as First Reviser, we select Carbonia as the correct original spelling.

\section{Carcelia Robineau-Desvoidy, 1830: 176.}

ORIGINAlly InCluded SPECIES: Carcelia lepida Robineau-Desvoidy, 1830; Carcelia bombylans RobineauDesvoidy, 1830; Carcelia festiva Robineau-Desvoidy, 1830; Musca puparum Fabricius, 1794; Carcelia vernalis Robineau-Desvoidy, 1830; Carcelia macroura Robineau-Desvoidy, 1830; Carcelia amoena Robineau-Desvoidy, 1830; Carcelia rubrella Robineau-Desvoidy, 1830; Carcelia distincta RobineauDesvoidy, 1830; Carcelia duponcheli Robineau-Desvoidy, 1830; Carcelia nigripes Robineau-Desvoidy, 1830; Carcelia plusiae Robineau-Desvoidy, 1830; Carcelia rapida Robineau-Desvoidy, 1830; Carcelia diversa Robineau-Desvoidy, 1830; Carcelia bombycivora Robineau-Desvoidy, 1830; Carcelia scutellaris Robineau-Desvoidy, 1830; Carcelia grisea Robineau-Desvoidy, 1830; Carcelia fuscipennis Robineau-Desvoidy, 1830; Carcelia aurifrons Robineau-Desvoidy, 1830; Carcelia velox RobineauDesvoidy, 1830; Carcelia flavescens Robineau-Desvoidy, 1830.

TYPE SPECIES: Carcelia bombylans Robineau-Desvoidy, 1830, by subsequent designation (Coquillett, 1910: 518).

CURRENT STATUS: Valid genus and subgenus [teste Herting \& Dely-Draskovits (1993: 212)].

FAMILY: TACHINIDAE.

REMARKS: Coquillett (1910: 518) stated that Robineau-Desvoidy (1863a: 220) designated "Tachina gnava MEIGEN (as bombylans, new species)". This is incorrect. Robineau-Desvoidy (1863a: 220) designated "Tachina gnava, Meig.", which was not one of the originally included species, and no included species in Robineau-Desvoidy (1830) were put into synonymy with it. Ironically, although specifically designating Tachina gnava Meigen as the type species of Carcelia, Robineau-Desvoidy (1863a: 239) clearly removed it altogether from the genus by stating under Carcelia bombylans: "C'est à tort que les diptèrologistes français l'ont rapportée au Tachina gnava de Meigen, qui n'appartient même pas à cette 
section, ainsi que je m'en suis assurê". Monceaux, in putting together the manuscript notes of the late Robineau-Desvoidy to produce the two-volume posthumous work, must have made an error in the species Robineau-Desvoidy actually intended for this genus. But in any case, by mentioning the designation of Carcelia bombylans (an originally included species) as the type, Coquillett (1910) is the earliest designation of an originally included species. Townsend (1916: 6) also designated Carcelia bombylans, but this was later.

87. Caricea Robineau-Desvoidy, 1830: 530.

ORIGINALLY INCLUDED SPECIES: Caricea femoralis Robineau-Desvoidy, 1830; Caricea communis RobineauDesvoidy, 1830; Caricea plumosula Robineau-Desvoidy, 1830; Caricea vulgaris Robineau-Desvoidy, 1830; Caricea analis Robineau-Desvoidy, 1830; Caricea paludosa Robineau-Desvoidy, 1830; Caricea vittata Robineau-Desvoidy, 1830; Caricea fusca Robineau-Desvoidy, 1830; Caricea littoralis RobineauDesvoidy, 1830; Caricea limpidipennis Robineau-Desvoidy, 1830; Caricea riparia Robineau-Desvoidy, 1830; Caricea pallipes Robineau-Desvoidy, 1830; Caricea tristis Robineau-Desvoidy, 1830; Caricea distincta Robineau-Desvoidy, 1830; Caricea lappae Robineau-Desvoidy, 1830; Caricea erythrocera Robineau-Desvoidy, 1830; Caricea tarsalis Robineau-Desvoidy, 1830; Caricea leucophaea RobineauDesvoidy, 1830.

TYPE SPECIES: Caricea communis Robineau-Desvoidy, 1830 [= Musca tigrina Fabricius, 1794], by subsequent designation (Duponchel in d'Orbigny, 1842b: 172).

CURRENT Status: Junior synonym of Coenosia Meigen, 1826 [teste Pont (1989: 695)].

FAMILY: MUSCIDAE.

REMARKS: Later type-species designations were by Rondani (1866: 76, 208) of Musca tigrina Fabricius, 1775 (but this was not one of the originally included species); Coquillett (1901: 135) of Caricea vulgaris Robineau-Desvoidy, 1830; Stein (1908: 11) of Caricea erythrocera Robineau-Desvoidy, 1830; Schnabl \& Dziedzicki (1911: 75) of Caricea communis Robineau-Desvoidy, 1830.

\section{Catilia Robineau-Desvoidy, 1830: 310.}

ORIGINALLY INCLUDED SPECIES: Catilia nitida Robineau-Desvoidy, 1830.

TYPE SPECIES: Catilia nitida Robineau-Desvoidy, 1830 [= Musca tigrina Fabricius, 1794], by monotypy. CURRENT STATUS: Placed in "Doubtful taxa in Tachinidae" by Herting \& Dely-Draskovits (1993: 435).

FAMILY: TACHINIDAE.

89. Celea Robineau-Desvoidy, 1863a: 273.

ORIGINALLY INCLUDED SPECIES: Phryxe flavipalpis Robineau-Desvoidy, 1830.

TYPE SPECIES: Phryxe flavipalpis Robineau-Desvoidy, 1830 [= Tachina affinis Fallén, 1810], by original designation.

CURRENT STATUS: Junior synonym of Hubneria Robineau-Desvoidy, 1848 [teste Herting \& Dely-Draskovits (1993: 211)].

FAMILY: TACHINIDAE.

90. Cephalemya Robineau-Desvoidy, 1830: 26.

CURRENT StATUS: Unjustified emendation of Cephalemyia Latreille, 1818; junior synonym of Oestrus Linnaeus, 1758, n. syn.

FAMILY: OESTRIDAE.

REMARKS: Made available as an emendation by virtue of similar spelling changes for two or more names from "myia to "mya" in the same work. 
91. Cephenemya Robineau-Desvoidy, 1830: 26.

CURRENT StATUS: Unjustified emendation of Cephenemyia Latreille, 1818; junior synonym of Cephenemyia Latreille, 1818, n. syn.

FAMILY: OESTRIDAE.

REMARKS: Made available as an emendation by virtue of similar spelling changes for two or more names from "myia to "mya" in the same work.

92. Cephysa Robineau-Desvoidy, 1863b: 677.

ORIGINALLY INCLUDED SPECIES: Cephysa muscidea Robineau-Desvoidy, 1863.

TYPE SPECIES: Cephysa muscidea Robineau-Desvoidy, 1863, by monotypy.

CURRENT Status: Junior synonym of Pollenia Robineau-Desvoidy, 1830 [teste Rognes (1991: 211)].

FAMILY: CALLIPHORIDAE.

\section{Ceranthia Robineau-Desvoidy, 1830: 88.}

ORIGINALLY INCLUDED SPECIES: Ceranthia fulvipes Robineau-Desvoidy, 1830; Ceranthia podacina Robineau-Desvoidy, 1830.

TYPE SPECIES: Ceranthia fulvipes Robineau-Desvoidy, 1830 [= Ceromya abdominalis Robineau-Desvoidy, 1830], by subsequent designation (Robineau-Desvoidy, 1863a: 685).

CURRENT Status: Valid subgenus of Siphona Meigen, 1803 [teste Tachi \& Shima (2005: 189)].

FAMILY: TACHINIDAE.

REMARKS: Coquillett (1910: 519) designated the same type species but this was later.

94. Ceromya Robineau-Desvoidy, 1830: 86.

ORIGINALly INCLUDED SPECIES: Ceromya erythrocera Robineau-Desvoidy, 1830; Ceromya abdominalis Robineau-Desvoidy, 1830; Ceromya rubrifrons Robineau-Desvoidy, 1830; Ceromya testacea RobineauDesvoidy, 1830; Ceromya microcera Robineau-Desvoidy, 1830.

TYPE SPECIES: Ceromya testacea Robineau-Desvoidy, 1830 [= Tachina bicolor Meigen, 1824], by subsequent designation (Coquillett, 1910: 520).

CURRENT STAtus: Valid genus [teste Herting \& Dely-Draskovits (1993: 327)].

FAMILY: TACHINIDAE.

EMENDATIONS: Ceromyia Agassiz, 1846a: 7 (unjustified).

95. Cerophora Robineau-Desvoidy, 1863a: 700.

ORIGINALLY INCLUDED SPECIES: Cerophora funesta Robineau-Desvoidy, 1863.

TYPE SPECIES: Cerophora funesta Robineau-Desvoidy, 1863 [= Tachina delecta Meigen, 1824], by original designation.

CURRENT STATUS: Preoccupied by d'Orbigny, 1836; junior synonym of Eloceria Robineau-Desvoidy, 1863 [teste Herting \& Dely-Draskovits (1993: 308)].

FAMILY: TACHINIDAE.

[Chariclaea] Robineau-Desvoidy, 1863a: 558.

CURRENT STATUS: Incorrect original spelling of Chariclea Robineau-Desvoidy, 1863a [teste Verrall in Scudder (1882: 67)].

FAMILY: TACHINIDAE. 
96. Chariclea Robineau-Desvoidy, 1863a: 557.

ORIGINALLY INCLUDED SPECIES: Chariclea coxalis Robineau-Desvoidy, 1863 (as "Chariclaea coxalis, R.D.").

TYPE SPECIES: Chariclea coxalis Robineau-Desvoidy, 1863 [= Tachina frontosa Meigen, 1824], by original designation (as “Chariclaea coxalis, R.-D.”).

CURRENT STATUs: Preoccupied by Curtis, 1825; Stephens, 1829; junior synonym of Bothria Rondani, 1856 [teste Herting \& Dely-Draskovits (1993: 237)].

FAMILY: TACHINIDAE.

REMARKS: There are two original spellings of this genus-group name in Robineau-Desvoidy (1863a): Chariclea (page 557) and Chariclaea (page 558). Acting as First Reviser, Verrall in Scudder (1882: 67) selected Chariclea as the correct original spelling.

\section{Chetocera Robineau-Desvoidy, 1830: 697.}

ORIGINALLY INCLUDED SPECIES: Chetocera claripennis Robineau-Desvoidy, 1830.

TYPE SPECIES: Chetocera claripennis Robineau-Desvoidy, 1830 [= Sciomyza albocostata Fallén, 1820], by monotypy.

CuRRENT StAtus: Valid subgenus of Pherbellia Robineau-Desvoidy, 1830 [teste Rozkošný \& Elberg (1984: 170)].

FAMILY: SCIOMYZIDAE.

EMENDATIONS: Chaetocera Agassiz, 1846b: 73, 80 (unjustified).

\section{Chione Robineau-Desvoidy, 1830: 679.}

ORIGINALLY INCLUDED SPECIES: Chione sepedonoidea Robineau-Desvoidy, 1830 (as "Chione sepedonoïdea. R. D."); Chione communis Robineau-Desvoidy, 1830; Chione ichneumonea Robineau-Desvoidy, 1830.

TYPE SPECIES: Chione sepedonoidea Robineau-Desvoidy, 1830 [= Musca albiseta Scopoli, 1763], by subsequent designation (Rozkošný \& Elberg, 1984: 184).

CURRENT STATUS: Preoccupied by Megerle, 1811; senior (but invalid) synonym of Knutsonia Verbeke, 1964 (subgenus of Ilione Haliday, 1837) [teste Steyskal et al. (2004: 563)].

FAMILY: SCIOMYZIDAE.

99. Chloe Robineau-Desvoidy, 1830: 560 (as Chloë).

ORIGINALly INCLUDED SPECIES: Chloe silvicola Robineau-Desvoidy, 1830 (as “Chloë silvicola. R. D.”).

TYPE SPECIES: Chloe silvicola Robineau-Desvoidy, 1830 (as “Chloë silvicola. R. D.”), by monotypy.

CURRENT Status: Preoccupied by Berthold, 1827; junior synonym of Hydrophoria Robineau-Desvoidy, 1830 [teste Griffiths (1998: 1884)].

FAMILY: ANTHOMYIIDAE.

REMARKS: Evenhuis \& Thompson (1990: 245) treated Chloe as questionably included in Anthomyiidae and Bisby et al. (2008) listed it as a junior synonym of Delia Robineau-Desvoidy, 1830. The only originally included nominal species, Chloe silvicola, was first interpreted by Barták et al. (1990: 443), who considered it as conspecific with Hydrophoria annulata auctt., nec Pandellé, 1899, and the name Hydrophoria silvicola (Robineau-Desvoidy, 1830) has subsequently been in use for that species. DelyDraskovits (1993) did not include Chloe and mistakenly referred to the sole included species as Hylemya silvicola Robineau-Desvoidy, 1830: 551 [a junior synonym of Hylemya nigrimana (Meigen, 1826)], rather than to Chloe silvicola Robineau-Desvoidy, 1830: 561. This mistake was corrected by Griffiths (1998: 1884), who also formally established the synonymy of Chloe with Hydrophoria. 
100. Chlorina Robineau-Desvoidy, 1830: 602.

ORIGINALLY INCLUDED SPECIES: Chlorina thoracica Robineau-Desvoidy, 1830; Chlorina phyllioidea Robineau-Desvoidy, 1830 (as "Chlorina phyllioïdea. R. D.”).

TYPE SPECIES: Chlorina thoracica Robineau-Desvoidy, 1830 [= Anthomyia solennis Meigen, 1826], by subsequent designation (Coquillett, 1910: 522).

CURRent Status: Junior synonym of Pegomya Robineau-Desvoidy, 1830 [teste Dely-Draskovits (1993: 76)].

FAMILY: ANTHOMYIIDAE.

101. Chlorophora Robineau-Desvoidy, 1830: 733.

ORIGINALLY INCLUDED SPECIES: Chlorophora liturata Robineau-Desvoidy, 1830.

TYPE SPECIES: Chlorophora liturata Robineau-Desvoidy, 1830, by monotypy.

CURRENT STATUS: Placed in "Unplaced Genera" by Steyskal (1968a: 13).

FAMILY: RICHARDIIDAE.

REMARKS: According to Hendel (1911a: 369, 1911b: 31), Chlorophora Robineau-Desvoidy, 1830 may be synonymous with Odontomera Macquart, 1843. If so, then Chlorophora would have priority and might upset stability of the more commonly used genus-group name Odontomera.

102. Chremia Robineau-Desvoidy, 1863b: 335.

ORIGINALly INCLUDED SPECIES: Chremia ciligera Robineau-Desvoidy, 1863.

TYPE SPECIES: Chremia ciligera Robineau-Desvoidy, 1863, by monotypy.

CURRENT STATUS: Placed in "Doubtful taxa in Tachinidae" by Herting \& Dely-Draskovits (1993: 435).

FAMILY: TACHINIDAE.

REMARKS: The type species is misspelled as Chremia diligera by Herting \& Dely-Draskovits (1993: 435).

103. Chryseria Robineau-Desvoidy, 1863b: 288.

ORIGINAlly InCluded SPECIES: Clytia cylindrica Robineau-Desvoidy, 1830; Clytia gentilis RobineauDesvoidy, 1830.

TYPE SPECIES: Clytia gentilis Robineau-Desvoidy, 1830 [= Musca helluo Fabricius, 1805], by subsequent designation (Coquillett, 1910: 523).

CURRENT STATUS: Junior synonym of Eliozeta Rondani, 1856 [teste Herting \& Dely-Draskovits (1993: 400)].

FAMILY: TACHINIDAE.

[Chrysomya] Robineau-Desvoidy MS name (Blainville et al., 1826: 11).

CURRENT STATUS: Unavailable name; suppressed by action of I.C.Z.N. (1990: 162 [Opinion 1601]); treated under Chrysomya Robineau-Desvoidy, 1830 [teste this work].

FAMILY: CALLIPHORIDAE.

104. Chrysomya Robineau-Desvoidy, 1830: 444.

ORIGINALly INCLUDED SPECIES: Chrysomya idioidea Robineau-Desvoidy, 1830 (as "Chrysomya Idioïdea. R. D.”); Chrysomya viridula Robineau-Desvoidy, 1830; Chrysomya affinis Robineau-Desvoidy, 1830; Chrysomya fulvicrura Robineau-Desvoidy, 1830; Chrysomya tibialis Robineau-Desvoidy, 1830; Chrysomya lherminieri Robineau-Desvoidy, 1830; Chrysomya alia Robineau-Desvoidy, 1830; Chrysomya coerulescens Robineau-Desvoidy, 1830; Chrysomya socia Robineau-Desvoidy, 1830; Chrysomya decora Robineau-Desvoidy, 1830; Chrysomya plaei Robineau-Desvoidy, 1830; Chrysomya 
lepida Robineau-Desvoidy, 1830; Chrysomya buccalis Robineau-Desvoidy, 1830; Chrysomya regalis Robineau-Desvoidy, 1830; Chrysomya dejeanii Robineau-Desvoidy, 1830; Chrysomya hyacinthina Robineau-Desvoidy, 1830; Chrysomya lalandii Robineau-Desvoidy, 1830; Chrysomya soror RobineauDesvoidy, 1830; Chrysomya duvaucelii Robineau-Desvoidy, 1830; Chrysomya gratiosa RobineauDesvoidy, 1830; Chrysomya brunicornis Robineau-Desvoidy, 1830; Chrysomya capensis RobineauDesvoidy, 1830.

TYPE SPECIES: Chrysomya regalis Robineau-Desvoidy, 1830 [= Musca marginalis Wiedemann, 1830], by subsequent designation (Coquillett, 1910: 523).

CURRENT STATUS: Valid genus [teste Rognes (2002: 11)].

FAMILY: CALLIPHORIDAE.

REMARKS: The I.C.Z.N. (1988b: 236 [Opinion 1507]) put the name Musca marginalis Wiedemann, 1830 on the Official List of Specific Names in Zoology and the name Chrysomya regalis Robineau-Desvoidy, 1830, which at that time was in general use as the valid name for this taxon (e.g., Pont 1980b: 789, Schumann 1986: 40) due to the preoccupation of Musca marginalis Wiedemann, 1830 by Geoffroy [often as Fourcroy], 1785 and Fallén, 1824 was put on the Official Index of Rejected and Invalid Species Names in Zoology. Musca marginalis Wiedemann, 1830 (as "Marginalis del Fabricius") was designated as the type of Chrysomya by Rondani (1863: 27), but this designation is invalid as it was of a species that was not originally included.

Emendations: Chrysomyia Macquart, 1835: 251 (unjustified), n. syn.; Chrysomyia Agassiz, 1846b: 85 (unjustified).

105. Chyromya Robineau-Desvoidy, 1830: 620.

ORIGINALLY INCLUDED SPECIES: Chyromya fenestrarum Robineau-Desvoidy, 1830.

TYPE SPECIES: Chyromya fenestrarum Robineau-Desvoidy, 1830 [= Musca flava Linnaeus, 1758], by monotypy.

CURRENT STATUS: Valid genus [teste Sabrosky (1999: 89)].

FAMILY: CHYROMYIDAE.

REMARKS: Placed on the Official List of Generic Names in Zoology by action of I.C.Z.N. (1968: 16 [Opinion 847]).

Emendations: Chiromyia Agassiz, 1846b: 81, 85 (unjustified); Chyromyia Schiner, 1864: 55 (unjustified), n. syn.

106. Clairvillia Robineau-Desvoidy, 1830: 234.

ORIGINALLY INCLUDED SPECIES: Ocyptera pusilla Meigen, 1824 (with Ocyptera cylindrica Fallén, 1815 [as “Ocyptera cylindrica. Fall. Ocyptera pusilla. Meig."] in synonymy).

TYPE SPECIES: Tachina biguttata Meigen, 1824, by fixation of O'Hara \& Wood (2004: 223) under ICZN Code Article 70.3.2, misidentified as Ocyptera pusilla Meigen, 1824 in the fixation by monotypy of Robineau-Desvoidy (1830: 234).

CURRENT Status: Valid genus [teste Herting \& Dely-Draskovits (1993: 421)].

FAMILY: TACHINIDAE.

107. Clelia Robineau-Desvoidy, 1830: 255.

ORIGINALly INCLUDED SPECIES: Clelia agilis Robineau-Desvoidy, 1830; Clelia rapida Robineau-Desvoidy, 1830; Clelia minor Robineau-Desvoidy, 1830; Clelia erythrocera Robineau-Desvoidy, 1830.

TYPE SPECIES: Clelia agilis Robineau-Desvoidy, 1830 [= Tachina tetraptera Meigen, 1824], by subsequent designation (Duponchel in d'Orbigny, 1843: 4).

CuRrent Status: Preoccupied by Fitzinger, 1826; junior synonym of Leucostoma Meigen, 1803 [teste Herting \& Dely-Draskovits (1993: 419)]. 
FAMILY: TACHINIDAE.

REMARKS: Robineau-Desvoidy (1863b: 62) designated Clelia agilis Robineau-Desvoidy, 1830 (in synonymy with Tachina tetraptera Meigen, 1824), but this was later.

EMENDATIONS: Cloelia Agassiz, 1846b: 90 (unjustified).

108. Clemelis Robineau-Desvoidy, 1863a: 481.

ORIGINALly INCLUDED SPECIES: Clemelis aurea Robineau-Desvoidy, 1863; Zenillia ciligera RobineauDesvoidy, 1830; Clemelis albifrons Robineau-Desvoidy, 1863; Clemelis flavifrons Robineau-Desvoidy, 1863; Clemelis cineraria Robineau-Desvoidy, 1863; Clemelis fuscifrons Robineau-Desvoidy, 1863.

TYPE SPECIES: Zenillia ciligera Robineau-Desvoidy, 1830 [= Tachina pullata Meigen, 1824], by original designation.

CURRENT Status: Valid genus [teste Herting \& Dely-Draskovits (1993: 231)].

FAMILY: TACHINIDAE.

109. Cleodora Robineau-Desvoidy, 1863a: 1047.

ORIGINALLY INCLUDED SPECIES: Cleodora ancilla Robineau-Desvoidy, 1863.

TYPE SPECIES: Cleodora ancilla Robineau-Desvoidy, 1863 [= Tachina rustica Fallén, 1810], by original designation.

CuRRent STAtus: Preoccupied by Péron \& Lesueur, 1810; Stephens, 1834; Mulsant, 1850; junior synonym of Adenia Robineau-Desvoidy, 1863 (subgenus of Exorista Meigen, 1803) [teste Herting (1984: 8)].

FAMILY: TACHINIDAE.

110. Cleonice Robineau-Desvoidy, 1863a: 1097.

ORIGINALLY INCLUDED SPECIES: Tachina grisea Fallén, 1810 (with “Tachina egena : Meig." in synonymy).

TYPE SPECIES: Tachina callida Meigen, 1824, by fixation of O'Hara \& Wood (2004: 334) under ICZN Code

Article 70.3.2, misidentified as Tachina grisea Fallén, 1810 in the original designation by RobineauDesvoidy (1863a: 1099).

CURRENT STATUS: Valid genus [teste O'Hara \& Wood (2004: 334)].

FAMILY: TACHINIDAE.

111. Clidonia Robineau-Desvoidy, 1830: 732.

ORIGINALly InCLUDED SPECIES: Clidonia basalis Robineau-Desvoidy, 1830.

TYPE SPECIES: Clidonia basalis Robineau-Desvoidy, 1830, by monotypy.

CURRENT StATUS: Valid genus [teste Evenhuis et al. (2009)].

FAMILY: UNPLACED ACALYPTRATAE.

REMARKS: No one has treated this genus-group name or its type species since Loew (1873:18), who gave the following remarks with regard to genera in "Ortalidae": "Clidonia is considered by the author himself to belong in a different family, in which we will not contradict him".

\section{Clytho Robineau-Desvoidy, 1830: 375.}

ORIGINAlly INCLUDED SPECIES: Clytho aurulenta Robineau-Desvoidy, 1830; Clytho argentea RobineauDesvoidy, 1830.

TYPE SPECIES: Clytho aurulenta Robineau-Desvoidy, 1830, by subsequent designation (Townsend, 1916: 6).

CURRENT STATUS: Treated as questionably included in Rhinophoridae [teste Evenhuis \& Thompson (1990: 252)]. 


\section{FAMILY: RHINOPHORIDAE.}

REMARKS: Macquart (1835: 232) placed Clytho Robineau-Desvoidy, 1830 in synonymy with Agria in the Sarcophagidae. Townsend (1939: 266) indicated it was like Halydaia [as "Halidaya"] Egger, 1856 [Tachinidae], which is where Bezzi \& Stein (1907: 463) treated it, but Herting \& Dely-Draskovits (1993: 390) indicated Bezzi \& Stein's (1907) use was in error. Neither the genus-group name nor its two included species are found in the Palaearctic catalogs of Rhinophoridae (Herting, 1993) or Tachinidae (Herting \& Dely-Draskovits, 1993); nor the world catalog of Sarcophagidae (Pape, 1996).

113. Clytia Robineau-Desvoidy, 1830: 287.

ORIGINALly INCluded SPECIES: Clytia dalmatica Robineau-Desvoidy, 1830; Clytia atra RobineauDesvoidy, 1830; Clytia cylindrica Robineau-Desvoidy, 1830; Clytia gentilis Robineau-Desvoidy, 1830; Musca continua Panzer, 1798 (as “Musca, Phasia continua. Fabr. Panz. Meig."); Clytia pratensis Robineau-Desvoidy, 1830; Clytia vaga Robineau-Desvoidy, 1830.

TYPe SPECIES: Musca continua Panzer, 1798 (as "T. continua F. Panz."), by subsequent designation (Westwood, 1840: 139).

CURRENT STATUS: Preoccupied by Lamouroux, 1812; senior (but invalid) synonym of Clytiomyia Rondani, 1861 [teste Herting \& Dely-Draskovits (1993: 401)].

FAMILY: TACHINIDAE.

REMARKS: An earlier type-species designation by Macquart (1837: 189) of Tachina pellucens Fallén, 1820 (as "Tachina pellucens, Meigen") is invalid as it was not originally included species.

114. Cnossia Robineau-Desvoidy, 1863a: 289.

ORIGINALLY INCLUDED SPECIES: Cnossia luteipalpis Robineau-Desvoidy, 1863.

TYPE SPECIES: Cnossia luteipalpis Robineau-Desvoidy, 1863 [= Tachina affinis Fallén, 1810], by original designation.

CURRENT StATUS: Junior synonym of Hubneria Robineau-Desvoidy, 1848 [teste Herting \& Dely-Draskovits (1993: 211)]

FAMILY: TACHINIDAE.

115. Coenia Robineau-Desvoidy, 1830: 800.

ORIGINALLY INCLUDED SPECIES: Coenia caricicola Robineau-Desvoidy, 1830.

TYPE SPECIES: Coenia caricicola Robineau-Desvoidy, 1830 [= Ephydra palustris Fallén, 1823], by monotypy.

CURRENT STATUS: Valid genus [teste Mathis \& Zatwarnicki (1995: 237)].

FAMILY: EPHYDRIDAE.

116. Conomya Robineau-Desvoidy, 1830: 384.

ORIGINALly InCludED SPECIES: Conomya tibialis Robineau-Desvoidy, 1830.

TYPE SPECIES: Conomya tibialis Robineau-Desvoidy, 1830, by monotypy.

CURRENT STATUS: Treated as incertae sedis in Sarcophaginae [teste Pape (1996: 442)].

FAMILY: SARCOPHAGIDAE.

EMENDATIONS: Conomyia Agassiz, 1846b: 97 (unjustified).

117. Coprina Robineau-Desvoidy, 1830: 810.

ORIGINALly INCLUDED SPECIES: Coprina bovina Robineau-Desvoidy, 1830. 
TYPE SPECIES: Coprina bovina Robineau-Desvoidy, 1830, by monotypy.

CURRENT STATUS: Placed in "Doubtful genera of Sphaeroceridae" [teste Roháček in Roháček (2001: 295)].

FAMILY: SPHAEROCERIDAE.

118. Cosmina Robineau-Desvoidy, 1830: 423.

ORIGINALly INCLUDED SPECIES: Cosmina fuscipennis Robineau-Desvoidy, 1830; Cosmina claripennis Robineau-Desvoidy, 1830; Cosmina arabica Robineau-Desvoidy, 1830.

TYPE SPECIES: Cosmina fuscipennis Robineau-Desvoidy, 1830, by subsequent designation (Townsend, 1916: 6).

CURRENT STATUS: Valid genus [teste Rognes (2002: 20)].

FAMILY: RHINIIDAE.

REMARKS: An earlier designation of the same type species by Brauer \& Bergenstamm (1893: 89) was considered invalid by the I.C.Z.N. (1956 [Direction 32]) as the designation given was not explicitly stated to be for the genus Cosmina.

119. Crameria Robineau-Desvoidy, 1830: 59.

ORIGINALly InCludED SPECIES: Crameria oestroidea Robineau-Desvoidy, 1830 (as "Crameria oestroïdea. R. D.”).

TYPE SPECIES: Crameria oestroidea Robineau-Desvoidy, 1830 (as “Crameria oeströ̈dea. R. D.”) [= Musca conspersus Harris, 1776], by monotypy.

CuRREnT Status: Preoccupied by Hübner, 1819; junior synonym of Trixa Meigen, 1824 [teste Herting \& Dely-Draskovits (1993: 355)].

FAMILY: TACHINIDAE.

120. Cuculla Robineau-Desvoidy, 1830: 523.

ORIGINAlly INCLUdED SPECIES: Cuculla cinerea Robineau-Desvoidy, 1830; Cuculla grisea RobineauDesvoidy, 1830; Cuculla palustris Robineau-Desvoidy, 1830.

TYPE SPECIES: Cuculla cinerea Robineau-Desvoidy, 1830 [= Musca triangula Fallén, 1825], by subsequent designation (Séguy, 1937: 253).

CURRENT STATUS: Preoccupied by Seba, 1761; junior synonym of Limnophora Robineau-Desvoidy, 1830 [teste Pont (1986b: 178)].

FAMILY: MUSCIDAE.

121. Curtisia Robineau-Desvoidy, 1849a: 440.

ORIGINALLY INCLUDED SPECIES: Curtisia regula Robineau-Desvoidy, 1849.

TYPE SPECIES: Curtisia regula Robineau-Desvoidy, 1849 [= Phryno nigripes Robineau-Desvoidy, 1830], by monotypy.

CURRENT StATUS: Junior synonym of Erythrocera Robineau-Desvoidy, 1849 [teste Herting \& DelyDraskovits (1993: 242)].

FAMILY: TACHINIDAE.

122. Cylidria Robineau-Desvoidy, 1830: 678.

ORIGINALLY INCLUDED SPECIES: Cylidria femorata Robineau-Desvoidy, 1830.

TYPE SPECIES: Cylidria femorata Robineau-Desvoidy, 1830, by monotypy.

CURRENT STATUS: Nomen dubium in Sciomyzidae [teste this work]. 
FAMILY: SCIOMYZIDAE.

REMARKS: The genus-group name and its included species have not been treated in any of the recent major regional Diptera catalogs. Robineau-Desvoidy (1830: 678) placed the genus between Sepedon Latreille and Chione Robineau-Desvoidy, and Schiner (1862: 61) placed the genus-group name under Limnia (all currently in Sciomyzidae).

EMENDATIONS: Cylindria Hendel, 1900: 323 (unjustified), n. syn.

[Cymnodia] Robineau-Desvoidy, 1863b: 883.

CURRENT STATUS: Incorrect original spelling of Gymnodia Robineau-Desvoidy, 1863 [teste this work].

FAMILY: MUSCIDAE.

123. Cynisca Robineau-Desvoidy, 1863a: 200.

ORIGINALLY INCLUDED SPECIES: Tachina arvicola Meigen, 1824.

TYPE SPECIES: Tachina arvicola Meigen, 1824 [= Tachina aemula Meigen, 1824], by original designation.

CURRENT StATUS: Preoccupied by Gray, 1844; Adams, 1854; junior synonym of Ethilla Robineau-Desvoidy,

1863 [teste Herting \& Dely-Draskovits (1993: 175)].

FAMILY: TACHINIDAE.

124. Cynomya Robineau-Desvoidy, 1830: 363.

ORIGINALLY INCLUDED SPECIES: Musca mortuorum Linnaeus, 1761; Musca chrysocephala De Geer, 1776;

Cynomya cadaverina Robineau-Desvoidy, 1830; Cynomya oceaniae Robineau-Desvoidy, 1830.

TYPE SPECIES: Musca mortuorum Linnaeus, 1761, by subsequent designation (Macquart, 1834b: 176).

CURRENT STATUS: Valid genus [teste Rognes (1991: 92)].

FAMILY: CALLIPHORIDAE.

REMARKS: Type designations by Westwood (1840: 141), Duponchel in d'Orbigny (1844a: 542), Rondani (1856: 89), and Brauer (1893: 500) are of the same nominal species but were later.

EMENDATIONS: Cynomyia Macquart, 1835: 232 (unjustified).

125. Cynthia Robineau-Desvoidy, 1863a: 769.

ORIGINALLY INCLUDED SPECIES: Cynthia pudica Robineau-Desvoidy, 1863.

TYPE SPECIES: Cynthia pudica Robineau-Desvoidy, 1863 [= Tachina spreta Meigen, 1824], by original designation.

CuRRENT STATUS: Preoccupied by Fabricius, 1807; Savigny, 1816; Thompson, 1829; junior synonym of Bithia Robineau-Desvoidy, 1863 [teste Herting \& Dely-Draskovits (1993: 338)].

FAMILY: TACHINIDAE.

126. Cyrillia Robineau-Desvoidy, 1863b: 31.

ORIGINALLY INCLUDED SPECIES: Blondelia fasciata Robineau-Desvoidy, 1830.

TYPE SPECIES: Blondelia fasciata Robineau-Desvoidy, 1830, by original designation.

CURRENT STATUS: Placed in "Doubtful taxa in Tachinidae" by Herting \& Dely-Draskovits (1993: 436).

FAMILY: TACHINIDAE.

127. Cytoria Robineau-Desvoidy, 1863a: 800.

ORIGINALly INCLUDED SPECIES: Nyctia servillei Robineau-Desvoidy, 1830; Cytoria monticola RobineauDesvoidy, 1863. 
TYPE SPECIES: Nyctia servillei Robineau-Desvoidy, 1830 [= Musca halterata Panzer, 1798], by subsequent designation (Townsend, 1916: 6).

CURRENT STATUS: Junior synonym of Nyctia Robineau-Desvoidy, 1830 [teste Pape (1996: 165)].

FAMILY: SARCOPHAGIDAE.

128. Cyzenis Robineau-Desvoidy, 1863a: 544.

ORIGINALly INCLUDED SPECIES: Cyzenis haemisphaerica Robineau-Desvoidy, 1863; Cyzenis vernalis Robineau-Desvoidy, 1863.

TYPE SPECIES: Cyzenis haemisphaerica Robineau-Desvoidy, 1863 [= Tachina albicans Fallén, 1810], by original designation.

CURRENT STATUS: Valid genus [teste Herting \& Dely-Draskovits (1993: 236)].

FAMILY: TACHINIDAE.

REMARKS: Robineau-Desvoidy (1863a: 545) listed "Phryno haemisphoerica : Rob. Desv." and "Eurigaster haemisphaerica : Rob. Desv." below the heading "Cyzenis haemisphaerica, R.-D." He removed the former from synonymy in the errata of the same work (Robineau-Desvoidy, 1863b: 918), writing "[page] 545, [lignes] 8 et 9, Phryno hoemisphoerica, supprimer ces deux lignes". Although this obstensibly left "Eurigaster haemisphaerica" in synonymy with Cyzenis haemisphaerica, Robineau-Desvoidy clearly intended this name to be deleted as well. "Eurigaster haemisphaerica" of Robineau-Desvoidy (1863a: 545 ) is a reference to an earlier work (Robineau-Desvoidy, 1848: 436) wherein this name is used as a changed combination for Phryno hemisphaerica Robineau-Desvoidy (1830: 144). Robineau-Desvoidy (1863a: 595) recognized Eurigaster Macquart, 1834 as a valid genus and included "Eurigaster haemisphaerica" as a valid (but misspelled) species with the original (but misspelled) combination of "Phryno hoemisphaerica" below it (page 597). Cyzenis haemisphaerica Robineau-Desvoidy, 1863 is therefore a new species-group name with no names in synonymy. The type species of Cyzenis was correctly cited as Cyzenis haemisphaerica [as "hemisphaerica"] by Herting \& Dely-Draskovits (1993: 236) but O'Hara \& Wood (2004: 169) overlooked the errata of Robineau-Desvoidy (1863b: 918) and cited Phryno hemisphaerica Robineau-Desvoidy, 1830 as the misidentified type species, then invoked ICZN Code Article 70.3.2 to fix Tachina albicans Fallén, 1810 as type species. There are two original spellings for the type species of Cyzenis Robineau-Desvoidy, 1863: haemisphaerica (RobineauDesvoidy, 1863a: 545) and hemisphoerica (Robineau-Desvoidy, 1863b: 875). Acting as First Reviser, we select haemisphaerica as the correct original spelling.

129. Dalmania Robineau-Desvoidy, 1853a: 146 [1853b: 66].

CuRRENT Status: Preoccupied by Emmrich, 1845; unjustified emendation of Dalmannia RobineauDesvoidy, 1830; junior synonym of Dalmannia Robineau-Desvoidy, 1830 [teste Sabrosky (1999: 106)].

FAMILY: CONOPIDAE.

REMARKS: Made available as an emendation by virtue of the original and changed spelling appearing in the same work and the changed spelling being adopted.

130. Dalmannia Robineau-Desvoidy, 1830: 248.

ORIGINAlly INCLUDED SPECIES: Myopa gemina Wiedemann, 1824; Dalmannia meridionalis RobineauDesvoidy, 1830; Myopa punctata Fabricius, 1794 (with Myopa virens Meigen, 1804 [as "Myopa virens. Fallén"] in synonymy); Myopa flavescens Meigen, 1824; Dalmannia stigma Robineau-Desvoidy, 1830; Dalmannia flavipes Robineau-Desvoidy, 1830.

TyPE SPECIES: Myopa gemina Wiedemann, 1824 [= Conops aculeatus Linnaeus, 1767], automatic [by designation of the same species (by subsequent designation of Desmarest in d'Orbigny, 1848: 798) for Stachynia Macquart, 1835]. 
CURRENT STATUS: Valid genus [teste Sabrosky (1999: 106)].

FAMILY: CONOPIDAE.

REMARKS: Previous catalogers [e.g., Chvála \& Smith (1988: 270), Sabrosky (1999: 106)] have listed the type designation for Dalmannia Robineau-Desvoidy, 1830 as Myopa punctata Fabricius, 1794 by subsequent designation of Rondani (1856: 59). However, Macquart (1835: 36) substituted [although unnecessarily] Stachynia Macquart, 1835 for Dalmannia Robineau-Desvoidy, which automatically takes the same type species. An earlier type-species designation for Stachynia of Myopa gemina Wiedemann, 1824 by Desmarest in d'Orbigny (1848: 798) was found by Evenhuis \& Thompson (1990), which thus becomes the type species for Dalmannia. Since Myopa gemina is currently treated in Dalmannia (Chvála \& Smith, 1988: 271), there is no change in generic concept with this change of type species.

EMENDATIONS: Dalmania Robineau-Desvoidy, 1853a: 146 (unjustified).

131. Damonia Robineau-Desvoidy, 1848a: 596.

ORIGINALly INCLUDED SPECIES: Damonia flavipalpis Robineau-Desvoidy, 1848.

TYPE SPECIES: Damonia flavipalpis Robineau-Desvoidy, 1848, by monotypy.

CURRENT STATUS: Placed in "Doubtful taxa in Tachinidae" by Herting \& Dely-Draskovits (1993: 436).

FAMILY: TACHINIDAE.

132. Dasyna Robineau-Desvoidy, 1830: 667.

ORIGINALly InCludED SPECIES: Dasyna fuscipennis Robineau-Desvoidy, 1830; Dasyna peleterii RobineauDesvoidy, 1830.

TYPE SPECIES: Dasyna fuscipennis Robineau-Desvoidy, 1830, by subsequent designation (Coquillett, 1910: 531).

CURRENT STATUs: Valid subgenus of Chyliza Fallén, 1820 [teste Soós (1984a: 35)].

FAMILY: PSILIDAE.

133. Dasyphora Robineau-Desvoidy, 1830: 409.

ORIGINALly InCludED SPECIES: Musca agilis Meigen, 1826; Dasyphora fervens Robineau-Desvoidy, 1830; Dasyphora viridula Robineau-Desvoidy, 1830; Dasyphora fuliginosa Robineau-Desvoidy, 1830.

TYPE SPECIES: Musca pratorum Meigen, 1826; hereby designated by fixation under ICZN Code Article 70.3.2; cited as Musca agilis Meigen, 1826 sensu Robineau-Desvoidy, 1830, in the subsequent (but invalid) designation by Hennig (1963b: 945).

CURRENT STATUS: Valid genus [teste Pont (1986b: 105)].

FAMILY: MUSCIDAE.

REMARKS: Previous catalogs have given the type-species designation as by Townsend (1916: 6). Townsend designated "Dasyphora agilis RD" but there unfortunately is no such originally included species. Robineau-Desvoidy (1830: 409) gave the species as "Dasyphora agilis." and placed "Musca agilis. Meig." in synonymy. Thus the included nominal species is a Meigen species and not one by RobineauDesvoidy. The first type designation to give the proper included species is by Hennig (1963b: 945) who stated “Gattungstypus von Dasyphora: 'D. agilis Meigen' sensu Rob.-Desv. (= pratorum Meigen: Bestimmung durch Townsend ...)”. Under the ICZN Code, a fixation of the taxonomic species involved in a misidentification is only valid when reference is made to Article 70.3.2. We are aware that 70.3.2 explicitly deals with earlier type designations, here interpreted as valid designations, but no other Article in the ICZN Code covers the present situation. 
134. Dejeania Robineau-Desvoidy, 1830: 33.

ORIGINALly INCLUded SPECIES: Dejeania brasiliensis Robineau-Desvoidy, 1830; Dejeania capensis Robineau-Desvoidy, 1830.

TYPE SPECIES: Dejeania capensis Robineau-Desvoidy, 1830 [= Stomoxys bombylans Fabricius, 1798], by subsequent designation (Coquillett, 1910: 531).

CURRENT STATUS: Valid genus [teste Crosskey (1980: 849)].

FAMILY: TACHINIDAE.

135. Delia Robineau-Desvoidy, 1830: 571.

ORIGinally InCluded SPECIES: Delia floricola Robineau-Desvoidy, 1830; Delia riparia RobineauDesvoidy, 1830; Delia vicina Robineau-Desvoidy, 1830; Delia deceptoria Robineau-Desvoidy, 1830; Delia agricola Robineau-Desvoidy, 1830; Delia arvicola Robineau-Desvoidy, 1830; Delia viatica Robineau-Desvoidy, 1830; Delia atrata Robineau-Desvoidy, 1830; Delia fuligo Robineau-Desvoidy, 1830; Delia maura Robineau-Desvoidy, 1830; Delia plumosula Robineau-Desvoidy, 1830; Delia liturata Robineau-Desvoidy, 1830; Delia cinerascens Robineau-Desvoidy, 1830; Delia vernalis Robineau-Desvoidy, 1830; Delia tessellata Robineau-Desvoidy, 1830; Delia campicola RobineauDesvoidy, 1830; Delia pratensis Robineau-Desvoidy, 1830; Musca chorea Fabricius, 1781; Delia calthae Robineau-Desvoidy, 1830; Delia palustris Robineau-Desvoidy, 1830; Delia herbicola RobineauDesvoidy, 1830; Delia soror Robineau-Desvoidy, 1830; Delia vulgaris Robineau-Desvoidy, 1830; Delia glabra Robineau-Desvoidy, 1830; Delia umbellatarum Robineau-Desvoidy, 1830; Delia anthophila Robineau-Desvoidy, 1830; Delia fuscipennis Robineau-Desvoidy, 1830; Delia germana RobineauDesvoidy, 1830; Delia littoralis Robineau-Desvoidy, 1830; Delia albipennis Robineau-Desvoidy, 1830. TYPE SPECIES: Delia floricola Robineau-Desvoidy, 1830, by subsequent designation (Coquillett, 1910: 531). CURRENT STATUS: Valid genus [teste Sabrosky (1999: 108)].

FAMILY: ANTHOMYIIDAE.

136. Delina Robineau-Desvoidy, 1830: 669.

Originally InCluded SPeCIES: Delina tibialis Robineau-Desvoidy, 1830; Delina dejeanii RobineauDesvoidy, 1830.

TYPE SPECIES: Delina dejeanii Robineau-Desvoidy, 1830 [= Cordylura nigrita Fallén, 1819], by subsequent designation (Séguy, 1952: 56).

CURRENT STATUS: Valid genus [teste Sabrosky (1999: 108)].

FAMILY: SCATHOPHAGIDAE.

137. Delphinia Robineau-Desvoidy, 1830: 719.

ORIGINALly INCLUDED SPECIES: Delphinia thoracica Robineau-Desvoidy, 1830.

TYPE SPECIES: Delphinia thoracica Robineau-Desvoidy, 1830 [= Musca picta Fabricius, 1781], by monotypy.

CURRENT STATUS: Valid genus [teste Steyskal (1965: 644)].

FAMILY: ULIDIIDAE.

138. Dichromya Robineau-Desvoidy, 1830: 707.

ORIGINALly INCLUDED SPECIES: Dichromya brasiliensis Robineau-Desvoidy, 1830.

TYPE SPECIES: Dichromya brasiliensis Robineau-Desvoidy, 1830, by monotypy.

CURRENT STATUS: Valid genus [teste McAlpine (1985: 219)].

FAMILY: HELEOMYZIDAE. 
REMARKS: Malloch (1933) did not study specimens, but treated Dichromya Robineau-Desvoidy (essentially as unplaced) in Ulidiidae simply to record the included species. Although it is not listed in the Neotropical catalogs of either the Heleomyzidae (Gill, 1968) or Otitidae (Steyskal, 1968b), McAlpine (1985) listed the Neotropical Dichromya among other genus-group names of Heleomyzidae in his review of Australian genera of Heleomyzidae, and we follow that treatment here.

\section{Dinera Robineau-Desvoidy, 1830: 307.}

ORIGINALly INCludED SPECIES: Dinera fulvipes Robineau-Desvoidy, 1830; Dinera grisea RobineauDesvoidy, 1830; Dinera punctata Robineau-Desvoidy, 1830; Dinera cylindrica Robineau-Desvoidy, 1830; Dinera pygmaea Robineau-Desvoidy, 1830.

TYPE SPECIES: Dinera grisea Robineau-Desvoidy, 1830 [= Musca carinifrons Fallén, 1817], by subsequent designation (Townsend, 1916: 6).

CURRENT Status: Valid genus [teste Herting \& Dely-Draskovits (1993: 361)].

FAMILY: TACHINIDAE.

140. Dionaea Robineau-Desvoidy, 1830: 253.

ORIGINAlly InCludED SPECIES: Dionaea forcipata Robineau-Desvoidy, 1830; Dionaea aurulans RobineauDesvoidy, 1830.

TYPE SPECIES: Dionaea forcipata Robineau-Desvoidy, 1830 [= Tachina aurifrons Meigen, 1824], by subsequent designation (Robineau-Desvoidy, 1863b: 54).

CurRent Status: Valid genus [teste Herting \& Dely-Draskovits (1993: 418)].

FAMILY: TACHINIDAE.

REMARKS: Robineau-Desvoidy (1863b: 54) designated as type species "Dionaea lineata, R.-D.", which was not an originally included species, but because Dionaea forcipata Robineau-Desvoidy, 1830 was placed in synonymy with $D$. lineata, the type designation is valid according to the ICZN Code Article 69.2.2. Dionaea was also proposed by Meigen (1800) [in Empididae], but this and other names in that work were suppressed by action of I.C.Z.N. (1963: 339 [Opinion 678]) and therefore does not preoccupy Dionaea Robineau-Desvoidy, 1830.

[Dorbinia] Robineau-Desvoidy, 1846a: 107.

CURRENT StATUS: Unavailable name; proposed without description or included species; treated under Winthemia Robineau-Desvoidy, 1830 [teste this work].

FAMILY: TACHINIDAE.

141. Dorbinia Robineau-Desvoidy, 1847: 272.

ORIGINALly INCluded SPECIES: Dorbinia ludibunda Robineau-Desvoidy, 1847; Dorbinia nitida RobineauDesvoidy, 1847; Dorbinia gentilis Robineau-Desvoidy, 1847; Carcelia aurifrons Robineau-Desvoidy, 1830.

TYPE SPECIES: Dorbinia ludibunda Robineau-Desvoidy, 1847 [= Musca quadripustulata Fabricius, 1794], by subsequent designation (Robineau-Desvoidy, 1863a: 217).

CURRENT StATus: Junior synonym of Winthemia Robineau-Desvoidy, 1830 [teste Herting \& DelyDraskovits (1993: 180)].

FAMILY: TACHINIDAE.

142. Drino Robineau-Desvoidy, 1863a: 250.

ORIGINALLY INCLUDED SPECIES: Drino volucris Robineau-Desvoidy, 1863. 
TYPE SPECIES: Drino volucris Robineau-Desvoidy, 1863 [= Tachina lota Meigen, 1824], by original designation.

CURRENT STATUS: Valid genus [teste Herting \& Dely-Draskovits (1993: 207)].

FAMILY: TACHINIDAE.

143. Dryope Robineau-Desvoidy, 1830: 618.

ORIGINALly INCLUDED SPECIES: Dryope communis Robineau-Desvoidy, 1830; Dryope liturata RobineauDesvoidy, 1830.

TYPE SPECIES: Dryope communis Robineau-Desvoidy, 1830 [= Musca flaveola Fabricius, 1794], by subsequent designation (Coquillett, 1910: 536).

CURRENT STATUS: Junior synonym of Dryomyza Fallén, 1820 [teste Pitkin \& Evenhuis (1989: 565)].

FAMILY: DRYOMYZIDAE.

REMARKS: Robineau-Desvoidy (1830: 619) tentatively listed the nominal species Musca flaveola Fabricius, 1794 in synonymy with Dryope communis Robineau-Desvoidy, 1830, but since it was doubtfully included in synonymy, it cannot be considered as an originally included nominal species according to the ICZN Code Article 67.2.5.

\section{Dryxo Robineau-Desvoidy, 1830: 787.}

ORIGINAlly InCluded SPECIES: Dryxo lispoidea Robineau-Desvoidy, 1830 (as “Dryxo lispoïdea. R. D."). TYPE SPECIES: Dryxo lispoidea Robineau-Desvoidy, 1830 (as “Dryxo lispoïdea. R. D.”), by monotypy.

CURRENT STATUS: Valid genus [teste Sabrosky (1999: 119)].

FAMILY: EPHYDRIDAE.

145. Dufouria Robineau-Desvoidy, 1830: 257.

ORIGinally InCluded Species: Dufouria aperta Robineau-Desvoidy, 1830; Dufouria nuda RobineauDesvoidy, 1830; Dufouria clausa Robineau-Desvoidy, 1830; Dufouria petiolata Robineau-Desvoidy, 1830.

TYPE SPECIES: Dufouria aperta Robineau-Desvoidy, 1830 [= Tachina chalybeata Meigen, 1824], by subsequent designation (Duponchel in d'Orbigny, 1844b: 143).

CURRENT STATUS: Valid genus [teste Herting \& Dely-Draskovits (1993: 393)].

FAMILY: TACHINIDAE.

REMARKS: Robineau-Desvoidy (1863b: 68) designated the same type species but this was later.

146. Dumerillia Robineau-Desvoidy, 1830: 38.

ORIGINALly INCLUDED SPECIES: Dumerillia rubida Robineau-Desvoidy, 1830.

TYPE SPECIES: Dumerillia rubida Robineau-Desvoidy, 1830, by monotypy.

CURRENT STATUS: Valid genus [teste Guimarães (1971: 53)].

FAMILY: TACHINIDAE.

EmEndations: Dumerilia Duponchel in d'Orbigny, 1844b: 145 (unjustified), n. syn.; Dumerilia Agassiz, 1846b: 131 (unjustified), n. syn.

147. Duponchelia Robineau-Desvoidy, 1863a: 531.

ORIGINALly INCLUDED SPECIES: Duponchelia silvestris Robineau-Desvoidy, 1863; Duponchelia infuscata Robineau-Desvoidy, 1863.

TYPE SPECIES: Duponchelia silvestris Robineau-Desvoidy, 1863, by subsequent designation (Townsend, 1916: 6). 
CuRrent Status: Preoccupied by Zeller, 1847; senior (but invalid) synonym of Parasetigena Brauer \& Bergenstamm, 1891 [teste Herting \& Dely-Draskovits (1993: 139)].

FAMILY: TACHINIDAE.

148. Duvaucelia Robineau-Desvoidy, 1830: 227.

ORIGINALLY INCLUDED SPECIES: Duvaucelia bicincta Robineau-Desvoidy, 1830.

TYPE SPECIES: Duvaucelia bicincta Robineau-Desvoidy, 1830, by monotypy.

CURRENT StATUs: Preoccupied by Risso, 1826; junior synonym of Lophosia Meigen, 1824 [teste Crosskey (1977: 594)].

FAMILY: TACHINIDAE.

149. Dyctia Robineau-Desvoidy, 1830: 692.

ORIGINALly INCLUDED SPECIES: Dyctia herbarum Robineau-Desvoidy, 1830; Dyctia claripennis RobineauDesvoidy, 1830; Dyctia nebulosa Robineau-Desvoidy, 1830; Dyctia palustris Robineau-Desvoidy, 1830; Dyctia nigricornis Robineau-Desvoidy, 1830; Dyctia paludosa Robineau-Desvoidy, 1830; Dyctia guerini Robineau-Desvoidy, 1830.

TYPE SPECIES: Dyctia herbarum Robineau-Desvoidy, 1830 [= Sciomyza cinerella Fallén, 1820], by present designation.

CURRENT STATUS: Junior synonym of Pherbellia Robineau-Desvoidy, 1830, n. syn.

FAMILY: SCIOMYZIDAE.

REMARKS: In listing this genus-group name, Robineau-Desvoidy (1830: 692) stated "Le genre Dyctie, établi et ensuite rejeté par M. Latreille". Latreille never published the name "Dyctia", so this comment may have referred to a label on a specimen that Robineau-Desvoidy saw, or to communication between Robineau-Desvoidy and Latreille since Latreille was one of the examiners of the 1826 manuscript of the 1830 work in which this name appeared. We disagree with the interpretation that this was a misspelling of the genus-group name Dictya Meigen, 1803 since the examiners of the 1826 manuscript informed Robineau-Desvoidy of Meigen's works and Robineau-Desvoidy subsequently tried to interpret Meigen's taxa as best he could, but he does not refer to Meigen anywhere in the section dealing with Dyctia and its included species. We follow Neave (1939: 170) in listing this as a proposal of a new genus-group name.

150. Edesia Robineau-Desvoidy, 1863a: 598.

ORIGINALly InCluded SPECIES: Edesia discreta Robineau-Desvoidy, 1863; Metopia rubrifrons Macquart, 1834.

TYPE SPECIES: Edesia discreta Robineau-Desvoidy, 1863 [= Phorocera rubrifrons Macquart, 1834], by original designation.

CURRENT STATUS: Junior synonym of Ceromasia Rondani, 1856 [teste Herting \& Dely-Draskovits (1993: 238)].

FAMILY: TACHINIDAE.

151. Edomya Robineau-Desvoidy, 1863a: 841.

ORIGINALLY INCLUDED SPECIES: Edomya agrestis Robineau-Desvoidy, 1863.

TYPE SPECIES: Edomya agrestis Robineau-Desvoidy, 1863 [= Musca muscaria Fallén, 1810], by original designation.

CURRENT STATUS: Junior synonym of Oswaldia Robineau-Desvoidy, 1863 [teste Herting \& Dely-Draskovits (1993: 162)].

FAMILY: TACHINIDAE.

EMENDATIONS: Edomyia Bezzi \& Stein, 1907: 302, n. syn. 
152. Egeria Robineau-Desvoidy, 1830: 555.

ORIGINALLY INCLUDED SPECIES: Egeria silvatica Robineau-Desvoidy, 1830; Egeria hortensis Robineau-

Desvoidy, 1830; Egeria testacea Robineau-Desvoidy, 1830.

TYPE SPECIES: Egeria silvatica Robineau-Desvoidy, 1830, by subsequent designation (Coquillett, 1910: 536).

CURRENT Status: Preoccupied by de Roissy, 1805; Leach, 1815; senior (but invalid) synonym of Botanophila Lioy, 1864 [teste Dely-Draskovits (1993: 23)].

FAMILY: ANTHOMYIIDAE.

EMENDATIONS: Aegeria Macquart, 1835: 321 (unjustified); Aegeria Agassiz, 1846b: 6 (unjustified), n. syn.

153. Eginia Robineau-Desvoidy, 1830: 502.

ORIGINALLY INCLUDED SPECIES: Eginia cylindrica Robineau-Desvoidy, 1830.

TYPE SPECIES: Eginia cylindrica Robineau-Desvoidy, 1830 [= Anthomyia ocypterata Meigen, 1826], by monotypy.

CuRRent Status: Valid genus [teste Pont (1986b: 111)].

FAMILY: MUSCIDAE.

EMENDATIONS: Aeginia Agassiz, 1846b: 8, 135 (unjustified).

\section{Egle Robineau-Desvoidy, 1830: 584.}

ORIGINALly INCLUDED SPECIES: Egle vulgaris Robineau-Desvoidy, 1830; Egle ludibunda RobineauDesvoidy, 1830; Egle campestris Robineau-Desvoidy, 1830; Egle agilis Robineau-Desvoidy, 1830; Egle anthophila Robineau-Desvoidy, 1830; Egle nitida Robineau-Desvoidy, 1830; Musca labiata Fabricius, 1787; Egle fabricii Robineau-Desvoidy, 1830; Egle autumnalis Robineau-Desvoidy, 1830; Egle floricola Robineau-Desvoidy, 1830; Egle pulverulenta Robineau-Desvoidy, 1830; Egle pallipes RobineauDesvoidy, 1830; Egle communis Robineau-Desvoidy, 1830; Egle viridescens Robineau-Desvoidy, 1830; Egle florum Robineau-Desvoidy, 1830; Egle festiva Robineau-Desvoidy, 1830; Egle flavescens Robineau-Desvoidy, 1830; Egle claripennis Robineau-Desvoidy, 1830; Egle praecox RobineauDesvoidy, 1830; Egle parva Robineau-Desvoidy, 1830; Egle nigra Robineau-Desvoidy, 1830; Egle exigua Robineau-Desvoidy, 1830.

TYPE SPECIES: Egle parva Robineau-Desvoidy, 1830, by subsequent designation (Coquillett, 1910: 536).

CURRENT STATUS: Valid genus [teste Michelsen (2009: 13)].

FAMILY: ANTHOMYIIDAE.

EMENDATIONS: Aegle Agassiz, 1846b: 9 (unjustified).

[Elaïmya] Robineau-Desvoidy MS name (Blainville et al., 1826: 17).

CURRENT STATUS: Unavailable name; suppressed by action of I.C.Z.N. (1990: 162 [Opinion 1601]) ; treated as a nomen dubium in Tephritidae [teste this work].

FAMILY: TEPHRITIDAE.

155. Elbaea Robineau-Desvoidy, 1863a: 286.

ORIGINALLY INCLUDED SPECIES: Elbaea montana Robineau-Desvoidy, 1863.

TYPE SPECIES: Elbaea montana Robineau-Desvoidy, 1863, by original designation.

CuRRENT STATUS: Placed in "Doubtful taxa in Tachinidae" by Herting \& Dely-Draskovits (1993: 436).

FAMILY: TACHINIDAE.

REMARKS: There are two original spellings of this genus-group name in Robineau-Desvoidy (1863a): Elbaea (page 286) and Elboea (page 1140). Acting as First Reviser, we select Elbaea as the correct original spelling. 
[Elboea] Robineau-Desvoidy, 1863a: 1140.

CURRENT STATUS: Incorrect original spelling of Elbaea Robineau-Desvoidy, 1863 [teste this work].

FAMILY: TACHINIDAE.

156. Eleone Robineau-Desvoidy, 1863b: 350.

ORIGINALLY INCLUDED SPECIES: Eleone haustellata Robineau-Desvoidy, 1863.

TYPE SPECIES: Eleone haustellata Robineau-Desvoidy, 1863 [= Stomoxys pedemontana Meigen, 1824], by monotypy.

CURRENT STATUS: Preoccupied by Massalongo, 1855; junior synonym of Rhamphina Macquart, 1835 [teste Herting \& Dely-Draskovits (1993: 392)].

FAMILY: TACHINIDAE.

[Elfia] Robineau-Desvoidy, 1849d: 158.

CURRENT STATUS: Unavailable name; proposed without description, indication, or included species; treated under Elfia Robineau-Desvoidy, 1850 [teste this work].

FAMILY: TACHINIDAE.

157. Elfia Robineau-Desvoidy, 1850b: 190.

ORIGINAlly INCLUDED SPECIES: Actia cingulata Robineau-Desvoidy, 1830; Elfia spathulata RobineauDesvoidy, 1850.

TYPE SPECIES: Actia cingulata Robineau-Desvoidy, 1830, by subsequent designation (Robineau-Desvoidy, 1863a: 672).

CURRENT StATUS: Junior synonym of Phytomyptera Rondani, 1845 [teste O'Hara \& Wood (2004: 252)].

FAMILY: TACHINIDAE.

REMARKS: Type species designation confirmed and Elfia placed on the Official List of Generic Names in Zoology by action of I.C.Z.N. (1987: 71 [Opinion 1432]). See REMARKS under Actia above for a summary of the history leading to the decision by the ICZN Commission.

158. Eloceria Robineau-Desvoidy, 1863a: 702.

ORIGINALLY INCLUDED SPECIES: Eloceria macrocera Robineau-Desvoidy, 1863 (as "Cerophora macrocera, R.-D.”).

TYPE SPECIES: Eloceria macrocera Robineau-Desvoidy, 1863 [= Tachina delecta Meigen, 1824], by original designation (as “Cerophora macrocera, R.-D.”).

CURRENT StATUS: Valid genus [teste Herting \& Dely-Draskovits (1993: 308)].

FAMILY: TACHINIDAE.

EMENDATIONS: Helocera Mik, 1883: 184 (unjustified).

159. Elodia Robineau-Desvoidy, 1863a: 936.

ORIGINALly INCLUDED SPECIES: Elodia gagatea Robineau-Desvoidy, 1863; Elodia fasciolata RobineauDesvoidy, 1863; Elodia pygmaea Robineau-Desvoidy, 1863; Meigenia nitida Robineau-Desvoidy, 1830.

TYPE SPECIES: Elodia gagatea Robineau-Desvoidy, 1863 [= Tachina morio Fallén, 1820], by original designation.

CURRENT Status: Valid genus [teste Herting \& Dely-Draskovits (1993: 246)].

FAMILY: TACHINIDAE. 
160. Elomya Robineau-Desvoidy, 1830: 296.

ORIGINALly INCLUDED SPECIES: Musca nebulosa Panzer, 1798; Elomya claripennis Robineau-Desvoidy, 1830; Elomya aurulans Robineau-Desvoidy, 1830; Elomya nigra Robineau-Desvoidy, 1830; Elomya abdominalis Robineau-Desvoidy, 1830.

TYPE SPECIES: Elomya claripennis Robineau-Desvoidy, 1830 [= Phasia lateralis Meigen, 1824], by subsequent designation (Townsend, 1916: 6).

CurRent Status: Valid genus [teste Herting \& Dely-Draskovits (1993: 408)].

FAMILY: TACHINIDAE.

EMENDATIOnS: Elomyia Macquart, 1834a: 204 (unjustified); Helomyia Meigen, 1838: 282 (unjustified); Helomyia Agassiz, 1846b: 176 (unjustified), n. syn.; Helomyia Verrall in Scudder, 1882: 121 (unjustified).

161. Elophoria Robineau-Desvoidy, 1830: 156.

ORIGINALly INCLUDED SPECIES: Elophoria myoidea Robineau-Desvoidy, 1830 (as "Elophoria myoïdea. R.

D.”); Elophoria flavisquamis Robineau-Desvoidy, 1830; Elophoria brunisquamis Robineau-Desvoidy, 1830.

TYPE SPECIES: Elophoria myoidea Robineau-Desvoidy, 1830, by subsequent designation (Townsend, 1916: 6).

CURRENT STATUS: Placed in "Doubtful taxa in Tachinidae" by Herting \& Dely-Draskovits (1993: 436).

FAMILY: TACHINIDAE.

EMENDATIONS: Helophoria Agassiz, 1846b: 176 (unjustified); Helophoria Bezzi \& Stein, 1907: 318 (unjustified), n. syn.

162. Elpe Robineau-Desvoidy, 1863a: 488.

ORIGINALLY INCLUDED SPECIES: Tachina inepta Meigen, 1824.

TYPE SPECIES: Tachina inepta Meigen, 1824, by original designation.

CURRENT StATUs: Junior synonym of Campylocheta Rondani, 1859 [teste Herting \& Dely-Draskovits (1993: 374)].

FAMILY: TACHINIDAE.

163. Elpigia Robineau-Desvoidy, 1863b: 149.

ORIGINALly INCLUDED SPECIES: Elpigia pellucida Robineau-Desvoidy, 1863; Elpigia punctata RobineauDesvoidy, 1863; Elpigia minuta Robineau-Desvoidy, 1863; Miltogramma heteroneura Meigen, 1830.

TYPE SPECIES: Elpigia pellucida Robineau-Desvoidy, 1863 [= Miltogramma heteroneura Meigen, 1830], by subsequent designation (Allen, 1926: 82).

CuRRent Status: Junior synonym of Taxigramma Perris, 1852 [teste Pape (1996: 151)].

FAMILY: SARCOPHAGIDAE.

REMARKS: Pape (1996) credited Verves (1986: 66) with the first type-species designation, but this is incorrect as Allen (1926) is earlier. Verves (1986) indicated incorrectly that Robineau-Desvoidy (1863b) designated Elpigia pellucida on page 150 of that work, but no such designation was made on that page or elsewhere in that work.

164. Ensina Robineau-Desvoidy, 1830: 751.

ORIGINALly INCLUDED SPECIES: Ensina chrysanthemi Robineau-Desvoidy, 1830; Ensina herbarum Robineau-Desvoidy, 1830; Ensina pratensis Robineau-Desvoidy, 1830; Ensina linariae Robineau- 
Desvoidy, 1830; Ensina scorzonerae Robineau-Desvoidy, 1830; Ensina doronici Robineau-Desvoidy, 1830.

TYPE SPECIES: Ensina scorzonerae Robineau-Desvoidy, 1830 [= Musca sonchi Linnaeus, 1767], by subsequent designation (Foote \& Freidberg, 1981: 30).

CURRENT STATUS: Valid genus [teste Norrbom et al. (1999: 143)].

FAMILY: TEPHRITIDAE.

REMARKS: Robineau-Desvoidy (1830: 753) included Musca sonchi Linnaeus, 1767 (as "An Musca Sonchi? Fabr.") in a tentative synonymy with Ensina scorzonerae Robineau-Desvoidy, 1830, but it was not an originally included species as it was doubtfully included. Westwood's (1840: 149) designation of Musca sonchi Linnaeus, 1767 is therefore invalid.

165. Enthenis Robineau-Desvoidy, 1863a: 199.

ORIGINALLY INCLUDED SPECIES: Enthenis ciligera Robineau-Desvoidy, 1863.

TYPE SPECIES: Enthenis ciligera Robineau-Desvoidy, 1863 [= Tachina dubia Fallén, 1810], by monotypy.

CURRENT StATUS: Junior synonym of Lypha Robineau-Desvoidy, 1830 [teste Herting \& Dely-Draskovits (1993: 292)].

FAMILY: TACHINIDAE.

166. Eperia Robineau-Desvoidy, 1863a: 316.

ORIGINALLY INCLUDED SPECIES: Eperia albicans Robineau-Desvoidy, 1863.

TYPE SPECIES: Eperia albicans Robineau-Desvoidy, 1863, by original designation.

CURRENT STATUS: Placed in "Doubtful taxa in Tachinidae" by Herting \& Dely-Draskovits (1993: 436).

FAMILY: TACHINIDAE.

167. Ephyra Robineau-Desvoidy, 1863b: 156.

Originally InCluded SPECIES: Phorinia micromera Robineau-Desvoidy, 1830; Ephyra laeta RobineauDesvoidy, 1863.

TYPE SPECIES: Phorinia micromera Robineau-Desvoidy, 1830 [= Tachina selecta Meigen, 1824], by original designation.

CuRRENT STATUS: Preoccupied by Péron \& Lesueur, 1810; Duponchel, 1829; Roux, 1831; junior synonym of Bessa Robineau-Desvoidy, 1863 [teste Herting \& Dely-Draskovits (1993: 142)].

FAMILY: TACHINIDAE.

REMARKS: When Monceaux put together Robineau-Desvoidy's 1863 work, he indicated in a footnote (1863b: 156) that the name Ephyra was not a good choice since it was preoccupied in the "Médusaires" (= Péron \& Lesueur, 1810), the "Lépidoptères" (= Duponchel, 1829), and the "Crustacés" (= Roux, 1831); but he refrained from taking the liberty of proposing a different name and kept the name in RobineauDesvoidy's manuscript.

168. Eratia Robineau-Desvoidy, 1863b: 237.

ORIGINALLY INCLUDED SPECIES: Eratia occlusa Robineau-Desvoidy, 1863.

TYPE SPECIES: Eratia occlusa Robineau-Desvoidy, 1863 [= Cistogaster celer Meigen, 1838], by monotypy.

CURRENT Status: Junior synonym of Strongygaster Macquart, 1834 [teste Herting \& Dely-Draskovits (1993: 416)].

FAMILY: TACHINIDAE. 
169. Erebia Robineau-Desvoidy, 1830: 207.

ORIGINALly INCLUDED SPECIES: Musca temula Scopoli, 1763 (as "Musca temula. Linn. Fabr. Vill. Scop. \&c."); Erebia flavipennis Robineau-Desvoidy, 1830.

TYPE SPECIES: Musca temula Scopoli, 1763, by subsequent designation [Macquart, 1855: 741 (as "Musca temula Linnaeus")].

CuRRent Status: Preoccupied by Dalman, 1816; senior (but invalid) synonym of Zophomyia Macquart, 1835 [teste Herting \& Dely-Draskovits (1993: 304)].

FAMILY: TACHINIDAE.

170. Eretria Robineau-Desvoidy, 1863a: 492.

ORIGINALly INCLUDED SPECIES: Eretria excitata Robineau-Desvoidy, 1863; Eretria albifrons RobineauDesvoidy, 1863; Eretria hilaris Robineau-Desvoidy, 1863; Eretria velox Robineau-Desvoidy, 1863; Eretria blanda Robineau-Desvoidy, 1863; Eretria obscurata Robineau-Desvoidy, 1863; Phorinia minor Robineau-Desvoidy, 1830; Eretria provocatrix Robineau-Desvoidy, 1863; Eretria agilis RobineauDesvoidy, 1863; Eretria festiva Robineau-Desvoidy, 1863; Eretria humilis Robineau-Desvoidy, 1863.

TYPE SPECIES: Eretria excitata Robineau-Desvoidy, 1863 [= Phorinia aurifrons Robineau-Desvoidy, 1830], by original designation.

CurRent Status: Junior synonym of Phorinia Robineau-Desvoidy, 1830 [teste Herting \& Dely-Draskovits (1993: 141)].

FAMILY: TACHINIDAE.

171. Eribea Robineau-Desvoidy, 1863a: 1033.

ORIGINALLY INCLUDED SPECIES: Eribea augur Robineau-Desvoidy, 1863; Eribea pagana RobineauDesvoidy, 1863; Eribea maculata Robineau-Desvoidy, 1863; Eribea honesta Robineau-Desvoidy, 1863; Eribea velox Robineau-Desvoidy, 1863; Eribea albiceps Robineau-Desvoidy, 1863; Eribea juvenilis Robineau-Desvoidy, 1863; Eribea morosa Robineau-Desvoidy, 1863; Eribea melancolica RobineauDesvoidy, 1863; Eribea cursoria Robineau-Desvoidy, 1863; Eribea schistacea Robineau-Desvoidy, 1863; Eribea ludibrina Robineau-Desvoidy, 1863; Eribea grossoria Robineau-Desvoidy, 1863; Eribea gagatina Robineau-Desvoidy, 1863; Eribea puella Robineau-Desvoidy, 1863; Eribea maesta RobineauDesvoidy, 1863.

TYPE SPECIES: Eribea augur Robineau-Desvoidy, 1863 [= Tachina rustica Fallén, 1810], by original designation.

CURRENT Status: Junior synonym of Adenia Robineau-Desvoidy, 1863 (subgenus of Exorista Meigen, 1803) [teste Herting (1984: 8)].

FAMILY: TACHINIDAE.

172. Erichsonia Robineau-Desvoidy, 1863b: 481.

ORIGINALLY INCLUDED SPECIES: Sarcophaga haemorrhoa Meigen, 1826 (with "Myophora aestivalis : Rob. Desv." in synonymy); Erichsonia valida Robineau-Desvoidy, 1863; Erichsonia pilosa RobineauDesvoidy, 1863; Erichsonia campestris Robineau-Desvoidy, 1863; Erichsonia chetalis RobineauDesvoidy, 1863; Erichsonia labialis Robineau-Desvoidy, 1863; Erichsonia oralis Robineau-Desvoidy, 1863; Erichsonia ambulatrix Robineau-Desvoidy, 1863; Erichsonia ardeacea Robineau-Desvoidy, 1863; Erichsonia fuliginosa Robineau-Desvoidy, 1863; Erichsonia musca Robineau-Desvoidy, 1863; Myophora albicans Robineau-Desvoidy, 1830; Erichsonia rustica Robineau-Desvoidy, 1863; Erichsonia umbripennis Robineau-Desvoidy, 1863; Erichsonia procax Robineau-Desvoidy, 1863; Erichsonia claripennis Robineau-Desvoidy, 1863; Erichsonia aestivalis Robineau-Desvoidy, 1863 (with "Myophora haemorrhoa : Rob. Desv." in synonymy); Erichsonia varinervis Robineau-Desvoidy, 1863; Erichsonia 
arborea Robineau-Desvoidy, 1863; Erichsonia inconstans Robineau-Desvoidy, 1863; Erichsonia contigua Robineau-Desvoidy, 1863; Erichsonia flavinervis Robineau-Desvoidy, 1863; Erichsonia anxia Robineau-Desvoidy, 1863; Myophora contempta Robineau-Desvoidy, 1830; Erichsonia nigricauda Robineau-Desvoidy, 1863; Erichsonia cursoria Robineau-Desvoidy, 1863; Erichsonia viatrix RobineauDesvoidy, 1863; Myophora arvensis Robineau-Desvoidy, 1830; Erichsonia hilaris Robineau-Desvoidy, 1863; Myophora nigricans Robineau-Desvoidy, 1830; Erichsonia infuscata Robineau-Desvoidy, 1863; Myophora fuscipennis Robineau-Desvoidy, 1830; Myophora grisella Robineau-Desvoidy, 1830; Myophora nana Robineau-Desvoidy, 1830; Erichsonia morio Robineau-Desvoidy, 1863.

TYPE SPECIES: Sarcophaga haemorrhoa Meigen, 1826, by original designation.

CURRENT StATus: Preoccupied by Westwood, 1849; Dana, 1849; senior (but invalid) synonym of Heteronychia Brauer \& Bergenstamm, 1889 (subgenus of Sarcophaga Meigen, 1826) [teste Pape (1996: 321)].

FAMILY: SARCOPHAGIDAE.

173. Erigone Robineau-Desvoidy, 1830: 65.

ORIGINALly INCLUDED SPECIES: Erigone anthophila Robineau-Desvoidy, 1830; Erigone scutellaris Robineau-Desvoidy, 1830; Erigone myophoroidaea Robineau-Desvoidy, 1830 (as "Erigone myophorö̈daea. R. D."); Erigone puparum Robineau-Desvoidy, 1830; Erigone flavipennis RobineauDesvoidy, 1830; Erigone tessellans Robineau-Desvoidy, 1830; Erigone viridulans Robineau-Desvoidy, 1830; Erigone dubia Robineau-Desvoidy, 1830.

TYPE SPECIES: Erigone anthophila Robineau-Desvoidy, 1830, by subsequent designation (Townsend, 1932: $42)$.

CURRENT Status: Preoccupied by Audouin, 1826; senior (but invalid) synonym of Eurithia RobineauDesvoidy, 1844 [teste Herting \& Dely-Draskovits (1993: 297)].

FAMILY: TACHINIDAE.

REMARKS: Robineau-Desvoidy (1863a: 151) designated “Musca radicum, Fabr.", but synonymized under that name were two originally included species (Erigone anthophila and E. scutellaris), thus this designation is invalid. Townsend (1932: 42) is the earliest valid designation of a single included nominal species. Townsend (1939: 250) designated the same species but this was later.

174. Erinia Robineau-Desvoidy, 1863a: 467.

ORIGINALLY INCLUDED SPECIES: Erinia silvatica Robineau-Desvoidy, 1863.

TYPE SPECIES: Erinia silvatica Robineau-Desvoidy, 1863 [= Exorista caudata Rondani, 1859], by monotypy. CURRENT STATUS: Junior synonym of Phryxe Robineau-Desvoidy, 1830 [teste Herting \& Dely-Draskovits (1993: 192)].

FAMILY: TACHINIDAE.

EMENDATIONS: Erinnia Bezzi \& Stein, 1907: 263 (unjustified), n. syn.

175. Ernestia Robineau-Desvoidy, 1830: 60.

ORIGINALLY INCLUDED SPECIES: Ernestia microcera Robineau-Desvoidy, 1830.

TYPE SPECIES: Ernestia microcera Robineau-Desvoidy, 1830 [= Tachina rudis Fallén, 1810], by monotypy. CuRrent Status: Junior synonym of Panzeria Robineau-Desvoidy, 1830 [teste O'Hara \& Wood (2004: 244)].

FAMILY: TACHINIDAE.

176. Ervia Robineau-Desvoidy, 1830: 225.

ORIGINALLY INCLUDED SPECIES: Ocyptera triquetra Olivier, 1811. 
TYPE SPECIES: Ocyptera triquetra Olivier, 1811, by monotypy.

CURRENT STATUS: Junior synonym of Icelia Robineau-Desvoidy, 1830 [teste O’Hara \& Wood (2004: 256)].

FAMILY: TACHINIDAE.

177. Erycia Robineau-Desvoidy, 1830: 146.

ORIGINALly INCLUDED SPECIES: Erycia grisea Robineau-Desvoidy, 1830; Erycia scutellaris RobineauDesvoidy, 1830; Erycia villica Robineau-Desvoidy, 1830; Erycia winthemi Robineau-Desvoidy, 1830; Erycia limpidipennis Robineau-Desvoidy, 1830.

TYPE SPECIES: Erycia grisea Robineau-Desvoidy, 1830 [= Tachina fatua Meigen, 1824], by subsequent designation (Townsend, 1916: 7).

CuRrent STATUS: Valid genus [teste Herting \& Dely-Draskovits (1993: 220)].

FAMILY: TACHINIDAE.

REMARKS: Robineau-Desvoidy (1863a: 902) designated "Tachina fatua, Meig.", but synonymized under that name were two originally included species (Erycia scutellaris and E. grisea), thus this designation is invalid.

178. Erynnia Robineau-Desvoidy, 1830: 125.

ORIGINALLY INCLUDED SPECIES: Erynnia nitida Robineau-Desvoidy, 1830.

TYPE SPECIES: Erynnia nitida Robineau-Desvoidy, 1830 [= Tachina ocypterata Fallén, 1810], by monotypy. CURRENT STATUS: Valid genus [teste Herting \& Dely-Draskovits (1993: 246)].

FAMILY: TACHINIDAE.

EMENDATIONS: Erinnya Agassiz, 1846b: 143 (unjustified).

179. Eryphe Robineau-Desvoidy, 1863a: 609.

ORIGINALLY INCLUDED SPECIES: Eryphe petiolata Robineau-Desvoidy, 1863.

TYPE SPECIES: Eryphe petiolata Robineau-Desvoidy, 1863, by monotypy.

CURRENT StATUS: Placed in "Doubtful taxa in Tachinidae" by Herting \& Dely-Draskovits (1993: 436).

FAMILY: TACHINIDAE.

180. Erytaea Robineau-Desvoidy, 1863a: 847.

ORIGINALLY INCLUDED SPECIES: Erytaea jucunda Robineau-Desvoidy, 1863.

TYPE SPECIES: Erytaea jucunda Robineau-Desvoidy, 1863 [= Tachina muscaria Fallén, 1810], by original designation.

CURRENT StATUs: Junior synonym of Oswaldia Robineau-Desvoidy, 1863 [teste Herting \& Dely-Draskovits (1993: 162)].

FAMILY: TACHINIDAE.

[Erythrocera] Robineau-Desvoidy, 1848b: 186.

CURRENT STATUS: Unavailable name; proposed without description or included species; treated under Erythrocera Robineau-Desvoidy, 1849 [teste this work].

FAMILY: TACHINIDAE.

181. Erythrocera Robineau-Desvoidy, 1849a: 436.

ORIGINALlY INCLUDED SPECIES: Erythrocera fulvipes Robineau-Desvoidy, 1849; Erythrocera flavipes Robineau-Desvoidy, 1849; Erythrocera scutellaris Robineau-Desvoidy, 1849; Erythrocera siphonoidea 
Robineau-Desvoidy, 1849 (as "Erythrocera siphonö̈dea" with "Phryno bucentö̈dea, R.-D." in synonymy); Erythrocera cinerea Robineau-Desvoidy, 1849; Phryno nigripes Robineau-Desvoidy, 1830; Erythrocera fulvescens Robineau-Desvoidy, 1849.

TYPE SPECIES: Phryno nigripes Robineau-Desvoidy, 1830, by subsequent designation [Robineau-Desvoidy, 1863a: 600 (as “Erythrocera nigripes, R.-D.")].

CURRENT Status: Valid genus [teste Herting \& Dely-Draskovits (1993: 242)].

FAMILY: TACHINIDAE.

182. Esila Robineau-Desvoidy, 1863a: 1063.

ORIGINALLY INCLUDED SPECIES: Esila arvorum Robineau-Desvoidy, 1863.

TYPE SPECIES: Esila arvorum Robineau-Desvoidy, 1863, by original designation.

CURRENT STATUS: Placed in "Doubtful taxa in Tachinidae" by Herting \& Dely-Draskovits (1993: 436).

FAMILY: TACHINIDAE.

183. Essenia Robineau-Desvoidy, 1863a: 193.

ORIGINALLY INCLUDED SPECIES: Essenia appendiculata Robineau-Desvoidy, 1863.

TYPE SPECIES: Essenia appendiculata Robineau-Desvoidy, 1863, by original designation.

CurRent STAtus: Placed in "Doubtful taxa" in the tribe Winthemiini by Herting \& Dely-Draskovits (1993: 183).

FAMILY: TACHINIDAE.

184. Estelia Robineau-Desvoidy, 1830: 635.

ORIGINALLY INCLUDED SPECIES: Estelia cinerea Robineau-Desvoidy, 1830; Estelia herbarum RobineauDesvoidy, 1830; Estelia impunctata Robineau-Desvoidy, 1830.

TYPE SPECIES: Estelia herbarum Robineau-Desvoidy, 1830, by subsequent designation (Coquillett, 1910: 540).

CuRRENT StATUS: Junior synonym of Chamaemyia Meigen, 1803 [teste Tanasijtshuk (1984: 222)].

FAMILY: CHAMAEMYIIDAE.

185. Estheria Robineau-Desvoidy, 1830: 305.

ORIGINALLY INCLUDED SPECIES: Estheria tibialis Robineau-Desvoidy, 1830; Estheria abdominalis Robineau-Desvoidy, 1830; Estheria imperatoriae Robineau-Desvoidy, 1830; Estheria floralis RobineauDesvoidy, 1830; Estheria vicina Robineau-Desvoidy, 1830.

TYPE SPECIES: Estheria imperatoriae Robineau-Desvoidy, 1830 [= Dexia cristata Meigen, 1826], by subsequent designation (Townsend, 1916: 7).

CURRENT STATUS: Valid genus [teste Herting \& Dely-Draskovits (1993: 362)].

FAMILY: TACHINIDAE.

REMARKS: Robineau-Desvoidy (1863b: 348) designated Dexia cristata Meigen (as "Estheria cristata. Meig.") but this was not an originally included species and the designation is therefore invalid. The statement by Robineau-Desvoidy (1863b: 349) "Le Dexia cristata de Zetterstedt n'est pas l'espèce Meigénienne, si l'on s'en rapporte à l'exacte description donnée par le naturaliste suédois qui a signalé une espèce entièrement nouvelle, à moins que ce ne soit notre Estheria vicina (Myod., p. 307, $n^{\circ}$ 5). $N$ 'ayant plus cet insecte à notre disposition, nous ne pouvons vérifier le fait' is conditional and does not place Estheria vicina in synonymy with Dexia cristata Meigen. The type-species designation by Townsend (1916) was confirmed and Estheria placed on the Official List of Generic Names in Zoology by action of I.C.Z.N. (1959: 225 [Opinion 553]). 
186. Etheria Robineau-Desvoidy, 1863b: 273.

ORIGINALLY INCLUDED SPECIES: Etheria pedicellata Robineau-Desvoidy, 1863; Etheria vernalis RobineauDesvoidy, 1863.

TYPE SPECIES: Etheria pedicellata Robineau-Desvoidy, 1863 [= Tachina globula Meigen, 1824], by subsequent designation (Townsend, 1916: 7).

CURRENT StATUS: Junior synonym of Strongygaster Macquart, 1834 [teste Herting \& Dely-Draskovits (1993: 416)].

FAMILY: TACHINIDAE.

187. Ethilla Robineau-Desvoidy, 1863a: 202.

ORIGINALLY INCLUDED SPECIES: Tachina aemula Meigen, 1824.

TYPE SPECIES: Tachina aemula Meigen, 1824, by original designation.

CURRENT STATUS: Valid genus [teste Herting \& Dely-Draskovits (1993: 175)].

FAMILY: TACHINIDAE.

EMENDATIONS: Ethyllia Mesnil, 1939: 32, 64 (unjustified).

188. Eudora Robineau-Desvoidy, 1863a: 623.

ORIGINALlY INCLUDED SPECIES: Eudora illustris Robineau-Desvoidy, 1863.

TYPE SPECIES: Eudora illustris Robineau-Desvoidy, 1863, by monotypy.

CURRENT Status: Preoccupied by Rafinesque, 1815; de Haan, 1833; Castelnau, 1840; senior (but invalid) synonym of Eudoromyia Bezzi, 1906 (subgenus of Tachina Meigen, 1803) [teste Herting \& DelyDraskovits (1993: 264)].

FAMILY: TACHINIDAE.

189. Eugenia Robineau-Desvoidy, 1863a: 919.

ORIGINAlly INCLUDED SPECIES: Eugenia fugax Robineau-Desvoidy, 1863; Eugenia silvatica RobineauDesvoidy, 1863.

TYPE SPECIES: Eugenia fugax Robineau-Desvoidy, 1863, by subsequent designation (Townsend, 1916: 7).

CURRENT STATUS: Preoccupied by Gould, 1855; Martens, 1860; no replacement name proposed; placed in

"Doubtful taxa in Tachinidae" by Herting \& Dely-Draskovits (1993: 436).

FAMILY: TACHINIDAE.

190. Eumea Robineau-Desvoidy, 1863a: 302.

ORIGINALly INCLUdED SPECIES: Eumea locuples Robineau-Desvoidy, 1863; Eumea vivida RobineauDesvoidy, 1863; Eumea luctuosa Robineau-Desvoidy, 1863; Eumea marginalis Robineau-Desvoidy, 1863; Eumea puberula Robineau-Desvoidy, 1863.

TYPE SPECIES: Eumea locuples Robineau-Desvoidy, 1863 [= Tachina linearicornis Zetterstedt, 1844], by original designation.

CurRent Status: Valid genus [teste Herting \& Dely-Draskovits (1993: 225)].

FAMILY: TACHINIDAE.

191. Euphemia Robineau-Desvoidy, 1830: 485.

ORIGINALLY INCLUDED SPECIES: Euphemia pratensis Robineau-Desvoidy, 1830; Euphemia claripennis

Robineau-Desvoidy, 1830; Euphemia tibialis Robineau-Desvoidy, 1830; Euphemia plumata Robineau-

Desvoidy, 1830. 
TYPE SPECIES: Euphemia pratensis Robineau-Desvoidy, 1830, by subsequent designation (Coquillett, 1901: 137).

CuRrent Status: Junior synonym of Phaonia Robineau-Desvoidy, 1830 [teste Pont (1986b: 116)].

FAMILY: MUSCIDAE

EMENDATION: Euphemyia Lioy, 1864a: 903 (unjustified), n. syn.

192. Euphoria Robineau-Desvoidy, 1863b: 799.

ORIGINALLY INCLUDED SPECIES: Euphoria nitidula Robineau-Desvoidy, 1863; Euphoria viridis RobineauDesvoidy, 1863; Euphoria polita Robineau-Desvoidy, 1863; Euphoria lauta Robineau-Desvoidy, 1863; Euphoria smaragda Robineau-Desvoidy, 1863; Euphoria aenea Robineau-Desvoidy, 1863; Euphoria venusta Robineau-Desvoidy, 1863; Euphoria viridula Robineau-Desvoidy, 1863; Lucilia calens Robineau-Desvoidy, 1830; Euphoria grata Robineau-Desvoidy, 1863; Euphoria vivida RobineauDesvoidy, 1863; Euphoria prompta Robineau-Desvoidy, 1863; Euphoria metallica Robineau-Desvoidy, 1863; Euphoria praecox Robineau-Desvoidy, 1863; Euphoria agilis Robineau-Desvoidy, 1863; Euphoria semiaurata Robineau-Desvoidy, 1863 (as "Euphoria semi-aurata, R.-D."); Lucilia viridescens Robineau-Desvoidy, 1830 (with "Lucilia caesarion : Macq." in synonymy); Euphoria chalybea Robineau-Desvoidy, 1863; Euphoria decora Robineau-Desvoidy, 1863; Musca caesarion Meigen, 1826; Euphoria scutellaris Robineau-Desvoidy, 1863; Euphoria floralis Robineau-Desvoidy, 1863; Euphoria frontalis Robineau-Desvoidy, 1863; Euphoria soror Robineau-Desvoidy, 1863; Euphoria viridicyanea Robineau-Desvoidy, 1863 (as "Euphoria viridi-cyanea, R.-D.”); Euphoria analis Robineau-Desvoidy, 1863; Euphoria puella Robineau-Desvoidy, 1863; Euphoria volucris Robineau-Desvoidy, 1863; Euphoria festiva Robineau-Desvoidy, 1863; Euphoria cyaneoviridis Robineau-Desvoidy, 1863 (as “Euphoria cyaneo-viridis, R.-D.”); Euphoria aurata Robineau-Desvoidy, 1863; Euphoria aestivalis Robineau-Desvoidy, 1863; Euphoria fulgida Robineau-Desvoidy, 1863; Euphoria caeruleifrons Robineau-Desvoidy, 1863; Euphoria autumnalis Robineau-Desvoidy, 1863; Euphoria ruralis RobineauDesvoidy, 1863; Euphoria corusca Robineau-Desvoidy, 1863; Euphoria gentilis Robineau-Desvoidy, 1863; Euphoria chrysorhoa Robineau-Desvoidy, 1863; Euphoria scintilla Robineau-Desvoidy, 1863; Euphoria chrysura Robineau-Desvoidy, 1863; Euphoria nitida Robineau-Desvoidy, 1863; Euphoria mollis Robineau-Desvoidy, 1863; Lucilia aurulans Robineau-Desvoidy, 1830 (with "Lucilia caesarion : Macq." in synonymy); Euphoria bella Robineau-Desvoidy, 1863; Euphoria blanda Robineau-Desvoidy, 1863; Euphoria jucunda Robineau-Desvoidy, 1863; Euphoria cunctans Robineau-Desvoidy, 1863; Musca splendida Meigen, 1826; Euphoria cyaneifrons Robineau-Desvoidy, 1863; Euphoria bicolor Robineau-Desvoidy, 1863 (as “ Euphoria bi-color, R.-D.”); Euphoria gratiosa Robineau-Desvoidy, 1863; Euphoria gloriosa Robineau-Desvoidy, 1863; Euphoria triumphalis Robineau-Desvoidy, 1863; Euphoria placida Robineau-Desvoidy, 1863; Euphoria callichroa Robineau-Desvoidy, 1863; Euphoria purpurea Robineau-Desvoidy, 1863; Euphoria adamantina Robineau-Desvoidy, 1863; Euphoria flamma Robineau-Desvoidy, 1863.

TYPE SPECIES: Euphoria nitidula Robineau-Desvoidy, 1863 [= Musca cornicina Fabricius, 1781], by subsequent designation (Townsend, 1916: 7).

CURRENT STATUS: Preoccupied by Burmeister, 1842; junior synonym of Neomyia Walker, 1859 [teste Pont (1986b: 96)].

FAMILY: MUSCIDAE.

REMARKS: Monceaux [in Robineau-Desvoidy (1863b: 800, footnote)] indicated that he was aware of the name Euphoria being preoccupied by Burmeister, 1842 in the Coleoptera; but he kept the name and did not offer a replacement name.

193. Eurithia Robineau-Desvoidy, 1844a: 24.

ORIGINALly INCLUDED SPECIES: Erigone puparum Robineau-Desvoidy, 1830 (with "Erigone tessellans. Rob. Desv.”, “Erigone viridulans. R. D.”, and "Erigone dubia. R. D.” in synonymy). 
TYPE SPECIES: Erigone puparum Robineau-Desvoidy, 1830, by monotypy.

CURRENT STATUS: Valid genus [teste Herting \& Dely-Draskovits (1993: 297)].

FAMILY: TACHINIDAE.

EMENDATIONS: Eurythia Brauer \& Bergenstamm, 1889: 18 (unjustified), n. syn.

194. Euryclea Robineau-Desvoidy, 1863a: 290.

ORIGINALly INCLUDED SPECIES: Euryclea tibialis Robineau-Desvoidy, 1863; Euryclea vivida RobineauDesvoidy, 1863.

TYPE SPECIES: Euryclea tibialis Robineau-Desvoidy, 1863, by original designation.

CURRENT StATUS: Valid subgenus of Carcelia Robineau-Desvoidy, 1830 [teste Herting \& Dely-Draskovits (1993: 216)].

FAMILY: TACHINIDAE.

195. Eurysthaea Robineau-Desvoidy, 1863a: 603.

ORIGINALLY INCLUDED SPECIES: Erythrocera scutellaris Robineau-Desvoidy, 1830.

TYPE SPECIES: Erythrocera scutellaris Robineau-Desvoidy, 1830, by original designation.

CURRENT STATUS: Valid genus [teste Herting \& Dely-Draskovits (1993: 245)].

FAMILY: TACHINIDAE.

EMENDATIONS: Euristhaea Marschall, 1873: 331 (unjustified), n. syn.

[Eurythia] Robineau-Desvoidy, 1843: 317.

CURRENT STATUS: Unavailable name; proposed without description or indication and no included species; treated under Eurithia Robineau-Desvoidy, 1844 [teste this work].

FAMILY: TACHINIDAE.

REMARKS: This name was published with this orthography before the original description of Eurithia Robineau-Desvoidy, 1844 (q.v.).

196. Eversmania Robineau-Desvoidy, 1863a: 181.

ORIGINALLY INCLUDED SPECIES: Eversmania ruficauda Robineau-Desvoidy, 1863.

TYPE SPECIES: Eversmania ruficauda Robineau-Desvoidy, 1863 [= Nemoraea erythrura Meigen, 1838], by original designation.

CuRREnT Status: Junior synonym of Winthemia Robineau-Desvoidy, 1830 [teste Herting \& DelyDraskovits (1993: 180)].

FAMILY: TACHINIDAE.

REMARKS: There are two original spellings of this genus-group name in Robineau-Desvoidy (1863a): Eversmania (page 181) and Evesrmania (page 1140). Acting as First Reviser, we select Eversmania as the correct original spelling.

EMENDATIONS: Eversmannia Marschall, 1873: 331 (unjustified).

[Evesrmania] Robineau-Desvoidy, 1863a: 1140.

CURRENT STATUS: Incorrect original spelling of Eversmania Robineau-Desvoidy, 1863 [teste this work].

FAMILY: TACHINIDAE.

197. Fabricia Robineau-Desvoidy, 1830: 42.

ORIGiNAlly InCluded SPECIES: Tachina ferox Panzer, 1809 (as "Musca ferox, Panz. CIV., 20. Tach. ferox, Meig. n. ${ }^{\circ}$ " with "Musca rotundata, Harris, tab. IX, fig. 2" in synonymy). 
TYPe SPECIES: Tachina ferox Panzer, 1809 (as “Musca ferox, Panz.)”, by monotypy.

CURRENT STATUS: Preoccupied by Blainville, 1828; Latreille, 1829; senior (but invalid) synonym of Nowickia Wachtl, 1894 (subgenus of Tachina Meigen, 1803) [teste O’Hara \& Wood (2004: 325)].

FAMILY: TACHINIDAE.

REMARKS: An unfortunate result of the presentation of the manuscript of Robineau-Desvoidy's Myodaires to the Académie des Sciences for their approval of publication and the resulting summary "Rapport" by Blainville et al. (1826) is that some of the information presented in the manuscript was noted by the committee members reviewing it and by their colleagues and then appeared in print in works published before Robineau-Desvoidy (1830). In those works, the new genera of Robineau-Desvoidy ended up with the authorship of others. One of these casualties was Fabricia.

198. Faedoria Robineau-Desvoidy, 1863a: 848.

ORIGINAlly INCLUDED SPECIES: Faedoria neglecta Robineau-Desvoidy, 1863; Faedoria campestris Robineau-Desvoidy, 1863.

TYPE SPECIES: Faedoria neglecta Robineau-Desvoidy, 1863, by original designation.

CURRENT STATUS: Placed in "Doubtful taxa in Tachinidae" by Herting \& Dely-Draskovits (1993: 436).

FAMILY: TACHINIDAE.

REMARKS: There are two original spellings of this genus-group name in Robineau-Desvoidy (1863a): Faedoria (page 848) and Foedoria (page 1140). Acting as First Reviser, we select Faedoria as the correct original spelling.

[Foedoria] Robineau-Desvoidy, 1863a: 1140.

CURRENT STATUS: Incorrect original spelling of Faedoria Robineau-Desvoidy, 1863a [teste this work].

FAMILY: TACHINIDAE.

[Fairemairia] Robineau-Desvoidy, 1853a: 111 [1853b: 31].

CURRENT StATUS: Incorrect original spelling of Fairmairia Robineau-Desvoidy, 1853 [teste this work].

FAMILY: CONOPIDAE.

199. Fairmairia Robineau-Desvoidy, 1853a: 111 [1853b: 31].

ORIGINALLY INCLUDED SPECIES: Myopa morio Meigen, 1804.

TYPE SPECIES: Myopa morio Meigen, 1804, by original designation.

CURRENT STATUS: Junior synonym of Myopa Fabricius, 1775 [teste Chvála \& Smith (1988: 262)].

FAMILY: CONOPIDAE.

REMARKS: There are two original spellings of this genus-group name in Robineau-Desvoidy (1853a):

Fairmairia and Fairemaria (both on page 111). Acting as First Reviser, we select Fairmairia as the correct original spelling.

200. Fannia Robineau-Desvoidy, 1830: 567.

ORIGINALLY INCLUDED SPECIES: Fannia saltatrix Robineau-Desvoidy, 1830.

TYPE SPECIES: Fannia saltatrix Robineau-Desvoidy, 1830 [= Musca scalaris Fabricius, 1794], by monotypy. CURRENT STATUS: Valid genus [teste Carvalho et al. (2003: 7)].

FAMILY: FANNIIDAE. 
201. Faunia Robineau-Desvoidy, 1830: 279.

TYPE SPECIES: Musca grossa Linnaeus, 1758, automatic [by designation of the same species (by subsequent designation of Brauer, 1893: 489) for Tachina Meigen, 1803].

CURRENT StATUS: Unnecessary new name for and junior synonym of Tachina Meigen, 1803 sensu stricto [teste Herting (1984: 84)].

FAMILY: TACHINIDAE.

202. Faurella Robineau-Desvoidy, 1830: 41.

ORIGINALLY INCLUDED SPECIES: Faurella meridionalis Robineau-Desvoidy, 1830.

TYPE SPECIES: Faurella meridionalis Robineau-Desvoidy, 1830, by monotypy.

CURREnT StAtus: Junior synonym of Peleteria Robineau-Desvoidy, 1830 [teste Herting \& Dely-Draskovits (1993: 276)].

FAMILY: TACHINIDAE.

203. Fausta Robineau-Desvoidy, 1830: 62.

ORIGINAlly INCLUDED SPECIES: Fausta nigra Robineau-Desvoidy, 1830; Fausta scutellaris RobineauDesvoidy, 1830; Fausta abdominalis Robineau-Desvoidy, 1830; Fausta viridescens Robineau-Desvoidy, 1830; Fausta florea Robineau-Desvoidy, 1830.

TYPE SPECIES: Fausta nigra Robineau-Desvoidy, 1830 [= Tachina nemorum Meigen, 1824], by subsequent designation (Townsend, 1916: 7).

CURRENT STATUS: Valid genus [teste Herting \& Dely-Draskovits (1993: 296)].

FAMILY: TACHINIDAE.

REMARKS: An earlier type-species designation by Robineau-Desvoidy (1863a: 158) of Tachina nemorum Meigen, 1824 is invalid because neither it nor its synonym Fausta lateralis Robineau-Desvoidy, 1846 were originally included species.

204. Feburia Robineau-Desvoidy, 1830: 256.

ORIGINALLY INCLUDED SPECIES: Feburia rapida Robineau-Desvoidy, 1830.

TYPE SPECIES: Feburia rapida Robineau-Desvoidy, 1830, by monotypy.

CURRENT STATUS: Placed in "Doubtful taxa in Tachinidae" by Herting \& Dely-Draskovits (1993: 436).

FAMILY: TACHINIDAE.

205. Fellaea Robineau-Desvoidy, 1830: 476.

ORIGINALly INCLUDED SPECIES: Fellaea fera Robineau-Desvoidy, 1830; Fellaea florea Robineau-Desvoidy, 1830; Fellaea nigripes Robineau-Desvoidy, 1830; Fellaea erratica Robineau-Desvoidy, 1830; Fellaea agilis Robineau-Desvoidy, 1830; Fellaea rubrifrons Robineau-Desvoidy, 1830; Fellaea nigrifrons Robineau-Desvoidy, 1830.

TYPE SPECIES: Fellaea fera Robineau-Desvoidy, 1830 [= Musca angelicae Scopoli, 1763], by subsequent designation (Coquillett, 1901: 137).

CuRRENT Status: Junior synonym of Phaonia Robineau-Desvoidy, 1830 [teste Pont (1986b: 116)].

FAMILY: MUSCIDAE.

EMENDATIONS: Fellea Agassiz, 1846b: 154 (unjustified), n. syn.

206. Feria Robineau-Desvoidy, 1830: 309.

ORIGINAlly InCLUdED SPECIES: Feria rubescens Robineau-Desvoidy, 1830; Feria nitida RobineauDesvoidy, 1830. 
TYPE SPECIES: Feria rubescens Robineau-Desvoidy, 1830, by subsequent designation (Townsend, 1916: 7). CURRENT STATUS: Placed in "Doubtful taxa in Tachinidae" by Herting \& Dely-Draskovits (1993: 436).

FAMILY: TACHINIDAE.

207. Fimetia Robineau-Desvoidy, 1830: 810.

ORIGINAlly InCluded SPECIES: Fimetia limpidipennis Robineau-Desvoidy, 1830; Fimetia cadaverina Robineau-Desvoidy, 1830; Fimetia atrata Robineau-Desvoidy, 1830.

TYPE SPECIES: Fimetia limpidipennis Robineau-Desvoidy, 1830, by present designation.

CURRENT STATUS: Placed in "Doubtful genera of Sphaeroceridae" by Roháček in Roháček (2001: 295).

FAMILY: SPHAEROCERIDAE.

\section{Fischeria Robineau-Desvoidy, 1830: 101.}

ORIGINALLY INCLUDED SPECIES: Fischeria bicolor Robineau-Desvoidy, 1830.

TYPE SPECIES: Fischeria bicolor Robineau-Desvoidy, 1830, by monotypy.

CurRent StATUS: Valid genus [teste Herting \& Dely-Draskovits (1993: 344)].

FAMILY: TACHINIDAE.

\section{Forellia Robineau-Desvoidy, 1830: 760.}

ORIGINALly InCLUdED SPECIES: Forellia onopordi Robineau-Desvoidy, 1830; Forellia dauci Robineau-

Desvoidy, 1830; Forellia brunithorax Robineau-Desvoidy, 1830.

TYPE SPECIES: Forellia onopordi Robineau-Desvoidy, 1830 [= Musca artemisiae Fabricius, 1794], by subsequent designation (Duponchel in d'Orbigny, 1845a: 676).

CURRENT STATUS: Junior synonym of Trypeta Meigen, 1803 [teste Norrbom et al. (1999: 238)].

FAMILY: TEPHRITIDAE.

\section{Freraea Robineau-Desvoidy, 1830: 285.}

ORIGINALLY INCLUDED SPECIES: Freraea gagatea Robineau-Desvoidy, 1830.

TYPE SPECIES: Freraea gagatea Robineau-Desvoidy, 1830, by monotypy.

CURRENT STATUS: Valid genus [teste Herting \& Dely-Draskovits (1993: 398)].

FAMILY: TACHINIDAE.

REMARKS: Robineau-Desvoidy (1830: 286) indicated that this genus-group name was dedicated to his friend, "le docteur Amand Frère". The spelling "Freraea" derives from the latinization of the name Frère to Freraeus.

EMENDATIONS: Frerea Agassiz, 1846b: 156 (unjustified), n. syn.

\section{Fucellia Robineau-Desvoidy, 1842c: 269.}

ORIGINALLY INCLUDED SPECIES: Fucellia arenaria Robineau-Desvoidy, 1842.

TYPE SPECIES: Fucellia arenaria Robineau-Desvoidy, 1842 [= Scathophaga maritima Haliday, 1838], by monotypy.

CURRENT STATUS: Valid genus [teste Sabrosky (1999: 140)].

FAMILY: ANTHOMYIIDAE.

212. Futilia Robineau-Desvoidy, 1863a: 1049.

ORIGINAlly InCluded SPECIES: Futilia flavicans Robineau-Desvoidy, 1863; Futilia teres RobineauDesvoidy, 1863; Futilia floralis Robineau-Desvoidy, 1863; Futilia hortorum Robineau-Desvoidy, 1863; 
Futilia nitida Robineau-Desvoidy, 1863; Futilia cursoria Robineau-Desvoidy, 1863; Futilia subtilis Robineau-Desvoidy, 1863; Futilia quieta Robineau-Desvoidy, 1863; Futilia irrorata RobineauDesvoidy, 1863; Futilia flavago Robineau-Desvoidy, 1863; Futilia lutescens Robineau-Desvoidy, 1863; Futilia incana Robineau-Desvoidy, 1863; Futilia munda Robineau-Desvoidy, 1863; Futilia rubrifrons Robineau-Desvoidy, 1863; Futilia olivacea Robineau-Desvoidy, 1863; Futilia inguinata RobineauDesvoidy, 1863; Futilia vesana Robineau-Desvoidy, 1863; Futilia lubrica Robineau-Desvoidy, 1863; Futilia pervia Robineau-Desvoidy, 1863; Futilia temerata Robineau-Desvoidy, 1863; Futilia blanda Robineau-Desvoidy, 1863; Futilia aurifacies Robineau-Desvoidy, 1863; Futilia obscurata RobineauDesvoidy, 1863.

TYPE SPECIES: Futilia floralis Robineau-Desvoidy, 1863 [= Tachina rustica Fallén, 1810], by original designation.

CURRENT STATUS: Junior synonym of Adenia Robineau-Desvoidy, 1863 (subgenus of Exorista Meigen, 1803) [teste Herting (1984: 8)].

FAMILY: TACHINIDAE.

213. Gaedartia Robineau-Desvoidy, 1863a: 565.

ORIGINALLY INCLUDED SPECIES: Gaedartia tibialis Robineau-Desvoidy, 1863; Gaedartia praecox RobineauDesvoidy, 1863.

TYPE SPECIES: Gaedartia tibialis Robineau-Desvoidy, 1863 [= Tachina praecox Meigen, 1824], by original designation.

CURRENT STATUS: Junior synonym of Campylocheta Rondani, 1859 [teste Herting \& Dely-Draskovits (1993: 374)].

FAMILY: TACHINIDAE.

EMENDATIONS: Goedartia Bezzi \& Stein, 1907: 318 (unjustified), n. syn.

214. Gaubilia Robineau-Desvoidy, 1863a: 1062.

ORIGINALLY INCLUDED SPECIES: Gaubilia dominula Robineau-Desvoidy, 1863.

TYPE SPECIES: Gaubilia dominula Robineau-Desvoidy, 1863 [= Tachina rustica Fallén, 1810], by original designation.

CURRENT StATUS: Junior synonym of Adenia Robineau-Desvoidy, 1863 (subgenus of Exorista Meigen, 1803) [teste Herting (1984: 8)].

FAMILY: TACHINIDAE.

215. Germaria Robineau-Desvoidy, 1830: 83.

ORIGINALLY INCLUDED SPECIES: Germaria latifrons Robineau-Desvoidy, 1830.

TYPE SPECIES: Germaria latifrons Robineau-Desvoidy, 1830 [= Tachina ruficeps Fallén, 1820], by monotypy.

CURRENT STATUS: Valid genus [teste Herting \& Dely-Draskovits (1993: 281)].

FAMILY: TACHINIDAE.

216. Gervaisia Robineau-Desvoidy, 1863b: 36.

ORIGINALLY INCLUDED SPECIES: Tachina piniariae Hartig, 1838.

TYPE SPECIES: Tachina piniariae Hartig, 1838, by original designation.

CURREnT Status: Preoccupied by Bonaparte, 1854; Waga, 1858; junior synonym of Blondelia Robineau-

Desvoidy, 1830 [teste Herting \& Dely-Draskovits (1993: 168)].

FAMILY: TACHINIDAE. 
217. Gesneria Robineau-Desvoidy, 1830: 378.

ORIGINALLY INCLUDED SPECIES: Gesneria erythrocera Robineau-Desvoidy, 1830; Gesneria brunicans Robineau-Desvoidy, 1830; Gesneria claripennis Robineau-Desvoidy, 1830; Gesneria cinerea RobineauDesvoidy, 1830; Gesneria rapida Robineau-Desvoidy, 1830; Gesneria campestris Robineau-Desvoidy, 1830; Gesneria grisea Robineau-Desvoidy, 1830; Gesneria riparia Robineau-Desvoidy, 1830; Gesneria albifrons Robineau-Desvoidy, 1830; Gesneria luteifrons Robineau-Desvoidy, 1830; Gesneria musca Robineau-Desvoidy, 1830; Gesneria floralis Robineau-Desvoidy, 1830; Gesneria aurifacies RobineauDesvoidy, 1830; Gesneria agrestis Robineau-Desvoidy, 1830; Gesneria fuliginosa Robineau-Desvoidy, 1830; Gesneria aurifrons Robineau-Desvoidy, 1830; Gesneria griseoflavescens Robineau-Desvoidy, 1830 (as “Gesneria griseo-flavescens. R. D.”).

TYPE SPECIES: Gesneria erythrocera Robineau-Desvoidy, 1830 [= Sarcophaga grisea Meigen, 1824], by subsequent designation (Townsend, 1916: 7).

CURRENT STATUS: Preoccupied by Hübner, 1825; senior (but invalid) synonym of Tephromyia Brauer \& Bergenstamm, 1891 (subgenus of Blaesoxipha Loew, 1861) [teste Pape (1996: 207)].

FAMILY: SARCOPHAGIDAE.

\section{Gimmenthalia Robineau-Desvoidy, 1863a: 851.}

ORIGINALLY INCLUDED SPECIES: Gimmenthalia officiosa Robineau-Desvoidy, 1863.

TYPE SPECIES: Gimmenthalia officiosa Robineau-Desvoidy, 1863, by monotypy.

CURRENT STATUS: Placed in "Doubtful taxa in Tachinidae" by Herting \& Dely-Draskovits (1993: 436).

FAMILY: TACHINIDAE.

EMENDATIONS: Gimmerthalia Bezzi \& Stein, 1907: 302 (unjustified), n. syn.

\section{Gouraldia Robineau-Desvoidy, 1851a: 150.}

ORIGINALly INCLUDED SPECIES: Gouraldia pupivora Robineau-Desvoidy, 1851 (with "Metopia fasciata" [from a Goureau label] in synonymy); Gouraldia binotata Robineau-Desvoidy, 1851 (as "Gouraldia binotata, Rob.-Desv."; with "Metopia fasciata" [from a Goureau label] in synonymy).

TYPE SPECIES: Gouraldia pupivora Robineau-Desvoidy, 1851 by subsequent designation (RobineauDesvoidy, 1863a: 916).

CURRENT STATUS: Questionable senior synonym of Eumea Robineau-Desvoidy, 1863 [teste Herting (1984: 63); cf. Herting \& Dely-Draskovits (1993: 226)].

FAMILY: TACHINIDAE.

REMARKS: A second (incorrect) original spelling of Gouraldia pupivora exists in the note below Gouraldia binotata (page 152): "Cet insecte pourrait être le mâle $d u \mathrm{G}$. pupurum : ce fait a besoin d'une confirmation péremptoire". Herting (1984: 63) considered G. pupivora as a questionable synonym of Tachina linearicornis Zetterstedt, 1844, the senior synonym of the type species of Eumea RobineauDesvoidy, 1863. Herting \& Dely-Draskovits (1993: 226) followed this interpretation by placing Gouraldia in "Doubtful taxa" after Eumea, but erred when they wrote "Type-species: Gouraldia pupivora Robineau-Desvoidy, $1851 \ldots=$ linearicornis (Zetterstedt, 1844)". Their intended meaning was clearly the same as that of Herting (1984) and their passage should have read "Type-species: Gouraldia pupivora Robineau-Desvoidy, 1851... = ?linearicornis (Zetterstedt, 1844)". Because the synonymy of these two nominal species is questionable, Gouraldia does not replace Eumea as the valid name of that genus.

220. Graphomya Robineau-Desvoidy, 1830: 403.

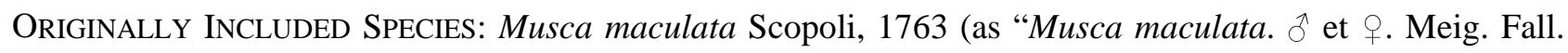
Musca maculata. ‥ Fabr. Musca vulpina. §ิ. Fabr.”); Graphomya vulpina Robineau-Desvoidy, 1830; 
Graphomya minor Robineau-Desvoidy, 1830; Graphomya americana Robineau-Desvoidy, 1830; Graphomya media Robineau-Desvoidy, 1830.

TyPE SPECIES: Musca maculata Scopoli, 1763, by subsequent designation (Duponchel in d'Orbigny, 1845b: 305).

CURRENT STATUS: Valid genus [teste Pont (1986b: 162)].

FAMILY: MUSCIDAE.

REMARKS: Rondani (1856: 91) and Brauer (1893: 508) designated the same type species but these were later. EMENDATIONS: Graphomyia Agassiz, $1846 \mathrm{~b}: 167$ (unjustified).

221. Gravenhorstia Robineau-Desvoidy, 1863a: 924.

ORIGINALly INCLUDED SPECIES: Gravenhorstia longicornis Robineau-Desvoidy, 1863; Tachina innoxia Meigen, 1824 (as “Masicera innoxia : Meig.").

TYPE SPECIES: Gravenhorstia longicornis Robineau-Desvoidy, 1863 [= Tachina grandicornis Zetterstedt, 1849], by original designation.

CURRENT StATUS: Preoccupied by Boie, 1836; senior (but invalid) synonym of Admontia Brauer \& Bergenstamm, 1889 [teste Herting \& Dely-Draskovits (1993: 160)].

FAMILY: TACHINIDAE.

\section{Guerinia Robineau-Desvoidy, 1830: 196.}

ORIGINAlly InCluded SPECIES: Guerinia festiva Robineau-Desvoidy, 1830; Guerinia musca RobineauDesvoidy, 1830; Guerinia vivax Robineau-Desvoidy, 1830; Guerinia winthemi Robineau-Desvoidy, 1830; Guerinia nana Robineau-Desvoidy, 1830; Guerinia gagatea Robineau-Desvoidy, 1830.

TYPE SPECIES: Guerinia festiva Robineau-Desvoidy, 1830, by subsequent designation (Coquillett, 1910: 548). CURRENT STATUS: Placed in "Doubtful taxa in Tachinidae" by Herting \& Dely-Draskovits (1993: 436).

FAMILY: TACHINIDAE.

223. Gymnocheta Robineau-Desvoidy, 1830: 371.

ORIGINALLY INCLUDED SPECIES: Tachina viridis Fallén, 1810 (as “Tachina viridis. Meig.”).

TYPE SPECIES: Tachina viridis Fallén, 1810 (as "Tachina viridis. Meig."), by monotypy.

CURRENT STATUS: Valid genus [teste Herting \& Dely-Draskovits (1993: 303)].

FAMILY: TACHINIDAE.

EMENDATIONS: Gymnochaeta Macquart, 1835: 149, 150 (unjustified).

[Gymnodia] Robineau-Desvoidy, 1830: 603.

CURRENT STATUS: Unavailable name; proposed in synonymy and not made available before 1961; treated under Pegomya Robineau-Desvoidy, 1830 [teste this work].

FAMILY: ANTHOMYIIDAE.

REMARKS: Mentioned under Chlorina phyllioidea Robineau-Desvoidy, 1830 with the notation “... j'en avais d'abord fait le genre Gymnodia; si un examen plus attentif découvre que le chète est villosule, elle devra rentrer dans le genre Phyllis." Here interpreted as proposed in synonymy with Chlorina RobineauDesvoidy, 1830 [= Pegomya Robineau-Desvoidy, 1830].

224. Gymnodia Robineau-Desvoidy, 1863b: 635.

ORIGINALLY INCLUDED SPECIES: Gymnodia pratensis Robineau-Desvoidy, 1863.

TYPE SPECIES: Gymnodia pratensis Robineau-Desvoidy, 1863 [= Anthomya polystigma Meigen, 1826], by monotypy. 
CURRENT STATUS: Valid genus [teste this work].

FAMILY: MUSCIDAE.

REMARKS: There are two original spellings of this genus-group name in Robineau-Desvoidy (1863b): Gymnodia (page 635) and Cymnodia (page 883). Acting as First Reviser, we select Gymnodia as the correct original spelling. This genus-group name is not preoccupied by Gymnodia Robineau-Desvoidy, 1830, which is an unavailable name (see above). Gymnodia Robineau-Desvoidy, 1863 is treated here as a senior synonym of Brontaea Kowarz, 1873. New status.

[Gymnomya] Robineau-Desvoidy, 1830: 692.

CURRENT STATUS: Unavailable name; name proposed in synonymy and not made available before 1961; treated as incertae sedis in Sciomyzidae [teste this work].

FAMILY: SCIOMYZIDAE.

REMARKS: Mentioned under Hydromya rubicunda Robineau-Desvoidy, 1830 with the notation “... j'en avais d'abord formé le genre Gymnomya, qu'il faudra peut-être rétablir'. Here interpreted as proposed in synonymy with Hydromya Robineau-Desvoidy, 1830 [incertae sedis in Sciomyzidae]. Gymnomyia is listed by Verrall in Scudder (1882: 150) as an emendation of Gymnomya, but unavailable names cannot be emended so it is itself a nomen nudum.

225. Haematobia Robineau-Desvoidy, 1830: 388.

ORIGINAlly INCLUdED SPECIES: Haematobia ferox Robineau-Desvoidy, 1830; Haematobia geniculata Robineau-Desvoidy, 1830; Haematobia serrata Robineau-Desvoidy, 1830; Haematobia tibialis Robineau-Desvoidy, 1830.

TYPE SPECIES: Haematobia ferox Robineau-Desvoidy, 1830 [= Conops irritans Linnaeus, 1758, n. syn.], by present designation.

CURRENT STATUS: Preoccupied by Le Peletier \& Serville, 1828; junior synonym of Haematobia Le Peletier \& Serville, 1828, n. syn.

FAMILY: MUSCIDAE.

REMARKS: Robineau-Desvoidy (1830: 388) proposed Haematobia as a new genus [which actually originated in his 1826 manuscript sent to the Académie des Sciences]. Le Peletier \& Serville (1828) had proposed the same name and generic concept two years earlier [and credited Robineau-Desvoidy with the name "Haematobia. Robin. ined."], no doubt based on seeing Robineau-Desvoidy's treatment of it in the Myodaires manuscript although it does not appear in the Blainville Rapport to the Académie des Sciences. But because there is no evidence in the Encyclopédie Méthodique itself that RobineauDesvoidy gave anything more than the name, it must take the authorship of Le Peletier \& Serville.

EMENDATIONS: Hoematobia Bigot, 1892: 192 (unjustified), n. syn.

[Haematomyza] Robineau-Desvoidy, 1863b: 391.

CURRENT STATUS: Unavailable name; proposed in synonymy and not made available before 1961; treated under Prosena Le Peletier \& Serville, 1828 [teste this work].

FAMILY: TACHINIDAE.

REMARKS: Monceaux, in preparing the manuscript for Robineau-Desvoidy's 1863 work, apparently misinterpreted Fallén's (1818) Haematomyzides-a name above the family-group and given in the Latin genitive declension ("Haematomyzidum") in the title of that work-as a genus-group name and put it in association with an available name (siberita; sensu Fallén). This is here interpreted as proposed in synonymy with Prosena Le Peletier \& Serville, 1828. 
226. Halesa Robineau-Desvoidy, 1863a: 315.

ORIGINALLY INCLUDED SPECIES: Halesa festinans Robineau-Desvoidy, 1863.

TYPE SPECIES: Halesa festinans Robineau-Desvoidy, 1863, by original designation.

CURRENT Status: Preoccupied by Walker, 1860; no new replacement name proposed; placed in "Doubtful taxa in Tachinidae" by Herting \& Dely-Draskovits (1993: 436).

FAMILY: TACHINIDAE.

227. Hamulia Robineau-Desvoidy, 1863b: 110.

ORIGINAlly INCLUdED SPECIES: Hamulia macquarti Robineau-Desvoidy, 1863; Miltogramma punctata

Meigen, 1824; Hamulia lateralis Robineau-Desvoidy, 1863 (as “Hamulia lateralis, Macq.").

TYPE SPECIES: Miltogramma punctata Meigen, 1824, by original designation.

CURRENT STATUS: Junior synonym of Miltogramma Meigen, 1803 [teste Pape (1996: 104)].

FAMILY: SARCOPHAGIDAE.

REMARKS: Robineau-Desvoidy (1830) attributed Hamulia lateralis to Macquart and gave a reference to "Miltogramma lateralis : Macq." as taken from a labeled specimen received from him. However, Macquart never described such a species and the nominal species is here attributed to RobineauDesvoidy, 1830 as "Hamulia lateralis".

228. Harrisia Robineau-Desvoidy, 1830: 323.

ORIGINAlly InCLUDED SPECIES: Harrisia scutellaris Robineau-Desvoidy, 1830; Harrisia brasiliensis Robineau-Desvoidy, 1830.

TYPE SPECIES: Harrisia scutellaris Robineau-Desvoidy, 1830, by subsequent designation (Townsend, 1916: 7).

CURRENT STATUS: Valid genus [teste Guimarães (1971: 185)].

FAMILY: TACHINIDAE.

\section{Hartigia Robineau-Desvoidy, 1863b: 521.}

ORIGINALly INCLUDED SPECIES: Hartigia strenua Robineau-Desvoidy, 1863; Hartigia sistoria RobineauDesvoidy, 1863; Hartigia flavescens Robineau-Desvoidy, 1863; Hartigia opaca Robineau-Desvoidy, 1863; Hartigia concolor Robineau-Desvoidy, 1863; Hartigia claripennis Robineau-Desvoidy, 1863; Hartigia villana Robineau-Desvoidy, 1863; Hartigia myoidea Robineau-Desvoidy, 1863 (as "Hartigia myoïdea, R.-D.”); Hartigia grisella Robineau-Desvoidy, 1863; Myophora obscuripennis RobineauDesvoidy, 1830; Hartigia socia Robineau-Desvoidy, 1863; Hartigia sordida Robineau-Desvoidy, 1863; Hartigia tristis Robineau-Desvoidy, 1863; Hartigia fuscipennis Robineau-Desvoidy, 1863; Phorella despecta Robineau-Desvoidy, 1830; Hartigia tenella Robineau-Desvoidy, 1863; Hartigia lugubris Robineau-Desvoidy, 1863.

TYPE SPECIES: Hartigia concolor Robineau-Desvoidy, 1863 [= Sarcophaga dissimilis Meigen, 1826], by original designation.

CURREnT StATUS: Preoccupied by Schiødte, 1838; senior (but invalid) synonym of Heteronychia Brauer \& Bergenstamm, 1889 (subgenus of Sarcophaga Meigen, 1826) [teste Pape (1996: 321)].

FAMILY: SARCOPHAGIDAE.

230. Haustellia Robineau-Desvoidy, 1853a: 127 [1853b: 47].

ORIGINALLY INCLUDED SPECIES: Myopa occulta Wiedemann, 1824.

TYPE SPECIES: Myopa occulta Wiedemann, 1824, by original designation.

CURRENT StATUS: Junior synonym of Myopa Fabricius, 1775 [teste Chvála \& Smith (1988: 262)].

FAMILY: CONOPIDAE. 
231. Haydaea Robineau-Desvoidy, 1863a: 563.

ORIGINALLY INCLUDED SPECIES: Haydaea frontalina Robineau-Desvoidy, 1863.

TYPE SPECIES: Haydaea frontalina Robineau-Desvoidy, 1863 [= Phorocera cinerea Macquart, 1850], by original designation.

CURRENT STATUS: Junior synonym of Istocheta Rondani, 1859 [teste Herting \& Dely-Draskovits (1993: 154)].

FAMILY: TACHINIDAE.

\section{Hebia Robineau-Desvoidy, 1830: 98.}

ORIGINALLY INCLUDED SPECIES: Hebia flavipes Robineau-Desvoidy, 1830.

TYPE SPECIES: Hebia flavipes Robineau-Desvoidy, 1830, by monotypy.

CURRENT STATUS: Valid genus [teste Herting \& Dely-Draskovits (1993: 253)].

FAMILY: TACHINIDAE.

233. Helina Robineau-Desvoidy, 1830: 493.

ORIGINALly INCLUDED SPECIES: Helina euphemioidea Robineau-Desvoidy, 1830 (as "Helina euphemiö̈dea.

R. D.”); Helina aricioidea Robineau-Desvoidy, 1830 (as “Helina ariciö̈dea. R. D.”); Helina bipunctata Robineau-Desvoidy, 1830; Helina claripennis Robineau-Desvoidy, 1830; Helina nigripes RobineauDesvoidy, 1830; Helina tibialis Robineau-Desvoidy, 1830; Helina rustica Robineau-Desvoidy, 1830.

TYPE SPECIES: Helina euphemioidea Robineau-Desvoidy, 1830 [= Anthomyia pertusa Meigen, 1826], by subsequent designation (Coquillett, 1901: 137).

CURRENT STATUS: Valid genus [teste Pont (1986b: 135)].

FAMILY: MUSCIDAE.

234. Hemithaea Robineau-Desvoidy, 1863a: 318.

ORIGINALLY INCLUDED SPECIES: Tachina erythrostoma Hartig, 1837.

TYPE SPECIES: Tachina erythrostoma Hartig, 1837, by original designation.

CURRENT StATUS: Junior synonym of Phryxe Robineau-Desvoidy, 1830 [teste Herting \& Dely-Draskovits (1993: 192)].

FAMILY: TACHINIDAE.

235. Hemyda Robineau-Desvoidy, 1830: 226.

ORIGINALLY INCLUDED SPECIES: Hemyda aurata Robineau-Desvoidy, 1830.

TYPE SPECIES: Hemyda aurata Robineau-Desvoidy, 1830, by monotypy.

CURRENT STATUS: Valid genus [teste Herting \& Dely-Draskovits (1993: 432)].

FAMILY: TACHINIDAE.

236. Heramya Robineau-Desvoidy, 1830: 709.

ORIGINALLY INCLUDED SPECIES: Oscinis nebulosa Olivier in Latreille, 1811 (as "Oscinis nebulosa. Latr.");

Heramya populicola Robineau-Desvoidy, 1830 (as "Oscinis populicola. Latr.”).

TYPE SPECIES: Oscinis nebulosa Olivier in Latreille, 1811, by subsequent designation (Hennig, 1939: 23).

CURRENT STATUS: Junior synonym of Otites Latreille, 1804 [teste Soós (1984c: 47)].

FAMILY: ULIDIIDAE.

REMARKS: "Oscinis populicola" was never described by Latreille, thus the nominal species included by

Robineau-Desvoidy is attributed to Robineau-Desvoidy, 1830 as "Heramya populicola".

EMENDATIONS: Heramyia Agassiz, 1846b: 178 (unjustified). 
237. Herbina Robineau-Desvoidy, 1830: 698.

ORIGINALLY INCLUDED SPECIES: Herbina suillioidea Robineau-Desvoidy, 1830 (as "Herbina suillioïdea. R.

D.”); Herbina rubetra Robineau-Desvoidy, 1830.

TYPE SPECIES: Herbina suillioidea Robineau-Desvoidy, 1830, by subsequent designation (Coquillett, 1910: $550)$.

CURRENT Status: Junior synonym of Suillia Robineau-Desvoidy, 1830 [teste Gorodkov (1984: 34)].

FAMILY: HELEOMYZIDAE.

\section{Herbstia Robineau-Desvoidy, 1851d: 184.}

ORIGINALLY INCLUDED SPECIES: Herbstia tibialis Robineau-Desvoidy, 1851.

TYPE SPECIES: Herbstia tibialis Robineau-Desvoidy, 1851, by monotypy.

CuRRent Status: Preoccupied by Edwards, 1834; senior (but invalid) synonym of Peribaea Robineau-

Desvoidy, 1863 [teste Herting \& Dely-Draskovits (1993: 331)].

FAMILY: TACHINIDAE.

REMARKS: Placed on the Official Index of Rejected and Invalid Generic Names in Zoology by action of I.C.Z.N. (1964: 343 [Opinion 712]).

\section{Herilla Robineau-Desvoidy, 1863a: 317.}

ORIGINALLY INCLUDED SPECIES: Herilla velox Robineau-Desvoidy, 1863.

TYPE SPECIES: Herilla velox Robineau-Desvoidy, 1863, by original designation.

CURRENT STATUS: Preoccupied by Adams, 1855; no new replacement name proposed; placed in "Doubtful taxa in Tachinidae" by Herting \& Dely-Draskovits (1993: 436).

FAMILY: TACHINIDAE.

\section{Herina Robineau-Desvoidy, 1830: 724.}

ORIGINAlly InCluded SPECIES: Herina violacea Robineau-Desvoidy, 1830; Herina sinensis RobineauDesvoidy, 1830; Herina marginata Robineau-Desvoidy, 1830; Herina thoracica Robineau-Desvoidy, 1830; Herina liturata Robineau-Desvoidy, 1830; Herina limbata Robineau-Desvoidy, 1830; Herina scutellaris Robineau-Desvoidy, 1830; Herina longicornis Robineau-Desvoidy, 1830; Herina fasciata Robineau-Desvoidy, 1830; Herina violascens Robineau-Desvoidy, 1830; Herina rufipes RobineauDesvoidy, 1830.

TYPE SPECIES: Herina liturata Robineau-Desvoidy, 1830 [= Ortalis nigrina Meigen, 1826], by subsequent designation (Hennig, 1939: 59).

CURRENT STATUS: Valid genus [teste Soós (1984c: 54)].

FAMILY: ULIDIIDAE.

REMARKS: Designations by Westwood $(1840)$ and Rondani $(1856,1869)$ are invalid because they are for nominal species that were not originally included. Previous workers have indicated Musca germinationis Rossi, 1790 as the senior synonym of Herina liturata Robineau-Desvoidy, 1830. However, Rossi's name is preoccupied by Musca germinationis Linnaeus, 1758. Strobl (1910: 188) was the first to indicate Ortalis nigrina Meigen, 1826 as the valid name for Musca germinationis Rossi, 1790, which we follow here. There are no new synonymies as all three names have been in synonymy previously.

\section{Hermya Robineau-Desvoidy, 1830: 226.}

Originally InCluded SPeCIES: Hermya afra Robineau-Desvoidy, 1830; Hermya hottentota RobineauDesvoidy, 1830.

TYPE SPECIES: Hermya afra Robineau-Desvoidy, 1830 [= Ocyptera diabolus Wiedemann, 1819], by subsequent designation (Townsend, 1916: 7). 
CURRENT Status: Valid genus [teste Herting \& Dely-Draskovits (1993: 400)].

FAMILY: TACHINIDAE.

EMENDATIONS: Hermyia Verrall in Scudder, 1882: 160 (unjustified), n. syn.

242. Hersilia Robineau-Desvoidy, 1863a: 499.

ORIGINAlly InCluded SPECIES: Hersilia cinerea Robineau-Desvoidy, 1863; Hersilia silvatica RobineauDesvoidy, 1863; Hersilia floralis Robineau-Desvoidy, 1863.

TYPE SPECIES: Hersilia cinerea Robineau-Desvoidy, 1863, by original designation.

CuRRENT STAtus: Preoccupied by Savigny, 1826; Dejean, 1835; Philippi, 1839; junior synonym of Oswaldia Robineau-Desvoidy, 1830 [teste Herting (1974: 13)].

FAMILY: TACHINIDAE.

REMARKS: Herting \& Dely-Draskovits (1993: 436) placed Hersilia in "Doubtful taxa in Tachinidae", but it is more properly placed in synonymy with Oswaldia as discussed by Herting (1974: 13), with its type species $H$. cinerea as a doubtful species of Oswaldia.

243. Hesione Robineau-Desvoidy, 1863a: 199.

ORIGINALLY INCLUDED SPECIES: Hesione microcera Robineau-Desvoidy, 1863.

TYPE SPECIES: Hesione microcera Robineau-Desvoidy, 1863, by original designation.

CuRRENT STATUS: Preoccupied by Rafinesque, 1815; Lamarck, 1818; Savigny, 1818; junior synonym of Macquartia Robineau-Desvoidy, 1830 [teste Herting \& Dely-Draskovits (1993: 312)].

FAMILY: TACHINIDAE.

244. Hesyquillia Robineau-Desvoidy, 1830: 708.

ORIGINALly INCLUdED SPECIES: Hesyquillia lugubris Robineau-Desvoidy, 1830; Musca seminationis Fabricius, 1775 (as "Musca seminationis. Linn.”).

TYPE SPECIES: Hesyquillia lugubris Robineau-Desvoidy, 1830, by subsequent designation (Coquillett, 1910: $551)$.

CuRRent Status: Junior synonym of Platystoma Meigen, 1803 [teste Soós (1984b: 40)].

FAMILY: PLATYSTOMATIDAE.

[Heterellia] Robineau-Desvoidy MS name (Stuke \& Clement, 2005: 4).

CURRENT StATUS: Unavailable name; manuscript name based on label data; treated as a nomen dubium in Conopidae [teste this work].

FAMILY: CONOPIDAE.

REMARKS: Stuke \& Clement (2005) reviewed the Conopidae taxa described by Robineau-Desvoidy (1830, 1853a,b) based on type specimens found in the Oxford University Museum of Natural History and listed this genus-group name.

245. Himera Robineau-Desvoidy, 1863a: 1124.

ORIGINALly INCLUDED SPECIES: Himera scutellaris Robineau-Desvoidy, 1863; Guerinia nana RobineauDesvoidy, 1830; Himera apicata Robineau-Desvoidy, 1863; Himera meigenii Robineau-Desvoidy, 1863 (with "Exorista hortulana : Meig." in synonymy).

TYPE SPECIES: Himera scutellaris Robineau-Desvoidy, 1863, by subsequent designation (Townsend, 1916:

$7)$. 
CuRrent STAtus: Preoccupied by Duponchel, 1829; no new replacement name proposed; placed in

"Doubtful taxa in Tachinidae" by Herting \& Dely-Draskovits (1993: 436).

FAMILY: TACHINIDAE.

246. Hubertia Robineau-Desvoidy, 1863b: 169.

ORIGINALLY INCLUDED SPECIES: Hubertia elegans Robineau-Desvoidy, 1863.

TYPE SPECIES: Hubertia elegans Robineau-Desvoidy, 1863, by original designation.

CURREnt Status: Junior synonym of Bessa Robineau-Desvoidy, 1863 [teste Herting \& Dely-Draskovits (1993: 142)].

FAMILY: TACHINIDAE.

\section{Hubneria Robineau-Desvoidy, 1848a: 601.}

ORIGINALly InCLUDED SPECIES: Hubneria gagatea Robineau-Desvoidy, 1848; Hubneria vivida RobineauDesvoidy, 1848; Carcelia distincta Robineau-Desvoidy, 1830; Carcelia duponcheli Robineau-Desvoidy, 1830; Carcelia nigripes Robineau-Desvoidy, 1830; Carcelia plusiae Robineau-Desvoidy, 1830; Hubneria caesia Robineau-Desvoidy, 1848; Hubneria apicalis Robineau-Desvoidy, 1848; Hubneria festiva Robineau-Desvoidy, 1848; Hubneria subaenea Robineau-Desvoidy, 1848; Hubneria nigrita Robineau-Desvoidy, 1848; Hubneria hilaris Robineau-Desvoidy, 1848; Hubneria rustica RobineauDesvoidy, 1848; Carcelia diversa Robineau-Desvoidy, 1830; Tachina alacris Meigen, 1824; Tachina arvicola Meigen, 1824; Hubneria campestris Robineau-Desvoidy, 1848; Hubneria pallidipes RobineauDesvoidy, 1848; Hubneria nitida Robineau-Desvoidy, 1848; Carcelia fuscipennis Robineau-Desvoidy, 1830; Hubneria nigripalpis Robineau-Desvoidy, 1848; Carcelia velox Robineau-Desvoidy, 1830.

TYPE SPECIES: Carcelia nigripes Robineau-Desvoidy, 1830 [= Tachina affinis Fallén, 1810], by subsequent designation (Robineau-Desvoidy, 1863a: 279).

CURRENT STATUS: Valid genus [teste Herting \& Dely-Draskovits (1993: 211)].

FAMILY: TACHINIDAE.

REMARKS: Robineau-Desvoidy (1863a: 279) designated "Tachina affinis, Fall." which was not an originally included species; however, according to the ICZN Code Article 69.2.2, the designation is valid by the listing of a single originally included species (Carcelia nigripes Robineau-Desvoidy, 1830) in synonymy.

EMENDATIONS: Huebneria Marschall, 1873: 334 (as Hübneria) (unjustified).

248. Hyalomya Robineau-Desvoidy, 1830: 298.

ORIGINAlly INCluded SPECIES: Phasia atropurpurea Meigen, 1824 (as "Phasia atro-pupurea. Meig."); Phasia hamata Meigen, 1824 (as "Phasia hamata. Latr. Meig."); Thereva obesa Fabricius, 1798; Hyalomya basalis Robineau-Desvoidy, 1830; Phasia albipennis Meigen, 1824; Hyalomya nebulosa Robineau-Desvoidy, 1830; Hyalomya carbonaria Robineau-Desvoidy, 1830; Phasia pusilla Meigen, 1824 (as "Phasia pusilla. Hoff. Meig."); Hyalomya corinna Robineau-Desvoidy, 1830; Phasia hyalipennis Meigen, 1824 (as "Phasia hyalipennis. Fallén. Meig,”); Phasia semicinerea Meigen, 1824 (as "Phasia semi-cinerea. Meig.").

TYPE SPECIES: Phasia semicinerea Meigen, 1824 [= Phasia pusilla Meigen, 1824], by subsequent designation (Westwood, 1840: 140).

CURRENT Status: Junior synonym of Phasia Meigen, 1824 [teste O’Hara \& Wood (2004: 227)].

FAMILY: TACHINIDAE.

REMARKS: Duponchel in d'Orbigny (1845b: 721) and Robineau-Desvoidy (1863b: 241) designated as type species Phasia atropurpurea Meigen, 1824 (the first of the twelve originally included species), but these were later.

EMENDATIONS: Hyalomyia Macquart, 1834a: 205 (unjustified). 
249. Hydrellia Robineau-Desvoidy, 1830: 790.

ORIGINALly INCLUDED SPECIES: Hydrellia communis Robineau-Desvoidy, 1830; Hydrellia aurifacies Robineau-Desvoidy, 1830; Hydrellia nitida Robineau-Desvoidy, 1830; Hydrellia chrysina RobineauDesvoidy, 1830; Hydrellia chrysella Robineau-Desvoidy, 1830; Hydrellia viridula Robineau-Desvoidy, 1830; Hydrellia brunifacies Robineau-Desvoidy, 1830; Hydrellia fuliginosa Robineau-Desvoidy, 1830; Hydrellia nigripes Robineau-Desvoidy, 1830; Hydrellia viridescens Robineau-Desvoidy, 1830; Hydrellia argyria Robineau-Desvoidy, 1830.

TYPE SPECIES: Hydrellia communis Robineau-Desvoidy, 1830 [= Notiphila griseola Fallén, 1813], by subsequent designation (Duponchel in d'Orbigny, 1845b: 743).

CURRENT STATUS: Valid genus [teste Sabrosky (1999: 161)].

FAMILY: EPHYDRIDAE.

REMARKS: The I.C.Z.N. (1985: 177 [Opinion 1321]), in dealing with the nomenclatural precedence of two family-group names in Ephydridae, placed Hydrellia on the Official List of Generic Names in Zoology and at the time thought that the earliest type-species designation was Hydrellia aurifacies RobineauDesvoidy, 1830 by Coquillett (1910: 553). The earlier designation of Hydrellia communis RobineauDesvoidy, 1830 by Duponchel in d'Orbigny (1845: 743) does not change the nomenclature or taxonomy of the genus-group name and is accepted here.

250. Hydrina Robineau-Desvoidy, 1830: 794

ORIGINAlly INCLUded SPECIES: Hydrina nitida Robineau-Desvoidy, 1830; Hydrina aurata RobineauDesvoidy, 1830; Hydrina vernalis Robineau-Desvoidy, 1830; Hydrina nigricruris Robineau-Desvoidy, 1830; Hydrina maculipennis Robineau-Desvoidy, 1830; Hydrina viridula Robineau-Desvoidy, 1830; Hydrina cardaminis Robineau-Desvoidy, 1830.

TYPE SPECIES: Hydrina vernalis Robineau-Desvoidy, 1830 [= Notiphila guttata Fallén, 1813], by subsequent designation (Coquillett, 1910: 553).

CURRENT STATUS: Preoccupied by Rafinesque, 1815; senior (but invalid) synonym of Hyadina Haliday, 1837 [teste Sabrosky (1999: 162)].

FAMILY: EPHYDRIDAE.

251. Hydromya Robineau-Desvoidy, 1830: 691.

ORIGINALLY INCLUDED SPECIES: Hydromya caeruleipennis Robineau-Desvoidy, 1830; Hydromya rubicunda Robineau-Desvoidy, 1830.

TYPE SPECIES: Hydromya caeruleipennis Robineau-Desvoidy, 1830 [= Musca dorsalis Fabricius, 1775], by subsequent designation (Rozkošný \& Elberg, 1984: 183).

CURRENT STATUS: Valid genus [teste Rozkošný \& Elberg (1984: 183)].

FAMILY: SCIOMYZIDAE.

Emendations: Hydromyia Curtis, 1837: 280 (unjustified), n. syn.; Hydromyia Agassiz, 1846b: 188 (unjustified).

252. Hydrophoria Robineau-Desvoidy, 1830: 503.

ORIGINALly INCLUDED SPECIES: Hydrophoria potamogeti Robineau-Desvoidy, 1830; Hydrophoria nymphaeae Robineau-Desvoidy, 1830; Hydrophoria trapae Robineau-Desvoidy, 1830; Hydrophoria fuliginosa Robineau-Desvoidy, 1830; Hydrophoria tibialis Robineau-Desvoidy, 1830; Hydrophoria sagittariae Robineau-Desvoidy, 1830; Hydrophoria nymphaeicola Robineau-Desvoidy, 1830; Hydrophoria testacea Robineau-Desvoidy, 1830; Hydrophoria littoralis Robineau-Desvoidy, 1830.

TYPE SPECIES: Musca lancifer Harris, 1780, by subsequent designation (I.C.Z.N., 1996: 62 [Opinion 1827]). CURRENT STATUS: Valid genus [teste Sabrosky (1999: 162)]. 
FAMILY: ANTHOMYIIDAE.

REMARKS: Coquillett (1910: 554) stated "Type, Musca vespertina FALLEN (as littoralis, new species), the last species, by designation of WESTWOOD, Intr., vol. 2, Synops., 1840, p. 142 (as nigrita Fallén)”. Westwood (1840) did not designate the species Hydrophoria littoralis. He stated (1840: 142) "HYDROPHORIA Mcq. Aricinae Littorales R. D. 31 sp. M. nigrita Fall.”. Coquillett confused Robineau-Desvoidy's group "Aricines littorales" with the species Hydrophoria littoralis. By using an originally included species, Coquillett (1910: 554) is a valid type species designation for Hydrophoria, but its acceptance would place the genus-group name in Muscidae and upset common usage of it in the Anthomyiidae (and of the name Hebecnema Schnabl, 1889 in the Muscidae). Therefore, Griffiths (1994) applied to the ICZN Commission to set aside all previous designations for Hydrophoria and designate Musca lancifer Harris, 1780 as the type species. Thus, Dely-Draskovits (1993: 60) is in error when stating that the type species of Hydrophoria is "Hydrophoria littoralis ROBINEAU-DESVOIDY, 1830 ... [= lancifer (HARRIS [1780])]".

\section{Hydrotaea Robineau-Desvoidy, 1830: 509.}

ORIGINALLY INCLUDED SPECIES: Hydrotaea monacantha Robineau-Desvoidy, 1830; Hydrotaea nebulosa Robineau-Desvoidy, 1830; Hydrotaea inermis Robineau-Desvoidy, 1830; Hydrotaea claripennis Robineau-Desvoidy, 1830; Hydrotaea nymphaeae Robineau-Desvoidy, 1830; Hydrotaea flavifacies Robineau-Desvoidy, 1830; Hydrotaea cinerea Robineau-Desvoidy, 1830; Hydrotaea riparia RobineauDesvoidy, 1830; Hydrotaea fuliginosa Robineau-Desvoidy, 1830; Hydrotaea palpalis RobineauDesvoidy, 1830; Musca meteorica Linnaeus, 1758; Hydrotaea gracilis Robineau-Desvoidy, 1830; Hydrotaea velutina Robineau-Desvoidy, 1830; Hydrotaea gagatea Robineau-Desvoidy, 1830; Hydrotaea nitida Robineau-Desvoidy, 1830; Hydrotaea atrata Robineau-Desvoidy, 1830; Hydrotaea floralis Robineau-Desvoidy, 1830.

TYPE SPECIES: Musca meteorica Linnaeus, 1758, by subsequent designation (Curtis, 1839: pl. 768).

CuRRent Status: Valid genus [teste Pont (1986b: 74)].

FAMILY: MUSCIDAE.

REMARKS: Later designations were by Westwood (1840: 142) of Musca meteorica Linnaeus, 1758; Rondani (1856: 94) of Musca dentipes Fabricius, 1805; and Karl (1928: 39) of Hydrotaea velutina RobineauDesvoidy, 1830; but none of these species were among the originally included species, so the designations are invalidly proposed.

EMENDATIONS: Hydrothea Pandellé, 1898: 7 (unjustified).

254. Hylemya Robineau-Desvoidy, 1830: 550.

ORIGINALly InCLUded SPeCIES: Hylemya strenua Robineau-Desvoidy, 1830; Hylemya plebeia RobineauDesvoidy, 1830 (as “Musca plebeia. Fabric. Anthomyia Meig."); Hylemya silvicola Robineau-Desvoidy, 1830; Hylemya luteipes Robineau-Desvoidy, 1830; Hylemya hyemalis Robineau-Desvoidy, 1830; Hylemya tibialis Robineau-Desvoidy, 1830; Hylemya vivida Robineau-Desvoidy, 1830; Hylemya nigripes Robineau-Desvoidy, 1830; Hylemya rustica Robineau-Desvoidy, 1830; Hylemya liturata Robineau-Desvoidy, 1830; Hylemya flavipennis Robineau-Desvoidy, 1830; Hylemya florea RobineauDesvoidy, 1830; Hylemya autumnalis Robineau-Desvoidy, 1830; Hylemya flavoptera RobineauDesvoidy, 1830; Hylemya arvensis Robineau-Desvoidy, 1830; Hylemya agrestis Robineau-Desvoidy, 1830.

TyPE SPeCIES: Hylemya strenua Robineau-Desvoidy, 1830 [= Musca vagans Panzer, 1798], by subsequent designation (Rondani, 1866: 74).

CURRENT STATUS: Valid genus [teste Sabrosky (1999: 163)].

FAMILY: ANTHOMYIIDAE.

REMARKS: Rondani (1856: 96) designated Musca strigosa "Fall." but this was not a valid designation because it was not an originally included species. Rondani (1866: 74) was the first valid designation by including 
Hylemya strenua Robineau-Desvoidy, 1830 as a synonym of Musca strigosa Fabricius, 1794. DelyDraskovits (1993: 63) mistakenly gave the type species as Musca vagans Panzer, 1798 as designated by Rondani (1866: 74). Musca vagans Panzer, 1798 is the oldest taxonomically valid species because Musca strigosa Fabricius, 1794 is preoccupied by Musca strigosa Gmelin, 1790, but it was not the nominal species name designated by Rondani (1866: 74). Although Robineau-Desvoidy (1830: 551) described a "Hylemya plebeia. Fabr." and cited under it "Musca plebeia. Fabric. Anthomyia Meig.", there is no nominal species that either Fabricius or Meigen described that could be considered the same as Robineau-Desvoidy's species. Coquillett (1910: 554) stated that Hylemya plebeia Robineau-Desvoidy, 1830 was a new species and we treat it as such here.

EMENDATIONs: Hylemyia Macquart, 1835: 315 (unjustified); Hylemia Marié, 1930: 227 (unjustified), n. syn.

\section{Hyperaea Robineau-Desvoidy, 1863b: 379.}

ORIGINALLY INCLUDED SPECIES: Hyperaea abdominalis Robineau-Desvoidy, 1863.

TYPE SPECIES: Hyperaea abdominalis Robineau-Desvoidy, 1863 [= Tachina femoralis Meigen, 1824], by monotypy.

CURRENT STATUS: Valid genus [teste Herting \& Dely-Draskovits (1993: 348)].

FAMILY: TACHINIDAE.

\section{Hyria Robineau-Desvoidy, 1863a: 1100.}

ORIGINALly INCLUDED SPECIES: Tachina tibialis Fallén, 1810 (as “Macquartia tibialis, Meig.”).

TYPE SPECIES: Tachina tibialis Fallén, 1810, by original designation (as "Macquartia tibialis, Meig.").

CURRENT Status: Preoccupied by Lamarck, 1819; Stephens, 1829; senior (but invalid) synonym of

Pelatachina Meade, 1894 [teste Herting \& Dely-Draskovits (1993: 311)].

FAMILY: TACHINIDAE.

\section{Icelia Robineau-Desvoidy, 1830: 224.}

ORIGINALly InCLUDED SPECIES: Icelia flavescens Robineau-Desvoidy, 1830; Icelia brasiliensis RobineauDesvoidy, 1830.

TYPE SPECIES: Icelia flavescens Robineau-Desvoidy, 1830, by subsequent designation (Townsend, 1916: 7).

CURRENT STATUS: Valid genus [teste O'Hara \& Wood (2004: 256)].

FAMILY: TACHINIDAE.

258. Ida Robineau-Desvoidy, 1863b: 389.

ORIGINALLY INCLUDED SPECIES: Ida petiolata Robineau-Desvoidy, 1863.

TYPE SPECIES: Ida petiolata Robineau-Desvoidy, 1863 [= Musca rustica Fabricius, 1775], by monotypy.

CuRREnT STATUS: Preoccupied by Jay, 1836; Gray, 1838; junior synonym of Dexia Meigen, 1826 [teste Herting \& Dely-Draskovits (1993: 365)].

FAMILY: TACHINIDAE.

\section{Ilaesa Robineau-Desvoidy, 1863a: 498.}

ORIGINAlly InCluded SPECIES: Ilaesa flavisquamis Robineau-Desvoidy, 1863; Phorocera delecta Meigen, 1838.

TYPE SPECIES: Ilaesa flavisquamis Robineau-Desvoidy, 1863 [= Anthomya polystigma Meigen, 1826], by original designation.

CURRENT STATUS: Placed in "Doubtful taxa in Tachinidae" by Herting \& Dely-Draskovits (1993: 436).

FAMILY: TACHINIDAE. 
260. Illigeria Robineau-Desvoidy, 1830: 273.

ORIGINALLY INCLUDED SPECIES: Illigeria atra Robineau-Desvoidy, 1830; Illigeria minor RobineauDesvoidy, 1830.

TYPE SPECIES: Illigeria atra Robineau-Desvoidy, 1830 [= Musca roralis Linnaeus, 1758], by subsequent designation (Townsend, 1916: 7).

CURRENT StATUS: Junior synonym of Melanophora Meigen, 1803 [teste Herting (1993: 116)].

FAMILY: RHINOPHORIDAE.

\section{Ismenia Robineau-Desvoidy, 1863a: 585.}

ORIGINALly INCLUDED SPECIES: Erycia villica Robineau-Desvoidy, 1830; Ismenia aestivalis RobineauDesvoidy, 1863; Ismenia campestris Robineau-Desvoidy, 1863; Ismenia conducta Robineau-Desvoidy, 1863; Ismenia pratensis Robineau-Desvoidy, 1863.

TYPE SPECIES: Erycia villica Robineau-Desvoidy, 1830, by subsequent designation (Townsend, 1916: 7).

CURRENT STATUS: Preoccupied by King, 1850; no new replacement name proposed; placed in "Doubtful taxa in Tachinidae" by Herting \& Dely-Draskovits (1993: 436).

FAMILY: TACHINIDAE.

REMARKS: Robineau-Desvoidy's (1863a: 584) type-species designation of Erycia villica Robineau-Desvoidy, 1830 was not for the genus Ismenia but for the tribe "Ismenidae". Sabrosky (1999: 167) contended that "Since there is only one genus in the tribe, that is virtually a designation for the genus also, and I have accepted it as such". We do not agree with this interpretation, and accept Townsend's (1916) designation as the earliest one for the genus.

\section{Isomera Robineau-Desvoidy, 1851f: 315.}

ORIGINALly INCLUDED SPECIES: Reaumuria blondeli Robineau-Desvoidy, 1830; Isomera parisiaca Robineau-Desvoidy, 1851.

TYPE SPECIES: Reaumuria blondeli Robineau-Desvoidy, 1830, by subsequent designation (Coquillett, 1910: 556; as "Reaumeria blondeli DESVOIDY").

CurRent Status: Placed in "Doubtful taxa in Tachinidae" by Herting \& Dely-Draskovits (1993: 436).

FAMILY: TACHINIDAE.

REMARKS: Herting (1974: 20-21) established the type species Reaumuria blondeli as a nomen dubium, and this interpretation was followed by Herting (1984: 189, note 68). Herting \& Dely-Draskovits (1993: 436) listed Isomera in "Doubtful taxa in Tachinidae" but cited Reaumuria blondeli as a synonym of Spallanzania picea [as Gonia picea] Robineau-Desvoidy, 1830, in error.

\section{Javetia Robineau-Desvoidy, 1863a: 1115.}

ORIGINALLY INCLUDED SPECIES: Macquartia germanica Robineau-Desvoidy, 1830; Javetia flavipalpis Robineau-Desvoidy, 1863; Macquartia viridescens Robineau-Desvoidy, 1830 (with Macquartia "atrata : Meig." in synonymy).

TYPE SPECIES: Macquartia germanica Robineau-Desvoidy, 1830 [= Tachina chalconota Meigen, 1824], by subsequent designation (Townsend, 1916: 7).

CURRENT StAtus: Junior synonym of Macquartia Robineau-Desvoidy, 1830 [teste Herting \& DelyDraskovits (1993: 312)].

FAMILY: TACHINIDAE.

264. Jurinia Robineau-Desvoidy, 1830: 34.

ORIGINALLY INCLUDED SPECIES: Jurinia metallica Robineau-Desvoidy, 1830; Jurinia brasiliensis RobineauDesvoidy, 1830; Jurinia aterrima Robineau-Desvoidy, 1830; Jurinia boscii Robineau-Desvoidy, 1830; 
Jurinia gagatea Robineau-Desvoidy, 1830; Jurinia indica Robineau-Desvoidy, 1830; Jurinia scutellaris Robineau-Desvoidy, 1830; Jurinia chrysiceps Robineau-Desvoidy, 1830; Jurinia leucostoma RobineauDesvoidy, 1830; Jurinia fulviventris Robineau-Desvoidy, 1830; Jurinia testacea Robineau-Desvoidy, 1830; Jurinia aurifacies Robineau-Desvoidy, 1830.

TYPE SPECIES: Jurinia gagatea Robineau-Desvoidy, 1830, by subsequent designation (Coquillett, 1910: 556). CURRENT StATUS: Valid genus [teste O'Hara \& Wood (2004: 314)].

FAMILY: TACHINIDAE.

265. Keratocera Robineau-Desvoidy, 1830: 788.

ORIGINALly INCLUDED SPECIES: Keratocera palustris Robineau-Desvoidy, 1830; Keratocera stagnicola Robineau-Desvoidy, 1830; Keratocera trapae Robineau-Desvoidy, 1830; Keratocera fulvicornis Robineau-Desvoidy, 1830; Keratocera brunipes Robineau-Desvoidy, 1830; Keratocera nigra RobineauDesvoidy, 1830; Keratocera viridescens Robineau-Desvoidy, 1830.

TYPE SPECIES: Keratocera stagnicola Robineau-Desvoidy, 1830, by subsequent designation (Cogan, 1984: 144).

CURRENT STATUS: Junior synonym of Notiphila Fallén, 1810 [teste Mathis \& Zatwarnicki (1995: 103)].

FAMILY: EPHYDRIDAE.

EMENDATION: Ceratocera Agassiz, 1846b: 73 (unjustified).

266. Kirbya Robineau-Desvoidy, 1830: 267.

ORIGINALLY INCLUDED SPECIES: Kirbya vernalis Robineau-Desvoidy, 1830; Kirbya hiemalis RobineauDesvoidy, 1830.

TYPE SPECIES: Kirbya vernalis Robineau-Desvoidy, 1830, [nomen oblitum; = Tachina moerens Meigen, 1830, nomen protectum], by subsequent designation (Robineau-Desvoidy, 1863a: 817).

CURRENT STATUS: Valid genus [teste Herting \& Dely-Draskovits (1993: 381)].

FAMILY: TACHINIDAE.

REMARKS: Previous listings of the type species have it as a junior synonym of Tachina moerens Meigen, 1830, but this is incorrect. Robineau-Desvoidy's 1830 paper came out 6 June while Meigen's 1830 paper came out six months later on 1 December [see References in this paper for dating of the former and Evenhuis (1997: 533) for dating of the latter]. However, Kirbya vernalis has not been used as a valid name after 1899 , whereas T. moerens is in prevailing usage as the valid name for the taxon. Tachina moerens has appeared as a valid name in the genus Kirbya in at least 25 publications by more than 10 authors during the past 50 years including the following: Cerretti (2001, 2004); Cerretti \& Ziegler (2004); Draber-Mońko (1982); Herting (1974, 1984); Herting \& Dely-Draskovits (1993); Herting \& Tschorsnig (1997); Hubenov (2008); Mesnil (1974); O’Hara \& Wood (2004); Pape et al. (1995); Richter (1987); Sabrosky (1999); Tschorsnig (1983, 1985, 1992); Tschorsnig \& Brechtel (1999); Tschorsnig \& Herting (1994a, 1994b, 1998, 2000); Tschorsnig \& Schmid-Egger (1993); Tschorsnig \& Ziegler (1999); Zangheri (1969). In the interests of nomenclatural stability, we hereby invoke reversal of precedence (ICZN Code Article 23.9.2) and declare Kirbya vernalis Robineau-Desvoidy, 1830 a nomen oblitum and Tachina moerens Meigen, 1830 a nomen protectum.

\section{Klugia Robineau-Desvoidy, 1863a: 787.}

ORIGINALly INCLUDED SPECIES: Tachina marginata Meigen, 1824; Klugia palpalis Robineau-Desvoidy, 1863; Klugia albiseta Robineau-Desvoidy, 1863.

TYPE SPECIES: Tachina marginata Meigen, 1824, by original designation.

CURRENT STATUS: Valid genus [teste Herting \& Dely-Draskovits (1993: 385)].

FAMILY: TACHINIDAE. 
268. Kockia Robineau-Desvoidy, 1863a: 818.

ORIGINALLY INCLUDED SPECIES: Kockia claripennis Robineau-Desvoidy, 1863; Kockia ebennia RobineauDesvoidy, 1863.

TYPE SPECIES: Kockia claripennis Robineau-Desvoidy, 1863 [= Cirillia angustifrons Rondani, 1856], by subsequent designation (Coquillett, 1910: 557).

CURRENT Status: Junior synonym of Phyto Robineau-Desvoidy, 1830 [teste Herting (1993: 110)].

FAMILY: RHINOPHORIDAE.

269. Lalage Robineau-Desvoidy, 1863a: 559.

ORIGINALLY INCLUDED SPECIES: Salia erythrocera Robineau-Desvoidy, 1830; Lalage bigotina RobineauDesvoidy, 1863; Lalage parisiaca Robineau-Desvoidy, 1863; Salia metallica Robineau-Desvoidy, 1830; Salia blondeli Robineau-Desvoidy, 1830; Salia nigricornis Robineau-Desvoidy, 1830; Musca bifasciata Fabricius, 1775.

TYPE SPECIES: Lalage bigotina Robineau-Desvoidy, 1863 [= Chetogena acuminata Rondani, 1859], by original designation.

CURRENT Status: Junior synonym of Chetogena Rondani, 1856 [teste Herting \& Dely-Draskovits (1993: 135)].

FAMILY: TACHINIDAE.

270. Lambertia Robineau-Desvoidy, 1863b: 30.

ORIGINALLY INCLUDED SPECIES: Blondelia pallidipalpis Robineau-Desvoidy, 1830.

TYPE SPECIES: Blondelia pallidipalpis Robineau-Desvoidy, 1830, by original designation.

CURRENT StATUS: Placed in "Doubtful taxa in Tachinidae" by Herting \& Dely-Draskovits (1993: 436).

FAMILY: TACHINIDAE.

271. Latreillia Robineau-Desvoidy, 1830: 104.

ORIGINALly INCLUDED SPECIES: Musca bifasciata Fabricius, 1775; Latreillia unifasciata RobineauDesvoidy, 1830; Latreillia campestris Robineau-Desvoidy, 1830; Latreillia albifrons RobineauDesvoidy, 1830; Latreillia lalandii Robineau-Desvoidy, 1830; Latreillia testacea Robineau-Desvoidy, 1830; Latreillia hirta Robineau-Desvoidy, 1830; Latreillia cuculliae Robineau-Desvoidy, 1830; Latreillia silvestris Robineau-Desvoidy, 1830; Latreillia minor Robineau-Desvoidy, 1830.

TYPE SPECIES: Musca bifasciata Fabricius, 1775, by subsequent designation (Coquillett, 1910: 558).

CuRRent Status: Preoccupied by Roux, 1830 (see below under Remarks); junior synonym of Belvosia Robineau-Desvoidy, 1830 [teste O'Hara \& Wood (2004: 160)].

FAMILY: TACHINIDAE.

REMARKS: Latreillia Robineau-Desvoidy, 1830 was published on 6 June 1830; however, no accurate date of publication has yet been found for Latreillia Roux, 1830. The I.C.Z.N. (1964: 343 [Opinion 712]) ruled Latreillia Roux, 1830 to have priority and placed Latreillia Robineau-Desvoidy, 1830 on the Official Index of Rejected and Invalid Names in Zoology.

272. Leiophora Robineau-Desvoidy, 1863a: 930.

ORIGINALLY INCLUDED SPECIES: Leiophora nitida Robineau-Desvoidy, 1863.

TYPE SPECIES: Leiophora nitida Robineau-Desvoidy, 1863 [= Tachina innoxia Meigen, 1824], by original designation.

CURRENT STATUS: Valid genus [teste Herting \& Dely-Draskovits (1993: 159)].

FAMILY: TACHINIDAE.

EMENDATIONS: Liophora Bezzi \& Stein, 1907: 282 (unjustified), n. syn. 
273. Lentiphora Robineau-Desvoidy, 1830: 656.

ORIGINALLY INCLUDED SPECIES: Lentiphora flaveola Robineau-Desvoidy, 1830.

TYPE SPECIES: Lentiphora flaveola Robineau-Desvoidy, 1830, by monotypy.

CURRENT STATUS: Valid genus [teste Gorodkov (1984: 42)].

FAMILY: HELEOMYZIDAE.

EMENDATIONS: Lentiophora Agassiz, 1846b: 204 (unjustified).

274. Leria Robineau-Desvoidy, 1830: 653.

ORIGINALLY INCLUDED SPECIES: Leria fungivora Robineau-Desvoidy, 1830; Leria humida RobineauDesvoidy, 1830; Leria domestica Robineau-Desvoidy, 1830; Leria fenestrarum Robineau-Desvoidy, 1830; Leria cuniculorum Robineau-Desvoidy, 1830; Leria subterranea Robineau-Desvoidy, 1830; Leria peleterii Robineau-Desvoidy, 1830.

TYPE SPECIES: Leria domestica Robineau-Desvoidy, 1830, by subsequent designation (Rondani, 1866: 5).

CURRENT Status: Junior synonym of Heleomyza Fallén, 1810 [teste Sabrosky (1999: 175)].

FAMILY: HELEOMYZIDAE.

\section{Leschenaultia Robineau-Desvoidy, 1830: 324}

ORIGINALly INCLUDED SPECIES: Leschenaultia cilipes Robineau-Desvoidy, 1830; Leschenaultia hirta Robineau-Desvoidy, 1830.

TYPE SPECIES: Leschenaultia cilipes Robineau-Desvoidy, 1830, by subsequent designation (Townsend, 1916: 7).

CURRENT STATUS: Valid genus [teste O'Hara \& Wood (2004: 187)].

FAMILY: TACHINIDAE.

REMARKS: The type species has been listed as a junior synonym of Tachina leucophrys Wiedemann, 1830 in previous catalogs and works, but this is incorrect. Robineau-Desvoidy's 1830 paper came out on 6 June while Wiedemann's 1830 paper came out less than three months later in September [see References in this paper for dating of the former and Evenhuis (1997: 822) for dating of the latter].

276. Leskia Robineau-Desvoidy, 1830: 100.

ORIGINALLY INCLUDED SPECIES: Leskia flavescens Robineau-Desvoidy, 1830.

TYPE SPECIES: Leskia flavescens Robineau-Desvoidy, 1830 [= Tachina aurea Fallén, 1820], by monotypy. Current Status: Valid genus [teste Herting \& Dely-Draskovits (1993: 342)].

FAMILY: TACHINIDAE.

277. Lespesia Robineau-Desvoidy, 1863a: 567.

ORIGINALLY INCLUDED SPECIES: Erycia ciliata Macquart, 1834 (as “Masicera ciliata : Macq.").

TYPE SPECIES: Achaetoneura anisotae Webber, 1930, by subsequent designation (I.C.Z.N., 1983: 97 [Opinion 1255]).

CURRENT STATUS: Valid genus [teste O'Hara \& Wood (2004: 123)].

FAMILY: TACHINIDAE.

REMARKS: Erycia ciliata Macquart, 1834 was the only species listed but Robineau-Desvoidy misidentified it. The I.C.Z.N. (1983: 97 [Opinion 1255]) designated Achaetoneura anisotae Webber, 1930 as the type species and at the same time placed Lespesia on the Official List of Generic Names in Zoology. 
278. Leucophora Robineau-Desvoidy, 1830: 562.

ORIGINALLY INCLUDED SPECIES: Leucophora grisea Robineau-Desvoidy, 1830; Leucophora floralis Robineau-Desvoidy, 1830; Leucophora cinerea Robineau-Desvoidy, 1830; Leucophora sericea Robineau-Desvoidy, 1830; Leucophora gagatea Robineau-Desvoidy, 1830.

TYPE SPECIES: Leucophora cinerea Robineau-Desvoidy, 1830, by subsequent designation (Coquillett, 1901: 138).

CURRENT STATUS: Valid genus [teste Dely-Draskovits (1993: 69)].

FAMILY: ANTHOMYIIDAE.

279. Ligeria Robineau-Desvoidy, 1863a: 935.

ORIGINALLY INCLUDED SPECIES: Ligeria petiolata Robineau-Desvoidy, 1863.

TYPE SPECIES: Ligeria petiolata Robineau-Desvoidy, 1863 [= Scopolia angusticornis Loew, 1847], by original designation.

CURRENT STATUS: Valid genus [teste Herting \& Dely-Draskovits (1993: 165)].

FAMILY: TACHINIDAE.

\section{Lilaea Robineau-Desvoidy, 1863b: 160.}

ORIGINALLY INCLUDED SPECIES: Lilaea aurozonata Robineau-Desvoidy, 1863 (as "Lilaea auro-zonata, R.D."); Phorinia macquarti Robineau-Desvoidy, 1830 (with "Tachina Tenthredinum : Hartig" in synonymy); Lilaea hilarella Robineau-Desvoidy, 1863; Lilaea trepida Robineau-Desvoidy, 1863; Lilaea amaena Robineau-Desvoidy, 1863; Lilaea cognata Robineau-Desvoidy, 1863; Phorinia gracilis Robineau-Desvoidy, 1830; Phorinia borealis Robineau-Desvoidy, 1830.

TYPE SPECIES: Lilaea aurozonata Robineau-Desvoidy, 1830 [= Tachina selecta Meigen, 1824], by subsequent designation (Townsend, 1916: 7).

CURRENT STATUS: Preoccupied by Walker, 1850; junior synonym of Bessa Robineau-Desvoidy, 1863 [teste Herting \& Dely-Draskovits (1993: 142)].

FAMILY: TACHINIDAE.

REMARKS: Previous catalogs have indicated the type species fixation for Lilaea Robineau-Desvoidy, 1863 as Phorinia macquarti Robineau-Desvoidy, 1830, by original designation. This is incorrect. RobineauDesvoidy (1863b: 160) designated two species "Phorinia Macquarti et Ph. gracilis, R.-D.", thereby invaliding both. The first valid type-species designation is of Lilaea aurozonata Robineau-Desvoidy, 1863, by Townsend (1916: 7). With this designation there is no change in the taxonomy of the genus as Lilaea aurozonata is currently placed in Bessa Robineau-Desvoidy, 1863.

281. Limnia Robineau-Desvoidy, 1830: 685.

ORIGINALly INCLUDED SPECIES: Limnia limbata Robineau-Desvoidy, 1830 (as “Musca limbata. Fabr.”); Limnia claripennis Robineau-Desvoidy, 1830; Limnia marginalis Robineau-Desvoidy, 1830; Musca marginata Fabricius, 1775; Limnia dejeanii Robineau-Desvoidy, 1830.

TYPE SPECIES: Limnia limbata Robineau-Desvoidy, 1830 [= Musca unguicornis Scopoli, 1763], by subsequent designation (Cresson, 1920: 75).

CURRENT StATUs: Valid genus [teste Rozkošný \& Elberg (1984: 185)].

FAMILY: SCIOMYZIDAE.

REMARKS: It cannot be excluded that Robineau-Desvoidy (1830: 685) was referring to Musca limbata Gmelin, 1790 (which is an objective synonym of Musca marginata Fabricius, 1775 and now treated in Coremacera Rondani, 1856 [Sciomyzidae]), when he wrote: "Musca limbata Fabr.". On page 686, Robineau-Desvoidy stated: "Cette espèce [Limnia marginata] et le L. limbata sont vrais Muscae marginata et limbata, ainsi nommés par M. Bosc, et ainsi étiquetés par lui dans sa collection, où Fabricius en prit la description. Je rapporte ce fait, parce que, dans une autre collection de Paris, j'ai vu 
le Musca marginata étiqueté du nom de Musca limbata. L'étiquette était de la main même de Fabricius." This change of the type species to Musca limbata Gmelin, 1790 would upset the stability of the two genera.

\section{Limnophora Robineau-Desvoidy, 1830: 517.}

ORIGINALLY INCLUDED SPECIES: Limnophora bengalensis Robineau-Desvoidy, 1830; Limnophora palustris Robineau-Desvoidy, 1830; Limnophora analis Robineau-Desvoidy, 1830; Limnophora obscura Robineau-Desvoidy, 1830; Limnophora macei Robineau-Desvoidy, 1830; Limnophora rivularis Robineau-Desvoidy, 1830; Limnophora fluviatilis Robineau-Desvoidy, 1830; Limnophora soror Robineau-Desvoidy, 1830; Limnophora littoralis Robineau-Desvoidy, 1830; Limnophora vicina Robineau-Desvoidy, 1830; Limnophora trapae Robineau-Desvoidy, 1830; Limnophora punctatella Robineau-Desvoidy, 1830; Limnophora frontalis Robineau-Desvoidy, 1830; Limnophora nitida Robineau-Desvoidy, 1830; Limnophora aquatica Robineau-Desvoidy, 1830; Limnophora coenosa Robineau-Desvoidy, 1830; Limnophora atra Robineau-Desvoidy, 1830; Limnophora gentilis RobineauDesvoidy, 1830; Limnophora brunicosa Robineau-Desvoidy, 1830; Limnophora meridionalis RobineauDesvoidy, 1830.

TYPE SPECIES: Limnophora palustris Robineau-Desvoidy, 1830 [= Anthomyia maculosa Meigen, 1826], by subsequent designation (Coquillett, 1910: 561).

CURRENT STATUS: Valid genus [teste Pont (1986b: 178)].

FAMILY: MUSCIDAE.

REMARKS: Later designations were by Westwood (1840: 142) of Anthomyia compuncta Wiedemann, 1817; Rondani (1856: 974) of Musca consimilis Fallén, 1825; and Karl (1928: 94) of Musca notata Fallén, 1823 ; but none of these were of originally included species so the designations are invalid.

\section{Limosia Robineau-Desvoidy, 1830: 535.}

ORIGINALLY INCLUDED SPECIES: Limosia scirporum Robineau-Desvoidy, 1830; Limosia rubripes RobineauDesvoidy, 1830; Limosia brunipes Robineau-Desvoidy, 1830; Limosia cinerea Robineau-Desvoidy, 1830; Limosia albidula Robineau-Desvoidy, 1830; Limosia campestris Robineau-Desvoidy, 1830; Limosia agrestis Robineau-Desvoidy, 1830; Limosia riparia Robineau-Desvoidy, 1830; Limosia nitens Robineau-Desvoidy, 1830; Limosia stagnalis Robineau-Desvoidy, 1830; Limosia rivularis RobineauDesvoidy, 1830; Limosia littoralis Robineau-Desvoidy, 1830; Limosia albida Robineau-Desvoidy, 1830; Limosia ripicola Robineau-Desvoidy, 1830; Limosia paludosa Robineau-Desvoidy, 1830; Limosia obscura Robineau-Desvoidy, 1830; Limosia picena Robineau-Desvoidy, 1830; Limosia fuliginosa Robineau-Desvoidy, 1830; Limosia hydrocotylis Robineau-Desvoidy, 1830; Limosia alismatis Robineau-Desvoidy, 1830; Limosia juncorum Robineau-Desvoidy, 1830; Limosia limpidipennis Robineau-Desvoidy, 1830; Limosia nigripes Robineau-Desvoidy, 1830; Limosia immaculata RobineauDesvoidy, 1830; Limosia palustris Robineau-Desvoidy, 1830; Limosia nigrifacies Robineau-Desvoidy, 1830; Limosia pallidipes Robineau-Desvoidy, 1830; Limosia nitidula Robineau-Desvoidy, 1830.

TYPE SPECIES: Limosia campestris Robineau-Desvoidy, 1830, by subsequent designation (Coquillett, 1901: 138).

CURRENT Status: Junior synonym of Coenosia Meigen, 1826 [teste Pont (1986b: 199)].

FAMILY: MUSCIDAE.

284. Linnaemya Robineau-Desvoidy, 1830: 52.

ORIGINALly INCLUDED SPECIES: Linnaemya sophia Robineau-Desvoidy, 1830; Linnaemya silvestris Robineau-Desvoidy, 1830; Linnaemya heraclaei Robineau-Desvoidy, 1830; Linnaemya analis Robineau-Desvoidy, 1830; Linnaemya distincta Robineau-Desvoidy, 1830; Linnaemya aestivalis Robineau-Desvoidy, 1830; Linnaemya borealis Robineau-Desvoidy, 1830. 
TYPE SPECIES: Linnaemya silvestris Robineau-Desvoidy, 1830 [= Tachina vulpina Fallén, 1810], by subsequent designation (Robineau-Desvoidy, 1863a: 131).

CURRENT STATUS: Valid genus and subgenus [teste Herting \& Dely-Draskovits (1993: 284)].

FAMILY: TACHINIDAE.

EMENDATIONS: Linnemyia Macquart, 1835: 81 (unjustified); Linnaemyia Aldrich, 1905: 451, 674 (unjustified), n. syn.

285. Lisella Robineau-Desvoidy, 1830: 649.

ORIGINALLY INCLUDED SPECIES: Lisella flava Robineau-Desvoidy, 1830.

TYPE SPECIES: Lisella flava Robineau-Desvoidy, 1830, by monotypy.

CURRENT STATUS: Junior synonym of Chyromya Robineau-Desvoidy, 1830 [teste Soós (1984d: 58)].

FAMILY: CHYROMYIDAE.

286. Listeria Robineau-Desvoidy, 1863b: 600.

ORIGINALLY INCLUDED SPECIES: Listeria agrestis Robineau-Desvoidy, 1863.

TYPE SPECIES: Listeria agrestis Robineau-Desvoidy, 1830, by monotypy.

CuRRENT StATUS: Junior synonym of Sarcophaga Meigen, 1826 [teste Pape (1996: 420)].

FAMILY: SARCOPHAGIDAE.

REMARKS: The type species is here considered as unrecognized in Sarcophaga. Verves (1986: 124, 125) has Listeria campestris Robineau-Desvoidy, 1863 as a valid name in Blaesoxipha Loew, 1861 (in synonymy with Musca lineata Fallén, 1817 of authors and Sarcophaga redempta Pandellé, 1896), but this was an error for Listeria agrestis Robineau-Desvoidy, 1863 according to Verves (1985: 380) [that this correction predates the error was caused by the considerable delay in publishing the Palaearctic Catalogue (i.e., Verves, 1986)]. See further discussion of Listeria Robineau-Desvoidy, 1863 in Pape (1994: 37).

287. Loevia Robineau-Desvoidy, 1863a: 896.

ORIGINALly INCLUDED SPECIES: Loevia maga Robineau-Desvoidy, 1863; Loevia cinerella RobineauDesvoidy, 1863; Phryno agilis Robineau-Desvoidy, 1830; Phryno brunea Robineau-Desvoidy, 1830.

TYPE SPECIES: Loevia maga Robineau-Desvoidy, 1863 [= Phorocera rubrifrons Macquart, 1834], by original designation (as “Loëvia maga, R.-D.”).

CuRRENT Status: Junior synonym of Ceromasia Rondani, 1856 [teste Herting \& Dely-Draskovits (1993: 238)].

FAMILY: TACHINIDAE.

EMENDATIONS: Loewia Herting, 1974: 25 (unjustified), n. syn.

288. Lonchopalpus Robineau-Desvoidy, 1853a: 115 [1853b: 35].

ORIGINALly INCLUDED SPECIES: Myopa dorsalis Fabricius, 1794 (with "Myopa ephippium : Fabr." and "Myopa fasciata : Meig." in synonymy).

TYPE SPECIES: Myopa dorsalis Fabricius, 1794, by original designation.

CURRENT STATUS: Junior synonym of Myopa Fabricius, 1775 [teste Chvála \& Smith (1988: 262)].

FAMILY: CONOPIDAE.

289. Lordatia Robineau-Desvoidy, 1830: 808.

ORIGINALLY INCLUDED SPECIES: Lordatia merdarum Robineau-Desvoidy, 1830; Lordatia stercoraria Robineau-Desvoidy, 1830; Lordatia cadaverina Robineau-Desvoidy, 1830; Lordatia coprina RobineauDesvoidy, 1830; Lordatia necrophaga Robineau-Desvoidy, 1830. 
TYPE SPECIES: Lordatia coprina Robineau-Desvoidy, 1830, by subsequent designation (Coquillett, 1910: $563)$.

CURRENT STATUS: Placed in "Doubtful genera of Sphaerocerinae" by Roháček, Quiros \& Marshall in Roháček (2001: 108).

FAMILY: SPHAEROCERIDAE.

290. Lucasia Robineau-Desvoidy, 1863b: 409.

ORIGINALLY INCLUDED SPECIES: Lucasia cyrrhata Robineau-Desvoidy, 1863.

TYPE SPECIES: Lucasia cyrrhata Robineau-Desvoidy, 1863, by monotypy.

CURRENT STATUS: Placed in "Doubtful taxa in Tachinidae" by Herting \& Dely-Draskovits (1993: 436).

FAMILY: TACHINIDAE.

291. Lucilia Robineau-Desvoidy, 1830: 452.

ORIGINALly INCLUdED SPECIES: Musca caesar Linnaeus, 1758; Lucilia lepida Robineau-Desvoidy, 1830; Lucilia dorsalis Robineau-Desvoidy, 1830; Lucilia amica Robineau-Desvoidy, 1830; Lucilia indica Robineau-Desvoidy, 1830; Lucilia pubescens Robineau-Desvoidy, 1830; Lucilia modesta RobineauDesvoidy, 1830; Lucilia limpidipennis Robineau-Desvoidy, 1830; Lucilia soror Robineau-Desvoidy, 1830; Lucilia azurea Robineau-Desvoidy, 1830; Lucilia germana Robineau-Desvoidy, 1830; Lucilia peruviana Robineau-Desvoidy, 1830; Lucilia usta Robineau-Desvoidy, 1830; Lucilia vicina RobineauDesvoidy, 1830; Lucilia eximia Robineau-Desvoidy, 1830; Musca cornicina Fabricius, 1781; Musca splendida Meigen, 1826; Lucilia carolinensis Robineau-Desvoidy, 1830; Lucilia compar RobineauDesvoidy, 1830; Lucilia viridescens Robineau-Desvoidy, 1830; Lucilia aurulans Robineau-Desvoidy, 1830; Lucilia elegans Robineau-Desvoidy, 1830; Lucilia virgo Robineau-Desvoidy, 1830; Lucilia calens Robineau-Desvoidy, 1830; Lucilia fervida Robineau-Desvoidy, 1830; Lucilia brunicosa RobineauDesvoidy, 1830; Lucilia cuprea Robineau-Desvoidy, 1830; Lucilia bengalensis Robineau-Desvoidy, 1830; Lucilia timorensis Robineau-Desvoidy, 1830; Lucilia peronii Robineau-Desvoidy, 1830; Lucilia rostrellum Robineau-Desvoidy, 1830; Lucilia pallipes Robineau-Desvoidy, 1830; Lucilia sapphirea Robineau-Desvoidy, 1830; Lucilia delicatula Robineau-Desvoidy, 1830; Lucilia fulvicornis RobineauDesvoidy, 1830; Lucilia hyacinthina Robineau-Desvoidy, 1830; Lucilia smaragdula RobineauDesvoidy, 1830.

TYPE SPECIES: Musca caesar Linnaeus, 1758, by subsequent designation (Macquart, 1834b: 162).

CURRENT STATUS: Valid genus [teste Rognes (1991: 147)].

FAMILY: CALLIPHORIDAE.

REMARKS: The designation of a type species for Lucilia by Macquart (1834b: 162) has been accepted by all the major Diptera catalogs although he used the vernacular name rather than the scientific: "Ce genre, dont le type est la mouche César de Linnê". The next valid type designation for Lucilia is by Westwood (1840: 141), which is also of Musca caesar Linnaeus, 1758.

292. Lupia Robineau-Desvoidy, 1863a: 910.

ORIGINALLY INCLUDED SPECIES: Lupia nitida Robineau-Desvoidy, 1863.

TYPE SPECIES: Lupia nitida Robineau-Desvoidy, 1863, by original designation.

CURRENT StATUS: Placed in "Doubtful taxa in Tachinidae" by Herting \& Dely-Draskovits (1993: 437).

FAMILY: TACHINIDAE.

293. Lycia Robineau-Desvoidy, 1830: 637.

ORIGINALly INCLUDED SPECIES: Lycia flava Robineau-Desvoidy, 1830; Lycia quatuorpunctata RobineauDesvoidy, 1830 (as “Lycia quatuor-punctata. R. D.”); Lycia sexpunctata Robineau-Desvoidy, 1830 (as 
“Lycia sex-punctata. R. D.”); Lycia impunctata Robineau-Desvoidy, 1830; Lycia femoralis RobineauDesvoidy, 1830; Lycia umbraculata Robineau-Desvoidy, 1830; Lycia nigricornis Robineau-Desvoidy, 1830; Lycia thoracica Robineau-Desvoidy, 1830; Lycia liturella Robineau-Desvoidy, 1830; Lycia liturata Robineau-Desvoidy, 1830.

TYPE SPECIES: Lycia flava Robineau-Desvoidy, 1830 [= Sapromyza rorida Fallén, 1820], by subsequent designation (Coquillett, 1910: 563).

CURRENT STATUS: Preoccupied by Hübner, 1823; senior (but invalid) synonym of Meiosimyza Hendel, 1925.

FAMILY: LAUXANIIDAE.

REMARKS: Hendel (1908: 3) designated Sapromyza rorida Fallén, 1820 as type species, but this was not an originally included species. Noting the name was preoccupied, Collin (1948: 237) replaced Lycia with Lyciella; but oddly enough in that same paper (page 225) Collin synonymized Meiosimyza. As Meiosimyza Hendel, 1925, new status, is the next available name, and as it is not preoccupied, it is treated as valid here. Lyciella, new status, is thus treated here as an invalid junior synonym of Meiosimyza Hendel, 1925.

294. Lydella Robineau-Desvoidy, 1830: 112.

ORIGINALLY INCLUDED SPECIES: Lydella grisescens Robineau-Desvoidy, 1830; Lydella agrestis RobineauDesvoidy, 1830; Lydella nitida Robineau-Desvoidy, 1830; Lydella dubia Robineau-Desvoidy, 1830; Lydella hydrocampae Robineau-Desvoidy, 1830; Lydella campestris Robineau-Desvoidy, 1830; Lydella myoidaea Robineau-Desvoidy, 1830 (as "Lydella myoüdaea. R. D.”); Lydella pallidipalpis RobineauDesvoidy, 1830; Lydella nigricornis Robineau-Desvoidy, 1830; Lydella floricola Robineau-Desvoidy, 1830; Lydella fulvipes Robineau-Desvoidy, 1830; Lydella scutellaris Robineau-Desvoidy, 1830.

TYPE SPECIES: Lydella grisescens Robineau-Desvoidy, 1830, by subsequent designation (RobineauDesvoidy, 1863a: 855).

CURRENT STATUS: Valid genus [teste Herting \& Dely-Draskovits (1993: 203)].

FAMILY: TACHINIDAE.

295. Lydina Robineau-Desvoidy, 1830: 124.

ORIGINALly INCLUDED SPECIES: Lydina nitida Robineau-Desvoidy, 1830; Lydina cuprea RobineauDesvoidy, 1830; Lydina macromera Robineau-Desvoidy, 1830.

TyPE SPECIES: Lydina nitida Robineau-Desvoidy, 1830 [= Tachina aenea Meigen, 1824], by subsequent designation (Robineau-Desvoidy, 1863a: 111).

CURRENT STATUS: Valid genus [teste Herting \& Dely-Draskovits (1993: 291)].

FAMILY: TACHINIDAE.

296. Lylibaea Robineau-Desvoidy, 1863a: 551.

ORIGINALLY INCLUDED SPECIES: Lylibaea temeraria Robineau-Desvoidy, 1863; Lylibaea tomentosa Robineau-Desvoidy, 1863.

TYPE SPECIES: Lylibaea temeraria Robineau-Desvoidy, 1863 [= Tachina rufiscutellaris Zetterstedt, 1859], by original designation.

CuRrent Status: Junior synonym of Nilea Robineau-Desvoidy, 1863 [teste Herting \& Dely-Draskovits (1993: 188)].

FAMILY: TACHINIDAE.

REMARKS: There are two original spellings of this genus-group name in Robineau-Desvoidy (1863a): Lylibaea (page 551) and Lyliboea (page 1141). Acting as First Reviser, we select Lylibaea as the correct original spelling. 
[Lyliboea] Robineau-Desvoidy, 1863a: 1141.

CURRENT STATUS: Incorrect original spelling of Lylibaea Robineau-Desvoidy, 1863 [teste this work].

FAMILY: TACHINIDAE.

\section{Lypha Robineau-Desvoidy, 1830: 141.}

ORIGINAlly InCluded SPECIES: Tachina dubia Fallén, 1810 (as “Tachina dubia? Meig.”); Lypha silvatica Robineau-Desvoidy, 1830.

TYPE SPECIES: Tachina dubia Fallén, 1810, by subsequent designation (Robineau-Desvoidy, 1863a: 196).

CURRENT STATUS: Valid genus [teste Herting \& Dely-Draskovits (1993: 292)].

FAMILY: TACHINIDAE.

REMARKS: The header for the first species reads "Lypha dubia", without attribution but with "Tachina dubia? Meig." listed below it. Robineau-Desvoidy (1830: 141) wrote after the description: "Cette espèce a été envoyée par M. von Winthem sous le nom de Tachina dubia. Meig." Meigen (1824: 360) attributed the species to Fallén, and we accept the commonly held view that Robineau-Desvoidy's (1830: 141) "Lypha dubia" is Tachina dubia Fallén, 1810.

298. Lythia Robineau-Desvoidy, 1863a: 707.

ORIGINALLY INCLUDED SPECIES: Lythia flavicornis Robineau-Desvoidy, 1863.

TYPE SPECIES: Lythia flavicornis Robineau-Desvoidy, 1863 [= Tachina exigua Meigen, 1824], by original designation.

CuRRENT STATUS: Junior synonym of Microsoma Macquart, 1855 [teste Herting \& Dely-Draskovits (1993: 397)].

FAMILY: TACHINIDAE.

299. Macquartia Robineau-Desvoidy, 1830: 204.

ORIGINALly INCLUDED SPECIES: Macquartia flavescens Robineau-Desvoidy, 1830; Tachina egens Meigen, 1826; Macquartia rubripes Robineau-Desvoidy, 1830; Macquartia germanica Robineau-Desvoidy, 1830; Macquartia viridescens Robineau-Desvoidy, 1830; Macquartia microcera Robineau-Desvoidy, 1830; Macquartia brachycera Robineau-Desvoidy, 1830; Macquartia grisescens Robineau-Desvoidy, 1830.

TYPE SPECIES: Macquartia rubripes Robineau-Desvoidy, 1830 [= Tachina dispar Fallén, 1820], by subsequent designation (Townsend, 1916: 7).

CurRent Status: Valid genus [teste Herting \& Dely-Draskovits (1993: 312)].

FAMILY: TACHINIDAE.

REMARKS: The designation by Robineau-Desvoidy (1863a: 1104) of Macquartia viridana RobineauDesvoidy, 1863 is invalid as it was not of an originally included species.

300. Macromya Robineau-Desvoidy, 1830: 322.

ORIGINALly INCLUDED SPECIES: Macromya depressa Robineau-Desvoidy, 1830; Macromya analis Robineau-Desvoidy, 1830.

TYPE SPECIES: Macromya depressa Robineau-Desvoidy, 1830, by subsequent designation (Townsend, 1916: 7).

CURRENT Status: Valid genus [teste O'Hara \& Wood (2004: 280)].

FAMILY: TACHINIDAE.

EMENDATIOns: Macromyia Agassiz, 1846b: 220 (unjustified), n. syn. 
301. Macrosoma Robineau-Desvoidy, 1830: 402.

ORIGINALly INCLUded SPECIES: Musca lardaria Fabricius, 1781 (as "Macrosôma lardaria. Musca. Fabr. Gmel. Fall. Anthomyia. Meig."); Macrosoma floralis Robineau-Desvoidy, 1830 (as "Macrosôma floralis, R. D.").

TYPE SPECIES: Musca lardaria Fabricius, 1781, by subsequent designation (Coquillett, 1901: 139).

CURRENT STATUS: Preoccupied by Hübner, 1818; Leach, 1819; de Haan, 1826; senior (but invalid) synonym of Polietes Rondani, 1866 [teste Pont (1986b: 85)].

FAMILY: MUSCIDAE.

302. Maculia Robineau-Desvoidy, 1863b: 279.

ORIGINALLY INCLUDED SPECIES: Maculia punctata Robineau-Desvoidy, 1863.

TYPE SPECIES: Maculia punctata Robineau-Desvoidy, 1863 [= probably Amobia sp.], by monotypy.

CURRENT STAtus: Junior synonym of Amobia Robineau-Desvoidy, 1830 [teste Herting (1974: 37) and this work].

FAMILY: SARCOPHAGIDAE.

REMARKS: The genus was not treated in either the Palaearctic Tachinidae catalog (Herting \& DelyDraskovits, 1993) or the world Sarcophagidae catalog (Pape, 1996). However, Herting (1974: 37) stated: "Maculia R.D. 1863 (II), p. 279, nur ${ }^{+}$punctata R.D. Von BEZZI $(1907$, p. 569) fälschlich in die Gattung Tamiclea gestellt. Der vorhandene Typus ist gar keine Tachine, sondern eine Sarcophagide (Pachyophthalmus oder ähnliche Art)". We are here relying on Herting's tentative identification and Robineau-Desvoidy's mention of a row of hair-like proclinate orbital bristles (autapomorphic for the genus Amobia; see Pape 1996), a large lower calypter and abdominal spots. Robineau-Desvoidy (1863b: 279) described both the male and the female sex in the generic description, but for the single included species he claimed to have seen only a single male: "Nous ne possédons qu'un Mâle de cette rare espèce".

303. Marshamia Robineau-Desvoidy, 1830: 57.

ORIGINALLY INCLUDED SPECIES: Marshamia analis Robineau-Desvoidy, 1830; Marshamia nigripes Robineau-Desvoidy, 1830.

TYPE SPECIES: Marshamia analis Robineau-Desvoidy, 1830 [= Tachina comta Fallén, 1810], by subsequent designation (Townsend, 1916: 7).

CURRENT STATUS: Junior synonym of Linnaemya Robineau-Desvoidy, 1830 sensu stricto [teste O'Hara \& Wood (2004: 241)].

FAMILY: TACHINIDAE.

[Marsilia] Monceaux in Robineau-Desvoidy, 1863b: 535.

CURRENT STATUS: Unavailable name; proposed in synonymy with Onesia Robineau-Desvoidy, 1830 and not made available before 1961; treated under Onesia Robineau-Desvoidy, 1830 [teste this work].

FAMILY: CALLIPHORIDAE.

REMARKS: The name Marsilia appears in a footnote explicitly written by Monceaux on his own behalf, and this is why we attribute the name to Monceaux and not to Robineau-Desvoidy. The use here is not a subsequent usage of Marsillia Rondani, 1861 since Robineau-Desvoidy died in 1857 and his manuscript was prepared from his notes after his death by Monceaux. Although Townsend (1916: 11) indicated the type species as being the same as that for Onesia, he did not remove the name from synonymy with Onesia, thus Marsilia remains unavailable. 
304. Meckelia Robineau-Desvoidy, 1830: 714.

ORIGINALly INCLUDED SPECIES: Otites elegans Latreille, 1805 (as “Oscinis elegans. Latr. Dict. d'Hist. natur.”); Meckelia philadelphica Robineau-Desvoidy, 1830.

TYPE SPECIES: Otites elegans Latreille, 1805 [= Musca formosa Panzer, 1798], by subsequent designation [Coquillett, 1910: 565 (as “Oscinis elegans DESVOIDY”)].

CURRENT STATUS: Preoccupied by Leuckart, 1828; junior synonym of Otites Latreille, 1804, n. syn.

FAMILY: ULIDIIDAE.

REMARKS: Soós (1984c: 53) listed the type species of Meckelia Robineau-Desvoidy, 1830 as "Oscinis elegans Robineau-Desvoidy" and, in the list of species below it, placed it as a junior synonym of Musca hortulana Rossi, 1790. However, Musca hortulana Rossi, 1790 is preoccupied by Scopoli, 1763, and Musca hyalinata Panzer, 1798 is the next available name and therefore the valid name (in the genus Otites). This latter synonymy was noted by Evenhuis (1989: 479). Moreover, Robineau-Desvoidy (1830: 714) did not propose a new species but was clearly referring to the "Oscinis elegans" of Latreille. Latreille originally described elegans in Otites in 1805 and then placed it in Oscinis (Latreille, 1811: 565) when he wrote the "Oscinis" entry for the genus-group name in the Encyclopédie Méthodique. The reprinted Laporte edition of this work carried the title "Dictionnaire Encyclopédique de l'Histoire Naturelle", hence Robineau Desvoidy's attribution as "Latr. Dict. d'Hist. natur." [see Evenhuis (2003a) for details on the publication and editions of this series]. Soós (1984c: 48) alleged that Otites elegans Latreille, 1805 was unavailable because it was proposed in synonymy with Musca formosa Panzer, 1798, but Latreille (1811) made the name available by treating it as valid in his entry for "Oscinis" in the Encyclopédie Méthodique. The change in type species over what has been considered in previous catalogs places Meckelia in synonymy with Otites rather than with Ceroxys Macquart, 1835.

305. Medina Robineau-Desvoidy, 1830: 138.

ORIGINALLY INCLUDED SPECIES: Medina elongata Robineau-Desvoidy, 1830; Medina cylindrica RobineauDesvoidy, 1830; Medina carceli Robineau-Desvoidy, 1830; Medina winthemi Robineau-Desvoidy, 1830 (with "Tachina pilipennis. Meig." in synonymy); Medina tristis Robineau-Desvoidy, 1830; Tachina luctuosa Meigen, 1824.

TYPE SPECIES: Medina cylindrica Robineau-Desvoidy, 1830 [= Tachina collaris Fallén, 1820], by subsequent designation (Coquillett, 1910: 565).

CURRENT STATUS: Valid genus [teste Herting \& Dely-Draskovits (1993: 151)].

FAMILY: TACHINIDAE.

306. Medoria Robineau-Desvoidy, 1830: 266.

ORIGINALLY INCLUDED SPECIES: Medoria agilis Robineau-Desvoidy, 1830.

TYPE SPECIES: Medoria agilis Robineau-Desvoidy, 1830, by monotypy.

CuRrent STATUS: Placed in "Doubtful taxa in Tachinidae" by Herting \& Dely-Draskovits (1993: 437).

FAMILY: TACHINIDAE.

307. Megaera Robineau-Desvoidy, 1830: 94.

ORIGINAlly InCLuded SPECIES: Megaera dira Robineau-Desvoidy, 1830 (with "Miltogramma conica. Meig." in synonymy); Megaera atrox Robineau-Desvoidy, 1830; Megaera crudelis Robineau-Desvoidy, 1830; Megaera fera Robineau-Desvoidy, 1830; Megaera inimica Robineau-Desvoidy, 1830.

TYPE SPECIES: Megaera atrox Robineau-Desvoidy, 1830, by subsequent designation (Robineau-Desvoidy, 1863b: 142).

CURRENT STATUS: Preoccupied by Wagler, 1830; senior (but invalid) synonym of Senotainia Macquart, 1846

[teste Pape (1996: 133)]. 
FAMILY: SARCOPHAGIDAE.

REMARKS: The type-species designation by Robineau-Desvoidy (1863b: 142) of Tachina conica Fallén, 1810 (as "Miltogramma conica, Fall.") is valid as "Megaera atrox : Rob. Desv." is listed as the only originally included species in synonymy under Megaera conica (Robineau-Desvoidy, 1863b: 143).

308. Megarhinus Robineau-Desvoidy, 1827: 412.

ORIGINALLY INCLUDED SPECIES: Culex haemorrhoidalis Fabricius, 1787.

TYPE SPECIES: Culex haemorrhoidalis Fabricius, 1787, by monotypy.

CURRENT STATUS: Preoccupied by Rafinesque, 1820; senior (but invalid) synonym of Lynchiella Lahille,

1904 (subgenus of Toxorhynchites Theobald, 1901) [teste Sabrosky (1999: 190)].

FAMILY: CULICIDAE.

EMENDATIONS: Megarhina Osten Sacken, 1881: 398 (unjustified), n. syn.

309. Megerlea Robineau-Desvoidy, 1830: 266.

ORIGINAlly INCLUdED SPECIES: Megerlea nitida Robineau-Desvoidy, 1830; Megerlea picea Robineau-

Desvoidy, 1830; Megerlea claripennis Robineau-Desvoidy, 1830.

TYPE SPECIES: Megerlea nitida Robineau-Desvoidy, 1830 [= Musca halterata Panzer, 1798], by subsequent designation (Robineau-Desvoidy, 1863b: 813).

CURRENT STATUS: Junior synonym of Nyctia Robineau-Desvoidy, 1830 [teste Pape (1996: 165)].

FAMILY: SARCOPHAGIDAE.

310. Meigenia Robineau-Desvoidy, 1830: 198.

ORIGINALly INCLUDED SPECIES: Meigenia cylindrica Robineau-Desvoidy, 1830; Meigenia grisescens Robineau-Desvoidy, 1830; Meigenia borealis Robineau-Desvoidy, 1830; Tachina bisignata Meigen, 1824; Meigenia immaculata Robineau-Desvoidy, 1830; Meigenia flavescens Robineau-Desvoidy, 1830; Meigenia vernalis Robineau-Desvoidy, 1830; Tachina floralis Meigen, 1824; Meigenia parva RobineauDesvoidy, 1830; Meigenia nitida Robineau-Desvoidy, 1830; Meigenia nana Robineau-Desvoidy, 1830.

TYPE SPECIES: Meigenia cylindrica Robineau-Desvoidy, 1830, by subsequent designation (Desmarest in d'Orbigny, 1849a: 318).

CURRENT STATUS: Valid genus [teste O'Hara \& Wood (2004: 96)].

FAMILY: TACHINIDAE.

REMARKS: The type species Meigenia cylindrica is listed in "Doubtful species" of Meigenia by Herting \& Dely-Draskovits (1993: 147). We do not propose any change to the well-established concept and prevailing usage of Meigenia.

[Melania] Robineau-Desvoidy MS name (Blainville et al., 1826: 12).

CURRENT STATUS: Unavailable name; suppressed by action of I.C.Z.N. (1990: 162 [Opinion 1601]); treated as a nomen dubium in Tachinidae [teste this work].

FAMILY: TACHINIDAE.

311. Melanosoma Robineau-Desvoidy, 1853a: 122 [1853b: 42].

ORIGINALly INCLUDED SPECIES: Myopa nigra Meigen, 1824 (with "Myopa bicolor : Meig." in synonymy); Myopa distincta Wiedemann, 1824 (as "Myopa distincta : Meig."); Melanosoma nigripes RobineauDesvoidy, 1853; Melanosoma brunipes Robineau-Desvoidy, 1853; Melanosoma zetterstedti RobineauDesvoidy, 1853. 
TYPE SPECIES: Myopa nigra Meigen, 1824, by original designation.

CURRENT STATUS: Valid genus [teste Chvála \& Smith (1988: 266)].

FAMILY: CONOPIDAE.

\section{Melia Robineau-Desvoidy, 1830: 101.}

ORIGINALly INCLUDED SPECIES: Melia albipennis Robineau-Desvoidy, 1830.

TYPE SPECIES: Melia albipennis Robineau-Desvoidy, 1830 [= Tachina leucoptera Meigen, 1824], by monotypy.

CuRrent Status: Preoccupied by Bosc, 1813; Billberg, 1820; Latreille, 1827; Curtis, 1828; Fischer de Waldheim, 1829; senior (but invalid) synonym of Melisoneura Rondani, 1861 [teste Herting \& DelyDraskovits (1993: 351)].

FAMILY: TACHINIDAE.

\section{Melibaea Robineau-Desvoidy, 1848a: 613.}

ORIGINALLY INCLUDED SPECIES: Carcelia lepida Robineau-Desvoidy, 1830; Melibaea aurulenta RobineauDesvoidy, 1848; Phryxe subrotundata Robineau-Desvoidy, 1830 (with "Phryxe microcera, R.-D." in synonymy); Phryxe blondeli Robineau-Desvoidy, 1830; Melibaea gagatea Robineau-Desvoidy, 1848; Melibaea zonaria Robineau-Desvoidy, 1848.

TYPE SPECIES: Melibaea aurulenta Robineau-Desvoidy, 1848 [= Tachina glauca Meigen, 1824], by subsequent designation (Robineau-Desvoidy, 1863a: 287).

CURRENT STATUS: Junior synonym of Phebellia Robineau-Desvoidy, 1846 [teste Herting \& Dely-Draskovits (1993: 184)].

FAMILY: TACHINIDAE.

314. Melieria Robineau-Desvoidy, 1830: 715.

ORIGINALly INCLUdED SPECIES: Musca gangraenosa Panzer, 1798 (as “Otites gangraenosa. Latr."); Melieria rubrella Robineau-Desvoidy, 1830; Melieria obscura Robineau-Desvoidy, 1830; Melieria pallipes Robineau-Desvoidy, 1830; Melieria pulicaria Robineau-Desvoidy, 1830.

TYPE SPECIES: Musca gangraenosa Panzer, 1798 [= Musca crassipennis Fabricius, 1794], by subsequent designation (Rondani, 1869: 8).

CURRENT STATUS: Valid genus [teste Soós (1984c: 51)].

FAMILY: ULIDIIDAE.

REMARKS: Rondani (1869: 8) designated "Scatophaga crassipennis Fabr.", which was not an originally included species, but he placed "gangraenosa Robineau-Desvoidy" in synonymy with it (page 9) and thus the designation is valid according to ICZN Code Article 69.2.2. Robineau-Desvoidy (1830: 716) described gangraenosa and stated in a note below the description that he had seen it in the Muséum National d'Histoire Naturelle, Paris under the label "Otites gangraenosa" by Latreille. Latreille was clearly using gangraenosa of Panzer as it was the only ulidiid with that name at the time.

315. Melina Robineau-Desvoidy, 1830: 695.

ORIGINALLY INCLUDED SPECIES: Melina riparia Robineau-Desvoidy, 1830.

TYPE SPECIES: Melina riparia Robineau-Desvoidy, 1830 [= Sciomyza dubia Fallén, 1820], by monotypy.

CuRrent Status: Preoccupied by Retzius, 1788; junior synonym of Pherbellia Robineau-Desvoidy, 1830 [teste Rozkošný \& Elberg (1984: 170)].

FAMILY: SCIOMYZIDAE. 
316. Melinda Robineau-Desvoidy, 1830: 439.

ORIGINALLY INCLUDED SPECIES: Melinda caerulea Robineau-Desvoidy, 1830 (with "An Musca caerulea ? Meig." in doubtful synonymy); Melinda albiceps Robineau-Desvoidy, 1830; Melinda leucocera Robineau-Desvoidy, 1830; Melinda azurea Robineau-Desvoidy, 1830; Melinda coelestis RobineauDesvoidy, 1830; Melinda soror Robineau-Desvoidy, 1830; Melinda gentilis Robineau-Desvoidy, 1830.

TYPE SPECIES: Melinda caerulea Robineau-Desvoidy, 1830, by subsequent designation (Townsend, 1916: 7). CURRENT STATUS: Valid genus [teste Rognes (1991: 199)].

FAMILY: CALLIPHORIDAE.

REMARKS: Schumann (1986: 31) and Rognes (1991: 199) attributed the type designation to Hendel (1901a: 32), who gave “Typ. caerulea Mg.”. However, Musca caerulea Meigen, 1826 was doubtfully included (in doubtful synonymy with Melinda caerulea Robineau-Desvoidy), thus type designations of this nominal species are invalid. The first valid type designation is of Melinda caerulea Robineau-Desvoidy, 1830 by Townsend (1916: 7).

\section{Meriania Robineau-Desvoidy, 1830: 69.}

ORIGINALLY INCLUDED SPECIES: Meriania silvatica Robineau-Desvoidy, 1830; Meriania borealis RobineauDesvoidy, 1830; Meriania capensis Robineau-Desvoidy, 1830.

TYPE SPECIES: Meriania silvatica Robineau-Desvoidy, 1830 [= Musca puparum Fabricius, 1794], by subsequent designation (Robineau-Desvoidy, 1863a: 168).

CURRENT Status: Junior synonym of Panzeria Robineau-Desvoidy, 1830 [teste this work].

FAMILY: TACHINIDAE.

REMARKS: Robineau-Desvoidy (1863a: 168) designated Musca puparum Fabricius, 1794, which was not one of the originally included species; however by including Meriania silvatica as the only originally included species in synonymy with Musca puparum, the designation is valid according to ICZN Code Article 69.2.2. Herting \& Dely-Draskovits (1993: 293) treated Meriania as a junior synonym of Ernestia Robineau-Desvoidy, 1830 and O'Hara \& Wood (2004: 244) treated Ernestia as a junior synonym of Panzeria Robineau-Desvoidy, 1830.

\section{Mericia Robineau-Desvoidy, 1830: 64.}

ORIGINALLY INCLUDED SPECIES: Mericia erigonea Robineau-Desvoidy, 1830.

TYPE SPECIES: Mericia erigonea Robineau-Desvoidy, 1830, by monotypy.

CURRENT STATUS: Placed in "Doubtful taxa in Tachinidae" by Herting \& Dely-Draskovits (1993: 437).

FAMILY: TACHINIDAE.

REMARKs: Treated as a junior synonym of Panzeria Robineau-Desvoidy, 1830 by Wood (1987: 1261) and O’Hara \& Wood (2004: 244), based on Mericia Robineau-Desvoidy, 1830 sensu Sabrosky \& Arnaud (1965: 1007).

319. Microptera Robineau-Desvoidy, 1830: 212.

ORIGINALLY INCLUDED SPECIES: Microptera nitida Robineau-Desvoidy, 1830.

TYPE SPECIES: Microptera nitida Robineau-Desvoidy, 1830 [= Tachina innoxia Meigen, 1824], by monotypy. CURRENT STAtUS: Preoccupied by Fleming, 1822; senior (but invalid) synonym of Leiophora RobineauDesvoidy, 1863 [teste Herting \& Dely-Draskovits (1993: 159)].

FAMILY: TACHINIDAE.

320. Minella Robineau-Desvoidy, 1830: 209.

ORIGINALly InCludED SPECIES: Minella nitida Robineau-Desvoidy, 1830. 
TYPE SPECIES: Minella nitida Robineau-Desvoidy, 1830 [= Tachina tenebricosa Meigen, 1824], by monotypy.

CURRENT StATus: Junior synonym of Macquartia Robineau-Desvoidy, 1830 [teste Herting \& DelyDraskovits (1993: 312)].

FAMILY: TACHINIDAE.

321. Minettia Robineau-Desvoidy, 1830: 646.

ORIGINAlly InCluded SPECIES: Minettia luctuosa Robineau-Desvoidy, 1830; Minettia claripennis Robineau-Desvoidy, 1830; Minettia nemorosa Robineau-Desvoidy, 1830; Minettia palustris RobineauDesvoidy, 1830; Minettia testacea Robineau-Desvoidy, 1830; Minettia flavipes Robineau-Desvoidy, 1830; Minettia flava Robineau-Desvoidy, 1830.

TYPe SPECIES: Minettia nemorosa Robineau-Desvoidy, 1830 [= Sapromyza rivosa Meigen, 1826], by subsequent designation (Westwood, 1840: 150).

CURRENT STATUS: Valid genus [teste Sabrosky (1999: 200)].

FAMILY: LAUXANIIDAE.

REMARKS: Westwood (1840: 150) designated Sapromyza rivosa Meigen, 1826, which was not an originally included species; however, by placing Minettia nemorosa Robineau-Desvoidy, 1830 in synonymy with Sapromyza rivosa on page 151, the designation is valid according to ICZN Code Article 69.2.2.

Hendel (1908: 28) designated Musca longipennis Fabricius, 1794 as the type species for Minettia Robineau-Desvoidy, 1830, which was followed by Frey (1927: 22). However, this designation is invalid as the nominal species was not originally included. Noting the error, Collin (1948) erected the subgenus Frendelia (a combination of the surnames "Frey" and "Hendel") to facetiously "honor" both authors for their respective mistakes and designated Musca longipennis Fabricius, 1794 as its type species.

Coquillett (1910: 570) designated Minettia luctuosa Robineau-Desvoidy, 1830, but this was later. If this designation was accepted, it would change the accepted classification of the genus because $M$. luctuosa is currently treated within the subgenus Frendelia Collin, 1948 in Minettia.

Noting that a type species had been designated for Terenia Robineau-Desvoidy, 1830 that would fall within the generic concept of Minettia, Sabrosky (1999: 200-201) placed Terenia as a synonym of Minettia. Acting as First Reviser, he chose Minettia as having priority over Terenia, thereby avoiding possible instability of nomenclature and taxonomy in having the lesser known name as having priority over a more common name.

322. Mintho Robineau-Desvoidy, 1830: 216.

ORIGINALly INCLUDED SPECIES: Musca compressa Fabricius, 1787; Mintho obscuripennis RobineauDesvoidy, 1830; Mintho capensis Robineau-Desvoidy, 1830.

TYPE SPECIES: Musca compressa Fabricius, 1787 (as “Dexia compressa Meigen”), by subsequent designation (Rondani, 1856: 79).

CURRENT STATUS: Valid genus [teste Herting \& Dely-Draskovits (1993: 345)].

FAMILY: TACHINIDAE.

\section{Misellia Robineau-Desvoidy, 1863b: 146.}

ORIGinally InCluded SPECIES: Megaera dira Robineau-Desvoidy, 1830; Misellia brunicosa RobineauDesvoidy, 1863; Miltogramma siphonina Zetterstedt, 1844.

TYPE SPECIES: Megaera dira Robineau-Desvoidy, 1830, by original designation.

CURRENT STATUS: Junior synonym of Taxigramma Perris, 1852 [teste Pape (1996: 151)].

FAMILY: SARCOPHAGIDAE.

REMARKS: Previous listings of the type species have it as a junior synonym of Miltogramma stictica Meigen, 1830, but this is incorrect. Robineau-Desvoidy's 1830 paper came out 6 June while Meigen's 1830 paper 
came out six months later on 1 December [see References in this paper for dating of the former and Evenhuis (1997: 533) for dating of the latter].

EMENDATIONS: Misella Brauer \& Bergenstamm, 1893: 145 (unjustified), n. syn.

324. Mollia Robineau-Desvoidy, 1863a: 949.

ORIGINALly INCLUDED SPECIES: Mollia obscurella Robineau-Desvoidy, 1863; Mollia delicatula RobineauDesvoidy, 1863.

TYPE SPECIES: Mollia obscurella Robineau-Desvoidy, 1863, by subsequent designation (Townsend, 1916: 7).

CURRENT STATUS: Preoccupied by Lamouroux, 1816; junior synonym of Medina Robineau-Desvoidy, 1830 [teste Herting \& Dely-Draskovits (1993: 151)].

FAMILY: TACHINIDAE.

325. Morellia Robineau-Desvoidy, 1830: 405.

ORIGINALly INCLUDED SPECIES: Morellia agilis Robineau-Desvoidy, 1830; Morellia horticola RobineauDesvoidy, 1830; Morellia concolor Robineau-Desvoidy, 1830; Morellia aenescens Robineau-Desvoidy, 1830.

TYPE SPECIES: Morellia agilis Robineau-Desvoidy, 1830 [= Musca hortorum Fallén, 1817], by subsequent designation (Townsend, 1916: 8).

CuRrent Status: Valid genus [teste Pont (1986b: 94)].

FAMILY: MUSCIDAE.

326. Moretia Robineau-Desvoidy, 1863b: 366.

ORIGINALLY INCLUDED SPECIES: Moretia sinophtalma Robineau-Desvoidy, 1863.

TYPE SPECIES: Moretia sinophtalma Robineau-Desvoidy, 1863, by monotypy.

CURRENT STATUS: Nomen dubium [teste this work].

FAMILY: CALLIPHORIDAE.

REMARKS: Although originally placed in the current concept of the family Tachinidae, Herting (1974: 41) stated that Moretia is obviously a Calliphoridae. Until a type specimen of the type species is found, Moretia is best regarded as a nomen dubium in Calliphoridae.

327. Morinia Robineau-Desvoidy, 1830: 264.

ORIGINAlly InCluded SPECIES: Morinia velox Robineau-Desvoidy, 1830; Morinia fuscipennis RobineauDesvoidy, 1830; Morinia parva Robineau-Desvoidy, 1830; Morinia rubescens Robineau-Desvoidy, 1830; Morinia claripennis Robineau-Desvoidy, 1830.

TYPE SPECIES: Morinia velox Robineau-Desvoidy, 1830 [= Musca doronici Scopoli, 1763], by subsequent designation (Rondani, 1862: 159).

CURRENT STATUS: Valid genus [teste Rognes (1991: 209)].

FAMILY: CALLIPHORIDAE.

328. Moschusa Robineau-Desvoidy, 1863b: 139.

ORIGINALLY INCLUDED SPECIES: Tachina polyodon Meigen, 1824.

TYPE SPECIES: Tachina polyodon Meigen, 1824, by original designation.

CURRENT STATUS: Junior synonym of Macronychia Rondani, 1859 [teste Pape (1996: 94); sometimes treated as a valid subgenus, e.g., Verves \& Khrokalo (2006)].

FAMILY: SARCOPHAGIDAE. 
329. Mosina Robineau-Desvoidy, 1830: 670.

ORIGINALLY INCLUDED SPECIES: Musca pubera Linnaeus, 1758 (as “Musca pubera. Linn. Fabr.”); Mosina nigra Robineau-Desvoidy, 1830; Mosina dejeanii Robineau-Desvoidy, 1830; Mosina latreillii RobineauDesvoidy, 1830; Mosina connexa Robineau-Desvoidy, 1830 (as “Dyctia connexa. Latreill.”); Mosina fulva Robineau-Desvoidy, 1830; Mosina filipes Robineau-Desvoidy, 1830.

TYPE SPECIES: Musca pubera Linnaeus, 1758, by subsequent designation (Westwood, 1840: 144).

CURRENT StATUS: Junior synonym of Cordilura Fallén, 1810 [teste Gorodkov (1986: 13)].

FAMILY: SCATHOPHAGIDAE.

330. Mufetia Robineau-Desvoidy, 1830: 431.

ORIGINALly INCLUDED SPECIES: Mufetia autissiodorensis Robineau-Desvoidy, 1830.

TYPE SPECIES: Mufetia autissiodorensis Robineau-Desvoidy, 1830, by monotypy.

CURRENT STATUS: Nomen dubium in Calliphorinae [teste Schumann (1986: 37)].

FAMILY: CALLIPHORIDAE.

331. Mulsantia Robineau-Desvoidy, 1863b: 557.

ORIGINALLY INCLUDED SPECIES: Mulsantia campestris Robineau-Desvoidy, 1863; Mulsantia lauta Robineau-Desvoidy, 1863; Myophora rustica Robineau-Desvoidy, 1830; Mulsantia tenebricosa Robineau-Desvoidy, 1863; Mulsantia atra Robineau-Desvoidy, 1863; Myophora abdominalis RobineauDesvoidy, 1830; Myophora obscura Robineau-Desvoidy, 1830; Mulsantia praeceps Robineau-Desvoidy, 1863; Myophora camporum Robineau-Desvoidy, 1830; Mulsantia nigrifrons Robineau-Desvoidy, 1863; Mulsantia tristis Robineau-Desvoidy, 1863; Musca striata Fabricius, 1794; Mulsantia cucullata Robineau-Desvoidy, 1863; Mulsantia albida Robineau-Desvoidy, 1863; Myophora sericea RobineauDesvoidy, 1830; Mulsantia praticola Robineau-Desvoidy, 1863; Myophora arvicola RobineauDesvoidy, 1830; Myophora albidipennis Robineau-Desvoidy, 1830; Mulsantia consobrina RobineauDesvoidy, 1863; Mulsantia vaga Robineau-Desvoidy, 1863; Myophora flavescens Robineau-Desvoidy, 1830; Mulsantia blanda Robineau-Desvoidy, 1863; Mulsantia flavidula Robineau-Desvoidy, 1863; Mulsantia caesia Robineau-Desvoidy, 1863; Mulsantia incauta Robineau-Desvoidy, 1863; Mulsantia reginaldi Robineau-Desvoidy, 1863; Mulsantia sollicitata Robineau-Desvoidy, 1863; Mulsantia pellex Robineau-Desvoidy, 1863; Mulsantia spreta Robineau-Desvoidy, 1863; Mulsantia meditata RobineauDesvoidy, 1863.

TYPE SPECIES: Mulsantia campestris Robineau-Desvoidy, 1863, by subsequent designation (Coquillett, 1910: 571).

CURRENT Status: Preoccupied by Reichenbach, 1854; junior synonym of Sarcophaga Meigen, 1826 but subgenerically unplaced [teste Pape (1996: 420)].

FAMILY: SARCOPHAGIDAE.

332. Muscina Robineau-Desvoidy, 1830: 406.

ORIGINALly INCLUDED SPECIES: Musca pabulorum Fallén, 1817 (as "Musca pabulorum. Fall. Meig."); Musca stabulans Fallén, 1817 (as “Musca stabulans. Fall. Meig."); Muscina grisea Robineau-Desvoidy, 1830; Muscina picaena Robineau-Desvoidy, 1830; Muscina concolor Robineau-Desvoidy, 1830; Muscina fungivora Robineau-Desvoidy, 1830.

TYPE SPECIES: Musca stabulans Fallén, 1817, by subsequent designation (Coquillett, 1910: 571).

CURRENT STATUS: Valid genus [teste Pont (1986b: 59)].

FAMILY: MUSCIDAE. 
333. Mycetia Robineau-Desvoidy, 1830: 805.

ORIGINALLY INCLUDED SPECIES: Mycetia communis Robineau-Desvoidy, 1830; Mycetia tibialis RobineauDesvoidy, 1830; Mycetia claripennis Robineau-Desvoidy, 1830; Mycetia vulgaris Robineau-Desvoidy, 1830; Mycetia pallipes Robineau-Desvoidy, 1830.

TYPE SPECIES: Mycetia vulgaris Robineau-Desvoidy, 1830, by subsequent designation (Coquillett, 1910: 572).

CURRENT STATUS: Placed in "Doubtful genera of Sphaeroceridae" [teste Roháček in Roháček (2001: 295)].

FAMILY: SPHAEROCERIDAE.

\section{Myconia Robineau-Desvoidy, 1863a: 306.}

ORIGINALLY INCLUDED SPECIES: Myconia appendiculata Robineau-Desvoidy, 1863.

TYPE SPECIES: Myconia appendiculata Robineau-Desvoidy, 1863, by original designation.

CURRENT STATUS: Placed in "Doubtful taxa in Tachinidae" by Herting \& Dely-Draskovits (1993: 437).

FAMILY: TACHINIDAE.

\section{Mydaea Robineau-Desvoidy, 1830: 479.}

ORIGINALly INCLUDED SPECIES: Mydaea floricola Robineau-Desvoidy, 1830; Mydaea pellucida RobineauDesvoidy, 1830; Mydaea suillorum Robineau-Desvoidy, 1830; Mydaea rapida Robineau-Desvoidy, 1830; Mydaea scutellaris Robineau-Desvoidy, 1830; Mydaea humeralis Robineau-Desvoidy, 1830; Mydaea musca Robineau-Desvoidy, 1830; Mydaea liturata Robineau-Desvoidy, 1830; Mydaea nigripes Robineau-Desvoidy, 1830.

TYPE SPECIES: Mydaea scutellaris Robineau-Desvoidy, 1830 [= Musca corni Scopoli, 1763], by subsequent designation (Coquillett, 1901: 139).

CuRRENT STATUS: Valid genus [teste Pont (1986b: 155)].

FAMILY: MUSCIDAE.

336. Mydina Robineau-Desvoidy, 1830: 495.

ORIGINALly INCLUdED SPECIES: Mydina fulvicornis Robineau-Desvoidy, 1830; Mydina analis RobineauDesvoidy, 1830; Mydina impunctata Robineau-Desvoidy, 1830; Mydina dispar Robineau-Desvoidy, 1830; Mydina nigricans Robineau-Desvoidy, 1830; Mydina communis Robineau-Desvoidy, 1830; Mydina claripennis Robineau-Desvoidy, 1830; Mydina limpidipennis Robineau-Desvoidy, 1830; Mydina nigripes Robineau-Desvoidy, 1830; Mydina vernalis Robineau-Desvoidy, 1830; Mydina fuliginosa Robineau-Desvoidy, 1830; Mydina cinerascens Robineau-Desvoidy, 1830; Mydina ludibunda RobineauDesvoidy, 1830; Mydina campestris Robineau-Desvoidy, 1830; Mydina pellucida Robineau-Desvoidy, 1830; Mydina laetifica Robineau-Desvoidy, 1830; Mydina nitens Robineau-Desvoidy, 1830; Mydina soror Robineau-Desvoidy, 1830; Mydina maura Robineau-Desvoidy, 1830; Mydina nigra RobineauDesvoidy, 1830; Mydina palustris Robineau-Desvoidy, 1830; Mydina mollis Robineau-Desvoidy, 1830.

TYPE SPECIES: Mydina dispar Robineau-Desvoidy, 1830 [= Musca quadrum Fabricius, 1805], by subsequent designation (Coquillett, 1901: 139).

Current Status: Junior synonym of Helina Robineau-Desvoidy, 1830 [teste Pont (1986b: 136)].

FAMILY: MUSCIDAE.

337. Myennis Robineau-Desvoidy, 1830: 717.

ORIGINALLY INCLUDED SPECIES: Myennis fasciata Robineau-Desvoidy, 1830.

TYPE SPECIES: Myennis fasciata Robineau-Desvoidy, 1830, by monotypy.

CURRENT STATUS: Valid genus [teste Soós (1984c: 58)].

FAMILY: ULIDIIDAE. 
338. Myobia Robineau-Desvoidy, 1830: 98.

ORIGINALly INCLUDED SPECIES: Myobia fragilis Robineau-Desvoidy, 1830; Myobia sublutea RobineauDesvoidy, 1830; Myobia ruficrus Robineau-Desvoidy, 1830 (with "Dexia ruficrus. Meig." in synonymy); Myobia cinerascens Robineau-Desvoidy, 1830; Myobia nana Robineau-Desvoidy, 1830; Myobia nitens Robineau-Desvoidy, 1830; Myobia testacea Robineau-Desvoidy, 1830.

TYPE SPECIES: Myobia fragilis Robineau-Desvoidy, 1830 [= Tachina inanis Fallén, 1810], by subsequent designation (Townsend, 1916: 8).

CURRENT STATUS: Preoccupied by Heyden, 1826; senior (but invalid) synonym of Solieria RobineauDesvoidy, 1849 [teste Herting \& Dely-Draskovits (1993: 342)].

FAMILY: TACHINIDAE.

REMARKS: Dexia ruficrus was never described by Meigen (and there is no other "ruficrus" in Tachinidae prior to 1830), thus the new species "Myobia ruficrus" is attributable to Robineau-Desvoidy, 1830.

EMENDATIONS: Myiobia Mik, 1890: 155 (unjustified), n. syn.

339. Myocera Robineau-Desvoidy, 1830: 328.

ORIGINALly INCLUDED SPECIES: Myocera longipes Robineau-Desvoidy, 1830; Myocera calcium RobineauDesvoidy, 1830; Myocera anthophila Robineau-Desvoidy, 1830; Myocera fera Robineau-Desvoidy, 1830; Myocera nomada Robineau-Desvoidy, 1830; Myocera grisescens Robineau-Desvoidy, 1830.

TYPE SPECIES: Myocera longipes Robineau-Desvoidy, 1830 [= Musca ferina Fallén, 1817], by subsequent designation (Townsend, 1916: 8).

CURRENT STATUS: Junior synonym of Dinera Robineau-Desvoidy, 1830 [teste Herting \& Dely-Draskovits (1993: 361)].

FAMILY: TACHINIDAE.

EMENDATIONS: Myiocera Brauer \& Bergenstamm, 1889: 58 (unjustified), n. syn.

340. Myodina Robineau-Desvoidy, 1830: 727.

ORIGINALly InCludED SPECIES: Musca urticae Fabricius, 1794 (as “Musca; Tephrytis urticae. Fabr. Latr.”). TYPE SPECIES: Musca urticae Fabricius, 1794 (as “Musca; Tephrytis urticae. Fabr. Latr.”) [= Musca vibrans Linnaeus, 1758], by monotypy.

CURRENT STATUS: Junior synonym of Seioptera Kirby \& Spence, 1817 [teste Sabrosky (1999: 207)].

FAMILY: ULIDIIDAE.

EMENDATIONS: Myiodina Agassiz, 1846b: 242 (unjustified).

341. Myopella Robineau-Desvoidy, 1853a: 98 [1853b: 18].

ORIGINALLY INCLUDED SPECIES: Myopella puncticeps Robineau-Desvoidy, 1853; Myopella florea RobineauDesvoidy, 1853; Myopella marginalis Robineau-Desvoidy, 1853; Myopella punctigera RobineauDesvoidy, 1853; Conops buccata Linnaeus, 1758 (as “Conops buccatus : Linn.”); Myopa pictipennis Robineau-Desvoidy, 1830; Myopella nigrodorsata Robineau-Desvoidy, 1853 (as "Myopella nigrodorsata. Rob.-Desv."); Myopella flavipennis Robineau-Desvoidy, 1853 (as “Myopella flavi-pennis. Rob.Desv.”); Myopella scutellaris Robineau-Desvoidy, 1853.

TYPE SPECIES: Conops buccata Linnaeus, 1758, by original designation (as "Conops buccatus : Linn"). CURRENT StATUS: Junior synonym of Myopa Fabricius, 1775 [teste I.C.Z.N. (1997: 133 [Opinion 1870])]. FAMILY: CONOPIDAE.

REMARKS: Placed on the Official Index of Rejected and Invalid Names in Zoology by action of I.C.Z.N. (1997: 133 [Opinion 1870]). 
342. Myophora Robineau-Desvoidy, 1830: 337.

ORIGINALLY INCLUDED SPECIES: Myophora brasiliensis Robineau-Desvoidy, 1830; Myophora humboldti Robineau-Desvoidy, 1830; Myophora chrysella Robineau-Desvoidy, 1830; Myophora flaveola Robineau-Desvoidy, 1830; Myophora lherminieri Robineau-Desvoidy, 1830; Myophora argyrostoma Robineau-Desvoidy, 1830; Musca haemorrhoidalis Fallén, 1817 (as "Sarcophaga haemorrhoïdalis. Fall. Meig."); Myophora villica Robineau-Desvoidy, 1830; Myophora cognata Robineau-Desvoidy, 1830; Myophora fervida Robineau-Desvoidy, 1830; Myophora peronii Robineau-Desvoidy, 1830; Myophora fulvicornis Robineau-Desvoidy, 1830; Myophora viridescens Robineau-Desvoidy, 1830; Myophora cubensis Robineau-Desvoidy, 1830; Myophora squalida Robineau-Desvoidy, 1830; Myophora crocata Robineau-Desvoidy, 1830; Myophora rubiginosa Robineau-Desvoidy, 1830; Myophora contempta Robineau-Desvoidy, 1830; Myophora agilis Robineau-Desvoidy, 1830; Myophora blondeli RobineauDesvoidy, 1830; Sarcophaga haemorrhoa Meigen, 1826 (as "Sarcophaga haemorrhoa. Fall. Meig."); Myophora aestivalis Robineau-Desvoidy, 1830; Myophora consobrina Robineau-Desvoidy, 1830; Myophora riparia Robineau-Desvoidy, 1830; Myophora albicans Robineau-Desvoidy, 1830; Myophora collinaris Robineau-Desvoidy, 1830; Myophora limpidipennis Robineau-Desvoidy, 1830; Myophora hemisphaerica Robineau-Desvoidy, 1830; Myophora horticola Robineau-Desvoidy, 1830; Myophora campestris Robineau-Desvoidy, 1830; Musca carnaria Linnaeus, 1758; Myophora soror RobineauDesvoidy, 1830; Myophora capensis Robineau-Desvoidy, 1830; Myophora brunifacies RobineauDesvoidy, 1830; Myophora carceli Robineau-Desvoidy, 1830; Myophora rustica Robineau-Desvoidy, 1830; Myophora intermedia Robineau-Desvoidy, 1830; Myophora fuliginosa Robineau-Desvoidy, 1830; Myophora nigra Robineau-Desvoidy, 1830; Myophora atrata Robineau-Desvoidy, 1830; Myophora sordida Robineau-Desvoidy, 1830; Myophora livida Robineau-Desvoidy, 1830; Myophora livens Robineau-Desvoidy, 1830; Myophora duvaucelii Robineau-Desvoidy, 1830; Myophora villana Robineau-Desvoidy, 1830; Myophora squamosa Robineau-Desvoidy, 1830; Myophora florea RobineauDesvoidy, 1830; Myophora sericea Robineau-Desvoidy, 1830; Musca striata Fabricius 1794 (as "Musca. Fabr. Sarcophaga striata. Meig."); Myophora squamigera Robineau-Desvoidy, 1830; Myophora grisea Robineau-Desvoidy, 1830; Myophora agricola Robineau-Desvoidy, 1830; Myophora depressa Robineau-Desvoidy, 1830; Myophora obscuricauda Robineau-Desvoidy, 1830; Myophora aurifacies Robineau-Desvoidy, 1830; Myophora abdominalis Robineau-Desvoidy, 1830; Myophora compar Robineau-Desvoidy, 1830; Myophora arvicola Robineau-Desvoidy, 1830; Myophora aprilis RobineauDesvoidy, 1830; Myophora conica Robineau-Desvoidy, 1830; Myophora cinerea Robineau-Desvoidy, 1830; Myophora vernalis Robineau-Desvoidy, 1830; Myophora specialis Robineau-Desvoidy, 1830; Myophora squalens Robineau-Desvoidy, 1830; Myophora affinis Robineau-Desvoidy, 1830; Myophora peregrina Robineau-Desvoidy, 1830; Myophora subrotunda Robineau-Desvoidy, 1830; Myophora laticrus Robineau-Desvoidy, 1830; Myophora obscura Robineau-Desvoidy, 1830; Myophora maialis Robineau-Desvoidy, 1830 (as “Myophora maïalis. R. D.”); Myophora arvensis Robineau-Desvoidy, 1830; Myophora subnitens Robineau-Desvoidy, 1830; Myophora vivida Robineau-Desvoidy, 1830; Myophora albidipennis Robineau-Desvoidy, 1830; Myophora obscuripennis Robineau-Desvoidy, 1830; Myophora rubescens Robineau-Desvoidy, 1830; Myophora grisescens Robineau-Desvoidy, 1830; Myophora camporum Robineau-Desvoidy, 1830; Myophora musca Robineau-Desvoidy, 1830; Myophora rapida Robineau-Desvoidy, 1830; Myophora atra Robineau-Desvoidy, 1830; Myophora nigricans Robineau-Desvoidy, 1830; Myophora fuscipennis Robineau-Desvoidy, 1830; Myophora arvorum Robineau-Desvoidy, 1830; Myophora flavescens Robineau-Desvoidy, 1830; Myophora grisea Robineau-Desvoidy, 1830; Myophora nana Robineau-Desvoidy, 1830.

TYPE SPECIES: Musca carnaria Linnaeus, 1758, by subsequent designation (Coquillett, 1910: 573).

CURRENT StATUS: Junior synonym of Sarcophaga Meigen, 1826 sensu stricto [teste Pape (1996: 387)].

FAMILY: SARCOPHAGIDAE.

REMARKS: Verves (1986: 180) indicated incorrectly that Robineau-Desvoidy (1830: 349) designated Myophora fuliginosa Robineau-Desvoidy, 1830 as type species. An examination of that work shows that 
no designation was made anywhere by Robineau-Desvoidy. The earliest valid designation of an included species is of Musca carnaria Linnaeus, 1758 by Coquillett (1910: 573).

EMENDAtions: Myiophora Agassiz, 1846b: 242 (unjustified); Myiophora Bezzi \& Stein, 1907: 469, n. syn.

[Myophore] Robineau-Desvoidy MS name (Blainville et al., 1826: 11).

CURRENT STATUS: Unavailable name; suppressed by action of I.C.Z.N. (1990: 162 [Opinion 1601]); treated under Sarcophaga Meigen, 1826 sensu stricto [teste this work].

REMARKS: This was likely a typesetter's error in misreading the handwriting of what was intended to be Myophora.

FAMILY: SARCOPHAGIDAE.

343. Myopina Robineau-Desvoidy, 1830: 675.

ORIGINALLY INCLUDED SPECIES: Myopina reflexa Robineau-Desvoidy, 1830.

TYPE SPECIES: Myopina reflexa Robineau-Desvoidy, 1830 [= Musca myopina Fallén, 1824], by monotypy.

CURRENT STATUS: Valid genus [teste Sabrosky (1999: 208)].

FAMILY: ANTHOMYIIDAE.

344. Myopina Robineau-Desvoidy, 1853a: 119 [1853b: 39].

ORIGINALly InCluded SPECIES: Myopa variegata Meigen, 1804; Myopa maculata Macquart, 1835.

TYPE SPECIES: Myopa variegata Meigen, 1804, by original designation.

CURRENT STATUS: Preoccupied by Robineau-Desvoidy, 1830; junior synonym of Myopa Fabricius, 1775

[teste Chvála \& Smith (1988: 262)].

FAMILY: CONOPIDAE.

345. Myorhina Robineau-Desvoidy, 1830: 383.

ORIGINALLY INCLUDED SPECIES: Myorhina campestris Robineau-Desvoidy, 1830.

TYPE SPECIES: Myorhina campestris Robineau-Desvoidy, 1830 [= Sarcophaga nigriventris Meigen, 1826], by monotypy.

CURRENT STATUS: Valid subgenus of Sarcophaga Meigen, 1826 [teste Pape (1996: 364)].

FAMILY: SARCOPHAGIDAE.

EMENDATIONS: Myiorrhina Bezzi \& Stein, 1907: 467 (unjustified).

346. Myoris Robineau-Desvoidy, 1830: 711.

ORIGINALLY INCLUDED SPECIES: Myoris silvatica Robineau-Desvoidy, 1830.

TYPE SPECIES: Myoris silvatica Robineau-Desvoidy, 1830, by monotypy.

CURRENT STATUS: Junior synonym of Otites Latreille, 1804 [teste Soós (1984: 47)].

FAMILY: ULIDIIDAE.

REMARKS: Previous listings of the type species have it as a junior synonym of Ortalis guttata Meigen, 1830, but this is incorrect. Robineau-Desvoidy's 1830 paper came out 6 June while Meigen's 1830 paper came out six months later on 1 December [see References in this paper for dating of the former and Evenhuis (1997: 533) for dating of the latter].

EMENDATIOns: Myorhis Agassiz, 1846b: 243 (unjustified); Myorrhis Verrall in Scudder, 1882: 219 (unjustified). 
347. Myostoma Robineau-Desvoidy, 1830: 327.

ORIGINALLY INCLUDED SPECIES: Myostoma microcera Robineau-Desvoidy, 1830; Myostoma scutellaris Robineau-Desvoidy, 1830.

TYPE SPECIES: Myostoma microcera Robineau-Desvoidy, 1830, by subsequent designation (Rondani, 1856: $83)$.

CURRENT StATUS: Junior synonym of Estheria Robineau-Desvoidy, 1830 [teste Herting \& Dely-Draskovits (1993: 363)].

FAMILY: TACHINIDAE.

EMENDATIONS: Myiostoma Brauer \& Bergenstamm, 1889: 50 (unjustified), n. syn.

348. Myrmecomya Robineau-Desvoidy, 1830: 721.

ORIGINALlY INCLUDED SPECIES: Myrmecomya formicaria Robineau-Desvoidy, 1830; Myrmecomya micropezoidea Robineau-Desvoidy, 1830 (as “Myrmecomya micropezoïdea. R. D.”).

TYPE SPECIES: Myrmecomya formicaria Robineau-Desvoidy, 1830 [= Cephalia rufipes Meigen, 1826], by subsequent designation (Coquillett, 1910: 573).

CURRENT STATUS: Junior synonym of Cephalia Meigen, 1826 [teste Sabrosky (1999: 208)].

FAMILY: ULIDIIDAE.

EMENDATIONS: Myrmecomyia Agassiz, 1846b: 243 (unjustified); Myrmecomyia Loew, 1873: 18 (unjustified).

349. Myrsina Robineau-Desvoidy, 1863b: 158.

ORIGINALly INCLUDED SPECIES: Myrsina ambulatrix Robineau-Desvoidy, 1863; Myrsina ardeacea Robineau-Desvoidy, 1863; Myrsina pusilla Robineau-Desvoidy, 1863.

TYPE SPECIES: Myrsina ambulatrix Robineau-Desvoidy, 1863 [= Tachina selecta Meigen, 1824], by original designation.

CURRENT Status: Junior synonym of Bessa Robineau-Desvoidy, 1830 [teste Herting \& Dely-Draskovits (1993: 142)].

FAMILY: TACHINIDAE.

350. Napaea Robineau-Desvoidy, 1830: 799.

ORIGINALlY INCLUDED SPECIES: Napaea stagnicola Robineau-Desvoidy, 1830; Napaea pygmaea RobineauDesvoidy, 1830.

TYPE SPECIES: Napaea stagnicola Robineau-Desvoidy, 1830, by subsequent designation (Coquillett, 1910: 573).

CuRrent Status: Preoccupied by Hübner, 1819; senior (but invalid) synonym of Parydra Stenhammar, 1844 [teste Sabrosky (1999: 210)].

FAMILY: EPHYDRIDAE.

\section{Neaera Robineau-Desvoidy, 1830: 84.}

ORIGINALLY INCLUDED SPECIES: Neaera immaculata Robineau-Desvoidy, 1830.

TYPE SPECIES: Neaera immaculata Robineau-Desvoidy, 1830 [= Tachina laticornis Meigen, 1824], by monotypy.

CuRrent Status: Valid genus [teste Herting \& Dely-Draskovits (1993: 320)].

FAMILY: TACHINIDAE. 
352. Nemopoda Robineau-Desvoidy, 1830: 743.

ORIGINALly INCLUDED SPECIES: Nemopoda putris Robineau-Desvoidy, 1830; Nemopoda ferruginea Robineau-Desvoidy, 1830; Nemopoda stercoraria Robineau-Desvoidy, 1830; Nemopoda brunicosa Robineau-Desvoidy, 1830; Nemopoda gagatea Robineau-Desvoidy, 1830.

TYPE SPECIES: Nemopoda putris Robineau-Desvoidy, 1830 [= Sepsis nitidula Fallén, 1820], by subsequent designation (d'Orbigny, 1846: 611).

Current Status: Valid genus [teste Pont \& Meier (2002: 117)].

FAMILY: SEPSIDAE.

REMARKS: Because there are no author's initials for this type-species designation, it is attributed to the editor, d'Orbigny, as explained by Evenhuis \& Thompson (1990: 237).

EMENDATIONS: Nematopoda Agassiz, 1846b: 247 (unjustified); Nematopoda Rye, 1873: 396 (unjustified).

\section{Nemoraea Robineau-Desvoidy, 1830: 71}

ORIGINAlly INCLUDED SPECIES: Nemoraea bombylans Robineau-Desvoidy, 1830; Nemoraea affinis Robineau-Desvoidy, 1830; Nemoraea fulva Robineau-Desvoidy, 1830; Nemoraea scutellata RobineauDesvoidy, 1830; Nemoraea obliqua Robineau-Desvoidy, 1830; Nemoraea brunicornis RobineauDesvoidy, 1830.

TYPE SPECIES: Nemoraea bombylans Robineau-Desvoidy, 1830 [= Tachina pellucida Meigen, 1824], by subsequent designation (Townsend, 1916: 8).

CURRENT StATUS: Valid genus [teste Herting \& Dely-Draskovits (1999: 282)].

FAMILY: TACHINIDAE.

REMARKS: An earlier type-species designation by Robineau-Desvoidy (1863a: 173) of Tachina pellucida Meigen, 1824 is invalid as it was not an originally included species and more than one originally included species were placed in synonymy with it.

\section{Nemorhina Robineau-Desvoidy, 1830: 389}

ORIGINALLY INCLUDED SPECIES: Nemorhina palpalis Robineau-Desvoidy, 1830.

TYPE SPECIES: Nemorhina palpalis Robineau-Desvoidy, 1830, by monotypy.

CuRrent Status: Treated under Glossina Wiedemann, 1830 [teste Pont (1980a: 764)].

FAMILY: GLOSSINIDAE.

REMARKS: Nemorhina Robineau-Desvoidy, 1830 is occasionally used as a valid subgenus, e.g., Sutton \& Carlson (1997) or included species are referred to as the Glossina palpalis group, e.g., Mohamed-Ahmed \& Mihok (1999). Robineau-Desvoidy (1830) came out on 6 June while Wiedemann's 1830 paper came out less than three months later in September [see References in this paper for dating of the former and Evenhuis (1997: 822) for dating of the latter], which means Nemorhina Robineau-Desvoidy, 1830 predates Glossina Wiedemann, 1830. However, acceptance of this priority would upset the nomenclature of the medically important Glossina, for which there is an extensive literature. Thus, application to the ICZN Commission for reverse priority between Nemorhina and Glossina when treated in synonymy is in preparation.

EMENDATIONS: Nematorhina Agassiz, 1846b: 247 (unjustified).

\section{Nerea Robineau-Desvoidy, 1830: 802.}

ORIGINAlly INCLUDED SPECIES: Nerea riparia Robineau-Desvoidy, 1830; Nerea stercoraria RobineauDesvoidy, 1830; Nerea nitida Robineau-Desvoidy, 1830.

TYPE SPECIES: Nerea riparia Robineau-Desvoidy, 1830, by subsequent designation (Coquillett, 1910: 575).

CURRENT STATUS: Placed in "Doubtful genera of Limosininae" [teste Roháček in Roháček (2001: 295)].

FAMILY: SPHAEROCERIDAE. 
356. Neria Robineau-Desvoidy, 1830: 736.

CURRENT STATUS: Unjustified emendation of Nerius Fabricius, 1805; junior synonym of Nerius Fabricius, 1805 [teste Neave, 1940: 318)].

FAMILY: NERIIDAE.

REMARKS: Robineau-Desvoidy (1830: 736) was clearly emending Nerius Fabricius when he proposed Neria by placing it in the heading with "Nerius. Fabr." in synonymy underneath it. He further stated under the diagnosis of Neria: "Fabricius, ainsi que j'en possède une étiquette de sa main avait nommé Nerius le genre d'insectes dont il est question." Despite this, Coquillett (1910: 575) recognized "Nerius FABRICIUS" and "Neria ROBINEAU-DESVOIDY" as separate genera and designated Nerius pilifer Fabricius, 1805 as type species of Nerius [currently in Neriidae] and Neria inulae Robineau-Desvoidy, 1830 as type species of Neria [currently in Micropezidae]. Neave (1940: 318) correctly cited Neria as an emendation of Nerius but other authors have followed the designations of Coquillett (1910). Currently, there are two species treated in Neria (Soós, 1984: 23). These species now fall under the next available name for the genus, Paracalobata Hendel, 1922: Paracalobata ephippium (Fabricius, 1794), n. comb.; Paracalobata octoannulata (Strobl, 1899), n. comb.

357. Nerina Robineau-Desvoidy, 1830: 557.

ORIGINALly INCLUDED SPECIES: Nerina cinerea Robineau-Desvoidy, 1830; Nerina albipennis RobineauDesvoidy, 1830; Nerina flavescens Robineau-Desvoidy, 1830; Nerina dejeanii Robineau-Desvoidy, 1830; Nerina prompta Robineau-Desvoidy, 1830.

TYPE SPECIES: Nerina albipennis Robineau-Desvoidy, 1830 [= Musca cinerella Fallén, 1825], by subsequent designation (Coquillett, 1910: 575; as "Nerina albipennis FALLEN").

CURRENT STATUS: Preoccupied by Deshayes, 1827; junior synonym of Adia Robineau-Desvoidy, 1830 [teste Dely-Draskovits (1993: 13)].

FAMILY: ANTHOMYIIDAE.

358. Nicaea Robineau-Desvoidy, 1863b: 399.

ORIGINALly INCLUDED SPECIES: Nicaea palpata Robineau-Desvoidy, 1863.

TYPE SPECIES: Nicaea palpata Robineau-Desvoidy, 1863 [= Dexia irrorata Meigen, 1826], by monotypy.

CURRENT Status: Junior synonym of Billaea Robineau-Desvoidy, 1830 [teste Herting \& Dely-Draskovits (1993: 357)].

FAMILY: TACHINIDAE.

EMENDATIONS: Nicea Marschall, 1873: 339 (unjustified), n. syn.

[Nigria] Robineau-Desvoidy MS name (Blainville et al., 1826: 12).

CURRENT STATUS: Unavailable name; suppressed by action of I.C.Z.N. (1990: 162 [Opinion 1601]); treated as a nomen dubium in Tachinidae [teste this work].

FAMILY: TACHINIDAE.

359. Nigrina Robineau-Desvoidy, 1863a: 932.

ORIGINALLY INCLUDED SPECIES: Nigrina flavipalpis Robineau-Desvoidy, 1863.

TYPE SPECIES: Nigrina flavipalpis Robineau-Desvoidy, 1863, by original designation.

CURRENT STATUS: Placed in "Doubtful taxa in Tachinidae" by Herting \& Dely-Draskovits (1993: 437).

FAMILY: TACHINIDAE. 
360. Nilea Robineau-Desvoidy, 1863a: 275.

ORIGINALLY INCLUDED SPECIES: Nilea innoxia Robineau-Desvoidy, 1863.

TYPE SPECIES: Nilea innoxia Robineau-Desvoidy, 1863, by original designation.

CurRent Status: Valid genus [teste Herting \& Dely-Draskovits (1993: 188)].

FAMILY: TACHINIDAE.

361. Nitellia Robineau-Desvoidy, 1830: 417.

ORIGINALly INCLUDED SPECIES: Musca vespillo Fabricius, 1794 (as “Musca vespillo. Fabr. Meig.”); Nitellia nana Robineau-Desvoidy, 1830.

TYPE SPECIES: Musca vespillo Fabricius, 1794 sensu Robineau-Desvoidy, 1830 [misidentification cf. Rognes

(1991: 215); = Musca atramentaria Meigen, 1826], by subsequent designation (Coquillett, 1910: 576).

CURRENT Status: Junior synonym of Pollenia Robineau-Desvoidy, 1830 [teste Rognes (1991: 212, 215)].

FAMILY: CALLIPHORIDAE.

362. Noeeta Robineau-Desvoidy, 1830: 778.

ORIGINALly INCLUDED SPECIES: Noeeta flavipes Robineau-Desvoidy, 1830; Noeeta brunicosa RobineauDesvoidy, 1830.

TYPE SPECIES: Noeeta flavipes Robineau-Desvoidy, 1380 [= Tephritis pupillata Fallén, 1814], by subsequent designation (White, 1986: 146).

CURRENT STATUS: Valid genus [teste Norrbom et al. (1999: 173)].

FAMILY: TEPHRITIDAE.

REMARKS: An earlier type-species designation by Hendel (1927: 206) of Tephritis pupillata Fallén, 1814 is invalid because it was not an originally included species.

363. Norellia Robineau-Desvoidy, 1830: 673.

ORIGINAlly INCLUDED SPECIES: Norellia pseudonarcissi Robineau-Desvoidy, 1830 (as "Norellia pseudonarcissi. R. D.”).

TYPE SPECIES: Norellia pseudonarcissi Robineau-Desvoidy, 1830 (as "Norellia pseudo-narcissi. R. D.”) [= Musca tipularia Fabricius, 1794], by monotypy.

CURRENT STATUS: Valid genus [teste Sabrosky (1999: 216)].

FAMILY: SCATHOPHAGIDAE.

364. Novia Robineau-Desvoidy, 1830: 210.

ORIGINALly INCLUDED SPECIES: Novia cylindrica Robineau-Desvoidy, 1830.

TYPE SPECIES: Novia cylindrica Robineau-Desvoidy, 1830, by monotypy.

CURRENT STATUS: Placed in "Doubtful taxa in Tachinidae" by Herting \& Dely-Draskovits (1993: 437).

FAMILY: TACHINIDAE.

365. Nupharia Robineau-Desvoidy, 1830: 785.

ORIGINALLY INCLUDED SPECIES: Nupharia rivularis Robineau-Desvoidy, 1830.

TYPE SPECIES: Nupharia rivularis Robineau-Desvoidy, 1830 [= Musca livens Fabricius, 1794], by monotypy.

CURRENT STATUS: Junior synonym of Hydromyza Fallén, 1813 [teste Gorodkov (1986: 36)].

FAMILY: SCATHOPHAGIDAE.

REMARKS: Gorodkov (1986: 36) incorrectly ascribed Hydromyza to Fallén (1823: 1). 
366. Nyctia Robineau-Desvoidy, 1830: 262.

ORIGINALly INCLUDED SPECIES: Nyctia carceli Robineau-Desvoidy, 1830; Nyctia servillei RobineauDesvoidy, 1830; Nyctia claripennis Robineau-Desvoidy, 1830; Nyctia trifaria Robineau-Desvoidy, 1830; Nyctia nitida Robineau-Desvoidy, 1830; Nyctia rubescens Robineau-Desvoidy, 1830; Nyctia pusilla Robineau-Desvoidy, 1830.

TYPE SPECIES: Nyctia carceli Robineau-Desvoidy, 1830 [= Musca halterata Panzer, 1798], by subsequent designation (Townsend, 1916: 8).

CURRENT STATUS: Valid genus [teste Pape (1996: 165)].

FAMILY: SARCOPHAGIDAE.

REMARKS: Pape (1996: 165) noted that the oldest name for the type species is Musca nigrita Scopoli, 1763, but in the interests of stability, he maintained Musca halterata Panzer, 1798. To our knowledge, the former name has not been used as valid after 1899, and the latter name has been used (consistently as Nyctia halterata) in more than 25 works by well over 10 authors published during the past 50 years: Čepelák \& Čepelák (1988); Chandler (1988); Draber-Mońko (1971); Dumčius \& Pakalniškis (2004); Gosseries (1991); Kara \& Pape (2002); Mihályi (1979, 1981); Pape (1987, 1996, 1998a); Pape et al. (1995, 2002); Petersen \& Pape (2001); Povolný (1997); Povolný \& Verves (1997); Rognes (2006); Irwin (2006); Velterop \& Sijstermans (2002); Verves (1982, 1986, 2000, 2001); Whitmore et al. (2008); Wills (1968); and Ziegler \& Lange (2001). In the interests of nomenclatural stability, we hereby invoke reversal of precedence (ICZN Code Article 23.9.2) and declare Musca nigrita Scopoli, 1763 a nomen oblitum and Musca halterata Panzer, 1798 a nomen protectum. Lehrer (2005: 56) erred when designating his Nyctia gilbochaeta Lehrer, 2005 [here interpreted as Nyctia lugubris Macquart, 1843 as revised by Pape (1996)] as a replacement type species of Nyctia. The designation of Townsend (1916: 8) stands, and Lehrer (2005: 56) was in error in invoking ICZN Code Article 70.3.2 as this relates exclusively to Townsend's concept of Nyctia halterata.

367. Obeida Robineau-Desvoidy, 1863b: 167.

ORIGINALLY INCLUDED SPECIES: Obeida obscurata Robineau-Desvoidy, 1863.

TYPE SPECIES: Obeida obscurata Robineau-Desvoidy, 1863, by original designation.

CURRENT StATUS: Placed in "Doubtful taxa in Tachinidae" by Herting \& Dely-Draskovits (1993: 437).

FAMILY: TACHINIDAE.

368. Oblicia Robineau-Desvoidy, 1830: 620.

ORIGINALLY INCLUDED SPECIES: Oblicia testacea Robineau-Desvoidy, 1830.

TYPE SPECIES: Oblicia testacea Robineau-Desvoidy, 1830 [= Musca fimetaria Linnaeus, 1761], by monotypy.

CurRent Status: Junior synonym of Psila Meigen, 1803 [teste Soós (1984a: 28)].

FAMILY: PSILIDAE.

369. Ocalea Robineau-Desvoidy, 1863a: 810.

ORIGINALLY INCLUDED SPECIES: Ocalea heterocera Robineau-Desvoidy, 1863 (as "Scopolia heterocera : Macq.").

TYPE SPECIES: Ocalea heterocera Robineau-Desvoidy, 1863 (as "Scopolia heterocera : Macq."), by monotypy.

CuRRENT STATUS: Preoccupied by Erichson, 1837; junior synonym of Wagneria Robineau-Desvoidy, 1830 [teste Herting \& Dely-Draskovits (1993: 380)].

FAMILY: TACHINIDAE. 
370. Occemya Robineau-Desvoidy, 1853a: 130 [1853b: 50].

ORIGINALLY INCLUDED SPECIES: Myopa sundewalli Zetterstedt, 1844 (as "Myopa sunderwalli : Zetterst."); Occemya femoralis Robineau-Desvoidy, 1853; Occemya nitidula Robineau-Desvoidy, 1853; Occemya fulvifrons Robineau-Desvoidy, 1853; Occemya meigeni Robineau-Desvoidy, 1853; Myopa pallipes Wiedemann, 1824 (as "Myopa pallipes : Megerle"); Occemya dufouri Robineau-Desvoidy, 1853; Occemya grisea Robineau-Desvoidy, 1853; Myopa atra Fabricius, 1775 (with "Myopa femorata : Fabr." in synonymy); Occemya lamarckii Robineau-Desvoidy, 1853; Occemya macquarti Robineau-Desvoidy, 1853; Occemya guerini Robineau-Desvoidy, 1853; Occemya bigoti Robineau-Desvoidy, 1853; Occemya brunipes Robineau-Desvoidy, 1853; Occemya lucasi Robineau-Desvoidy, 1853; Myopa pusilla Meigen, 1824; Myopa nana Robineau-Desvoidy, 1830.

TYPE SPECIES: Myopa atra Fabricius, 1775, by original designation.

CURRENT STATUS: Junior synonym of Thecophora Rondani, 1845 [teste Chvála \& Smith (1988: 267)].

FAMILY: CONOPIDAE.

EMENDATIONS: Occemyia Schiner, 1861a: 381 (unjustified), n. syn.; Occemyia Marschall, 1873: 339 (unjustified).

371. Odinia Robineau-Desvoidy, 1830: 648.

ORIGINALly INCLUDED SPECIES: Odinia trinotata Robineau-Desvoidy, 1830 (as “Odinia tri-notata. R. D.”); Odinia peleterii Robineau-Desvoidy, 1830.

TYPE SPECIES: Odinia trinotata Robineau-Desvoidy, 1830, by subsequent designation (Rondani, 1875: 167).

CURRENT StATUS: Valid genus [teste Sabrosky (1999: 219)].

FAMILY: ODINIIDAE.

REMARKS: Previous listings of the type species have it as a junior synonym of Milichia maculata Meigen, 1830 , but this is incorrect. Robineau-Desvoidy's 1830 paper came out 6 June while Meigen's 1830 paper came out six months later on 1 December [see References in this paper for dating of the former and Evenhuis (1997: 533) for dating of the latter].

\section{Oebalia Robineau-Desvoidy, 1863b: 414.}

ORIGINALLY INCLUDED SPECIES: Oebalia anacantha Robineau-Desvoidy, 1863.

TYPE SPECIES: Oebalia anacantha Robineau-Desvoidy, 1863 [= Tachina cylindrica Fallén, 1810], by monotypy.

CuRRENT STATUS: Valid genus [teste Pape (1996: 119)].

FAMILY: SARCOPHAGIDAE.

REMARKS: There are two original spellings of this genus in Robineau-Desvoidy (1863b): Oebalia (page 414) and Aebalia (page 347). Acting as First Reviser, Pape (1996: 119) selected Oebalia as the correct original spelling.

373. Olbya Robineau-Desvoidy, 1863a: 170.

ORIGINALly INCLUDED SPECIES: Olbya brunisquamis Robineau-Desvoidy, 1863.

TYPE SPECIES: Olbya brunisquamis Robineau-Desvoidy, 1863 [= Tachina tessellum Meigen, 1824], by monotypy.

CURRENT Status: Junior synonym of Macquartia Robineau-Desvoidy, 1830 [teste Herting \& DelyDraskovits (1993: 312)].

FAMILY: TACHINIDAE. 
374. Olina Robineau-Desvoidy, 1830: 812.

ORIGINALLY INCLUDED SPECIES: Olina clavipes Robineau-Desvoidy, 1830; Olina capensis RobineauDesvoidy, 1830; Olina hirtipes Robineau-Desvoidy, 1830; Olina pallipes Robineau-Desvoidy, 1830; Olina nudipes Robineau-Desvoidy, 1830.

TYPE SPECIES: Olina hirtipes Robineau-Desvoidy, 1830, by subsequent designation (Macquart, 1835: 571).

CURRENT STATUS: Valid genus; removed from Sphaeroceridae to Heleomyzidae [teste Roháček in Roháček (2001: 39)]; treated as incertae sedis in Heleomyzidae [teste this work].

FAMILY: HELEOMYZIDAE.

REMARKS: Roháček in Roháček (2001: 39) noted that because of the size and other characteristics of the type species, it and other nominal species described by Robineau-Desvoidy in Olina should probably be removed to Heleomyzidae.

375. Olinda Robineau-Desvoidy, 1830: 116.

ORIGINALLY INCLUDED SPECIES: Olinda brasiliensis Robineau-Desvoidy, 1830.

TYPE SPECIES: Olinda brasiliensis Robineau-Desvoidy, 1830, by monotypy.

CURRENT STATUS: Valid genus [teste Guimarães (1971: 217)].

FAMILY: TACHINIDAE.

376. Olivieria Robineau-Desvoidy, 1830: 228.

ORIGINALLY INCLUDED SPECIES: Musca lateralis Fabricius, 1775 (as “Ocyptera lateralis. Oliv. Fabr.”).

TYPE SPECIES: Musca lateralis Fabricius, 1775 (as "Ocyptera lateralis. Oliv. Fabr.") [preoccupied by Musca lateralis Linnaeus, 1758; = Musca rufomaculata De Geer, 1776], by monotypy.

CURRENT STATUS: Junior synonym of Eriothrix Meigen, 1803 [teste Herting \& Dely-Draskovits (1993: 371)]. FAMILY: TACHINIDAE.

EMENDATIONS: Oliviera Lioy, 1864b: 66 (unjustified), n. syn.

377. Onesia Robineau-Desvoidy, 1830: 365.

ORIGINALly INCLUDED SPECIES: Onesia floralis Robineau-Desvoidy, 1830; Onesia riparia RobineauDesvoidy, 1830; Onesia claripennis Robineau-Desvoidy, 1830; Onesia viarum Robineau-Desvoidy, 1830; Onesia vulgaris Robineau-Desvoidy, 1830; Onesia cuprea Robineau-Desvoidy, 1830; Onesia lepida Robineau-Desvoidy, 1830; Onesia viridicyanea Robineau-Desvoidy, 1830 (as "Onesia viridicyanea. R. D.”); Onesia viridula Robineau-Desvoidy, 1830; Onesia tessellata Robineau-Desvoidy, 1830; Onesia viridulans Robineau-Desvoidy, 1830; Onesia caerulea Robineau-Desvoidy, 1830; Onesia prompta Robineau-Desvoidy, 1830; Onesia velox Robineau-Desvoidy, 1830; Onesia cyanea RobineauDesvoidy, 1830; Onesia gentilis Robineau-Desvoidy, 1830.

TYPE SPECIES: Onesia floralis Robineau-Desvoidy, 1830, by subsequent designation (Townsend, 1916: 8).

CURRENT STATUS: Valid genus [teste Rognes (1991: 97)].

FAMILY: CALLIPHORIDAE.

\section{Opesia Robineau-Desvoidy, 1863b: 276.}

ORIGINALly INCLUDED SPECIES: Opesia gagatea Robineau-Desvoidy, 1863; Opesia adspersa RobineauDesvoidy, 1863; Opesia florilega Robineau-Desvoidy, 1863; Opesia grisea Robineau-Desvoidy, 1863; Opesia occlusa Robineau-Desvoidy, 1863.

TYPE SPECIES: Opesia gagatea Robineau-Desvoidy, 1863 [= Phasia cana Meigen, 1824], by subsequent designation (Townsend, 1916: 8).

CURRENT STATUS: Valid genus [teste Herting \& Dely-Draskovits (1993: 407)].

FAMILY: TACHINIDAE. 
379. Ophelia Robineau-Desvoidy, 1830: 120.

ORIGINALly INCLUDED SPECIES: Ophelia agilis Robineau-Desvoidy, 1830; Ophelia festiva RobineauDesvoidy, 1830; Ophelia gracilis Robineau-Desvoidy, 1830; Ophelia lutescens Robineau-Desvoidy, 1830; Ophelia cinerea Robineau-Desvoidy, 1830.

TYPE SPECIES: Ophelia gracilis Robineau-Desvoidy, 1830 [= Tachina campestris Fallén, 1810], by subsequent designation (Townsend, 1916: 8).

CurRent Status: Preoccupied by Savigny, 1822; junior synonym of Metopia Meigen, 1803 [teste Pape (1996: 97)].

FAMILY: SARCOPHAGIDAE.

380. Ophina Robineau-Desvoidy, 1863a: 298.

ORIGINALLY INCLUDED SPECIES: Ophina fulvipes Robineau-Desvoidy, 1863.

TYPE SPECIES: Ophina fulvipes Robineau-Desvoidy, 1863 [= Tachina picta Meigen, 1824], by original designation.

CURRENT STATUS: Valid subgenus of Linnaemya Robineau-Desvoidy, 1830 [teste Herting \& Dely-Draskovits (1993: 286)].

FAMILY: TACHINIDAE.

381. Ophyra Robineau-Desvoidy, 1830: 516.

ORIGINALLY INCLUDED SPECIES: Ophyra nitida Robineau-Desvoidy, 1830; Ophyra pubescens RobineauDesvoidy, 1830; Ophyra rutilans Robineau-Desvoidy, 1830; Ophyra viridescens Robineau-Desvoidy, 1830.

TyPE SPECIES: Ophyra nitida Robineau-Desvoidy, 1830 [= Musca ignava Harris, 1780], by subsequent designation (Rondani, 1866: 70).

CURRENT Status: Junior synonym of Hydrotaea Robineau-Desvoidy, 1830 [teste Pont (1986b: 74)].

FAMILY: MUSCIDAE.

REMARKS: Later designations by Westwood (1840: 142) and De Galdo (1856) of Anthomyia leucostoma Wiedemann, 1817 are invalid as this species was not an originally included species.

EMENDATIONS: Ophira Bigot, 1892: 203 (unjustified), n. syn.

382. Oppia Robineau-Desvoidy, 1863a: 309.

ORIGINALly INCLUDED SPECIES: Hubneria nigripalpis Robineau-Desvoidy, 1848; Oppia tristis RobineauDesvoidy, 1863; Carcelia fuscipennis Robineau-Desvoidy, 1830; Oppia albibarbis Robineau-Desvoidy, 1863; Oppia muscidea Robineau-Desvoidy, 1863; Oppia floralis Robineau-Desvoidy, 1863.

TYPE SPECIES: Hubneria nigripalpis Robineau-Desvoidy, 1848, by fixation of O'Hara \& Wood (2004: 137) under ICZN Code Article 70.3.2, misidentified as Carcelia fuscipennis Robineau-Desvoidy, 1830 in the original designation by Robineau-Desvoidy (1863a: 310).

CurRent Status: Preoccupied by Koch, 1835; senior (but invalid) synonym of Prooppia Townsend, 1926 [teste O'Hara \& Wood (2004: 137)].

FAMILY: TACHINIDAE.

383. Oppia Robineau-Desvoidy, 1863b: 404.

ORIGINALLY INCLUDED SPECIES: Oppia ciligera Robineau-Desvoidy, 1863.

TYPE SPECIES: Oppia ciligera Robineau-Desvoidy, 1863 [= Tachina devia Fallén, 1820], by monotypy.

CURRENT STATUS: Preoccupied by Koch, 1835; junior synonym of Brachicoma Rondani, 1856 [teste Pape (1996: 160)].

FAMILY: SARCOPHAGIDAE. 
384. Orbellia Robineau-Desvoidy, 1830: 656.

ORIGINALLY INCLUDED SPECIES: Orbellia myopiformis Robineau-Desvoidy, 1830; Orbellia ornithivora Robineau-Desvoidy, 1830.

TYPE SPECIES: Orbellia myopiformis Robineau-Desvoidy, 1830, by subsequent designation (Coquillett, 1910: $580)$.

CURRENT STATUS: Valid genus [teste Sabrosky (1999: 225)].

FAMILY: HELEOMYZIDAE.

385. Orellia Robineau-Desvoidy, 1830: 765.

ORIGINALLY INCLUDED SPECIES: Orellia flavicans Robineau-Desvoidy, 1830.

TYPE SPECIES: Orellia flavicans Robineau-Desvoidy, 1830 [= Musca stictica Gmelin, 1790], by monotypy. CURRENT STATUS: Valid genus [teste Norrbom et al. (1999: 177)].

FAMILY: TEPHRITIDAE.

386. Oresbia Robineau-Desvoidy, 1863b: 411.

ORIGINALLY INCLUDED SPECIES: Oresbia arenaria Robineau-Desvoidy, 1863.

TYPE SPECIES: Oresbia arenaria Robineau-Desvoidy, 1863, by monotypy.

CURRENT STATUS: Nomen dubium [teste this work].

FAMILY: SARCOPHAGIDAE.

REMARKS: Although originally placed in the current concept of the family Tachinidae, Herting (1974: 41) stated that the description of Oresbia places it in the Miltogramminae of the Sarcophagidae. Until a type specimen of the type species is found, Oresbia is best regarded as a nomen dubium in Miltogramminae.

387. Oria Robineau-Desvoidy, 1863a: 274.

ORIGINALLY INCLUDED SPECIES: Oria fugitiva Robineau-Desvoidy, 1863.

TYPE SPECIES: Oria fugitiva Robineau-Desvoidy, 1863, by original designation.

CURRENT STATUS: Preoccupied by Hübner, 1821; Guenée, 1852; no new replacement name proposed; placed in "Doubtful taxa in Tachinidae" by Herting \& Dely-Draskovits (1993: 437).

FAMILY: TACHINIDAE.

388. Orillia Robineau-Desvoidy, 1849a: 474.

ORIGINALly INCLUDED SPECIES: Orillia curvinervis Robineau-Desvoidy, 1849; Orillia rectinervis RobineauDesvoidy, 1849; Orillia pellucida Robineau-Desvoidy, 1849.

TYPE SPECIES: Orillia rectinervis Robineau-Desvoidy, 1849, by subsequent designation (Coquillett, 1910: $580)$.

CURRENT STATUS: Placed in "Doubtful taxa in Tachinidae" by Herting \& Dely-Draskovits (1993: 437).

FAMILY: TACHINIDAE.

389. Orizia Robineau-Desvoidy, 1863b: 678.

ORIGINAlly INCLUdED SPECIES: Orizia conjuncta Robineau-Desvoidy, 1863; Orizia caesia RobineauDesvoidy, 1863; Orizia rubricornis Robineau-Desvoidy, 1863; Orizia rufella Robineau-Desvoidy, 1863;

Orizia arvorum Robineau-Desvoidy, 1863; Orizia gagatea Robineau-Desvoidy, 1863.

TYPE SPECIES: Orizia conjuncta Robineau-Desvoidy, 1863, by subsequent designation (Townsend, 1916: 8).

CURRENT Status: Junior synonym of Pollenia Robineau-Desvoidy, 1830 [teste Rognes (1991: 212)].

FAMILY: CALLIPHORIDAE. 
REMARKS: Schumann (1986: 48) mistakenly listed Orizia as an "Unavailable name", and Rognes (1991: 212) stated that no type species had been designated among the six nominal species originally included. The type species was listed under "Nomina dubia of the subfamily Polleniinae" by Schumann (1986: 49).

\section{Ormia Robineau-Desvoidy, 1830: 428.}

ORIGINALLY INCLUDED SPECIES: Ormia punctata Robineau-Desvoidy, 1830.

TYPE SPECIES: Ormia punctata Robineau-Desvoidy, 1830, by monotypy.

CURRENT STATUS: Valid genus [teste O'Hara \& Wood (2004: 281)].

FAMILY: TACHINIDAE.

EMENDATIONS: Hormia Agassiz, 1846b: 186 (unjustified).

\section{Orthellia Robineau-Desvoidy, $1863 \mathrm{~b}: 837$.}

ORIGINALly INCLUDED SPECIES: Orthellia rectinervis Robineau-Desvoidy, 1863; Orthellia mollis RobineauDesvoidy, 1863; Orthellia hyemalis Robineau-Desvoidy, 1863; Orthellia lubrica Robineau-Desvoidy, 1863.

TYPE SPECIES: Orthellia rectinervis Robineau-Desvoidy, 1863 [= Lucilia viridescens Robineau-Desvoidy, 1830], by subsequent designation (Townsend, 1916: 8).

CURRENT STATUS: Junior synonym of Neomyia Walker, 1859 [teste Pont (1986b: 96)].

FAMILY: MUSCIDAE.

392. Osmaea Robineau-Desvoidy, 1830: 84.

ORIGINALLY INCLUDED SPECIES: Osmaea grisea Robineau-Desvoidy, 1830.

TYPE SPECIES: Osmaea grisea Robineau-Desvoidy, 1830 [= Tachina setipennis Fallén, 1810], by monotypy.

CURRENT STATUS: Junior synonym of Triarthria Stephens, 1829 [teste Herting \& Dely-Draskovits (1993: 318)].

FAMILY: TACHINIDAE.

393. Osmina Robineau-Desvoidy, 1863b: 166.

ORIGINALLY INCLUDED SPECIES: Osmina lubrica Robineau-Desvoidy, 1863.

TYPE SPECIES: Osmina lubrica Robineau-Desvoidy, 1863 [= Tachina parallela Meigen, 1824], by original designation.

CURRENT Status: Junior synonym of Bessa Robineau-Desvoidy, 1830 [teste Herting \& Dely-Draskovits (1993: 142)].

FAMILY: TACHINIDAE.

394. Oswaldia Robineau-Desvoidy, 1863a: 840.

ORIGINALLY INCLUDED SPECIES: Oswaldia muscaria Robineau-Desvoidy, 1863; Oswaldia flavipennis Robineau-Desvoidy, 1863.

TYPE SPECIES: Oswaldia muscaria Robineau-Desvoidy, 1863 [= Tachina muscaria Fallén, 1810], by original designation.

CurRent Status: Valid genus [teste Herting \& Dely-Draskovits (1993: 161)].

FAMILY: TACHINIDAE. 
395. Oxyna Robineau-Desvoidy, 1830: 755.

ORIGINALLY INCLUDED SPECIES: Oxyna cinerea Robineau-Desvoidy, 1830; Oxyna flavescens RobineauDesvoidy, 1830; Oxyna femoralis Robineau-Desvoidy, 1830; Oxyna maculata Robineau-Desvoidy, 1830.

TYPE SPECIES: Oxyna flavescens Robineau-Desvoidy, 1830 [preoccupied by Musca flavescens Fabricius, 1798; = Trypeta flavipennis Loew, 1844], by subsequent designation (Hendel, 1914: 96).

CURRENT STATUS: Valid genus [teste Norrbom et al. (1999: 179)].

FAMILY: TEPHRITIDAE.

REMARKS: Loew (1844: 368) proposed the replacement name Trypeta flavipennis for Oxyna flavescens Robineau-Desvoidy, 1830 because, at that time, both Robineau-Desvoidy's name and Musca flavescens Fabricius, 1798 were being treated in the genus Trypeta; they were thus secondary homonyms and the Fabrician name had priority. The ICZN Code Article 59.3 states: "A junior secondary homonym replaced before 1961 is permanently invalid unless the substitute name is not in use and the relevant taxa are no longer considered congeneric." Although the nominal taxa are no longer congeneric, the replacement name Oxyna flavipennis (Loew) is currently in use so it cannot be replaced by the original name Oxyna flavescens Robineau-Desvoidy, 1830. Orthographic note: Fabricius (1798: 565) used the spelling "Musca flauescens" for this nominal species. However, Fabricius (1799: 33) used the spelling "Musca flavescens", which has since been the common spelling for the nominal species. Normally, virtually any intentional change in spelling from the original (apart from changes in gender endings caused by new combinations) is deemed an unjustified emendation. However, the ICZN Code Article 33.2.3.1 states that "when an unjustified emendation is in prevailing usage and is attributed to the original author and date, it is deemed to be a justified emendation". Fabricius (1799) emended his flauescens to flavescens and retained the authorship and date. Because flavescens is in prevailing usage, it is deemed a justified emendation.

396. Oxyphora Robineau-Desvoidy, 1830: 757.

ORIGINALLY INCLUDED SPECIES: Oxyphora pyrethri Robineau-Desvoidy, 1830; Oxyphora cardui RobineauDesvoidy, 1830.

TYPE SPECIES: Oxyphora pyrethri Robineau-Desvoidy, 1830, by subsequent designation (Foote \& Freidberg, 1981: 32).

CURRENT STATUS: Valid genus [teste Norrbom et al. (1999: 180)].

FAMILY: TEPHRITIDAE.

397. Pales Robineau-Desvoidy, 1830: 154.

Originally Included SPeCIES: Pales florea Robineau-Desvoidy, 1830; Pales petrosa Robineau-Desvoidy, 1830; Pales vernalis Robineau-Desvoidy, 1830; Pales blondeli Robineau-Desvoidy, 1830; Pales viridescens Robineau-Desvoidy, 1830; Pales brunicans Robineau-Desvoidy, 1830.

TyPE SPECIES: Pales florea Robineau-Desvoidy, 1830 [= Tachina pavida Meigen, 1824], by subsequent designation (Coquillett, 1910: 582).

CURRENT Status: Valid genus [teste Herting \& Dely-Draskovits (1993: 233)].

FAMILY: TACHINIDAE.

REMARKS: Robineau-Desvoidy (1863a: 519) designated "Pales strenua, R.-D." as type species but as this was not an originally included species, the designation is invalid. Pales was also proposed by Meigen (1800) [in Tipulidae], but this and other names in that work were suppressed by action of I.C.Z.N. (1963: 339 [Opinion 678]) and therefore does not preoccupy Pales Robineau-Desvoidy, 1830. 
398. Pallasia Robineau-Desvoidy, 1830: 239.

ORIGINALly INCLUDED SPECIES: Syrphus globosus Fabricius, 1775 (as "Syrphus globosus. Panz. Tachina globosa. Fabric.”; with "Gymnosoma dispar. Fall.” in synonymy); Pallasia ovata Robineau-Desvoidy, 1830.

TYPE SPECIES: Syrphus globosus Fabricius, 1775, by subsequent designation [Coquillett, 1910: 582 (as "Musca globosa Fabricius")].

CURRENT StATus: Junior synonym of Cistogaster Latreille, 1829 [teste Herting \& Dely-Draskovits (1993: 406)].

FAMILY: TACHINIDAE.

EMENDATIONS: Palassa Lioy, 1864d: 74 (unjustified), n. syn.

399. Palpomya Robineau-Desvoidy, 1830: 708.

ORIGINALLY INCLUDED SPECIES: Palpomya lalandii Robineau-Desvoidy, 1830.

TYPE SPECIES: Palpomya lalandii Robineau-Desvoidy, 1830 [= Platystoma asphaltina Wiedemann, 1824], by monotypy.

CURRENT STATUS: Valid genus [teste Steyskal (1980: 569)].

FAMILY: PLATYSTOMATIDAE.

Emendations: Palpomyia Macquart, 1835: 445 (unjustified), n. syn.; Palpomyia Loew, 1873: 17 (unjustified).

400. Palpostoma Robineau-Desvoidy, 1830: 429.

ORIGINALLY INCLUDED SPECIES: Palpostoma testacea Robineau-Desvoidy, 1830.

TYPE SPECIES: Palpostoma testaceum Robineau-Desvoidy, 1830 (as "Palpostoma testacea. R. D."), by monotypy.

CURRENT STATUS: Valid genus [teste Cantrell \& Crosskey (1989: 748)].

FAMILY: TACHINIDAE.

401. Palusia Robineau-Desvoidy, 1830: 542.

ORIGINALly INCLUDED SPECIES: Palusia menyanthidis Robineau-Desvoidy, 1830; Palusia flavescens

Robineau-Desvoidy, 1830; Palusia acuta Robineau-Desvoidy, 1830; Palusia flavicornis Robineau-

Desvoidy, 1830; Palusia brunicornis Robineau-Desvoidy, 1830; Palusia sagittariae Robineau-

Desvoidy, 1830; Palusia punctata Robineau-Desvoidy, 1830; Palusia testacea Robineau-Desvoidy, 1830; Palusia pallidicornis Robineau-Desvoidy, 1830; Palusia femoralis Robineau-Desvoidy, 1830.

TYPE SPECIES: Palusia testacea Robineau-Desvoidy, 1830, by subsequent designation (Coquillett, 1901: 139).

CuRrent Status: Junior synonym of Coenosia Meigen, 1826 [teste Pont (1986b: 199)].

FAMILY: MUSCIDAE.

402. Panzeria Robineau-Desvoidy, 1830: 68.

ORIGINALly INCLUDED SPECIES: Panzeria lateralis Robineau-Desvoidy, 1830.

TYPE SPECIES: Panzeria lateralis Robineau-Desvoidy, 1830 [= Tachina rudis Fallén, 1810], by monotypy. Current Status: Valid genus [teste O'Hara \& Wood (2004: 244)].

FAMILY: TACHINIDAE. 
403. Parthenia Robineau-Desvoidy, 1830: 231.

ORIGINALly INCLUDED SPECIES: Ocyptera cylindrica Fabricius, 1805; Parthenia carolinae RobineauDesvoidy, 1830; Parthenia boscii Robineau-Desvoidy, 1830 (with "Ocyptera radicum. Fabr." in synonymy).

TyPE SPECIES: Parthenia boscii Robineau-Desvoidy, 1830 [= Musca brassicaria Fabricius, 1775], by subsequent designation (Coquillett, 1910: 585).

CuRrent Status: Junior synonym of Cylindromyia Meigen, 1803 sensu stricto [teste Herting \& DelyDraskovits (1993: 426)].

FAMILY: TACHINIDAE.

404. Paykullia Robineau-Desvoidy, 1830: 270.

ORIGINAlly InCluded SPeCIES: Paykullia rubricornis Robineau-Desvoidy, 1830; Paykullia riparia Robineau-Desvoidy, 1830; Paykullia fuliginosa Robineau-Desvoidy, 1830.

TYPE SPECIES: Paykullia rubricornis Robineau-Desvoidy, 1830 [= Ocyptera maculata Fallén, 1815], by subsequent designation (Coquillett, 1910: 585).

CURRENT STATUS: Valid genus [teste Herting (1993: 114)].

FAMILY: RHINOPHORIDAE.

405. Peckia Robineau-Desvoidy, 1830: 335.

Originally InCluded SPECIES: Peckia imperialis Robineau-Desvoidy, 1830; Peckia lamanensis RobineauDesvoidy, 1830; Peckia aterrima Robineau-Desvoidy, 1830; Peckia plumipes Robineau-Desvoidy, 1830; Peckia lanipes Robineau-Desvoidy, 1830.

TYPE SPECIES: Peckia imperialis Robineau-Desvoidy, 1830, by subsequent designation (Coquillett, 1910: $585)$.

CURRENT STATUS: Valid genus and subgenus [teste Pape (1996: 273)].

FAMILY: SARCOPHAGIDAE.

REMARKS: The type species has been listed as a junior synonym of Sarcophaga praeceps Wiedemann, 1830 in previous catalogs and works, but this is incorrect. Robineau-Desvoidy's 1830 paper came out on 6 June while Wiedemann's 1830 paper came out less than three months later in September [see References in this paper for dating of the former and Evenhuis (1997: 822) for dating of the latter]. The name Peckia imperialis Robineau-Desvoidy, 1830 appears to fulfill the requirements for a nomen oblitum, but as we do not see Sarcophaga praeceps Wiedemann, 1830 fulfilling the conditions for a nomen protectum, we are following strict priority.

406. Pegomya Robineau-Desvoidy, 1830: 598.

ORIGINALly InCludED SPECIES: Anthomyia hyoscyami Panzer, 1809 (as "Musca hyoscami. Fabr. Anthomyia.

Meig."); Pegomya brunicornis Robineau-Desvoidy, 1830; Pegomya cerasi Robineau-Desvoidy, 1830;

Pegomya brunicosa Robineau-Desvoidy, 1830; Pegomya brunea Robineau-Desvoidy, 1830; Pegomya flavipes Robineau-Desvoidy, 1830.

TYPE SPECIES: Anthomyia hyoscyami Panzer, 1809, by subsequent designation [Coquillett, 1901: 140 (as "Musca hyoscyami Fabr.")].

CURRENT STATUS: Valid genus [teste Sabrosky (1999: 236)].

FAMILY: ANTHOMYIIDAE.

EMENDATIOns: Pegomyia Curtis, 1837: 264 (unjustified), n. syn. 
407. Peleteria Robineau-Desvoidy, 1830: 39.

ORIGINALly INCluded SPECIES: Peleteria javanica Robineau-Desvoidy, 1830; Peleteria leschenaldi Robineau-Desvoidy, 1830; Peleteria lalandii Robineau-Desvoidy, 1830; Peleteria abdominalis Robineau-Desvoidy, 1830.

TYPE SPECIES: Peleteria abdominalis Robineau-Desvoidy, 1830, by subsequent designation (Coquillett, 1910: 586).

CuRRent Status: Valid genus [teste Herting \& Dely-Draskovits (1993: 276)].

FAMILY: TACHINIDAE.

REMARKS: An earlier type-species designation by Macquart (1845: 262) of Echinomyia rubescens RobineauDesvoidy, 1830 is invalid as it was not an originally included species. Robineau-Desvoidy (1863a: 615) designated Tachina prompta Meigen, 1824, but that designation is also invalid because Tachina prompta was not an originally included species and had no originally included species listed in synonymy.

EMENDATIONS: Peletieria Bezzi, 1906: 54 (unjustified).

408. Peribaea Robineau-Desvoidy, 1863a: 720.

ORIGinally InCluded SPECIES: Peribaea apicalis Robineau-Desvoidy, 1863; Peribaea flavicornis Robineau-Desvoidy, 1863; Peribaea minuta Robineau-Desvoidy, 1863.

TYPE SPECIES: Peribaea apicalis Robineau-Desvoidy, 1863 [= Herbstia tibialis Robineau-Desvoidy, 1851], by subsequent designation (Coquillett, 1910: 587).

CURRENT STATUS: Valid genus [teste Herting \& Dely-Draskovits (1993: 331)].

FAMILY: TACHINIDAE.

409. Peronia Robineau-Desvoidy, 1830: 517.

ORIGINALLY INCLUDED SPECIES: Peronia rostrata Robineau-Desvoidy, 1830.

TYPE SPECIES: Peronia rostrata Robineau-Desvoidy, 1830, by monotypy.

CURRENT Status: Preoccupied by Fleming, 1822; senior (but invalid) synonym of Australophyra Malloch, 1923 [teste Savage \& Wheeler (2004: 273)].

FAMILY: MUSCIDAE.

410. Phaedima Robineau-Desvoidy, 1863a: 842.

ORIGINALly INCLUDED SPECIES: Phaedima honesta Robineau-Desvoidy, 1863; Phaedima aestivalis Robineau-Desvoidy, 1863; Phaedima flavipalpis Robineau-Desvoidy, 1863; Phaedima nebulosa Robineau-Desvoidy, 1863; Phaedima excitata Robineau-Desvoidy, 1863; Phaedima vesana RobineauDesvoidy, 1863; Phaedima lauta Robineau-Desvoidy, 1863; Phaedima ruralis Robineau-Desvoidy, 1863; Phaedima solers Robineau-Desvoidy, 1863.

TYPE SPECIES: Phaedima aestivalis Robineau-Desvoidy, 1863 [= Tachina spectabilis Meigen, 1824], by original designation.

CURRENT STATUS: Junior synonym of Oswaldia Robineau-Desvoidy, 1863 [teste Herting \& Dely-Draskovits (1993: 162)].

FAMILY: TACHINIDAE.

411. Phaenicia Robineau-Desvoidy, 1863b: 750.

ORIGINALly INCLUDED SPECIES: Phaenicia coccinea Robineau-Desvoidy, 1863; Phaenicia purpurea Robineau-Desvoidy, 1863; Phaenicia scintilla Robineau-Desvoidy, 1863; Phaenicia favilla RobineauDesvoidy, 1863; Phaenicia flavipalpis Robineau-Desvoidy, 1863; Phaenicia obscuripalpis RobineauDesvoidy, 1863; Phaenicia pyrochroa Robineau-Desvoidy, 1863; Phaenicia gratiosa Robineau- 
Desvoidy, 1863; Phaenicia ignea Robineau-Desvoidy, 1863; Phaenicia calidula Robineau-Desvoidy, 1863; Phaenicia pyrois Robineau-Desvoidy, 1863 (as “Phaenicia pyroïs, R.-D.”); Phaenicia ardens Robineau-Desvoidy, 1863; Phaenicia erythrea Robineau-Desvoidy, 1863; Phaenicia ignita RobineauDesvoidy, 1863; Phaenicia viva Robineau-Desvoidy, 1863; Phaenicia decora Robineau-Desvoidy, 1863; Phaenicia flammea Robineau-Desvoidy, 1863; Phaenicia amaena Robineau-Desvoidy, 1863; Phaenicia flammula Robineau-Desvoidy, 1863; Phaenicia aestuans Robineau-Desvoidy, 1863; Phaenicia corusca Robineau-Desvoidy, 1863; Phaenicia hilaris Robineau-Desvoidy, 1863; Phaenicia pumicea RobineauDesvoidy, 1863; Phaenicia propinqua Robineau-Desvoidy, 1863; Phaenicia dulcis Robineau-Desvoidy, 1863; Phaenicia pauperata Robineau-Desvoidy, 1863; Phaenicia pretiosa Robineau-Desvoidy, 1863; Phaenicia locuples Robineau-Desvoidy, 1863; Phaenicia labialis Robineau-Desvoidy, 1863; Phaenicia rubrella Robineau-Desvoidy, 1863; Phaenicia vaga Robineau-Desvoidy, 1863; Phaenicia dimidiata Robineau-Desvoidy, 1863; Phaenicia praestans Robineau-Desvoidy, 1863; Phaenicia dorsalis Robineau-Desvoidy, 1863; Phaenicia viridulans Robineau-Desvoidy, 1863; Phaenicia insignita Robineau-Desvoidy, 1863; Phaenicia fulgens Robineau-Desvoidy, 1863; Phaenicia impatiens RobineauDesvoidy, 1863; Phaenicia scutellaris Robineau-Desvoidy, 1863; Phaenicia pratensis RobineauDesvoidy, 1863; Phaenicia lepida Robineau-Desvoidy, 1863; Phaenicia venusta Robineau-Desvoidy, 1863; Phaenicia prompta Robineau-Desvoidy, 1863; Phaenicia nitida Robineau-Desvoidy, 1863; Phaenicia blanda Robineau-Desvoidy, 1863; Phaenicia aurata Robineau-Desvoidy, 1863; Phaenicia floralis Robineau-Desvoidy, 1863; Phaenicia bicolor Robineau-Desvoidy, 1863; Phaenicia campestris Robineau-Desvoidy, 1863; Phaenicia concinna Robineau-Desvoidy, 1863; Phaenicia deses RobineauDesvoidy, 1863; Phaenicia docilis Robineau-Desvoidy, 1863; Phaenicia gemmula Robineau-Desvoidy, 1863; Phaenicia metallica Robineau-Desvoidy, 1863; Phaenicia subtilis Robineau-Desvoidy, 1863; Phaenicia dolosa Robineau-Desvoidy, 1863; Phaenicia tenera Robineau-Desvoidy, 1863; Phaenicia thoracica Robineau-Desvoidy, 1863; Phaenicia inclyta Robineau-Desvoidy, 1863; Phaenicia rutila Robineau-Desvoidy, 1863; Phaenicia micans Robineau-Desvoidy, 1863; Phaenicia foetida RobineauDesvoidy, 1863; Phaenicia benigna Robineau-Desvoidy, 1863; Phaenicia teres Robineau-Desvoidy, 1863; Phaenicia notata Robineau-Desvoidy, 1863; Phaenicia pruinosa Robineau-Desvoidy, 1863; Phaenicia exilis Robineau-Desvoidy, 1863; Phaenicia fulvicornis Robineau-Desvoidy, 1863; Phaenicia flagrans Robineau-Desvoidy, 1863; Phaenicia azurea Robineau-Desvoidy, 1863; Phaenicia cyanella Robineau-Desvoidy, 1863; Phaenicia sumptuosa Robineau-Desvoidy, 1863; Phaenicia umbrosa Robineau-Desvoidy, 1863; Phaenicia elegans Robineau-Desvoidy, 1863; Phaenicia polita RobineauDesvoidy, 1863; Phaenicia chrysella Robineau-Desvoidy, 1863; Phaenicia viridula Robineau-Desvoidy, 1863; Phaenicia cognata Robineau-Desvoidy, 1863; Phaenicia marginalis Robineau-Desvoidy, 1863; Phaenicia consobrina Robineau-Desvoidy, 1863; Phaenicia nuptialis Robineau-Desvoidy, 1863; Phaenicia cinctella Robineau-Desvoidy, 1863; Phaenicia facilis Robineau-Desvoidy, 1863; Phaenicia lenis Robineau-Desvoidy, 1863; Phaenicia glabrata Robineau-Desvoidy, 1863; Phaenicia pulchella Robineau-Desvoidy, 1863; Phaenicia fulvifrons Robineau-Desvoidy, 1863.

TYPE SPECIES: Phaenicia concinna Robineau-Desvoidy, 1863 [= Musca sericata Meigen, 1826], by subsequent designation (Townsend, 1916: 8).

CURRENT STATUS: Junior synonym of Lucilia Robineau-Desvoidy, 1830 [teste Rognes (1991: 147)].

FAMILY: CALLIPHORIDAE.

REMARKS: There are two original spellings of this genus-group name in Robineau-Desvoidy (1863b): Phaenicia (page 750) and Phoenicia (page 900). Acting as First Reviser, we select Phaenicia as the correct original spelling.

\section{Phanemya Robineau-Desvoidy, 1830: 254.}

ORIGINALLY INCLUDED SPECIES: Phanemya musca Robineau-Desvoidy, 1830.

TYPE SPECIES: Phanemya musca Robineau-Desvoidy, 1830 [= Tachina biguttata Meigen, 1824], by monotypy. 
CURRENT STATUS: Junior synonym of Clairvillia Robineau-Desvoidy, 1830 [teste Herting \& Dely-Draskovits (1993: 421)].

FAMILY: TACHINIDAE.

Emendations: Phanemyia Agassiz, 1846b: 283 (unjustified); Phaniomyia Brauer \& Bergenstamm, 1889: 76 (unjustified), n. syn.

413. Phantasma Robineau-Desvoidy, 1830: 739.

ORIGINALLY INCLUDED SPECIES: Musca filiformis Fabricius, 1794; Phantasma thoracicum RobineauDesvoidy, 1830.

TYPE SPECIES: Musca filiformis Fabricius, 1794 [= Musca corrigiolata Linnaeus, 1767], by subsequent designation (Coquillett, 1910: 587).

CURRENT STATUS: Junior synonym of Micropeza Robineau-Desvoidy, 1830 [teste Soós (1984: 19)].

FAMILY: MICROPEZIDAE.

414. Phaonia Robineau-Desvoidy, 1830: 482.

ORIGINALly INCLUDED SPECIES: Phaonia viarum Robineau-Desvoidy, 1830; Phaonia vagatoria RobineauDesvoidy, 1830; Phaonia aricioidea Robineau-Desvoidy, 1830 (as "Phaonia ariciö̈dea. R. D.”); Phaonia villana Robineau-Desvoidy, 1830; Phaonia canaliculata Robineau-Desvoidy, 1830.

TYPE SPECIES: Phaonia viarum Robineau-Desvoidy, 1830 [= Musca validus Harris, 1780], by subsequent designation (Coquillett, 1901: 140).

CurRent STAtus: Valid genus [teste Pont (1986b: 116)].

FAMILY: MUSCIDAE.

[Phebellia] Robineau-Desvoidy, 1845: 109.

CURRENT STATUS: Unavailable name; proposed without description or included species; treated under Phebellia Robineau-Desvoidy, 1846 [teste this work].

FAMILY: TACHINIDAE.

415. Phebellia Robineau-Desvoidy, 1846b: 37.

ORIGINALLY INCLUDED SPECIES: Phebellia aestivalis Robineau-Desvoidy, 1846.

TYPE SPECIES: Phebellia aestivalis Robineau-Desvoidy, 1846 [= Tachina villica Zetterstedt, 1838], by monotypy.

CuRrent STATUS: Valid genus [teste Herting \& Dely-Draskovits (1993: 184)].

FAMILY: TACHINIDAE.

416. Phegea Robineau-Desvoidy, 1863a: 908.

ORIGINALLY INCLUDED SPECIES: Erycia limpidipennis Robineau-Desvoidy, 1830; Phegea ruficornis Robineau-Desvoidy, 1863.

TYPE SPECIES: Erycia limpidipennis Robineau-Desvoidy, 1830 [= Tachina cinerea Fallén, 1810], by original designation.

CURRENT STATUS: Preoccupied by Gistel, 1848; junior synonym of Zaira Robineau-Desvoidy, 1830 [teste Herting \& Dely-Draskovits (1993: 148)].

FAMILY: TACHINIDAE. 
417. Phenicellia Robineau-Desvoidy, 1863a: 802.

ORIGINALLY INCLUDED SPECIES: Tachina nigra Hartig, 1838.

TYPE SPECIES: Tachina nigra Hartig, 1838 [preoccupied by Tachina nigra Robineau-Desvoidy, 1830; =

Tachina haematodes Meigen, 1824], by monotypy.

CURRENT STATUs: Junior synonym of Thelaira Robineau-Desvoidy, 1830 [teste Herting \& Dely-Draskovits (1993: 389)].

FAMILY: TACHINIDAE.

EMENDATIONS: Phoenicella Mik \& Wachtl, 1895: 219 (unjustified).

418. Pherbellia Robineau-Desvoidy, 1830: 695.

ORIGINALly INCLUDED SPECIES: Pherbellia vernalis Robineau-Desvoidy, 1830.

TYPE SPECIES: Pherbellia vernalis Robineau-Desvoidy, 1830 [= Sciomyza schoenherri Fallén, 1826], by monotypy.

CURRENT STATUS: Valid genus [teste Sabrosky (1999: 242)].

FAMILY: SCIOMYZIDAE.

419. Pherbina Robineau-Desvoidy, 1830: 687.

ORIGINALly INCLUDED SPECIES: Pherbina gentilis Robineau-Desvoidy, 1830; Pherbina flavescens Robineau-Desvoidy, 1830; Musca reticulata Fabricius, 1781 (as "Tetanocera reticulata. Dumér."); Pherbina cayennensis Robineau-Desvoidy, 1830; Pherbina communis Robineau-Desvoidy, 1830; Pherbina vicina Robineau-Desvoidy, 1830; Pherbina limbata Robineau-Desvoidy, 1830; Pherbina boscii Robineau-Desvoidy, 1830; Pherbina soror Robineau-Desvoidy, 1830; Pherbina germana Robineau-Desvoidy, 1830; Pherbina paludosa Robineau-Desvoidy, 1830.

TYPE SPECIES: Musca reticulata Fabricius, 1781 [= Musca coryleti Scopoli, 1763], by subsequent designation

[Coquillett, 1910: 588 (as "Musca coryleta Scopoli (as Tetanocera reticulata DUMERIL)")].

CURRENT STATUS: Valid genus [teste Rozkošný \& Elberg (1984: 186)].

FAMILY: SCIOMYZIDAE.

420. Pherecida Robineau-Desvoidy, 1863a: 1118.

ORIGINALly INCLUDED SPECIES: Tachina egens Fallén, 1810 (with "Macquartia flavescens : Rob. Desv." and

"Tachina grisea : Meig." in synonymy); Pherecida agraria Robineau-Desvoidy, 1863.

TYPE SPECIES: Tachina egens Fallén, 1810 [= Tachina grisea Fallén, 1810], by original designation.

CURRENT STATUS: Junior synonym of Macquartia Robineau-Desvoidy, 1830 [teste Herting \& DelyDraskovits (1993: 312)].

FAMILY: TACHINIDAE.

421. Phericia Robineau-Desvoidy, 1863b: 70.

ORIGINALLY INCLUDED SPECIES: Dufouria clausa Robineau-Desvoidy, 1830.

TYPE SPECIES: Dufouria clausa Robineau-Desvoidy, 1830 [= Tachina nigrita Fallén, 1810], by original designation.

CuRRENT Status: Junior synonym of Dufouria Robineau-Desvoidy, 1830 [teste Herting \& Dely-Draskovits (1993: 393)].

FAMILY: TACHINIDAE.

422. Philea Robineau-Desvoidy, 1863a: 314.

ORIGINALLY INCLUDED SPECIES: Philea cursoria Robineau-Desvoidy, 1863. 
TYPE SPECIES: Philea cursoria Robineau-Desvoidy, 1863 [= Tachina alacris Meigen, 1824], by original designation.

CurRent Status: Preoccupied by Zetterstedt, 1838; senior (but invalid) synonym of Ptesiomyia Brauer \& Bergenstamm, 1893 [teste Herting \& Dely-Draskovits (1993: 188)].

FAMILY: TACHINIDAE.

423. Philinta Robineau-Desvoidy, 1830: 568.

ORIGINALLY INCLUDED SPECIES: Musca canicularis Linnaeus, 1761 (as “Musca canicularis. Linn. Fabr."); Philinta pallipes Robineau-Desvoidy, 1830; Philinta testacea Robineau-Desvoidy, 1830; Philinta flava Robineau-Desvoidy, 1830.

TYPE SPECIES: Musca canicularis Linnaeus, 1761, by subsequent designation (Coquillett, 1901: 140). CURRENT StATUS: Junior synonym of Fannia Robineau-Desvoidy, 1830 [teste Pont (1986a: 43)].

FAMILY: FANNIIDAE.

EMENDATIOns: Philintha Agassiz, 1846a: 30 (unjustified), n. syn.

[Phoenicia] Robineau-Desvoidy, 1863b: 900.

CURRENT STATUS: Incorrect original spelling of Phaenicia Robineau-Desvoidy, 1863 [teste this work].

FAMILY: CALLIPHORIDAE.

424. Pholoe Robineau-Desvoidy, 1863a: 297.

ORIGINALLY INCLUDED SPECIES: Melibaea zonaria Robineau-Desvoidy, 1830.

TYPE SPECIES: Melibaea zonaria Robineau-Desvoidy, 1830 [= Tachina heraclei Meigen, 1824], by original designation.

CURRENT STATUS: Preoccupied by Johnston, 1839; junior synonym of Phryxe Robineau-Desvoidy, 1830 [teste Herting \& Dely-Draskovits (1993: 192)].

FAMILY: TACHINIDAE.

425. Phoraea Robineau-Desvoidy, 1830: 600.

ORIGINALly INCLUDED SPECIES: Phoraea flavescens Robineau-Desvoidy, 1830; Phoraea scutellaris Robineau-Desvoidy, 1830; Phoraea myoidea Robineau-Desvoidy, 1830 (as “Phoraea myoïdea. R. D.”); Phoraea humeralis Robineau-Desvoidy, 1830.

TYPE SPECIES: Phoraea flavescens Robineau-Desvoidy, 1830 [= Musca testacea De Geer, 1776], by subsequent designation (Coquillett, 1910: 588).

CURRENT Status: Junior synonym of Pegomya Robineau-Desvoidy, 1830 [teste Dely-Draskovits (1993: 76)].

FAMILY: ANTHOMYIIDAE.

REMARKS: Previous listings of the type species have it as a junior synonym of Anthomyia silacea Meigen, 1830, but this is incorrect. Robineau-Desvoidy's 1830 paper came out 6 June while Meigen's 1830 paper came out six months later on 1 December [see References in this paper for dating of the former and Evenhuis (1997: 533) for dating of the latter]. Dely-Draskovits (1993: 85, 87) listed flavescens as a junior synonym of silacea but also had a separate entry where silacea was listed as a junior synonym of testacea. All three nominal taxa are now considered synonymous (Michelsen, 2006). 
426. Phorbia Robineau-Desvoidy, 1830: 559.

ORIGINAlly InCluded SPeCIES: Phorbia musca Robineau-Desvoidy, 1830; Phorbia rustica RobineauDesvoidy, 1830; Phorbia claripennis Robineau-Desvoidy, 1830; Phorbia arvensis Robineau-Desvoidy, 1830; Phorbia grisea Robineau-Desvoidy, 1830.

TYPE SPECIES: Phorbia musca Robineau-Desvoidy, 1830 [= Anthomyia sepia Meigen, 1826], by subsequent designation (Coquillett, 1910: 589).

CURRENT Status: Valid genus [teste Sabrosky (1999: 243)].

FAMILY: ANTHOMYIIDAE.

427. Phorcida Robineau-Desvoidy, 1863a: 251.

ORIGINALly INCLUDED SPECIES: Hubneria acronita Robineau-Desvoidy, 1850 (as "Hubneria acronyctae :

Rob. Desv."); Phorcida scutellaris Robineau-Desvoidy, 1863; Phorcida subglabra Robineau-Desvoidy, 1863; Phorcida rectinervis Robineau-Desvoidy, 1863; Phorcida campephaga Robineau-Desvoidy, 1863; Tachina lota Meigen, 1824.

TYPE SPECIES: Hubneria acronita Robineau-Desvoidy, 1850 [= Tachina hortulana Meigen, 1824], by original designation (as "Hubneria acronyctae, R.-D.").

CURRENT STATUS: Junior synonym of Nilea Robineau-Desvoidy, 1863 [teste Herting \& Dely-Draskovits (1993: 189)].

FAMILY: TACHINIDAE.

428. Phorella Robineau-Desvoidy, 1830: 362.

ORIGINALly INCLUDED SPECIES: Phorella arvensis Robineau-Desvoidy, 1830; Phorella florum RobineauDesvoidy, 1830; Phorella squalida Robineau-Desvoidy, 1830; Phorella atrata Robineau-Desvoidy, 1830; Phorella despecta Robineau-Desvoidy, 1830; Phorella morio Robineau-Desvoidy, 1830.

TYPE SPECIES: Phorella arvensis Robineau-Desvoidy, 1830 [= Musca variegata Scopoli, 1763], by subsequent designation (Robineau-Desvoidy, 1863b: 530).

CURRENT STATUS: Junior synonym of Sarcophaga Meigen, 1826 sensu stricto [teste Pape (1996: 387)].

FAMILY: SARCOPHAGIDAE.

429. Phorinia Robineau-Desvoidy, 1830: 118.

Originally InCluded SPECIES: Phorinia aurifrons Robineau-Desvoidy, 1830; Phorinia gracilis RobineauDesvoidy, 1830; Phorinia micromera Robineau-Desvoidy, 1830; Phorinia macquarti RobineauDesvoidy, 1830; Phorinia minor Robineau-Desvoidy, 1830; Phorinia borealis Robineau-Desvoidy, 1830.

TYPE SPECIES: Phorinia aurifrons Robineau-Desvoidy, 1830, by subsequent designation (RobineauDesvoidy, 1863a: 491).

CURRENT STATUS: Valid genus [teste Herting \& Dely-Draskovits (1993: 141)].

FAMILY: TACHINIDAE.

430. Phormia Robineau-Desvoidy, 1830: 465.

ORIGINALly InCludED SPECIES: Phormia caerulea Robineau-Desvoidy, 1830; Musca regina Meigen, 1826; Phormia philadelphica Robineau-Desvoidy, 1830; Phormia fulvifacies Robineau-Desvoidy, 1830; Phormia cuprea Robineau-Desvoidy, 1830; Phormia terraenovae Robineau-Desvoidy, 1830 (as "Phormia Terrae-Novae. R. D."); Phormia vittata Robineau-Desvoidy, 1830; Phormia squalens Robineau-Desvoidy, 1830.

TYPE SPECIES: Musca regina Meigen, 1826, by subsequent designation (Robineau-Desvoidy, 1849f: v). 
CURRENT STATUS: Valid genus [teste Rognes (1991: 105)].

FAMILY: CALLIPHORIDAE.

431. Phorocera Robineau-Desvoidy, 1830: 131.

ORIGINALly INCLUDED SPECIES: Phorocera agilis Robineau-Desvoidy, 1830; Phorocera rapida RobineauDesvoidy, 1830; Phorocera scutellaris Robineau-Desvoidy, 1830; Phorocera apricans RobineauDesvoidy, 1830; Phorocera fera Robineau-Desvoidy, 1830; Phorocera noctuarum Robineau-Desvoidy, 1830; Phorocera nitens Robineau-Desvoidy, 1830; Phorocera prorsae Robineau-Desvoidy, 1830; Phorocera antiopis Robineau-Desvoidy, 1830; Phorocera iovora Robineau-Desvoidy, 1830; Phorocera pygerae Robineau-Desvoidy, 1830; Phorocera caiae Robineau-Desvoidy, 1830; Phorocera myoidea Robineau-Desvoidy, 1830 (as "Phorocera myö̈dea. R. D."); Phorocera bombycivora RobineauDesvoidy, 1830; Phorocera flavipennis Robineau-Desvoidy, 1830; Phorocera gracilis RobineauDesvoidy, 1830; Phorocera vernalis Robineau-Desvoidy, 1830; Phorocera velox Robineau-Desvoidy, 1830; Phorocera limpidipennis Robineau-Desvoidy, 1830; Phorocera cylindrica Robineau-Desvoidy, 1830; Phorocera botyvora Robineau-Desvoidy, 1830; Phorocera cirrata Robineau-Desvoidy, 1830.

TYPE SPECIES: Phorocera agilis Robineau-Desvoidy, 1830 [= Tachina assimilis Fallén, 1810], by subsequent designation (Robineau-Desvoidy, 1863a: 509).

CURRENT STATUS: Valid genus [teste Herting \& Dely-Draskovits (1993: 139)].

FAMILY: TACHINIDAE.

REMARKS: Robineau-Desvoidy (1863a: 509) designated as type species "Tachina assimilis, Meig.", which was not an originally included species. However, by including Phorocera agilis Robineau-Desvoidy, 1830 in synonymy with Tachina assimilis, the designation is valid according to ICZN Code Article 69.2.2.

[Phorophylla] Robineau-Desvoidy MS name (Blainville et al., 1826: 10, 22).

CURRENT STATUS: Unavailable name; suppressed by action of I.C.Z.N. (1990: 162 [Opinion 1601]); treated as a nomen dubium in Tachinidae [teste this work].

FAMILY: TACHINIDAE.

432. Phorophylla Robineau-Desvoidy, 1830: 213.

ORIGINALly INCLUDED SPECIES: Phorophylla palpata Robineau-Desvoidy, 1830.

TYPE SPECIES: Phorophylla palpata Robineau-Desvoidy, 1830, by monotypy.

CurRent Status: Placed in "Doubtful taxa in Tachinidae" by Herting \& Dely-Draskovits (1993: 437).

FAMILY: TACHINIDAE.

433. Phorosia Robineau-Desvoidy, 1853a: 109 [1853b: 29].

ORIGINALLY INCLUDED SPECIES: Conops testaceus Linnaeus, 1767 (with "Myopa pellucida : Rob.-Desv." in synonymy).

TYPE SPECIES: Conops testaceus Linnaeus, 1767, by original designation.

CURRENT StATUS: Junior synonym of Myopa Fabricius, 1775 [teste Chvála \& Smith (1988: 262)].

FAMILY: CONOPIDAE.

434. Phorostoma Robineau-Desvoidy, 1830: 326.

ORIGINALly InCluded SPECIES: Phorostoma subrotunda Robineau-Desvoidy, 1830.

TYPE SPECIES: Phorostoma subrotunda Robineau-Desvoidy, 1830 [= Musca ferina Fallén, 1817], by monotypy. 
CuRRENT Status: Junior synonym of Dinera Robineau-Desvoidy, 1830 [teste Herting \& Dely-Draskovits (1993: 361)].

FAMILY: TACHINIDAE.

435. Phrosia Robineau-Desvoidy, 1830: 668.

ORIGINALly INCLUDED SPECIES: Phrosia scirpi Robineau-Desvoidy, 1830.

TYPE SPECIES: Phrosia scirpi Robineau-Desvoidy, 1830 [= Ocyptera albilabris Fabricius, 1805], by monotypy.

CURRENT STATUS: Valid genus [teste Gorodkov (1986: 37)].

FAMILY: SCATHOPHAGIDAE.

[Phrosina] Robineau-Desvoidy, 1863b: 101.

CURRENT STATUS: Incorrect original spelling of Phrosinella Robineau-Desvoidy, 1863 [teste Townsend (1938: 136)].

FAMILY: SARCOPHAGIDAE.

436. Phrosinella Robineau-Desvoidy, 1863b: 82.

ORIGINALLY INCLUDED SPECIES: Phrosina argyrina Robineau-Desvoidy, 1863.

TYPE SPECIES: Phrosina argyrina Robineau-Desvoidy, 1863 [= Tachina nasuta Meigen, 1824], by monotypy. CURRENT STATUS: Valid genus [teste Pape (1996: 122)].

FAMILY: SARCOPHAGIDAE.

REMARKS: There are two original spellings of this genus in Robineau-Desvoidy (1863b): Phrosinella (page 82) and Phrosina (page 101). Acting as First Reviser, Townsend (1938: 136), selected Phrosinella as the correct original spelling.

437. Phryno Robineau-Desvoidy, 1830: 143.

Originally Included SpeCies: Phryno rustica Robineau-Desvoidy, 1830; Phryno agilis RobineauDesvoidy, 1830; Phryno brunea Robineau-Desvoidy, 1830; Phryno nigripes Robineau-Desvoidy, 1830; Phryno hemisphaerica Robineau-Desvoidy, 1830; Phryno bucentoidea Robineau-Desvoidy, 1830 (as "Phryno bucentoïdea. R. D.").

TYPE SPECIES: Phryno agilis Robineau-Desvoidy, 1830 [= Tachina vetula Meigen, 1824], by subsequent designation (Townsend 1916: 8).

CurRent Status: Valid genus [teste Herting \& Dely-Draskovits (1993: 235)].

FAMILY: TACHINIDAE.

REMARKS: An earlier type-species designation by Robineau-Desvoidy (1863a: 541) of "Tachina vetula, Meig." is invalid as it was not an originally included species.

438. Phryxe Robineau-Desvoidy, 1830: 158.

Originally InCluded SPeCies: Phryxe zonata Robineau-Desvoidy, 1830; Phryxe servillii RobineauDesvoidy, 1830; Phryxe sabulosa Robineau-Desvoidy, 1830; Phryxe ciliata Robineau-Desvoidy, 1830; Phryxe palesioidea Robineau-Desvoidy, 1830 (as Phryxe palesioüdea. R. D.”); Phryxe rotundata Robineau-Desvoidy, 1830; Phryxe consobrina Robineau-Desvoidy, 1830; Phryxe subrotundata Robineau-Desvoidy, 1830; Phryxe microcera Robineau-Desvoidy, 1830; Phryxe blondeli RobineauDesvoidy, 1830; Phryxe villica Robineau-Desvoidy, 1830; Phryxe prompta Robineau-Desvoidy, 1830; Phryxe scutellaris Robineau-Desvoidy, 1830; Phryxe depressa Robineau-Desvoidy, 1830; Phryxe 
grisescens Robineau-Desvoidy, 1830; Phryxe macquarti Robineau-Desvoidy, 1830; Phryxe coarctata Robineau-Desvoidy, 1830; Phryxe superba Robineau-Desvoidy, 1830; Phryxe lasiocampae RobineauDesvoidy, 1830; Phryxe sphingivora Robineau-Desvoidy, 1830; Phryxe pavoniae Robineau-Desvoidy, 1830; Phryxe bombycivora Robineau-Desvoidy, 1830; Phryxe tiphaecola Robineau-Desvoidy, 1830; Phryxe larvicola Robineau-Desvoidy, 1830; Phryxe florida Robineau-Desvoidy, 1830; Phryxe scutellata Robineau-Desvoidy, 1830; Phryxe agilis Robineau-Desvoidy, 1830; Phryxe athaliae RobineauDesvoidy, 1830; Phryxe punctata Robineau-Desvoidy, 1830; Phryxe cinerascens Robineau-Desvoidy, 1830; Phryxe velox Robineau-Desvoidy, 1830; Phryxe similis Robineau-Desvoidy, 1830; Phryxe arvensis Robineau-Desvoidy, 1830; Phryxe flavipalpis Robineau-Desvoidy, 1830; Phryxe frontalis Robineau-Desvoidy, 1830; Phryxe carceli Robineau-Desvoidy, 1830.

TYPE SPECIES: Phryxe athaliae Robineau-Desvoidy, 1830 [= Tachina vulgaris Fallén, 1810], by subsequent designation [Robineau-Desvoidy, 1863a: 329 (as “Tachina vulgaris, Fall.”)].

CURRENT Status: Valid genus [teste Herting \& Dely-Draskovits (1993: 192)].

FAMILY: TACHINIDAE.

REMARKS: Robineau-Desvoidy (1863a: 329) designated as type species "Tachina vulgaris, Fall.", which was not an originally included species. However, by including Phryxe athaliae Robineau-Desvoidy, 1830 in synonymy with Tachina vulgaris, the designation is valid according to ICZN Code Article 69.2.2.

\section{Phumosia Robineau-Desvoidy, 1830: 427.}

ORIGinally InCluded SPeCIES: Phumosia abdominalis Robineau-Desvoidy, 1830; Phumosia pallidula Robineau-Desvoidy, 1830; Phumosia belvosii Robineau-Desvoidy, 1830.

TYPE SPECIES: Phumosia abdominalis Robineau-Desvoidy, 1830, by subsequent designation (Townsend, 1916: 8).

CURRENT STATUS: Valid genus [teste Sabrosky (1999: 246)].

FAMILY: CALLIPHORIDAE.

EMENDATIONS: Phymatusa Agassiz, 1846b: 290 (unjustified).

\section{Phyllis Robineau-Desvoidy, 1830: 603.}

ORIGINALly InCluded SPeCIES: Phyllis flava Robineau-Desvoidy, 1830; Phyllis plumosula RobineauDesvoidy, 1830; Phyllis rohrelliformis Robineau-Desvoidy, 1830; Phyllis brunea Robineau-Desvoidy, 1830.

TyPE SPECIES: Phyllis flava Robineau-Desvoidy, 1830 [= Anthomyia diaphana Wiedemann, 1817], by subsequent designation (Coquillett, 1901: 140).

CURRENT STATUS: Nomen oblitum; senior synonym of Thricops Rondani, 1856 (nomen protectum), but here treated as a nomen oblitum through the ICZN Code principle of the Reversal of Precedence [teste this work].

FAMILY: MUSCIDAE.

REMARKS: Griffiths (1982: 8) and Dely-Draskovits (1993: 77) incorrectly considered the type species as Phyllis brunea Robineau-Desvoidy, 1830 [by subsequent designation of Hennig (1962a: 630)], which is currently treated in the genus Pegomya Robineau-Desvoidy, 1830 [Anthomyiidae]. Coquillett's (1901) designation of Phyllis flava is earlier, but this designation has been overlooked by recent workers on the Muscidae (Hennig, 1962a; Huckett, 1965b; Pont, 1986b; Gregor et al., 2002; Savage, 2003). Since Phyllis flava is now treated as a junior synonym of Anthomyia diaphana Wiedemann, 1817 (now in Thricops), the name Phyllis is an older generic name for Thricops Rondani, 1856. However, whereas Phyllis has not been used as a valid name for a taxon since 1830, Thricops is in prevailing usage as the valid name for a group of flies that is speciose, widespread and frequently abundant in the temperate Holarctic Region. Thricops has appeared as the valid name in at least 25 works by well over 10 authors, published during the past 50 years, including the following: Chandler (1998); Gregor (1988, 1997, 2004); 
Gregor \& Rozkošný (2007); Gregor et al. (2002); Hennig (1962, 1965); Hofmans (1991); Huckett et al. (1987); Kozuharova et al. (2006); Papp (2001); Pont (1971, 1975, 1986, 1993, 1995); Pont \& Ackland (1995); Pont \& Horsfield (1992); Pont \& Merz (1998); Prijs (2002); Sabrosky (1999); Savage (2003); Savage \& Wheeler (2004); Savage et al. (2004); Skidmore (1985); Teschner (1999); Xue \& Chao (1998); Zimin \& El'berg (1970). In the interests of nomenclatural stability, we hereby invoke reversal of precedence (ICZN Code Article 23.9.2) and declare Phyllis Robineau-Desvoidy, 1830 a nomen oblitum and Thricops Rondani, 1856 a nomen protectum.

441. Phyllomya Robineau-Desvoidy, 1830: 213.

ORIGINALLY INCLUDED SPECIES: Musca volvulus Fabricius, 1794.

TYPE SPECIES: Musca volvulus Fabricius, 1794, by monotypy.

CurRent Status: Valid genus [teste Herting \& Dely-Draskovits (1993: 387; as "Phyllomyia")].

FAMILY: TACHINIDAE.

REMARKS: Placed on the Official List of Generic Names in Zoology by action of I.C.Z.N. (1988a: 74 [Opinion 1475]).

EMENDATions: Phyllomyia Agassiz, 1846a: 31 (unjustified), n. syn.

[Phyto] Robineau-Desvoidy MS name (Blainville et al., 1826: 22).

CURRENT STATUS: Unavailable name; suppressed by action of I.C.Z.N. (1990: 162 [Opinion 1601]); treated under Phyto Robineau-Desvoidy, 1830 [teste this work].

FAMILY: RHINOPHORIDAE.

442. Phyto Robineau-Desvoidy, 1830: 218.

ORIGINALly InCluded SPECIES: Phyto nigra Robineau-Desvoidy, 1830; Phyto nigrogrisescens RobineauDesvoidy, 1830 (as “Phyto nigro-grisescens. R. D.”); Phyto palpalis Robineau-Desvoidy, 1830; Phyto prompta Robineau-Desvoidy, 1830.

TYPE SPECIES: Phyto nigra Robineau-Desvoidy, 1830 [= Tachina melanocephala Meigen, 1824], by subsequent designation (Townsend, 1916: 8).

CURRENT STATUS: Valid genus [teste Herting (1993: 107)].

FAMILY: RHINOPHORIDAE.

443. Picconia Robineau-Desvoidy, 1863b: 33.

ORIGINALly InCluded SPeCIES: Picconia bipartita Robineau-Desvoidy, 1863 (as "Picconia bi-partita, R.D.”).

TYPE SPECIES: Picconia bipartita Robineau-Desvoidy, 1863 (as "Picconia bi-partita, R.-D.”) [= Tachina incurva Zetterstedt, 1844], by monotypy.

CURRENT STATUS: Valid genus [teste Herting \& Dely-Draskovits (1993: 166)].

FAMILY: TACHINIDAE.

444. Pictinia Robineau-Desvoidy, 1853a: 95 [1853b: 15].

ORIGINAlly InCluded SPECIES: Pictinia fulvipalpis Robineau-Desvoidy, 1853 (with "Myopa buccata. Rob.-

Desv." in synonymy).

TYPE SPECIES: Myopa buccata Robineau-Desvoidy, 1830, by original designation.

CURRENT STATUS: Junior synonym of Myopa Fabricius, 1775 [teste Chvála \& Smith (1988: 261)].

FAMILY: CONOPIDAE. 
REMARKs: Only one species was included in the genus, Pictinia fulvipalpis Robineau-Desvoidy, 1853, with Myopa buccata. Rob.-Desv." [= Conops buccata Linnaeus, 1758, sensu Robineau-Desvoidy] in synonymy.

445. Pierretia Robineau-Desvoidy, 1863b: 422.

Originally InCluded SPECIES: Pierretia aestivalis Robineau-Desvoidy, 1863; Pierretia praecox RobineauDesvoidy, 1863; Myophora vivida Robineau-Desvoidy, 1830; Pierretia rusticana Robineau-Desvoidy, 1863; Myophora atra Robineau-Desvoidy, 1830; Pierretia maura Robineau-Desvoidy, 1863; Pierretia limpida Robineau-Desvoidy, 1863; Pierretia vernalis Robineau-Desvoidy, 1863; Pierretia squalens Robineau-Desvoidy, 1863; Pierretia parva Robineau-Desvoidy, 1863; Myophora agilis RobineauDesvoidy, 1830; Myophora arvorum Robineau-Desvoidy, 1830; Pierretia melanura Robineau-Desvoidy, 1863; Pierretia sordida Robineau-Desvoidy, 1863.

TYPE SPECIES: Pierretia praecox Robineau-Desvoidy, 1863 [= Sarcophaga nigriventris Meigen, 1826], by subsequent designation (Townsend, 1916: 8).

CURRENT StATUS: Junior synonym of Myorhina Robineau-Desvoidy, 1830 (subgenus of Sarcophaga Meigen, 1826) [teste Pape (1996: 364)].

FAMILY: SARCOPHAGIDAE.

446. Pissemya Robineau-Desvoidy, 1851f: 318.

ORIGINALLY INCLUDED SPECIES: Gonia atra Meigen, 1826.

TYPE SPECIES: Gonia atra Meigen, 1826, by monotypy.

CURRENT StATUS: Junior synonym of Gonia Meigen, 1803 [teste Herting \& Dely-Draskovits (1993: 257)].

FAMILY: TACHINIDAE.

EMENDATIONS: Pissemyia Schiner, 1861a: 440 (unjustified), n. syn.

447. Pitthaea Robineau-Desvoidy, 1863a: 188.

Originally InCluded Species: Pitthaea nebulosa Robineau-Desvoidy, 1863; Pitthaea morosa RobineauDesvoidy, 1863; Pitthaea tristis Robineau-Desvoidy, 1863.

TyPE SPECIES: Pitthaea nebulosa Robineau-Desvoidy, 1863 [= Tachina floralis Fallén, 1810], by original designation.

CURRENT STATUS: Junior synonym of Nemorilla Rondani, 1856 [teste Herting \& Dely-Draskovits (1993: 182)].

FAMILY: TACHINIDAE.

448. Platymya Robineau-Desvoidy, 1830: 116.

ORIGINALly INCLUDED SPECIES: Platymya rubescens Robineau-Desvoidy, 1830; Platymya aestivalis Robineau-Desvoidy, 1830; Platymya blondeli Robineau-Desvoidy, 1830.

TYPE SPECIES: Platymya aestivalis Robineau-Desvoidy, 1830 [= Tachina fimbriata Meigen, 1824], by subsequent designation (Robineau-Desvoidy, 1863a: 191).

CurRent Status: Valid genus [teste Herting \& Dely-Draskovits (1993: 224)].

FAMILY: TACHINIDAE.

EMENDATIONS: Platymyia Agassiz, 1846b: 296 (unjustified).

449. Plaxemya Robineau-Desvoidy, 1830: 392.

ORIGINALLY INCLUDED SPECIES: Plaxemya sugillatrix Robineau-Desvoidy, 1830. 
TYPE SPECIES: Plaxemya sugillatrix Robineau-Desvoidy, 1830, by monotypy.

CURRENT STATUS: Junior synonym of Musca Linnaeus, 1758 sensu stricto [teste Pont (1986b: 87)].

FAMILY: MUSCIDAE.

EMENDATIONS: Placomyia Agassiz, 1846b: 293, 297 (unjustified); Plaxemyia Schiner, 1861b: 593 (unjustified), n. syn.; Placomyia Brauer \& Bergenstamm, 1889: 88 (unjustified).

[Pollenia] Robineau-Desvoidy MS name (Blainville et al., 1826: 11).

CURRENT STATUS: Unavailable name; suppressed by action of I.C.Z.N. (1990: 162 [Opinion 1601]); treated under Pollenia Robineau-Desvoidy, 1830 [teste this work].

FAMILY: CALLIPHORIDAE.

450. Pollenia Robineau-Desvoidy, 1830: 412.

ORIGINAlly InCluded SPECIES: Pollenia fulvicornis Robineau-Desvoidy, 1830; Pollenia viatica RobineauDesvoidy, 1830; Pollenia vivida Robineau-Desvoidy, 1830; Musca rudis Fabricius, 1794 (as "Musca rudis. Fabr. Meig."); Pollenia cyanescens Robineau-Desvoidy, 1830; Pollenia pumila RobineauDesvoidy, 1830; Pollenia autumnalis Robineau-Desvoidy, 1830; Pollenia agilis Robineau-Desvoidy, 1830; Pollenia floralis Robineau-Desvoidy, 1830; Pollenia bicolor Robineau-Desvoidy, 1830; Pollenia vernalis Robineau-Desvoidy, 1830; Pollenia tomentosa Robineau-Desvoidy, 1830; Pollenia micans Robineau-Desvoidy, 1830; Pollenia pubescens Robineau-Desvoidy, 1830; Pollenia atrata RobineauDesvoidy, 1830; Pollenia metallica Robineau-Desvoidy, 1830.

TYPE SPECIES: Musca rudis Fabricius, 1794, by original designation.

CURRENT STATUS: Valid genus [teste Rognes (1991: 212)].

FAMILY: CALLIPHORIDAE.

451. Polystodes Robineau-Desvoidy, 1830: 722.

ORIGINALly InCLUDED SPECIES: Polystodes ichneumoneus Robineau-Desvoidy, 1830.

TYPE SPECIES: Polystodes ichneumoneus Robineau-Desvoidy, 1830, by monotypy.

CURRENT STATUS: Valid genus [teste Steyskal (1980: 571)].

FAMILY: PLATYSTOMATIDAE.

EMENDATIOns: Polistodes Speiser, 1915: 99 (unjustified), n. syn.

452. Potamia Robineau-Desvoidy, 1830: 507.

Originally InCluded Species: Potamia littoralis Robineau-Desvoidy, 1830; Potamia pallipes RobineauDesvoidy, 1830.

TYPE SPECIES: Potamia littoralis Robineau-Desvoidy, 1830, by subsequent designation (Séguy, 1937: 329).

CURRENT STATUS: Valid genus [teste Pont (1986b: 83)].

FAMILY: MUSCIDAE.

453. Prionella Robineau-Desvoidy, 1830: 759.

ORIGINAlly InCluded SPECIES: Prionella beauvoisii Robineau-Desvoidy, 1830; Prionella villosa RobineauDesvoidy, 1830.

TYPE SPECIES: Prionella beauvoisii Robineau-Desvoidy, 1830, by subsequent designation (Coquillett, 1910: 594).

CURRENT STATUS: Valid genus [teste Steyskal (1965: 654)].

FAMILY: ULIDIIDAE. 
454. Priophora Robineau-Desvoidy, 1863b: 611.

ORIGINALLY INCLUDED SPECIES: Haematobia serrata Robineau-Desvoidy, 1830.

TYPE SPECIES: Haematobia serrata Robineau-Desvoidy, 1830 [= Conops irritans Linnaeus, 1758], by monotypy.

CURRENT Status: Junior synonym of Haematobia Le Peletier \& Serville, 1828 [teste Pont (1986b: 109)].

FAMILY: MUSCIDAE.

455. Psorophora Robineau-Desvoidy, 1827: 412.

ORiginally InCluded SPECIES: Culex ciliata Fabricius, 1794 (as "Culex ciliatus. Fabr.-Wied."); Psorophora boscii Robineau-Desvoidy, 1827; Culex molesta Wiedemann, 1820 (as "Culex molestus. Wied.").

TYPE SPECIES: Culex ciliata Fabricius, 1794, by subsequent designation (Theobald, 1901: 263).

CURRENT Status: Valid genus [teste Sabrosky (1999: 262)].

FAMILY: CULICIDAE.

456. Pterella Robineau-Desvoidy, 1863b: 121.

ORIGINAlly InCluded SPECIES: Miltogramma grisea Meigen, 1824; Pterella mellifrons RobineauDesvoidy, 1863; Pterella florea Robineau-Desvoidy, 1863; Tachina oestracea Fallén, 1820; Miltogramma murina Meigen, 1824.

TYPE SPECIES: Miltogramma grisea Meigen, 1824, by original designation.

CURRENT STATUS: Valid genus [teste Pape (1996: 131)].

FAMILY: SARCOPHAGIDAE.

457. Ptilocera Robineau-Desvoidy, 1830: 221.

Originally InCluded Species: Ptilocera palpalis Robineau-Desvoidy, 1830; Ptilocera conica RobineauDesvoidy, 1830.

TYPE SPECIES: Ptilocera palpalis Robineau-Desvoidy, 1830 [= Zeuxia cinerea Meigen, 1826], by subsequent designation (Townsend, 1916: 8).

CuRREnT Status: Preoccupied by Wiedemann, 1820; Henning, 1832; junior synonym of Zeuxia Meigen, 1826 [teste Herting \& Dely-Draskovits (1993: 368)].

FAMILY: TACHINIDAE.

458. Ptilopsis Robineau-Desvoidy, 1863b: 272.

ORIGinally InCluded SPECIES: Ptilopsis sexmaculata Robineau-Desvoidy, 1863 (as "Ptilopsis sexmaculata, R.-D.”).

TYPE SPECIES: Ptilopsis sexmaculata Robineau-Desvoidy, 1863 (as "Ptilopsis sex-maculata, R.-D.”), by monotypy.

CURRENT STATUS: Preoccupied by Kaup, 1848; no new replacement name proposed; placed in "Doubtful taxa in Tachinidae" by Herting \& Dely-Draskovits (1993: 437).

FAMILY: TACHINIDAE.

459. Purpurella Robineau-Desvoidy, 1853a: 117 [1853b: 37].

ORIGINALly INCLUDED SPECIES: Purpurella nobilis Robineau-Desvoidy, 1853.

TYPE SPECIES: Purpurella nobilis Robineau-Desvoidy, 1853, by monotypy.

CurRent Status: Junior synonym of Myopa Fabricius, 1775 [teste Chvála \& Smith (1988: 262)].

FAMILY: CONOPIDAE. 
460. Pyrellia Robineau-Desvoidy, 1830: 462.

ORIGINAlly InCluded SPECIES: Pyrellia violacea Robineau-Desvoidy, 1830; Pyrellia vivida RobineauDesvoidy, 1830; Pyrellia usta Robineau-Desvoidy, 1830; Pyrellia cuprea Robineau-Desvoidy, 1830; Pyrellia ignita Robineau-Desvoidy, 1830; Pyrellia calida Robineau-Desvoidy, 1830; Pyrellia littoralis Robineau-Desvoidy, 1830; Pyrellia bicolor Robineau-Desvoidy, 1830; Pyrellia fervida RobineauDesvoidy, 1830.

TYPE SPECIES: Pyrellia vivida Robineau-Desvoidy, 1830, by subsequent designation (Townsend, 1916: 8). CuRRENT STAtus: Valid genus [teste Pont (1986b: 101)].

FAMILY: MUSCIDAE.

EMENDATIONS: Pyrelia Am Stein, 1857: 106 (unjustified), n. syn.

461. Ramburia Robineau-Desvoidy, 1851d: 189.

ORIGINALLY INCLUDED SPECIES: Tachina setipennis Fallén, 1810.

TYPE SPECIES: Tachina setipennis Fallén, 1810, by monotypy.

CURRENT STATUS: Junior synonym of Triarthria Stephens, 1829 [teste Herting \& Dely-Draskovits (1993: 318)].

FAMILY: TACHINIDAE.

462. Ramonda Robineau-Desvoidy, 1863a: 790.

ORIGINAlly INCludED SPECIES: Ramonda fasciata Robineau-Desvoidy, 1863; Ramonda cuculliae Robineau-Desvoidy, 1863; Ramonda flavisquamis Robineau-Desvoidy, 1863.

TYPE SPECIES: Ramonda fasciata Robineau-Desvoidy, 1863 [= Tachina spathulata Fallén, 1820], by original designation.

CuRrent Status: Valid subgenus of Periscepsia Gistel, 1848 [teste O’Hara \& Wood (2004: 65)].

FAMILY: TACHINIDAE.

463. Ravinia Robineau-Desvoidy, 1863b: 434.

ORIGINALly INCLUDED SPECIES: Ravinia aurea Robineau-Desvoidy, 1863; Sarcophaga haematodes Meigen, 1826 (with "Myophora limpidipennis : Rob. Desv.", "Myophora haemispherica : Rob. Desv." and "Myophora horticola : Rob. Desv." in synonymy); Ravinia hebes Robineau-Desvoidy, 1863; Ravinia sulcata Robineau-Desvoidy, 1863.

TYPE SPECIES: Sarcophaga haematodes Meigen, 1826 [= Musca pernix Harris, 1780], by original designation.

CURRENT StATUS: Valid genus [teste Pape (1996: 284)].

FAMILY: SARCOPHAGIDAE.

464. Reaumuria Robineau-Desvoidy, 1830: 79.

ORIGINALly INCLUDED SPECIES: Reaumuria lalandii Robineau-Desvoidy, 1830; Musca capitata De Geer, 1776; Reaumuria blondeli Robineau-Desvoidy, 1830; Reaumuria melanura Robineau-Desvoidy, 1830; Reaumuria timorensis Robineau-Desvoidy, 1830; Reaumuria olivieri Robineau-Desvoidy, 1830; Reaumuria desvoidyi Robineau-Desvoidy, 1830.

TYPE SPECIES: Musca capitata De Geer, 1776, by subsequent designation (Robineau-Desvoidy, 1863a: 733). CURRENT STATUS: Junior synonym of Gonia Meigen, 1803 [teste Herting \& Dely-Draskovits (1993: 257)]. FAMILY: TACHINIDAE. 
465. Retellia Robineau-Desvoidy, 1830: 683.

ORIGINALLY INCLUDED SPECIES: Retellia claropunctata Robineau-Desvoidy, 1830 (as "Retellia claropunctata. R. D.").

TYPE SPECIES: Retellia claropunctata Robineau-Desvoidy, 1830 (as “Retellia claro-punctata. R. D.”), by monotypy.

CURRENT STATUS: Valid genus [teste this work].

FAMILY: SCIOMYZIDAE.

REMARKS: Knutson et al. (1976: 7) preferred to recognize Dictyodes Malloch, 1933 over Retellia RobineauDesvoidy, 1830, explaining that reversing this would upset stability. Retellia is not preoccupied and is a senior synonym of Dictyodes Malloch, 1933. We do not agree that giving priority to Retellia would upset stability since neither genus-group name is pervasive in the literature. There seems to be about the same amount of usage (about ten published works each), with Dictyodes being used more recently. We also note that Retellia claropunctata Robineau-Desvoidy, 1830 has priority over Tetanocera dictyodes Wiedemann, 1830, which is a change from previous catalog listings [see References in this paper for dating of Robineau-Desvoidy (1830) and Evenhuis (1997: 822) for dating of Wiedemann (1830)]. Macquart (1844: 336) was the first to note the synonymy.

466. Rhedia Robineau-Desvoidy, 1830: 74.

ORIGINAlly INCLUDED SPECIES: Rhedia vernalis Robineau-Desvoidy, 1830; Rhedia testacea RobineauDesvoidy, 1830; Rhedia vicina Robineau-Desvoidy, 1830; Rhedia sicula Robineau-Desvoidy, 1830; Rhedia bombylans Robineau-Desvoidy, 1830; Rhedia capensis Robineau-Desvoidy, 1830; Rhedia diversa Robineau-Desvoidy, 1830; Rhedia fulva Robineau-Desvoidy, 1830; Rhedia atra RobineauDesvoidy, 1830.

TYPE SPECIES: Rhedia vicina Robineau-Desvoidy, 1830 [= Gonia atra Meigen, 1826], by subsequent designation (Coquillett, 1910: 600).

CURRENT STATUs: Junior synonym of Gonia Meigen, 1803 [teste Herting \& Dely-Draskovits (1993: 257)].

FAMILY: TACHINIDAE.

REMARKS: Coquillett (1897: 132) stated that Robineau-Desvoidy (1830: 74) proposed Rhedia as a replacement name thinking Gonia Meigen was preoccupied in mollusks. A check of Robineau-Desvoidy (1830) shows this not to be the case. Robineau-Desvoidy's actual words when proposing this genusgroup name are "Ce genre, que je consacre à la mémoire de Fr. Rhédi, fait partie du G. Gonia des entomologistes allemands". Thus, he was proposing it for only a part of the genus Gonia and not replacing it. Robineau-Desvoidy's (1851f: 309) spelling of "Redia" is not an emendation but an incorrect subsequent spelling of Rhedia.

Emendations: Redia Bezzi \& Stein, 1907: 362 (unjustified), n. syn.

\section{Rhinia Robineau-Desvoidy, 1830: 422.}

ORIGINALLY INCLUDED SPECIES: Rhinia testacea Robineau-Desvoidy, 1830.

TYPE SPECIES: Rhinia testacea Robineau-Desvoidy, 1830, by monotypy.

CURRENT STATUS: Valid genus [teste Rognes (2002: 27)].

FAMILY: RHINIIDAE.

REMARKS: The type species has been listed as a junior synonym of Idia apicalis Wiedemann, 1830 in previous catalogs and works, but this is incorrect. Robineau-Desvoidy's 1830 paper came out 6 June while Wiedemann's 1830 paper came out less than three months later in September [see References in this paper for dating of the former and Evenhuis (1997: 822) for dating of the latter]. The name Rhinia testacea Robineau-Desvoidy, 1830 does not fulfill the requirements for a nomen oblitum (used as valid by, e.g., Senior-White et al. 1924), and we are therefore following strict priority. 
468. Rhinomya Robineau-Desvoidy, 1830: 123.

ORIGINALLY INCLUDED SPECIES: Rhinomya gagatea Robineau-Desvoidy, 1830.

TYPE SPECIES: Rhinomya gagatea Robineau-Desvoidy, 1830, by monotypy.

CurRent Status: Placed in "Doubtful taxa in Tachinidae" by Herting \& Dely-Draskovits (1993: 437).

FAMILY: TACHINIDAE.

EMENDAtions: Rhinomyia Agassiz, 1846b: 324 (unjustified), n. syn.; Rhynomyia Schiner, 1861b: 494, 549 (unjustified), n. syn.

469. Rhinophora Robineau-Desvoidy, 1830: 258.

ORIGINALLY INCLUDED SPECIES: Rhinophora nigripennis Robineau-Desvoidy, 1830; Rhinophora gagatea Robineau-Desvoidy, 1830; Rhinophora metallica Robineau-Desvoidy, 1830; Rhinophora tessellata Robineau-Desvoidy, 1830; Rhinophora hottentota Robineau-Desvoidy, 1830; Rhinophora pusilla Robineau-Desvoidy, 1830.

TYPE SPECIES: Rhinophora gagatea Robineau-Desvoidy, 1830, by subsequent designation (Townsend, 1916: $8)$.

CURRENT StATUS: Valid genus [teste Herting (1993: 114)].

FAMILY: RHINOPHORIDAE.

REMARKS: An earlier type-species designation by Robineau-Desvoidy (1863b: 5) of "Rhinophora gagatina, Meig." [= Tachina gagatina Meigen, 1824] is invalid because it was not an originally included species and more than one originally included species were listed in synonymy with it. Herting (1993: 117) placed Tachina gagatina Meigen, 1824 in his list of "Nomina dubia in Rhinophoridae".

470. Rhyncomya Robineau-Desvoidy, 1830: 424.

ORIGINALLY INCLUDED SPECIES: Musca felina Fabricius, 1794.

TYPE SPECIES: Musca felina Fabricius, 1794, by monotypy.

CURRENT STATUS: Valid genus [teste Rognes (2002: 28)].

FAMILY: RHINIIDAE.

EMENDATIONS: Rhynchomyia Agassiz, 1846b: 326 (unjustified), n. syn.

471. Richardia Robineau-Desvoidy, 1830: 728.

ORIGINALLY INCLUDED SPECIES: Richardia saltatoria Robineau-Desvoidy, 1830.

TYPE SPECIES: Richardia saltatoria Robineau-Desvoidy, 1830 [= Dacus podagricus Fabricius, 1805], by monotypy.

CURRENT STATUS: Valid genus [teste Sabrosky (1999: 270)].

FAMILY: RICHARDIIDAE.

472. Rivellia Robineau-Desvoidy, 1830: 729.

ORIGINALLY INCLUDED SPECIES: Rivellia herbarum Robineau-Desvoidy, 1830; Rivellia viridulans RobineauDesvoidy, 1830; Rivellia boscii Robineau-Desvoidy, 1830.

TYPE SPECIES: Rivellia herbarum Robineau-Desvoidy, 1830 [= Musca syngenesiae Fabricius, 1781], by subsequent designation (Rondani, 1869: 8).

CuRRENT Status: Valid genus [teste Sabrosky (1999: 271)].

FAMILY: PLATYSTOMATIDAE.

EMENDATIONS: Revellia Agassiz, 1846b: 322 (unjustified), n. syn. 
473. Roeselia Robineau-Desvoidy, 1830: 145 (as "Roëselia").

ORIGINALLY INCLUDED SPECIES: Roeselia arvensis Robineau-Desvoidy, 1830; Roeselia agrestis RobineauDesvoidy, 1830; Roeselia cylindrica Robineau-Desvoidy, 1830; Roeselia silvatica Robineau-Desvoidy, 1830.

TYPE SPECIES: Roeselia arvensis Robineau-Desvoidy, 1830 [= Tachina pallipes Fallén, 1820], by subsequent designation (Townsend, 1916: 8).

CURRENT STATUS: Preoccupied by Hübner, 1825; senior (but invalid) synonym of Ocytata Gistel, 1848 [teste Herting \& Dely-Draskovits (1993: 240)].

FAMILY: TACHINIDAE.

REMARKS: An earlier type-species designation by Robineau-Desvoidy (1863a: 605) of "Tachina pallipes, Zetterst." is invalid because it was not an originally included species and no originally included species were listed in synonymy with it.

474. Rohrella Robineau-Desvoidy, 1830: 489.

ORIGINALly INCLUDED SPECIES: Musca testacea Fabricius, 1781; Rohrella soror Robineau-Desvoidy, 1830; Rohrella blondeli Robineau-Desvoidy, 1830; Rohrella humeralis Robineau-Desvoidy, 1830; Rohrella cognata Robineau-Desvoidy, 1830; Rohrella dorsalis Robineau-Desvoidy, 1830; Rohrella fragilis Robineau-Desvoidy, 1830; Rohrella germana Robineau-Desvoidy, 1830; Rohrella punctata RobineauDesvoidy, 1830.

TYPE SPECIES: Rohrella fragilis Robineau-Desvoidy, 1830 [= Musca pallida Fabricius, 1787], by subsequent designation (Coquillett, 1901: 141).

CurRent Status: Junior synonym of Phaonia Robineau-Desvoidy, 1830 [teste Pont (1986b: 116)].

FAMILY: MUSCIDAE.

REMARKS: Enderlein (1927: 53) designated Musca testacea Fabricius, 1781, but this was later.

\section{Rondania Robineau-Desvoidy, 1850a: 192.}

ORIGINALLY INCLUDED SPECIES: Rondania cucullata Robineau-Desvoidy, 1850.

TYPE SPECIES: Rondania cucullata Robineau-Desvoidy, 1850, by monotypy.

CuRrent Status: Valid genus [teste Herting \& Dely-Draskovits (1993: 395)].

FAMILY: TACHINIDAE.

[Roudania] Robineau-Desvoidy, 1849d: 158.

CURRENT STATUS: Unavailable name; proposed without description, indication, or included species; treated under Rondania Robineau-Desvoidy, 1850 [teste this work].

FAMILY: TACHINIDAE.

476. Rutilia Robineau-Desvoidy, 1830: 319.

ORIGINALly InCludED SPECIES: Musca leonina Fabricius, 1776; Rutilia formosa Robineau-Desvoidy, 1830 (as "Musca formosa. Donavan"); Tachina vivipara Fabricius, 1805; Rutilia durvillei Robineau-Desvoidy, 1830.

TYPE SPECIES: Tachina vivipara Fabricius, 1805, by subsequent designation (Crosskey, 1967: 26).

CURRENT StATUs: Valid genus [teste Cantrell \& Crosskey (1989: 744)].

FAMILY: TACHINIDAE.

REMARKS: Townsend (1916: 8) had long been considered as the earliest subsequent designation for Rutilia Robineau-Desvoidy, but the species "Rutilia vivipara RD" designated by Townsend does not exist and is ambiguous because it could either mean a species misidentified by Robineau-Desvoidy as Tachina 
vivipara Fabricius, 1805 or the true Tachina vivipara Fabricius, 1805. Noting that Townsend's (1916) and other previous type-species designations were invalid, Crosskey (1967: 26) clearly laid out the nomenclatural history of Rutilia Robineau-Desvoidy, 1830 and designated Tachina vivipara Fabricius, 1805 as the type species.

477. Sabethes Robineau-Desvoidy, 1827: 411.

ORIGINALly INCLUDED SPECIES: Sabethes locuples Robineau-Desvoidy, 1827; Culex longipes Fabricius, 1805 (as “Culex longipes. Fabr. Wiedem.").

TYPE SPECIES: Sabethes locuples Robineau-Desvoidy, 1827 [= Culex cyaneus Fabricius, 1805], by subsequent designation (Le Peletier \& Serville, 1828: 658).

CURRENT Status: Valid genus [teste Sabrosky (1999: 272)].

FAMILY: CULICIDAE.

REMARKS: Coquillett (1910: 602) designated the same type species but this was later.

EMENDATIONS: Sabettus Agassiz, 1846b: 329 (unjustified).

478. Sagaris Robineau-Desvoidy, 1863a: 486.

ORIGINALly INCLUDED SPECIES: Sagaris laevigata Robineau-Desvoidy, 1863; Sagaris marginalis RobineauDesvoidy, 1863; Sagaris cinerea Robineau-Desvoidy, 1863.

TYPE SPECIES: Sagaris laevigata Robineau-Desvoidy, 1863, by original designation.

CuRRENT STATUS: Preoccupied by Panzer, 1806; Walker, 1854; no new replacement name proposed; placed in "Doubtful taxa in Tachinidae" by Herting \& Dely-Draskovits (1993: 437).

FAMILY: TACHINIDAE.

479. Salia Robineau-Desvoidy, 1830: 108.

ORIGINALly INCLUDED SPECIES: Salia bombycivora Robineau-Desvoidy, 1830; Salia velox RobineauDesvoidy, 1830; Salia echinura Robineau-Desvoidy, 1830; Salia cirrata Robineau-Desvoidy, 1830; Salia erythrocera Robineau-Desvoidy, 1830; Salia nigricornis Robineau-Desvoidy, 1830; Salia blondeli Robineau-Desvoidy, 1830; Salia metallica Robineau-Desvoidy, 1830.

TYPE SPECIES: Salia echinura Robineau-Desvoidy, 1830 [= Tachina obliquata Fallén, 1810], by subsequent designation (Robineau-Desvoidy, 1863a: 553).

CURRENT STATUS: Preoccupied by Hübner, 1818; senior (but invalid) synonym of Chetogena Rondani, 1856 [teste Herting \& Dely-Draskovits (1993: 135)].

FAMILY: TACHINIDAE.

480. Saltella Robineau-Desvoidy, 1830: 746.

ORIGINALLY INCLUDED SPECIES: Saltella longipes Robineau-Desvoidy, 1830; Saltella nigripes Robineau-

Desvoidy, 1830; Saltella nana Robineau-Desvoidy, 1830.

TYPE SPECIES: Saltella nigripes Robineau-Desvoidy, 1830, by subsequent designation (Westwood, 1840: 147).

Current Status: Valid genus [teste Pont \& Meier (2002: 107)].

FAMILY: SEPSIDAE.

481. Salticella Robineau-Desvoidy, 1830: 683.

ORIGINALLY INCLUDED SPECIES: Salticella saltatrix Robineau-Desvoidy, 1830.

TYPE SPECIES: Salticella saltatrix Robineau-Desvoidy, 1830, by monotypy. 
CURRENT Status: Valid genus [teste Sabrosky (1999: 272)].

FAMILY: SCIOMYZIDAE.

REMARKS: Previous listings of the type species have it as a junior synonym of Lucina fasciata Meigen, 1830, but this is incorrect. Robineau-Desvoidy's 1830 paper came out 6 June while Meigen's 1830 paper came out six months later on 1 December [see References in this paper for dating of the former and Evenhuis (1997: 533) for dating of the latter].

482. Saphaea Robineau-Desvoidy, 1830: 796.

ORIGINALLY INCLUDED SPECIES: Saphaea proboscidea Robineau-Desvoidy, 1830.

TYPE SPECIES: Saphaea proboscidea Robineau-Desvoidy, 1830, by monotypy.

CURRENT STATUS: Valid genus [teste Becker (1896: 253)]; treated as incertae sedis in Ephydridae [teste this work].

FAMILY: EPHYDRIDAE.

REMARKS: This nominal genus was treated in Ephydridae in the revisionary work of Becker (1896) but it is missing from both the Palaearctic (Cogan, 1984) and world (Mathis \& Zatwarnicki, 1995) catalogs of Ephydridae.

\section{Sargella Robineau-Desvoidy, 1830: 674.}

ORIGINALly INCLUDED SPECIES: Sargella cylindrica Robineau-Desvoidy, 1830.

TYPE SPECIES: Sargella cylindrica Robineau-Desvoidy, 1830, by monotypy.

CURRENT STATUS: Treated under "Genres et espèces douteux" [teste Séguy (1952: 7)].

FAMILY: SCATHOPHAGIDAE.

REMARKS: This genus-group name has not been studied since its original description. Séguy (1952: 7) placed it as doubtfully included in Scathophagidae, but it was omitted from the Palaearctic catalog (Gorodkov, 1986).

484. Scaligeria Robineau-Desvoidy, 1863b: 476.

ORIGINALLY INCLUDED SPECIES: Scaligeria myoidaea Robineau-Desvoidy, 1863 (as Scaligera myoïdaea, R.D.”); Myophora maialis Robineau-Desvoidy, 1830 (as “Myophora maïalis, R.-D.”); Scaligeria ardeacea Robineau-Desvoidy, 1863; Scaligeria humilis Robineau-Desvoidy, 1863; Scaligeria brunisquamis Robineau-Desvoidy, 1863; Scaligeria fuligo Robineau-Desvoidy, 1863; Scaligeria praeceps RobineauDesvoidy, 1863; Scaligeria pervia Robineau-Desvoidy, 1863; Scaligeria fugax Robineau-Desvoidy, 1863.

TYPE SPECIES: Myophora maialis Robineau-Desvoidy, 1830, by original designation (as "Myophora maïalis, R.-D.").

CURRENT STATUS: Junior synonym of Sarcophaga Meigen, 1826, but subgenerically unplaced [teste Pape (1996: 420)].

FAMILY: SARCOPHAGIDAE.

485. Scatella Robineau-Desvoidy, 1830: 801.

ORIGINAlly InCluded SPECIES: Scatella buccata Robineau-Desvoidy, 1830; Scatella cinerea RobineauDesvoidy, 1830.

TYPE SPECIES: Scatella buccata Robineau-Desvoidy, 1830, [= Ephydra stagnalis Fallén, 1813], by subsequent designation (Coquillett, 1910: 603).

CURRENT STATUS: Valid genus [teste Mathis \& Zatwarnicki (1995: 263)].

FAMILY: EPHYDRIDAE. 
486. Scatina Robineau-Desvoidy, 1830: 629.

ORIGINALLY INCLUDED SPECIES: Scatina claripennis Robineau-Desvoidy, 1830.

TYPE SPECIES: Scatina claripennis Robineau-Desvoidy, 1830, by monotypy.

CURRENT STATUS: Junior synonym of Scathophaga Meigen, 1803 [teste Gorodkov (1986: 29)].

FAMILY: SCATHOPHAGIDAE.

REMARKS: Gorodkov (1986: 29) synonymized the genus-group name with Scathophaga but left its only species, Scatina claripennis, in "Doubtful genera and species" of Scathophagidae (Gorodkov, 1986: 39).

487. Scatophora Robineau-Desvoidy, 1830: 811.

ORIGINALly INCLUDED SPECIES: Scatophora merdaria Robineau-Desvoidy, 1830; Scatophora carolinensis Robineau-Desvoidy, 1830.

TYPE SPECIES: Scatophora carolinensis Robineau-Desvoidy, 1830, by subsequent designation (Spuler, 1924: 378).

CURRENT StATUS: Placed in "Doubtful genera of Sphaeroceridae" [teste Roháček in Roháček (2001: 295)].

FAMILY: SPHAEROCERIDAE.

REMARKS: The genus is currently considered a nomen dubium by Roháček in Roháček (2001: 296) because the type species is unrecognizable, and it may not even belong in Sphaeroceridae.

488. Schaumia Robineau-Desvoidy, 1863b: 43.

ORIGINALLY INCLUDED SPECIES: Tachina bimaculata Hartig, 1838 (as "Tachina bi-maculata : Hartig").

TYPE SPECIES: Tachina inclusa Hartig, 1838 by fixation of O'Hara et al. (2009: 46) under ICZN Code Article 70.3.2, misidentified as Tachina bimaculata Hartig, 1838 (as "Tachina bi-maculata : Hartig") in the fixation by monotypy of Robineau-Desvoidy (1863b: 43).

CURRENT STAtus: Junior synonym of Blondelia Robineau-Desvoidy, 1830 [teste Herting \& Dely-Draskovits (1993: 168)].

FAMILY: TACHINIDAE.

489. Scopolia Robineau-Desvoidy, 1830: 268.

ORIGINALLY INCLUDED SPECIES: Scopolia rupestris Robineau-Desvoidy, 1830; Musca carbonaria Panzer, 1798; Scopolia viatica Robineau-Desvoidy, 1830; Scopolia rufipes Robineau-Desvoidy, 1830; Scopolia parasita Robineau-Desvoidy, 1830.

TYPE SPECIES: Musca carbonaria Panzer, 1798, by subsequent designation (Zetterstedt, 1844: 1239).

CurRent Status: Preoccupied by Hübner, 1825; senior (but invalid) synonym of Periscepsia Gistel, 1848 sensu stricto [teste O'Hara \& Wood (2004: 64)].

FAMILY: TACHINIDAE.

490. Scotia Robineau-Desvoidy, 1863a: 255.

ORIGINALly INCLUDED SPECIES: Scotia placida Robineau-Desvoidy, 1863; Scotia saturniae RobineauDesvoidy, 1863.

TYPE SPECIES: Scotia placida Robineau-Desvoidy, 1863, by original designation.

CURRENT STATUS: Preoccupied by Hübner, 1821; Leuckart, 1849; replaced by Neoscotia Townsend, 1919; placed in "Doubtful taxa in Tachinidae" by Herting \& Dely-Draskovits (1993: 437).

FAMILY: TACHINIDAE. 
491. Scyphella Robineau-Desvoidy, 1830: 650.

ORIGINALLY INCLUDED SPECIES: Scyphella flavicornis Robineau-Desvoidy, 1830; Scyphella nigricornis Robineau-Desvoidy, 1830.

TYPE SPECIES: Scyphella flavicornis Robineau-Desvoidy, 1830 [= Musca flava Linnaeus, 1758], by subsequent designation (Bezzi, 1904: 178).

CURRENT StATUS: Junior synonym of Chyromya Robineau-Desvoidy, 1830 [teste Soós (1984d: 58)].

FAMILY: CHYROMYIDAE.

492. Servaisia Robineau-Desvoidy, 1863b: 429.

ORIGINALLY INCLUDED SPECIES: Sarcophaga erythrura Meigen, 1826 (as "Sarcophaga erythrocera : Meig."; with "Myophora riparia : Rob. Desv." and "Myophora collinaris : Rob. Desv." in synonymy); Servaisia egena Robineau-Desvoidy, 1863; Servaisia gagatea Robineau-Desvoidy, 1863; Servaisia luteicauda Robineau-Desvoidy, 1863; Myophora crocata Robineau-Desvoidy, 1830.

TYPE SPECIES: Sarcophaga erythrura Meigen, 1826, by original designation (as "Sarcophaga erythrocera : Meig.").

CURRENT STATUS: Valid subgenus of Blaesoxipha Loew, 1861 [teste Pape (1996: 202)].

FAMILY: SARCOPHAGIDAE.

493. Servillia Robineau-Desvoidy, 1830: 49.

ORIGINALly INCLUDED SPECIES: Tachina ursina Meigen, 1824; Servillia pilosa Robineau-Desvoidy, 1830; Servillia subpilosa Robineau-Desvoidy, 1830.

TYPE SPECIES: Tachina ursina Meigen, 1824, by subsequent designation (Robineau-Desvoidy, 1863a: 644).

CURRENT STATUS: Junior synonym of Tachina Meigen, 1803 sensu stricto [teste O'Hara et al. (2009: 175)].

FAMILY: TACHINIDAE.

EMENDATIONS: Servilia Am Stein, 1857: 103 (unjustified), n. syn.

494. Setellia Robineau-Desvoidy, 1830: 732.

ORIGINALLY INCLUDED SPECIES: Setellia afra Robineau-Desvoidy, 1830.

TYPE SPECIES: Setellia afra Robineau-Desvoidy, 1830, by monotypy.

CURRENT STATUS: Valid genus [teste Sabrosky (1999: 281)].

FAMILY: RICHARDIIDAE.

\section{Setulia Robineau-Desvoidy, 1863b: 124.}

ORIGINAlly InCluded SPECIES: Setulia cerceridis Robineau-Desvoidy, 1863 (as "Setulia Cerceridis, Guérin"); Setulia flavescens Robineau-Desvoidy, 1863; Setulia aurifrons Robineau-Desvoidy, 1863; Miltogramma grisea Meigen, 1824; Miltogramma intricata Meigen, 1824; Miltogramma incompta Meigen, 1830; Miltogramma tessellata Meigen, 1824; Miltogramma germari Meigen, 1824; Miltogramma algira Macquart, 1843 (as "Setulia Algira, Macq." with "Miltogramma Algira : Meig." in synonymy).

TYPE SPECIES: Setulia cerceridis Robineau-Desvoidy, 1863 [= Miltogramma grisea Meigen, 1824], by original designation (as "Setulia Cerceridis, Guérin").

Current Status: Junior synonym of Pterella Robineau-Desvoidy, 1863 [teste Pape (1996: 131)].

FAMILY: SARCOPHAGIDAE. 
496. Silbermania Robineau-Desvoidy, 1863b: 73.

ORIGINALLY INCLUDED SPECIES: Dufouria petiolata Robineau-Desvoidy, 1830.

TYPE SPECIES: Dufouria petiolata Robineau-Desvoidy, 1830 [= Tachina nigrita Fallén, 1810], by monotypy.

CURRENT STATUS: Junior synonym of Dufouria Robineau-Desvoidy, 1830 [teste Herting \& Dely-Draskovits (1993: 393; as "Silbermannia")].

FAMILY: TACHINIDAE.

EMENDATIONS: Silbermannia Marschall, 1873: 346 (unjustified), n. syn.

497. Sironia Robineau-Desvoidy, 1863a: 305.

ORIGINALLY INCLUDED SPECIES: Melibaea gagatea Robineau-Desvoidy, 1830.

TYPE SPECIES: Melibaea gagatea Robineau-Desvoidy, 1830, by original designation.

CurRent Status: Preoccupied by Hübner, 1823; Clemens, 1860; no new replacement name proposed; placed in "Doubtful taxa in Tachinidae" by Herting \& Dely-Draskovits (1993: 437).

FAMILY: TACHINIDAE.

498. Sitarea Robineau-Desvoidy, 1830: 763.

ORIGINALLY INCLUDED SPECIES: Sitarea scorzonerae Robineau-Desvoidy, 1830 (with "An Musca Doronici ?

De Geer" in doubtful synonymy); Sitarea dejeanii Robineau-Desvoidy, 1830.

TYPE SPECIES: Sitarea scorzonerae Robineau-Desvoidy, 1830, by subsequent designation (Desmarest in d'Orbigny, 1848a: 643).

CURRENT STATUS: Junior synonym of Orellia Robineau-Desvoidy, 1830 [teste Norrbom et al. (1999: 177)].

FAMILY: TEPHRITIDAE.

499. Smidtia Robineau-Desvoidy, 1830: 183.

ORIGINALly INCLUDED SPECIES: Smidtia vernalis Robineau-Desvoidy, 1830; Smidtia myoidea RobineauDesvoidy, 1830 (as “Smidtia myö̈dea. R. D.”).

TYPE SPECIES: Smidtia vernalis Robineau-Desvoidy, 1830 [= Tachina conspersa Meigen, 1824], by subsequent designation (Desmarest in d'Orbigny, 1848a: 649).

CURRENT STATUS: Valid genus [teste Herting \& Dely-Draskovits (1993: 178)].

FAMILY: TACHINIDAE.

REMARKS: Robineau-Desvoidy (1863a: 294) designated the same type species but this was later.

[Soleria] Robineau-Desvoidy, 1849a: 464.

CURRENT STATUS: Incorrect original spelling of Solieria Robineau-Desvoidy, 1849a [teste this work].

FAMILY: TACHINIDAE.

[Solieria] Robineau-Desvoidy, 1848b: 186.

CURRENT STATUS: Unavailable name; proposed without description or included species; treated under Solieria Robineau-Desvoidy, 1849 [teste this work].

FAMILY: TACHINIDAE.

500. Solieria Robineau-Desvoidy, 1849a: 461

ORIGINALly InCLUdED SPECIES: Solieria binotata Robineau-Desvoidy, 1849; Solieria festiva Robineau-

Desvoidy, 1849; Solieria gagatea Robineau-Desvoidy, 1849; Solieria immaculata Robineau-Desvoidy, 
1849; Soleria rustica Robineau-Desvoidy, 1849; Solieria elongata Robineau-Desvoidy, 1849; Myobia ruficrus Robineau-Desvoidy, 1830; Solieria vicina Robineau-Desvoidy, 1849; Solieria modesta Robineau-Desvoidy, 1849; Solieria germana Robineau-Desvoidy, 1849; Tachina inanis Fallén, 1810; Solieria fuscana Robineau-Desvoidy, 1849; Solieria femoralis Robineau-Desvoidy, 1849; Solieria brunicosa Robineau-Desvoidy, 1849; Solieria cinerea Robineau-Desvoidy, 1849; Myobia cinerascens Robineau-Desvoidy, 1830; Solieria pulverulenta Robineau-Desvoidy, 1849; Solieria dimidiata Robineau-Desvoidy, 1849; Solieria nigra Robineau-Desvoidy, 1849; Myobia nana Robineau-Desvoidy, 1830; Myobia nitens Robineau-Desvoidy, 1830; Myobia lateralis Robineau-Desvoidy, 1830; Tachina pacifica Meigen, 1824.

TYPE SPECIES: Tachina inanis Fallén, 1810, by subsequent designation (Coquillett, 1910: 606).

CURRENT Status: Valid genus [teste Herting \& Dely-Draskovits (1993: 342)].

FAMILY: TACHINIDAE.

REMARKS: Townsend (1916: 7) designated Solieria brunicosa Robineau-Desvoidy, 1849 as type species but this was later. Both Tachina inanis and Solieria brunicosa are currently treated in Solieria. There are two original spellings of this genus-group name in Robineau-Desvoidy (1849a): Solieria (page 461) and Soleria (page 464). Acting as First Reviser, we select Solieria as the correct original spelling.

501. Sophia Robineau-Desvoidy, 1830: 317.

ORIGINALly INCLUDED SPECIES: Sophia pellucida Robineau-Desvoidy, 1830; Sophia punctata RobineauDesvoidy, 1830; Sophia gagatea Robineau-Desvoidy, 1830; Sophia filipes Robineau-Desvoidy, 1830.

TYPE SPECIES: Sophia filipes Robineau-Desvoidy, 1830, by subsequent designation (Townsend, 1916: 9). CURRENT STATUS: Valid genus [teste Guimarães (1971: 112)].

FAMILY: TACHINIDAE.

REMARKS: Lamarck (1816) listed the Péron manuscript name "Sophia" in synonymy [under Calliantra Péron \& Lesueur, 1810] as did Blainville (1830) [under Beroe Gronovius, 1760], and the name was not made available before 1961 so it is unavailable and does not enter into homonymy with Sophia RobineauDesvoidy, 1830. Guimarães (1971: 112) treated Sophia as a valid genus but left its type species, Sophia filipes, as "Unrecognized".

502. Spallanzania Robineau-Desvoidy, 1830: 78.

ORIGINALly InCluded SPeCIES: Spallanzania picea Robineau-Desvoidy, 1830; Spallanzania gallica Robineau-Desvoidy, 1830.

TYPE SPECIES: Spallanzania gallica Robineau-Desvoidy, 1830 [= Tachina hebes Fallén, 1820], by subsequent designation (Coquillett, 1910: 606).

CURRENT STATUS: Valid genus [teste Herting \& Dely-Draskovits (1993: 262)].

FAMILY: TACHINIDAE.

503. Sphenella Robineau-Desvoidy, 1830: 773.

ORIGINALLY INCLUDED SPECIES: Sphenella linariae Robineau-Desvoidy, 1830.

TYPE SPECIES: Sphenella linariae Robineau-Desvoidy, 1830 [= Tephritis marginata Fallén, 1814], by monotypy.

CURRENT STATUS: Valid genus [teste Norrbom et al. (1999: 208)].

FAMILY: TEPHRITIDAE.

504. Sphora Robineau-Desvoidy, 1830: 394.

ORIGINALly INCLUDED SPECIES: Sphora nigricans Robineau-Desvoidy, 1830; Sphora australis Robineau-

Desvoidy, 1830. 
TYPE SPECIES: Sphora nigricans Robineau-Desvoidy, 1830 [= Musca domestica Linnaeus, 1758], by subsequent designation (Townsend, 1916: 9).

CURRENT STATUS: Junior synonym of Musca Linnaeus, 1758 [teste Pont (1973: 136)].

FAMILY: MUSCIDAE.

505. Spinolia Robineau-Desvoidy, 1863b: 41.

ORIGINALLY INCLUDED SPECIES: Tachina inclusa Hartig, 1838.

TYPE SPECIES: Tachina inclusa Hartig, 1838, by monotypy.

CuRRENT STATUs: Preoccupied by Dahlbom, 1854; Costa, 1858; junior synonym of Blondelia Robineau-

Desvoidy, 1830 [teste Herting \& Dely-Draskovits (1993: 168)].

FAMILY: TACHINIDAE.

506. Staegeria Robineau-Desvoidy, 1863a: 972.

ORIGINALly INCLUDED SPECIES: Staegeria lateralis Robineau-Desvoidy, 1863; Staegeria chrysella Robineau-Desvoidy, 1863; Staegeria boscorum Robineau-Desvoidy, 1863; Staegeria camporum Robineau-Desvoidy, 1863; Staegeria muscidea Robineau-Desvoidy, 1863; Staegeria elongata RobineauDesvoidy, 1863; Staegeria egena Robineau-Desvoidy, 1863; Staegeria servula Robineau-Desvoidy, 1863; Staegeria floralis Robineau-Desvoidy, 1863; Staegeria flavida Robineau-Desvoidy, 1863; Staegeria aestivalis Robineau-Desvoidy, 1863; Staegeria schistella Robineau-Desvoidy, 1863; Tachina pratensis Robineau-Desvoidy, 1830; Staegeria cinerea Robineau-Desvoidy, 1863; Staegeria villana Robineau-Desvoidy, 1863; Staegeria canescens Robineau-Desvoidy, 1863; Staegeria ardelio RobineauDesvoidy, 1863; Staegeria occlusa Robineau-Desvoidy, 1863; Staegeria rusticana Robineau-Desvoidy, 1863; Staegeria nigrita Robineau-Desvoidy, 1863; Staegeria atrata Robineau-Desvoidy, 1863; Staegeria faedata Robineau-Desvoidy, 1863.

TYPE SPECIES: Tachina pratensis Robineau-Desvoidy, 1830 [probably = Tachina mimula Meigen, 1824], by original designation.

CURRENT StATUS: Preoccupied by Rondani, 1856; junior synonym of Adenia Robineau-Desvoidy, 1863 (subgenus of Exorista Meigen, 1803) [teste Herting (1984: 228)].

FAMILY: TACHINIDAE.

REMARKS: Herting (1974: 28) wrote that the type species Tachina pratensis "gehört wahrscheinlich zur Exorista rustica-Gruppe". Herting (1984: 228) was more specific and suggested that T. pratensis "Probably = Exorista (Adenia) mimula Meigen", and we follow this interpretation. Herting \& DelyDraskovits (1993: 133) were essentially in agreement in placing T. pratensis in "Doubtful species" of Exorista (Adenia), but erred in placing Staegeria Robineau-Desvoidy, 1863 in "Doubtful taxa in Tachinidae" and contradicted this placement by citing T. pratensis as a synonym of "Exorista (Adenia) mimula Meigen” (page 438).

507. Stagnia Robineau-Desvoidy, 1830: 508.

ORIGINALly INCLUDED SPECIES: Stagnia nymphaearum Robineau-Desvoidy, 1830; Stagnia potamogeti Robineau-Desvoidy, 1830.

TYPE SPECIES: Stagnia nymphaearum Robineau-Desvoidy, 1830, by subsequent designation (Hennig, 1963a: 773).

CuRrent Status: Junior synonym of Phaonia Robineau-Desvoidy, 1830 [teste Pont (1986b: 116)].

FAMILY: MUSCIDAE.

508. Stephania Robineau-Desvoidy, 1863a: 807.

ORIGINALLY INCLUDED SPECIES: Stephania meridionalis Robineau-Desvoidy, 1863. 
TYPE SPECIES: Stephania meridionalis Robineau-Desvoidy, 1863 [= Tachina setipennis Fallén, 1810], by monotypy.

CuRRENT STATUS: Preoccupied by Guenée, 1850; junior synonym of Triarthria Stephens, 1829 [teste Herting \& Dely-Draskovits (1993: 319)].

FAMILY: TACHINIDAE.

[Stephenia] Robineau-Desvoidy, 1863b: 910.

CURRENT STATUS: Incorrect original spelling of Stephensia Robineau-Desvoidy, 1863b [teste this work].

FAMILY: TACHINIDAE.

509. Stephensia Robineau-Desvoidy, 1863b: 17.

ORIGINALLY INCLUDED SPECIES: Stephensia ciligera Robineau-Desvoidy, 1863.

TYPE SPECIES: Stephensia ciligera Robineau-Desvoidy, 1863 [= Tachina exigua Meigen, 1824], by monotypy.

CuRRENT Status: Preoccupied by Stainton, 1858; junior synonym of Microsoma Macquart, 1855 [teste Herting \& Dely-Draskovits (1993: 397)].

FAMILY: TACHINIDAE.

REMARKS: There are two original spellings of this genus-group name in Robineau-Desvoidy (1863b): Stephensia (page 17) and Stephenia (page 910). Acting as First Reviser, we select Stephensia as the correct original spelling.

510. Stevenia Robineau-Desvoidy, 1830: 220.

ORIGINALly InCluded SPECIES: Stevenia tomentosa Robineau-Desvoidy, 1830; Stevenia nitens RobineauDesvoidy, 1830; Stevenia nigripennis Robineau-Desvoidy, 1830; Stevenia velox Robineau-Desvoidy, 1830.

TYPE SPECIES: Stevenia tomentosa Robineau-Desvoidy, 1830 [= Tachina atramentaria Meigen, 1824], by subsequent designation (Desmarest in d'Orbigny, 1848b: 32).

CURRENT STATUS: Valid genus [teste Herting (1993: 111)].

FAMILY: RHINOPHORIDAE.

REMARKS: Robineau-Desvoidy (1863b: 378) designated the same type species but this was later.

511. Stomina Robineau-Desvoidy, 1830: 411.

ORIGINALLY INCLUDED SPECIES: Stomina rubricornis Robineau-Desvoidy, 1830.

TYPE SPECIES: Stomina rubricornis Robineau-Desvoidy, 1830 [= Musca tachinoides Fallén, 1817], by monotypy.

CURRENT STATUS: Valid genus [teste Herting \& Dely-Draskovits (1993: 391)].

FAMILY: TACHINIDAE.

512. Strauzia Robineau-Desvoidy, 1830: 718.

ORIGINALly INCLUDED SPECIES: Strauzia inermis Robineau-Desvoidy, 1830; Strauzia armata RobineauDesvoidy, 1830.

TYPE SPECIES: Strauzia inermis Robineau-Desvoidy, 1830, by subsequent designation (Foote, 1965: 676).

CURRENT STATUS: Valid genus [teste Norrbom et al. (1999: 211)].

FAMILY: TEPHRITIDAE.

REMARKS: Robineau-Desvoidy's 1830 paper came out on 6 June while Wiedemann's 1830 paper came out less than three months later in September [see References in this paper for dating of the former and 
Evenhuis (1997: 822) for dating of the latter]. The type species is therefore a senior synonym of Trypeta longipennis Wiedemann, 1830, but as we have found no use of the name Strauzia inermis RobineauDesvoidy, 1830 as valid after 1899, it appears to fulfill the requirements for a nomen oblitum. Trypeta longipennis Wiedemann, 1830 is in prevailing usage and has appeared as a valid name in the genus Strauzia in at least 25 publications by more than 10 authors during the past 50 years including the following: Arnett (1985), Bisby et al. (2008), Bridges (1972), Charlet et al. (1987), Charlet et al. (1992), Denoroy (1996), Eaton \& Kaufman (2007), Fast (1966), Foote (1964, 1965), Foote \& Steyskal (1987), Foote et al. (1993), Frost (1964), Hilgendorf \& Goeden (1981), Hill (1987), Hoes \& Huang (1975), Kays \& Nottingham (2008), Marshall (2006), Norrbom et al. (1999), Organisation for Economic Co-operation and Development (2006), Steck \& Sutton (2001), Steyskal (1972, 1986), Stoltzfus (1988), Sutton \& Steck (2005), Westdal \& Barrett (1960, 1962), Williams et al. (1998). In the interests of nomenclatural stability, we hereby invoke reversal of precedence (ICZN Code Article 23.9.2) and declare Strauzia inermis Robineau-Desvoidy, 1830 a nomen oblitum and Trypeta longipennis Wiedemann, 1830 a nomen protectum. Norrbom et al. (1999: 211) alleged that the emendation by Loew (1873: 243) to Straussia was justified, but they rejected it in the interests of stability. The same emendation by Agassiz (1846b: 354) is earlier, but the dedication by Robineau-Desvoidy (1830: 718) stating for whom the genus-group Strauzia is being named is "M. Straus", not "Strauss", thus the emendations listed below are all unjustified (and would be so in any case as Robineau-Desvoidy's repeated use of "Strauzia" is a clear indication that this was the intended spelling). Loew (1873: 17) remarked that Strauzia was named for Hercule "StraussDürkheim" [also spelled as "Straus-Dürkheim"] (an anatomy professor at the Collège de Paris). Loew may have been correct but there is no etymology of this name in Robineau-Desvoidy (1830) other than the aforementioned dedication to "M. Straus, anatomiste".

EmEndations: Straussia Agassiz, 1846b: 354 (unjustified); Straussia Loew, 1873: 17, 243 (unjustified); Straxissia Verrall in Scudder, 1882: 319 (unjustified), n. syn.

513. Sturmia Robineau-Desvoidy, 1830: 171.

ORIGINALLY INCLUDED SPECIES: Sturmia atropivora Robineau-Desvoidy, 1830; Sturmia vanessae RobineauDesvoidy, 1830; Sturmia floricola Robineau-Desvoidy, 1830; Sturmia concolor Robineau-Desvoidy, 1830.

TYPE SPECIES: Sturmia atropivora Robineau-Desvoidy, 1830, by subsequent designation (Desmarest in d'Orbigny, 1848b: 76-77).

CURRENT STATUS: Valid genus [teste Herting \& Dely-Draskovits (1993: 247)].

FAMILY: TACHINIDAE.

REMARKS: Robineau-Desvoidy (1863a: 888) designated Sturmia vanessae Robineau-Desvoidy, 1830 as type species, but this was later. Acceptance of the earlier designation causes problems with stability of nomenclature and taxonomy of Sturmia, making it a synonym of Drino Robineau-Desvoidy, 1830. An application to the ICZN Commission to conserve the designation of Robineau-Desvoidy (1863a: 888) and suppress any earlier designations is in preparation.

[Stygia] Robineau-Desvoidy MS name (Blainville et al., 1826: 11).

CURRENT STATUS: Unavailable name; suppressed by action of I.C.Z.N. (1990: 162 [Opinion 1601]); treated as a nomen dubium in Muscidae [teste this work].

FAMILY: MUSCIDAE.

514. Stygina Robineau-Desvoidy, 1863a: 798.

ORIGINALly INCLUDED SPECIES: Nyctia carceli Robineau-Desvoidy, 1830; Stygina obscurata RobineauDesvoidy, 1863; Stygina usta Robineau-Desvoidy, 1863. 
TYPE SPECIES: Nyctia carceli Robineau-Desvoidy, 1830 [= Musca halterata Panzer, 1798], by original designation.

CurRent Status: Preoccupied by Salter, 1853; junior synonym of Nyctia Robineau-Desvoidy, 1830 [teste Pape (1996: 165)].

FAMILY: SARCOPHAGIDAE.

\section{Stylia Robineau-Desvoidy, 1830: 754.}

ORIGINALly INCLUDED SPECIES: Stylia maculata Robineau-Desvoidy, 1830; Stylia mentharum RobineauDesvoidy, 1830; Stylia bidentis Robineau-Desvoidy, 1830.

TYPE SPECIES: Stylia maculata Robineau-Desvoidy, 1830, by subsequent designation (Desmarest in d'Orbigny, 1848b: 78).

CURRENT STATUS: Treated as "Unrecognized” [teste Norrbom et al. (1999: 212)].

FAMILY: TEPHRITIDAE.

516. Stylophora Robineau-Desvoidy, 1830: 723.

ORIGINALLY INCLUDED SPECIES: Stylophora zonata Robineau-Desvoidy, 1830.

TYPE SPECIES: Stylophora zonata Robineau-Desvoidy, 1830, by monotypy.

CURRENT STATUS: Preoccupied by Schweigger, 1819; no new replacement name proposed; treated as nomen dubium in Acalyptratae [teste this work].

FAMILY: UNPLACED ACALYPTRATAE.

517. Suillia Robineau-Desvoidy, 1830: 642.

ORIGINALLY INCLUDED SPECIES: Suillia tuberivora Robineau-Desvoidy, 1830; Suillia fungorum RobineauDesvoidy, 1830; Suillia claripennis Robineau-Desvoidy, 1830; Suillia communis Robineau-Desvoidy, 1830 (with "Musca suilla. Fabr." in synonymy); Suillia liturata Robineau-Desvoidy, 1830; Suillia sexnotata Robineau-Desvoidy, 1830 (as "Suillia sex-notata. R. D.”); Suillia fragilis Robineau-Desvoidy, 1830; Suillia cinerea Robineau-Desvoidy, 1830; Suillia flaveola Robineau-Desvoidy, 1830; Suillia lineata Robineau-Desvoidy, 1830; Suillia violascens Robineau-Desvoidy, 1830; Suillia arcuata Robineau-Desvoidy, 1830.

TYPE SPECIES: Suillia fungorum Robineau-Desvoidy, 1830, by subsequent designation (Coquillett, 1910: $610)$.

CURRENT STATUS: Valid genus [teste Gorodkov (1984: 34)].

FAMILY: HELEOMYZIDAE.

REMARKS: If no type material can be found for type species Suillia fungorum Robineau-Desvoidy, 1830, then the designation of a neotype should be considered to fix its identity and uphold the concept of the genus. For the present, we are accepting Gorodkov's (1984: 41) tentative suggestion of considering Suillia fungorum Robineau-Desvoidy, 1830 as a senior synonym of Helomyza variegata Loew, 1862, and we treat the former as the valid species.

518. Sylvia Robineau-Desvoidy, 1830: 636.

ORIGINALly INCLUDED SPECIES: Sylvia apicalis Robineau-Desvoidy, 1830; Sylvia punctata RobineauDesvoidy, 1830; Sylvia quatuorpunctata Robineau-Desvoidy, 1830 (as "Sylvia quatuor-punctata. R. D.”); Sylvia cinerea Robineau-Desvoidy, 1830; Sylvia mollis Robineau-Desvoidy, 1830.

TYPE SPECIES: Sylvia apicalis Robineau-Desvoidy, 1830, by subsequent designation (Coquillett, 1910: 610).

CURRENT StATUS: Preoccupied by Scopoli, 1769; junior synonym of Sapromyza Fallén, 1810 [teste Coquillett (1910: 610)]; listed as nomen dubium in Lauxaniidae [teste Papp (1984: 216)]. 
FAMILY: LAUXANIIDAE.

REMARKS: Hendel (1908: 3) designated Sapromyza pallidiventris Fallén, 1820, but this was not an originally included species. Papp (1984: 216) placed Sylvia in his list of "Nomina dubia" along with four of the five originally included species. The fifth originally included species, Sylvia quatuorpunctata RobineauDesvoidy, 1830, was omitted by Papp (1984) and has not been listed in any regional Diptera catalog.

\section{Talmonia Robineau-Desvoidy, 1863a: 704.}

ORIGINALLY INCLUDED SPECIES: Talmonia tibialis Robineau-Desvoidy, 1863.

TYPE SPECIES: Talmonia tibialis Robineau-Desvoidy, 1863 [= Tachina rubricosa Meigen, 1824], by original designation.

CURRENT STATUS: Junior synonym of Tricogena Rondani, 1856 [teste Herting (1993: 110)].

FAMILY: RHINOPHORIDAE.

520. Temesia Robineau-Desvoidy, 1863a: 292.

ORIGINALly InCludED SPECIES: Temesia obsequiosa Robineau-Desvoidy, 1863.

TYPE SPECIES: Temesia obsequiosa Robineau-Desvoidy, 1863, by original designation.

CURRENT STATUS: Preoccupied by Leach, 1852; no new replacement name proposed; placed in "Doubtful taxa in Tachinidae" by Herting \& Dely-Draskovits (1993: 438).

FAMILY: TACHINIDAE.

521. Terellia Robineau-Desvoidy, 1830: 758.

ORIGINALLY INCLUDED SPECIES: Terellia palpata Robineau-Desvoidy, 1830; Terellia luteola RobineauDesvoidy, 1830.

TYPE SPECIES: Terellia palpata Robineau-Desvoidy, 1830 [= Musca serratulae Linnaeus, 1758], by subsequent designation (Cogan \& Munro, 1980: 552).

CURRENT STATUS: Valid genus [teste Norrbom et al. (1999: 221, 222)].

FAMILY: TEPHRITIDAE.

REMARKS: Earlier type designations by Westwood (1840: 149), Desmarest in d'Orbigny (1849b: 472), Rondani (1856: 114), and Coquillett (1910: 613) are invalid because they are of species not originally included by Robineau-Desvoidy (1830).

522. Terenia Robineau-Desvoidy, 1830: 640.

ORIGINALly INCLUDED SPECIES: Terenia suillorum Robineau-Desvoidy, 1830; Terenia laticrus RobineauDesvoidy, 1830; Terenia grossipes Robineau-Desvoidy, 1830; Terenia delicatula Robineau-Desvoidy, 1830.

TYPE SPECIES: Terenia suillorum Robineau-Desvoidy, 1830, by subsequent designation (Coquillett, 1910: 613).

CURRENT STATUS: Junior synonym of Minettia Robineau-Desvoidy, 1830 [teste Sabrosky (1999: 201, 302)].

FAMILY: LAUXANIIDAE.

REMARKS: See No. 321 Minettia above for details on the type-species designations of both genus-group names and Sabrosky's (1999) action to maintain nomenclatural and taxonomic stability of Minettia.

EMENDATIONS: Terennia Schiner, 1868: 227 (unjustified), n. syn.

523. Thalpia Robineau-Desvoidy, 1863b: 169.

ORIGINAlly InCLUded SPECIES: Thalpia mera Robineau-Desvoidy, 1863; Thalpia caesia RobineauDesvoidy, 1863; Thalpia humilis Robineau-Desvoidy, 1863. 
TYPE SPECIES: Thalpia mera Robineau-Desvoidy, 1863 [= Tachina parallela Meigen, 1824], by original designation.

Current Status: Junior synonym of Bessa Robineau-Desvoidy, 1863 [teste Herting \& Dely-Draskovits (1993: 142)].

FAMILY: TACHINIDAE.

524. Thapsia Robineau-Desvoidy, 1863a: 689.

ORIGINALLY INCLUDED SPECIES: Tachina albicollis Meigen, 1824.

TYPE SPECIES: Tachina albicollis Meigen, 1824 [= Tachina laticornis Meigen, 1824], by original designation.

CURRENT STATUS: Preoccupied by Martens, 1860; junior synonym of Neaera Robineau-Desvoidy, 1830 [teste Herting \& Dely-Draskovits (1993: 320)].

FAMILY: TACHINIDAE.

525. Theano Robineau-Desvoidy, 1863a: 256.

ORIGINALLY INCLUDED SPECIES: Theano cinerea Robineau-Desvoidy, 1863.

TYPE SPECIES: Theano cinerea Robineau-Desvoidy, 1863, by original designation.

CURRENT STATUS: Placed in "Doubtful taxa in Tachinidae" by Herting \& Dely-Draskovits (1993: 438).

FAMILY: TACHINIDAE.

526. Thelaira Robineau-Desvoidy, 1830: 214 (as "Thelaïra").

ORIGINALLY INCLUDED SPECIES: Thelaira abdominalis Robineau-Desvoidy, 1830; Thelaira bifasciata Robineau-Desvoidy, 1830 (with “An Dexia bifasciata? Meig.” in doubtful synonymy).

TYPE SPECIES: Thelaira abdominalis Robineau-Desvoidy, 1830 [= Musca solivagus Harris, 1780], by subsequent designation (Townsend, 1916: 9).

CURRENT STATUS: Valid genus [teste Herting \& Dely-Draskovits (1993: 389)].

FAMILY: TACHINIDAE.

REMARKS: Dexia bifasciata Meigen, 1826 was doubtfully synonymized, so it cannot be considered as an originally included species, thus the nominal species Thelaira bifasciata is attributable to RobineauDesvoidy.

[Thelesina] Monceaux in Robineau-Desvoidy, 1863b: 535.

CURRENT STATUS: Unavailable name; proposed in synonymy with Onesia Robineau-Desvoidy, 1830 and not made available before 1961; treated under Onesia Robineau-Desvoidy, 1830 [teste this work].

FAMILY: CALLIPHORIDAE.

REMARKS: The name Thelesina appears in a footnote explicitly written by Monceaux on his own behalf, and this is why we attribute the name to him and not to Robineau-Desvoidy. Townsend (1916: 11) referred to his discussion of Marsilia to indicate his same presumptions for Thelesina. Although Townsend stated that the type species was the same as that for Onesia, he did not remove the name from synonymy with Onesia, thus Thelesina remains unavailable.

527. Thelida Robineau-Desvoidy, 1830: 655.

ORIGINALLY INCLUDED SPECIES: Thelida filiformis Robineau-Desvoidy, 1830.

TYPE SPECIES: Thelida filiformis Robineau-Desvoidy, 1830, by monotypy.

CURRENT STATUS: Junior synonym of Heteromyza Fallén, 1820 [teste Gorodkov (1984: 42)].

FAMILY: HELEOMYZIDAE. 
[Thelipus] Robineau-Desvoidy, 1830: 655.

CURRENT STATUS: Unavailable name; proposed in synonymy and not made available before 1961; treated as unplaced in Heleomyzidae [teste this work].

FAMILY: HELEOMYZIDAE.

REMARKS: Thelipus Robineau-Desvoidy, 1830 appeared in this statement under Leria peleterii: “J'en avais d'abord fait un genre sous le nom de Thelipus." By associating Thelipus with Leria peleterii, RobineauDesvoidy effectively proposed Thelipus in synonymy with Leria. Leria peleterii Robineau-Desvoidy, 1830 was treated by Gorodkov (1984: 45) in "Nomina dubia" in Heleomyzidae.

528. Themira Robineau-Desvoidy, 1830: 745.

ORIGINALly INCLUDED SPECIES: Themira pilosa Robineau-Desvoidy, 1830; Themira phantasma RobineauDesvoidy, 1830.

TYPE SPECIES: Themira pilosa Robineau-Desvoidy, 1830 [= Musca putris Linnaeus, 1758], by subsequent designation (Rondani, 1874: 170).

CURRENT STATUS: Valid genus [teste Pont \& Meier (2002: 49)].

FAMILY: SEPSIDAE.

529. Theone Robineau-Desvoidy, 1863b: 401.

ORIGINALly INCLUdED SPECIES: Theone trifaria Robineau-Desvoidy, 1863; Theone villana RobineauDesvoidy, 1863.

TYPE SPECIES: Theone trifaria Robineau-Desvoidy, 1863 [= Tachina polyodon Meigen, 1824], by subsequent designation (Townsend, 1916: 9).

CurRent STAtus: Preoccupied by Gistel, 1857; junior synonym of Macronychia Rondani, 1859 [teste Pape (1996: 94)].

FAMILY: SARCOPHAGIDAE.

530. Theresia Robineau-Desvoidy, 1830: 325.

ORIGINALLY INCLUDED SPECIES: Theresia tandrec Robineau-Desvoidy, 1830.

TYPE SPECIES: Theresia tandrec Robineau-Desvoidy, 1830 [= Musca rutilans Fabricius, 1781], by monotypy. CURRENT STATUS: Junior synonym of Billaea Robineau-Desvoidy, 1830 [teste O'Hara \& Wood (2004: 22)].

FAMILY: TACHINIDAE.

531. Theria Robineau-Desvoidy, 1830: 337.

ORIGINALly INCLUDED SPECIES: Theria palpalis Robineau-Desvoidy, 1830.

TYPE SPECIES: Theria palpalis Robineau-Desvoidy, 1830, by monotypy.

CURRENT StATUS: Preoccupied by Hübner, 1825; senior (but invalid) synonym of Eurychaeta Brauer \&

Bergenstamm, 1891 [teste Rognes (1991: 141)].

FAMILY: CALLIPHORIDAE.

532. Thyella Robineau-Desvoidy, 1863a: 183.

ORIGINALly INCLUDED SPECIES: Tachina pabulina Meigen, 1824.

TYPE SPECIES: Tachina pabulina Meigen, 1824 [= Tachina floralis Fallén, 1810], by original designation.

CURRENT StATUS: Preoccupied by Wallengren, 1858; junior synonym of Nemorilla Rondani, 1856 [teste

Herting \& Dely-Draskovits (1993: 182)].

FAMILY: TACHINIDAE. 
533. Tilesia Robineau-Desvoidy, 1863b: 364.

ORIGINALLY INCLUDED SPECIES: Tilesia frontalis Robineau-Desvoidy, 1863.

TYPE SPECIES: Tilesia frontalis Robineau-Desvoidy, 1863, by monotypy.

CURRENT STATUS: Preoccupied by Lamouroux, 1821; Swainson, 1838; no new replacement name proposed; treated as nomen dubium [teste this work].

FAMILY: SARCOPHAGIDAE.

REMARKS: Although originally proposed in our current concept of the family Tachinidae, which placement was followed by Bezzi \& Stein (1907), Herting (1974: 41) stated that the description of Tilesia places it the Miltogramminae of the Sarcophagidae, possibly because of the statement "Viviparisme constate". Until a specimen of the type species is recognized, we treat it as a nomen dubium.

534. Timavia Robineau-Desvoidy, 1863a: 257.

ORIGINALLY INCLUDED SPECIES: Smidtia flavipalpis Robineau-Desvoidy, 1848.

TYPE SPECIES: Smidtia flavipalpis Robineau-Desvoidy, 1848 [= Tachina amoena Meigen, 1824], by original designation.

CuRRent Status: Junior synonym of Smidtia Robineau-Desvoidy, 1848 [teste O'Hara \& Wood (2004: 205)].

FAMILY: TACHINIDAE.

REMARKS: Although Robineau-Desvoidy (1863a: 258) labeled the only included species as "Sp. ined." he cited "Smidtia flavipalpis : Rob. Desv." in synonymy, thus the "Sp. inedit." must have been an error that the editor Monceaux failed to correct.

535. Tlephusa Robineau-Desvoidy, 1863a: 307.

ORIGINALLY INCLUDED SPECIES: Tlephusa aurifrons Robineau-Desvoidy, 1863; Tlephusa honesta RobineauDesvoidy, 1863; Tlephusa noctuarum Robineau-Desvoidy, 1863.

TYPE SPECIES: Tlephusa aurifrons Robineau-Desvoidy, 1863, by original designation.

CuRrent Status: Valid genus [teste Herting \& Dely-Draskovits (1993: 190)].

FAMILY: TACHINIDAE.

536. Tomella Robineau-Desvoidy, 1830: 630.

ORIGINALLY INCLUDED SPECIES: Tomella guerini Robineau-Desvoidy, 1830.

TYPE SPECIES: Tomella guerini Robineau-Desvoidy, 1830, by monotypy.

CURRENT StATUS: Valid genus; treated as "tentatively placed" in Scathophagidae [teste Evenhuis (2007)].

FAMILY: SCATHOPHAGIDAE.

537. Trennia Robineau-Desvoidy, 1830: 484.

ORIGINALLY INCLUDED SPECIES: Trennia nigricornis Robineau-Desvoidy, 1830.

TYPE SPECIES: Trennia nigricornis Robineau-Desvoidy, 1830 [= Anthomyia errans Meigen, 1826], by monotypy.

CURREnT Status: Junior synonym of Phaonia Robineau-Desvoidy, 1830 [teste Pont (1986b: 116)].

FAMILY: MUSCIDAE.

538. Umbrina Robineau-Desvoidy, 1830: 649.

ORIGINALLY INCLUDED SPECIES: Odinia peleterii Robineau-Desvoidy, 1830.

TYPE SPECIES: Odinia peleterii Robineau-Desvoidy, 1830, by monotypy. 
CuRRENT STATUS: Preoccupied by Cuvier, 1816; originally proposed in synonymy with Odinia RobineauDesvoidy, 1830, but made available by subsequent adoption as a genus of Milichiidae by Scudder (1882: 348); currently incertae sedis in Acalyptratae [teste Gaimari \& Mathis (in press)].

FAMILY: UNPLACED ACALYPTRATAE.

REMARKS: Robineau-Desvoidy (1830: 649) proposed Umbrina under Odinia peleterii Robineau-Desvoidy, 1830 [unplaced Acalyptratae], which is here interpreted as a proposal in synonymy with Odinia Robineau-Desvoidy, 1830.

\section{Uramya Robineau-Desvoidy, 1830: 215.}

ORIGINALLY INCLUDED SPECIES: Uramya producta Robineau-Desvoidy, 1830.

TYPE SPECIES: Uramya producta Robineau-Desvoidy, 1830, by monotypy.

CURRENT STATUS: Valid genus [teste O'Hara \& Wood (2004: 49)].

FAMILY: TACHINIDAE.

EMEndations: Uramyia Agassiz, 1846a: 41 (unjustified), n. syn.; Uromyia Brauer \& Bergenstamm, 1893: 151 (unjustified), n. syn.

\section{Urellia Robineau-Desvoidy, 1830: 774.}

ORIGINALLY INCLUDED SPECIES: Urellia calcitrapae Robineau-Desvoidy, 1830; Urellia parisiensis Robineau-Desvoidy, 1830.

TYPE SPECIES: Urellia calcitrapae Robineau-Desvoidy, 1830 [= Musca stellata Fuessley, 1775], by subsequent designation (Foote, 1965: 666).

CURRENT STATUS: Junior synonym of Trupanea Macquart, 1827 [teste Norrbom et al. (1999: 230)].

FAMILY: TEPHRITIDAE.

REMARKS: The designation by Coquillett (1910: 618) mentioned by Norrbom et al. (1999: 230) is invalid. Coquillett (1910) did not designate Urellia calcitrapae Robineau-Desvoidy, 1830 as the type species of Urellia, but instead designated Musca stellata Fuessley, 1775, which was not an originally included species. Foote (1965: 666) listed an originally included species (Urellia calcitrapae) as the type species, and this is the first valid subsequent designation for Urellia Robineau-Desvoidy, 1830.

\section{Urophora Robineau-Desvoidy, 1830: 769.}

ORIGINALLY INCLUDED SPECIES: Musca cardui Linnaeus, 1758 (as "Tephrytis cardui. Fabr."); Urophora solstitialis Robineau-Desvoidy, 1830 (with "Musca solstitialis? Fabr." in doubtful synonymy); Urophora femoralis Robineau-Desvoidy, 1830; Urophora reaumurii Robineau-Desvoidy, 1830; Urophora liturata Robineau-Desvoidy, 1830; Urophora sonchi Robineau-Desvoidy, 1830; Musca centauri Fabricius, 1794 (as "Musca centaureae. Fabr. n. ${ }^{\circ}$ 199."); Urophora brunicornis Robineau-Desvoidy, 1830; Urophora dejeanii Robineau-Desvoidy, 1830.

TYPE SPECIES: Musca cardui Linnaeus, 1758, by subsequent designation [Westwood, 1840: 149 (as "Tephrytis cardui. Fabr.")].

CURRENT STATUS: Valid genus [teste Norrbom et al. (1999: 241)].

FAMILY: TEPHRITIDAE.

REMARKS: The listing of "Musca solstitialis ? Fabr." under Urophora solstitialis Robineau-Desvoidy, 1830 cannot be considered as an originally included species as it was doubtfully included.

[Vafrellia] Robineau-Desvoidy, 1849d: 158.

CURRENT STATUS: Unavailable name; proposed without description, indication, or included species; treated under Vafrellia Robineau-Desvoidy, 1850 [teste this work].

FAMILY: TACHINIDAE. 
542. Vafrellia Robineau-Desvoidy, 1850: 191.

ORIGINALLY INCLUDED SPECIES: Ceranthia podacina Robineau-Desvoidy, 1830 (as "Carenthia podacina, R. D.").

TYPE SPECIES: Ceranthia podacina Robineau-Desvoidy, 1830 (as "Carenthia podacina, R. D.”), by monotypy.

CURRENT STATUS: Placed in "Doubtful taxa in Tachinidae" by Herting \& Dely-Draskovits (1993: 438).

FAMILY: TACHINIDAE.

543. Vanzemia Robineau-Desvoidy, 1863a: 941.

ORIGINALLY INCLUDED SPECIES: Vanzemia flavipalpis Robineau-Desvoidy, 1863.

TYPE SPECIES: Vanzemia flavipalpis Robineau-Desvoidy, 1863 [= Tachina ocypterata Fallén, 1810], by original designation.

CURRENT STATUS: Junior synonym of Erynnia Robineau-Desvoidy, 1830 [teste Herting \& Dely-Draskovits (1993: 246)].

FAMILY: TACHINIDAE.

544. Velocia Robineau-Desvoidy, 1863a: 950.

ORIGINALly InCluded SPECIES: Velocia cursoria Robineau-Desvoidy, 1863; Velocia pavida RobineauDesvoidy, 1863.

TYPE SPECIES: Velocia cursoria Robineau-Desvoidy, 1863, by subsequent designation (Townsend, 1916: 9).

CURRENT STATUS: Junior synonym of Medina Robineau-Desvoidy, 1830 [teste Herting \& Dely-Draskovits (1993: 151)].

FAMILY: TACHINIDAE.

REMARKS: The type species Velocia cursoria is listed in "Doubtful species" of Medina by Herting \& DelyDraskovits (1993: 152).

545. Verreauxia Robineau-Desvoidy, 1863a: 893.

ORIGINALLY INCLUDED SPECIES: Verreauxia auripilis Robineau-Desvoidy, 1863.

TYPE SPECIES: Verreauxia auripilis Robineau-Desvoidy, 1863, by original designation.

CURRENT STATUS: Preoccupied by Hartlaub, 1856; junior synonym of Blepharipa Rondani, 1856 [teste Cantrell \& Crosskey (1989: 776)].

FAMILY: TACHINIDAE.

546. Vidalia Robineau-Desvoidy, 1830: 719.

ORIGINALLY INCLUDED SPECIES: Vidalia impressifrons Robineau-Desvoidy, 1830.

TYPE SPECIES: Vidalia impressifrons Robineau-Desvoidy, 1830, by monotypy.

CURRENT STATUS: Valid genus [teste Norrbom et al. (1999: 245)].

FAMILY: TEPHRITIDAE.

[Voidia] Robineau-Desvoidy MS name (Blainville et al., 1826: 10).

CURRENT STATUS: Unavailable name; suppressed by action of I.C.Z.N. (1990: 162 [Opinion 1601]); treated as a nomen dubium in Calyptratae [teste this work].

FAMILY: UNPLACED CALYPTRATAE. 
547. Volusia Robineau-Desvoidy, 1830: 674.

ORIGINALLY INCLUDED SPECIES: Volusia nitida Robineau-Desvoidy, 1830.

TYPE SPECIES: Volusia nitida Robineau-Desvoidy, 1830 [= Tanypeza longimana Fallén, 1820], by monotypy. CURRENT StATUS: Junior synonym of Tanypeza Fallén, 1820 [teste Hendel (1910: 308)].

FAMILY: TANYPEZIDAE.

\section{Voria Robineau-Desvoidy, 1830: 195.}

ORIGINALLY INCLUDED SPECIES: Voria latifrons Robineau-Desvoidy, 1830.

TYPE SPECIES: Voria latifrons Robineau-Desvoidy, 1830 [= Tachina ruralis Fallén, 1810], by monotypy.

CurRent Status: Valid genus [teste Herting \& Dely-Draskovits (1993: 383)].

FAMILY: TACHINIDAE.

\section{Wagneria Robineau-Desvoidy, 1830: 126.}

ORIGINALLY INCLUDED SPECIES: Wagneria gagatea Robineau-Desvoidy, 1830.

TYPE SPECIES: Wagneria gagatea Robineau-Desvoidy, 1830, by monotypy.

CURRENT STATUS: Valid genus [teste Herting \& Dely-Draskovits (1993: 380)].

FAMILY: TACHINIDAE.

550. Walkeria Robineau-Desvoidy, 1863a: 995 (as "Walkéria").

ORIGINALLY INCLUDED SPECIES: Walkeria lauta Robineau-Desvoidy, 1863; Tachina claripennis RobineauDesvoidy, 1830; Walkeria anceps Robineau-Desvoidy, 1863; Walkeria musca Robineau-Desvoidy, 1863; Walkeria cyanescens Robineau-Desvoidy, 1863; Walkeria contraria Robineau-Desvoidy, 1863; Walkeria contenta Robineau-Desvoidy, 1863; Tachina festiva Robineau-Desvoidy, 1830; Walkeria lateralis Robineau-Desvoidy, 1863; Tachina aurifrons Robineau-Desvoidy, 1830; Tachina albida Robineau-Desvoidy, 1830; Tachina campestris Robineau-Desvoidy, 1830; Walkeria exilis RobineauDesvoidy, 1863; Walkeria flavicans Robineau-Desvoidy, 1863; Walkeria jejuna Robineau-Desvoidy, 1863; Walkeria scutellaris Robineau-Desvoidy, 1863; Walkeria hilaris Robineau-Desvoidy, 1863; Tachina diversa Robineau-Desvoidy, 1830; Walkeria laevis Robineau-Desvoidy, 1863; Walkeria regula Robineau-Desvoidy, 1863; Walkeria cunctata Robineau-Desvoidy, 1863; Walkeria monita RobineauDesvoidy, 1863; Walkeria hilarella Robineau-Desvoidy, 1863; Walkeria compar Robineau-Desvoidy, 1863; Tachina atrata Robineau-Desvoidy, 1830; Walkeria emissa Robineau-Desvoidy, 1863; Walkeria ardeacea Robineau-Desvoidy, 1863; Walkeria munda Robineau-Desvoidy, 1863; Tachina florum Robineau-Desvoidy, 1830; Walkeria cita Robineau-Desvoidy, 1863; Walkeria pauperata RobineauDesvoidy, 1863; Walkeria limpida Robineau-Desvoidy, 1863; Walkeria misera Robineau-Desvoidy, 1863; Tachina distincta Robineau-Desvoidy, 1830; Walkeria insidiosa Robineau-Desvoidy, 1863; Walkeria agrorum Robineau-Desvoidy, 1863; Walkeria gentilis Robineau-Desvoidy, 1863; Walkeria ventralis Robineau-Desvoidy, 1863; Walkeria vivida Robineau-Desvoidy, 1863; Walkeria blandula Robineau-Desvoidy, 1863; Walkeria delicatula Robineau-Desvoidy, 1863; Tachina aurea RobineauDesvoidy, 1830; Walkeria fausta Robineau-Desvoidy, 1863; Walkeria discreta Robineau-Desvoidy, 1863; Musca larvarum Linnaeus, 1758; Walkeria grossoria Robineau-Desvoidy, 1863; Walkeria quieta Robineau-Desvoidy, 1863; Tachina abdominalis Robineau-Desvoidy, 1830; Walkeria pacifica Robineau-Desvoidy, 1863; Walkeria fulvicornis Robineau-Desvoidy, 1863.

TYPE SPECIES: Walkeria lauta Robineau-Desvoidy, 1863 [= Tachina rustica Fallén, 1810], by subsequent designation (Townsend, 1916: 9).

CURRENT StATUS: Preoccupied by Fleming, 1823; junior synonym of Adenia Robineau-Desvoidy, 1863 (subgenus of Exorista Meigen, 1803) [teste Herting (1984: 8)].

FAMILY: TACHINIDAE. 
551. Weberia Robineau-Desvoidy, 1830: 233.

ORIGINALLY INCLUDED SPECIES: Weberia appendiculata Robineau-Desvoidy, 1830.

TYPE SPECIES: Weberia appendiculata Robineau-Desvoidy, 1830 [= Tachina digramma Meigen, 1824], by monotypy.

CURRENT STATUS: Valid genus [teste Herting \& Dely-Draskovits (1993: 423)].

FAMILY: TACHINIDAE.

552. Westwodia Robineau-Desvoidy, 1863a: 940.

ORIGINALLY INCLUDED SPECIES: Westwodia atra Robineau-Desvoidy, 1863; Westwodia flavisquamis Robineau-Desvoidy, 1863.

TYPE SPECIES: Westwodia atra Robineau-Desvoidy, 1863 [= Tachina morio Fallén, 1810], by original designation.

CURRENT Status: Junior synonym of Elodia Robineau-Desvoidy, 1863 [teste Herting \& Dely-Draskovits (1993: 247; as "Westwoodia")].

FAMILY: TACHINIDAE.

REMARKS: There is no evidence in Robineau-Desvoidy (1863a) that the spelling of his Westwodia is "an inadvertent error", which means that this is the correct original spelling. This genus-group name is therefore not preoccupied by Westwoodia Brullé, 1846 or Westwoodia Bate, 1857. Subsequent workers have commonly misspelled the Robineau-Desvoidy name as "Westwoodia" thereby leading to the errant assumption that it is preoccupied. However, the orthography in Herting (1974: 26) is an intentional (although unjustified) emendation.

EMENDATIONS: Westwoodia Herting, 1974: 26 (unjustified), n. syn.

553. Wiedmania Robineau-Desvoidy, 1863a: 928.

ORIGINALLY INCLUDED SPECIES: Wiedmania hirtella Robineau-Desvoidy, 1863.

TYPE SPECIES: Wiedmania hirtella Robineau-Desvoidy, 1863 [= Tachina innoxia Meigen, 1824], by monotypy.

CURRENT STATUS: Junior synonym of Leiophora Robineau-Desvoidy, 1863 [teste Herting \& Dely-Draskovits (1993: 159)].

FAMILY: TACHINIDAE.

REMARKS: There is no evidence in Robineau-Desvoidy (1863a) that the spelling of his Wiedmania is "an inadvertent error", which means that this is the correct original spelling. Herting \& Dely-Draskovits (1993: 159) attributed this genus-group name to Robineau-Desvoidy, 1863 but considered it a "misidentification and misspelling of Wiedemannia, MEIGEN, 1838".

\section{Winthemia Robineau-Desvoidy, 1830: 173.}

ORIGINALLY INCLUDED SPECIES: Winthemia ciligera Robineau-Desvoidy, 1830; Tachina variegata Meigen, 1824 (as "Musca variegata. Fabr. Tachina variegata. Fall. Meig."); Winthemia flavescens RobineauDesvoidy, 1830; Winthemia catocalae Robineau-Desvoidy, 1830; Winthemia nobilis RobineauDesvoidy, 1830; Winthemia viarum Robineau-Desvoidy, 1830; Musca quadripustulata Fabricius, 1794; Winthemia vinulae Robineau-Desvoidy, 1830.

TYPE SPECIES: Musca quadripustulata Fabricius, 1794, by subsequent designation [Desmarest in d'Orbigny, 1849c: 301 (as “W. quadripustulata Rob.-Desv., Fabr., Pall., Meig.”)].

CURRENT STATUS: Valid genus [teste O'Hara \& Wood (2004: 205)].

FAMILY: TACHINIDAE. 
555. Xyphosia Robineau-Desvoidy, 1830: 762.

ORIGINALLY INCLUDED SPECIES: Xyphosia cirsiorum Robineau-Desvoidy, 1830; Xyphosia lappae RobineauDesvoidy, 1830; Xyphosia arvensis Robineau-Desvoidy, 1830.

TYPE SPECIES: Xyphosia cirsiorum Robineau-Desvoidy, 1830 [= Musca miliaria Schrank, 1781], by subsequent designation (Desmarest in d'Orbigny, 1849d: 332).

CURRENT STATUS: Valid genus [teste Norrbom et al. (1999: 248)].

FAMILY: TEPHRITIDAE.

EMENDATIONS: Xiphosia Agassiz, 1846a: 41 (unjustified), n. syn.

556. Zabia Robineau-Desvoidy, 1830: 600.

ORIGINALLY INCLUDED SPECIES: Zabia longipes Robineau-Desvoidy, 1830.

TYPE SPECIES: Zabia longipes Robineau-Desvoidy, 1830 [= Anthomyia bicolor Wiedemann, 1817], by monotypy.

CURRENT Status: Junior synonym of Pegomya Robineau-Desvoidy, 1830 [teste Dely-Draskovits (1993: 77)].

FAMILY: ANTHOMYIIDAE.

557. Zaida Robineau-Desvoidy, 1830: 150 (as "Zaïda").

ORIGINAlly INCLUDED SPECIES: Zaida agilis Robineau-Desvoidy, 1830; Zaida crataegellae RobineauDesvoidy, 1830; Zaida falculae Robineau-Desvoidy, 1830.

TYPE SPECIES: Zaida agilis Robineau-Desvoidy, 1830, by subsequent designation (Townsend, 1916: 9).

CURRENT STATUS: Junior synonym of Meigenia Robineau-Desvoidy, 1830 [teste Herting \& Dely-Draskovits (1993: 145)].

FAMILY: TACHINIDAE.

REMARKS: The type species Zaida agilis is listed in "Doubtful species" of Meigenia by Herting \& DelyDraskovits (1993: 147).

558. Zaira Robineau-Desvoidy, 1830: 150 (as “Zaïra").

ORIGINALLY INCLUDED SPECIES: Zaira agrestis Robineau-Desvoidy, 1830.

TYPE SPECIES: Zaira agrestis Robineau-Desvoidy, 1830 [= Tachina cinerea Fallén, 1810], by monotypy.

CURRENT STATUS: Valid genus [teste Herting \& Dely-Draskovits (1993: 148)].

FAMILY: TACHINIDAE.

559. Zaphne Robineau-Desvoidy, 1830: 527.

ORIGINALLY INCLUDED SPECIES: Zaphne hylemyoidea Robineau-Desvoidy, 1830 (as "Zaphne hylemyoüdea.

R. D.”); Zaphne egerioidea Robineau-Desvoidy, 1830 (as “Zaphne egeriö̈dea. R. D.”).

TYPE SPECIES: Zaphne hylemyoidea Robineau-Desvoidy, 1830 [= Anthomyia divisa Meigen, 1826], by subsequent designation (Hennig, 1969: 251).

CuRrent Status: Valid genus [teste Dely-Draskovits (1993: 96)].

FAMILY: ANTHOMYIIDAE.

\section{Zelia Robineau-Desvoidy, 1830: 314.}

ORIGINALly INCLUDED SPECIES: Zelia rostrata Robineau-Desvoidy, 1830; Zelia strenua RobineauDesvoidy, 1830; Zelia analis Robineau-Desvoidy, 1830; Zelia apicalis Robineau-Desvoidy, 1830; Zelia velox Robineau-Desvoidy, 1830. 
TYPE SPECIES: Zelia rostrata Robineau-Desvoidy, 1830 [= Dexia vertebrata Say, 1829], by subsequent designation (Coquillett, 1910: 621).

CURRENT STATUS: Valid genus [teste O'Hara \& Wood (2004: 37)].

FAMILY: TACHINIDAE.

561. Zelinda Robineau-Desvoidy, 1863a: 485.

ORIGINALLY INCLUDED SPECIES: Zelinda aurulenta Robineau-Desvoidy, 1863.

TYPE SPECIES: Zelinda aurulenta Robineau-Desvoidy, 1863, by original designation.

CURRENT STATUS: Placed in "Doubtful taxa in Tachinidae" by Herting \& Dely-Draskovits (1993: 438).

FAMILY: TACHINIDAE.

562. Zelleria Robineau-Desvoidy, 1863a: 984.

ORIGINALly InCluded SPECIES: Zelleria jocax Robineau-Desvoidy, 1863; Zelleria verax RobineauDesvoidy, 1863; Zelleria rapax Robineau-Desvoidy, 1863; Zelleria capax Robineau-Desvoidy, 1863; Zelleria nugax Robineau-Desvoidy, 1863; Zelleria minax Robineau-Desvoidy, 1863; Zelleria pugnax Robineau-Desvoidy, 1863; Zelleria tenax Robineau-Desvoidy, 1863; Zelleria fugax Robineau-Desvoidy, 1863; Zelleria fallax Robineau-Desvoidy, 1863; Zelleria procax Robineau-Desvoidy, 1863; Zelleria ferax Robineau-Desvoidy, 1863; Zelleria vivax Robineau-Desvoidy, 1863; Zelleria audax RobineauDesvoidy, 1863; Zelleria sagax Robineau-Desvoidy, 1863; Zelleria sequax Robineau-Desvoidy, 1863; Zelleria mendax Robineau-Desvoidy, 1863; Zelleria edax Robineau-Desvoidy, 1863; Zelleria valida Robineau-Desvoidy, 1863.

TYPE SPECIES: Zelleria verax Robineau-Desvoidy, 1863 [= Tachina mimula Meigen, 1824], by original designation.

CURRENT STATUS: Preoccupied by Stainton, 1849; Duchassaing, 1850; Egger, 1856; junior synonym of Adenia Robineau-Desvoidy, 1863 (subgenus of Exorista Meigen, 1803) [teste Herting (1984: 8)].

FAMILY: TACHINIDAE.

563. Zenais Robineau-Desvoidy, 1830: 148 (as "Zenaïs").

ORIGINALLY INCLUDED SPECIES: Zenais silvestris Robineau-Desvoidy, 1830; Zenais fera RobineauDesvoidy, 1830; Zenais sicula Robineau-Desvoidy, 1830.

TYPE SPECIES: Zenais silvestris Robineau-Desvoidy, 1830, by subsequent designation (Desmarest in d'Orbigny, 1849d: 346).

CURRENT STATUS: Nomen dubium [teste this work].

FAMILY: TACHINIDAE.

REMARKS: Robineau-Desvoidy (1863a: 912) designated Zenais fera Robineau-Desvoidy, 1830 as type species, but this was later. Herting \& Dely-Draskovits (1993: 145) listed Zenais Robineau-Desvoidy, 1830 as a synonym of Meigenia Robineau-Desvoidy, 1830 (with Zenais fera as type species), but placed Zenais silvestris Robineau-Desvoidy, 1830 in "Doubtful taxa in Tachinidae" (page 456).

\section{Zenillia Robineau-Desvoidy, 1830: 152.}

ORIGINALly INCLUDED SPECIES: Zenillia lepida Robineau-Desvoidy, 1830; Musca libatrix Panzer, 1798 (as

"Musca libatrix. Fabr. Tachina libatrix. Meig."); Zenillia ciligera Robineau-Desvoidy, 1830.

TYPE SPECIES: Musca libatrix Panzer, 1798, by subsequent designation (Robineau-Desvoidy, 1863a: 471).

CURRENT STATUS: Valid genus [teste Herting \& Dely-Draskovits (1993: 229)].

FAMILY: TACHINIDAE. 
565. Zetterstedtia Robineau-Desvoidy, 1863a: 1024.

ORIGINALLY INCLUDED SPECIES: Zetterstedtia albifrons Robineau-Desvoidy, 1863; Zetterstedtia sordida Robineau-Desvoidy, 1863; Zetterstedtia fulvida Robineau-Desvoidy, 1863; Zetterstedtia pumila Robineau-Desvoidy, 1863; Zetterstedtia vernalis Robineau-Desvoidy, 1863; Zetterstedtia amica Robineau-Desvoidy, 1863; Zetterstedtia nitens Robineau-Desvoidy, 1863; Zetterstedtia spinulosa Robineau-Desvoidy, 1863; Tachina germana Robineau-Desvoidy, 1830; Zetterstedtia volucris Robineau-Desvoidy, 1863; Zetterstedtia facialis Robineau-Desvoidy, 1863; Zetterstedtia inanis Robineau-Desvoidy, 1863; Zetterstedtia caesia Robineau-Desvoidy, 1863; Tachina diaphanipennis Robineau-Desvoidy, 1830 (with "Tachina rustica : Rob. Desv.", "Tachina ruralis : Fall." and "Tachina larvarum: Meig." in synonymy).

TYPE SPECIES: Tachina germana Robineau-Desvoidy, 1830 [= Tachina rustica Fallén, 1810], by original designation.

Current Status: Preoccupied by Rondani, 1845; junior synonym of Adenia Robineau-Desvoidy, 1863 (subgenus of Exorista Meigen, 1803) [teste Herting (1984: 8)].

FAMILY: TACHINIDAE.

566. Zorella Robineau-Desvoidy, 1863a: 918

ORIGINALly INCLUDED SPECIES: Zorella pavida Robineau-Desvoidy, 1863.

TYPE SPECIES: Zorella pavida Robineau-Desvoidy, 1863 [= Tachina stabulans Meigen, 1824], by monotypy. CuRRENT Status: Junior synonym of Lydella Robineau-Desvoidy, 1863 [teste Herting \& Dely-Draskovits (1993: 203)].

FAMILY: TACHINIDAE. 


\section{Diptera Genus-Group Names Incorrectly Attributed to Robineau-Desvoidy}

The following names have been found in two nomenclators and one article as attributed to RobineauDesvoidy, but a thorough search has failed to find them among his publications. The ones listed by Brullé (1831) may have derived from manuscript names that Robineau-Desvoidy intended to use in his 1830 work but did not.

[Antidulina]

SOURCE: Brullé (1831: 267).

CURRENT STATUS: Unavailable name; proposed without description, indication, or included species.

[Chiomyza]

SOURCE: Agassiz (1846a: 8).

CURRENT STATUS: Unavailable name; proposed without description, indication, or included species.

[Corella]

SOURCE: Brullé (1831: 267).

CURRENT STATUS: Unavailable name; proposed without description, indication, or included species.

[Hypogaea]

SOURCE: Agassiz (1846a: 19).

CURRENT STATUS: Unavailable name; proposed without description, indication, or included species.

[Malacomyia]

SOURCE: Agassiz (1846a: 22).

CURRENT STATUS: Unavailable name; proposed without description, indication, or included species.

[Ocyptera]

SOURCE: Robineau-Desvoidy (1830: 229).

CURRENT STATUS: Error for Ocyptera Latreille, 1804.

FAMILY: TACHINIDAE.

REMARKS: Robineau-Desvoidy (1830: 229) wrote "Ocyptera. R. D." for the genus. This has been interpreted as an error for Ocyptera Latreille, 1804 by subsequent authors, and we concur.

[Phyllodromya]

SOURCE: Verrall in Scudder (1882: 262).

CURRENT STATUS: Unavailable name; proposed without description, indication, or included species.

REMARKS: Stated by Scudder (1882) to date from Robineau-Desvoidy (1830) and to be an emendation of Phyllodromia Zetterstedt, 1837 [Empididae], which is clearly impossible from a chronological standpoint, but also because Robineau-Desvoidy did not work on Empididae.

[Phyllophaga]

SOURCE: Agassiz (1846a: 31). 
CURRENT STATUS: Unavailable name; proposed without description, indication, or included species.

[Plumosia]

SOURCE: Agassiz (1846a: 32).

CURRENT STATUS: Unavailable name; proposed without description, indication, or included species.

[Scintillia]

SOURCE: Brullé (1831: 267).

CURRENT STATUS: Unavailable name; proposed without description, indication, or included species.

[Sphaera]

SOURCE: Agassiz (1846a: 36).

CURRENT STATUS: Unavailable name; proposed without description, indication, or included species. 
Format of typeface of families below follows that of the catalog.

Anthomyinde: Adia, Anthomya, Chloe, Chlorina, Delia, Egeria, Egle, Fucellia, [Gymnodia], Hydrophoria, Hylemya, Leucophora, Myopina, Nerina, Pegomya, Phoraea, Phorbia, Zabia, Zaphne

CAlliphoridae: Amenia, Bellardia, Bengalia, [Calliphora], Calliphora, Cephysa, [Chrysomya], Chrysomya, Cynomya, Lucilia, [Marsilia], Melinda, Moretia, Morinia, Mufetia, Nitellia, Onesia, Orizia, Phaenicia, [Phoenicia], Phormia, Phumosia, [Pollenia], Pollenia, [Thelesina], Theria

CHAMAEMYIIDAE: Estelia

Chyromyidae: Chyromya, Lisella, Scyphella

Conopidae: Dalmania, Dalmannia, [Fairemairia], Fairmairia, Haustellia, [Heterella], Lonchopalpus,

Melanosoma, Myopella, Myopina, Occemya, Phorosia, Pictinia, Purpurella

Culicidae: Megarhinus, Psorophora, Sabethes

DROSOPHILIDAE: Bacchis

DRYOMYZIDAE: Dryope

EPHYDRIDAE: Coenia, Dryxo, Hydrellia, Hydrina, Keratocera, Napaea, Saphaea, Scatella

FANNIIDAE: Aminta, Fannia, Philinta

GLOSSINIDAE: Nemorhina

Heleomyzidae: Dichromya, Herbina, Lentiphora, Leria, Olina, Orbellia, Suillia, Thelida, [Thelipus]

LAUXANIIDAE: Lycia, Minettia, Sylvia, Terenia

MICROPEZIDAE: Phantasma

Muscidae: Alina, Aricia, Azelia, [Biomye], Blainvillia, Blissonia, Byomya, Camilla, Caricea, Cuculla,

[Cymnodia], Dasyphora, Eginia, Euphemia, Euphoria, Fellaea, Graphomya, Gymnodia, Haematobia,

Helina, Hydrotaea, Limnophora, Limosia, Macrosoma, Morellia, Muscina, Mydaea, Mydina, Ophyra, Orthellia, Palusia, Peronia, Phaonia, Phyllis, Plaxemya, Potamia, Priophora, Pyrellia,

Rohrella, Sphora, Stagnia, [Stygia], Trennia

NERIIDAE: Neria

OESTRIDAE: Cephalemya, Cephenemya

ODINIIDAE: Odinia

Platystomatidae: Boisduvalia, Hesyquillia, Palpomya, Polystodes, Rivellia

PSILIDAE: Dasyna, Oblicia

RHINIIDAE: Beria, Cosmina, Rhinia, Rhyncomya

Rhinophoridae: Clytho, Illigeria, Kockia, Paykullia, [Phyto], Phyto, Rhinophora, Stevenia, Talmonia

RICHARDIIDAE: Chlorophora, Richardia, Setellia

Sarcophagidae: [Aebalia], Agria, Amobia, Anicia, Araba, Arabella, Argyrella, Argyria, Bellieria,

Bercaea, Calyptia, Conomya, Cytoria, Elpigia, Erichsonia, Gesneria, Hamulia, Hartigia, Listeria,

Maculia, Megaera, Megerlea, Misellia, Moschusa, Mulsantia, Myophora, [Myophore], Myorhina,

Nyctia, Oebalia, Ophelia, Oppia, Oresbia, Peckia, Phorella, [Phrosina], Phrosinella, Pierretia,

Pterella, Ravinia, Scaligeria, Servaisia, Setulia, Stygina, Theone, Tilesia

Scathophagidae: Amina, Delina, Mosina, Norellia, Nupharia, Phrosia, Sargella, Scatina, Tomella

SciomyZidae: Arina, Chetocera, Chione, Cylidria, Dyctia, [Gymnomya], Hydromya, Limnia, Melina,

Pherbellia, Pherbina, Retellia, Salticella

SEPSIDAE: Nemopoda, Saltella, Themira

SPHAERoCERIDAE: Coprina, Fimetia, Lordatia, Mycetia, Nerea, Scatophora

Tachinidae: Acemya, Actia, Adenia, [Adenia], Aetylia, [Afrella], Afrellia, Afzelia, Ahrensia, Albinia,

Alophora, Amedea, Amesia, Amphisa, Amyclaea, Andrina, Anemya, Anetia, Aphria, Aplomya, Arenia, 
Arge, Aria, Arisbaea, [Arisbea], Arraltia, Asbella, Ateria, Athrycia, Atilia, Atrania, Aubaea, Aubaea, Bebricia, Belida, Bellina, Belvosia, Beraldia, Bessa, Besseria, Bigotia, Billaea, Bithia, Blondelia, Blumia, Bohemania, Bonellia, Bonnetia, Brachelia, Bremia, Brullaea, [Buquetia], Buquetia, Caenis, [Calyptia], Calyptidia, [Carbonaria], Carbonia, Carcelia, Catilia, Celea, Ceranthia, Ceromya, Cerophora, [Chariclaea], Chariclea, Chremia, Chryseria, Clairvillia, Clelia, Clemelis, Cleodora, Cleonice, Clytia, Cnossia, Crameria, Curtisia, Cynisca, Cynthia, Cyrillia, Cyzenis, Damonia, Dejeania, Dinera, Dionaea, [Dorbinia], Dorbinia, Drino, Dufouria, Dumerillia, Duponchelia, Duvaucelia, Edesia, Edomya, Elbaea, [Elboea], Eleone, [Elfia], Elfia, Eloceria, Elodia, Elomya, Elophoria, Elpe, Enthenis, Eperia, Ephyra, Eratia, Erebia, Eretria, Eribea, Erigone, Erinia, Ernestia, Ervia, Erycia, Erynnia, Eryphe, Erytaea, [Erythrocera], Erythrocera, Esila, Essenia, Estheria, Etheria, Ethilla, Eudora, Eugenia, Eumea, Eurithia, Euryclea, Eurysthaea, [Eurythia], Eversmania, [Evesrmania], Fabricia, Faedoria, Faunia, Faurella, Fausta, Feburia, Feria, Fischeria, [Foedoria], Freraea, Futilia, Gaedartia, Gaubilia, Germaria, Gervaisia, Gimmenthalia, Gouraldia, Gravenhorstia, Guerinia, Gymnocheta, [Haematomyza], Halesa, Harrisia, Haydaea, Hebia, Hemithaea, Hemyda, Herbstia, Herilla, Hermya, Hersilia, Hesione, Himera, Hubertia, Hubneria, Hyalomya, Hyperaea, Hyria, Icelia, Ida, Ilaesa, Ismenia, Isomera, Javetia, Jurinia, Kirbya, Klugia, Lalage, Lambertia, Latreillia, Leiophora, Leschenaultia, Leskia, Lespesia, Ligeria, Lilaea, Linnaemya, Loevia, Lucasia, Lupia, Lydella, Lydina, Lylibaea, [Lyliboea], Lypha, Lythia, Macquartia, Macromya, Marshamia, Medina, Medoria, Meigenia, [Melania], [Melanophora], Melia, Melibaea, Meriania, Mericia, Microptera, Minella, Mintho, Mollia, Myconia, Myobia, Myocera, Myostoma, Myrsina, Neaera, Nemoraea, Nicaea, [Nigria], Nigrina, Nilea, Novia, Obeida, Ocalea, Olbya, Olinda, Olivieria, Opesia, Ophina, Oppia, Oria, Orillia, Ormia, Osmaea, Osmina, Oswaldia, Pales, Pallasia, Palpostoma, Panzeria, Parthenia, Peleteria, Peribaea, Phaedima, Phanemya, [Phebellia], Phebellia, Phegea, Phenicellia, Pherecida, Phericia, Philea, Pholoe, Phorcida, Phorinia, Phorocera, [Phorophylla], Phorophylla, Phorostoma, Phryno, Phryxe, Phyllomya, Picconia, Pissemya, Pitthaea, Platymya, Ptilocera, Ptilopsis, Ramburia, Ramonda, Reaumuria, Rhedia, Rhinomya, Roeselia, Rondania, [Roudania], Rutilia, Sagaris, Salia, Schaumia, Scopolia, Scotia, Servillia, Silbermania, Sironia, Smidtia, [Soleria], [Solieria], Solieria, Sophia, Spallanzania, Spinolia, Staegeria, Stephania, [Stephenia], Stephensia, Stomina, Sturmia, Temesia, Thalpia, Thapsia, Theano, Thelaira, Theresia, Thyella, Timavia, Tlephusa, Uramya, [Vafrellia], Vafrellia, Vanzemia, Velocia, Verreauxia, Voria, Wagneria, Walkeria, Weberia, Westwodia, Wiedmania, Winthemia, Zaida, Zaira,

Zelia, Zelinda, Zelleria, Zenais, Zenillia, Zetterstedtia, Zorella

TANYPEZIDAE: Volusia

TePhritidae: Acidia, Acinia, Aciura, [Elaïmya], Ensina, Forellia, Noeeta, Orellia, Oxyna, Oxyphora, Sitarea, Sphenella, Strauzia, Stylia, Terellia, Urellia, Urophora, Vidalia, Xyphosia

UlididDAE: Blainvillia, Delphinia, Heramya, Herina, Meckelia, Melieria, Myennis, Myodina, Myoris, Myrmecomya, Prionella

UnPLACED ACALYPTRATAE: Clidonia, Stylophora, Umbrina

UNPLACED CALYPTRATAE: [Voidia] 


\section{Index of Diptera Species-Group Names Proposed by Robineau-Desvoidy}

Date letters correspond to the date-letter combination in the references below.

abdominalis, Aphria, 1830: 89

abdominalis, Aplomya, 1863a: 461

abdominalis, Blondelia, 1830: 122

abdominalis, Ceromya, 1830: 87

abdominalis, Elomya, 1830: 297

abdominalis, Estheria, 1830: 306

abdominalis, Fausta, 1830: 63

abdominalis, Hyperaea, 1863b: 380

abdominalis, Myophora, 1830: 354

abdominalis, Peleteria, 1830: 41

abdominalis, Phumosia, 1830: 427

abdominalis, Tachina, 1830: 190

abdominalis, Tephrytis, 1830: 768

abdominalis, Thelaira, 1830: 215

acetosae, Pegomya, 1851c: 232

acronita, Hubneria, 1850a: 167

activa, Phryxe, 1863a: 352

aculeata, Stomoxis, 1830: 386

acuta, Palusia, 1830: 543

adamantina, Euphoria, 1863b: 836

adspersa, Opesia, 1863b: 277

aenea, Euphoria, 1863b: 803

aenea, Hyalomya, 1863b: 257

aenescens, Morellia, 1830: 406

aestiva, Phryxe, 1863a: 335 [as “oestiva" 1863b: 902]

aestivalis, Erichsonia, 1863b: 490

aestivalis, Euphoria, 1863b: 820

aestivalis, Ismenia, 1863a: 585

aestivalis, Linnaemya, 1830: 54

aestivalis, Meigenia, 1863a: 1079

aestivalis, Myobia, 1863b: 308

aestivalis, Myophora, 1830: 344

aestivalis, Phaedima, 1863a: 843

aestivalis, Phebellia, 1846b: 38

aestivalis, Pierretia, 1863b: 422

aestivalis, Platymya, 1830: 117

aestivalis, Staegeria, 1863a: 978

aestuans, Lucilia, 1863b: 740

aestuans, Phaenicia, 1863b: 763

affinis, Chrysomya, 1830: 445

affinis, Lucilia, 1863b: 735

affinis, Myophora, 1830: 356

affinis, Nemoraea, 1830: 72

affinis, Tachina, 1830: 193

afra, Hermya, 1830: 227

afra, Sarcophaga, 1863b: 464

afra, Setellia, 1830: 733

agilis, Azelia, 1830: 594

agilis, Bercaea, 1863b: 552 agilis, Clelia, 1830: 255

agilis, Egle, 1830: 585

agilis, Eretria, 1863a: 497

agilis, Euphoria, 1863b: 809

agilis, Fellaea, 1830: 478

agilis, Lucilia, 1863b: 723

agilis, Medoria, 1830: 266

agilis, Meigenia, 1863a: 1074

agilis, Morellia, 1830: 405

agilis, Myophora, 1830: 343

agilis, Ophelia, 1830: 120

agilis, Phorocera, 1830: 132

agilis, Phryno, 1830: 143

agilis, Phryxe, 1830: 167

agilis, Pollenia, 1830: 415

agilis, Sarcophaga, 1863b: 455

agilis, Zaida, 1830: 151

agnita, Phryxe, 1863a: 425

agraria, Bercaea, 1863b: 554

agraria, Myophora, 1863b: 515

agraria, Pherecida, 1863a: 1120

agrestis, Bonellia, 1863a: 138

agrestis, Edomya, 1863a: 841

agrestis, Gesneria, 1830: 382

agrestis, Hylemya, 1830: 555

agrestis, Limosia, 1830: 537

agrestis, Listeria, 1863b: 600

agrestis, Lydella, 1830: 113

agrestis, Phasia, 1830: 292

agrestis, Roeselia, 1830: 146

agrestis, Zaira, 1830: 150

agricola, Delia, 1830: 573

agricola, Myophora, 1830: 353

agricola, Phasia, 1863b: 221

agricola, Phryxe, 1863a: 398

agrorum, Exorista, 1863a: 262

agrorum, Walkeria, 1863a: 1013

albibarbis, Oppia, 1863a: 312

albicans, Eperia, 1863a: 316

albicans, Myophora, 1830: 345

albiceps, Eribea, 1863a: 1036

albiceps, Erigone, 1863a: 154

albiceps, Melinda, 1830: 440

albida, Dinera, 1863b: 355

albida, Limosia, 1830: 538

albida, Mulsantia, 1863b: 567

albida, Phryxe, 1863a: 440

albida, Sarcophaga, 1863b: 446

albida, Tachina, 1830: 192 
albidipennis, Myophora, 1830: 358

albidula, Limosia, 1830: 537

albidula, Myophora, 1863b: 513

albifacies, Phasia, 1830: 293

albifrons, Clemelis, 1863a: 483

albifrons, Eretria, 1863a: 493

albifrons, Gesneria, 1830: 381

albifrons, Latreillia, 1830: 105

albifrons, Phasia, 1863b: 225

albifrons, Zetterstedtia, 1863a: 1024

albipennis, Delia, 1830: 581

albipennis, Melia, 1830: 102

albipennis, Nerina, 1830: 557

albiseta, Klugia, 1863a: 789

albisquamis, Myobia, 1863b: 312

algira, Peleteria, 1863a: 621

alia, Chrysomya, 1830: 447

alismatis, Limosia, 1830: 540

amaena, Lilaea, 1863b: 163

amaena, Phaenicia, 1863b: 762 [as “amoena” 1863b: 900]

amaena, Pyrellia, 1863b: 841

ambigua, Phryxe, 1863a: 390

ambulans, Atilia, 1863a: 477

ambulatoria, Sarcophaga, 1863b: 471

ambulatrix, Erichsonia, 1863b: 486

ambulatrix, Myrsina, 1863b: 158

americana, Graphomya, 1830: 404

amica, Lucilia, 1830: 453

amica, Zetterstedtia, 1863a: 1027

amoena, Carcelia, 1830: 179

amphion, Carcelia, 1863a: 237

anacantha, Oebalia, 1863b: 415

analis, Caricea, 1830: 532

analis, Euphoria, 1863b: 816

analis, Limnophora, 1830: 519

analis, Linnaemya, 1830: 54

analis, Macromya, 1830: 322

analis, Marshamia, 1830: 58

analis, Mydina, 1830: 496

analis, Zelia, 1830: 315

anceps, Bercaea, 1863b: 557

anceps, Phryxe, 1863a: 342

anceps, Walkeria, 1863a: 996

ancilla, Cleodora, 1863a: 1047

ancilla, Phryxe, 1863a: 403

andana, Jurinia, 1863a: 657

angustata, Myophora, 1863b: 506

anilis, Phryxe, 1863a: 455

annulata, Acinia, 1830: 777

annulipes, Myopa, 1830: 246

anthophila, Delia, 1830: 580

anthophila, Egle, 1830: 586

anthophila, Erigone, 1830: 66 anthophila, Myocera, 1830: 330

anthropophaga, Thyreophora, 1830: 623

antiopis, Phorocera, 1830: 134

anxia, Erichsonia, 1863b: 493

anxia, Phryxe, 1863a: 415

aperta, Dufouria, 1830: 258

apicalis, Carcelia, 1851a: 148

apicalis, Hubneria, 1848a: 605

apicalis, Hyalomya, 1863b: 246

apicalis, Myocera, 1863b: 394

apicalis, Peribaea, 1863a: 721

apicalis, Phorocera, 1863a: 513

apicalis, Solieria, 1863b: 321

apicalis, Sylvia, 1830: 636

apicalis, Zelia, 1830: 316

apicata, Himera, 1863a: 1125

appellata, Phryxe, 1863a: 391

appendiculata, Essenia, 1863a: 194

appendiculata, Myconia, 1863a: 307

appendiculata, Phryxe, 1863a: 340

appendiculata, Weberia, 1830: 233

aprica, Phryxe, 1863a: 337

apricans, Phorocera, 1830: 133

apricata, Bercaea, 1863b: 556

apricata, Bonellia, 1863a: 139

aprilina, Phryxe, 1863a: 349

aprilis, Myophora, 1830: 355

aquatica, Limnophora, 1830: 521

aquaticus, Sepedon, 1830: 678

aquilegiae, Phytomyza, 1851e: 397

arabica, Cosmina, 1830: 424

arborea, Erichsonia, 1863b: 491

arborea, Phorocera, 1863a: 514

arctii, Acinia, 1830: 777

arcuata, Onesia, 1863b: 545

arcuata, Suillia, 1830: 645

ardeacea, Amobia, 1863b: 132

ardeacea, Doria, 1863a: 538

ardeacea, Erichsonia, 1863b: 486

ardeacea, Meigenia, 1863a: 1085

ardeacea, Myrsina, 1863b: 159

ardeacea, Phryxe, 1863a: 433

ardeacea, Scaligeria, 1863b: 478 [as "ardeacaea" 1863b: 908]

ardeacea, Walkeria, 1863a: 1009

ardelio, Staegeria, 1863a: 981

ardens, Phaenicia, 1863b: 758

ardua, Meigenia, 1863a: 1070

arenaria, Fucellia, 1842c: 272

arenaria, Oresbia, 1863b: 413

argentea, Clytho, 1830: 376

argentea, Myopa, 1830: 246

arguta, Carcelia, 1847: 279 
argyria, Hydrellia, 1830: 793

argyrina, Phrosina, 1863b: 102

argyritarsis, Anopheles, 1827: 411

argyrostoma, Myophora, 1830: 340

aricioidea, Helina, 1830: 493

aricioidea, Phaonia, 1830: 483

arida, Dinera, 1863b: 355

arion, Carcelia, 1847: 275

armata, Strauzia, 1830: 719

arrogans, Lucilia, 1863b: 723

arvensis, Hylemya, 1830: 554

arvensis, Lucilia, 1863b: 714

arvensis, Myophora, 1830: 358

arvensis, Phasia, 1830: 292

arvensis, Phorbia, 1830: 560

arvensis, Phorella, 1830: 362

arvensis, Phryxe, 1830: 169

arvensis, Roeselia, 1830: 145

arvensis, Sarcophaga, 1863b: 454

arvensis, Tachina, 1830: 194

arvensis, Xyphosia, 1830: 763

arvicola, Delia, 1830: 573

arvicola, Meigenia, 1863a: 1079

arvicola, Myophora, 1830: 355

arvorum, Esila, 1863a: 1063

arvorum, Exorista, 1863a: 266

arvorum, Myophora, 1830: 361

arvorum, Orizia, 1863b: 680

assimilis, Araba, 1830: 130

aterrima, Jurinia, 1830: 35

aterrima, Peckia, 1830: 336

athaliae, Phryxe, 1830: 167

atra, Aplomya, 1863a: 463

atra, Arraltia, 1863b: 73

atra, Clytia, 1830: 288

atra, Gymnosoma, 1830: 238

atra, Hyalomya, 1863b: 261

atra, Illigeria, 1830: 274

atra, Limnophora, 1830: 522

atra, Melanophora, 1830: 272

atra, Mulsantia, 1863b: 560

atra, Myophora, 1830: 360

atra, Neaera, 1850b: 189

atra, Rhedia, 1830: 78

atra, Tachina, 1830: 194

atra, Westwodia, 1863a: 941

atrabilis, Phryxe, 1863a: 399

atrata, Delia, 1830: 574

atrata, Fimetia, 1830: 810

atrata, Hyalomya, 1863b: 261

atrata, Hydrotaea, 1830: 514

atrata, Meigenia, 1863a: 1075

atrata, Myophora, 1830: 349 atrata, Phorella, 1830: 363

atrata, Phryxe, 1863a: 399

atrata, Phryxe, 1863a: 457

atrata, Pollenia, 1830: 417

atrata, Staegeria, 1863a: 983

atrata, Tachina, 1830: 195

atratella, Phasia, 1863b: 224

atripalpis, Fabricia, 1863a: 627

atripes, Micropeza, 1830: 743

atripes, Neria, 1830: 738

atriplicia, Pegomya, 1851c: 230

atropivora, Sturmia, 1830: 171

atrox, Megaera, 1830: 95

audax, Zelleria, 1863a: 992

augur, Eribea, 1863a: 1034

aurata, Euphoria, 1863b: 820

aurata, Hemyda, 1830: 226

aurata, Hydrina, 1830: 794

aurata, Lucilia, 1863b: 713

aurata, Myobia, 1863b: 310

aurata, Phaenicia, 1863b: 776

aurata, Sarcophaga, 1863b: 445

aurea, Aplomya, 1863a: 464

aurea, Clemelis, 1863a: 481

aurea, Clytia, 1863b: 281

aurea, Phryxe, 1863a: 348

aurea, Ravinia, 1863b: 434

aurea, Tachina, 1830: 191

aurea, Zenillia, 1863a: 471

auriceps, Orillia, 1863b: 336

aurifacies, Amobia, 1863b: 138

aurifacies, Beraldia, 1863a: 906

aurifacies, Futilia, 1863a: 1061

aurifacies, Gesneria, 1830: 382

aurifacies, Hydrellia, 1830: 791

aurifacies, Jurinia, 1830: 38

aurifacies, Musca, 1830: 396

aurifacies, Myophora, 1830: 354

aurifacies, Phryxe, 1863a: 329

aurifacies, Stomoxis, 1863b: 607

auriflua, Sarcophaga, 1863b: 457

aurifrons, Carcelia, 1830: 182

aurifrons, Erycia, 1863a: 904

aurifrons, Gesneria, 1830: 383

aurifrons, Ophelia, 1863b: 98

aurifrons, Phorinia, 1830: 118

aurifrons, Phryxe, 1863a: 349

aurifrons, Setulia, 1863b: 125

aurifrons, Tachina, 1830: 190

aurifrons, Tlephusa, 1863a: 308

auripes, Conops, 1836: 688

auripilis, Verreauxia, 1863a: 894

aurocincta, Phryxe, 1863a: 364 
aurozonata, Lilaea, 1863b: 161 aurulans, Calliphora, 1830: 437 aurulans, Dionaea, 1830: 254 aurulans, Elomya, 1830: 297 aurulans, Lispa, 1830: 526 aurulans, Lucilia, 1830: 458 aurulans, Musca, 1830: 397 aurulenta, Aubaea, 1863a: 187 aurulenta, Clytho, 1830: 376 aurulenta, Melibaea, 1848a: 615 aurulenta, Miltogramma, 1863b: 115 aurulenta, Phryno, 1849a: 434 aurulenta, Phryxe, 1863a: 341 aurulenta, Phryxe, 1863a: 348 aurulenta, Winthemia, 1847: 269 aurulenta, Zelinda, 1863a: 485 austera, Phryxe, 1863a: 366 australis, Sphora, 1830: 394 autissiodorensis, Mufetia, 1830: 431 autumnalis, Egle, 1830: 587 autumnalis, Euphoria, 1863b: 822 autumnalis, Hylemya, 1830: 554 autumnalis, Pollenia, 1830: 414 avida, Phryxe, 1863a: 437 azurea, Lucilia, 1830: 455 azurea, Melinda, 1830: 440 azurea, Phaenicia, 1863b: 788 basalis, Clidonia, 1830: 732 basalis, Hyalomya, 1830: 299 basalis, Phryxe, 1863a: 418 beauvoisii, Prionella, 1830: 760 bella, Euphoria, 1863b: 828 bellierella, Pales, 1863a: 519 bellierella, Phryxe, 1863a: 382 belvosii, Phumosia, 1830: 428 bengalensis, Idia, 1830: 421 bengalensis, Limnophora, 1830: 518 bengalensis, Lucilia, 1830: 460 benigna, Phaenicia, 1863b: 784 berceella, Phryxe, 1863a: 384 bercei, Carcelia, 1850a: 164 bercei, Phorocera, 1850a: 177 biciliata, Amobia, 1863b: 137 bicincta, Belvosia, 1830: 103 bicincta, Duvaucelia, 1830: 228 bicolor, Euphoria, 1863b: 832 bicolor, Fischeria, 1830: 101 bicolor, Phaenicia, 1863b: 777 bicolor, Pollenia, 1830: 415 bicolor, Pyrellia, 1830: 465 bidentis, Stylia, 1830: 755 bifasciata, Thelaira, 1830: 215 bigoti, Occemya, 1853a: 142 [1853b: 62] bigotina, Lalage, 1863a: 560 bigotinus, Micropalpus, 1863a: 120 biguttata, Aricia, 1830: 488 binotata, Dionaea, 1863b: 56 binotata, Gouraldia, 1851a: 151 binotata, Meigenia, 1863a: 1090 binotata, Phryxe, 1863a: 364 binotata, Solieria, 1849a: 462 bipartita, Picconia, 1863b: 34 bipunctata, Agria, 1830: 377 bipunctata, Helina, 1830: 493 bipunctatus, Culex, 1827: 405 bisbinotata, Aricia, 1830: 489 blanda, Bessa, 1863b: 165 blanda, Eretria, 1863a: 495 blanda, Euphoria, 1863b: 828 blanda, Futilia, 1863a: 1060 blanda, Mulsantia, 1863b: 572 blanda, Phaenicia, 1863b: 775 blandula, Walkeria, 1863a: 1016 blondeli, Myophora, 1830: 344 blondeli, Pales, 1830: 156 blondeli, Phryxe, 1830: 161 blondeli, Platymya, 1830: 118 blondeli, Reaumuria, 1830: 80 blondeli, Rohrella, 1830: 491 blondeli, Salia, 1830: 110 boisduvalii, Sarcophaga, 1863b: 465 bombycivora, Carcelia, 1830: 181 bombycivora, Phorocera, 1830: 136 bombycivora, Phryxe, 1830: 165 bombycivora, Salia, 1830: 108 bombylans, Carcelia, 1830: 177 bombylans, Nemoraea, 1830: 72 bombylans, Rhedia, 1830: 76 borealis, Linnaemya, 1830: 54 borealis, Meigenia, 1830: 199 borealis, Meriania, 1830: 70 borealis, Phorinia, 1830: 120 boscii, Jurinia, 1830: 36 boscii, Parthenia, 1830: 232 boscii, Pherbina, 1830: 690 boscii, Psorophora, 1827: 413 boscii, Rivellia, 1830: 730 boscorum, Phryxe, 1863a: 398 boscorum, Staegeria, 1863a: 974 botyvora, Phorocera, 1830: 138 bovina, Coprina, 1830: 810 bovina, Musca, 1830: 398 brachycera, Macquartia, 1830: 206 brasiliensis, Dejeania, 1830: 33 brasiliensis, Dichromya, 1830: 707 brasiliensis, Harrisia, 1830: 324 
brasiliensis, Icelia, 1830: 224

brasiliensis, Jurinia, 1830: 35

brasiliensis, Illigeria, 1863a: 1138

brasiliensis, Myophora, 1830: 338

brasiliensis, Olinda, 1830: 116

brassicae, Pegomya, 1851c: 235

brunea, Pegomya, 1830: 599

brunea, Phryno, 1830: 144

brunea, Phyllis, 1830: 605

brunibarbis, Calliphora, 1830: 434

brunicans, Gesneria, 1830: 379

brunicans, Pales, 1830: 156

brunicornis, Chrysomya, 1830: 451

brunicornis, Nemoraea, 1830: 73

brunicornis, Palusia, 1830: 543

brunicornis, Pegomya, 1830: 599

brunicornis, Urophora, 1830: 772

brunicosa, Limnophora, 1830: 522

brunicosa, Lucilia, 1830: 459

brunicosa, Misellia, 1863b: 148

brunicosa, Nemopoda, 1830: 745

brunicosa, Noeeta, 1830: 779

brunicosa, Pegomya, 1830: 599

brunicosa, Solieria, 1849a: 470

brunicosa, Solieria, 1863b: 333

brunifacies, Hydrellia, 1830: 792

brunifacies, Myophora, 1830: 348

brunipennis, Aminta, 1830: 570

brunipes, Keratocera, 1830: 789

brunipes, Limosia, 1830: 536

brunipes, Melanosoma, 1853a: 126 [1853b: 46]

brunipes, Occemya, 1853a: 143 [1853b: 63]

brunisquamis, Elophoria, 1830: 157

brunisquamis, Olbya, 1863a: 170

brunisquamis, Scaligeria, 1863b: 479

brunithorax, Forellia, 1830: 762

buccalis, Albinia, 1830: 210

buccalis, Chrysomya, 1830: 448

buccalis, Pollenia, 1863b: 672

buccata, Scatella, 1830: 801

bucentoidea, Phryno, 1830: 144

cadaverina, Cynomya, 1830: 365

cadaverina, Fimetia, 1830: 810

cadaverina, Lordatia, 1830: 809

caepicola, Anthomia, 1851c: 234

caerulea, Lucilia, 1863b: 731

caerulea, Melinda, 1830: 439

caerulea, Onesia, 1830: 369

caerulea, Phormia, 1830: 466

caeruleifrons, Euphoria, 1863b: 822 [as "coeruleifrons" 1863b: 880]

caeruleipennis, Hydromya, 1830: 691

caerulescens, Hyalomya, 1863b: 245 [as "coerulescens" 1863b: 884]

caerulescens, Pales, 1863a: 529

caesia, Blissonia, 1863b: 648

caesia, Hubneria, 1848a: 605

caesia, Lucilia, 1863b: 747

caesia, Mulsantia, 1863b: 573

caesia, Ophelia, 1863b: 96

caesia, Orizia, 1863b: 678

caesia, Phryxe, 1863a: 384

caesia, Pollenia, 1863b: 670

caesia, Sarcophaga, 1863b: 452

caesia, Thalpia, 1863b: 171

caesia, Zetterstedtia, 1863a: 1032

caiae, Phorocera, 1830: 135

calcitrans, Culex, 1827: 409

calcitrapae, Urellia, 1830: 774

calcium, Myocera, 1830: 329

calens, Lucilia, 1830: 459

calida, Pyrellia, 1830: 464

calidula, Dasyphora, 1863b: 651

calidula, Phaenicia, 1863b: 756

callichroa, Euphoria, 1863b: 835

callimorphae, Carcelia, 1863a: 230

calthae, Delia, 1830: 577

campephaga, Phorcida, 1863a: 254

campestris, Aplomya, 1863a: 462

campestris, Aubaea, 1863a: 186

campestris, Egle, 1830: 585

campestris, Erichsonia, 1863b: 484

campestris, Erycia, 1863a: 905

campestris, Faedoria, 1863a: 849

campestris, Gesneria, 1830: 380

campestris, Hubneria, 1848a: 610

campestris, Ismenia, 1863a: 586

campestris, Latreillia, 1830: 105

campestris, Limosia, 1830: 537

campestris, Lydella, 1830: 114

campestris, Masicera, 1863a: 873

campestris, Meigenia, 1863a: 1077

campestris, Mulsantia, 1863b: 558

campestris, Musca, 1830: 395

campestris, Mydina, 1830: 499

campestris, Myophora, 1830: 346

campestris, Myorhina, 1830: 383

campestris, Onesia, 1863b: 547

campestris, Phaenicia, 1863b: 777

campestris, Phasia, 1830: 292

campestris, Solieria, 1863b: 322

campestris, Tachina, 1830: 190

campicola, Delia, 1830: 576

campicola, Musca, 1863b: 627

campicola, Pales, 1863a: 523

camporum, Myophora, 1830: 359 
camporum, Phryxe, 1863a: 344 camporum, Staegeria, 1863a: 974 canaliculata, Phaonia, 1830: 484 canescens, Staegeria, 1863a: 981 canora, Carcelia, 1863a: 235 cantans, Carcelia, 1863a: 223 capax, Zelleria, 1863a: 987 capensis, Chrysomya, 1830: 451 capensis, Dejeania, 1830: 34 capensis, Dexia, 1830: 314 capensis, Meriania, 1830: 71 capensis, Mintho, 1830: 217 capensis, Myophora, 1830: 347 capensis, Olina, 1830: 812 capensis, Rhedia, 1830: 77 capensis, Scatophaga, 1830: 625 carbonaria, Hyalomya, 1830: 300 carbonaria, Macquartia, 1863a: 1108 carbunculus, Lucilia, 1863b: 712 carceli, Calyptia, 1863b: 576 carceli, Medina, 1830: 140 carceli, Myophora, 1830: 348 carceli, Nyctia, 1830: 263 carceli, Phryxe, 1830: 170 carceli, Zodion, 1830: 251 cardaminis, Hydrina, 1830: 796 cardui, Oxyphora, 1830: 757 caricicola, Coenia, 1830: 800 carnifex, Byomya, 1830: 393 carolinae, Parthenia, 1830: 232 carolinensis, Lucilia, 1830: 457 carolinensis, Neria, 1830: 738 carolinensis, Scatophaga, 1830: 629 carolinensis, Scatophora, 1830: 811 catocalae, Winthemia, 1830: 174 cauta, Dufouria, 1863b: 70 cauta, Lydella, 1863a: 863 cauta, Phryxe, 1863a: 375 cayennensis, Neria, 1830: 737 cayennensis, Pherbina, 1830: 689 cellarum, Bacchis, 1830: 804 cerasi, Pegomya, 1830: 599 cerceridis, Setulia, 1863b: 124 chalybea, Euphoria, 1863b: 811 chetalis, Erichsonia, 1863b: 484 chorea, Anthomya, 1830: 582 chorea, Hyalomya, 1863b: 266 chrysanthemi, Ensina, 1830: 752 chrysella, Hydrellia, 1830: 792 chrysella, Lucilia, 1863b: 746 chrysella, Myophora, 1830: 339 chrysella, Phaenicia, 1863b: 792 chrysella, Staegeria, 1863a: 973 chrysiceps, Jurinia, 1830: 37 chrysifacies, Myophora, 1863b: 506 chrysigastris, Lucilia, 1863b: 736 chrysina, Fausta, 1863a: 161 chrysina, Hydrellia, 1830: 792 chrysis, Lucilia, 1863b: 711 chrysocephala, Stomoxis, 1863b: 604 chrysorhoa, Euphoria, 1863b: 824 chrysura, Euphoria, 1863b: 825 ciliata, Phryxe, 1830: 159 ciliatula, Sarcophaga, 1863b: 455 ciligera, Chremia, 1863b: 335 ciligera, Enthenis, 1863a: 199 ciligera, Oppia, 1863b: 406 ciligera, Stephensia, 1863b: 19 ciligera, Winthemia, 1830: 173 ciligera, Zenillia, 1830: 154 cilipes, Leschenaultia, 1830: 325 cincta, Dexia, 1830: 312 cinctella, Phaenicia, 1863b: 796 cineraria, Clemelis, 1863a: 484 cinerascens, Delia, 1830: 575 cinerascens, Mydina, 1830: 499 cinerascens, Myobia, 1830: 99 cinerascens, Phryxe, 1830: 168 cinerea, Amobia, 1863b: 132 cinerea, Blondelia, 1863b: 28 cinerea, Cuculla, 1830: 523 cinerea, Dinera, 1863b: 357 cinerea, Erycia, 1863a: 904 cinerea, Erythrocera, 1849a: 439 cinerea, Estelia, 1830: 635 cinerea, Gesneria, 1830: 379 cinerea, Hebia, 1863a: 579 cinerea, Hersilia, 1863a: 501 cinerea, Hydrotaea, 1830: 511 cinerea, Idia, 1830: 422 cinerea, Leucophora, 1830: 563 cinerea, Limosia, 1830: 536 cinerea, Lydella, 1863a: 861 cinerea, Myophora, 1830: 355 cinerea, Nerina, 1830: 557 cinerea, Ophelia, 1830: 121 cinerea, Oxyna, 1830: 755 cinerea, Phryxe, 1863a: 387 cinerea, Pollenia, 1863b: 669 cinerea, Sagaris, 1863a: 487 cinerea, Scatella, 1830: 801 cinerea, Solieria, 1849a: 470 cinerea, Staegeria, 1863a: 980 cinerea, Suillia, 1830: 644 cinerea, Sylvia, 1830: 637 cinerea, Theano, 1863a: 257 
cinerea, Winthemia, 1847: 270

cinereicollis, Hubneria, 1863a: 280

cinereifrons, Amobia, 1863b: 133

cinerella, Arabella, 1863b: 91

cinerella, Loevia, 1863a: 897

cinerella, Megaera, 1863b: 144

cinerella, Meigenia, 1863a: 1066

cinerella, Phasia, 1863b: 223

cinerella, Phryxe, 1863a: 360

cingulata, Actia, 1830: 86

cirrata, Phorocera, 1830: 138

cirrata, Salia, 1830: 109

cirsiorum, Xyphosia, 1830: 762

cita, Aubaea, 1863a: 186

cita, Melibaea, 1863a: 288

cita, Phryxe, 1863a: 395

cita, Walkeria, 1863a: 1010

claripennis, Acinia, 1830: 778

claripennis, Aricia, 1830: 488

claripennis, Carcelia, 1863a: 222

claripennis, Chetocera, 1830: 697

claripennis, Cosmina, 1830: 423

claripennis, Dyctia, 1830: 693

claripennis, Egle, 1830: 590

claripennis, Elomya, 1830: 297

claripennis, Erichsonia, 1863b: 490

claripennis, Euphemia, 1830: 486

claripennis, Gesneria, 1830: 379

claripennis, Hartigia, 1863b: 524

claripennis, Helina, 1830: 494

claripennis, Hyalomya, 1863b: 260

claripennis, Hydrotaea, 1830: 510

claripennis, Kockia, 1863a: 819

claripennis, Limnia, 1830: 685

claripennis, Megerlea, 1830: 267

claripennis, Minettia, 1830: 646

claripennis, Morinia, 1830: 266

claripennis, Mycetia, 1830: 806

claripennis, Mydina, 1830: 497

claripennis, Myophora, 1863b: 509

claripennis, Nyctia, 1830: 263

claripennis, Onesia, 1830: 367

claripennis, Phorbia, 1830: 560

claripennis, Phryxe, 1863a: 372

claripennis, Sarcophaga, 1863b: 456

claripennis, Scatina, 1830: 629

claripennis, Scatophaga, 1830: 628

claripennis, Stomoxis, 1863b: 604

claripennis, Suillia, 1830: 643

claripennis, Tachina, 1830: 192

claripennis, Tetanocera, 1830: 682

claripennis, Thelaira, 1863a: 781

claripennis, Thryptocera, 1863a: 716 claropunctata, Retellia, 1830: 683

clausa, Anemya, 1863a: 850

clausa, Dufouria, 1830: 258

clausa, Syphona, 1850b: 209

clavipes, Olina, 1830: 812

coarctata, Phryxe, 1830: 163

coccinea, Phaenicia, 1863b: 750

coelestis, Lucilia, 1863b: 710 [as “caelestis" 1863b: 886]

coelestis, Melinda, 1830: 440 [as “caelestis" 1863b: 890]

coenosa, Limnophora, 1830: 522

coerulescens, Chrysomya, 1830: 447

coffeana, Pollenia, 1863b: 675

cognata, Lilaea, 1863b: 163

cognata, Myophora, 1830: 341

cognata, Phaenicia, 1863b: 793

cognata, Phryxe, 1863a: 408

cognata, Rohrella, 1830: 491

collinaris, Elomya, 1863b: 236

collinaris, Myophora, 1830: 345

collinaris, Myophora, 1863b: 516

commendata, Myophora, 1863b: 516

commota, Phryxe, 1863a: 422

communis, Caricea, 1830: 531

communis, Chione, 1830: 680

communis, Dryope, 1830: 619

communis, Egle, 1830: 588

communis, Hydrellia, 1830: 791

communis, Mycetia, 1830: 805

communis, Mydina, 1830: 497

communis, Pherbina, 1830: 689

communis, Sphaerocera, 1830: 807

communis, Suillia, 1830: 643

compar, Lucilia, 1830: 457

compar, Myophora, 1830: 354

compar, Walkeria, 1863a: 1008

compos, Phryxe, 1863a: 409

compressa, Calliphora, 1830: 438

compta, Phryxe, 1863a: 438

concessa, Phryxe, 1863a: 414

concinna, Phaenicia, 1863b: 778

concolor, Beraldia, 1863a: 908

concolor, Culex, 1827: 405

concolor, Hartigia, 1863b: 523

concolor, Meigenia, 1863a: 1067

concolor, Morellia, 1830: 406

concolor, Muscina, 1830: 408

concolor, Phryxe, 1863a: 334

concolor, Sturmia, 1830: 172

conducta, Ismenia, 1863a: 586

conducta, Phryxe, 1863a: 393

confusa, Phryxe, 1863a: 441

conica, Amobia, 1830: 96

conica, Myophora, 1830: 355 
conica, Ptilocera, 1830: 222

conjuncta, Orizia, 1863b: 678

connexa, Meigenia, 1863a: 1080

connexa, Mosina, 1830: 672

conscia, Phryxe, 1863a: 353

consecuta, Phryxe, 1863a: 339

consentanea, Phryxe, 1863a: 400

consimilis, Syphona, 1850b: 205

consobrina, Mulsantia, 1863b: 570

consobrina, Myophora, 1830: 344

consobrina, Phaenicia, 1863b: 795

consobrina, Phryxe, 1830: 160

consobrinus, Culex, 1827: 408

contempta, Myophora, 1830: 343

contempta, Pollenia, 1863b: 676

contenta, Phryxe, 1863a: 417

contenta, Walkeria, 1863a: 998

contigua, Erichsonia, 1863b: 492

continua, Musca, 1863b: 628

contraria, Walkeria, 1863a: 997

convicta, Zenais, 1863a: 913

coprina, Lordatia, 1830: 809

coprivora, Sphaerocera, 1830: 807

corinna, Hyalomya, 1830: 301

corusca, Euphoria, 1863b: 823

corusca, Phaenicia, 1863b: 763

corusca, Phormia, 1863b: 849

coxalis, Chariclaea, 1863a: 558

coxalis, Micropalpus, 1863a: 127

crassicornis, Winthemia, 1847: 266

crataegellae, Zaida, 1830: 151

crocata, Myophora, 1830: 343

crudelis, Megaera, 1830: 95

cubensis, Myophora, 1830: 342

cuculliae, Echinomya, 1830: 49

cucullata, Mulsantia, 1863b: 567

cucullata, Rondania, 1850b: 193

cuculliae, Baumhaueria, 1863a: 757

cuculliae, Hubneria, 1850a: 166

cuculliae, Latreillia, 1830: 107

cuculliae, Masicera, 1863a: 878

cuculliae, Phorocera, 1850a: 177

cuculliae, Ramonda, 1863a: 791

cunctans, Euphoria, 1863b: 830

cunctans, Stomoxis, 1863b: 607

cunctata, Phryxe, 1863a: 353

cunctata, Walkeria, 1863a: 1006

cuniculorum, Leria, 1830: 655

cupraea, Smidtia, 1848a: 595

cuprea, Lucilia, 1830: 459

cuprea, Lydina, 1830: 125

cuprea, Onesia, 1830: 368

cuprea, Phormia, 1830: 467 cuprea, Pyrellia, 1830: 464

cursitana, Myophora, 1863b: 518

cursoria, Eribea, 1863a: 1038

cursoria, Erichsonia, 1863b: 494

cursoria, Futilia, 1863a: 1051

cursoria, Lydella, 1863a: 866

cursoria, Miltogramma, 1863b: 116

cursoria, Philea, 1863a: 314

cursoria, Sarcophaga, 1863b: 458

cursoria, Velocia, 1863a: 951

curvinervis, Orillia, 1849a: 475

cyanea, Boisduvalia, 1830: 731

cyanea, Lucilia, 1863b: 733

cyanea, Onesia, 1830: 370

cyaneifrons, Euphoria, 1863b: 831

cyanella, Phaenicia, 1863b: 789

cyaneoviridis, Euphoria, 1863b: 819

cyanescens, Pollenia, 1830: 414

cyanescens, Walkeria, 1863a: 997

cylindrica, Clytia, 1830: 288

cylindrica, Dinera, 1830: 308

cylindrica, Eginia, 1830: 503

cylindrica, Lucilia, 1863b: 730

cylindrica, Lydella, 1863a: 859

cylindrica, Medina, 1830: 139

cylindrica, Meigenia, 1830: 199

cylindrica, Novia, 1830: 211

cylindrica, Phorocera, 1830: 137

cylindrica, Roeselia, 1830: 146

cylindrica, Sargella, 1830: 675

cylindrica, Tephrytis, 1830: 767

cyrrhata, Lucasia, 1863b: 411

dalmatica, Clytia, 1830: 287

dauci, Forellia, 1830: 761

debita, Phryxe, 1863a: 380

deceptoria, Delia, 1830: 573

decidua, Phryxe, 1863a: 417

decipiens, Hubneria, 1863a: 282

decora, Chrysomya, 1830: 448

decora, Euphoria, 1863b: 812

decora, Phaenicia, 1863b: 760

dejeanii, Chrysomya, 1830: 449

dejeanii, Delina, 1830: 670

dejeanii, Dexia, 1830: 312

dejeanii, Limnia, 1830: 687

dejeanii, Mosina, 1830: 671

dejeanii, Nerina, 1830: 558

dejeanii, Sitarea, 1830: 764

dejeanii, Urophora, 1830: 772

delata, Phryxe, 1863a: 361

delicata, Orillia, 1863b: 339

delicatula, Lucilia, 1830: 461

delicatula, Mollia, 1863a: 949 
delicatula, Tachina, 1863a: 971

delicatula, Terenia, 1830: 641

delicatula, Walkeria, 1863a: 1016

delusa, Phryxe, 1863a: 431

demens, Aetylia, 1863a: 272

demissa, Anicia, 1863b: 100

demissa, Hubneria, 1863a: 281

demissa, Ophelia, 1863b: 98

depressa, Macromya, 1830: 322

depressa, Myophora, 1830: 353

depressa, Phryxe, 1830: 162

deses, Phaenicia, 1863b: 778

despecta, Amobia, 1863b: 135

despecta, Phorella, 1830: 363

desvoidyi, Reaumuria, 1830: 275

devicta, Meigenia, 1863a: 1072

diaphanipennis, Tachina, 1830: 190

diffusa, Lucilia, 1863b: 743

diligens, Phryxe, 1863a: 454

dimidiata, Aplomya, 1863a: 465

dimidiata, Hyalomya, 1863b: 241

dimidiata, Phaenicia, 1863b: 769

dimidiata, Solieria, 1849a: 471

dimidiata, Winthemya, 1863a: 215

dira, Megaera, 1830: 95

dira, Stomoxis, 1830: 387

discolor, Jurinia, 1863a: 660

discolor, Lucilia, 1863b: 733

discreta, Edesia, 1863a: 599

discreta, Nemoraea, 1863a: 175

discreta, Phryxe, 1863a: 413

discreta, Walkeria, 1863a: 1018

discretus, Micropalpus, 1863a: 125

dispar, Macquartia, 1863a: 1106

dispar, Mydina, 1830: 497

dissimilis, Argyrella, 1863b: 87

distincta, Carcelia, 1830: 179

distincta, Caricea, 1830: 534

distincta, Linnaemya, 1830: 54

distincta, Melanophora, 1830: 273

distincta, Phryxe, 1863a: 435

distincta, Tachina, 1830: 191

diversa, Carcelia, 1830: 181

diversa, Lucilia, 1863b: 704

diversa, Rhedia, 1830: 77

diversa, Tachina, 1830: 193

dives, Lucilia, 1863b: 715

docilis, Phaenicia, 1863b: 779

dolosa, Phaenicia, 1863b: 781

dolosa, Phryxe, 1863a: 332

domestica, Leria, 1830: 654

dominula, Gaubilia, 1863a: 1062

doronici, Ensina, 1830: 753 dorsalis, Azelia, 1830: 593

dorsalis, Lucilia, 1830: 453

dorsalis, Phaenicia, 1863b: 770

dorsalis, Phasia, 1863b: 212

dorsalis, Rohrella, 1830: 491

dorsalis, Tephrytis, 1830: 766

dubia, Erigone, 1830: 68

dubia, Lydella, 1830: 113

dufouri, Occemya, 1853a: 136 [1853b: 56]

dulcis, Phaenicia, 1863b: 766

dumeti, Myophora, 1863b: 508

dumetorum, Phryxe, 1863a: 418

duodecimpunctata, Azelia, 1830: 593

duponcheli, Carcelia, 1830: 179

durvillei, Rutilia, 1830: 321

duvaucelii, Chrysomya, 1830: 451

duvaucelii, Myophora, 1830: 351

ebennia, Kockia, 1863a: 819

echinura, Salia, 1830: 109

edax, Zelleria, 1863a: 994

educata, Bonellia, 1863a: 140

educata, Phryxe, 1863a: 381

edwarsella, Phryxe, 1863a: 438

egena, Phryxe, 1863a: 447

egena, Servaisia, 1863b: 431

egena, Staegeria, 1863a: 976

egerioidea, Zaphne, 1830: 527

electa, Phryxe, 1863a: 420

elegans, Hubertia, 1863b: 169

elegans, Lucilia, 1830: 458

elegans, Phaenicia, 1863b: 791

elliptica, Phryxe, 1863a: 419

elongata, Medina, 1830: 139

elongata, Solieria, 1849a: 465

elongata, Staegeria, 1863a: 975

emissa, Walkeria, 1863a: 1009

erigonea, Mericia, 1830: 65

errans, Echinomya, 1830: 45

errans, Myophora, 1863b: 514

erratica, Fellaea, 1830: 478

erratica, Linnemya, 1863a: 132

erratica, Sarcophaga, 1863b: 447

erucastri, Pales, 1863a: 526

erucastri, Phryxe, 1863a: 412

eruceti, Hubneria, 1850a: 165

erythrea, Phaenicia, 1863b: 758

erythrocera, Athrycia, 1830: 111

erythrocera, Caricea, 1830: 534

erythrocera, Ceromya, 1830: 87

erythrocera, Clelia, 1830: 256

erythrocera, Gesneria, 1830: 378

erythrocera, Salia, 1830: 109

euphemioidea, Helina, 1830: 493 
exacta, Phryxe, 1863a: 351

excitata, Eretria, 1863a: 492

excitata, Phaedima, 1863a: 844

excitata, Phryxe, 1863a: 356

exigua, Afzelia, 1863a: 582

exigua, Egle, 1830: 591

exilis, Meigenia, 1863a: 1068

exilis, Phaenicia, 1863b: 787

exilis, Phryxe, 1863a: 446

exilis, Walkeria, 1863a: 1002

eximia, Lucilia, 1830: 456

extrema, Phryxe, 1863a: 442

fabricii, Egle, 1830: 587

facialis, Aplomya, 1863a: 463

facialis, Lucilia, 1863b: 726

facialis, Masicera, 1863a: 872

facialis, Zetterstedtia, 1863a: 1030

facilis, Phaenicia, 1863b: 796

faedata, Staegeria, 1863a: 984

falculae, Zaida, 1830: 151

fallax, Phryxe, 1863a: 447

fallax, Sarcophaga, 1863b: 475

fallax, Zelleria, 1863a: 990

famula, Phryxe, 1863a: 430

fasciata, Blondelia, 1830: 123

fasciata, Herina, 1830: 727

fasciata, Myennis, 1830: 717

fasciata, Ramonda, 1863a: 790

fasciata, Wagneria, 1863a: 794

fasciolata, Aplomya, 1863a: 462

fasciolata, Elodia, 1863a: 938

fastuosa, Lucilia, 1863b: 712

fatua, Phryxe, 1863a: 374

fausta, Lucilia, 1863b: 742

fausta, Phryxe, 1863a: 416

fausta, Walkeria, 1863a: 1017

favilla, Phaenicia, 1863b: 752

femoralis, Aciura, 1830: 773

femoralis, Ahrensia, 1863b: 16

femoralis, Caricea, 1830: 531

femoralis, Hyalomya, 1863b: 259

femoralis, Lycia, 1830: 639

femoralis, Occemya, 1853: 132 [1853b: 52]

femoralis, Oxyna, 1830: 756

femoralis, Palusia, 1830: 545

femoralis, Solieria, 1849a: 469

femoralis, Urophora, 1830: 770

femorata, Cylidria, 1830: 679

fenestrarum, Chyromya, 1830: 621

fenestrarum, Leria, 1830: 654

fera, Fellaea, 1830: 477

fera, Megaera, 1830: 95

fera, Myocera, 1830: 330 fera, Phorocera, 1830: 133

fera, Zenais, 1830: 149

ferax, Zelleria, 1863a: 991

ferox, Haematobia, 1830: 388

ferrugata, Phryxe, 1863a: 452

ferruginea, Alophora, 1830: 295

ferruginea, Nemopoda, 1830: 744

fervens, Dasyphora, 1830: 410

fervida, Lucilia, 1830: 459

fervida, Myophora, 1830: 341

fervida, Pyrellia, 1830: 465

festinans, Argyrella, 1863b: 88

festinans, Halesa, 1863a: 315

festinans, Lydella, 1863a: 862

festinata, Tachina, 1863a: 970

festiva, Carcelia, 1830: 177

festiva, Egle, 1830: 589

festiva, Eretria, 1863a: 497

festiva, Euphoria, 1863b: 818

festiva, Guerinia, 1830: 196

festiva, Hubneria, 1848a: 606

festiva, Melanophora, 1830: 272

festiva, Myophora, 1863b: 512

festiva, Onesia, 1863b: 543

festiva, Ophelia, 1830: 121

festiva, Pales, 1863a: 523

festiva, Solieria, 1849a: 462

festiva, Tachina, 1830: 192

fida, Phryxe, 1863a: 416

filiformis, Thelida, 1830: 656

filipes, Mosina, 1830: 673

filipes, Sophia, 1830: 318

flagrans, Phaenicia, 1863b: 788

flamma, Euphoria, 1863b: 836

flamma, Lucilia, 1863b: 706

flammea, Phaenicia, 1863b: 761

flammula, Phaenicia, 1863b: 762

flava, Lisella, 1830: 649

flava, Lycia, 1830: 638

flava, Minettia, 1830: 648

flava, Philinta, 1830: 569

flava, Phyllis, 1830: 604

flavago, Futilia, 1863a: 1054

flaveola, Dufouria, 1863b: 69

flaveola, Lentiphora, 1830: 656

flaveola, Myophora, 1830: 339

flaveola, Suillia, 1830: 644

flavescens, Anthomya, 1830: 582

flavescens, Athrycia, 1830: 112

flavescens, Carcelia, 1830: 183

flavescens, Egle, 1830: 590

flavescens, Hartigia, 1863b: 522

flavescens, Icelia, 1830: 224 
flavescens, Leskia, 1830: 100

flavescens, Macquartia, 1830: 204

flavescens, Meigenia, 1830: 200

flavescens, Myophora, 1830: 361

flavescens, Nerina, 1830: 558

flavescens, Oxyna, 1830: 756

flavescens, Palusia, 1830: 543

flavescens, Pherbina, 1830: 688

flavescens, Phoraea, 1830: 601

flavescens, Roeselia, 1849a: 445

flavescens, Setulia, 1863b: 125

flavescens, Solieria, 1863b: 329

flavescens, Stomoxis, 1863b: 605

flavescens, Winthemia, 1830: 174

flavibarbis, Phryxe, 1863a: 385

flavicans, Amobia, 1863b: 136

flavicans, Futilia, 1863a: 1049

flavicans, Orellia, 1830: 765

flavicans, Walkeria, 1863a: 1003

flavicornis, Lythia, 1863a: 708

flavicornis, Palusia, 1830: 543

flavicornis, Peribaea, 1863a: 721

flavicornis, Scyphella, 1830: 650

flavicornis, Trichopoda, 1830: 284

flavida, Onesia, 1863b: 543

flavida, Staegeria, 1863a: 977

flavidula, Mulsantia, 1863b: 573

flavifacies, Hydrotaea, 1830: 511

flavifrons, Clemelis, 1863a: 484

flavifrons, Phorocera, 1851a: 152

flavinervis, Erichsonia, 1863b: 493

flavipalpis, Athrycia, 1863a: 832

flavipalpis, Belida, 1863b: 45

flavipalpis, Damonia, 1848a: 597

flavipalpis, Javetia, 1863a: 1116

flavipalpis, Nigrina, 1863a: 933

flavipalpis, Onesia, 1863b: 545

flavipalpis, Phaedima, 1863a: 843

flavipalpis, Phaenicia, 1863b: 753

flavipalpis, Phryxe, 1830: 169

flavipalpis, Smidtia, 1848a: 596

flavipalpis, Vanzemia, 1863a: 942

flavipennis, Erebia, 1830: 208

flavipennis, Erigone, 1830: 67

flavipennis, Hylemya, 1830: 553

flavipennis, Myopella, 1853a: 107 [1853b: 27]

flavipennis, Oswaldia, 1863a: 841

flavipennis, Phorocera, 1830: 136

flavipes, Ceranthia, 1850b: 200

flavipes, Dalmannia, 1830: 250

flavipes, Erythrocera, 1849a: 438

flavipes, Hebia, 1830: 98

flavipes, Idia, 1830: 420 flavipes, Minettia, 1830: 648

flavipes, Neria, 1830: 738

flavipes, Noeeta, 1830: 778

flavipes, Pegomya, 1830: 600

flavisquamis, Aplomya, 1863a: 465

flavisquamis, Atilia, 1863a: 476

flavisquamis, Dionaea, 1863b: 57

flavisquamis, Elophoria, 1830: 157

flavisquamis, Ilaesa, 1863a: 498

flavisquamis, Myobia, 1863b: 312

flavisquamis, Phryxe, 1863a: 368

flavisquamis, Ramonda, 1863a: 791

flavisquamis, Roeselia, 1849a: 445

flavisquamis, Thryptocera, 1851a: 149

flavisquamis, Westwodia, 1863a: 941

flavocincta, Salia, 1863a: 554

flavoptera, Hylemya, 1830: 554

flavovirens, Culex, 1827: 410

floralis, Aminta, 1830: 571

floralis, Anthomya, 1830: 583

floralis, Dufouria, 1863b: 68

floralis, Erycia, 1863a: 905

floralis, Estheria, 1830: 307

floralis, Euphoria, 1863b: 814

floralis, Futilia, 1863a: 1050

floralis, Gesneria, 1830: 381

floralis, Hersilia, 1863a: 502

floralis, Hydrotaea, 1830: 514

floralis, Leucophora, 1830: 563

floralis, Lucilia, 1863b: 744

floralis, Macrosoma, 1830: 403

floralis, Musca, 1830: 397

floralis, Onesia, 1830: 366

floralis, Oppia, 1863a: 313

floralis, Phaenicia, 1863b: 777

floralis, Pollenia, 1830: 415

floralis, Staegeria, 1863a: 977

floralis, Zenillia, 1863a: 474

florea, Azelia, 1830: 592

florea, Fausta, 1830: 64

florea, Fellaea, 1830: 477

florea, Hylemya, 1830: 554

florea, Myopella, 1853a: 100 [1853b: 20]

florea, Myophora, 1830: 352

florea, Pales, 1830: 155

florea, Pterella, 1863b: 122

floricola, Delia, 1830: 572

floricola, Egle, 1830: 587

floricola, Lydella, 1830: 115

floricola, Mydaea, 1830: 479

floricola, Sturmia, 1830: 172

florida, Onesia, 1863b: 548

florida, Phryxe, 1830: 166 
florida, Sarcophaga, 1863b: 454

floridula, Bercaea, 1863b: 551

florilega, Opesia, 1863b: 277

florilega, Zenillia, 1863a: 474

florivaga, Lydella, 1863a: 864

florum, Egle, 1830: 589

florum, Phorella, 1830: 362

florum, Tachina, 1830: 192

fluviatilis, Limnophora, 1830: 520

fluviatilis, Lispa, 1830: 525

foetida, Phaenicia, 1863b: 784

fontinalis, Nitellia, 1863b: 683

forcipata, Clairvillia, 1863b: 186

forcipata, Dionaea, 1830: 253

formicaria, Myrmecomya, 1830: 722

formosa, Rutilia, 1830: 320

fragilis, Myobia, 1830: 98

fragilis, Rohrella, 1830: 492

fragilis, Suillia, 1830: 644

frater, Culex, 1827: 408

frontalina, Haydaea, 1863a: 564

frontalis, Euphoria, 1863b: 815

frontalis, Limnophora, 1830: 521

frontalis, Myophora, 1863b: 507

frontalis, Phryxe, 1830: 169

frontalis, Pollenia, 1863b: 671

frontalis, Sarcophaga, 1863b: 468

frontalis, Tilesia, 1863b: 366

fugax, Eugenia, 1863a: 920

fugax, Myophora, 1863b: 512

fugax, Scaligeria, 1863b: 481

fugax, Zelleria, 1863a: 989

fugitiva, Amobia, 1863b: 133

fugitiva, Lydella, 1863a: 865

fugitiva, Oria, 1863a: 275

fugitiva, Phryxe, 1863a: 411

fulgens, Bonellia, 1863a: 139

fulgens, Phaenicia, 1863b: 771

fulgida, Euphoria, 1863b: 821

fulgida, Micropeza, 1830: 742

fuliginosa, Calyptia, 1863b: 577

fuliginosa, Dasyphora, 1830: 410

fuliginosa, Erichsonia, 1863b: 487

fuliginosa, Gesneria, 1830: 382

fuliginosa, Gymnosoma, 1830: 237

fuliginosa, Hydrellia, 1830: 793

fuliginosa, Hydrophoria, 1830: 505

fuliginosa, Hydrotaea, 1830: 512

fuliginosa, Limosia, 1830: 540

fuliginosa, Lydella, 1863a: 863

fuliginosa, Morellia, 1863b: 638

fuliginosa, Mydina, 1830: 498

fuliginosa, Myophora, 1830: 349 fuliginosa, Paykullia, 1830: 271

fuliginosa, Prosena, 1863b: 392

fuliginosa, Sphaerocera, 1830: 807

fuliginosum, Zodion, 1853a: 156 [1853b: 76]

fuligo, Delia, 1830: 574

fuligo, Scaligeria, 1863b: 479

fulva, Araba, 1830: 131

fulva, Mosina, 1830: 672

fulva, Nemoraea, 1830: 72

fulva, Nemoraea, 1863a: 175

fulva, Ocyptera, 1830: 230

fulva, Rhedia, 1830: 77

fulvescens, Erythrocera, 1849a: 440

fulvescens, Musca, 1830: 397

fulvescens, Myophora, 1863b: 504

fulvibarbis, Calliphora, 1830: 434

fulvicorne, Zodion, 1853a: 158 [1853b: 78]

fulvicornis, Dasiphora, 1863b: 653

fulvicornis, Keratocera, 1830: 789

fulvicornis, Lucilia, 1830: 462

fulvicornis, Lucilia, 1863b: 745

fulvicornis, Lydella, 1863a: 855

fulvicornis, Mydina, 1830: 496

fulvicornis, Myophora, 1830: 341

fulvicornis, Phaenicia, 1863b: 787

fulvicornis, Pollenia, 1830: 413

fulvicornis, Walkeria, 1863a: 1023

fulvicrura, Chrysomya, 1830: 446

fulvicrus, Aria, 1830: 309

fulvida, Zetterstedtia, 1863a: 1025

fulvifacies, Phormia, 1830: 467

fulvifrons, Gesneria, 1863b: 597

fulvifrons, Occemya, 1853a: 134 [1853b: 54]

fulvifrons, Phaenicia, 1863b: 799

fulvipalpis, Megaera, 1863b: 144

fulvipalpis, Myobia, 1849a: 456

fulvipalpis, Phorella, 1863b: 532

fulvipalpis, Pictinia, 1853a: 96 [1853b: 16]

fulvipalpis, Sarcophaga, 1863b: 442

fulvipes, Ceranthia, 1830: 88

fulvipes, Dinera, 1830: 308

fulvipes, Erythrocera, 1849a: 437

fulvipes, Lydella, 1830: 115

fulvipes, Myopa, 1830: 246

fulvipes, Ophina, 1863a: 299

fulvipes, Zodion, 1853a: 157 [1853b: 77]

fulviventris, Jurinia, 1830: 37

funebris, Adenia, 1863a: 1042

funebris, Myophora, 1863b: 500

funebris, Pollenia, 1863b: 664

funesta, Cerophora, 1863a: 701

fungivora, Leria, 1830: 653

fungivora, Muscina, 1830: 408 
fungorum, Suillia, 1830: 642

fusca, Caricea, 1830: 533

fusca, Phorocera, 1863a: 512

fuscana, Camilla, 1863b: 642

fuscana, Hyalomya, 1863b: 254

fuscana, Phasia, 1863b: 213

fuscana, Solieria, 1849a: 468

fuscicornis, Phasia, 1863b: 223

fuscicornis, Syphona, 1850b: 205

fuscifacies, Myophora, 1863b: 520

fuscifrons, Clemelis, 1863a: 485

fuscifrons, Phryxe, 1863a: 377

fuscipennis, Carcelia, 1830: 182

fuscipennis, Cosmina, 1830: 423

fuscipennis, Dasyna, 1830: 668

fuscipennis, Delia, 1830: 580

fuscipennis, Hartigia, 1863b: 528

fuscipennis, Morinia, 1830: 265

fuscipennis, Myophora, 1830: 361

fuscipennis, Reaumuria, 1863a: 742

fuscipes, Phasia, 1863b: 219

futilis, Phryxe, 1863a: 441

gagatea, Anthomya, 1830: 583

gagatea, Elodia, 1863a: 938

gagatea, Freraea, 1830: 286

gagatea, Guerinia, 1830: 198

gagatea, Hubneria, 1848a: 602

gagatea, Hydrotaea, 1830: 514

gagatea, Jurinia, 1830: 36

gagatea, Leucophora, 1830: 564

gagatea, Melibaea, 1848a: 616

gagatea, Morellia, 1863b: 638

gagatea, Nemopoda, 1830: 745

gagatea, Opesia, 1863b: 276

gagatea, Orizia, 1863b: 680

gagatea, Phyto, 1863b: 49

gagatea, Rhinomya, 1830: 124

gagatea, Rhinophora, 1830: 259

gagatea, Servaisia, 1863b: 431

gagatea, Solieria, 1849a: 463

gagatea, Sophia, 1830: 318

gagatea, Wagneria, 1830: 126

gagatina, Eribea, 1863a: 1040

gallica, Spallanzania, 1830: 79

gemma, Lucilia, 1863b: 738

gemmula, Lucilia, 1863b: 705

gemmula, Phaenicia, 1863b: 779

geniculata, Bacchis, 1830: 805

geniculata, Haematobia, 1830: 388

gentilis, Azelia, 1830: 592

gentilis, Clytia, 1830: 288

gentilis, Dorbinia, 1847: 273

gentilis, Euphoria, 1863b: 824 gentilis, Limnophora, 1830: 522

gentilis, Melinda, 1830: 441

gentilis, Onesia, 1830: 370

gentilis, Onesia, 1863b: 542

gentilis, Pherbina, 1830: 688

gentilis, Walkeria, 1863a: 1014

geoffroyi, Sarcophaga, 1863b: 458

geometra, Neria, 1830: 736

germana, Delia, 1830: 580

germana, Lucilia, 1830: 455

germana, Myophora, 1863b: 511

germana, Onesia, 1863b: 545

germana, Pherbina, 1830: 690

germana, Phryxe, 1863a: 334

germana, Rohrella, 1830: 492

germana, Solieria, 1849a: 467

germana, Tachina, 1830: 191

germanica, Macquartia, 1830: 205

gesnerioidea, Agria, 1830: 378

glabra, Delia, 1830: 579

glabrata, Hyalomya, 1863b: 258

glabrata, Phaenicia, 1863b: 797

glabrata, Phryxe, 1863a: 450

glabratus, Micropalpus, 1863a: 122

glaucescens, Bonellia, 1863a: 136

gloriosa, Euphoria, 1863b: 833

gnava, Dasyphora, 1863b: 650

gouraldi, Elophoria, 1851a: 152

gouraldi, Pegomya, 1851c: 231

gracilis, Carcelia, 1863a: 225

gracilis, Dexia, 1830: 313

gracilis, Hydrotaea, 1830: 513

gracilis, Ophelia, 1830: 121

gracilis, Phorinia, 1830: 119

gracilis, Phorocera, 1830: 136

grandis, Tachina, 1863a: 963

grata, Dufouria, 1863b: 69

grata, Euphoria, 1863b: 806

grata, Meigenia, 1863a: 1073

grata, Phryxe, 1863a: 402

gratella, Hyalomya, 1863b: 244

gratiosa, Chrysomya, 1830: 451

gratiosa, Euphoria, 1863b: 833

gratiosa, Phaenicia, 1863b: 755

grisea, Agria, 1830: 377

grisea, Aminta, 1830: 570

grisea, Araba, 1830: 131

grisea, Billaea, 1830: 328

grisea, Carcelia, 1830: 182

grisea, Ceromya, 1850b: 198

grisea, Cuculla, 1830: 523

grisea, Dexia, 1830: 314

grisea, Dinera, 1830: 308 
grisea, Erycia, 1830: 147

grisea, Gesneria, 1830: 380

grisea, Guerinia, 1863a: 1123

grisea, Leucophora, 1830: 562

grisea, Lypha, 1863a: 197

grisea, Muscina, 1830: 408

grisea, Myophora, 1830: 353

grisea, Myophora, 1830: 361

grisea, Occemya, 1853a: 137 [1853b: 57]

grisea, Opesia, 1863b: 278

grisea, Osmoea, 1830: 84

grisea, Phorbia, 1830: 560

grisea, Tachina, 1830: 195

grisella, Erichsonia, 1863b: 498

grisella, Gesneria, 1863b: 595

grisella, Hartigia, 1863b: 525

grisella, Meigenia, 1863a: 1069

grisella, Musca, 1863b: 622

grisella, Phryxe, 1863a: 339

grisella, Pollenia, 1863b: 661

griseoflavescens, Gesneria, 1830: 383

grisescens, Agria, 1830: 377

grisescens, Lydella, 1830: 112

grisescens, Macquartia, 1830: 206

grisescens, Meigenia, 1830: 199

grisescens, Myocera, 1830: 330

grisescens, Myophora, 1830: 359

grisescens, Phryxe, 1830: 163

grossipes, Terenia, 1830: 641

grossoria, Eribea, 1863a: 1039

grossoria, Walkeria, 1863a: 1021

guerinella, Phryxe, 1863a: 456

guerini, Acidia, 1830: 721

guerini, Dyctia, 1830: 695

guerini, Occemya, 1853a: 141 [1853b: 61]

guerini, Phorocera, 1850a: 178

guerini, Tomella, 1830: 630

hadenae, Phorocera, 1851a: 153

haemathura, Bercaea, 1863b: 551

haemisphaerica, Cyzenis, 1863a: 545 [as "hemisphoerica" 1863b: 875 ]

haitensis, Trichopoda, 1830: 285

haustellata, Eleone, 1863b: 351

hebes, Ravinia, 1863b: 436

hemisphaerica, Myophora, 1830: 346

hemisphaerica, Phryno, 1830: 144

heraclaei, Linnaemya, 1830: 53

herbaria, Myophora, 1863b: 508

herbaria, Sarcophaga, 1863b: 459

herbarum, Dyctia, 1830: 693

herbarum, Ensina, 1830: 752

herbarum, Estelia, 1830: 635

herbarum, Oscinis, 1830: 712 herbarum, Rivellia, 1830: 729

herbicola, Delia, 1830: 578

heterocera, Ocalea, 1863a: 811

hiemalis, Kirbya, 1830: 268

hilarella, Lilaea, 1863b: 162

hilarella, Walkeria, 1863a: 1007

hilaris, Argyria, 1863b: 85

hilaris, Carcelia, 1847: 280

hilaris, Eretria, 1863a: 494

hilaris, Erichsonia, 1863b: 496

hilaris, Hubneria, 1848a: 607

hilaris, Meigenia, 1863a: 1082

hilaris, Onesia, 1863b: 544

hilaris, Phaenicia, 1863b: 764

hilaris, Phryxe, 1863a: 345

hilaris, Sarcophaga, 1863b: 471

hilaris, Walkeria, 1863a: 1004

hirta, Latreillia, 1830: 106

hirta, Leschenaultia, 1830: 325

hirtella, Wiedmania, 1863a: 929

hirtipes, Olina, 1830: 812

hispida, Tachina, 1830: 189

holosericea, Phasia, 1863b: 211

honesta, Eribea, 1863a: 1035

honesta, Phaedima, 1863a: 842

honesta, Phryxe, 1863a: 357

honesta, Tlephusa, 1863a: 308

hortensis, Egeria, 1830: 556

hortensis, Phryxe, 1863a: 371

hortensis, Pollenia, 1863b: 665

horticola, Morellia, 1830: 405

horticola, Myophora, 1830: 346

hortorum, Futilia, 1863a: 1050

hortorum, Meigenia, 1863a: 1076

hortulana, Amobia, 1863b: 136

hortulana, Phytomyza, 1851e: 403

hottentota, Hermya, 1830: 227

hottentota, Musca, 1830: 399

hottentota, Neria, 1830: 737

hottentota, Rhinophora, 1830: 260

humboldti, Myophora, 1830: 338

humeralis, Micropalpus, 1863a: 119

humeralis, Mydaea, 1830: 481

humeralis, Phoraea, 1830: 602

humeralis, Rohrella, 1830: 491

humeralis, Syphona, 1850b: 207

humeralis, Thryptocera, 1851a: 149

humida, Leria, 1830: 654

humilis, Argyria, 1863b: 86

humilis, Eretria, 1863a: 498

humilis, Pales, 1863a: 526

humilis, Sarcophaga, 1863b: 473

humilis, Scaligeria, 1863b: 479 
humilis, Scatophaga, 1830: 628

humilis, Thalpia, 1863b: 171

hyacinthina, Chrysomya, 1830: 450

hyacinthina, Lucilia, 1830: 462

hyalinata, Atrania, 1863a: 815

hyalinata, Pales, 1863a: 525

hyalinata, Panzeria, 1863a: 147

hyalipennis, Sarcophaga, 1863b: 473

hydrocampae, Lydella, 1830: 113

hydrocotylis, Limosia, 1830: 540

hyemalis, Hylemya, 1830: 552

hyemalis, Orthellia, 1863b: 838

hylemyoidea, Zaphne, 1830: 527

ichneumonea, Chione, 1830: 680

ichneumoneus, Polystodes, 1830: 723

idioidea, Chrysomya, 1830: 445

ignea, Phaenicia, 1863b: 755

ignita, Phaenicia, 1863b: 759

ignita, Pyrellia, 1830: 464

ignota, Phryxe, 1863a: 333

illustris, Eudora, 1863a: 623

immaculata, Limosia, 1830: 541

immaculata, Meigenia, 1830: 200

immaculata, Neaera, 1830: 85

immaculata, Solieria, 1849a: 464

impatiens, Carbonia, 1863a: 809

impatiens, Fausta, 1863a: 163

impatiens, Meigenia, 1863a: 1071

impatiens, Phaenicia, 1863b: 772

impatiens, Phryxe, 1863a: 453

impavida, Aplomya, 1863a: 464

imperatoriae, Estheria, 1830: 306

imperfecta, Macquartia, 1863a: 1107

imperialis, Amenia, 1830: 443

imperialis, Peckia, 1830: 335

impressifrons, Vidalia, 1830: 719

imprudens, Phryxe, 1863a: 434

impunctata, Aricia, 1830: 487

impunctata, Estelia, 1830: 636

impunctata, Lycia, 1830: 639

impunctata, Mydina, 1830: 496

impunctata, Tephrytis, 1830: 767

inanis, Zetterstedtia, 1863a: 1031

incana, Amobia, 1863b: 135

incana, Futilia, 1863a: 1055

incauta, Mulsantia, 1863b: 573

inclyta, Lucilia, 1863b: 703

inclyta, Phaenicia, 1863b: 782

inclyta, Sarcophaga, 1863b: 443

inconspecta, Fausta, 1863a: 166

inconstans, Erichsonia, 1863b: 491

indica, Jurinia, 1830: 36

indica, Lucilia, 1830: 453 indica, Neria, 1830: 737

inermis, Hydrotaea, 1830: 510

inermis, Strauzia, 1830: 718

iners, Sarcophaga, 1863b: 439

infesta, Stomoxis, 1830: 387

infima, Meigenia, 1863a: 1069

inflata, Beria, 1830: 418

infuscata, Duponchelia, 1863a: 532

infuscata, Erichsonia, 1863b: 497

infuscata, Zaira, 1863b: 370

infuscata, Zenillia, 1863a: 474

ingenua, Lucilia, 1863b: 714

inguinata, Futilia, 1863a: 1057

inimica, Megaera, 1830: 96

inimica, Stomoxis, 1830: 387

innocua, Meigenia, 1863a: 1083

innoxia, Nilea, 1863a: 276

innoxia, Phryxe, 1863a: 397

inops, Fausta, 1863a: 163

inops, Phryxe, 1863a: 386

insidiosa, Anicia, 1863b: 100

insidiosa, Calliphora, 1863b: 695

insidiosa, Phryxe, 1863a: 338

insidiosa, Walkeria, 1863a: 1013

insignis, Lucilia, 1863b: 716

insignita, Phaenicia, 1863b: 771

intacta, Adenia, 1863a: 1044

intacta, Meigenia, 1863a: 1072

intacta, Phryxe, 1863a: 451

integra, Aplomya, 1863a: 463

integra, Hubneria, 1863a: 281

integra, Hyalomya, 1863b: 257

integra, Myophora, 1863b: 520

integra, Pales, 1863a: 527

integra, Phryxe, 1863a: 360

intermedia, Echinomya, 1830: 47

intermedia, Loxocera, 1830: 667

intermedia, Myophora, 1830: 349

interrupta, Echinomya, 1863a: 636

interrupta, Roeselia, 1849a: 444

intersecta, Hyalomya, 1863b: 248

inulae, Neria, 1830: 738

iovora, Phorocera, 1830: 135

iraeos, Agromyza, 1851e: 393

irrequieta, Pales, 1863a: 529

irrorata, Adenia, 1863a: 1042

irrorata, Futilia, 1863a: 1053

jaceae, Acinia, 1830: 776

jaceae, Tephrytis, 1830: 766

javanensis, Sepedon, 1830: 677

javanica, Peleteria, 1830: 40

jejuna, Walkeria, 1863a: 1003

jocax, Zelleria, 1863a: 985 
jocosa, Arabella, 1863b: 92

jucunda, Blainvillia, 1830: 714

jucunda, Erytaea, 1863a: 848

jucunda, Euphoria, 1863b: 829

judicata, Phryxe, 1863a: 401

juncorum, Limosia, 1830: 540

jussa, Phryxe, 1863a: 396

juvenilis, Eribea, 1863a: 1037

juvenilis, Micropalpus, 1863a: 128

labialis, Erichsonia, 1863b: 485

labialis, Phaenicia, 1863b: 768

labialis, Phorella, 1863b: 532

labialis, Pollenia, 1863b: 671

labiata, Bengalia, 1830: 426

laeta, Aetylia, 1863a: 271

laeta, Amobia, 1863b: 133

laeta, Bercaea, 1863b: 555

laeta, Ephyra, 1863b: 157

laeta, Macquartia, 1863a: 1110

laeta, Phryxe, 1863a: 368

laeta, Tachina, 1830: 193

laeta, Tachina, 1863a: 965

laetatoria, Lucilia, 1863b: 718

laetifica, Mydina, 1830: 500

laevigata, Carcelia, 1863a: 229

laevigata, Pales, 1847: 285

laevigata, Phryxe, 1863a: 385

laevigata, Sagaris, 1863a: 486

laevigatus, Micropalpus, 1863a: 123

laevis, Lucilia, 1863b: 743

laevis, Walkeria, 1863a: 1005

lalandii, Chrysomya, 1830: 450

lalandii, Latreillia, 1830: 106

lalandii, Palpomya, 1830: 708

lalandii, Peleteria, 1830: 40

lalandii, Reaumuria, 1830: 80

lamanensis, Peckia, 1830: 335

lamarckii, Occemya, 1853a: 140 [1853b: 60]

lamberti, Rhinomyia, 1851b: xxvii

lanipes, Peckia, 1830: 336

lapilaei, Echinomya, 1830: 44

lappae, Caricea, 1830: 534

lappae, Phytomyza, 1851e: 399

lappae, Xyphosia, 1830: 763

larvicola, Phryxe, 1830: 166

lasiocampae, Phryxe, 1830: 164

lateralis, Arisbaea, 1863b: 291

lateralis, Bonellia, 1830: 57

lateralis, Echinomya, 1830: 49

lateralis, Fausta, 1846b: 32

lateralis, Hamulia, 1863b: 114

lateralis, Meigenia, 1863a: 1075

lateralis, Pales, 1863a: 529 lateralis, Panzeria, 1830: 69

lateralis, Phryxe, 1863a: 342

lateralis, Staegeria, 1863a: 972

lateralis, Walkeria, 1863a: 999

laticornis, Amphisa, 1863b: 130

laticrus, Myophora, 1830: 357

laticrus, Terenia, 1830: 641

latifrons, Germaria, 1830: 83

latifrons, Voria, 1830: 196

latreillii, Gymnosoma, 1830: 237

latreillii, Mesembrina, 1830: 401

latreillii, Mosina, 1830: 671

latreillii, Xysta, 1830: 286

lauta, Euphoria, 1863b: 802

lauta, Mulsantia, 1863b: 558

lauta, Phaedima, 1863a: 845

lauta, Walkeria, 1863a: 995

lavata, Phryxe, 1863a: 407

lefebvrei, Echinomya, 1830: 45

lenis, Phaenicia, 1863b: 797

lepida, Carcelia, 1830: 177

lepida, Chrysomya, 1830: 448

lepida, Lucilia, 1830: 453

lepida, Onesia, 1830: 368

lepida, Phaenicia, 1863b: 773

lepida, Phryxe, 1863a: 440

lepida, Zenillia, 1830: 153

leschenaldi, Peleteria, 1830: 40

leucocera, Melinda, 1830: 440

leucophaea, Caricea, 1830: 535

leucoptera, Bacchis, 1830: 804

leucostoma, Jurinia, 1830: 37

levis, Phryxe, 1863a: 331

lherminieri, Chrysomya, 1830: 446

lherminieri, Myophora, 1830: 339

libatrix, Stomoxis, 1830: 387

libera, Lucilia, 1863b: 741

libera, Phryxe, 1863a: 423

liliorum, Herbina, 1842b: 267

limbata, Herina, 1830: 726

limbata, Lucilia, 1863b: 746

limbata, Pherbina, 1830: 690

limpida, Pierretia, 1863b: 425

limpida, Walkeria, 1863a: 1011

limpidipennis, Caricea, 1830: 533

limpidipennis, Erycia, 1830: 148

limpidipennis, Fimetia, 1830: 810

limpidipennis, Hyalomya, 1863b: 259

limpidipennis, Limosia, 1830: 540

limpidipennis, Lucilia, 1830: 454

limpidipennis, Mydina, 1830: 498

limpidipennis, Myophora, 1830: 346

limpidipennis, Phorocera, 1830: 137 
linariae, Ensina, 1830: 753

linariae, Sphenella, 1830: 774

lineata, Dionaea, 1863b: 55

lineata, Phasia, 1863b: 214

lineata, Suillia, 1830: 645

lispoidea, Dryxo, 1830: 787

littoralis, Calliphora, 1830: 435

littoralis, Caricea, 1830: 533

littoralis, Delia, 1830: 580

littoralis, Hydrophoria, 1830: 506

littoralis, Limnophora, 1830: 520

littoralis, Limosia, 1830: 538

littoralis, Potamia, 1830: 507

littoralis, Pyrellia, 1830: 464

littoralis, Tachina, 1830: 189

liturata, Chlorophora, 1830: 733

liturata, Delia, 1830: 575

liturata, Dryope, 1830: 619

liturata, Herina, 1830: 725

liturata, Hylemya, 1830: 553

liturata, Lycia, 1830: 640

liturata, Mydaea, 1830: 481

liturata, Suillia, 1830: 643

liturata, Urophora, 1830: 771

liturella, Lycia, 1830: 640

livens, Myophora, 1830: 350

livida, Myophora, 1830: 350

locuples, Argyria, 1863b: 86

locuples, Eumea, 1863a: 303

locuples, Phaenicia, 1863b: 767

locuples, Sabethes, 1827: 412

longicornis, Gravenhorstia, 1863a: 925

longicornis, Herina, 1830: 726

longipes, Bonnetia, 1830: 55

longipes, Myocera, 1830: 329

longipes, Neria, 1830: 737

longipes, Saltella, 1830: 747

longipes, Zabia, 1830: 600

longirostris, Myopa, 1830: 243

lonicerae, Phytomyza, 1851e: 396

lophyri, Sturmia, 1863a: 892

lubrica, Futilia, 1863a: 1059

lubrica, Orthellia, 1863b: 839

lubrica, Osmina, 1863b: 167

lubrica, Phryxe, 1863a: 332

lubrica, Zenillia, 1863a: 475

lucasi, Occemya, 1853a: 144 [1853b: 64]

luctuosa, Eumea, 1863a: 304

luctuosa, Meigenia, 1863a: 1074

luctuosa, Minettia, 1830: 646

luctuosa, Phryxe, 1863a: 340

ludibrina, Eribea, 1863a: 1039

ludibunda, Aminta, 1830: 570 ludibunda, Bacchis, 1830: 804

ludibunda, Ceromya, 1850b: 195

ludibunda, Dorbinia, 1847: 272

ludibunda, Egle, 1830: 585

ludibunda, Mydina, 1830: 499

ludifacies, Musca, 1863b: 621

lugubris, Hartigia, 1863b: 529

lugubris, Hesyquillia, 1830: 709

lugubris, Sarcophaga, 1863b: 476

luperinae, Wagneria, 1863a: 794

lusoria, Morellia, 1863b: 637

lusoria, Myophora, 1863b: 515

lusoria, Phryxe, 1863a: 387

lusoria, Sarcophaga, 1863b: 468

luteicauda, Servaisia, 1863b: 431

luteifrons, Gesneria, 1830: 381

luteipalpis, Cnossia, 1863a: 290

luteipalpis, Sturmia, 1847: 262

luteipes, Aricia, 1830: 488

luteipes, Hylemya, 1830: 551

luteola, Terellia, 1830: 759

lutescens, Futilia, 1863a: 1054

lutescens, Ophelia, 1830: 121

macei, Limnophora, 1830: 519

macquarti, Athrycia, 1863a: 833

macquarti, Hamulia, 1863b: 112

macquarti, Myophora, 1863b: 514

macquarti, Occemya, 1853a: 141 [1853b: 61]

macquarti, Phorinia, 1830: 119

macquarti, Phryxe, 1830: 163

macquartina, Lypha, 1863a: 198

macrocera, Eloceria, 1863a: 703

macrocera, Tachina, 1830: 189

macroglossae, Tachina, 1850a: 169

macromera, Lydina, 1830: 125

macroura, Carcelia, 1830: 178

maculata, Eribea, 1863a: 1035

maculata, Oxyna, 1830: 756

maculata, Pales, 1863a: 528

maculata, Phryxe, 1863a: 344

maculata, Solieria, 1863b: 324

maculata, Stylia, 1830: 754

maculata, Thelaira, 1863a: 777

maculipennis, Hydrina, 1830: 795

maculosa, Phasia, 1863b: 206

maesta, Eribea, 1863a: 1041

maesta, Lydella, 1863a: 866

maesta, Meigenia, 1863a: 1076

maesta, Phryxe, 1863a: 341 [as “moesta” 1863b: 903]

maga, Loevia, 1863a: 896

magnifica, Lucilia, 1863b: 710

maialis, Lucilia, 1863b: 715

maialis, Myophora, 1830: 357 
maialis, Phryxe, 1863a: 427

mandata, Phryxe, 1863a: 361

marginalis, Eumea, 1863a: 304

marginalis, Limnia, 1830: 685

marginalis, Lucilia, 1863b: 745

marginalis, Myopella, 1853a: 101 [1853b: 21]

marginalis, Phaenicia, 1863b: 794

marginalis, Phryxe, 1863a: 352

marginalis, Sagaris, 1863a: 487

marginalis, Tachina, 1863a: 971

marginata, Herina, 1830: 725

marginella, Miltogramma, 1863b: 118

marginella, Pollenia, 1863b: 666

marginella, Tetanocera, 1830: 682

maritima, Tetanocera, 1830: 683

maura, Delia, 1830: 574

maura, Mydina, 1830: 501

maura, Pierretia, 1863b: 425

media, Graphomya, 1830: 405

medicaginis, Agromyza, 1851e: 393

meditata, Mulsantia, 1863b: 576

meditata, Phryxe, 1863a: 423

meigeni, Miltogramma, 1863b: 116

meigeni, Occemya, 1853a: 135 [1853b: 55]

meigenii, Aplomya, 1863a: 466

meigenii, Himera, 1863a: 1126

meigenii, Sarcophaga, 1863b: 442

melancolica, Eribea, 1863a: 1038

melanocera, Bengalia, 1830: 426

melanocera, Dexia, 1830: 312

melanocera, Onesia, 1863b: 546

melanocera, Syphona, 1850b: 206

melanocolica, Tachina, 1863a: 970

melanopus, Trichopoda, 1830: 284

melanura, Bellina, 1863b: 195

melanura, Pierretia, 1863b: 428

melanura, Reaumuria, 1830: 81

melina, Leria, 1842a: 261

mellifrons, Pterella, 1863b: 121

mendax, Zelleria, 1863a: 993

mentharum, Stylia, 1830: 754

menyanthidis, Palusia, 1830: 542

mera, Thalpia, 1863b: 170

merdaria, Scatophora, 1830: 811

merdarum, Lordatia, 1830: 808

merdivora, Scatophaga, 1830: 628

meridionalis, Dalmannia, 1830: 249

meridionalis, Faurella, 1830: 41

meridionalis, Limnophora, 1830: 523

meridionalis, Stephania, 1863a: 808

metallica, Euphoria, 1863b: 808

metallica, Jurinia, 1830: 35

metallica, Phaenicia, 1863b: 780 metallica, Pollenia, 1830: 417

metallica, Rhinophora, 1830: 259

metallica, Salia, 1830: 110

mexicana, Dejeania, 1863a: 652

micans, Guerinia, 1863a: 1123

micans, Macquartia, 1863a: 1107

micans, Phaenicia, 1863b: 783

micans, Pollenia, 1830: 416

microcera, Ceromya, 1830: 88

microcera, Ernestia, 1830: 61

microcera, Gymnosoma, 1830: 238

microcera, Hesione, 1863a: 200

microcera, Macquartia, 1830: 206

microcera, Myostoma, 1830: 327

microcera, Phryxe, 1830: 161

micromera, Phorinia, 1830: 119

micropezoidea, Myrmecomya, 1830: 722

millefolii, Acinia, 1830: 777

minax, Zelleria, 1863a: 987

miniata, Phryxe, 1863a: 389

minor, Clelia, 1830: 256

minor, Graphomya, 1830: 404

minor, Illigeria, 1830: 274

minor, Latreillia, 1830: 107

minor, Phorinia, 1830: 119

minuta, Araba, 1830: 130

minuta, Aubaea, 1863a: 188

minuta, Elpigia, 1863b: 151

minuta, Gymnosoma, 1830: 238

minuta, Hyalomya, 1863b: 258

minuta, Peribaea, 1863a: 722

minuta, Phorella, 1863b: 533

minuta, Phryxe, 1863a: 347

minuta, Stomoxis, 1863b: 606

mirifica, Lucilia, 1863b: 706

misera, Myophora, 1863b: 504

misera, Onesia, 1863b: 547

misera, Phryxe, 1863a: 391

misera, Walkeria, 1863a: 1012

missa, Phryxe, 1863a: 445

mitis, Phryxe, 1863a: 414

mitis, Sarcophaga, 1863b: 466

modesta, Lucilia, 1830: 454

modesta, Lydella, 1863a: 858

modesta, Pales, 1863a: 530

modesta, Phryxe, 1863a: 426

modesta, Sarcophaga, 1863b: 472

modesta, Solieria, 1849a: 466

modesta, Solieria, 1863b: 325

modica, Lucilia, 1863b: 705

moesta, Sarcophaga, 1863b: 452

mollis, Anthomya, 1830: 583

mollis, Euphoria, 1863b: 826 
mollis, Mydina, 1830: 502

mollis, Orthellia, 1863b: 838

mollis, Sylvia, 1830: 637

monacantha, Hydrotaea, 1830: 509

monita, Walkeria, 1863a: 1007

monspeliaca, Calliphora, 1830: 436

montana, Elbaea, 1863a: 286

montana, Myophora, 1863b: 518

monticola, Cytoria, 1863a: 801

monticola, Myophora, 1863b: 517

moreti, Tachina, 1853c: 534

morio, Bercaea, 1863b: 555

morio, Erichsonia, 1863b: 499

morio, Phorella, 1830: 363

morosa, Eribea, 1863a: 1037

morosa, Phryxe, 1863a: 404

morosa, Pitthaea, 1863a: 189

mosquito, Culex, 1827: 407

munda, Futilia, 1863a: 1055

munda, Phryxe, 1863a: 417

munda, Walkeria, 1863a: 1009

muricola, Anicia, 1863b: 101

musca, Buquetia, 1847: 287

musca, Calliphora, 1830: 436

musca, Carcelia, 1863a: 227

musca, Erichsonia, 1863b: 487

musca, Gesneria, 1830: 381

musca, Guerinia, 1830: 197

musca, Mydaea, 1830: 481

musca, Myophora, 1830: 360

musca, Phanemya, 1830: 254

musca, Phorbia, 1830: 559

musca, Walkeria, 1863a: 996

musca, Zaira, 1863b: 371

muscaria, Oswaldia, 1863a: 840

muscaria, Phorella, 1863b: 533

muscidea, Cephysa, 1863b: 677

muscidea, Oppia, 1863a: 313

muscidea, Phryxe, 1863a: 392

muscidea, Staegeria, 1863a: 975

mustelina, Leria, 1842a: 261

myoidaea, Sarcophaga, 1863b: 459

myoidaea, Lydella, 1830: 114

myoidaea, Scaligeria, 1863b: 477

myoidea, Acemya, 1830: 203

myoidea, Calliphora, 1830: 436

myoidea, Elophoria, 1830: 157

myoidea, Hartigia, 1863b: 525

myoidea, Phoraea, 1830: 601

myoidea, Phorocera, 1830: 135

myoidea, Phryxe, 1863a: 450 [as "myoidoea" 1863b: 903]

myoidea, Smidtia, 1830: 184

myophoroidaea, Erigone, 1830: 67 myopiformis, Orbellia, 1830: 657

nana, Calliphora, 1830: 436

nana, Guerinia, 1830: 197

nana, Meigenia, 1830: 201

nana, Myobia, 1830: 100

nana, Myopa, 1830: 248

nana, Myophora, 1830: 361

nana, Nitellia, 1830: 418

nana, Phryxe, 1863a: 347

nana, Saltella, 1830: 747

nana, Solieria, 1863b: 328

narcissi, Herbina, 1836: 689

nebulosa, Azelia, 1830: 592

nebulosa, Dyctia, 1830: 694

nebulosa, Hyalomya, 1830: 300

nebulosa, Hydrotaea, 1830: 510

nebulosa, Myophora, 1863b: 511

nebulosa, Phaedima, 1863a: 844

nebulosa, Pitthaea, 1863a: 189

necrophaga, Lordatia, 1830: 809

neglecta, Faedoria, 1863a: 849

neglecta, Phryxe, 1863a: 393

nemorosa, Minettia, 1830: 647

nemorosa, Scatophaga, 1830: 625

nemorum, Phryxe, 1863a: 432

nervosa, Fausta, 1846b: 31

nicaeana, Echinomya, 1863a: 641

nicaeana, Onesia, 1863b: 546

nicaeensis, Dasyphora, 1863b: 653 [as "niceensis" 1863b: 876]

nigra, Egle, 1830: 591

nigra, Elomya, 1830: 297

nigra, Fausta, 1830: 63

nigra, Keratocera, 1830: 790

nigra, Mosina, 1830: 671

nigra, Mydina, 1830: 501

nigra, Myophora, 1830: 349

nigra, Panzeria, 1863a: 147

nigra, Phasia, 1830: 291

nigra, Phryxe, 1863a: 448

nigra, Phyto, 1830: 219

nigra, Solieria, 1849a: 472

nigra, Tachina, 1830: 195

nigribarbis, Panzeria, 1863a: 148

nigricans, Anthomya, 1830: 584

nigricans, Aricia, 1830: 488

nigricans, Mydina, 1830: 497

nigricans, Myophora, 1830: 360

nigricans, Sphora, 1830: 394

nigricauda, Erichsonia, 1863b: 494

nigricornis, Dyctia, 1830: 694

nigricornis, Echinomya, 1830: 45

nigricornis, Fausta, 1863a: 164 
nigricornis, Lycia, 1830: 639

nigricornis, Lydella, 1830: 115

nigricornis, Ptilocera, 1863b: 383

nigricornis, Salia, 1830: 110

nigricornis, Scatophaga, 1830: 627

nigricornis, Scyphella, 1830: 650

nigricornis, Trennia, 1830: 484

nigricruris, Hydrina, 1830: 795

nigrifacies, Limosia, 1830: 541

nigrifrons, Fellaea, 1830: 479

nigrifrons, Lucilia, 1863b: 719

nigrifrons, Mulsantia, 1863b: 563

nigrifrons, Phryxe, 1863a: 377

nigrifrons, Thryptocera, 1863a: 714

nigripalpis, Athrycia, 1863a: 835

nigripalpis, Hubneria, 1848a: 612

nigripalpis, Lypha, 1863a: 197

nigripalpis, Phormia, 1863b: 846

nigripalpis, Thryptocera, 1851d: 182

nigripalpis, Winthemia, 1847: 269

nigripennis, Rhinophora, 1830: 259

nigripennis, Stevenia, 1830: 221

nigripes, Carcelia, 1830: 180

nigripes, Fellaea, 1830: 477

nigripes, Helina, 1830: 494

nigripes, Hydrellia, 1830: 793

nigripes, Hylemya, 1830: 553

nigripes, Limosia, 1830: 541

nigripes, Marshamia, 1830: 58

nigripes, Melanosoma, 1853a: 125 [1853b: 45]

nigripes, Mydaea, 1830: 481

nigripes, Mydina, 1830: 498

nigripes, Phryno, 1830: 144

nigripes, Saltella, 1830: 747

nigrita, Afrellia, 1863a: 934

nigrita, Athrycia, 1863a: 832

nigrita, Aubaea, 1863a: 187

nigrita, Hubneria, 1848a: 607

nigrita, Masicera, 1863a: 875

nigrita, Phryxe, 1863a: 330

nigrita, Phryxe, 1863a: 443

nigrita, Sarcophaga, 1863b: 474

nigrita, Staegeria, 1863a: 983

nigriventris, Jurinia, 1863a: 659

nigrodorsata, Myopella, 1853a: 106 [1853b: 26]

nigrogrisescens, Phyto, 1830: 219

nitens, Aplomya, 1830: 185

nitens, Limosia, 1830: 538

nitens, Mydina, 1830: 500

nitens, Myobia, 1830: 100

nitens, Myophora, 1863b: 520

nitens, Phorocera, 1830: 134

nitens, Pollenia, 1863b: 664 nitens, Stevenia, 1830: 221

nitens, Zetterstedtia, 1863a: 1028

nitida, Ahrensia, 1863b: 17

nitida, Ateria, 1863a: 810

nitida, Blondelia, 1830: 122

nitida, Boisduvalia, 1830: 731

nitida, Catilia, 1830: 311

nitida, Dorbinia, 1847: 273

nitida, Egle, 1830: 586

nitida, Erynnia, 1830: 126

nitida, Euphoria, 1863b: 826

nitida, Feria, 1830: 310

nitida, Futilia, 1863a: 1051

nitida, Hubneria, 1848a: 611

nitida, Hyalomya, 1863b: 250

nitida, Hydrellia, 1830: 791

nitida, Hydrina, 1830: 794

nitida, Hydrotaea, 1830: 514

nitida, Leiophora, 1863a: 931

nitida, Limnophora, 1830: 521

nitida, Lupia, 1863a: 911

nitida, Lydella, 1830: 113

nitida, Lydina, 1830: 124

nitida, Megerlea, 1830: 267

nitida, Meigenia, 1830: 201

nitida, Micropeza, 1830: 743

nitida, Microptera, 1830: 212

nitida, Minella, 1830: 209

nitida, Myophora, 1863b: 510

nitida, Nerea, 1830: 803

nitida, Nyctia, 1830: 264

nitida, Ophyra, 1830: 516

nitida, Phaenicia, 1863b: 775

nitida, Platymya, 1863a: 192

nitida, Smidtia, 1863a: 295

nitida, Volusia, 1830: 674

nitida, Winthemya, 1863a: 214

nitidula, Euphoria, 1863b: 800

nitidula, Limosia, 1830: 542

nitidula, Lucilia, 1863b: 747

nitidula, Occemya, 1853a: 133 [1853b: 53]

nobilis, Meigenia, 1863a: 1091

nobilis, Oscinis, 1830: 713

nobilis, Purpurella, 1853a: 118 [1853b: 38]

nobilis, Tachina, 1863a: 965

nobilis, Winthemia, 1830: 175

noctuarum, Phorocera, 1830: 134

noctuarum, Phryxe, 1863a: 379

noctuarum, Tlephusa, 1863a: 309

nomada, Myocera, 1830: 330

notata, Phaenicia, 1863b: 786

notata, Phryxe, 1863a: 370

notata, Rondania, 1863a: 679 
notatum, Zodion, 1830: 251

nuda, Dufouria, 1830: 258

nudipes, Olina, 1830: 813

nudithorax, Micropalpus, 1863a: 125

nugax, Phryxe, 1863a: 422

nugax, Zelleria, 1863a: 987

nupta, Phryxe, 1863a: 383

nuptialis, Lucilia, 1863b: 749

nuptialis, Phaenicia, 1863b: 795

nymphaeae, Hydrophoria, 1830: 504

nymphaeae, Hydrotaea, 1830: 511

nymphaearum, Stagnia, 1830: 508

nymphaeicola, Hydrophoria, 1830: 506

nymphaeicola, Lispa, 1830: 524

objecta, Phryxe, 1863a: 365

oblata, Phryxe, 1863a: 434

obliqua, Gymnosoma, 1830: 237

obliqua, Nemoraea, 1830: 73

obliquata, Amobia, 1863b: 132

oblita, Phryxe, 1863a: 388

oblita, Phryxe, 1863a: 446

oblonga, Acemya, 1830: 202

oblonga, Phasia, 1830: 291

obscura, Araba, 1830: 130

obscura, Arina, 1830: 696

obscura, Azelia, 1830: 593

obscura, Limnophora, 1830: 519

obscura, Limosia, 1830: 539

obscura, Melieria, 1830: 716

obscura, Myophora, 1830: 357

obscura, Myophora, 1863b: 503

obscurata, Eretria, 1863a: 495

obscurata, Futilia, 1863a: 1062

obscurata, Melibaea, 1863a: 289

obscurata, Obeida, 1863b: 168

obscurata, Phryxe, 1863a: 453

obscurata, Stygina, 1863a: 799

obscurata, Winthemya, 1863a: 214

obscurella, Actia, 1851d: 187

obscurella, Lucilia, 1863b: 744

obscurella, Meigenia, 1863a: 1080

obscurella, Mollia, 1863a: 949

obscurella, Myophora, 1863b: 503

obscuricauda, Myophora, 1830: 354

obscuripalpis, Phaenicia, 1863b: 754

obscuripennis, Mintho, 1830: 217

obscuripennis, Myophora, 1830: 359

obscuripennis, Phasia, 1830: 293

obscuripes, Meigenia, 1863a: 1067

obscuripes, Solieria, 1863b: 334

obsequens, Phryxe, 1863a: 356

obsequiosa, Temesia, 1863a: 292

obtenta, Phryxe, 1863a: 404 occlusa, Anetia, 1863a: 868

occlusa, Atilia, 1863a: 475

occlusa, Blumia, 1863a: 468

occlusa, Calyptidia, 1863b: 60

occlusa, Eratia, 1863b: 238

occlusa, Opesia, 1863b: 278

occlusa, Staegeria, 1863a: 982

oceaniae, Calliphora, 1830: 438

oceaniae, Cynomya, 1830: 365

ocypteroidea, Brullaea, 1863a: 773

odyneri, Amobia, 1853c: 535

oenanthis, Bonnetia, 1830: 56

oestroidea, Crameria, 1830: 60

offensa, Phryxe, 1863a: 451

officiosa, Gimmenthalia, 1863a: 852

officiosa, Phryxe, 1863a: 359

olivacea, Futilia, 1863a: 1057

olivieri, Reaumuria, 1830: 82

onopordi, Forellia, 1830: 761

opaca, Hartigia, 1863b: 523

opaca, Meigenia, 1863a: 1078

opacina, Hyalomya, 1863b: 251

operata, Phryxe, 1863a: 351

operosa, Voria, 1863a: 827

opportuna, Phryxe, 1863a: 376

opposita, Phryxe, 1863a: 338

oralis, Adia, 1830: 558

oralis, Bercaea, 1863b: 554

oralis, Erichsonia, 1863b: 485

oralis, Pollenia, 1863b: 674

orgyae, Carcelia, 1863a: 237

orgyae, Phorocera, 1850a: 176

orgyae, Zenillia, 1850a: 168

ornithivora, Orbellia, 1830: 657

orthalidis, Phorocera, 1850a: 175

ostendata, Phryxe, 1863a: 378

ovata, Pallasia, 1830: 239

ovatrix, Lucilia, 1863b: 709

pabulina, Myophora, 1863b: 509

pabulina, Phryxe, 1863a: 455

pabulorum, Sarcophaga, 1863b: 441

pacifica, Meigenia, 1863a: 1084

pacifica, Phryxe, 1863a: 424

pacifica, Walkeria, 1863a: 1023

pagana, Eribea, 1863a: 1034

palesioidea, Phryxe, 1830: 160

palesioidea, Phryxe, 1863a: 337

pallens, Bengalia, 1830: 426

pallida, Dejeania, 1863a: 653

pallidicornis, Palusia, 1830: 544

pallidipalpis, Blondelia, 1830: 123

pallidipalpis, Lydella, 1830: 114

pallidipes, Hubneria, 1848a: 610 
pallidipes, Limosia, 1830: 542

pallidipes, Myophora, 1863b: 507

pallidula, Phumosia, 1830: 427

pallipes, Caricea, 1830: 533

pallipes, Culex, 1827: 410

pallipes, Egle, 1830: 588

pallipes, Hyalomya, 1863b: 264

pallipes, Lucilia, 1830: 461

pallipes, Melieria, 1830: 717

pallipes, Mycetia, 1830: 806

pallipes, Olina, 1830: 813

pallipes, Philinta, 1830: 569

pallipes, Potamia, 1830: 507

palpalis, Bessa, 1863b: 166

palpalis, Hydrotaea, 1830: 512

palpalis, Klugia, 1863a: 789

palpalis, Nemorhina, 1830: 390

palpalis, Phyto, 1830: 219

palpalis, Ptilocera, 1830: 222

palpalis, Solieria, 1863b: 326

palpalis, Theria, 1830: 337

palpalis, Wagneria, 1863a: 795

palpata, Blainvillia, 1830: 515

palpata, Nicaea, 1863b: 401

palpata, Phorophylla, 1830: 214

palpata, Terellia, 1830: 758

paludosa, Caricea, 1830: 532

paludosa, Dyctia, 1830: 694

paludosa, Limosia, 1830: 539

paludosa, Lispa, 1830: 526

paludosa, Myophora, 1863b: 500

paludosa, Pherbina, 1830: 691

paludosa, Tetanocera, 1830: 682

palustris, Cuculla, 1830: 523

palustris, Delia, 1830: 578

palustris, Dyctia, 1830: 694

palustris, Keratocera, 1830: 788

palustris, Limnophora, 1830: 518

palustris, Limosia, 1830: 541

palustris, Minettia, 1830: 647

palustris, Mydina, 1830: 502

paradoxa, Loxocera, 1830: 666

parasita, Miltogramma, 1830: 94

parasita, Scopolia, 1830: 270

parisiaca, Isomera, 1851f: 315

parisiaca, Lalage, 1863a: 561

parisiensis, Amina, 1830: 630

parisiensis, Trixa, 1863b: 374

parisiensis, Urellia, 1830: 775

parva, Egle, 1830: 590

parva, Meigenia, 1830: 201

parva, Morinia, 1830: 265

parva, Pierretia, 1863b: 427 parvula, Meigenia, 1863a: 1068

pascuorum, Myophora, 1863b: 505

pauperata, Meigenia, 1863a: 1088

pauperata, Phaenicia, 1863b: 766

pauperata, Phorella, 1863b: 531

pauperata, Phryxe, 1863a: 430

pauperata, Walkeria, 1863a: 1011

pavida, Melinda, 1863b: 698

pavida, Phryxe, 1863a: 345

pavida, Velocia, 1863a: 951

pavida, Zorella, 1863a: 918

pavoniae, Phryxe, 1830: 165

pedemontana, Peleteria, 1863a: 619

pedicellata, Etheria, 1863b: 275

pedicellatum, Zodion, 1830: 252

pediculariarum, Acinia, 1830: 776

peleterii, Dasyna, 1830: 668

peleterii, Leria, 1830: 655

peleterii, Odinia, 1830: 649

peleterii, Sepedon, 1830: 677

pellex, Mulsantia, 1863b: 575

pellucida, Elpigia, 1863b: 150

pellucida, Mydaea, 1830: 479

pellucida, Mydina, 1830: 500

pellucida, Myopa, 1830: 244

pellucida, Orillia, 1849a: 476

pellucida, Sophia, 1830: 318

penetrans, Culex, 1827: 407

pentatomae, Ocyptera, 1830: 229

peregrina, Myophora, 1830: 356

peronii, Lucilia, 1830: 460

peronii, Myophora, 1830: 341

peruviana, Calliphora, 1830: 438

peruviana, Lucilia, 1830: 455

pervia, Amobia, 1863b: 137

pervia, Futilia, 1863a: 1059

pervia, Scaligeria, 1863b: 480

petiolata, Dufouria, 1830: 258

petiolata, Eryphe, 1863a: 610

petiolata, Hebia, 1849a: 442

petiolata, Ida, 1863b: 389

petiolata, Ligeria, 1863a: 936

petiolata, Phyto, 1863b: 50

petrosa, Pales, 1830: 155

phantasma, Themira, 1830: 746

philadelphica, Meckelia, 1830: 715

philadelphica, Phormia, 1830: 466

philanthi, Araba, 1830: 129

phyllioidea, Chlorina, 1830: 603

picaena, Muscina, 1830: 408

picea, Echinomya, 1830: 44

picea, Megerlea, 1830: 267

picea, Spallanzania, 1830: 78 
picena, Limosia, 1830: 539

pictipennis, Myopa, 1830: 243

pieridis, Phorocera, 1850a: 179

pieridis, Phryxe, 1850a: 172

pilipennis, Actia, 1830: 86

pilipes, Alina, 1863b: 640

pilosa, Alophora, 1830: 295

pilosa, Erichsonia, 1863b: 483

pilosa, Servillia, 1830: 50

pilosa, Themira, 1830: 746

pilosula, Pollenia, 1863b: 674

placida, Carcelia, 1863a: 225

placida, Euphoria, 1863b: 834

placida, Phasia, 1863b: 218

placida, Phryxe, 1863a: 428

placida, Scotia, 1863a: 255

plaei, Chrysomya, 1830: 448

plantaginis, Phytomyza, 1851e: 404

plantaris, Acinia, 1830: 778

plumata, Euphemia, 1830: 486

plumipes, Peckia, 1830: 336

plumosula, Caricea, 1830: 532

plumosula, Delia, 1830: 575

plumosula, Phyllis, 1830: 604

plusiae, Carcelia, 1830: 180

podacina, Ceranthia, 1830: 89

polita, Bonellia, 1863a: 136

polita, Euphoria, 1863b: 801

polita, Phaenicia, 1863b: 792

populicola, Heramya, 1830: 710

potamogeti, Hydrophoria, 1830: 504

potamogeti, Lispa, 1830: 525

potamogeti, Stagnia, 1830: 509

potatoria, Adenia, 1863a: 1046

potatoria, Atilia, 1863a: 477

potatoria, Phryxe, 1863a: 445

potens, Servillia, 1863a: 645

praeceps, Atilia, 1863a: 478

praeceps, Lydella, 1863a: 861

praeceps, Mulsantia, 1863b: 562 [as “proeceps” 1863b: 892]

praeceps, Phryxe, 1863a: 367

praeceps, Scaligeria, 1863b: 480 [as “proeceps" 1863b: 908]

praecox, Amobia, 1863b: 138

praecox, Egle, 1830: 590

praecox, Euphoria, 1863b: 809

praecox, Gaedartia, 1863a: 567

praecox, Kirbya, 1863a: 818

praecox, Phryxe, 1863a: 371

praecox, Pierretia, 1863b: 423 [as “proecox" 1863b: 905]

praecox, Sarcophaga, 1863b: 465 [as “proecox” 1863b:

908] praecox, Stomoxis, 1863b: 608 [as “proecox” 1863b: 910]

praefixa, Phryxe, 1863a: 409

praegnata, Myobia, 1863b: 307 [as "proegnata” 1863b: 894]

praestans, Phaenicia, 1863b: 769

prasina, Lucilia, 1863b: 720

pratensis, Alina, 1863b: 640

pratensis, Clytia, 1830: 289

pratensis, Delia, 1830: 577

pratensis, Ensina, 1830: 752

pratensis, Euphemia, 1830: 485

pratensis, Gymnodia, 1863b: 635

pratensis, Hyalomya, 1863b: 256

pratensis, Ismenia, 1863a: 587

pratensis, Lucilia, 1863b: 717

pratensis, Meigenia, 1863a: 1090

pratensis, Myobia, 1863b: 309

pratensis, Phaenicia, 1863b: 772

pratensis, Phryxe, 1863a: 426

pratensis, Pollenia, 1863b: 668

pratensis, Sarcophaga, 1863b: 448

pratensis, Tachina, 1830: 194

praticola, Mulsantia, 1863b: 569

praticola, Myophora, 1863b: 502

pratorum, Hubneria, 1863a: 281

pretiosa, Lucilia, 1863b: 708

pretiosa, Phaenicia, 1863b: 767

primulae, Phytomyza, 1851e: 396

probata, Phryxe, 1863a: 452

proboscidea, Saphaea, 1830: 796

procax, Erichsonia, 1863b: 489

procax, Sarcophaga, 1863b: 453

procax, Zelleria, 1863a: 990

producta, Uramya, 1830: 216

profuga, Adenia, 1863a: 1046

profuga, Solieria, 1863b: 327

prompta, Caenis, 1863a: 676

prompta, Euphoria, 1863b: 807

prompta, Nerina, 1830: 558

prompta, Onesia, 1830: 370

prompta, Phaenicia, 1863b: 774

prompta, Phryxe, 1830: 162

prompta, Phyto, 1830: 219

properata, Phryxe, 1863a: 425

propinqua, Adenia, 1863a: 1043

propinqua, Phaenicia, 1863b: 765

propinqua, Sarcophaga, 1863b: 445

prorsae, Phorocera, 1830: 134

provida, Phryxe, 1863a: 355

provocatrix, Eretria, 1863a: 496

proxima, Phryxe, 1863a: 408

pruinosa, Meigenia, 1863a: 1083

pruinosa, Phaenicia, 1863b: 786 
pruinosa, Phryxe, 1863a: 448 pseudoacori, Sepedon, 1830: 677

pseudonarcissi, Norellia, 1830: 673

puberula, Eumea, 1863a: 304

pubescens, Lucilia, 1830: 454

pubescens, Ophyra, 1830: 516

pubescens, Pollenia, 1830: 416

pudica, Cynthia, 1863a: 769

puella, Carcelia, 1863a: 240

puella, Eribea, 1863a: 1040

puella, Euphoria, 1863b: 817

puella, Phryxe, 1863a: 380

puella, Tachina, 1863a: 966

pugnax, Zelleria, 1863a: 988

pulchella, Phaenicia, 1863b: 798

pulicaria, Melieria, 1830: 717

pulverulenta, Egle, 1830: 588

pulverulenta, Peleteria, 1863a: 615

pulverulenta, Solieria, 1849a: 471

pumicea, Phaenicia, 1863b: 765

pumila, Meigenia, 1863a: 1070

pumila, Peleteria, 1863a: 622

pumila, Pollenia, 1830: 414

pumila, Zetterstedtia, 1863a: 1026

punctata, Agria, 1830: 377

punctata, Dinera, 1830: 308

punctata, Echinomya, 1830: 48

punctata, Elpigia, 1863b: 150

punctata, Maculia, 1863b: 279

punctata, Ormia, 1830: 428

punctata, Palusia, 1830: 544

punctata, Phryxe, 1830: 167

punctata, Rohrella, 1830: 492

punctata, Sophia, 1830: 318

punctata, Sylvia, 1830: 636

punctatella, Limnophora, 1830: 521

punctatus, Cistogaster, 1863b: 193

puncticeps, Myopella, 1853a: 99 [1853b: 19]

punctigera, Myopella, 1853a: 102 [1853b: 22]

punctulata, Agria, 1830: 377

punctulata, Bonellia, 1863a: 136

pungens, Culex, 1827: 407

pungens, Stomoxis, 1830: 386

puparum, Erigone, 1830: 67

puparum, Eurithia, 1844a: 25

puparum, Masicera, 1863a: 881

pupivora, Gouraldia, 1851a: 151

pupivora, Phryxe, 1863a: 366

purpurea, Euphoria, 1863b: 835

purpurea, Hyalomya, 1863b: 244

purpurea, Lucilia, 1863b: 726

purpurea, Phaenicia, 1863b: 751

pusilla, Myrsina, 1863b: 159 pusilla, Nyctia, 1830: 264

pusilla, Phorocera, 1850a: 181

pusilla, Rhinophora, 1830: 260

pusilla, Syphona, 1830: 92

pusilla, Tephrytis, 1830: 766

putris, Bacchis, 1830: 804

putris, Nemopoda, 1830: 744

pygerae, Phorocera, 1830: 135

pygmaea, Dinera, 1830: 309

pygmaea, Elodia, 1863a: 939

pygmaea, Meigenia, 1863a: 1066

pygmaea, Micropeza, 1830: 743

pygmaea, Napaea, 1830: 800

pygmaea, Phryxe, 1863a: 429

pyralidis, Aubaea, 1863a: 186

pyrethri, Oxyphora, 1830: 757

pyrochroa, Phaenicia, 1863b: 754

pyrois, Phaenicia, 1863b: 757

pyropus, Lucilia, 1863b: 702

quadriguttata, Phryxe, 1863a: 378

quadrillum, Phryxe, 1863a: 439

quadrimaculata, Masicera, 1863a: 879

quadrimaculata, Zenais, 1863a: 912

quadrinotata, Phryxe, 1863a: 431

quadrinotata, Syphona, 1850b: 203

quadrisignata, Meigenia, 1863a: 1089

quaesita, Phryxe, 1863a: 336 [as “quoesita” 1863b: 904]

quatuorpunctata, Aricia, 1830: 489

quatuorpunctata, Lycia, 1830: 638

quatuorpunctata, Sylvia, 1830: 637

quieta, Futilia, 1863a: 1053

quieta, Lucilia, 1863b: 708

quieta, Meigenia, 1863a: 1077

quieta, Phryxe, 1863a: 433

quieta, Walkeria, 1863a: 1021

ramburii, Sarcophaga, 1863b: 466

ranunculi, Phytomyza, 1851e: 401

rapax, Zelleria, 1863a: 986

rapida, Carcelia, 1830: 180

rapida, Clelia, 1830: 255

rapida, Feburia, 1830: 257

rapida, Gesneria, 1830: 379

rapida, Mydaea, 1830: 480

rapida, Myophora, 1830: 360

rapida, Phorocera, 1830: 132

rapida, Phryxe, 1863a: 428

rapida, Tachina, 1830: 187

reaumurii, Urophora, 1830: 770

rectella, Phryxe, 1863a: 357

rectinervis, Baumhaueria, 1863a: 759

rectinervis, Myobia, 1863b: 307

rectinervis, Orillia, 1849a: 475

rectinervis, Orthellia, 1863b: 837 
rectinervis, Phorcida, 1863a: 253

reflexa, Besseria, 1830: 233

reflexa, Myopina, 1830: 676

regalis, Chrysomya, 1830: 449

reginaldi, Mulsantia, 1863b: 574

regula, Curtisia, 1849a: 441

regula, Walkeria, 1863a: 1006

relata, Phryxe, 1863a: 421

repanda, Lydella, 1863a: 859

retusa, Phryxe, 1863a: 439

riparia, Caricea, 1830: 533

riparia, Delia, 1830: 572

riparia, Gesneria, 1830: 380

riparia, Hydrotaea, 1830: 512

riparia, Limosia, 1830: 537

riparia, Lispa, 1830: 525

riparia, Melina, 1830: 695

riparia, Musca, 1830: 398

riparia, Myophora, 1830: 345

riparia, Myophora, 1863b: 517

riparia, Nerea, 1830: 802

riparia, Onesia, 1830: 366

riparia, Paykullia, 1830: 271

ripicola, Limosia, 1830: 539

rivularis, Aminta, 1830: 571

rivularis, Limnophora, 1830: 519

rivularis, Limosia, 1830: 538

rivularis, Musca, 1863b: 627

rivularis, Nupharia, 1830: 785

robinaldi, Phytomyza, 1851e: 405

rohrelliformis, Phyllis, 1830: 604

rostrata, Calliphora, 1830: 439

rostrata, Idia, 1830: 421

rostrata, Peronia, 1830: 517

rostrata, Zelia, 1830: 315

rostrellum, Lucilia, 1830: 460

rotundata, Phryxe, 1830: 160

rubescens, Echinomya, 1830: 46

rubescens, Feria, 1830: 310

rubescens, Melanophora, 1830: 273

rubescens, Morinia, 1830: 265

rubescens, Myophora, 1830: 359

rubescens, Nyctia, 1830: 264

rubescens, Platymya, 1830: 117

rubescens, Tachina, 1830: 188

rubetra, Herbina, 1830: 699

rubetra, Latreillia, 1863a: 574

rubetra, Ptilocera, 1863b: 382

rubicunda, Hydromya, 1830: 692

rubida, Dumerillia, 1830: 39

rubida, Elomya, 1863b: 230

rubidus, Culex, 1827: 404

rubiginosa, Bonellia, 1830: 57 rubiginosa, Myophora, 1830: 343

rubrella, Aplomya, 1863a: 466

rubrella, Carcelia, 1830: 179

rubrella, Melieria, 1830: 716

rubrella, Phaenicia, 1863b: 768

rubrella, Phryxe, 1863a: 390

rubricornis, Bellieria, 1863b: 433

rubricornis, Echinomya, 1830: 46

rubricornis, Lucilia, 1863b: 722

rubricornis, Orizia, 1863b: 679

rubricornis, Paykullia, 1830: 270

rubricornis, Stomina, 1830: 411

rubrifacies, Onesia, 1863b: 544

rubrifrons, Ceromya, 1830: 87

rubrifrons, Fellaea, 1830: 478

rubrifrons, Futilia, 1863a: 1056

rubrifrons, Myopa, 1830: 247

rubrifrons, Stomoxis, 1863b: 606

rubripes, Limosia, 1830: 536

rubripes, Macquartia, 1830: 205

rufella, Orizia, 1863b: 679

ruficauda, Eversmania, 1863a: 182

ruficornis, Asbella, 1863b: 399

ruficornis, Phegea, 1863a: 909

ruficrus, Myobia, 1830: 99

rufipes, Herina, 1830: 727

rufipes, Scopolia, 1830: 269

rufiventris, Micropalpus, 1863a: 124

rumicis, Pegomya, 1851c: 232

rupestris, Scopolia, 1830: 269

ruralis, Arabella, 1863b: 93

ruralis, Clytia, 1863b: 285

ruralis, Erythrocera, 1863a: 601

ruralis, Euphoria, 1863b: 823

ruralis, Exorista, 1863a: 266

ruralis, Gesneria, 1863b: 599

ruralis, Myobia, 1863b: 314

ruralis, Phaedima, 1863a: 846

ruralis, Phryxe, 1863a: 373

ruralis, Zenillia, 1863a: 473

ruricola, Sarcophaga, 1863b: 472

rustica, Aricia, 1830: 487

rustica, Blissonia, 1863b: 649

rustica, Erichsonia, 1863b: 488

rustica, Gesneria, 1863b: 599

rustica, Helina, 1830: 495

rustica, Hubneria, 1848a: 608

rustica, Hyalomya, 1863b: 252

rustica, Hylemya, 1830: 553

rustica, Musca, 1863b: 622

rustica, Myophora, 1830: 348

rustica, Phorbia, 1830: 559

rustica, Phryno, 1830: 143 
rustica, Phryxe, 1863a: 335

rustica, Pollenia, 1863b: 663

rustica, Soleria, 1849a: 464

rustica, Tachina, 1830: 189

rusticana, Bonellia, 1863a: 137

rusticana, Miltogramma, 1863b: 118

rusticana, Pierretia, 1863b: 424

rusticana, Staegeria, 1863a: 982

rutila, Phaenicia, 1863b: 782

rutilans, Boisduvalia, 1830: 730

rutilans, Ophyra, 1830: 516

rutilus, Micropalpus, 1863a: 126

sabulosa, Anicia, 1863b: 99

sabulosa, Phryxe, 1830: 159

saepium, Phryxe, 1863a: 455 [as “soepium” 1863b: 904]

sagax, Zelleria, 1863a: 992

sagittariae, Hydrophoria, 1830: 505

sagittariae, Palusia, 1830: 544

saltatoria, Richardia, 1830: 728

saltatrix, Fannia, 1830: 567

saltatrix, Salticella, 1830: 684

saphyrea, Pyrellia, 1863b: 843

sapphirea, Lucilia, 1830: 461

saturniae, Baumhaueria, 1863a: 757

saturniae, Scotia, 1863a: 256

schistacea, Eribea, 1863a: 1038

schistacea, Erycia, 1863a: 905

schistella, Staegeria, 1863a: 978

scintilla, Euphoria, 1863b: 825

scintilla, Lucilia, 1863b: 749

scintilla, Phaenicia, 1863b: 752

scintilla, Pyrellia, 1863b: 842

scirpi, Phrosia, 1830: 669

scirporum, Limosia, 1830: 536

scolopendri, Phytomyza, 1851e: 402

scorzonerae, Ensina, 1830: 753

scorzonerae, Sitarea, 1830: 764

scutellaris, Amedea, 1830: 207

scutellaris, Brachyopa, 1843: 317 [1844b: 39]

scutellaris, Carcelia, 1830: 181

scutellaris, Erigone, 1830: 66

scutellaris, Erycia, 1830: 147

scutellaris, Erythrocera, 1849a: 438

scutellaris, Euphoria, 1863b: 814

scutellaris, Fausta, 1830: 63

scutellaris, Harrisia, 1830: 324

scutellaris, Herina, 1830: 726

scutellaris, Himera, 1863a: 1125

scutellaris, Jurinia, 1830: 36

scutellaris, Lucilia, 1863b: 716

scutellaris, Lydella, 1830: 115

scutellaris, Mydaea, 1830: 480

scutellaris, Myopella, 1853a: 108 [1853b: 28] scutellaris, Myostoma, 1830: 327

scutellaris, Phaenicia, 1863b: 772

scutellaris, Phoraea, 1830: 601

scutellaris, Phorcida, 1863a: 252

scutellaris, Phorocera, 1830: 133

scutellaris, Phorocera, 1863a: 240

scutellaris, Phryxe, 1830: 162

scutellaris, Tachina, 1830: 188

scutellaris, Walkeria, 1863a: 1004

scutellata, Nemoraea, 1830: 73

scutellata, Phryxe, 1830: 166

secutrix, Bessa, 1863b: 165

secutrix, Hyalomya, 1863b: 264

secutrix, Phryxe, 1863a: 382

sedula, Erigone, 1863a: 153

sedula, Phryxe, 1863a: 376

selecta, Phryxe, 1863a: 432

semiaurata, Euphoria, 1863b: 810

senilis, Phryxe, 1863a: 355

sepedonoidea, Chione, 1830: 680

sepium, Smidtia, 1863a: 296

sequax, Zelleria, 1863a: 993

serena, Phryxe, 1863a: 406

sericea, Carcelia, 1863a: 224

sericea, Leucophora, 1830: 563

sericea, Myophora, 1830: 352

sericea, Phorocera, 1863a: 515

sericeus, Micropalpus, 1863a: 126

serrata, Haematobia, 1830: 389

serva, Amyclaea, 1863b: 404

serva, Phryxe, 1863a: 388

servillei, Nyctia, 1830: 263

servillii, Aphria, 1830: 90

servillii, Phryxe, 1830: 159

servula, Staegeria, 1863a: 977

setosa, Lydella, 1863a: 862

severa, Phryxe, 1863a: 420

sexmaculata, Ptilopsis, 1863b: 273

sexnotata, Suillia, 1830: 644

sexpunctata, Lycia, 1830: 638

sicula, Rhedia, 1830: 76

sicula, Zenais, 1830: 149

siculus, Culex, 1827: 406

signata, Phryxe, 1863a: 330

silvatica, Egeria, 1830: 555

silvatica, Erinia, 1863a: 467

silvatica, Eugenia, 1863a: 920

silvatica, Hersilia, 1863a: 502

silvatica, Lypha, 1830: 142

silvatica, Meriania, 1830: 70

silvatica, Myophora, 1863b: 519

silvatica, Myoris, 1830: 711

silvatica, Pollenia, 1863b: 664 
silvatica, Roeselia, 1830: 146

silvestris, Duponchelia, 1863a: 531

silvestris, Latreillia, 1830: 107

silvestris, Linnaemya, 1830: 53

silvestris, Phryxe, 1863a: 401

silvestris, Tachina, 1830: 194

silvestris, Zenais, 1830: 149

silvicola, Chloe, 1830: 561

silvicola, Hylemya, 1830: 551

similis, Ocyptera, 1830: 230

similis, Phryxe, 1830: 168

simplex, Hubneria, 1863a: 285

sinensis, Herina, 1830: 724

sinophtalma, Moretia, 1863b: 367

siphonoidea, Erythrocera, 1849a: 439

sistoria, Hartigia, 1863b: 522

smaragda, Euphoria, 1863b: 802

smaragdula, Lucilia, 1830: 462

smaragdula, Pyrellia, 1863b: 843

sobria, Phryxe, 1863a: 369

socia, Chrysomya, 1830: 447

socia, Hartigia, 1863b: 526

socia, Phryxe, 1863a: 354

socialis, Lucilia, 1863b: 724

sodalis, Phryxe, 1863a: 367

solata, Phryxe, 1863a: 427

solers, Lucilia, 1863b: 734

solers, Phaedima, 1863a: 847

solers, Phryxe, 1863a: 379

solidaginis, Calobata, 1830: 739

sollicitata, Mulsantia, 1863b: 574

solstitialis, Urophora, 1830: 769

sonans, Carcelia, 1863a: 234

sonchi, Phytomyza, 1851e: 400

sonchi, Urophora, 1830: 771

sonora, Carcelia, 1863a: 226

sophia, Linnaemya, 1830: 53

sordida, Hartigia, 1863b: 527

sordida, Myophora, 1830: 350

sordida, Sarcophaga, 1863b: 474

sordida, Zetterstedtia, 1863a: 1025

soror, Anthomya, 1830: 583

soror, Calobata, 1830: 739

soror, Chrysomya, 1830: 450

soror, Delia, 1830: 578

soror, Euphoria, 1863b: 815

soror, Limnophora, 1830: 520

soror, Lucilia, 1830: 455

soror, Melinda, 1830: 441

soror, Musca, 1830: 399

soror, Mydina, 1830: 501

soror, Myophora, 1830: 347

soror, Pherbina, 1830: 690 soror, Rohrella, 1830: 491

soror, Tachina, 1830: 191

sororella, Phryxe, 1863a: 381

sortita, Phryxe, 1863a: 396

spathulata, Elfia, 1850b: 190

specialis, Myophora, 1830: 356

spectabilis, Lucilia, 1863b: 707

sphingivora, Phryxe, 1830: 164

spinulosa, Zetterstedtia, 1863a: 1029

spitzbergensis, Calliphora, 1830: 435

spondylii, Phytomyza, 1851e: 400

sponsa, Bercaea, 1863b: 556

sponsa, Winthemya, 1863a: 209

spreta, Adenia, 1863a: 1042

spreta, Mulsantia, 1863b: 575

spreta, Phryxe, 1863a: 333

squalens, Myophora, 1830: 356

squalens, Phormia, 1830: 468

squalens, Pierretia, 1863b: 426

squalida, Myocera, 1863b: 396

squalida, Myophora, 1830: 342

squalida, Phorella, 1830: 362

squamigera, Myophora, 1830: 352

squamipallens, Araba, 1830: 130

squamosa, Myophora, 1830: 351

stagnalis, Limosia, 1830: 538

stagnicola, Keratocera, 1830: 789

stagnicola, Napaea, 1830: 799

stercoraria, Lordatia, 1830: 809

stercoraria, Nemopoda, 1830: 745

stercoraria, Nerea, 1830: 803

stigma, Dalmannia, 1830: 250

stimulans, Byomya, 1830: 393

stimulata, Phryxe, 1863a: 406

stomoxidea, Musca, 1830: 396

strenua, Bercaea, 1863b: 549

strenua, Hartigia, 1863b: 521

strenua, Hylemya, 1830: 550

strenua, Pales, 1847: 283

strenua, Pollenia, 1863b: 657

strenua, Servillia, 1863a: 649

strenua, Zelia, 1830: 315

stygina, Phryxe, 1863a: 443

subaenea, Hubneria, 1848a: 606

subglabra, Phorcida, 1863a: 252

sublutea, Myobia, 1830: 99

submetallica, Pollenia, 1863b: 676

subnitens, Myophora, 1830: 358

subpilosa, Servillia, 1830: 50

subrotunda, Acemya, 1830: 202

subrotunda, Myophora, 1830: 357

subrotunda, Phorostoma, 1830: 327

subrotundata, Phryxe, 1830: 161 
subterranea, Leria, 1830: 655 subtilis, Futilia, 1863a: 1052 subtilis, Phaenicia, 1863b: 780 subtilis, Phryxe, 1863a: 335 sugillatrix, Plaxemya, 1830: 392 sugillatrix, Stomoxis, 1830: 386 suillioidea, Herbina, 1830: 698 suillorum, Mydaea, 1830: 480 suillorum, Terenia, 1830: 641 sulcata, Ravinia, 1863b: 437 sumptuosa, Lucilia, 1863b: 748 sumptuosa, Phaenicia, 1863b: 789 superba, Phryxe, 1830: 164 susurrans, Carcelia, 1863a: 231 sylvatica, Syphona, 1850b: 208 syrphoidea, Idia, 1830: 421 tandrec, Theresia, 1830: 326 tardata, Tachina, 1863a: 968 tarsalis, Caricea, 1830: 534 tarsalis, Lispa, 1830: 526 tasmanica, Carcelia, 1863a: 240 temeraria, Lylibaea, 1863a: 551 temeraria, Myophora, 1863b: 519 temeraria, Phryxe, 1863a: 345 temerata, Futilia, 1863a: 1060 tenax, Zelleria, 1863a: 989 tenebricosa, Mulsantia, 1863b: 560 tenebricosa, Phryxe, 1863a: 375 tenebricosa, Sarcophaga, 1863b: 450 tenella, Hartigia, 1863b: 529 tenera, Phaenicia, 1863b: 781 tepida, Lucilia, 1863b: 736 teres, Futilia, 1863a: 1050 teres, Onesia, 1863b: 542 teres, Phaenicia, 1863b: 785 terminalis, Arge, 1863a: 183 terminalis, Exorista, 1863a: 261 terminata, Lydella, 1863a: 860 terraenovae, Phormia, 1830: 467 tessellans, Bonellia, 1830: 56 tessellans, Erigone, 1830: 67 tessellata, Delia, 1830: 576 tessellata, Nitellia, 1863b: 683 tessellata, Onesia, 1830: 369 tessellata, Phasia, 1863b: 214 tessellata, Rhinophora, 1830: 259 tessellata, Sarcophaga, 1863b: 467 testacea, Bengalia, 1830: 426 testacea, Boisduvalia, 1830: 730 testacea, Ceromya, 1830: 88 testacea, Echinomya, 1830: 48 testacea, Egeria, 1830: 556 testacea, Hydrophoria, 1830: 506 testacea, Jurinia, 1830: 38 testacea, Latreillia, 1830: 106 testacea, Minettia, 1830: 647 testacea, Myobia, 1830: 275 testacea, Myobia, 1849a: 458 testacea, Oblicia, 1830: 620 testacea, Palpostoma, 1830: 429 testacea, Palusia, 1830: 544 testacea, Philinta, 1830: 569 testacea, Rhedia, 1830: 75 testacea, Rhinia, 1830: 423 testacea, Syphona, 1850b: 207 testacea, Solieria, 1863b: 319 testacea, Winthemya, 1863a: 216 tetrella, Myophora, 1863b: 513 thamnicola, Oscinis, 1830: 712 thapsi, Phytomyza, 1851e: 398 thoracica, Bonellia, 1863a: 139 thoracica, Chlorina, 1830: 603 thoracica, Delphinia, 1830: 720 thoracica, Herina, 1830: 725 thoracica, Lycia, 1830: 639 thoracica, Micropeza, 1830: 742 thoracica, Phaenicia, 1863b: 781 thoracica, Scatophaga, 1830: 626 thoracicum, Phantasma, 1830: 740 thoracicus, Culex, 1827: 409 tibialis, Aciura, 1830: 773 tibialis, Azelia, 1830: 594 tibialis, Boisduvalia, 1830: 731 tibialis, Chrysomya, 1830: 446 tibialis, Conomya, 1830: 384 tibialis, Culex, 1827: 404 tibialis, Delina, 1830: 669 tibialis, Estheria, 1830: 306 tibialis, Euphemia, 1830: 486 tibialis, Eurigaster, 1849a: 435 tibialis, Euryclea, 1863a: 291 tibialis, Fausta, 1863a: 164 tibialis, Gaedartia, 1863a: 566 tibialis, Haematobia, 1830: 389 tibialis, Helina, 1830: 494 tibialis, Herbstia, 1851d: 185 tibialis, Hydrophoria, 1830: 505 tibialis, Hylemya, 1830: 552 tibialis, Macquartia, 1863a: 1111 tibialis, Mycetia, 1830: 806 tibialis, Talmonia, 1863a: 705 timida, Lydella, 1863a: 864 timida, Phryxe, 1863a: 395 timorensis, Lucilia, 1830: 460 timorensis, Reaumuria, 1830: 81 tiphaecola, Phryxe, 1830: 165 
tomentosa, Lucilia, 1863b: 719

tomentosa, Lylibaea, 1863a: 552

tomentosa, Pollenia, 1830: 416

tomentosa, Stevenia, 1830: 220

tranquilla, Aetylia, 1863a: 271

tranquilla, Phryxe, 1863a: 407

transita, Phryxe, 1863a: 436

trapae, Hydrophoria, 1830: 505

trapae, Keratocera, 1830: 789

trapae, Limnophora, 1830: 520

tremula, Carcelia, 1863a: 235

tremula, Phryxe, 1863a: 369

trepida, Lilaea, 1863b: 162

trepida, Phryxe, 1863a: 370

trifaria, Nyctia, 1830: 263

trifaria, Theone, 1863b: 402

trinotata, Odinia, 1830: 648

tristis, Caricea, 1830: 534

tristis, Hartigia, 1863b: 527

tristis, Medina, 1830: 140

tristis, Mulsantia, 1863b: 564

tristis, Oppia, 1863a: 311

tristis, Phryxe, 1863a: 389

tristis, Pitthaea, 1863a: 190

tristis, Sarcophaga, 1863b: 463

tristis, Syphona, 1850b: 203

triumphalis, Euphoria, 1863b: 834

tuberivora, Suillia, 1830: 642

umbellatarum, Delia, 1830: 579

umbraculata, Lycia, 1830: 639

umbrarum, Scatophaga, 1830: 626

umbripennis, Erichsonia, 1863b: 489

umbripennis, Myopa, 1830: 243

umbrosa, Phaenicia, 1863b: 790

unifasciata, Latreillia, 1830: 105

urbana, Phryxe, 1863a: 435

urens, Lucilia, 1863b: 742

urinaria, Scatella, 1849b: 94 [1849e: xciv]

usta, Lucilia, 1830: 456

usta, Pyrellia, 1830: 463

usta, Stygina, 1863a: 800

vaccina, Musca, 1863b: 625

vafra, Phryxe, 1863a: 403

vaga, Clytia, 1830: 289

vaga, Mulsantia, 1863b: 571

vaga, Myobia, 1849a: 457

vaga, Myocera, 1863b: 397

vaga, Myophora, 1863b: 504

vaga, Phaenicia, 1863b: 768

vaga, Phryxe, 1863a: 436

vagans, Myophora, 1863b: 516

vagans, Sarcophaga, 1863b: 463

vagans, Tachina, 1830: 187 vagatoria, Musca, 1830: 399

vagatoria, Phaonia, 1830: 483

vagus, Micropalpus, 1863a: 127

valida, Erichsonia, 1863b: 482

valida, Lucilia, 1863b: 732

valida, Phryxe, 1863a: 354

valida, Thelaira, 1863a: 776

valida, Zelleria, 1863a: 994

vanessae, Erycia, 1850a: 170

vanessae, Phryxe, 1850a: 171

vanessae, Sturmia, 1830: 172

varensis, Musca, 1863b: 627

varensis, Pollenia, 1863b: 663

variabilis, Amesia, 1863b: 364

varinervis, Erichsonia, 1863b: 491

velox, Carcelia, 1830: 182

velox, Eretria, 1863a: 494

velox, Eribea, 1863a: 1036

velox, Herilla, 1863a: 317

velox, Morinia, 1830: 265

velox, Onesia, 1830: 370

velox, Phorocera, 1830: 137

velox, Phryxe, 1830: 168

velox, Salia, 1830: 108

velox, Sarcophaga, 1863b: 459

velox, Stevenia, 1830: 221

velox, Zelia, 1830: 316

velutina, Hydrotaea, 1830: 513

ventralis, Micropalpus, 1863a: 125

ventralis, Walkeria, 1863a: 1015

venusta, Euphoria, 1863b: 804

venusta, Lucilia, 1863b: 727

venusta, Phaenicia, 1863b: 774

verax, Zelleria, 1863a: 986

vernalis, Azelia, 1830: 594

vernalis, Bellardia, 1863b: 548

vernalis, Carcelia, 1830: 178

vernalis, Clytia, 1863b: 285

vernalis, Cyzenis, 1863a: 545

vernalis, Dalmania, 1853a: 149 [1853b: 69]

vernalis, Delia, 1830: 576

vernalis, Echinomya, 1830: 48

vernalis, Etheria, 1863b: 275

vernalis, Haematobia, 1863b: 610

vernalis, Hydrina, 1830: 795

vernalis, Kirbya, 1830: 268

vernalis, Lucilia, 1863b: 724

vernalis, Meigenia, 1830: 200

vernalis, Mydina, 1830: 498

vernalis, Myophora, 1830: 356

vernalis, Pales, 1830: 156

vernalis, Peleteria, 1863a: 617

vernalis, Pherbellia, 1830: 696 
vernalis, Phorocera, 1830: 137

vernalis, Phryxe, 1863a: 411

vernalis, Pierretia, 1863b: 426

vernalis, Pollenia, 1830: 415

vernalis, Rhedia, 1830: 75

vernalis, Smidtia, 1830: 183

vernalis, Zenillia, 1863a: 473

vernalis, Zetterstedtia, 1863a: 1026

versicolor, Jurinia, 1863a: 662

vesana, Futilia, 1863a: 1058

vesana, Phaedima, 1863a: 845

vesana, Phryxe, 1863a: 392

vespertilionea, Thelida, 1842a: 262

vestita, Hyalomya, 1863b: 249

viarum, Onesia, 1830: 367

viarum, Phaonia, 1830: 482

viarum, Winthemia, 1830: 175

viatica, Delia, 1830: 574

viatica, Meigenia, 1863a: 1082

viatica, Pollenia, 1830: 413

viatica, Scopolia, 1830: 269

viatrix, Erichsonia, 1863b: 495

vicina, Calliphora, 1830: 435

vicina, Delia, 1830: 572

vicina, Estheria, 1830: 307

vicina, Limnophora, 1830: 520

vicina, Lucilia, 1830: 456

vicina, Pherbina, 1830: 689

vicina, Phryxe, 1863a: 361

vicina, Rhedia, 1830: 76

vicina, Solieria, 1849a: 466

vicina, Tachina, 1830: 193

victrix, Lucilia, 1863b: 704

vigil, Adenia, 1863a: 1045

vigil, Phryxe, 1863a: 413

vigilans, Arabella, 1863b: 92

villana, Clytia, 1863b: 286

villana, Hartigia, 1863b: 524

villana, Miltogramma, 1863b: 118

villana, Myobia, 1849a: 459

villana, Myophora, 1830: 351

villana, Phaonia, 1830: 484

villana, Phryxe, 1863a: 343

villana, Staegeria, 1863a: 980

villana, Theone, 1863b: 402

villica, Erycia, 1830: 147

villica, Macquartia, 1863a: 1111

villica, Myophora, 1830: 340

villica, Phryxe, 1830: 161

villica, Solieria, 1863b: 323

villica, Tachina, 1830: 188

villosa, Calliphora, 1830: 437

villosa, Plagia, 1863a: 824 villosa, Prionella, 1830: 760

villosus, Anopheles, 1827: 411

vinosa, Phryxe, 1863a: 421

vinulae, Winthemia, 1830: 176

violacea, Byomya, 1830: 393

violacea, Herina, 1830: 724

violacea, Melanophora, 1830: 272

violacea, Pyrellia, 1830: 463

violaceus, Culex, 1827: 403

violacina, Lucilia, 1863b: 729

violacina, Pollenia, 1863b: 675

violascens, Herina, 1830: 727

violascens, Suillia, 1830: 645

virescens, Reaumuria, 1863a: 741

virgo, Lucilia, 1830: 458

virgo, Meigenia, 1863a: 1073

virgo, Phryxe, 1863a: 446

virgo, Sarcophaga, 1863b: 469

viridana, Lucilia, 1863b: 742

viridana, Macquartia, 1863a: 1104

viridescens, Calliphora, 1830: 437

viridescens, Egle, 1830: 589

viridescens, Fausta, 1830: 64

viridescens, Hydrellia, 1830: 793

viridescens, Keratocera, 1830: 790

viridescens, Lucilia, 1830: 458

viridescens, Macquartia, 1830: 206

viridescens, Myophora, 1830: 342

viridescens, Ophyra, 1830: 517

viridescens, Pales, 1830: 156

viridicyanea, Euphoria, 1863b: 816

viridicyanea, Onesia, 1830: 368

viridis, Culex, 1827: 410

viridis, Euphoria, 1863b: 801

viridis, Lucilia, 1863b: 748

viridula, Chrysomya, 1830: 445

viridula, Dasyphora, 1830: 410

viridula, Euphoria, 1863b: 805

viridula, Hydrellia, 1830: 792

viridula, Hydrina, 1830: 795

viridula, Onesia, 1830: 369

viridula, Phaenicia, 1863b: 793

viridulans, Erigone, 1830: 68

viridulans, Onesia, 1830: 369

viridulans, Phaenicia, 1863b: 770

viridulans, Rivellia, 1830: 729

vittata, Aricia, 1830: 489

vittata, Caricea, 1830: 532

vittata, Phormia, 1830: 467

viva, Phaenicia, 1863b: 759

vivax, Guerinia, 1830: 197

vivax, Zelleria, 1863a: 991

vivida, Amobia, 1863b: 136 
vivida, Camilla, 1863b: 642

vivida, Ceromya, 1850b: 196

vivida, Eumea, 1863a: 303

vivida, Euphoria, 1863b: 807

vivida, Euryclea, 1863a: 291

vivida, Hubneria, 1848a: 602

vivida, Hylemya, 1830: 552

vivida, Masicera, 1863a: 874

vivida, Micropeza, 1830: 742

vivida, Myophora, 1830: 358

vivida, Nyctia, 1863a: 796

vivida, Pollenia, 1830: 413

vivida, Pyrellia, 1830: 463

vivida, Walkeria, 1863a: 1015

volatilis, Phryxe, 1863a: 362

volucris, Arenia, 1863b: 403

volucris, Drino, 1863a: 250

volucris, Euphoria, 1863b: 818

volucris, Zetterstedtia, 1863a: 1030

vomiturationis, Mydaea, 1849c: 157 [1849g: xviii]

vulgaris, Athrycia, 1863a: 834 vulgaris, Caricea, 1830: 532

vulgaris, Delia, 1830: 579

vulgaris, Egle, 1830: 584

vulgaris, Mycetia, 1830: 806

vulgaris, Onesia, 1830: 367

vulnerans, Stomoxis, 1863b: 605

vulpina, Graphomya, 1830: 404

vulpina, Leria, 1842a: 258

westermanni, Brachelia, 1830: 62

winthemi, Erycia, 1830: 148

winthemi, Guerinia, 1830: 197

winthemi, Medina, 1830: 140

xylostei, Phytomyza, 1851e: 398

zetterstedti, Melanosoma, 1853a: 126 [1853b: 46]

zetterstedtii, Dinera, 1863b: 353

zetterstedtii, Sarcophaga, 1863b: 439

zonaria, Melibaea, 1848a: 617

zonata, Aplomya, 1830: 185

zonata, Phryxe, 1830: 159

zonata, Stylophora, 1830: 723 


\section{Acknowledgments}

First and foremost we wish to thank Chris Thompson for his unstinting and unselfish work over several decades in conceiving, designing, and building the Biosystematic Database of World Diptera, which has become an indispensable resource for the entire community of dipterists and for many outside it, and which provided the initial stimulus for the present review. We thank the following for their help during this study: Shannon Henderson checked the accuracy of every name in the species index and cleaned and OCRed the Blainville Rapport. Steve Gaimari checked the acalypterate entries and added the fun fact regarding the origin of the genus-group name Frendelia Collin. Pierfilippo Cerretti and Hans-Peter Tschorsnig helped with Kirbya references. Daniel Whitmore and Knut Rognes kindly gave their opinion of the identity of Tilesia RobineauDesvoidy. Darren Mann generously scanned the J.O. Westwood copy of the Blainville Rapport, making it available for wider use. Eckhard Groll helped with dates of receipt of journals published by the Senckenberg Deutsches Entomologisches Institute. ACP thanks Mme Marguerite Boivin of Saint-Sauveur-en-Puisaye for a fascinating discussion of Robineau-Desvoidy during ACP's brief visit to Saint-Sauveur in 1997, and Benedictus Ceusters, the proprietor of "Les Renards" at that time, for his enthusiastic interest in Robineau-Desvoidy, for information about the house and its grounds, and for the photographs reproduced in Figs. 4-5. We also thank Christophe Daugeron for current information on the Robineau-Desvoidy collection of Diptera and Marcía Couri for the photographs of the Robineau-Desvoidy collection (Figs. 12-13). Last but not least we thank our reviewers (David McAlpine, Verner Michelsen, Monty Wood) whose input has led to many improvements in the quality and presentation of this paper.

\section{References}

As accurate as possible dates of publication were searched for and are listed in square brackets after the citation. If not annotated otherwise, they derive from the publication itself. Where no date other than the year could be found, the date is listed as "31 December+" to distinguish from actual 31 December publication dates. In the same fashion, if no date other than the month could be found, it should be construed as being available on the last day of that month until better evidence of publication issuance can be found.

Agassiz, L. (1846a) Nomina systematica generum Dipterorum, tam viventium quam fossilium, secundum ordinem alphabeticum disposita, adjectis auctoribus, libris in quibus reperiuntur, anno editionis, etymologia et familiis ad quas pertinent, [Pt. 4], [vi] + 42 pp. In: Agassiz, L., Nomenclator zoologicus continens nomina systematica generum animalium tam viventium quam fossilium, secundum ordinem alphabeticum disposita, adjectis auctoribus, libris, in quibus reperiuntur, anno editionis, etymologia et familias, ad quas pertinent, in singulis classibus. Fasc. IX/X: Titulum et praefationem operis, Mollusca, Lepidoptera, Strepsiptera, Diptera, Myriapoda, Thysanura, Thysanoptera, Suctoria, Epizoa et Arachnidas. Jent \& Gassman, Soloduri [= Solothurn, Switzerland]. [before 25 November]

[Recorded in the 25 November issue of Bibliographie de la France.]

Agassiz, L. (1846b) Nomenclatoris zoologici index universalis, continens nomina systematica classium, ordinum, familiarum et generum animalium omnium, tam viventium quam fossilium, secundum ordinem alphabeticum unicum disposita, adjectis homonymiis plantarum, nec non variis adnotationibus et emendationibus. [= Fasc. XII].

“1847”. Jent \& Gassman, Soloduri [= Solothurn, Switzerland]. viii + 393 pp. [29 December]

[For dating, see Evenhuis (1997: 50-52).]

Aldrich, J.M. (1905) A catalogue of the North American Diptera (or two-winged flies). Smithsonian Miscellaneous Collections, 46(2), 1-680. [before 25 May]

[Dated from Thompson et al. (1999).]

Allen, H.W. (1926) North American species of two-winged flies belonging to the tribe Miltogrammini. Proceedings of the United States National Museum, 68(9), 1-106. [27 May]

Am Stein, J.R. (1857) Bündner Dipteren. Verzeichniss der Sammlung des Hrn. Major Am Stein in Malans. Jahresbericht der Naturforschenden Gesellschaft Graubündens, (N.F.) 2, 89-111. [31 December+]

Arnett, R.H. (1985) American insects: a handbook of the insects of America north of Mexico. Van Nostrand Reinhold Co., New York. 850 pp. [February] 
Barták, M., Michelsen, V. \& Rozkošný, R. (1990) New records of Anthomyiidae from Czechoslovakia, with revised check list of Czechoslovak species (Diptera). Skripta Facultatis Scientiarium Naturalium Universitatis Purkyniensis Brunensis, 20, 439-449. [31 December+]

Becker, T. (1896) Dipterologische Studien IV. Ephydridae. Berliner Entomologische Zeitschrift, 41, 91-276. ["Mitte" December]

Bezzi, M. (1904) Intorno al generi Pelethophila Hangeb. e Chiromyia Rob. Desv (Ins., Dipt.). Atti della Società Italiana di Scienze Naturali di Milano, 43, 173-181. [before 12 July]

[Date received at BMNH.]

Bezzi, M. (1906) Noch einige neue Namen für Dipterengattungen. Zeitschrift für Systematische Hymenopterologie und Dipterologie, 6, 49-55. [1 January]

Bezzi, M. \& Stein, P. (1907) Cyclorrhapha Aschiza. Cyclorrhapha Schizophora: Schizometopa, pp. 1-747. In: Becker, T., Bezzi, M., Kertész, K. \& Stein, P. (eds.), Katalog der paläarktischen Dipteren. Band III. Budapest. 828 pp. [1 December]

[In this volume, Stein was responsible for the "Anthomyidae", which included the modern Fanniidae, Anthomyiidae, and much of the Muscidae; Bezzi was responsible for the remainder.]

Bigot, J.M.F. (1858) [M. J. Bigot donne lecture d'une notice nécrologique, qui lui avait été demandée par la Société, sur notre collègue M. le docteur Robineau-Desvoidy.] Annales de la Société Entomologique de France, (3) 5[1857], cxxxii-cxxxv. [24 February]

[Dated from C.D. Sherborn manuscript notes.]

Bigot, J.M.F. (1877) Notes et mélanges diptérologiques. Annales de la Société Entomologique de France, (5) 7, 260262. [30 October]

[Dated from C.D. Sherborn manuscript notes.]

Bigot, J.M.F. (1892) Catalogue of the Diptera of the Oriental Region. Part III. Journal of the Asiatic Society of Bengal, 61, 178-236. [23 July]

Bisby, F.A., Roskov, Y.R., Orrell, T.M., Nicolson D., Paglinawan, L.E., Bailly N., Kirk, P.M., Bourgoin, T., van Hertum, J. (eds.) (2008) Species 2000 \& ITIS Catalogue of Life: 2008 Annual Checklist. CD-ROM. Species 2000, Reading, U.K.

Blainville, H.M.D. de (1830) Zoophytes, pp. 1-546. In: Cuvier, G.-F., Dictionnaire des Sciences Naturelles, dans lequel on traite méthodiquement des différens êtres de la nature, considérés soit en eux-mêmes, d'après l'état actuel de nos connoissances, soit relativement a l'utilité qu'en peuvent retirer la médecine, l'agriculture, le commerce et les arts. Suivi d'une biographie des plus célèbres naturalistes. Ouvrage destiné aux médecins, aux agriculteurs, aux commercans, aux artistes, aux manufacturiers, et à tous ceux qui ont intérêt à connoître les productions de la nature, leurs caractères génériques et spécifiques, leur lieu natal, leurs propriétés et leurs usages. Tome soixantième. [ZOOPH-ZYT]. Levrault, Paris. [vi] + 631 pp. [June]

[Date recorded in Journal de Sçavans.]

Blainville, H.M.D. de, Latreille, P.-A. \& Duméril, A.M.C. (1826) Rapport sur les myodaires du Docteur Robineau Desvoidy. Académie Royale des Sciences, Paris. 24 pp. [26 October]

Boisduval, J.B.A.D. de (1846) Notice sur M. le Comte Dejean. Annales de la Société Entomologique de France (2), 3[1845], 499-520, 1 pl. [25 March] [Dated from C.D. Sherborn manuscript notes.]

Brauer, F. (1893) Vorarbeiten zu einer Monographie der Muscaria Schizometopa (exclusive Anthomyidae) von Prof. Dr. Fr. Brauer und Julius Edl. v. Bergenstamm. Verhandlungen der Kaiserlich-Königlichen Zoologisch-Botanischen Gesellschaft in Wien, 43, 447-525. ["Ende" December]

Brauer, F. \& Bergenstamm, J.E. von (1889) Die Zweiflügler des Kaiserlichen Museums zu Wien. IV. Vorarbeiten zu einer Monographie der Muscaria Schizometopa (exclusive Anthomyidae). Pars I. Denkschriften der Kaiserlichen Akademie der Wissenschaften, Wien, Mathematisch-Naturwissenschaftliche Classe, 56, 69-180, 11 pls. [March] [Date received by Naturae Novitates.]

Brauer, F \& Bergenstamm, J.E. von (1893) Die Zweiflügler des Kaiserlichen Museums zu Wien. VI. Vorarbeiten zu einer Monographie der Muscaria Schizometopa (exclusive Anthomyidae). Pars III. F. Tempsky, Wien [= Vienna]. 152 pp. [August] [Date received by Naturae Novitates.]

Bridges, R.G. (1972) Choline metabolism in insects. Advances in Insect Physiology, 9, 50-111. [15 January] [Dated from information from the publisher.]

Brullé, A. (1831) Coup d'oeil sur l'entomologie de la Morée. Annales des Sciences Naturelles, 23, 244-267. [May]

Cantrell, B.K. \& Crosskey, R.W. (1989) Family Tachinidae, pp. 733-784. In: Evenhuis, N.L. (ed.), Catalog of the Diptera of the Australasian and Oceanian Regions. Bishop Museum Press, Honolulu \& E.J. Brill, Leiden. 1155 pp. [23 August] [Dated from Evenhuis (2008b).] 
Carroll, L.E., Norrbom, A.L., Thompson, F.C. \& Evenhuis, N.L. (1999) Bibliography, pp. 303-492. In: Thompson, F.C. (ed.), Fruit Fly Expert Identification System and Systematic Information Database. Myia, 9[1998], vii + 524 pp. [22 February]

[Dated from information from the publisher.]

Carvalho, C.J.B. de, Pont, A.C., Couri, M.S. \& Pamplona, D. (2003) A catalogue of the Fanniidae (Diptera) of the Neotropical Region. Zootaxa, 219, 32 pp. [23 June]

Čepelák, J. \& Čepelák, C. (1988) Höhere Zweiflügler (Diptera, Brachycera) im Gebirge Biele Karpaty. II. Vršatské Bradlá. Biológia, 43, 567-574. [June]

Cerretti, P. (2001) I Tachinidi della Tenuta Presidenziale di Castelporziano (Diptera, Tachinidae). Bollettino dell'Associazione Romana di Entomologia, 56, 63-113. [31 December+]

Cerretti, P. (2004) I Tachinidi (Diptera, Tachinidae). In: Latella, L. (ed.), Il Monte Pastello. Memorie del Museo Civico di Storia Naturale di Verona (2 serie, Monografie Naturalistiche), 1, 225-243. [31 December+]

Cerretti, P. \& Ziegler, J. (2004) Chorologic data on tachinid flies from mainland Greece (Diptera, Tachinidae). Fragmenta Entomologica, 36, 275-317. [31 December+]

Chandler, P.J. (ed.) (1998) Checklists of insects of the British Isles (New Series), Part 1: Diptera (incorporating a list of Irish Diptera). Handbooks for the Identification of British Insects, 12, xx +234 pp. [13 November]

Charlet, L.D., Brewer, G.J. \& Beregovoy, V.H. (1992) Insect fauna of the heads and stems of native sunflowers (Asterales: Asteraceae) in eastern North Dakota. Environmental Entomology, 21, 493-500. [31 December+]

Charlet, L.D., Kopp, D.D. \& Oseto, C.Y. (1987) Sunflowers: their history and associated insect community in the northern Great Plains. Bulletin of the Entomological Society of America, 33, 69-75. ["Summer"]

Chvála, M. \& Smith, K.G.V. (1988) Family Conopidae, pp. 245-272. In: Soós, Á. \& Papp, L. (eds.), Catalogue of Palaearctic Diptera. Volume 8. Syrphidae-Conopidae. Akadémiai Kiadó, Budapest. 363 pp. [20 September] [Dated from Evenhuis (2008b).]

Cogan, B.H. (1984) Family Ephydridae, pp. 126-176. In: Sóos, Á. \& Papp, L. (eds.), Catalogue of Palaearctic Diptera. Volume 10. Clusiidae-Chloropidae. Akadémiai Kiadó, Budapest. 402 pp. [15 October]

[Dated from Evenhuis (2008b).]

Cogan, B.H. \& Munro, H.K. (1980) Family Tephritidae, pp. 518-554. In: Crosskey, R.W. (ed.), Catalogue of the Diptera of the Afrotropical Region. British Museum (Natural History), London. 1437 pp. [20 July]

[Dated from Evenhuis (2008b).]

[Colette, S.-G.] (1901) Claudine à Paris. Ollendorff, Paris. 321 pp. [31 December+] [Authored under the pseudonym "Willy".]

Collin, J.E. (1948) A short synopsis of the British Sapromyzidae (Diptera). Transactions of the Royal Entomological Society of London, 99, 225-242. [25 June]

Collin, J.E. (1960) The British species of Myopa (Dipt., Conopidae). Entomologist's Monthly Magazine, 95, $145-151$. [19 January]

Coquillett, D.W. (1897) Revision of the Tachinidae of America north of Mexico. United States Department of Agriculture, Division of Entomology, Technical Series, 7, 156 pp. [October]

[Date recorded as published by Hill (1898: 68).]

Coquillett, D.W. (1901) Types of anthomyid genera. Journal of the New York Entomological Society, 9, $134-146$. [September]

Coquillett, D.W. (1910) The type-species of the North American genera of Diptera. Proceedings of the United States National Museum, 37, 499-647. [4 August]

Cresson, E.T., Jr. (1920) A revision of the Nearctic Sciomyzidae (Diptera, Acalyptratae). Transactions of the American Entomological Society, 46, 27-89. [31 March]

Crosland, M. (1973) Colette, the difficulty of loving. A biography. Bobbs-Merrill Company, Indianapolis \& New York. xxx + 284 pp. [31 December+]

Crosskey, R.W. (1967) An index-catalogue of the genus-group names of Oriental and Australasian Tachinidae (Diptera) and their type-species. Bulletin of the British Museum (Natural History) Entomology, 20, 1-39. [17 April]

[Date of BMNH library stamp.]

Crosskey, R.W. (1977) Family Tachinidae, pp. 586-697. In: Delfinado, M.D. \& Hardy, D.E. (eds.), A catalog of the Diptera of the Oriental Region. Volume III. Suborder Cyclorrhapha (excluding Division Aschiza). University Press of Hawaii, Honolulu. $\mathrm{x}+854$ pp. [4 November] [Dated from Evenhuis (2008b).]

Crosskey, R.W. (1980) Family Tachinidae, pp. 822-882. In: Crosskey, R.W. (ed.), Catalogue of the Diptera of the Afrotropical Region. British Museum (Natural History), London. 1437 pp. [20 July]

[Dated from Evenhuis (2008b).]

Curtis, J. (1837) A guide to an arrangement of British insects; being a catalogue of all the named species hitherto discovered in Great Britain and Ireland. 2nd edition, greatly enlarged. J. Pigot \& Co., London. vi + 284 pp. [after June] 
[Date of preface.]

Curtis, J. (1839) British entomology; being illustrations and descriptions of the genera of insects found in Great Britain and Ireland: containing coloured figures from nature of the most rare and beautiful species, and in many instances of the plants upon which they are found. Volume 16. [Part 192. Pls. 766-769.] Privately published, London. [1 December]

[Dated from Evenhuis (1997: 166).]

De Galdo, D.M.M.J. (1856) Los tres reinos de la naturaleza. Museo pintoresco de historial natural. Descripcion completa de los animales, vegetales y minerales útiles y agradables; su forma, instituto, costumbres, virtudes ó aplicaciones á la agricultura, la medicina, y las artes en general, comprendiendo mayor número de géneros que en todas las obras publicadas hasta el dia, con un tratado de geologia, ó teorias actuales sobre las formacion y revolucion del globo, y un bosquejo historico de los progresos de ciencias naturales en general y en España: obre arreglada sobre las trabajos de los mas eminentes naturalistas de todos las paises, Buffon, Blanchard, Boitard, Bragniard, Cavanilles, los Cuvier, Daubenton, de Condolle, Humboldt, los Jussieu, Lacepede, Lagasca, Lamarck, Latreille, Lesson, Linneo d'orbigny, Rousseau, Saint Hilaire, saint Pierre, Virey, Werner, etc. con todos los descumbrimintos posteriores hasta el dia. Tomo VI. Zoologia. Gaspar y Roig, Madrid. 708 pp. [31 December+]

Dely-Draskovits, Á. (1993) Family Anthomyiidae, pp. 11-102. In: Soós, Á. \& Papp, L. (eds.), Catalogue of Palaearctic Diptera. Volume 13. Anthomyiidae-Tachinidae. Hungarian Natural History Museum, Budapest. 624 pp. [15 December]

[Dated from Evenhuis (2008b).]

Denoroy, P. (1996) The crop physiology of Helianthus tuberosus L.: a model oriented view. Biomass and Bioenergy, 11, 11-32. [31 December+]

Desmarest, E. (1847) [Note]. Annales de la Société Entomologique de France (2) 5, xiv. [26 May]

[No authorship is given for this note, but Desmarest was the secretary of the Sociéte and responsible for the minutes.]

Des Moulins, C. (1852) Rapport sur la maladie du raisin. Congrès Scientifique de France, 1851, 284-292. [31 December+]

Draber-Mońko, A. (1971) Einige Calyptrata (Diptera) vom Bieszczady-Gebirge. Fragmenta Faunistica, 17, $483-543$. [In Polish with Russian and German summaries.] [15 November]

Draber-Mońko, A. (1982) Tachinid flies (Diptera, Tachinidae) of Warsaw and Mazovia. Memorabilia Zoologica, 35[1981], 141-162. [before 17 September]

[Printed date on the article masthead is "1981", but the copyright for the issue is 1982; received at BPBM library on this date.]

Duché, E. (1859) Notice biographique sur le Docteur Robineau-Desvoidy. Congrès Scientifique de France, 23, 87-126. [31 December+]

[Also reprinted, pp. 3-40. In: Robineau-Desvoidy, J.-B. (1863a), Histoire naturelle des diptères des environs de Paris. Oeuvre posthume du $\mathrm{D}^{\mathrm{r}}$ Robineau-Desvoidy publiée par les soins de sa famille, sous la direction de M. H. Monceaux. Tome premier. Masson et fils, Paris, Wagner, Leipzig, and Williams \& Norgate, London. xvi + 1143 pp.]

Duda, L. (1938) Sphaeroceridae (Cypselidae). Die Fliegen der Palaearktischen Region, 57, 182 pp. [24 March-28 July] [Dated from Evenhuis (1997).]

Dumčius, O. \& Pakalniškis, S. (2004) New for Lithuania Diptera species found in 1993-2004. New and Rare for Lithuania Insect Species, Records and Descriptions, 16, 53-61. [31 December+]

Eaton, E.R. \& Kaufman, K. (2007) Kaufman field guide to insects of North America. The easiest guides for fast identification. Houghton Mifflin Harcourt, New York. 392 pp. [28 February]

Enderlein, G. (1927) Dipterologische Studien. XVII. Konowia, 6, 50-56. [22 March]

Evenhuis, N.L. (1990) Dating of livraisons and volumes of d'Orbigny's Dictionnaire Universel d'Histoire Naturelle. Bishop Museum Occasional Papers, 30, 219-225. [6 June]

[For dating of this journal, see Evenhuis (2008a).]

Evenhuis, N.L. (1994) The publication and dating of P.A. Wytsman's Genera Insectorum. Archives of Natural History, 21(1), 49-66. [21 January]

Evenhuis, N.L. (1997) Litteratura Taxonomica Dipterorum (1758-1930) being a selected list of the books and prints of Diptera taxonomy from the beginning of Linnaean nomenclature to the end of the year 1930; containing information on the biographies, bibliographies, types, collections, and patronymic genera of the authors listed in this work; including detailed information on publication dates, original and subsequent editions, and other ancillary data concerning the publications listed herein. 2 vols. Backhuys Publishers, Leiden. $\mathrm{x}+871 \mathrm{pp} .[10$ October]

[Date of mailing from publisher.]

Evenhuis, N.L. (2003a) Dating and publication of the Encyclopédie Méthodique (1782-1832), with special reference to the parts of the Histoire Naturelle and details on the Histoire Naturelle des Insectes. Zootaxa, 166, 1-48. [17 March]

Evenhuis, N.L. (2003b) Publication and dating of the journals forming the Annals and Magazine of Natural History and the Journal of Natural History. Zootaxa, 385, 1-68. [16 December] 
Evenhuis, N.L. (2007) Australasian/Oceanian Diptera Catalog_Web Version. Family Scathophagidae. Available from: http://hbs.bishopmuseum.org/aocat/scathophagidae.html. Last accessed on 4 December 2008.

Evenhuis, N.L. (2008a) Celebrating a centuria of volumes of the Bishop Museum Occasional Papers: history, contents, dates of publication, and author index. Bishop Museum Occasional Papers, 101, 1-76. [3 October]

Evenhuis, N.L. (2008b) Dates of publication of regional and world Diptera catalogs. Studia Dipterologica, 14, 397-403. [29 October]

Evenhuis, N.L., Pape, T., Pont, A.C. \& Thompson, F.C. (eds.) (2009) Biosystematic Database of World Diptera, Version 10.5. Available from: http://www.diptera.org/biosys.htm. Last accessed on 18 October 2009.

Evenhuis, N.L., Pape, T., Pont, A.C. \& Thompson, F.C. (2010) Flying after Linnaeus: Diptera names since Systema Naturae (1758), pp. 75-82. In: Polaszek, A. (ed.), Systema Naturae 250 - the Linnaean Ark. CRC Press, Boca Raton, Florida. 288 pp. [February]

Evenhuis, N.L. \& Thompson, F.C. (1990) Type designations of genus-group names of Diptera given in d'Orbigny's Dictionnaire Universel d'Histoire Naturelle. Bishop Museum Occasional Papers, 30, 226-258. [June]

Evenhuis, N.L., Thompson, F.C., Pont, A.C. \& Pyle, B.L. (1989) Literature cited, pp. 809-991. In: Evenhuis, N.L. (ed.), Catalog of the Diptera of the Australasian and Oceanian Regions. Bishop Museum Press, Honolulu \& E.J. Brill, Leiden. 1155 pp. [23 August]

[Dated from Evenhuis (2008b).]

Fabricius, J.C. (1798) Supplementum entomologiae systematicae. C.G. Proft et Storch, Hafniae [= Copenhagen]. [4] + 572 pp. [20 May]

[Dated from Evenhuis (1997: 248).]

Fabricius, J.C. (1799) Index alphabeticus in J.C. Fabricii Supplementum entomologiae systematicae. C.G. Proft et Storch, Hafniae [= Copenhagen]. $52+$ [2] pp. [12 December]

[Dated from Evenhuis (1997: 249).]

Fairmaire, L., Lucas, H., \& Sichel, J. (1858) Rapport sur un ouvrage manuscrit de Robineau-Desvoidy sur les diptères des environs de Paris. Annales de la Société Entomologique de France, (3) 6, 339-341. [8 September]

[Dated from C.D. Sherborn manuscript notes.]

Fallén, C.F. 1818. Monographia Haematomyzidum Sveciae. Quam Venia Ampliss. Fac. Philos. Lund. In Lyceo Carolino d. XVII Junii MDCCCXVIII. Berlingianus, Lundae [= Lund]. 16 pp. [17 June]

Fast, P.G. (1966) A comparative study of the phospholipids and fatty acids of some insects. Lipids, 1, 209-215. [May]

Férussac, A.S. (1828) [Review] Essai sur les myodaires; par M. Robineau-Desvoidy, D.M. Bulletin Universel des Sciences et de l'Industrie (Deuxième Section, Bulletin des Sciences Naturelles et Géologie), 10(2), 316-318. [April]

Foote, R.H. (1964) Notes on the Walker types of New World Tephritidae (Diptera). Journal of the Kansas Entomological Society, 37, 316-326. [21 October]

Foote, R.H. (1965) Family Tephritidae (Trypetidae, Trupaneidae), pp. 658-678. In: Stone, A., Sabrosky, C.W., Wirth, W.W., Foote, R.H. \& Coulson, J.R. (eds.), A catalog of the Diptera of America north of Mexico. United States Department of Agriculture, Agriculture Handbook, 276, iv + 1696 pp. [23 August]

[Dated from Evenhuis (2008b).]

Foote, R.H., Blanc, F.L. \& Norrbom, A.L. (1993) Handbook of the Fruit Flies (Diptera: Tephritidae) of America North of Mexico. Comstock Publishing Associates, Ithaca, New York. 571 pp. [June]

[Dated from information from the publisher.]

Foote, R.H. \& Freidberg, A. (1981) The taxonomy and nomenclature of some Palaearctic Tephritidae (Diptera). Journal of the Washington Academy of Sciences, 70[1980], 29-34. [16 February]

Foote, R.H. \& Steyskal, G.C. (1987) Tephritidae, pp. 66-831. In: McAlpine, J.F., Peterson, B.V., Shewell, G.E., Teskey, H.J., Vockeroth, J.R. \& Wood, D.M. (coordinators), Manual of Nearctic Diptera. Volume 2. Agriculture Canada, Monograph No. 28. Ottawa. vi + 675-1332 pp. [31 March]

[Dated from information from the publisher.]

Frey, R. (1927) Philippinische Dipteren. IV. Fam. Lauxaniidae. Acta Societatis pro Fauna et Flora Fennica, 56(8), 1-44. [31 December+]

Fries, B. (1834) Årsberättelse om nyare zoologiska arbeten och upptäckter, till Kongl. Vetenskaps-Academien afgiven den 31 mars 1834. P.A. Norstedt \& Söner, Stockholm. viii + 208 pp. [after March]

Frost, S.W. (1964) Insects taken in light traps at the Archbold Biological Station, Highlands County, Florida. The Florida Entomologist, 47, 129-161. [June]

Gaimari, S.D. \& Mathis, W.N. (in press) World catalog and conspectus of Odiniidae. Myia, 12.

Gerstaecker, A. (1865) [Review] Histoire naturelle des diptères des environs de Paris, oeuvre posthume du Dr. Robineau-Desvoidy, publiée par les soins de sa famille, sous la direction de M. H. Monceaux. Paris 1863. (2 Vol. in 8., 1143 und 920 pag.). Archiv für Naturgeschichte, 31(2), 515-518. [31 December+]

Giard, A. (1878) De l'influence néfaste des prix de l'Académie. Bulletin Scientifique du Département du Nord et des Pays Voisins, (2) 1, 214-217. [September] 
Gill, G.D. (1968) Family Heleomyzidae (Helomyzidae) including the Trixoscelididae (Trichoscelidae) (86). A Catalogue of the Diptera of the Americas South of the United States, 85, 1-13. [20 October]

Gorodkov, K.B. (1984) Family Heleomyzidae (Helomyzidae), pp. 15-45. In: Soós, Á. \& Papp, L. (eds.), Catalogue of Palaearctic Diptera. Volume 10. Clusiidae-Chloropidae. Elsevier Science Publishers, Amsterdam \& Akadémiai Kiadó, Budapest. 402 pp. [1 March]

[Dated from Evenhuis (2008b).]

Gorodkov, K.B. (1986) Family Scathophagidae, pp. 11-41. In: Soós, Á. \& Papp, L. (eds.), Catalogue of Palaearctic Diptera. Volume 11. Scathophagidae-Hypodermatidae. Akadémiai Kiadó, Budapest. 346 pp. [1 March] [Dated from Evenhuis (2008b).]

Gosseries, J. (1991) Sarcophagidae, pp. 195-198. In: Grootaert, P., De Bruyn, L. \& De Meyer, M. (eds.), Catalogue of the Diptera of Belgium. Studiedocumenten van het Koninklijk Belgisch Institut voor Natuurwetenschappen, 70, 338 pp. [29 November]

Gregor, F. (1988) Thricops tatricus sp.n. from the western Carpathians and Pyrenees (Diptera, Muscidae). Acta Entomologica Bohemoslovaca, 85, 447-456. [30 November]

Gregor, F. (1997) Muscidae, pp. 93-96. In: Chvála, M. (ed.), Check list of Diptera (Insecta) of the Czech and Slovak Republics. Karolinum, Charles University Press, Praha [= Prague]. 130 pp. [after 25 June]

[Date given in introduction.]

Gregor, F. (2004) Relative size of ommatidial facets and questions concerning dichoptism in selected Muscidae (Diptera). In: Bitušík, P. (ed.), Diptera bohemoslovaca. Vol. 12. Acta Facultatis Ecologiae, 12, Supplement 1, 43-48. [31 December+]

Gregor, F. \& Rozkošný, R. (2007) Thricops albibasalis (Zetterstedt, 1849) and some other records of interesting Fanniidae and Muscidae (Diptera) in Central Europe. Studia Dipterologica, 13[2006], 333-335. [12 July]

Gregor, F., Rozkošný, R., Barták, M. \& Vaňhara, J. (2002) The Muscidae (Diptera) of Central Europe. Folia Facultatis Scientiarum Naturalium Universitatis Masarykianae Brunensis, Biologia, 107, 280 pp. [31 December+]

Griffiths. G.C.D. (1982) Anthomyiidae [part]. Flies of the Nearctic Region. 8(2)(1), 1-160. [31 December+]

Griffiths, G.C.D. (1994) Case 2858. Hydrophoria Robineau-Desvoidy, 1830 (Insecta, Diptera): proposed designation of Musca lancifer Harris, [1780] as type species. Bulletin of Zoological Nomenclature, 51, 28-30. [March]

Griffiths, G.C.D. (1998) Anthomyiidae [part]. Flies of the Nearctic Region. 8(2)(12), 1873-2120. [31 December+]

Guérin, F.E. (1827) Myodaires, pp. 365-368. In: Bory de St.-Vincent, J.B.G.M., Dictionnaire classique d'histoire naturelle, par Messieurs Audouin, Isid. Bordon, Ad. Brongniart, De Candolle, Daudebard de Férussac, A. Desmoulins, Drapiez, Edwards, Flourens, Geoffroy de Saint-Hilaire, A. De Jussieu, Kunth, G. de Lafosse, Lamouroux, Latreille, Lucas fils, Presle-Duplessis, C. Prévost, A. Richard, Thiébaut de Berneaud, et Bory de Saint-Vincent. Ouvrage dirigé par ce dernier collaborateur, et dans lequel on a ajouté, pour le porter au niveau de la science, un grand nombre de mots qui n'avaient pu faire partie de la plupart des dictionnaires antérieurs. Tome onzième. Baudoin Frères, Paris. [January]

[For dating, see Evenhuis (1997: 111). The author changed his name to Guérin-Méneville in 1836.]

Guimarães, J.H. (1971) Family Tachinidae (Larvaevoridae). A Catalogue of the Diptera of the Americas South of the United States, 104, 1-333. [6 December]

Hendel, F. (1900) Untersuchung über die europäischen Arten der Gattung Tetanocera im Sinne Schiner's. Eine dipterologische Studie. Verhandlungen der Kaiserlich-Königlichen Zoologisch-Botanischen Gesellschaft in Wien, 50, 319-358. [24 August]

Hendel, F. (1901a) Beitrag zur Kenntnis der Calliphorinen (Dipt.). Wiener Entomologische Zeitung, 20, 28-33. [31 January]

Hendel, F. (1901b) Ueber einige neue oder weniger bekannte europäische Muscaria schizometopa. Verhandlungen der Kaiserlich-Königlichen Zoologisch-Botanischen Gesellschaft in Wien, 51, 198-211. [3 May]

Hendel, F. (1908) Diptera. Fam. Muscaridae. Subfam. Lauxaninae. Genera Insectorum, 68, 1-66. [23 June] [For dating of this series, see Evenhuis (1994).]

Hendel, F. (1910) Über die Nomenklatur der Acalypterengattungen nach Th. Beckers Katalog der palärktischen Dipteren, Bd. 4. Wiener Entomologische Zeitung, 29, 307-313. [15 December]

Hendel, F. (1911a) Die Arten der Dipteren-Subfamilie Richardiinae. Deutsche Entomologische Zeitschrift, 1911, 367396. [26 June]

Hendel, F. (1911b) Diptera. Fam. Muscaridae. Subfam. Richardiinae. Genera Insectorum, 113, 1-56. [9 September] [For dating of this series, see Evenhuis (1994).]

Hendel, F. (1914a) Die Gattungen der Bohrfliegen. (Analytische Übersicht aller bisher bekannten Gattungen der Tephritinae.). Wiener Entomologische Zeitung, 33, 73-98. [30 April]

Hendel, F. (1914b) Diptera. Fam. Muscaridae. Subfam. Platystominae. Genera Insectorum, 157, 1-179. [10 June] [For dating of this series, see Evenhuis (1994).]

Hendel, F. (1927) Trypetidae [part]. Die Fliegen der Palaearktischen Region, 49[Lieferung 19], 193-221. [29 June] [Dated from Evenhuis (1997).] 
Hennig, W. (1939) Otitidae. (46. Pterocallidae und 47. Ortalidae.). Die Fliegen der Palaearktischen Region, 46/ 47[Lieferung 126], 1-48. [10 May]

[Dated from Evenhuis (1997).]

Hennig, W. (1962a) Muscidae [part]. Die Fliegen der Palaearktischen Region, 63b[Lieferung 225], 625-672. [26 January] [Dated from Evenhuis (1997).]

Hennig, W. (1962b) Muscidae [part]. Die Fliegen der Palaearktischen Region, 63b[Lieferung 229], 721-768. [15 June] [Dated from Evenhuis (1997).]

Hennig, W. (1963a) Muscidae [part]. Die Fliegen der Palaearktischen Region, 63b[Lieferung 233], 769-816. [29 January]

[Dated from Evenhuis (1997).]

Hennig, W. (1963b) Muscidae [part]. Die Fliegen der Palaearktischen Region, 63b[Lieferung 242], 913-960. [19 November]

[Dated from Evenhuis (1997).]

Hennig, W. (1965) Vorarbeiten zu einem phylogenetischen System der Muscidae (Diptera: Cyclorrhapha). Stuttgarter Beiträge zur Naturkunde, 141, 100 pp. [1 June]

Hennig, W. (1969) Anthomyiidae [part]. Die Fliegen der Palaearktischen Region, 63a[Lieferung 278], 241-288. [24 November]

[Dated from Evenhuis (1997).]

Herting, B. (1974) Revision der von Robineau-Desvoidy beschriebenen europäischen Tachiniden und Rhinophoriden (Diptera). Stuttgarter Beiträge zur Naturkunde (A), 264, 46 pp. [1 June]

Herting, B. (1984) Catalogue of Palearctic Tachinidae (Diptera). Stuttgarter Beiträge zur Naturkunde (A), 369,228 pp. [30 November]

Herting, B. (1993) Family Rhinophoridae, pp. 102-117. In: Soós, Á. \& Papp, L. (eds.), Catalogue of Palaearctic Diptera. Volume 13. Anthomyiidae-Tachinidae. Hungarian Natural History Museum, Budapest. 624 pp. [15 December]

[Dated from Evenhuis (2008b).]

Herting, B. \& Dely-Draskovits, Á. (1993) Family Tachinidae, pp. 118-458. In: Soós, Á. \& Papp, L. (eds.), Catalogue of Palaearctic Diptera. Volume 13. Anthomyiidae-Tachinidae. Hungarian Natural History Museum, Budapest. 624 pp. [15 December] [Dated from Evenhuis (2008b).]

Herting, B. \& Tschorsnig, H.-P. (1997) Raupenfliegen (Diptera, Tachinidae) aus der Schweiz. Mitteilungen der Schweizerischen Entomologischen Gesellschaft, 70, 77-92. [31 December+]

Hilgendorf, J.H. \& Goeden, R.D. (1981) Phytophagous insects reported from cultivated and weedy varieties of the sunflower, Helianthus annuus L., in North America. Bulletin of the Entomological Society of America, 27, 102109. [31 December+]

Hill, D.S. (1987) Agricultural insect pests of temperate regions and their control. Cambridge University Press, Cambridge, U.K. 672 pp. [29 May]

[Dated from information from the publisher.]

Hill, G.W. (1898) Report of the chief of the Division of Publications, pp. 43-86. In: Wilson, J., Annual reports of the Department of Agriculture for the fiscal year ended June 30, 1898. Report of the Secretary of Agriculture. Government Printing Office, Washington, D.C. 1xii + 212 pp. [after 23 November] [Date of letter to President by the Secretary.]

Hoes, J.A. \& Huang, H.C. (1975) Sclerotinia sclerotiorum: Viability and separation of sclerotia from soil. Phytopathological Notes, 65, 1431-1432.

Hofmans, K. (1991) Muscidae, pp. 187-191. In: Grootaert, P., De Bruyn, L. \& De Meyer, M. (eds.), Catalogue of the Diptera of Belgium. Studiedocumenten van het Koninklijk Belgisch Institut voor Natuurwetenschappen, 70, 338 pp. [29 November]

Horn, W. \& Kahle, I. (1935) Ueber entomologische Sammlungen. (Ein Beitrag zur Geschichte der Entomo-Museologie.) Teil I. Entomologische Beihefte aus Berlin-Dahlem, 2, 1-160, pls. 1-16. [14 December]

Horn, W. \& Kahle, I. (1936) Ueber entomologische Sammlungen. (Ein Beitrag zur Geschichte der Entomo-Museologie.) Teil II. Entomologische Beihefte aus Berlin-Dahlem, 3, 161-296, pls. 17-26. [4 October]

Horn, W. \& Kahle, I. (1937) Ueber entomologische Sammlungen, Entomologen und Entomo-Museologie. (Ein Beitrag zur Geschichte der Entomologie.) Teil III. Entomologische Beihefte aus Berlin-Dahlem, 4, 297-536, i-vi, pls. 27-38. [1 August]

Horn, W., Kahle, I., Friese, G. \& Gaedicke, R. (1990a) Collectiones entomologicae. Ein Kompendium über den Verbleib entomologischer Sammlungen der Welt bis 1960. Teil I: A bis K. Akademie der Landwirtschaftswissenschaften, Berlin. Pp. 1-220, portrait. [31 December+] 
Horn, W., Kahle, I., Friese, G. \& Gaedicke, R. (1990b) Collectiones entomologicae. Ein Kompendium über den Verbleib entomologischer Sammlungen der Welt bis 1960. Teil II: L bis Z. Akademie der Landwirtschaftswissenschaften, Berlin. Pp. 223-573, 38 pls., 125 portraits. [31 December+]

Hubenov, Z. (2008) Composition and zoogeographical characteristics of the family Tachinidae (Insecta: Diptera) in the Balkan countries. Acta Zoologica Bulgarica, 60, 243-265. [31 December+]

Huckett, H.C. \& Vockeroth, J.R. (1987) Muscidae, pp. 1115-1131. In: McAlpine, J.F., Peterson, B.V., Shewell, G.E., Teskey, H.J., Vockeroth, J.R. \& Wood, D.M. (coordinators), Manual of Nearctic Diptera. Volume 2. Agriculture Canada Monograph, 28, i-vi, 675-1332. [31 March]

Huot, J.-J.-N. (1830) Muscides, pp. 419-420. In: Courtin, E.M.P.M.A., Encyclopédie moderne, ou dictionnaire abrégé des hommes et des choses, des sciences, des lettres et des arts, avec l'indication des ouvrages où les divers sujets sont développés et approfondis. Deuxième edition, revue, corrigée et augmentée. Tome sixième. T. Lejeune, Bruxelles. 479 pp. [31 May]

[Dated from Revue Bibliographique des Pays-Bays et des Étranger.]

International Commission on Zoological Nomenclature (1954) Opinion 274. Addition of the name Calliphora RobineauDesvoidy, 1830 (Class Insecta, Order Diptera) to the Official List of Generic Names in Zoology (Opinion supplementary to Opinion 82). Opinions and Declarations of the International Commission on Zoological Nomenclature, 6, 75-82. [10 September]

International Commission on Zoological Nomenclature (1956) Direction 32. Addition to the Official List of Works Approved as Available for Zoological Nomenclature of the titles of certain works dealt with in Opinions rendered in the period up to the end of 1936 and to the Official Index of Rejected and Invalid Works in Zoological Nomenclature of certain works similarly dealt with in the same period and matters incidental thereto. Opinions and Declarations of the International Commission on Zoological Nomenclature, 1C, 307-328. [17 May]

International Commission on Zoological Nomenclature (1959) Opinion 553. Addition of the generic name Cyzicus Audouin, 1837 (Class Crustacea, Order Conchostraca), to the Official List of Generic Names in Zoology and matters incidental thereto. Opinions and Declarations of the International Commission on Zoological Nomenclature, 20, 225-240. [14 April]

International Commission on Zoological Nomenclature (1963) Opinion 678. The suppression under the plenary powers of the pamphlet published by Meigen, 1800. Bulletin of Zoological Nomenclature, 20, 339-342. [21 October]

International Commission on Zoological Nomenclature (1964) Opinion 712. Forty-seven genera of decapod Crustacea: placed on the Official List. Bulletin of Zoological Nomenclature, 21, 336-351. [26 November]

International Commission on Zoological Nomenclature (1968) Opinion 847. Chamaemyia Meigen, 1803 (Insecta, Diptera): designation of a type-species under the Plenary Powers. Bulletin of Zoological Nomenclature, $25,16-17$. [24 May]

International Commission on Zoological Nomenclature (1983) Opinion 1255. Lespesia Robineau-Desvoidy, 1863 (Diptera, Tachinidae): designation of type species. Bulletin of Zoological Nomenclature, 40, 97-101. [15 July]

International Commission on Zoological Nomenclature (1985) Opinion 1321. Grant of nomenclatural precedence to Ephydridae Zetterstedt, 1837 over Hydrelliidae Robineau-Desvoidy, 1830 (Insecta, Diptera). Bulletin of Zoological Nomenclature, 42, 177-179. [27 June]

International Commission on Zoological Nomenclature (1987) Opinion 1432. Actia Robineau-Desvoidy, 1830 (Insecta, Diptera): Roeselia lamia Meigen, 1838, designated as type species. Bulletin of Zoological Nomenclature, 44, 7172. [23 March]

International Commission on Zoological Nomenclature (1988a) Opinion 1475. Dexia Meigen, 1826 (Insecta, Diptera): Musca rustica Fabricius, 1775 designated as the type species. Bulletin of Zoological Nomenclature, 45, 74-75. [25 March]

International Commission on Zoological Nomenclature (1988b) Opinion 1507. Musca marginalis Wiedemann, 1830 (currently Chrysomya marginalis; Insecta, Diptera): specific name conserved. Bulletin of Zoological Nomenclature, 45, 236. [23 September]

International Commission on Zoological Nomenclature (1990) Opinion 1601. Rapport sur les Myodaires du Docteur Robineau-Desvoidy (1826): suppressed for nomenclatural purposes. Bulletin of Zoological Nomenclature, 47, 162. [29 June]

International Commission on Zoological Nomenclature (1996) Opinion 1827. Hydrophoria Robineau-Desvoidy, 1830 (Insecta, Diptera): Musca lancifer Harris, [1780] designated as the type species, and a neotype designated for $M$. lancifer. Bulletin of Zoological Nomenclature, 53, 62-63. [29 March]

International Commission on Zoological Nomenclature (1997) Opinion 1870. Sicus Scopoli, 1763 and Myopa Fabricius, 1775 (Insecta, Diptera): conserved by the designation of Conops ferrugineus Linnaeus, 1761 and C. buccatus Linnaeus, 1758 as the respective type species; and Coenomyia Latreille, 1796 placed on the Official List. Bulletin of Zoological Nomenclature, 54, 133-135. [30 June]

International Commission on Zoological Nomenclature (1999) International Code of Zoological Nomenclature. Fourth edition. International Trust for Zoological Nomenclature, London. xxix +306 pp. 
Irwin, A.G. (2006) A note on proanepisternal hairs in Nyctia halterata (Panzer) (Diptera, Sarcophagidae). Dipterists Digest, (2) 12, 106. [6 February]

Jacot, A.P. (1929) Xenillus clypeator Robineau-Desvoidy and its identity. Psyche, 36, 125-128. [9 July]

Kara, K. \& Pape, T. (2002) Check list of Turkish Sarcophagidae (Insecta, Diptera) with new records. Entomologische Zeitschrift, 49, 291-295. [31 December+]

Karl, O. (1928) Zweiflügler oder Diptera. II: Muscidae. In: Dahl, F. (ed.), Die Tierwelt Deutschlands und der angrenzenden Meeresteile nach ihren Merkmalen und nach ihrer Lebensweise, 13, iv + 232 pp. [28 September] [Dated from Evenhuis (1997: 188).]

Kays, S.J. \& Nottingham, S.F. (2008) Biology and chemistry of Jerusalem artichoke: Helianthus tuberosus L. CRC Press, Boca Raton, Florida. 496 pp. [13 August]

[Dated from information from the publisher.]

Kozuharova, E., Lavchiev, V. \& Lennartsson, T. (2006) Flies of the genus Thricops (Muscidae, Diptera) as pollinators of plants with bell-shaped flowers in the mountains of Bulgaria. Studia Dipterologica, 12[2005], 409-418. [18 April]

Kurahashi, H. (1989) Family Calliphoridae, pp. 702-718. In: Evenhuis, N.L. (ed.), Catalog of the Diptera of the Australasian and Oceanian Regions. Bishop Museum Press, Honolulu \& E.J. Brill, Leiden. 1155 pp. [23 August] [Dated from Evenhuis (2008b).]

Lacombe, P. (1903) [Réponses] P. Saint-A., pseudonyme (224). La Correspondance Historique et Archéologique, 10, 176-177. [June]

Latreille, P.-A. (1811) Oscine, pp. 561-567. In: Encyclopédie méthodique, ou par un ordre de matières. Histoire naturelle. Insectes. Tome huitième [part 2]. H. Agasse, Paris. Pp. 361-722. [23 October]

[Dated from Evenhuis (2003a).]

Latreille, P.-A. (1825) Familles naturelles du règne animal. J.-B. Baillière, Paris. 570 pp. [16 May] [Dated from Evenhuis (1997: 411).]

Latreille, P.-A. (1829) Stomoxe, pp. 669-670. In: Bory de St.-Vincent, J.B.G.M., Dictionnaire classique d'histoire naturelle, par Messieurs Audouin, Isid. Bordon, Ad. Brongniart, De Candolle, Daudebard de Férussac, A. Desmoulins, Drapiez, Edwards, Flourens, Geoffroy de Saint-Hilaire, A. De Jussieu, Kunth, G. de Lafosse, Lamouroux, Latreille, Lucas fils, Presle-Duplessis, C. Prévost, A. Richard, Thiébaut de Berneaud, et Bory de Saint-Vincent. Ouvrage dirigé par ce dernier collaborateur, et dans lequel on a ajouté, pour le porter au niveau de la science, un grand nombre de mots qui n'avaient pu faire partie de la plupart des dictionnaires antérieurs. Tome quinzième. Baudoin Frères, Paris. [May]

[For dating, see Evenhuis (1997: 111).]

Latreille, P.-A. (1830) Tachine, pp. 7-10. In: Bory de St.-Vincent, J.B.G.M., Dictionnaire classique d'histoire naturelle, par Messieurs Audouin, Isid. Bordon, Ad. Brongniart, De Candolle, Daudebard de Férussac, A. Desmoulins, Drapiez, Edwards, Flourens, Geoffroy de Saint-Hilaire, A. De Jussieu, Kunth, G. de Lafosse, Lamouroux, Latreille, Lucas fils, Presle-Duplessis, C. Prévost, A. Richard, Thiébaut de Berneaud, et Bory de Saint-Vincent. Ouvrage dirigé par ce dernier collaborateur, et dans lequel on a ajouté, pour le porter au niveau de la science, un grand nombre de mots qui n'avaient pu faire partie de la plupart des dictionnaires antérieurs. Tome sixième. Baudoin Frères, Paris. [30 October] [For dating, see Evenhuis (1997: 111).]

Lehrer, A.Z. (2005) Révision du genre Nyctia Robineau-Desvoidy (Diptera, Sarcophagidae). Bulletin de la Société Entomologique de Mulhouse, 61, 55-63. [before 25 November] [Date copy sent from author.]

Le Peletier, A.L.M. \& Serville, J.G.A. (1828) Stomoxydes, pp. 499-500. In: Encyclopédie méthodique, ou par un ordre de matières. Histoire naturelle. Insectes. Tome dixième [part 2]. $\mathrm{M}^{\mathrm{me}}$ veuve Agasse, Paris. Pp. 345-832 + [1]. [13 December] [Dated from Evenhuis (2003a).]

Lioy, A. (1864a) I ditteri distribuiti secundo un nuovo metodo di classificazione naturale [part]. Atti della Reale Istituto Veneto di Scienze, Lettere et Arti, (3) 9, 879-910. [20 April] [Dated from Thompson et al. (1999).]

Lioy, A. (1864b) I ditteri distribuiti secundo un nuovo metodo di classificazione naturale [part]. Atti della Reale Istituto Veneto di Scienze, Lettere et Arti, (3) 10, 59-84. [November] [Dated from Thompson et al. (1999).]

Loew, H. (1844) Kritische Untersuchung der europäischen Arten des Genus Trypeta Meig. Zeitschrift für die Entomologie (Germar's), 5, 312-437. [before May] [Date recorded in Revue et Magasin de Zoologie Pure et Appliquée.]

Loew, H. (1856) Neue Beiträge zur Kenntnis der Dipteren. Vierter Beitrag, pp. 1-57. In: Loew, H., Schulnachrichten der Königlichen Realschule zu Meseritz womit zu der am 17. März 1856 stattfindenden öffentlichen Prüfung alle Gönner und Freunde der Anstalt, insbesondere die Eltern und Angehörigen sämmtlicher Schüler. F.W. Lorenz, Meseritz [= Międzyrzecz]. 65 pp. [17 March] 
[Also issued separately (without the academic material), published by Mittler \& Sohn, Berlin, 1856, pp. 1-57. The paper cited above consists of an address given by Loew at a program of examinations on the 17th of March. Since it is assumed this paper was given out on the date of the examinations, the date of publication is given as such.]

Loew, H. (1862) Monographs of the Diptera of North America. Part I. Smithsonian Miscellaneous Collections 6(1)[= No. 141], xxiv + 221 pp. [April]

Loew, H. (1866) Diptera americae septentrionalis indigena. Centuria septima. Berliner Entomologische Zeitschrift, 10, 1-54. [July]

Loew, H. (1873) Monographs of the Diptera of North America. Part III. Smithsonian Miscellaneous Collections 11[= No. 256], vii + $351+$ XIII pp. [December]

Macleay, W.S. (1831) Exposition de l'anatomie comparée du thorax dans les insectes ailés, suivie d'une revue de l'état actuel de la nomenclature de cette partie. (Accompagnée de notes par M. Audouin). Annales de Sciences Naturelles, 25, 95-151. [February]

Macquart, P.-J.-M. (1834a) Histoire naturelle des insectes. Diptères. Tome première. N.E. Roret, Paris. 578 pp. [31 March]

[Dated from Evenhuis (1997: 512).]

Macquart, P.-J.-M. (1834b) Insectes diptères du nord de la France. Tome V. Athéricères: créophiles, oestrides, myopaires, conopsaires, scénopiniens, céphalopsides. L. Danel, Paris. 232 pp. [21 July]

[Dated from Evenhuis (1997); the journal version in the Mémoires de la Société Royale des Sciences, de l'Agriculture et des Arts,

Lille, 1833, 137-168, came out a few months later; see Evenhuis (1997: 510) for dating.]

Macquart, P.-J.-M. (1835) Histoire naturelle des insectes. Diptères. Tome deuxième. N.E. Roret, Paris. 703 or 710 pp. [1 April]

[Dated from Evenhuis (1997: 512).]

Macquart, P.-J.-M. (1837) Description d'un nouveau genre d'insectes diptères de la famille des créophiles, tribu des tachinaires. Mémoires de la Société Royale des Sciences, de l'Agriculture et des Arts, Lille, 1835, 188-191. [21 October]

[Dated from Bibliographie de la France.]

Macquart, P.-J.-M. (1838) Diptères exotiques nouveaux ou peu connus. Tome premier.-1 $1^{\text {re }}$ partie. Roret, Paris. Pp. 5221, 25 pls. [17 September]

[The journal version in the Mémoires de la Société Royale des Sciences, de l'Agriculture et des Arts, Lille, 1838(2), 9-225, came out on 27 October; see Evenhuis (1997: 512) for dating.]

Macquart, P.-J.-M. (1842) Observations sur un mémoire et une notice de M. Robineau-Desvoidy, insérés dans les Annales de la Société entomologique de France, 1841, $4^{\mathrm{e}}$ trimestre. Annales de la Société Entomologique de France, 11, 165-171. [October]

[Dated from C.D. Sherborn manuscript notes.]

Macquart, P.-J.-M. (1844) Diptères exotiques nouveaux ou peu connus. Tome deuxième.-3e partie. “1843”. Roret, Paris. 304 pp., 36 pls. [February]

[The journal version in the Mémoires de la Société Royale des Sciences, de l'Agriculture et des Arts, Lille, 1842, 162-460, came out a few months later; see Evenhuis (1997: 513) for dating.]

Macquart, P.-J.-M. (1845) Nouvelles observations sur les insectes diptères de la tribu des tachinaires [part]. Annales de la Société Entomologique de France, (2) 3, 237-280. [22 October]

[Dated from C.D. Sherborn manuscript notes; the remaining pages of this article (pp. 281-296) came out on 22 December.]

Macquart, P.-J.-M. (1847) Observations sur une notice de M. le docteur Robineau-Desvoidy intitulée Coup d'oeil rétrospectif, \&. Annales de la Société Entomologique de France, (2) 5, 71-82. [26 May]

[Dated from C.D. Sherborn manuscript notes.]

Macquart, P.-J.-M. (1851) Diptères exotiques nouveaux ou peu connus. Suite du 4. ${ }^{\mathrm{e}}$ supplément publié dans les mémoires de 1849 [part]. Mémoires de la Société Royale des Sciences, de l'Agriculture et des Arts, Lille, 1850, 134-294. [5 April]

[Dated from Evenhuis (1997).]

Macquart, P.-J.-M. (1855) Nouvelles observations sur les diptères d'Europe de la tribu des tachinaires (suite). Annales de la Société Entomologique de France, (3) 2[1854], 733-754. [11 April]

[Dated from C.D. Sherborn manuscript notes.]

Malloch, J.R. (1933) Acalyptrata [Heleomyzidae, Trypetidae, Sciomyzidae, Sapromyzidae, etc.]. Diptera of Patagonia and South Chile, 6, 177-391. [25 November]

Marié, P. (1930) Contribution à l'étude et à la recherche des arthropodes commensaux de la marmotte des Alpes. Annales de Sciences Naturelles (Zoologie), (10) 13, 185-233. [September]

Marschall, A.F. de (1873) Nomenclator zoologicus continens nomina systematica generum animalium tam viventium quam fossilium, secundum ordinem alphabeticum disposita. C. Ueberreuter, Vindobonae [= Vienna]. v + $482 \mathrm{pp}$. [after 20 October]

[Dated from Evenhuis (1997).] 
Marshall, S.A. (2006) Insects. Their natural history and diversity. With a photographic guide to insects of eastern North America. Firefly Books. 718 pp. [June]

[Dated from information from the publisher.]

Matile, L. (1974) Découverte des dessins inédits du diptériste J.W. Meigen. Bulletin de la Société Entomologique de France, 79, 104. [10 October]

McAlpine, D.K. (1985) The Australian genera of Heleomyzidae (Diptera: Schizophora) and a reclassification of the family into tribes. Records of the Australian Museum, 36, 203-251. [11 June]

Meigen, J.W. (1800) Nouvelle classification des mouches à deux ailes (Diptera L.) d'après un plan tout nouveau. Par J.G. Meigen. “An VIII (1800 v.s.)”. J.J. Fuchs, Paris. 40 pp. [before 22 September] [Dated from Thompson et al. (1999: 476).]

Meigen, J.W. (1824) Systematische Beschreibung der bekannten europäischen zweiflügeligen Insekten. Vierter Theil. Schultz-Wundermann, Hamm. xii + 428 pp. [after 24 September] [Date of preface.]

Meigen, J.W. (1826) Systematische Beschreibung der bekannten europäischen zweiflügeligen Insekten. Fünfter Theil. Schulz, Hamm. xii + 412 pp. [after 1 August] [Date of preface.]

Meigen, J.W. (1838) Systematische Beschreibung der bekannten europäischen zweiflügeligen Insekten. Siebenter Theil oder Supplementband. Schulz, Hamm. xii + 434 pp., pls. 67-74. [21 September]

[Dated from the Allgemeine Bibliographie für Deutschland.]

Mesnil, L.P. (1939) Essai sur les tachinaires (Larvaevoridae). Monographies publiées par les Stations et Laboratoires de Recherches Agronomiques, 7, 1-67 + v pp. [31 December+]

Mesnil, L.P. (1974) Larvaevorinae (Tachininae). Die Fliegen der Palaearktischen Region, 64g[Lieferung 304], 12331304. [19 July]

[Dated from Evenhuis (1997).]

Michaud, J.F. \& Poujoulat, B. (1833) Correspondance d'Orient 1830-1831. III. “1834”. Ducollet, Paris. 503 pp. [21 December]

[Although it has "1834" on the title page, it was recorded on this date by Bibliographie de la France. The entire work covers 6 volumes from 1833-1835.]

Michelsen, V. (2006) A new European species of Pegomya Robineau-Desvoidy (Diptera: Anthomyiidae) near $P$. testacea (De Geer). Zootaxa, 1260, 37-46. [14 July]

Michelsen, V. (2009) Revision of the willow catkin flies, genus Egle Robineau-Desvoidy (Diptera: Anthomyiidae), in Europe and neighbouring areas. Zootaxa, 2043, 1-76. [16 March]

Mihályi, F. (1979) Fémeslegyek-Húslegyek. Calliphoridae-Sarcophagidae. Fauna Hungarica, 135, 1-152. [31 December+]

Mihályi, F. (1981) Results of the expedition to Albania in 1961 by Deutsches Entomologisches Institut. Diptera, Calliphoridae, Sarcophagidae, Rhinophoridae. Beiträge zur Entomologie, 30(2)[1980], 333-341. [28 January]

[Date received by the Senckenberg Deutsches Entomologisches Institut library.]

Mik, J. (1883) Dipterologische Bemerkungen I, II. Verhandlungen der Kaiserlich-Königlichen Zoologisch-Botanischen Gesellschaft in Wien, 33, 181-192. ["Ende" August]

Mik, J. (1889) Literatur. Wiener Entomologische Zeitung, 8, 164-168. [30 April]

Mik, J. (1890) Dipterologische Miscellen. Wiener Entomologische Zeitung, 9, 153-158. [25 June]

Mik, J. (1894) Dipterologische Miscellen (2. Serie). IV. Wiener Entomologische Zeitung, 13, 49-54. [28 February]

Mik, J. \& Wachtl, F.A. (1895) Commentar zu den Arbeiten von Hartig und Ratzeburg über Raupenfliegen (Tachiniden). Auf Grund einer Revision der Hartig'schen Tachiniden-Sammlung. Wiener Entomologische Zeitung, 14, $213-250$. [25 August]

Mohamed-Ahmed, M.M. \& Mihok, S. (1999) Responses of Glossina fuscipes fuscipes (Diptera: Glossinidae) and other Diptera to carbon dioxide in linear and dense forests. Bulletin of Entomological Research, 89, 177-184. [April]

Monceaux, H. (1858) Notice sur un ouvrage manuscrit du Docteur J.-B. Robineau-Desvoidy intitulé: Diptères des environs de Paris. Annales de la Société Entomologique de France, (3) 6, 331-338. [8 September] [Dated from C.D. Sherborn manuscript notes.]

Monceaux, H. (1863) Introduction, pp. iii-xii. In: Robineau-Desvoidy, J-B., Histoire naturelle des diptères des environs de Paris. Oeuvre posthume du $\mathrm{D}^{\mathrm{r}}$ Robineau-Desvoidy publiée par les soins de sa famille, sous la direction de M. H. Monceaux. Tome premier. Masson et fils, Paris, Wagner, Leipzig, and Williams \& Norgate, London. xvi +1143 pp. [11 January]

Morge, G. (1975) Dipteren-Farbtafeln nach den bisher nicht veröffentlichten Original-Handzeichnungen Meigens : „Johann Wilhelm Meigen : Abbildung der europaeischen zweiflügeligen Insecten, nach der Natur“. Pars I. Vorwort des Herausgebers-Faksimiles von Original-Legenden und -Index: „Erklaerung der Fig.“ und „Alphabetisches Verzeichniss der Gattungen und Arten“-Farbtafeln I-LXXX. Beiträge zur Entomologie, 25, 383-500, pls. ilxxxi. [31 December+] 
Morge, G. (1976a) Dipteren-Farbtafeln nach den bisher nicht veröffentlichten Original-Handzeichnungen Meigens : „Johann Wilhelm Meigen : Abbildung der europaeischen zweiflügeligen Insecten, nach der Natur“. Pars II: Farbtafeln LXXXI-CLX. Beiträge zur Entomologie, 26, 441, pls. 1xxxi-clxi. [31 December+]

Morge, G. (1976b) Dipteren-Farbtafeln nach den bisher nicht veröffentlichten Original-Handzeichnungen Meigens : „Johann Wilhelm Meigen : Abbildung der europaeischen zweiflügeligen Insecten, nach der Natur“. Pars III: Farbtafeln CLXI-CCCV. Beiträge zur Entomologie, 26, 543, pls. clxi-cccv. [31 December+]

Neave, S.A. (1939) Nomenclator zoologicus. A list of the names of genera and subgenera in zoology from the tenth edition of Linnaeus 1758 to the end of 1935. In four volumes. Vol. II. D-L. Zoological Society of London. [ii] + 1025 pp. [31 December+]

Neave, S.A. (1940) Nomenclator zoologicus. A list of the names of genera and subgenera in zoology from the tenth edition of Linnaeus 1758 to the end of 1935. In four volumes. Vol. III. M-P. Zoological Society of London. xiv + 957 pp. [19 June]

[Date of receipt at BMNH.]

Norrbom, A.L., Carroll, L.E., Thompson, F.C., White, I.M. \& Freidberg, A. (1999) Systematic database of names, pp. 65-251. In: F.C. Thompson (ed.), Fruit Fly Expert Identification System and Systematic Information Database. Myia, 9[1998], vii + 524 pp. [22 February]

[Dated from information from the publisher.]

O’Hara, J.E. (1985) Actia Robineau-Desvoidy, 1830 (Insecta, Diptera): request for designation of type species. Z.N. (S.) 2491. Bulletin of Zoological Nomenclature, 42, 93-97. [2 April]

O'Hara, J.E., Shima, H. \& Zhang C.-T. (2009) Annotated catalogue of the Tachinidae (Insecta: Diptera) of China. Zootaxa, 2190, 236 pp. [6 August]

O'Hara, J.E. \& Wood, D.M. (2004) Catalogue of the Tachinidae (Diptera) of America north of Mexico. Memoirs on Entomology, International, 18 , iv +410 pp. [3 January]

Orbigny, C.V.D. d' (ed.) (1839) [Livraison 2], pp. 49-96. Dictionnaire universel d'histoire naturelle résumant et complétant tous les faits présentés par les encyclopédies, les anciens dictionnaires scientifiques, les oeuvres complètes de Buffon, et les meilleurs traités spéciaux sur les diverses branches des sciences naturelles; - donnant la description des êtres et des divers phénomènes de la nature, l'étymologie et la définition des noms scientifiques, les principales applications des corps organiques et inorganiques à l'agriculture, à la médecine, aux arts industriels, etc.; dirigé par M. Charles d'Orbigny, et enrichi d'un magnifique atlas de 288 planches gravées sur acier. Tome premier. C. Renard, Paris. [August]

[For dating, see Evenhuis (1990).]

Orbigny, C.V.D. d' (ed.) (1842a) [Livraisons 18-23], pp. 321-720. Dictionnaire universel d'histoire naturelle résumant et complétant tous les faits présentés par les encyclopédies, les anciens dictionnaires scientifiques, les oeuvres complètes de Buffon, et les meilleurs traités spéciaux sur les diverses branches des sciences naturelles; - donnant la description des êtres et des divers phénomènes de la nature, l'étymologie et la définition des noms scientifiques, les principales applications des corps organiques et inorganiques à l'agriculture, à la médecine, aux arts industriels, etc.; dirigé par M. Charles d'Orbigny, et enrichi d'un magnifique atlas de 288 planches gravées sur acier. Tome deuxième. C. Renard, Paris. [24 January-20 June]

[These livraisons cover the type designations for Bacchis, Bengalia, Blondelia, and Boisduvalia; for dating, see Evenhuis (1990); exact livraisons and dates of issue for each genus-group entry listed here are unknown and cover more than one livraison and date of issue.]

Orbigny, C.V.D. d' (ed.) (1842b) [Livraison 27], pp. 177-240. Dictionnaire universel d'histoire naturelle résumant et complétant tous les faits présentés par les encyclopédies, les anciens dictionnaires scientifiques, les oeuvres complètes de Buffon, et les meilleurs traités spéciaux sur les diverses branches des sciences naturelles; - donnant la description des êtres et des divers phénomènes de la nature, l'étymologie et la définition des noms scientifiques, les principales applications des corps organiques et inorganiques à l'agriculture, à la médecine, aux arts industriels, etc.; dirigé par M. Charles d'Orbigny, et enrichi d'un magnifique atlas de 288 planches gravées sur acier. Tome troisième. C. Renard, Paris. [7 November]

[For dating, see Evenhuis (1990).]

Orbigny, C.V.D. d' (ed.) (1843) [Livraison 37], pp. 1-48. Dictionnaire universel d'histoire naturelle résumant et complétant tous les faits présentés par les encyclopédies, les anciens dictionnaires scientifiques, les oeuvres complètes de Buffon, et les meilleurs traités spéciaux sur les diverses branches des sciences naturelles; - donnant la description des êtres et des divers phénomènes de la nature, l'étymologie et la définition des noms scientifiques, les principales applications des corps organiques et inorganiques à l'agriculture, à la médecine, aux arts industriels, etc.; dirigé par M. Charles d'Orbigny, et enrichi d'un magnifique atlas de 288 planches gravées sur acier. Tome quatrième. C. Renard, Paris. [31 July]

[For dating, see Evenhuis (1990).]

Orbigny, C.V.D. d' (ed.) (1844a) Cynomye. Cynomya, pp. 542-543. Dictionnaire universel d'histoire naturelle résumant et complétant tous les faits présentés par les encyclopédies, les anciens dictionnaires scientifiques, les 
oeuvres complètes de Buffon, et les meilleurs traités spéciaux sur les diverses branches des sciences naturelles; donnant la description des êtres et des divers phénomènes de la nature, l'étymologie et la définition des noms scientifiques, les principales applications des corps organiques et inorganiques à l'agriculture, à la médecine, aux arts industriels, etc.; dirigé par M. Charles d'Orbigny, et enrichi d'un magnifique atlas de 288 planches gravées sur acier. Tome quatrième. C. Renard, Paris. [19 February-8 April]

[For dating, see Evenhuis (1990); exact livraison and date of issue unknown]

Orbigny, C.V.D. d' (ed.) (1844b) [Livraison 51], pp. 129-206. Dictionnaire universel d'histoire naturelle résumant et complétant tous les faits présentés par les encyclopédies, les anciens dictionnaires scientifiques, les oeuvres complètes de Buffon, et les meilleurs traités spéciaux sur les diverses branches des sciences naturelles; - donnant la description des êtres et des divers phénomènes de la nature, l'étymologie et la définition des noms scientifiques, les principales applications des corps organiques et inorganiques à l'agriculture, à la médecine, aux arts industriels, etc.; dirigé par M. Charles d'Orbigny, et enrichi d'un magnifique atlas de 288 planches gravées sur acier. Tome cinquième. C. Renard, Paris. [12 August]

[For dating, see Evenhuis (1990).]

Orbigny, C.V.D. d' (ed.) (1845a) [Livraison 59], pp. 651-728. Dictionnaire universel d'histoire naturelle résumant et complétant tous les faits présentés par les encyclopédies, les anciens dictionnaires scientifiques, les oeuvres complètes de Buffon, et les meilleurs traités spéciaux sur les diverses branches des sciences naturelles; - donnant la description des êtres et des divers phénomènes de la nature, l'étymologie et la définition des noms scientifiques, les principales applications des corps organiques et inorganiques à l'agriculture, à la médecine, aux arts industriels, etc.; dirigé par M. Charles d'Orbigny, et enrichi d'un magnifique atlas de 288 planches gravées sur acier. Tome cinquième. C. Renard, Paris. [17 February]

[For dating, see Evenhuis (1990).]

Orbigny, C.V.D. d' (ed.) (1845b) Dictionnaire universel d'histoire naturelle résumant et complétant tous les faits présentés par les encyclopédies, les anciens dictionnaires scientifiques, les oeuvres complètes de Buffon, et les meilleurs traités spéciaux sur les diverses branches des sciences naturelles; - donnant la description des êtres et des divers phénomènes de la nature, l'étymologie et la définition des noms scientifiques, les principales applications des corps organiques et inorganiques à l'agriculture, à la médecine, aux arts industriels, etc.; dirigé par M. Charles d'Orbigny, et enrichi d'un magnifique atlas de 288 planches gravées sur acier. Tome sixième. C. Renard, Paris. [22 December]

[For dating, see Evenhuis (1990).]

Orbigny, C.V.D. d' (ed.) (1846) [Livraisons 91-92], pp. 513-640. Dictionnaire universel d'histoire naturelle résumant et complétant tous les faits présentés par les encyclopédies, les anciens dictionnaires scientifiques, les oeuvres complètes de Buffon, et les meilleurs traités spéciaux sur les diverses branches des sciences naturelles; - donnant la description des êtres et des divers phénomènes de la nature, l'étymologie et la définition des noms scientifiques, les principales applications des corps organiques et inorganiques à l'agriculture, à la médecine, aux arts industriels, etc.; dirigé par M. Charles d'Orbigny, et enrichi d'un magnifique atlas de 288 planches gravées sur acier. Tome huitième. C. Renard, Paris. [14 December]

[For dating, see Evenhuis (1990).]

Orbigny, C.V.D. d' (ed.) (1848a) [Livraisons 127-132], pp. 417-816. Dictionnaire universel d'histoire naturelle résumant et complétant tous les faits présentés par les encyclopédies, les anciens dictionnaires scientifiques, les oeuvres complètes de Buffon, et les meilleurs traités spéciaux sur les diverses branches des sciences naturelles; donnant la description des êtres et des divers phénomènes de la nature, l'étymologie et la définition des noms scientifiques, les principales applications des corps organiques et inorganiques à l'agriculture, à la médecine, aux arts industriels, etc.; dirigé par M. Charles d'Orbigny, et enrichi d'un magnifique atlas de 288 planches gravées sur acier. Tome onzième. C. Renard, Paris. [9 September]

[For dating, see Evenhuis (1990).]

Orbigny, C.V.D. d' (ed.) (1848b) [Livraison 134], pp. 65-128. Dictionnaire universel d'histoire naturelle résumant et complétant tous les faits présentés par les encyclopédies, les anciens dictionnaires scientifiques, les oeuvres complètes de Buffon, et les meilleurs traités spéciaux sur les diverses branches des sciences naturelles; - donnant la description des êtres et des divers phénomènes de la nature, l'étymologie et la définition des noms scientifiques, les principales applications des corps organiques et inorganiques à l'agriculture, à la médecine, aux arts industriels, etc.; dirigé par M. Charles d'Orbigny, et enrichi d'un magnifique atlas de 288 planches gravées sur acier. Tome douzième. C. Renard, Paris. [9 September]

[For dating, see Evenhuis (1990).]

Orbigny, C.V.D. d' (ed.) (1849a) [Livraisons 138-139], pp. 312-478. Dictionnaire universel d'histoire naturelle résumant et complétant tous les faits présentés par les encyclopédies, les anciens dictionnaires scientifiques, les oeuvres complètes de Buffon, et les meilleurs traités spéciaux sur les diverses branches des sciences naturelles; donnant la description des êtres et des divers phénomènes de la nature, l'étymologie et la définition des noms scientifiques, les principales applications des corps organiques et inorganiques à l'agriculture, à la médecine, aux 
arts industriels, etc.; dirigé par M. Charles d'Orbigny, et enrichi d'un magnifique atlas de 288 planches gravées sur acier. Tome douzième. C. Renard, Paris. [2 January]

[For dating, see Evenhuis (1990).]

Orbigny, C.V.D. d' (ed.) (1849b) [Livraisons 140-141], pp. 479-522. Dictionnaire universel d'histoire naturelle résumant et complétant tous les faits présentés par les encyclopédies, les anciens dictionnaires scientifiques, les oeuvres complètes de Buffon, et les meilleurs traités spéciaux sur les diverses branches des sciences naturelles; donnant la description des êtres et des divers phénomènes de la nature, l'étymologie et la définition des noms scientifiques, les principales applications des corps organiques et inorganiques à l'agriculture, à la médecine, aux arts industriels, etc.; dirigé par M. Charles d'Orbigny, et enrichi d'un magnifique atlas de 288 planches gravées sur acier. Tome douzième. C. Renard, Paris. [26 March]

[For dating, see Evenhuis (1990).]

Orbigny, C.V.D. d' (ed.) (1849c) [Livraisons 148-149], pp. 193-320. Dictionnaire universel d'histoire naturelle résumant et complétant tous les faits présentés par les encyclopédies, les anciens dictionnaires scientifiques, les oeuvres complètes de Buffon, et les meilleurs traités spéciaux sur les diverses branches des sciences naturelles; donnant la description des êtres et des divers phénomènes de la nature, l'étymologie et la définition des noms scientifiques, les principales applications des corps organiques et inorganiques à l'agriculture, à la médecine, aux arts industriels, etc.; dirigé par M. Charles d'Orbigny, et enrichi d'un magnifique atlas de 288 planches gravées sur acier. Tome treizième. C. Renard, Paris. [10 September]

[For dating, see Evenhuis (1990).]

Orbigny, C.V.D. d' (ed.) (1849d) [Livraison 150], pp. 321-384. Dictionnaire universel d'histoire naturelle résumant et complétant tous les faits présentés par les encyclopédies, les anciens dictionnaires scientifiques, les oeuvres complètes de Buffon, et les meilleurs traités spéciaux sur les diverses branches des sciences naturelles; —donnant la description des êtres et des divers phénomènes de la nature, l'étymologie et la définition des noms scientifiques, les principales applications des corps organiques et inorganiques à l'agriculture, à la médecine, aux arts industriels, etc.; dirigé par M. Charles d'Orbigny, et enrichi d'un magnifique atlas de 288 planches gravées sur acier. Tome treizième. C. Renard, Paris. [5 November]

[For dating, see Evenhuis (1990).]

Organisation for Economic Co-operation and Development (ed.) (2006) Safety Assessment of Transgenic Organisms: OECD Consensus Documents. Vols. 1 and 2. OECD Publishing, Paris. 744 pp. [24 July] [Dated from information from the publisher.]

Osten Sacken, C.R. (1881) Enumeration of the Diptera of the Malay Archipelago collected by Prof. Odoardo Beccari, M. ${ }^{r}$ L. M. d'Albertis and others. Annali del Museo Civico di Storia Naturale "Giacomo Doria", 16, 393-492. [16 February]

Osten Sacken, C.R. (1894) Two critical remarks about the recently-published third part of the Muscaria Schizometopa of MM. Brauer and Bergenstamm; also a notice on Robineau-Desvoidy. Berliner Entomologische Zeitschrift, 38[1893], 380-386. [January]

Osten Sacken, C.R. (1903) Notice on the circumstances of the publication of two principal works (1830, 1863) of Robineau-Desvoidy, pp. 180-192. In his: My life work in entomology. Parts I and II. Cambridge, Massachusetts. [before 9 December]

[Date recorded by the Société Entomologique de France.]

Pandellé, L. (1894) Études sur les muscides de France. $\mathrm{II}^{\mathrm{e}}$ partie [part]. Revue d'Entomologie (Caen), $13,1-113$. [Publication split: pp. 1-52 (January); pp. 53-84 (March); pp. 85-113 (May).]

Pape, T. (1987) The Sarcophagidae (Diptera) of Fennoscandia and Denmark. Fauna Entomologica Scandinavica, 19, 1203, 2 pls. [31 December+]

Pape, T. (1994) The world Blaesoxipha Loew, 1861 (Diptera: Sarcophagidae). Entomologica Scandinavica Supplement, 45, 1-247. [31 December+]

Pape, T. (1996) Catalogue of the Sarcophagidae of the world (Insecta: Diptera). Memoirs on Entomology, International, 8,558 pp. [30 July]

Pape, T. (1998a) Sarcophagidae, pp. 649-678. In: Papp, L. \& Darvas, B. (eds.), Contributions to a manual of Palaearctic Diptera (with special reference to flies of economic importance). Volume 3. Higher Brachycera. Science Herald, Budapest. 880 pp. [30 November]

Pape, T. (1998b) Rhinophoridae, pp. 679-689. In: Papp, L. \& Darvas, B. (eds.), Contributions to a manual of Palaearctic Diptera (with special reference to flies of economic importance). Volume 3. Higher Brachycera. Science Herald, Budapest. 880 pp. [30 November]

Pape, T., González-Mora, D., Peris, S.V. \& Baez, M. (2002) Sarcophagidae, pp. 218-221. In: Carles-Tolrá HjorthAndersen, M. (ed.), Catálogo de los Diptera de España, Portugal y Andorra. Boletín de la Sociedad Entomológica Aragonesa, Monografia, 8, 323 pp. [31 December] 
Pape, T., Richter, V., Rivosecchi, L. \& Rognes, K. (1995) Diptera Hippoboscoidea, Oestroidea. In: Minelli, A., Ruffo, S. \& La Posta, S. (eds.), Checklist delle specie della fauna italiana. Fascicolo 78. Calderini, Bologna. 36 pp. [31 December+]

Papp, L. (2001) Muscidae, pp. 406-420. In: Papp, L. (ed.), Checklist of the Diptera of Hungary. Hungarian Natural History Museum, Budapest. 550 pp. [30 November]

Petersen, J.F.T. \& Pape, T. (2001) Sarcophagidae. In: Petersen, J.F.T. \& Meier, R. (eds.), A preliminary list of the Diptera of Denmark. Steenstrupia, 26[2000], 214-216. [December]

Peyerimhoff, P. de (1932) La Société Entomologique de France (1832-1931), pp. 1-86. In: Société Entomologique de France. Livre du Centenaire. Société Entomologique de France, Paris. xii + 729 pp. [30 June]

Pitkin, B.R. \& Evenhuis, N.L. (1989) 85. Family Dryomyzidae, pp. 565. In: Evenhuis, N.L. (ed.), Catalog of the Diptera of the Australasian and Oceanian Regions. Bishop Museum Press, Honolulu \& E.J. Brill, Leiden. 1155 pp. [23 August] [Dated from Evenhuis (2008b).]

Pont, A.C. (1971) Some Muscidae (Diptera) from Lake Tarraure, Lule Lappmark, Sweden, with notes on collecting in other parts of Scandinavia. Entomologisk Tidskrift, 92, 100-122. [4 June]

Pont, A.C. (1973) Studies on Australian Muscidae (Diptera). IV. A revision of the subfamilies Muscinae and Stomoxyinae. Australian Journal of Zoology, Supplement Series, 21, 129-296. [30 August]

Pont, A.C. (1975) Himalayan Muscidae (Diptera). II. New species of Hydrotaeini. Opuscula Zoologica München, 139,13 pp. [1 December]

Pont, A.C. (1980a) Family Glossinidae, pp. 762-765. In: Crosskey, R.W. (ed.), Catalogue of the Diptera of the Afrotropical Region. British Museum (Natural History), London. 1437 pp. [20 July] [Dated from Evenhuis (2008b).]

Pont, A.C. (1980b) Family Calliphoridae, pp. 779-800. In: Crosskey, R.W. (ed.), Catalogue of the Diptera of the Afrotropical Region. British Museum (Natural History), London. 1437 pp. [20 July] [Dated from Evenhuis (2008b).]

Pont, A.C. (1986a) Family Fanniidae, pp. 41-57. In: Soós, Á. \& Papp, L. (eds.), Catalogue of Palaearctic Diptera. Volume 11. Scathophagidae-Hypodermatidae. Akadémiai Kiadó, Budapest. 346 pp. [15 February] [Dated from Evenhuis (2008b).]

Pont, A.C. (1986b) Family Muscidae, pp. 57-215. In: Soós, Á. \& Papp, L. (eds.), Catalogue of Palaearctic Diptera. Volume 11. Scathophagidae-Hypodermatidae. Akadémiai Kiadó, Budapest. 346 pp. [15 February] [Dated from Evenhuis (2008b).]

Pont, A.C. (1989) Family Muscidae, pp. 675-699. In: Evenhuis, N.L. (ed.), Catalog of the Diptera of the Australasian and Oceanian Regions. Bishop Museum Press, Honolulu \& E.J. Brill, Leiden. 1155 pp. [23 August] [Dated from Evenhuis (2008b).]

Pont, A.C. (1993) Observations on anthophilous Muscidae and other Diptera (Insecta) in Abisko National Park, Sweden. Journal of Natural History, 27, 631-643. [28 June]

Pont, A.C. (1995) Muscidae from above the tree-line in the Upper Ötz Valley (Tyrol, Austria) (Insecta, Diptera). Bericht des Naturwissenschaftlich-Medizinischen Vereins Innsbruck, 82, 311-318. [October]

Pont, A.C. \& Ackland, D.M. (1995) Fanniidae, Muscidae and Anthomyiidae associated with burrows of the Alpine Marmot (Marmota marmota Linnaeus) in the Upper Ötz Valley (Tyrol, Austria) (Insecta, Diptera). Bericht des Naturwissenschaftlich-Medizinischen Vereins Innsbruck, 82, 319-324. [October]

Pont, A.C. \& Horsfield, D. (1992) Thricops genarum (Zetterstedt, 1838) (Dipt., Muscidae), confirmed as a British species. Entomologist's Monthly Magazine, 128, 109-110. [31 July]

Pont, A.C. \& Meier, R. (2002) The Sepsidae (Diptera) of Europe. Fauna Entomologica Scandinavica, 37,221 pp., 2 pls. [August]

Pont, A.C. \& Merz, B. (1998) Muscidae, pp. 321-329. In: Merz, B., Bächli, G., Haenni, J.-P. \& Gonseth, Y. (eds.), Fauna Helvetica, 1. Diptera-Checklist. Centre Suisse de Cartographie de la Faune, and Schweizerische Entomologische Gesellschaft, Neuchâtel. 369 pp. [18 August]

Pont, A.C. \& Xue W.-Q. (2007) The publication date of "Flies of China". Studia Dipterologica, 14, 159-160. [20 December]

Povolný, D. (1997) Sarcophagidae, pp. 98-100. In: Chvála, M. (ed.), Check list of Diptera (Insecta) of the Czech and Slovak Republics. Karolinum, Charles University Press, Praha [= Prague]. 130 pp. [after 25 June] [Date given in introduction.]

Povolný, D. \& Verves, Y.G. (1997) The flesh-flies of Central Europe (Insecta, Diptera, Sarcophagidae). Spixiana Supplement, 24, 260 pp. [15 October]

Prijs, H.J. (2002) Family Muscidae, pp. 323-333. In: Beuk, P.L.T. (ed.), Checklist of the Diptera of The Netherlands. KNNV Uitgeverij, Utrecht. 448 pp. [31 December+]

Quantin, M. (1851) Troisième séance du 16 juin, pp. 37-46. In: Congrès Archéologique de France. Séances générales tenues à Auxerre, à Cluny \& à Clermont-Ferrand, en 1850 par la Société Française pour la Conservation des Monuments Historiques. Derache, Paris. 308 pp. [31 December+] 
Quantin, M. (1858) [M. Quantin informe la Société de la mort regrettable de M. le docteur Robineau-Desvoidy.] Bulletin de la Société des Sciences Historiques et Naturelles de l'Yonne, 11, 296. [31 December+]

Richter, V.A. (1987) Morphological parallelisms in the Tachinidae (Diptera). Entomologicheskoe Obozrenie, 66, 66-86. [In Russian.] [June]

[English translation in Entomological Review, 66(4), 35-55, 1987.]

Robineau-Desvoidy, J.-B. (1823) Propositions sur divers objets de médecine. Présentées et publiquement soutenues à la Faculté de Médecine de Montpellier le 22 Avril 1823. Pour obtenir le grade de Docteur en Médecine. J. Martel, Montpellier. 2 pp. [23 April]

Robineau-Desvoidy, J.-B. (1827) Essai sur la tribu des culicidés. Mémoires de la Société d'Histoire Naturelle, Paris, 3, 390-413, 1 plate. [October]

Robineau-Desvoidy, J.-B. (1828) Recherches sur l'organisation vertébrale des crustacés, des arachnides et des insectes. Compère Jeune, Paris. lxxviii + 228 pp., folding plate with 9 figs. [4 February]

[Dated from Procès-Verbaux des Séances de l'Académie des Sciences, Paris.]

Robineau-Desvoidy, J.-B. (1830) Essai sur les myodaires. Mémoires présentés par divers savans à l'Académie Royale des Sciences de l'Institut de France (Sciences Mathématiques et Physiques), (2) 2, 813 pp. [6 June]

[Date recorded as received in Bibliographie de la France.]

Robineau-Desvoidy, J.-B. (1838) Essai statistique sur le canton de Saint Sauveur-en-Puisaye. Gratiot, Paris. 112 pp. [31 December+]

Robineau-Desvoidy, J.-B. (1839) [Note: la nullité des progrès de la science dans l'étude des mouches, depuis la publication de [mon] essai sur les Myodaires.] Bulletin de la Société Entomologique de France, 8, vi. [June] [Dated from C.D. Sherborn manuscript notes.]

Robineau-Desvoidy, J.-B. (1842a) Mémoire sur trois espèces nouvelles de malacomydes. Annales de la Société Entomologique de France, 10[1841], 251-262. [January] [Dated from C.D. Sherborn manuscript notes.]

Robineau-Desvoidy, J.-B. (1842b) Notice sur l'herbine des lis, Herbina liliorum. Annales de la Société Entomologique de France, 10[1841], 263-268. [January]

[Dated from C.D. Sherborn manuscript notes.]

Robineau-Desvoidy, J.-B. (1842c) Notice sur le genre fucellie, Fucellia, R. D., et en particulier sur le Fucellia arenaria. Annales de la Société Entomologique de France, 10[1841], 269-272. [January]

[Dated from C.D. Sherborn manuscript notes.]

Robineau-Desvoidy, J.-B. (1843) [Sociétés Savantes: M. Saint-Martin ... donne lecture à un mémoire de M. RobineauDesvoidy intitulé: Études sur les myodaires des environs de Paris.] Revue Zoologique par la Société Cuvierienne, 6, 317. [early November]

Robineau-Desvoidy, J.-B. (1844a) Études sur les myodaires des environs de Paris. Annales de la Société Entomologique de France, (2) 2, 5-38. [5 June] [Dated from C.D. Sherborn manuscript notes.]

Robineau-Desvoidy, J.-B. (1844b) Description d'une nouvelle espèce du genre Brachyopa. Annales de la Société Entomologique de France, (2) 2, 39-40. [5 June] [Dated from C.D. Sherborn manuscript notes.]

Robineau-Desvoidy, J.-B. (1845) [Sociétés Savantes: M. Robineau-Desvoidy envoie un troisième mémoire sur les myodaires des environs de Paris.] Revue Zoologique par la Société Cuvierienne, 8, 108-109. [early April]

Robineau-Desvoidy, J.-B. (1846a) [Sociétés Savantes: Il est donné lecture d'un nouveau mémoire de M. le docteur Robineau-Desvoidy sur les myodaires des environs de Paris.] Revue Zoologique par la Société Cuvierienne, 8, 107. [early April]

Robineau-Desvoidy, J.-B. (1846b) Myodaires des environs de Paris (suite). Annales de la Société Entomologique de France, (2) 4, 17-38. [8 July]

[Dated from C.D. Sherborn manuscript notes.]

Robineau-Desvoidy, J.-B. (1846c) Coup d'oeil rétrospectif sur quelques points de l'entomologie actuelle. Annales de la Société Entomologique de France, (2) 4, 347-358. [23 December]

[Dated from C.D. Sherborn manuscript notes.]

Robineau-Desvoidy, J.-B. (1847) Myodaires des environs de Paris (suite). Annales de la Société Entomologique de France, (2) 5, 255-287. [25 August]

[Dated from C.D. Sherborn manuscript notes.]

Robineau-Desvoidy, J.-B. (1848a) Études sur les myodaires des environs de Paris. Annales de la Société Entomologique de France, (2) 5[1847], 591-617. [14 June]

[Dated from C.D. Sherborn manuscript notes.]

Robineau-Desvoidy, J.-B. (1848b) [Sociétés Savantes: On communique une nouvelle suite de mémoires de M. Robineau-Desvoidy sur les myodaires des environs de Paris.] Revue Zoologique par la Société Cuvierienne, 10, 185-186. [early July] 
Robineau-Desvoidy, J.-B. (1849a) Myodaires des environs de Paris (suite). Annales de la Société Entomologique de France, (2) 6[1848], 429-477. [9 May]

[Dated from C.D. Sherborn manuscript notes.]

Robineau-Desvoidy, J.-B. (1849b) [Sociétés Savantes: sur le Teichomyza muraria.] Revue et Magasin de Zoologie Pure et Appliquée, (2) 1, 94. [early March]

Robineau-Desvoidy, J.-B. (1849c) [Sociétés Savantes: M. Robineau-Desvoidy s'occupe des larves de diverses myodaires qui ont vécu aux dépens de l'homme.] Revue et Magasin de Zoologie Pure et Appliquée, (2) 1, 157. [early April]

Robineau-Desvoidy, J.-B. (1849d) [Sociétés Savantes: M. Robineau-Desvoidy donne lecture d'un mémoire sur plusieurs espèces de myodaires entomobies des environs de Paris.] Revue et Magasin de Zoologie Pure et Appliquée, (2) 1, 158. [early April]

Robineau-Desvoidy, J.-B. (1849e) [Note: sur le Teichomyza muraria.] Bulletin de la Société Entomologique de France, (2) $6[1848]$, xciv-xcv. [9 May]

[Dated from C.D. Sherborn manuscript notes.]

Robineau-Desvoidy, J.-B. (1849f) [Note: un mémoire où M. Leon Dufour donne la description de la larve et des moeurs d'une muscide, larve qui vit du sang de petites hirondelles.] Bulletin de la Société Entomologique de France, (2) 7 , iv-v. [11 July]

[Dated from C.D. Sherborn manuscript notes.]

Robineau-Desvoidy, J.-B. (1849g) [Note: sur les larves de diverses myodaires qui ont vécu aux dépens de l'homme.] Bulletin de la Société Entomologique de France, (2) 7, xvii-xix. [11 July]

[Dated from C.D. Sherborn manuscript notes.]

Robineau-Desvoidy, J.-B. (1849h) Découverte d'une statue de Vénus Anadyomène dans les ferriers de Menzilles. Bulletin de la Société des Sciences Historiques et Naturelles de l'Yonne, 3, 393-394, pl. 25. [31 December+]

Robineau-Desvoidy, J.-B. (1850a) Mémoire sur plusieurs espèces de myodaires-entomobies. Annales de la Société Entomologique de France, (2) 8, 157-181. [June]

[Dated from C.D. Sherborn manuscript notes.]

Robineau-Desvoidy, J.-B. (1850b) Myodaires des environs de Paris (suite). Annales de la Société Entomologique de France, (2) 8, 183-209. [June]

[Dated from C.D. Sherborn manuscript notes.]

Robineau-Desvoidy, J.-B. (1851a) Sur les éclosions de dix espèces d'entomobies obtenues par M. le colonel Goureau. Revue et Magasin de Zoologie Pure et Appliquée, (2) 3, 147-155. [early April]

Robineau-Desvoidy, J.-B. (1851b) [Note: description d'une nouvelle espèce de myodaire.] Bulletin de la Société Entomologique de France, (2) 9, xxvi-xxviii. [23 April]

[Dated from C.D. Sherborn manuscript notes.]

Robineau-Desvoidy, J.-B. (1851c) Description de plusieurs espèces de myodaires dont les larves sont mineuses des feuilles de végétaux. Revue et Magasin de Zoologie Pure et Appliquée (2) 3, 229-236. [early June]

Robineau-Desvoidy, J.-B. (1851d) Myodaires des environs de Paris (suite). Annales de la Société Entomologique de France, (2) 9, 177-190. [23 July]

[Dated from C.D. Sherborn manuscript notes.]

Robineau-Desvoidy, J.-B. (1851e) Description d'agromyzes et de phytomyzes écloses chez M. le colonel Goureau. Revue et Magasin de Zoologie Pure et Appliquée, (2) 3, 391-405. [August]

Robineau-Desvoidy, J.-B. (1851f) Myodaires des environs de Paris (suite). Annales de la Société Entomologique de France, (2) 9, 305-321. [1 November]

[Dated from C.D. Sherborn manuscript notes.]

Robineau-Desvoidy, J.-B. (1853a) Diptères des environs de Paris. Famille des myopaires. Bulletin de la Société des Sciences Historiques et Naturelles de l'Yonne, 7, 83-160. [November]

Robineau-Desvoidy, J.-B. (1853b) Diptères des environs de Paris. Famille des myopaires. Gens myopariarum. Perriquet, Auxerre, France. $80+[2]$ pp.

[This is a separately paginated reprint of the preceding article.]

Robineau-Desvoidy, J.-B. (1853c) Sur les éclosions de plusieurs de diptères, obtenues par le docteur Moret, médecin à Auxerre. Bulletin de la Société des Sciences Historiques et Naturelles de l'Yonne, 7, 531-536.

Robineau-Desvoidy, J.-B. (1863a) Histoire naturelle des diptères des environs de Paris. Oeuvre posthume du $\mathrm{D}^{\mathrm{r}}$ Robineau-Desvoidy publiée par les soins de sa famille, sous la direction de M. H. Monceaux. Tome premier. Masson et fils, Paris, Wagner, Leipzig, and Williams \& Norgate, London. xvi + 1143 pp. [11 January]

[Announced as published by Monceaux at the 11 January meeting of the Société.]

Robineau-Desvoidy, J.-B. (1863b) Histoire naturelle des diptères des environs de Paris. Oeuvre posthume du $\mathrm{D}^{\mathrm{r}}$ Robineau-Desvoidy publiée par les soins de sa famille, sous la direction de M. H. Monceaux. Tome second. Masson et fils, Paris, Wagner, Leipzig, and Williams \& Norgate, London. 920 pp. [11 January]

[Announced as published by Monceaux at the 11 January meeting of the Société.] 
Rocher, J.-P. (2003) Quelques érudits du XIX siècle, leurs alliances familiales et leur milieu. Bulletin de la Société des Sciences Historiques et Naturelles de l'Yonne, 134[2002], 149-170. [31 December+]

Rognes, K. (1991) Blowflies (Diptera, Calliphoridae) of Fennoscandia and Denmark. Fauna Entomologica Scandinavica, 24, 1-272. [before 21 February]

Rognes, K. (2002) Blowflies (Diptera, Calliphoridae) of Israel and adjacent areas with a new species from Tunisia. Entomologica Scandinavica Supplement, 59, 1-148. [before 19 September]

Rognes, K. (2006) Bengalomania - A review of Andy Z. Lehrer's book on Bengalia Robineau-Desvoidy, 1830 and related works (Diptera, Calliphoridae). Studia Dipterologica, 12[2005], 443-471. [18 April]

Roháček, J. (ed.) (2001) World catalog of Sphaeroceridae (Diptera). Slezské zemské Muzeum, Opava, Czech Republic. 414 pp. [15 December]

Rondani, C. (1856) Dipterologiae Italicae prodromus. Vol: I. Genera Italica ordinis dipterorum ordinatim disposita et distincta et in familias et stirpes aggregata. A. Stoccih, Parmae [= Parma]. $226+$ [2] pp. [10 September]

[Dated from advertisement from publisher in Stettiner Entomologische Zeitung.]

Rondani, C. (1859) Dipterologiae Italicae prodromus. Vol: III. Species Italicae ordinis dipterorum in genera characteribus definita, ordinatim, collectae, methodo analitica distinctae, et novis vel minus cognitis descriptis. Pars secunda. Muscidae, Siphoninae et (partim) Tachininae. A. Stocchi, Parmae [= Parma]. $243+$ [1] pp. [31 December+]

Rondani, C. (1861) Dipterologiae Italicae prodromus. Vol. IV. Species Italicae ordinis dipterorum in genera characteribus definita, ordinatim collectae, methodo analatica distinctae, et novis vel minus cognitis descriptis. Pars tertia. Muscidae Tachininarum complementum. A. Stocchi, Parmae [= Parma]. 174 pp. [31 December+]

Rondani, C. (1862) Dipterologiae Italicae prodromus. Vol. V. Species Italicae ordinis dipterorum in genera characteribus definita, ordinatim collectae, methodo analitica distinctae, et novis vel minus cognitis descriptis. Pars quarta. Muscidae. Phasiinae-Dexiinae-Muscinae-Stomoxidinae. P. Grazioli, Parmae [= Parma]. 239 pp. [31 December+]

Rondani, C. (1863) Diptera exotica revisa et annotata novis nonnullis descriptis. E. Soliani, Modena. 99 pp. [31 December+]

Rondani, C. (1866) Anthomyinae Italicae collectae distinctae et in ordinem dispositae. Dipterorum stirps XVII. Anthomyinae Rndn. Atti della Società Italiana di Scienze Naturali, 9, 68-217. [June]

Rondani, C. (1870) Ortalidinae Italicae collectae, distinctae et in ordinem dispositae. Dipterologiae Italicae prodromus. Pars VII. Fasc. 4 (sect. 1). Bulletino della Società Entomologica Italiana, 2, 5-34, 105-133. [Publication split: pp. 5-34 (March); pp. 105-33 (June).]

Rondani, C. (1871) Ortalidinae Italicae collectae, distinctae et in ordinem dispositae. Dipterologiae Italicae prodromia Pars VII-Fasc. 4. Bulletino della Società Entomologica Italiana, 3, 3-24, 161-188. [Publication split: pp. 3-24 (March); pp. 161-88 (June).]

Rondani, C. (1874) Species Italicae ordinis Dipterorum (Muscaria Rndn.). Stirps XXI.-Tanipezinae Rndn. Bulletino della Società Entomologica Italiana, 6, 167-182. [September]

Royer, M. (1929) Une excursion à Saint-Sauveur-en-Puisaye (Yonne). La tombe de Robineau-Desvoidy dans le parc du château. Bulletin de l'Association des Naturalistes de la Vallée du Loing, 11[1928], 178-195. [13 April]

Royer, M. (1931) La collection de diptères de Robineau-Desvoidy retrouvée et transportée au Muséum national d'Histoire naturelle. Bulletin de l'Association des Naturalistes de la Vallée du Loing, 14, 44-48. [17 September]

Rozkošný, R. \& Elberg, K. (1984) Family Sciomyzidae (Tetanoceridae), pp. 167-192. In: Sóos, Á. \& Papp, L. (eds.), Catalogue of Palaearctic Diptera. Volume 9. Micropezidae-Agromyzidae. Akadémiai Kiadó, Budapest. 460 pp. [15 October]

[Dated from Evenhuis (2008b).]

Rye, E.C. (1873) Diptera, p. 211-444 [of Insecta section]. In: Newton, A. (ed.), The zoological record for 1871; being volume ninth of the record of zoological literature. Zoological Record Association, London. [19 February] [Dated from back cover.]

Sabrosky, C.W. (1974) In defense of Robineau-Desvoidy. Mosquito Systematics, 6, 220-221. [September]

Sabrosky, C.W. (1988) Case 2654. Rapport sur les Myodaires du Docteur Robineau Desvoidy, (1826): proposed nomenclatural suppression. Bulletin of Zoological Nomenclature, 45, 283-287. [December]

Sabrosky, C.W. (1999) Family group names in Diptera. An annotated catalog. Myia, 10, 1-360. [before 4 April]

Sabrosky, C.W. \& Arnaud, P.H., Jr. (1965) Family Tachinidae (Larvaevoridae), pp. 961-1108. In: Stone, A., Sabrosky, C.W., Wirth, W.W., Foote, R.H. \& Coulson, J.R. (eds.), A catalog of the Diptera of America north of Mexico. United States Department of Agriculture, Agriculture Handbook, 276, iv + 1696 pp. [23 August] [Dated from Evenhuis (2008b).]

Savage, J. (2003) Revision of the genus Thricops Rondani (Diptera: Muscidae). Insect Systematics and Evolution, Supplement, 61, 143 pp. [31 December+]

Savage, J. \& Wheeler, T.A. (2004) Phylogeny of the Azeliini (Diptera: Muscidae). Studia Dipterologica, 11, 259-299. [15 November] 
Savage, J., Wheeler, T.A. \& Wiegmann, B.M. (2004) Phylogenetic analysis of the genus Thricops Rondani (Diptera: Muscidae) based on molecular and morphological characters. Systematic Entomology, 29, 395-414. [16 June]

Schiner, J.R. (1861a) Fauna austriaca. Die Fliegen (Diptera). Nach der analytischen Methode bearbeitet von J. Rudolf Schiner. Mit der Charakteristik sämmtlicher europäischer Gattungen, der Beschreibung aller in Deutschland vorkommenden Arten und der Aufzählung aller bisher beschriebenen europäischen Arten. II. Theil. [Heft 5], pp. 369-440. C. Gerold's Sohn, Wien [= Vienna]. [April-November]

[Dated from Evenhuis (1997: 695).]

Schiner, J.R. (1861b) Fauna austriaca. Die Fliegen (Diptera). Nach der analytischen Methode bearbeitet von J. Rudolf Schiner. Mit der Charakteristik sämmtlicher europäischer Gattungen, der Beschreibung aller in Deutschland vorkommenden Arten und der Aufzählung aller bisher beschriebenen europäischen Arten. II. Theil. [Heft 6/7], pp. 441-656. "1862". C. Gerold's Sohn, Wien [= Vienna]. [4 December]

[Dated from Evenhuis (1997: 695).]

Schiner, J.R. (1862) Fauna austriaca. Die Fliegen (Diptera). Nach der analytischen Methode bearbeitet von J. Rudolf Schiner. Mit der Charakteristik sämmtlicher europäischer Gattungen, der Beschreibung aller in Deutschland vorkommenden Arten und der Aufzählung aller bisher beschriebenen europäischen Arten. II. Theil. [Heft 8; concl.], pp. 1-80. “1864”. C. Gerold's Sohn, Wien [= Vienna]. [2 July]

[Dated from Evenhuis (1997: 695).]

Schiner, J.R. (1864a) Catalogus systematicus Dipterorum Europae. Societatis Zoologico-Botanicae, Vindobonae [= Vienna]. 115 pp. [after May]

[Date of preface.]

Schiner, J.R. (1864b) Fauna austriaca. Die Fliegen (Diptera). Nach der analytischen Methode bearbeitet, mit der Charakteristik sämmtlicher europäischer Gattungen, der Beschreibung aller in Deutschland vorkommenden Arten und der Aufzählung aller bisher beschriebenen europäischen Arten. II. Theil. [Heft 11/12], pp. 289-480. C. Gerold's Sohn, Wien [= Vienna]. [4 November]

[Dated from Evenhuis (1997: 695).]

Schnabl, J. \& Dziedzicki, H. (1911) Die Anthomyiden. Nova Acta Academiae Caesarea Leopoldino-Carolinae Germanicum Naturae Curiosorum, 95, 55-358. [31 December+]

Schumann, H. (1986) Calliphoridae, pp. 11-58. In: Soós, Á. \& Papp, L. (eds.), Catalogue of Palaearctic Diptera. Volume 12. Calliphoridae—Sarcophagidae. Akadémiai Kiadó, Budapest. 265 pp. [1 March] [Dated from Evenhuis (2008b).]

Scudder, S.H. (1882) Nomenclator zoologicus. An alphabetical list of all generic names that have been employed by naturalists for recent and fossil names from the earliest times to the close of the year 1879. I. Supplemental list of genera in zoology. List of generic names employed in zoology and paleontology to the close of the year 1879, chiefly supplemental to those catalogued by Agassiz and Marschall, or indexed in the Zoological Record. Bulletin of the United States National Museum, 19[i], i-xxi, 1-376. [August]

[Recorded in the August issue of American Journal of Science.]

Scudder, S.H. (1884) Nomenclator zoologicus. An alphabetical list of all generic names that have been employed by naturalists for recent and fossil names from the earliest times to the close of the year 1879. II. Universal index to genera in zoology. Complete list of generic names employed in zoology and paleontology to the close of the year 1879, as contained in the nomenclators of Agassiz, Marschall and Scudder, and in the Zoological Record. Bulletin of the United States National Museum, 19[ii], 340 pp. [31 December+]

[Dated from Evenhuis (1997: 724).]

Séguy, E. (1925) Études sur les anthomyides. $1^{\text {re }}$ note. Encyclopédie Entomologique (B) (II), Diptera, 1, 125-136. [31 January]

Séguy, E. (1937) Diptera. Fam. Muscidae. Genera Insectorum, 205, 1-604. [9 August]

[For dating of this series, see Evenhuis (1994).]

Séguy, E. (1952) Diptera. Fam. Scatophagidae. Genera Insectorum, 209, 1-107. [14 March]

[For dating of this series, see Evenhuis (1994).]

Senior-White, R.A., Aubertin, D. \& Smart, J. (1940) Diptera. Family Calliphoridae. In: Sewell, R.B.S. (ed.), The fauna of British India, including the remainder of the Oriental Region. Vol. VI. Taylor and Francis, Ltd., London. xiii + 288 pp. [28 March]

[Dated from Evenhuis (1997: 734).]

Skidmore, P. (1985) The biology of the Muscidae of the world. Series Entomologica, 29, xiv + 550 pp. [February] [Dated from Evenhuis et al. (1989: 961).]

Soós, Á. (1984) Family Micropezidae, pp. 19-24. In: Soós, Á. \& Papp, L. (eds.), Catalogue of Palaearctic Diptera. Volume 9. Micropezidae-Agromyzidae. Akadémiai Kiadó, Budapest. 460 pp. [15 October] [Dated from Evenhuis (2008b).]

Soós, Á. (1984a) Family Psilidae, pp. 28-35. In: Soós, Á. \& Papp, L. (eds.), Catalogue of Palaearctic Diptera. Volume 9. Micropezidae-Agromyzidae. Akadémiai Kiadó, Budapest. 460 pp. [15 October] 
[Dated from Evenhuis (2008b).]

Soós, Á. (1984b) Family Platystomatidae, pp. 38-45. In: Soós, Á. \& Papp, L. (eds.), Catalogue of Palaearctic Diptera. Volume 9. Micropezidae-Agromyzidae. Akadémiai Kiadó, Budapest. 460 pp. [15 October]

[Dated from Evenhuis (2008b).]

Soós, Á. (1984c) Family Otitidae, pp. 45-59. In: Soós, Á. \& Papp, L. (eds.), Catalogue of Palaearctic Diptera. Volume 9. Micropezidae-Agromyzidae. Akadémiai Kiadó, Budapest. 460 pp. [15 October] [Dated from Evenhuis (2008b).]

Soós, Á. (1984d) Family Chyromyidae, pp. 56-60. In: Soós, Á. \& Papp, L. (eds.), Catalogue of Palaearctic Diptera. Volume 10. Clusiidae-Chloropidae. Akadémiai Kiadó, Budapest. 460 pp. [15 October]

[Dated from Evenhuis (2008b).]

Speiser, P. (1915) Beiträge zur Dipterenfauna von Kamerun. III. Deutsche Entomologische Zeitschrift, 1915, 91-106. [15 April]

Spuler, A. (1924) North American genera and subgenera of the dipterous family Borboridae. Proceedings of the Academy of Natural Sciences, Philadelphia, 75, 369-378. [19 February]

Steck, G.J. \& Sutton, B.D. (2001) New records for Tephritidae (Diptera) in Great Smoky Mountains National Park. Insecta Mundi, 14[2000], 255-256. [21 August]

Stein, P. (1908) Analytische Übersicht aller mir bekannten breitstirnigen Anthomyiden-Männchen mit Ausschluss der Gattungen Lispa und Fucellia (Dipt.). Wiener Entomologische Zeitung, 27, 1-15. [1 January]

Steyskal, G.C. (1965) Family Otitidae (Ortalidae, Ulidiidae, Pterocallidae), pp. 642-654. In: Stone, A., Sabrosky, C.W., Wirth, W.W., Foote, R.H. \& Coulson, J.R., (eds.), A catalog of the Diptera of America north of Mexico. United States Department of Agriculture, Agriculture Handbook, 276, iv + 1696 pp. [23 August]

[Dated from Evenhuis (2008b).]

Steyskal, G.C. (1968a) Family Richardiidae. A Catalogue of the Diptera of the Americas South of the United States, 53, 1-26. [21 April]

Steyskal, G.C. (1968b) Family Otitidae (Ortalidae; including Pterocallidae, Ulidiidae). A Catalogue of the Diptera of the Americas South of the United States, 54, 1-31. [21 April]

Steyskal, G.C. (1972) A new species of Myoleja with a key to North American species (Diptera: Tephritidae). The Florida Entomologist, 55, 207-211. [11 September]

Steyskal, G.C. (1977) Family Platystomatidae, pp. 135-164. In: Delfinado, M.D. \& Hardy, D.E. (eds.), A catalog of the Diptera of the Oriental Region. Volume III. Suborder Cyclorrhapha (excluding Division Aschiza). University Press of Hawaii, Honolulu. $\mathrm{x}+854$ pp. [4 November]

[Dated from Evenhuis (2008b).]

Steyskal, G.C. (1980) Family Platystomatidae, pp. 563-574. In: Crosskey, R.W. (ed.), Catalogue of the Diptera of the Afrotropical Region. British Museum (Natural History), London. 1437 pp. [20 July] [Dated from Evenhuis (2008b).]

Steyskal, G.C. (1986) Taxonomy of the adults of the genus Strauzia Robineau-Desvoidy (Diptera, Tephritidae). Insecta Mundi, 1, 101-117. [2 March]

Steyskal, G.C., Thompson, F.C., Mathis, W.N. \& Knutson, L.V. (2004) The type species of Ilione (Diptera: Sciomyzidae). Studia Dipterologica, 10[2003], 559-564. [19 May]

Stoltzfus, W.B. (1988) The taxonomy and biology of Strauzia (Diptera: Tephritidae). Journal of the Iowa Academy of Sciences, 96, 117-126.

Strobl, G. (1910) Die Dipteren von Steiermark. [V.] II. Nachtrag. Mitteilungen des Naturwissenschaftlichen Vereins für Steiermark, 46[1909], 45-293. [before 17 October]

[Dated from Carroll et al., 1998: 468.]

Stuke, J.-H. \& Clements, D.K. (2005) The interpretation of some Conopidae (Diptera) described by Robineau-Desvoidy. Zootaxa, 886, 1-12. [7 March]

Sutton, B.D. \& Carlson, D.A. (1997) Cuticular hydrocarbons of Glossina, III: Subgenera Glossina and Nemorhina. Journal of Chemical Ecology, 23, 1291-1320. [31 December+]

Sutton, B. D. \& Steck, G. J. (2005) An annotated checklist of the Tephritidae (Diptera) of Florida. Insecta Mundi, 19, 227-245. [December]

Swainson, W. (1840) Taxidermy, bibliography, and biography. In: Lardner, D., The Cabinet Cyclopaedia. Natural History. Longman, Orme, Brown, Green \& Longmans \& Taylor, London. 392 pp. [before 8 August] [Date reviewed in the Literary Gazette.]

Tachi, T. \& Shima, H. (2005) Revision of the subgenus Ceranthia Robineau-Desvoidy of the genus Siphona Meigen of Japan (Diptera: Tachinidae). Entomological Science, 8, 189-200. [30 June]

Tanasijtshuk, V.N. (1984) Family Chamaemyiidae (Ochthiphilidae), pp. 220-232. In: Soós, Á. \& Papp, L. (eds.), Catalogue of Palaearctic Diptera. Volume 9. Micropezidae-Agromyzidae. Akadémiai Kiadó, Budapest. 460 pp. [15 October]

[Dated from Evenhuis (2008b).] 
Teschner, D. (1999) Muscidae, pp. 161-166. In: Schumann, H., Bährmann, R. \& Stark, A. (eds.), Checkliste der Dipteren Deutschlands. Studia Dipterologica Supplement, 2, 354 pp. [10 February]

Theobald, F.W. (1901) A monograph of the Culicidae or mosquitoes. Mainly compiled from the collections received at the British Museum from various parts of the world in connection with the investigation into the cause of malaria conducted by the Colonial Office and the Royal Society. Volume I. British Museum (Natural History), London. $\mathrm{xviii}+424$ pp. [23 November]

[Dated from Evenhuis (1997).]

Thompson, F.C., Evenhuis, N.L. \& Sabrosky, C.W. (1999) Bibliography of the family-group names of Diptera. Myia, 10, 363-556. [before 7 April]

[Date sent by senior author.]

Townsend, C.H.T. (1915) Correction of the misuse of the generic name Musca, with description of two new genera. Journal of the Washington Academy of Sciences, 5, 433-436. [19 June]

Townsend, C.H.T. (1916) Designations of muscoid genotypes, with new genera and species. Insecutor Inscitiae Menstruus, 4, 4-12. [31 March]

Townsend, C.H.T. (1932) Notes on Old-World oestromuscoid types. Part II. Annals and Magazine of Natural History, (10) 9, 33-57. [1 January]

[Dated from Evenhuis (2003b).]

Townsend, C.H.T. (1938) Manual of myiology in twelve parts. Part VI. Muscoid generic diagnoses and data. Stephanostomatini to Moriniini. Privately published, Itaquaquecetuba, São Paulo. 246 pp. [April]

Townsend, C.H.T. (1939) Manual of myiology in twelve parts. Part VIII. Oestroid diagnoses and data. Microtropesini to Voriini. Privately published, Itaquaquecetuba, São Paulo. 308 pp. [before 1 September]

Tschorsnig, H.-P. (1983) Untersuchungen zur Ökologie der Raupenfliegen (Dipt., Tachinidae) im Mooswald, am Kaiserstuhl und in Rhein-Trockenwald. Mitteilungen des Badischen Landesvereins für Naturkunde und Naturschutz, (N.F.) 13, 213-236. [31 December]

Tschorsnig, H.-P. (1985) Taxonomie forstlich wichtiger Parasiten: Untersuchungen zur Struktur des männlichen Postabdomens der Raupenfliegen (Diptera, Tachinidae). Stuttgarter Beiträge zur Naturkunde (A), 383, 137 pp. [29 November]

Tschorsnig, H.-P. (1992) Tachinidae (Diptera) from the Iberian Peninsula and Mallorca. Stuttgarter Beiträge zur Naturkunde (A), 472, 76 pp. [15 April]

Tschorsnig, H.-P. \& Brechtel, F. (1999) Raupenfliegen (Diptera: Tachinidae) aus dem Bienwald (Rheinland-Pfalz). Mitteilungen der Pollichia, 86, 127-138. [31 December+]

Tschorsnig, H.-P. \& Herting, B. (1994a) Die Raupenfliegen (Diptera: Tachinidae) Mitteleuropas: Bestimmungstabellen und Angaben zur Verbreitung und Ökologie der einzelnen Arten. Stuttgarter Beiträge zur Naturkunde (A), 506, 170 pp. [4 September]

Tschorsnig, H.-P. \& Herting, B. (1994b) Die Raupenfliegen (Diptera: Tachinidae) des "Pferdstrieb" bei Sandhausen. Beihefte zu den Veröffentlichungen für Naturschutz und Landschaftspflege Baden-Württemberg, 80, 211-222. [31 December+]

Tschorsnig, H.-P. \& Herting, B. (1998) Tachinidae, pp. 343-356. In: Merz, B., Bächli, G., Haenni, J.-P. \& Gonseth, Y. (eds.), Fauna Helvetica, 1. Diptera-Checklist. Centre Suisse de Cartographie de la Faune, and Schweizerische Entomologische Gesellschaft, Neuchâtel. 369 pp. [18 August]

Tschorsnig, H.-P. \& Herting, B. (2000) Raupenfliegen (Diptera, Tachinidae) aus der Trockenaue am südbadischen Oberrhein. Naturschutz-Spectrum, Themen, 92, 233-241. [31 December+]

Tschorsnig, H.-P. \& Schmid-Egger, C. (1993) Raupenfliegen (Diptera, Tachinidae) von extensiv genutzten oder aufgelassenen Weinbergen im Enztal und im Stromberg (Baden-Württemberg). Jahreshefte der Gesellschaft für Naturkunde in Württemberg, 148, 209-220. [15 December]

Tschorsnig, H.-P. \& Ziegler, J. (1999) Tachinidae, pp. 204-214. In: Schumann, H., Bährmann, R. \& Stark, A. (eds.), Checkliste der Dipteren Deutschlands. Studia Dipterologica Supplement, 2, 354 pp. [10 February]

Velterop, J.H.C. \& Sijstermans, L.E.N. (2002) Family Sarcophagidae, pp. 344-349. In: Beuk, P.L.T. (ed.), Checklist of the Diptera of The Netherlands. KNNV Uitgeverij, Utrecht. 448 pp. [31 December+]

Verves, Y.G. (1982) Sarcophaginae [part]. Die Fliegen der Palaearktischen Region, 64h[Lieferung 327], $235-296$. [before 27 August]

[Dated from Evenhuis (1997: 470).]

Verves, Y.G. (1985) Sarcophaginae [part]. Die Fliegen der Palaearktischen Region, 64h[Lieferung 330], 297-440. [31 December+]

Verves, Y.G. (1986) Sarcophagidae, pp. 58-193. In: Soós, Á. \& Papp, L. (eds.), Catalogue of Palaearctic Diptera. Volume 12. Calliphoridae-Sarcophagidae. Akadémiai Kiadó, Budapest. 265 pp. [1 March]

[Dated from Evenhuis (2008b).]

Verves, Y.G. (2000) A checklist of Ukrainian Sarcophagidae (Diptera), with a description of a new species. Journal of the Ukrainian Entomological Society, 4(3-4), 49-57. [January] 
[Dated from information supplied by the author.]

Verves, Y.G. (2001) The composition of Paramacronychiinae (Sarcophagidae, Diptera) with the descriptions of two new genera. International Journal of Dipterological Research, 12, 145-149. [29 November]

[Dated from information from the editor.]

Verves, Y.G. \& Khrokalo, L.A. (2006) Review of Macronychiinae (Diptera, Sarcophagidae) of the world. Vestnik Zoologii, 40, 219-239. [March-April]

Wainwright, C.J. (1928) The British Tachinidae (Diptera). Transactions of the Entomological Society of London, 76, 139-254. [August]

Westdal, P.H. \& Barrett, C.F. (1960) Life-history and habits of the sunflower maggot, Strauzia longipennis (Wied.) (Diptera: Trypetidae), in Manitoba. The Canadian Entomologist, 92, 481-488. [29 July]

Westdal, P.H. \& Barrett, C.F. (1962) Injury by the sunflower maggot, Strauzia longipennis (Wied.) (Diptera: Trypetidae), to sunflowers in Manitoba. The Canadian Journal of Plant Science, 42, 11-14.

Westwood, J.O. (1840) Order XIII. Diptera Aristotle. (Antliata Fabricius. Halteriptera Clairv.), pp. 125-154. In his: An introduction to the modern classification of insects; founded on the natural habits and corresponding organisation of the different families. Synopsis of the genera of British insects. Longman, Orme, Brown, Green \& Longmans, London. 158 pp. [June]

[Dated from Evenhuis (1997).]

White, I.M. (1986) A new species of Paroxyna Hendel and notes on the nomenclature of other British Tephritidae (Diptera). Entomologist's Monthly Magazine, 122, 145-163. [11 August]

Whitmore, D., Richet, R. \& Pape, T. (2008) Sarcophagidae, pp. 229-237. In: Ziegler, J. (ed.), Diptera Stelviana. A dipterological perspective on a changing alpine landscape. Volume 1. Studia Dipterologica Supplement, 16, 395 pp. [22 December]

Wiedemann, C.R.W. (1830) Aussereuropäische zweiflügelige Insekten. Als Fortsetzung des Meigenschen Werkes. Erster Theil. Schulz, Hamm. xii + 684 pp. [1 September]

[Dated from Evenhuis (1997).]

Williams, R.H., Whipps, J.M. \& Cooke, R.C. (1998) Role of soil mesofauna in dispersal of Coniothyrium minitans: transmission to sclerotia of Sclerotinia sclerotiorum. Soil Biology and Biochemistry, 30, 1929-1935. [31 December+]

Williston, S.W. (1908) Manual of North American Diptera. Third edition. J.T. Hathaway, New Haven, Connecticut. 405 pp. [28 August]

[From information supplied in a review of the book by J.M. Aldrich, 1909 in Science.]

Wills, H.J. (1968) Diptera from Monks Wood National Nature Reserve. Entomological Record and Journal of Variation, 80, 115-119, 134-140. [31 December+]

Wood, D.M. (1987) Tachinidae, pp. 1193-1269. In: McAlpine, J.F., Peterson, B.V., Shewell, G.E., Teskey, H.J., Vockeroth, J.R. \& Wood, D.M. (coordinators), Manual of Nearctic Diptera. Volume 2. Agriculture Canada Monograph, 28, i-vi, 675-1332. [15 March]

[Dating information supplied by the publisher.]

Xue W.-Q. \& Chao C.-M. (eds.) (1998) Flies of China. Volume 1. Liaoning Science and Technology Press, Shenyang. $2+7$ $+6+14+1365$ pp. [May]

[For dating, see Pont \& Xue (2007).]

Zangheri, P. (1969) Repertorio sistematico e topografico della flora e della fauna vivente e fossile della Romagna. In base ai materiali contenuti nel Museo Zangheri (nel Museo Civico di Storia Naturale di Verona). Con cenni sull'ambiente naturale ed una sintesi biogeografica. Saggio d'illustrazione naturalistica d'una regione italiana. Tomo III. Museo Civico di Storia Naturale di Verona, Memorie fuori serie N. 1, 1166-1179. [31 December+]

Zetterstedt, J.W. (1845) Diptera Scandinaviae disposita et descripta. Tomus tertius. [Part 2] Officina Lundbergiana, Lundae [= Lund]. Pp. 1013-1280.

[This volume published in two parts (1844-1845). For dating and pagination, see Evenhuis (1997: 840).]

Ziegler, J. \& Lange, C. (2001) Asselfliegen, Fleischfliegen, und Raupenfliegen (Rhinophoridae, Sarcophagidae, Tachinidae) aus Südtirol (Italien). Gredleriana, 1, 133-170. [31 December]

Zimin, L.S. \& El'berg, K.Ya. (1970) [Family Muscidae-true flies], pp. 511-595. In: Shtakel'berg, A.A. \& Narchuk, E.P. (eds.), [Keys to the insects of the European part of the USSR in five volumes (general editor G.Ya. Bey-Bienko). V, flies, fleas, second part]. Nauka, Leningrad [= St. Petersburg]. 943 pp. [October] [In Russian; English translation published, Washington, D.C., 1988, pp. 839-974.]

[Dated from information from publisher.] 


\section{APPENDIX I. Complete Bibliography of André-Jean-Baptiste Robineau-Desvoidy}

[surname found in the literature as "Robineau-Desvoidy", "Robineau des Voidy", "Robineau-Duvoidy", "Robineau-Devoidy", "Robineau des Voidis", "Robineau-Desvoidis", "Robineau-Desvoisdy"; "Robineau",

"Robinot-Desvoidy"]

\section{8}

Épître à mon ami P. Pietresson-Saint-Aubin (de Saint-Sauveur), auteur du "Dictionnaire historique, topographique et militaire de tous les environs de Paris »; des «Cimetières et Catacombes de Paris »; et des «Victoires, conquêtes, revers, désastres et guerres civiles des Français de 1792 à 1815 ». Doublet, Paris. 12 pp. [March]

[Dated from Lacombe (1903); the author on the pamphlet is given as "Robineau-Duvoidy (Saint-Sauveur), étudiant de médecine". According to Lacombe (1903) only 50 copies were printed and it was not intended for the trade.]

\section{3}

Propositions sur divers objets de médecine. Présentées et publiquement soutenues à la Faculté de Médecine de Montpellier le 22 Avril 1823. Pour obtenir le grade de Docteur en Médecine. J. Martel, Montpellier. 2 pp. [23 April]

\section{6}

Sur l'organe de l'odorat dans les crustacés. Nouveau Bulletin de la Société Philomatique, 1826, 192. [31 December+]

[Author given as "Robineau des Voidy".]

Sur l'harmonie des espèces de coléoptères tétramères avec le règne végétal. Nouveau Bulletin de la Société Philomatique, 1826, 192-193. [31 December+]

[Author given as "Robineau des Voidy".]

[Séance du 28 août 1826: un ouvrage manuscrit sur le "Genre mouche de Linnaeus", dont il fait une famille sous le nom de myodaires.] Procès Verbaux de l'Académie des Sciences de Paris, 8, 420.

[This records that the manuscript was sent to a publication committee consisting of Duméril, Latreille and

Blainville. The author is here given as "Robinot-Desvoidy".]

\section{7}

Essai sur la tribu des culicidés. Mémoires de la Société d'Histoire Naturelle, Paris, 3, 390-413, 1 pl. [October]

[Séance du 5 février 1827: M. de Blainville donne communication d'une lettre de M. Robineau-Desvoidy sur l'organe de l'olfaction dans les crustacés.] Procès-Verbaux des Séances de l'Académie des Sciences de Paris, 8, 488.

[Séance du 26 mars 1827: MM. Raspail et Robineau-Desvoidy lisent un mémoire intitulé Recherches sur l'histoire naturelle de l'Alcyonella stagnorum (Lamarck).] Procès-Verbaux des Séances de l'Académie des Sciences de Paris, 8, 511.

[Séance du 18 juin 1827: M. Robineau-Desvoidy remet une lettre contenant les principaux résultats qu'il a obtenus sur les organes buccaux des hyménoptères, des diptères, des lépidoptères et hémiptères.] ProcèsVerbaux des Séances de l'Académie des Sciences de Paris, 8, 551. 
[Séance du 25 juin 1827: MM. Raspail et Robineau-Desvoidy annoncent posséder des myriades d'alcyonelles, et adressent un paquet cacheté qu'ils demandent à l'Académie de faire déposer au Sécretariat.] Procès-Verbaux des Séances de l'Académie des Sciences de Paris, 8, 557.

[Séance du 24 septembre 1827: M. Robineau-Desvoidy lit un mémoire intitulé Recherches sur l'organisation vertébrale des animaux des classes inférieures.] Procès-Verbaux des Séances de l'Académie des Sciences de Paris, 8, 594.

[Séance du 22 octobre 1827: M. Robineau-Desvoidy lit un mémoire intitulé Recherches sur l'organisation vertébrale des crustacés, des arachnides et des insectes.] Procès-Verbaux des Séances de l'Académie des Sciences de Paris, 8, 608.

Organe de l'olfaction des crustacés (communication faite à l'Acad. roy. des sc. le 5 fév. 1827). Bulletin Universel des Sciences et de l'Industrie (2, Bulletin des Sciences Naturelles et de Géologie), 11, 135137. [29 December]

Usage des balanciers des diptères. Bulletin Universel des Sciences et de l'Industrie (2, Bulletin des Sciences Naturelles et de Géologie), 11, 159. [29 December]

\section{8}

Recherches sur l'organisation vertébrale des crustacés, des arachnides et des insectes. Compère Jeune, Paris. lxxviii + 228 pp., folding plate with 9 figs. [4 February]

[Dated from Procès-Verbaux des Séances de l'Académie des Sciences, Paris.]

[Séance du 22 décembre 1828: M. Robineau-Desvoidy adresse un essai manuscrit sur la Théorie de la coquille des animaux mollusques.] Procès-Verbaux des Séances de l'Académie des Sciences de Paris, 9, 165.

\section{9}

Le 17 mars 1815. Robert, Auxerre. 11 pp. [20 March]

Lettre à M. le gérant responsable du "Mémorial de l'Yonne". Tastu, Paris. 16 pp. [18 April]

[Dated from Bibliographie de la France.]

[Séance du 19 octobre 1829: Il est donné lecture d'une lettre de M. Robineau-Desvoidy qui communique deux observations qu'il a faites récemment: $1^{\mathrm{e}}$ Dans un terrain argilo-sablonneux ... $2^{\mathrm{e}}$ Ayant fait l'anatomie d'une vipère ... sous le nom de Serpents rouges.] Procès-Verbaux des Séances de l'Académie des Sciences de Paris, 9, 330.

\section{0}

Sur un nouveau genre des parasites. Annales de Sciences d'Observation, 3, 122-127. [January]

Correspondance. Annales de Sciences d'Observation, 3, 143-144. [January]

Sur la composition organique de la coquille des animaux-mollusques. Annales de Sciences d'Observation, 3 , 251-277. [February]

On the organic composition of the shell of molluscous animals. Edinburgh Journal of Natural and Geographical Science, 2, 222-228. [June]

[English translation of the previous article.]

Essai sur les myodaires. Mémoires présentés par divers savans à l'Académie Royale des Sciences de l'Institut de France (Sciences Mathématiques et Physiques), (2) 2, 813 pp. [6 June]

[An older spelling of "Savants" was "Savans". The former spelling was used in the name of the journal on the blue cover of this work, while the latter spelling was used in the journal name on two subsequent title pages. One or more of these pages are often missing from bound copies of this work. "Savans" was also used in a shortened journal title "Savans étrangers" in the footer on the first page of each signature.] 
The first seven marked * were read in title only, and are the titles of Robineau-Desvoidy manuscripts presented by Blainville.

*[Note: mémoire sur deux espèces d'osmie qui font leur nid dans des coquilles d'helice, et sur un eulophe dont le larve vit aux dépens de ces osmies.] Comptes Rendus Hebdomadaires des Séances de l'Académie des Sciences, Paris, 3, 174. [after 16 August]

[Date of séance.]

*[Note: mémoire pour servir à l'histoire des sapyges.] Comptes Rendus Hebdomadaires des Séances de l'Académie des Sciences, Paris, 3, 174. [after 16 August]

[Date of séance.]

*[Note: sur plusieurs insectes parasites du blaireau.] Comptes Rendus Hebdomadaires des Séances de l'Académie des Sciences, Paris, 3, 174. [after 16 August]

[Date of séance.]

*[Note: sur les mouches qui vivent dans les excréments du blaireau, de la chauve-souris et de la belette.] Comptes Rendus Hebdomadaires des Séances de l'Académie des Sciences, Paris, 3, 174. [after 16 August]

[Date of séance.]

*[Note: sur un nouvel ennemi de l'abeille domestique, l'asylus diadema.] Comptes Rendus Hebdomadaires des Séances de l'Académie des Sciences, Paris, 3, 174. [after 16 August]

[Date of séance.]

*[Note: sur le conops auripes.] Comptes Rendus Hebdomadaires des Séances de l'Académie des Sciences, Paris, 3, 174. [after 16 August]

[Date of séance.]

*[Note: sur une nouvelle espèce de mouche (herbina narcissi) qui vit dans les liliacées.] Comptes Rendus Hebdomadaires des Séances de l'Académie des Sciences, Paris, 3, 174. [after 16 August]

[Date of séance.]

[Note: mémoire sur une espèce de chenille qui a vécu dans les intestins humains.] Comptes Rendus Hebdomadaires des Séances de l'Académie des Sciences, Paris, 3, 442. [after 10 October] [Date of séance. Title only.]

Note sur le Ptinus carinatus. Comptes Rendus Hebdomadaires des Séances de l'Académie des Sciences, Paris, 3, 442-443. [after 10 October]

[Date of the séance. Contains an abstract in addition to the title.]

Complément au mémoire sur des chenilles qui ont vécu dans les intestins humains. Comptes Rendus Hebdomadaires des Séances de l'Académie des Sciences, Paris, 3, 764. [after 19 December]

[Date of the séance.]

\section{7}

Mémoires et notices sur diverses espèces des insectes. Bibliothèque Universelle de Genève, 12, $433-434$. [November]

\section{8}

Essai statistique sur le canton de Saint Sauveur-en-Puisaye. Gratiot, Paris. 112 pp. [31 December+] [Published in a facsimile edition in 2002 in the series "Monographies des villes et villages de France", edited by M.-G. Micberth, Le Livre d'Histoire-Lorisse, Paris. 100 pp.] 
[Note: la nullité des progrès de la science dans l'étude des mouches, depuis la publication de [mon] essai sur les Myodaires.] Bulletin de la Société Entomologique de France, 8, vi. [June]

Mémoire sur le Xenillus clypeator, coléoptère nouveau. Annales de la Société Entomologique de France, 8 , 455-472. [November]

[Robineau-Desvoidy's article is on pp. 455-462. Pages 463-472 are a "Rapport sur le mémoire de M. Robineau-

Desvoidy" by M. Démary, which is signed at its conclusion by H. Lucas and Démary.]

\section{2}

Mémoire sur trois espèces nouvelles de malacomydes. Annales de la Société Entomologique de France, 10[1841], 251-262. [January]

Notice sur l'herbine des lis, Herbina liliorum. Annales de la Société Entomologique de France, 10[1841], 263-268. [January]

Notice sur le genre fucellie, Fucellia, R.-D., et en particulier sur le Fucellia arenaria. Annales de la Société Entomologique de France, 10[1841], 269-272. [January]

Notice sur le Thyreophora cynophila. Annales de la Société Entomologique de France, 10[1841], 273. [January]

Notice sur le Phasia crassipennis. Annales de la Société Entomologique de France, 10[1841], 274. [January]

Lettre de Jean Baptiste Robineau-Desvoidy, électeur et caporal de la garde nationale de son commune rurale, à M. Ambroise, candidat à la députation, simple chasseur de l'ex-garde nationale d'Auxerre, vendeur de paroles par profession, et non encore décoré. Saint Sauveur, ce $1^{e}$ juillet 1842. Cardon, Troyes. 4 pp. [6 July]

Sur l'usage réel des antennes chez les insectes. Bulletin de la Société Entomologique de France, 11, xxiiixxvii. [October]

\section{3}

[Sociétés Savantes: M. Saint-Martin ... donne lecture à un mémoire de M. Robineau-Desvoidy intitulé: Études sur les myodaires des environs de Paris.] Revue Zoologique par la Société Cuvierienne, 6, 317. [3 November]

[Sociétés Savantes: Dans une seconde note, M. Robineau-Desvoidy décrit une nouvelle espèce de diptères qu'il a découverte aux environs de Saint-Sauveur (Yonne).] Revue Zoologique par la Société Cuvierienne, 6, 317. [3 November]

1844

Études sur les myodaires des environs de Paris. Annales de la Société Entomologique de France, (2) 2, 5-38. [5 June]

Description d'une nouvelle espèce du genre Brachyopa. Annales de la Société Entomologique de France, (2) 2, 39-40. [5 June]

\section{5}

[Sociétés Savantes: M. Robineau-Desvoidy envoie un troisième mémoire sur les myodaires des environs de Paris.] Revue Zoologique par la Société Cuvierienne, 8, 108-109. [3 April]

[M. L. Buquet donne lecture d'une lettre de M. Robineau-Desvoidy qui accompagne l'envoi d'un nouveau mémoire sur les myodaires.] Bulletin de la Société Entomologique de France, (2) 3, xii. [August] 
[Note: sur l'origine des grès micacés et des silex qu'on retrouve à la surface de la formation jurassique.] Bulletin de la Société Géologique de France, (2) 2, 696-697. [31 December+]

[Note: sur une explication que les grès observés près de Chatel-Censoir ... .] Bulletin de la Société Géologique de France, (2) 2, 697-698. [31 December+]

[Note: sur la formation de la brèche à la violence d'un courant diluvien.] Bulletin de la Société Géologique de France, (2) 2, 721, 723. [31 December+]

\section{6}

[Sociétés Savantes: Il est donné lecture d'un nouveau mémoire de M. le docteur Robineau-Desvoidy sur les myodaires des environs de Paris.] Revue Zoologique par la Société Cuvierienne, 8, 107. [3 April]

Myodaires des environs de Paris (suite). Annales de la Société Entomologique de France, (2) 4, 17-38. [8 July]

Coup d'oeil rétrospectif sur quelques points de l'entomologie actuelle. Annales de la Société Entomologique de France, (2) 4, 347-358. [23 December]

Description et explication raisonnée des grottes ou cryptes de l'église de l'abbaye de St.-Germain d'Auxerre. Cardon, Troyes. [31 December+]

\section{7}

[Note: sur la vie d'une muscine et d'une delie dans le vinaigre de Colchique.] Annales de la Société Entomologique de France, (2) 4[1846], lxxxi-lxxxii. [24 March]

Myodaires des environs de Paris (suite). Annales de la Société Entomologique de France, (2) 5, 255-287. [25 August]

\section{8}

Études sur les Myodaires des environs de Paris. Annales de la Société Entomologique de France, (2) 5[1847], 591-617. [14 June]

[Sociétés Savantes: On communique une nouvelle suite de mémoires de M. Robineau-Desvoidy sur les Myodaires des environs de Paris.] Revue Zoologique par la Société Cuvierienne, 11, 185-186. [3 July]

[M. Robineau lit des observations au sujet d'un Mémoire de M. Déy, sur Bléneau.] Bulletin de la Société des Sciences Historiques et Naturelles de l'Yonne, 2, 438-441. [31 December+]

[M. Robineau annonce qu'il a découvert ... la plante connue sous le nom de Jusquiane blanc.] Bulletin de la Société des Sciences Historiques et Naturelles de l'Yonne, 2, 442. [31 December+]

Réponse au mémoire de M. Colteau, sur l'origine des blocs quartzeux et siliceux de Magny. Bulletin de la Société des Sciences Historiques et Naturelles de l'Yonne, 2, 579-589. [31 December+]

\section{9}

Myodaires des environs de Paris (suite). Annales de la Société Entomologique de France, (2) 6[1848], 429477. [9 May]

[Sociétés Savantes: sur le Teichomyza muraria.] Revue et Magasin de Zoologie Pure et Appliquée, (2) 1, 94. [3 March]

[Sociétés Savantes: M. Robineau-Desvoidy s'occupe des larves de diverses myodaires qui ont vécu aux dépens de l'homme.] Revue et Magasin de Zoologie Pure et Appliquée, (2) 1, 157. [3 April] 
[Sociétés Savantes: M. Robineau-Desvoidy donne lecture d'un mémoire sur plusieurs espèces de myodaires entomobies des environs de Paris.] Revue et Magasin de Zoologie Pure et Appliquée, (2) 1, 158. [3 April]

[Note: sur le mémoire récemment envoyé par M. Léon Dufour, où notre infatigable collègue annonce que les phasies à l'état de larve vivent dans le corps des hémiptères.] Bulletin de la Société Entomologique de France, (2) 6[1848], xciv. [9 May]

[Note: sur le Teichomyza muraria.] Bulletin de la Société Entomologique de France, (2) 6[1848], xciv-xcv. [9 May]

[Note: un mémoire où M. Léon Dufour donne la description de la larve et des moeurs d'une muscide, larve qui vit du sang de petites hirondelles.] Bulletin de la Société Entomologique de France, (2) 7, iv-v. [11 July]

[Note: sur les moeurs du Thyreophora cynophila.] Bulletin de la Société Entomologique de France, (2) 7, vvi. [11 July]

[Observations sur les ravages causés par les chenilles de l'Orgyia pudibunda dans les environs de SaintSauveur.] Bulletin de la Société Entomologique de France, (2) 7, xiv. [11 July]

[Observations sur les ravages causés par les chenilles de Pyralis viridana dans les environs de Saint-Sauveur.] Bulletin de la Société Entomologique de France, (2) 7, xv. [11 July]

[Note: sur les larves de diverses myodaires qui ont vécu aux dépens de l'homme.] Bulletin de la Société Entomologique de France, (2) 7, xvii-xix. [11 July]

Mémoire sur les crustacés du terrain néocomien de Saint-Sauveur-en-Puisaye (Yonne). Annales de la Société Entomologique de France, (2) 7, 95-141. [10 October]

[M. Robineau-Desvoidy lit un mémoire sur les sauriens du kimmeridge-clay de Saint-Sauveur (lu à l'Académie des Sciences le 27 février 1849.] Bulletin de la Société des Sciences Historiques et Naturelles de l'Yonne, 3, 118-119. [31 December+]

Notice sur les sauriens du Kimméridge-Clay de Saint-Sauveur-en-Puisaye. Bulletin de la Société des Sciences Historiques et Naturelles de l'Yonne, 3, 134-136. [31 December+]

[M. le docteur Robineau-Desvoidy annonce qu'il a été fait deux trouvailles de monnaies du $\mathrm{XVI}^{\mathrm{e}}$ siècle.] Bulletin de la Société des Sciences Historiques et Naturelles de l'Yonne, 3, 275. [31 December+]

Découverte d'une statue de Vénus Anadyomène dans les ferriers de Menzilles. Bulletin de la Société des Sciences Historiques et Naturelles de l'Yonne, 3, 393-394, pl. 25. [31 December+]

\section{0}

[Note: sur la place que doit occuper le genre Trixa.] Bulletin de la Société Entomologique de France, (2) 8, viii-ix. [March]

Mémoire sur plusieurs espèces de myodaires-entomobies. Annales de la Société Entomologique de France, (2) $8,157-181$. [June]

Myodaires des environs de Paris (suite). Annales de la Société Entomologique de France, (2) 8, 183-209. [June]

Recherches sur l'étymologie des noms des cours d'eau de l'ancien diocèse d'Auxerre. Bulletin de la Société des Sciences Historiques et Naturelles de l'Yonne, 4, 461-469, pl. 4. [31 December+]

\section{1}

Sur les éclosions de dix espèces d'entomobies obtenues par M. le colonel Goureau. Revue et Magasin de Zoologie Pure et Appliquée, (2) 3, 147-155. [early April]

Description de plusieurs espèces de myodaires dont les larves sont mineuses des feuilles de végétaux. Revue et Magasin de Zoologie Pure et Appliquée, (2) 3, 229-236. [early April] 
Notice des sauriens du Kimméridge-Clay de Saint-Sauveur-en-Puisaye. Revue et Magasin de Zoologie Pure et Appliquée, (2) 3, 247-250. [early April]

[Note: description d'une nouvelle espèce de myodaire.] Bulletin de la Société Entomologique de France, (2) 9, xxvi-xxviii. [23 April]

Myodaires des environs de Paris (suite). Annales de la Société Entomologique de France, (2) 9, 177-190. [23 July]

[Note: M. Robineau des Voidis dit qu'il y a en Puisaye, près de Treigny, un dolmen ... .] Congrès Archéologique de France, 1850, 15. [26 July]

[Dated from Bibliographie de la France.]

[Note: M. Robineau des Voidis rapport que la Puisaie renferme énormément de tumulus ... .] Congrès Archéologique de France, 1850, 16. [26 July]

[Dated from Bibliographie de la France.]

[Note: M. Robineau-Desvoidis indique comme se rattachant à la fois à l'époque celtique et à l'occupation romaine ... .] Congrès Archéologique de France, 1850, 28. [26 July]

[Dated from Bibliographie de la France.]

[Note: M. Robineau-Desvoidis donne quelques explications ingénieuses sur les deux statues de Cerès et de Noë ... .] Congrès Archéologique de France, 1850, 38. [26 July] [Dated from Bibliographie de la France.]

[Note: M. Robineau-Desvoidis prend la parole pour expliquer sa pensée sur les cryptes de l'église de St.Germain que le Congrès vient de visiter ...]. Congrès Archéologique de France, 1850, 39-40. [26 July] [Dated from Bibliographie de la France.]

[Note: M. Robineau-Desvoidis cite la tour de St.-Sauveur, énorme donjon de forme elliptique.] Congrès Archéologique de France, 1850, 52. [26 July]

[Dated from Bibliographie de la France.]

[Note: M. Robineau cite le château de Ratilly comme une construction du milieu du XIII ${ }^{\mathrm{e}}$. siècle.] Congrès Archéologique de France, 1850, 54. [26 July]

[Dated from Bibliographie de la France.]

Description d'agromyzes et de phytomyzes écloses chez M. le colonel Goureau. Revue et Magasin de Zoologie Pure et Appliquée, (2) 3, 391-405. [August]

Mémoire sur la maladie de la vigne et sur celle de la pomme de terre. Revue et Magasin de Zoologie Pure et Appliquée, (2) 3, 454-462. [September]

Myodaires des environs de Paris (suite). Annales de la Société Entomologique de France, (2) 9, 305-321. [1 November]

[Note: présentation d'un Mémoire sur les sables et grès ferrugineux de la Haute-Puisaye.] Bulletin de la Société des Sciences Historiques et Naturelles de l'Yonne, 5, 320. [31 December+]

[Note: réponse sur les observations de M. Cotteau.] Bulletin de la Société des Sciences Historiques et Naturelles de l'Yonne, 5, 321. [31 December+]

Notice sur un ichthyosaure trouvé dans la craie, à Saint-Saveur. Bulletin de la Société des Sciences Historiques et Naturelles de l'Yonne, 5, 403-408, pls 7, 8. [31 December+]

Mémoire sur les sables et grès ferrugineux de la Haute-Puisaye. Bulletin de la Société des Sciences Historiques et Naturelles de l'Yonne, 5, 409-420. [31 December+]

Mémoire sur un gisement de calcaire d'eau douce à Saint-Martin-sur-Ouanne (Yonne). Bulletin de la Société des Sciences Historiques et Naturelles de l'Yonne, 5, 455-459. [31 December+]

1852

Mémoire sur les gallinsectes de l'olivier, du citronnier, de l'oranger, du laurier-rose, et sur les maladies qu'ils y occasionnent dans la province de Nice et dans le département du Var. [Extract.] Comptes Rendus Hebdomadaires de l'Académie des Sciences, Paris, 35, 183-185. [after 2 August]

[Date of séance.] 
[Note: M Robineau-Desvoidis fait remarquer que M. Baudoin pourrait entretenir le congrès ... .] Congrès Archéologique de France, 1851, 130. [after June]

[Note: M. Robineau-Desvoidis s'étend longuement sur la découverte faite à la Chapelle, près de SaintSauveur-en-Puisaye ... .] Congrès Archéologique de France, 1851, 132. [after June]

[Note: M. Robineau-Desvoidis confirme cette observation de M. Morellet par les observations qu'il a faites lui-même ... .] Congrès Archéologique de France, 1851, 133. [after June]

[Note: M. Robineau-Desvoidis prétend que l'abbaye de St.-Germain a été le point précis ... .] Congrès Archéologique de France, 1851, 137. [after June]

[Note: M. Robineau-Desvoidis partage l'avis de M. Gallois ... .] Congrès Archéologique de France, 1851, 175. [after June]

Note: mémoire sur les gallinsectes de l'olivier, du citronnier, du laurier rose, et sur les maladies qu'ils y occasionnent dans la province de Nice et dans le département du Var. Revue et Magasin de Zoologie Pure et Appliquée, (2) 4, 380. [August]

Notice sur deux fléaux qui attaquent le blé. Revue et Magasin de Zoologie Pure et Appliquée, (2) 4, 397-400. [August]

Mémoire sur les grès ferrugineux tertiaires de la commune de Tannerre (Yonne). Bulletin de la Société des Sciences Historiques et Naturelles de l'Yonne, 6, 97-104. [31 December+]

[Note: sur la vente des belles verrières de St.-Florentin.] Bulletin Monumental, (2) 8, 168. [31 December+] [co-authored with Challe, C.-M.-A.]

[Note: M. Robineau-Desvoidy fait remarquer qu'il ne peut y avoir de flore spéciale à une localité ... .] Congrès Scientifique de France, 1851, 57.

[Note: M. Robineau-Desvoidy fait connaître à la section que des recherches actives qu'il a faites dans l'Yonne ...lui ont donné une explication complète du mode de formation des sables de la Loire... .] Congrès Scientifique de France, 1851, 58.

[Note: M. Robineau-Desvoidy a étudié également les allures de ce sarcopte, surtout sur le charme, le chêne et l'érable ... .] Congrès Scientifique de France, 1851, 59.

[Note: M. Robineau-Desvoidy cite pour exemple la Salle des Fées à Druy-les-belles-Fontaines département de l'Yonne ... .] Congrès Scientifique de France, 1851, 64.

[Note: La parole est à M. Robineau-Desvoidy, pour une motion d'ordre ... Il est admis, dit M. RobineauDesvoidy, que le département de l'Yonne ne possède pas de calcaire d'eau douce ... .] Congrès Scientifique de France, 1851, 65.

[Note: M. Robineau-Desvoidy annonce qu'il s'est livré à de nouvelles recherches sur la question agitée ... .] Congrès Scientifique de France, 1851, 73.

[Note: M. Robineau-Desvoidy demande à donner communication d'un travail sur la maladie du raisin et celle des pommes de terre .... .] Congrès Scientifique de France, 1851, 74-75.

\section{3}

Diptères des environs de Paris. Famille des myopaires. Bulletin de la Société des Sciences Historiques et Naturelles de l'Yonne, 7, 83-160. [November]

Diptères des environs de Paris. Famille des myopaires. Gens myopariarum. Perriquet, Auxerre, France. $80+$ [2] pp. [31 December+]

[This is a separately paginated reprint of the preceding article.]

Notice sur la caverne ossifère d'Arcy-sur-Cure, Yonne. Comptes Rendus Hebdomadaires de l'Académie des Sciences, Paris, 37, 453-455. [after 19 September]

[Date of séance.]

Catalogue des insectes coléoptères du canton de Saint-Sauveur-en-Puisaye (Yonne) [part]. Bulletin de la Société des Sciences Historiques et Naturelles de l'Yonne, 7, 335-368. [31 December+]

Sur les éclosions de plusieurs espèces de diptères, obtenues par le docteur Moret, médecin à Auxerre. Bulletin de la Société des Sciences Historiques et Naturelles de l'Yonne, 7, 531-536. [31 December+] 
Notice sur des médailles trouvées aux environs de Briare et à Rogny. Bulletin de la Société des Sciences Historiques et Naturelles de l'Yonne, 8, 19-34. [31 December+]

Catalogue des coléoptères du canton Saint-Sauveur-en-Puisaye (Yonne) [concl.]. Bulletin de la Société des Sciences Historiques et Naturelles de l'Yonne, 8, 251-284. [31 December+]

\section{6}

Mémoire sur les gale-insectes de l'olivier, du citronnier, de l'oranger, du laurier-rose, et sur les maladies qu'ils y occasionnent dans la province de Nice et dans le département du Var. Revue et Magasin de Zoologie Pure et Appliquée, (2) 8, 121-128. [March]

Mémoire sur les gale-insectes de l'olivier, du citronnier, de l'oranger, du laurier-rose, et sur les maladies qu'ils y occasionnent dans la province de Nice et dans le département du Var. Revue et Magasin de Zoologie Pure et Appliquée, (2) 8, 180-188. [April]

Mémoire sur les gale-insectes de l'olivier, du citronnier, de l'oranger, du laurier-rose, et sur les maladies qu'ils y occasionnent dans la province de Nice et dans le département du Var. Revue et Magasin de Zoologie Pure et Appliquée, (2) 8, 277-284. [June]

Mémoire sur les gale-insectes de l'olivier, du citronnier, de l'oranger, du laurier-rose, et sur les maladies qu'ils y occasionnent dans la province de Nice et dans le département du Var. Revue et Magasin de Zoologie Pure et Appliquée, (2) 8, 387-393. [August]

\section{3}

Histoire naturelle des diptères des environs de Paris. Oeuvre posthume du $\mathrm{D}^{\mathrm{r}}$ Robineau-Desvoidy publiée par les soins de sa famille, sous la direction de M. H. Monceaux. Tome premier. Masson et fils, Paris, Wagner, Leipzig, and Williams \& Norgate, London. xvi + 1143 pp. [11 January]

[Announced as published by Monceaux at the 11 January meeting of the Société des Sciences Historiques et Naturelles de l'Yonne.]

Histoire naturelle des diptères des environs de Paris. Oeuvre posthume du $\mathrm{D}^{\mathrm{r}}$ Robineau-Desvoidy publiée par les soins de sa famille, sous la direction de M. H. Monceaux. Tome second. Masson et fils, Paris, Wagner, Leipzig, and Williams \& Norgate, London. 920 pp. [11 January]

[Announced as published by Monceaux at the 11 January meeting of the Société des Sciences Historiques et Naturelles de l'Yonne.] 


\section{APPENDIX II. Robineau-Desvoidy's Collections, Collectors, and Localities}

\section{The Robineau-Desvoidy Collections}

Robineau-Desvoidy's personal collection of Diptera was bequeathed, together with all his other collections (insects, fossils, geology, archaeology) to the Société des Sciences Historiques et Naturelles de l'Yonne. It appears that when the 170 boxes of Diptera arrived at the Muséum d'Histoire Naturelle in Auxerre around 1860, they were stored in an attic under the rafters and, without a curator to care for them, they soon began to suffer from seasonal variations in temperature and humidity and from the depredations of mice, beetles and moths. In the late 1920s there was a resurgence of interest in Robineau-Desvoidy, and not only was his grave located (Royer, 1929; see also Fig. 9) but the remains of his Diptera collection were found (Royer, 1931) and were moved to the Muséum National d'Histoire Naturelle in Paris where they were curated by the dipterist Eugène Séguy (1890-1985). The greater part of the Diptera collection was lost. What survived, consisting mainly of Tachinidae, comprised 2000 specimens representing some 738 species that are now arranged in 17 large glass-topped cartons (Séguy in Royer, 1931; updated by C. Daugeron (pers. comm.); see example in Figs. 12-13). A type-written index of the species names was made by Séguy.

His collection must originally have been vast, especially if he kept and pinned all the specimens that he discussed. For example, he wrote:

\footnotetext{
Heureux l'Entomologiste qui fixera avec certitude l'espèce de Phorocère. Nous avons opéré sur des milliers d'individus et nous sommes convaincu que l'hybridisme mettra un obstacle constant à de rigoreuses délimitations. [Happy the Entomologist who will define with certainty the Phorocera species. We have studied thousands of specimens (our italics) and we are convinced that hybridization will form a continual barrier to precise definitions.] (Robineau-Desvoidy, 1863a: 508).
}

Robineau-Desvoidy did not always state where the specimens that he studied were collected. The vast majority were collected around Paris, his home town of Saint-Sauveur-en-Puisaye, and, in his last decade, around Nice in the south of France where he probably went for his health. All this material was in his personal collection, and material that was given to him by other entomologists clearly suffered the same fate as the rest of his collection. Some of the private collections that were made available to him may have survived, whilst others are known with certainty to have been destroyed. There are other difficulties involved in determining where his specimens may have been deposited (and where they may still survive). For example, in 1830 a number of flies were described from Philadelphia: some were stated to be in Dejean's collection and some in the "Muséum" (i.e., the Muséum National d'Histoire Naturelle in Paris), while others have no information beyond the locality, and their original or ultimate location must be a matter of guesswork. A number of types were destroyed even during Robineau-Desvoidy's own lifetime, for example Aplomya sabulosa RobineauDesvoidy (1863a: 461, "J'ai perdu l'exemplaire typique" - "I have lost the typical specimen").

Below we give a list of the collectors who supplied Robineau-Desvoidy with material together with what is known of the location of their material, and then follow this with a list of the collections that RobineauDesvoidy studied for his 1830 and 1863 books and for other descriptive papers.

\section{Collectors}

The following acronyms are used for museums and institutes mentioned in this section:

ANSP Academy of Natural Sciences, Philadelphia, USA [also holds the collections of the American Entomological Society]

BMNH Natural History Museum, London, UK

MHNL Musée d'Histoire Naturelle, Lille, France

MNHNP Muséum National d'Histoire Naturelle, Paris, France 

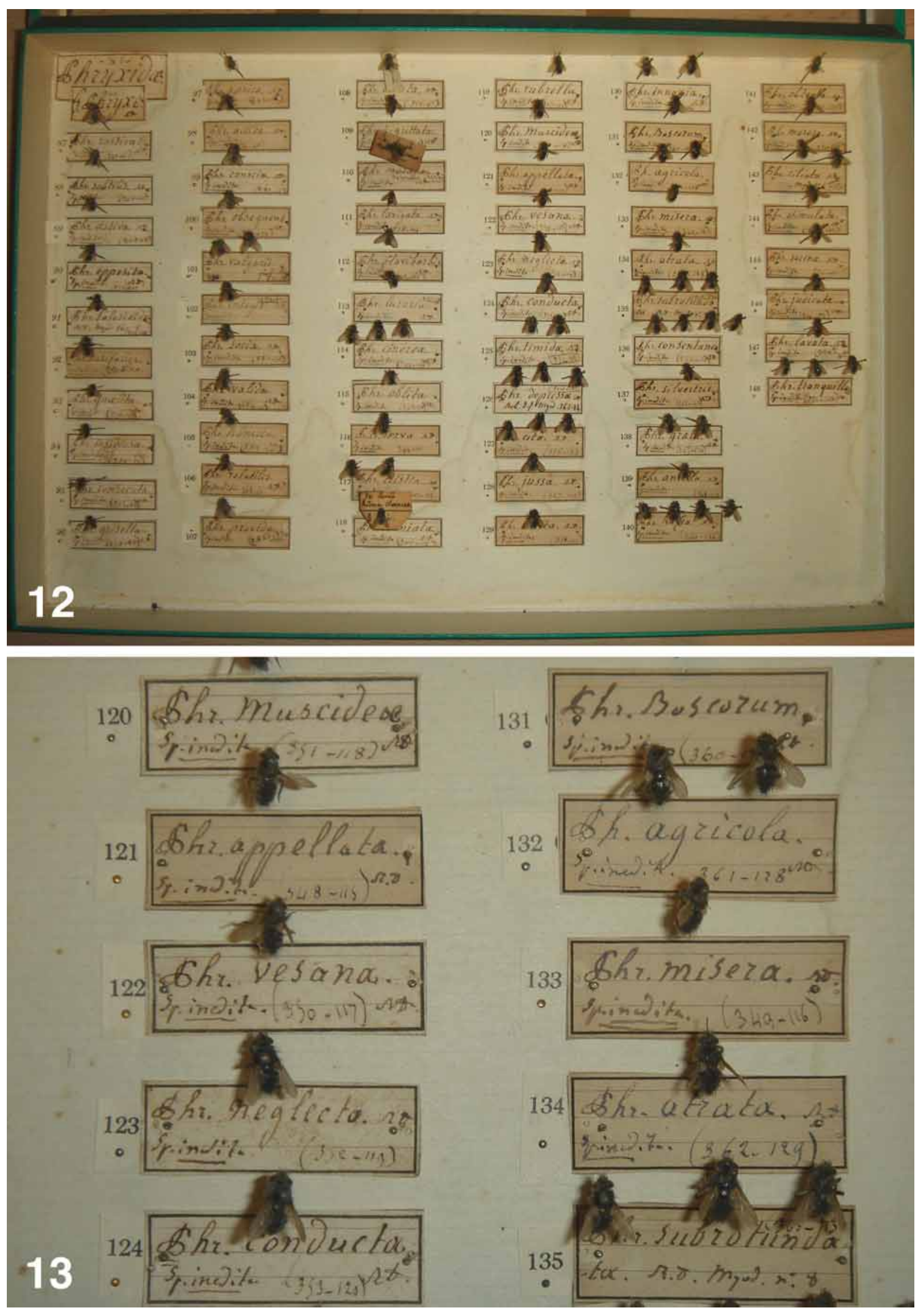

FIGURES 12-13. 12. Drawer of specimens of the genus Phryxe Robineau-Desvoidy (Tachinidae) of the Robineau-Desvoidy collection in the Muséum National d'Histoire Naturelle, Paris. 13. Detail of a portion of the drawer of specimens in Fig. 12. Photos: Marcía Couri. 
MRSNT Museo Regionale di Scienze Naturali, Turin, Italy

NMW Naturhistorisches Museum, Vienna, Austria

NNML National Natuurhistorische Museum, Leiden, Netherlands

OUMNH Oxford University Museum of Natural History, Oxford, UK

SLBV Société Linnéenne de Bordeaux, Bordeaux, France

ZMUC Natural History Museum of Denmark, Copenhagen, Denmark

The localities mentioned here are written as given by Robineau-Desvoidy, and their modern names are given, where different or necessary for clarification, in the "Localities" list that follows the list of collectors and collections.

Audinet-Serville - see under "Collections" below. [1830]

Bagriot. Not mentioned by Horn \& Kahle (1935) or Horn et al. (1990a). [1850a; 1 species]

Bellier de la Chavignerie, Jean Baptiste Eugène (1819-1888). His insect collection went to Charles Oberthür (Horn \& Kahle, 1935: 17; Horn et al., 1990a: 35) and the fate of his Diptera is not known. One species reared by Bellier de la Chavignerie is stated to be in Bigot's collection (Robineau-Desvoidy, 1863a: 516), and perhaps others will also be found there. [1850a, 1863a, 1863b; 56 species of Tachinidae reared from Lepidoptera hosts]

Bercé, Jean Etienne (1802-1879). Not mentioned by Horn \& Kahle (1935) or Horn et al. (1990a). [1850a, 1863a; 11 species of Tachinidae, reared from Lepidoptera hosts]

Bigot, Jacques Marie Frangile (1818-1893). His Diptera collection was purchased by G.H. Verrall, who donated some groups to the BMNH and bequeathed the rest to his nephew J.E. Collin, who in turn bequeathed it to OUMNH (Horn \& Kahle, 1935: 19; Horn et al., 1990a: 40). [1853, 1863a, 1863b; 24 species of Conopidae and Tachinidae, many from Piedmont, Italy, also from Sennart forest and Corsica]

Blondel - see under "Collections" below. [1830]

Boisduval, Jean Baptiste Alphonse Dechauffour de (1799-1879). His collection was divided up after his death, but no mention is made of any Diptera (Horn \& Kahle, 1935: 24; Horn et al., 1990a: 46). [1830; 1 species, reared from a lepidopteran host]

Bosc - see under "Collections" below. [1830]

Carcel - see under "Collections" below. [1830]

Dejean - see under "Collections" below. [1830]

Diard, Pierre Médard (1794-1863). His insects from India collected pre-1826 went to MNHNP, and those collected later from the then Dutch East Indies went to NNML (Horn \& Kahle, 1935: 56; Horn et al., 1990a: 92). [1830; 1 species, "Bengale"]

Duponchel, Philogène Auguste Joseph (1774-1846). His Lepidoptera collection went to MNHNP (Horn \& Kahle, 1935: 64; Horn et al., 1990a: 102). [1830, France, 4 species, and 1863, 1 species, all reared from Lepidoptera hosts]

Duvaucel, Alfred (1792-1824). His insects from India collected pre-1824 went to MNHNP (Horn \& Kahle, 1935: 64; Horn et al., 1990a: 103). [1830; 5 species, "Bengale"]

Goureau, Claude Charles (1790-1879). Not mentioned by Horn \& Kahle (1935) or Horn et al. (1990a). [1851, 4 papers, reared Tachinidae (10 species), Anthomyiidae (4 species), Agromyzidae (13 species); and 2 species in 1863a]

Guérin [after 1836, Guérin-Méneville], Félix Edouard (1799-1874). His collection was divided up after his death and the Diptera went in part to MNHNP (Horn \& Kahle, 1935: 99; Horn et al., 1990a: 150). Material used by Robineau-Desvoidy in 1830 was given to him by Guérin-Méneville. [1830; 20 species, "Baie des Chiens-Marins", "Brésil", "Cap de Bonne-Espérance", "Cayenne", "Surinam", "Coromandel", "Indes Orientales", "Lamana", “Amérique septentrionale", "Port-au-Prince”, "Port Jackson”, "Amérique méridionale", "Sumatra"; 1849, 1 species; 1850a, 7 species; 1863, 4 species]

Hodgard. Not mentioned by Horn \& Kahle (1935) or Horn et al. (1990a). [1830; 1 species, "Haïti"] 
Joanny. Not mentioned by Horn \& Kahle (1935) or Horn et al. (1990a). [1863b; 1 species, France, Nice, given to Robineau-Desvoidy]

Lalande, Pierre Antoine de (1787-1828). Not mentioned by Horn \& Kahle (1935) or Horn et al. (1990b). He accompanied Saint-Hilaire on his travels in South America, and was naturalist on a voyage of the Coquille. His Diptera are in MNHNP [1830; 5 species, "Cap de Bonne-Espérance", and 2 species, "Brésil"]

Lambert, Paul. Not mentioned by Horn \& Kahle (1935) or Horn et al. (1990a). [1 species, reared from a beetle]

Lapilaye, Bachelot de [also as La Pylaye] (1786-1856). Not mentioned by Horn \& Kahle (1935) or Horn et al. (1990a). [1830; 1 species, "Terre-Neuve", actually in MNHNP according to Robineau-Desvoidy (1863a: 642)]

Latreille, Pierre André (1762-1833). No Diptera are mentioned specifically, but his first entomological collection went in 1826 to Dejean (see under "Collections" below). [1830]

Lefebvre, Alexandre (1798-1868). According to Horn \& Kahle (1935: 151) and Horn et al. (1990b: 229), his collection was auctioned after his death and its fate is unknown. [1827, 1830; 10 species including one mosquito, Sicily]

Le Peletier - see under "Collections" below. [1830]

Leschenault de la Tour, Jean-Baptiste-Louis-Claude-Theodore [also as Leschenauld] (1773-1826). His pre1826 collections from Guyana went to MNHNP (Horn \& Kahle, 1935: 153; Horn et al., 1990b: 233). [1830; 1 species, "Surinam", in Robineau-Desvoidy's own collection according to Robineau-Desvoidy (1863a: 658)]

Lesueur, Charles Alexander (1778-1846). His collections from North America pre-1826 and from the voyage of the Géographe went to MNHNP (Horn \& Kahle, 1935: 154, and 1937: 367; Horn et al., 1990b: 234). [1830; 2 species, "Philadelphie" and "Amérique du Nord"]

Lherminier, Felix Louis [also as L'Herminier] (1779-1833). Not mentioned by Horn \& Kahle (1935) or Horn et al. (1990b). [1830; 4 species, "Caroline"] His son, Ferdinand L'Herminier (1802-1866), was also a naturalist, and is actually the L'Herminier listed by Horn \& Kahle (1935: 155) and Horn et al. (1990b: 236).

Macé. Not mentioned by Horn \& Kahle (1935) or Horn et al. (1990b), but his Diptera are in MNHNP. [1830; 2 species, "Bengale"]

Macquart, Justin Pierre Marie (1778-1855). His personal Diptera collection is in MHNL, many exotic species are in MNHNP whilst those described from the Bigot collection are partly in OUMNH and partly in BMNH (Horn \& Kahle, 1936: 163; Horn et al., 1990b: 247). [1830; Lille, on several occasions "elle m'a été communiquée par M. Macquart de Lille"]

Milbert, Jean-Baptiste-Gabriel-Marie (1747-1833). Insects from North America collected before 1826 are in MNHNP (Horn \& Kahle, 1936: 177; Horn et al., 1990b: 263). Some Diptera were also in the Dejean collection (see under "Collections" below). [1830; 1 species, "Philadelphie"]

Moret. Not mentioned by Horn \& Kahle (1936) or Horn et al. (1990b). A physician friend of RobineauDesvoidy and a resident of Auxerre. [1853, 2 species; 1863, 3 species]

Olivier, Guillaume Antoine (1756-1814). Collections from his Oriental travels went to MNHNP; his private collection was partly destroyed and partly divided (Horn \& Kahle, 1936: 197; Horn et al., 1990b: 289), but no Diptera are mentioned. [1830; 2 species, "Égypte” (MNHNP) and “île de Scio"]

Palisot de Beauvois, Ambroise Marie François Joseph (1752-1820). No Diptera collections are mentioned, but some Coleoptera went via Dejean (see under "Collections" below) and others to BMNH (Horn \& Kahle, 1936: 201; Horn et al., 1990b: 294). [1830; 16 species, "Caroline", "Amérique septentrionale", "États Unis d'Amérique", "Haïti"; 2 of these (Jurinia chrysiceps Robineau-Desvoidy, 1830 and Jurinia aterrima Robineau-Desvoidy, 1830) were stated to be in Robineau-Desvoidy's own collection according to Robineau-Desvoidy (1863a: 656, 660)]

Péron, François (1775-1810). Insects from the voyage of the "Géographe" went to MNHNP (Horn \& Kahle, 1936: 205; Horn et al., 1990b: 300). [1830; 9 species, “Timor", "Port Jackson”] 
Plée, Auguste (1787-1825). His collection went to MNHNP (Horn \& Kahle, 1936: 210; Horn et al., 1990b: 306). [1830; 1 species, "Antilles"]

Poey y Aloy, Felipe (1798-1891). His insects from Cuba (but no Diptera mentioned) went to the American Entomological Society in Philadelphia, now in ANSP (Horn \& Kahle, 1936: 211; Horn et al., 1990b: 307). [1827, 1830; 5 species including one mosquito, "Havana"]

Roux, Polydore Le (1792-1833). Not mentioned by Horn \& Kahle (1935) or Horn et al. (1990b). [1830; 1 species, no locality given]

Saint-Hilaire, Augustin François César Prouvançal de, [also known as Auguste] (1779-1853). Insects from Minas Gerais and Montevideo pre-1826 to MNHNP (Horn \& Kahle, 1936: 236; Horn et al., 1990b: 339). [1830; 17 species, "Capitainerie de Rio-Grande", "Capitainerie de Missiones", "Capitainerie de Guaratuba", "Goyaz", "Monte-Video"]

Urville, Jules Sébastien César Dumont d' (1790-1842). Insects including Diptera from the voyages of the Astrolabe and the Zélée went to MNHNP (Horn \& Kahle, 1936: 285; Horn et al., 1990b: 401). [1830; 3 species, "Nouvelle-Hollande"]

Verreaux, Jules Pierre (1807-1873). One of a family of travelers and collectors who supplied material for MNHNP (Horn \& Kahle, 1936: 288-289; Horn et al., 1990b). The father was a natural history dealer in Paris, while his three sons traveled and supplied him with material for sale. The Diptera were largely described by Macquart. Verreaux collected in Australia in 1842/1847, and in Tasmania in 1843. [1863b; 1 species, Tasmania]

Westermann, Bernt Wilhelm (1781-1868). His collections are in ZMUC (Horn \& Kahle, 1937: 301; Horn et al., 1990b: 420) but some specimens were sent to MNHNP. [1830; 1 species, "Batavia"]

Winthem, Wilhelm von (1799-1847). His Diptera, which were studied by Johann Wilhelm Meigen (17641845), mostly went to NMW (Horn \& Kahle, 1937: 305; Horn et al., 1990b: 425) but a few are in MNHNP. [1830; “... envoyée à Paris par M. von Winthem”, Meigen species and some with MS names]

\section{Principal collections}

Robineau-Desvoidy (1830: 3-4) listed the collections that he studied, in addition to his own: Le Peletier de Saint-Fargeau, Audinet-Serville, Blondel, all from the Paris area; Carcel from the French provinces of Dauphiné and Anjou; Dejean (which included Latreille material); and exotic species in the Muséum du Jardin du Roi, Paris (which is now MNHNP).

Audinet-Serville, Jean Guillaume (1775-1858). His collection was broken up and dispersed after his death. Horn \& Kahle (1935: 8) and Horn et al. (1990a: 22) make no mention of the fate of his Diptera. One species of mosquito was described from his collection by Robineau-Desvoidy (1827), and a further 15 species of Diptera in the Essai sur les Myodaires (1830).

Blondel, Hippolyte. The Blondel collection was destroyed (Herting, 1974: 10, 20). Blondel accompanied Carcel on an expedition to Smyrna (Turkey), where the latter died in 1831. Horn \& Kahle (1935: 22) and Horn et al. (1990a: 44) make no mention of any Diptera; his Coleoptera went to the SLBV (Horn \& Kahle, l.c.). Twenty-one species of Diptera were described from his collection by Robineau-Desvoidy (1830).

Bosc d'Antic, Louis Auguste Guillaume (1759-1828). The Bosc Diptera, from Carolina and Java, were destroyed (Herting, 1974: 10, 20), but his Hymenoptera went to MNHNP (Horn \& Kahle, 1935: 26; Horn et al., 1990a: 48). No Diptera are mentioned by Horn \& Kahle (l.c.). One species of mosquito was described from his collection by Robineau-Desvoidy (1827), and a further 17 species of Diptera in the Essai sur les Myodaires (1830). 
Carcel (??-1831). Carcel is not listed by Horn \& Kahle (1935) or Horn et al. (1990a), and his collection is regarded as destroyed (Herting, 1974: 10, 20). All that we have been able to discover is that he made a large collection of insects and shells in Turkey, where he was accompanied by Blondel and where he died young in 1831 (Michaud \& Poujoulat, 1834: 365). One mosquito was described from his collection by RobineauDesvoidy in 1827, and 49 species of Diptera in 1830.

Dejean, Pierre François Marie Auguste, Comte de (1780-1845). His collection was broken up and sold upon his death, as no institute could afford to buy it in its entirety (see the advertisement for the sale of this collection in Germar's Magazin der Entomologie 2(2), 448-449, 1840). No mention is made of Diptera by Horn \& Kahle (1935: 52-53; 1937: 331), but Horn et al. (1990a: 87) stated that his Diptera went to F. de Brême whose collection, or at least the Coleoptera, went to MRSNT (Horn et al., 1990a: 54). In fact, Dejean's Diptera went to Bigot (Robineau-Desvoidy, 1863a: 1114), and what remains is in OUMNH (where ACP has seen both Dejean and Latreille specimens). Robineau-Desvoidy (1830: 4) wrote particularly warmly about Dejean's collection: "Enfin la plus riche et la plus intéressante collection de Myodaires que je connaisse, celle que M. Latreille aimait à augmenter depuis tant d'années, la collection de M. le comte Dejean, m'a été ouverte avec cette complaisance et des attentions particulières qui font tant d'honneur à ce célèbre entomologiste." [Finally, the richest and most interesting collection of the Myodaires that I know, and the one which Monsieur Latreille was delighted to augment over so many years, that of the Count Dejean, has been opened to me with that kindness and with the special attentiveness that does this celebrated entomologist so much honor.] Robineau-Desvoidy described specimens labeled by Latreille in Dejean's collection: some were published names, while others were manuscript names that now have to be credited to Robineau-Desvoidy. Six species of mosquitoes were described from his collection by Robineau-Desvoidy (1827), and 159 myodaires by Robineau-Desvoidy (1830). Localities include France, Germany, Dalmatia, the Greek Archipelago, East Indies, Java, Cape of Good Hope, Senegal, Nova Scotia, Philadelphia, Brazil, Peru, Cayenne.

Le Peletier, Amédée Louis Michel, Comte de Saint-Fargeau (1770-1845). Horn \& Kahle (1936: 204) and Horn et al. (1990b: 299) stated that his Hymenoptera went to MNHNP and to MRSNT, but no mention is made of his Diptera. In retirement he lived in Saint-Germain-en-Laye. One of his sons was an army officer and collected insects for his father, hence Robineau-Desvoidy's (1830) references both to M. le comte de Saint-Fargeau (or M. Le Peletier de Saint-Fargeau, or Comte Amédée de Saint-Fargeau) and to M. Amédée de Saint-Fargeau fils [= son]. Fifty-six species were described from his collection by Robineau-Desvoidy (1830).

Muséum. The Muséum National d'Histoire Naturelle in Paris. Robineau-Desvoidy's exotic types survive and are in the Collection Exotique together with Macquart's types. Forty-three species were described from the Muséum by Robineau-Desvoidy (1830). In the same work a further 73 were described from extra-European localities without any collection mentioned, and may well also be in the Muséum. Three species of mosquito were described in 1827 and 5 myodaires in 1863a, b.

Vienna. Brauer (1893: 451) wrote on the subject of the Brauer \& Bergenstamm monograph: "die Sammlung E. v. Bergenstamm's enthielt viele Typen Rondani's und Robineau-Desvoidy's". It is possible that there are syntypes in Vienna, but we think that this statement means "typical examples" not "syntypes".

\section{LOCALITIES}

EUROPE: FRANCE

les Alpes

d'Arcy-sur-Eure 
Angers

Auxerre

Beauvais

Bois de Boulogne

Bondi

Bordeaux (Landes)

Havre, port du

Cap de la Hève

Forêt de Saint-Germain

Galliâ

Gentilly

Hyères, les dunes de Hyères (Var).

Jardin de Luxembourg

La Forterre (Puisaye)

Lainsecq

La Lozère

La Rochelle

Lille

Lyon

Menton, sur les collines de

Menton, contre un ruisseau

midi de la France

Montmorency

Montmorency, l'étang de

Montpellier

Nice, in numerous habitats (les collines, les hautes collines, les montagnes, dans un bois, dans un pré, dans les prés humides, les champs, sur les bords de la mer)

Paris, Parisiis

Picardie

Provence

Rogny (Yonne).

grotte de Saint-André, près de Nice

Saint-Germain-en-Laye

Saint-Gratien

Saint-Hospice, comté de Nice

Saint-Sauveur, in several habitats, mainly les étangs, les marais and les marais tourbeux

Seine, bords de la

Sennart, forêt de

[Toussaint, environs de la Toussaint and avant et aprês la Toussaint.] Not a locality, but the festival of All Saints Day, celebrated by western Christianity annually on 1 November

Var

Var, les bords du

Var, à l'embouchure du

Var, dans les champs voisins du

Versailles

Ville d'Avray 
Ville-d'Avray, étang de

Yonne river

\section{ELSEWHERE IN EUROPE/NORTH AFRICA:}

\section{Locality}

Algérie

Allemande

l'Archipel Grec

Corse

Dalmatie

Égypte

Espagne

Germaniâ

Ile de Scio

Italie

Piémont

Sicilie. Siciliâ

Spitzberg

Zurich, canton de

\section{AFROTROPICAL:}

\section{Locality}

Afrique

Afrique intérieure

Arabie

Cap de Bonne-Éspérance

Congo

Île de France

Îles de la mer d'Afrique

Sénégal

\section{ORIENTAL:}

\section{Locality}

Batavia

Bengale

Coromandel

Inde

Indes orientales

Java

Sumatra

Timor

\section{Contemporary Equivalent}

ALGERIA

GERMANY

The Greek Archipelago, i.e., ISLANDS OF THE AEGEAN SEA

FRANCE, Corsica

CROATIA, Dalmatia

EGYPT

SPAIN

GERMANY

GREECE, island of Chios

ITALY

ITALY, Piedmont

ITALY, Sicily

SVALBARD ISLANDS, Spitsbergen

SWITZERLAND, Zurich

\section{Contemporary Equivalent}

AFRICA

INNER AFRICA

ARABIA, probably a reference to the Arabian Peninsula

SOUTH AFRICA, Cape of Good Hope

Probably the Congo River, in the DEMOCRATIC REPUBLIC OF CONGO

MAURITIUS

ISLANDS OF THE AFRICAN SEA, which could be either the Atlantic Ocean or the Indian Ocean

SENEGAL

\section{Contemporary Equivalent}

INDONESIA, Java, now called Jakarta, the capital of Indonesia

INDIA, West Bengal and BANGLADESH

INDIA, the Coromandel Coast, which runs along the southern half of the east coast of India

INDIA

EAST INDIES, i.e., mostly INDONESIA

INDONESIA, Java

INDONESIA, Sumatra

INDONESIA, Timor 
AUSTRALASIAN:

\section{Locality}

Baie des Chiens Marins

Îles de l'Océanie

Nouvelle Hollande

Port Jackson

Tasmanie

\section{NEARCTIC:}

\section{Locality}

Amérique boréale

Amérique du Nord

Amérique septentrionale

Caroline, Carolinâ

États Unis d'Amérique

Nouvelle-Écosse

Pensylvaniâ

Philadelphie

Terre-Neuve

Virginie

\section{NEOTROPICAL:}

\section{Locality}

Amérique méridionale

Antilles

Brasil, Brasiliâ, Brésil

Cayenne

Cuba

Goyaz

Guaratuba, Capitainerie de

Haïti

Havana

Îles Malouines

in insulis Americae

Lamana

Missiones, Capitainerie de

Monte-Video

Pérou

Port au Prince

Rio Grande, Capitainerie de

St Domingue

Surinam

\section{Contemporary Equivalent}

AUSTRALIA, Bass Strait, King Island

ISLANDS OF THE PACIFIC OCEAN

AUSTRALIA

AUSTRALIA, New South Wales, Sydney Harbour

AUSTRALIA, Tasmania

\section{Contemporary Equivalent \\ BOREAL AMERICA \\ NORTH AMERICA \\ NORTHERN AMERICA}

UNITED STATES OF AMERICA, North and South Carolina UNITED STATES OF AMERICA

CANADA, Nova Scotia

UNITED STATES OF AMERICA, Pennsylvania

UNITED STATES OF AMERICA, Philadelphia

CANADA, Newfoundland

UNITED STATES OF AMERICA, Virginia

\section{Contemporary Equivalent}

SOUTH AMERICA

ANTILLES, islands of the Caribbean Sea

BRAZIL

FRENCH GUIANA, Cayenne

CUBA

BRAZIL, Goyaz state

BRAZIL, Paraná state, Guaratuba

HAITI

CUBA, Havana

FALKLAND ISLANDS

the islands of America, probably of the Caribbean

FRENCH GUIANA, La Mana

ARGENTINA, Missiones province

URUGUAY, Montevideo

PERU

HAITI, Port-au-Prince

BRAZIL, Rio Grande do Sul province

DOMINICAN REPUBLIC, Santo Domingo

SURINAME 
Reproduced on the following pages is the Rapport sur les Essai des Myodaires by Henri Ducrotay de Blainville (1826), which was submitted to the Académie des Sciences in Paris on 2 October 1826. It contains the results of the examination of Robineau-Desvoidy's manuscript.

\section{RAPPORT}

SUR

\section{LES MYODAIRES}

\section{DU DOCTEUR ROBINEAU DESVOIDY,}

I.t DANS LA SÉANCE DE L'ACADÉMIE ROYALE DES SCIENCES,

LE 2 OCTOBRE 1826 .

LAcadémiz nous a chargés, MM. Latreille, Duméril et moi, d'examiner un travail sur les Myodaires ou sur les différentes espèces de Mouches, que lui a présenté M. le docteur Robineau Desvoidy, dans sa séance du 28 août dernier, et de lui faire connaître le jugement que nous en aurions porté. C'est ce que nous allons faire avec quelques détails, l'auteur n'ayant pu, par la nalure même de son travail, en lire que quelques considérations générales à l'Académic.

$\mathrm{Si}$, malgré les travaur nombreur qui se sont succédés depuis le commencement du dernier siècle jusqu'ici, l'entomologie est encore en général très-peu avancée, et appelle de toutes parts des collaborateurs qui s'occupent spécialement de chacune des divisions plus ou moins tranchées qu'on y a établies, il faut convenir qu'aucune de ces parties n'est peut-être dans une confusion aussi grande que l'histoire des Insectes hexapodes, que l'on désigne d'une manière tranchée par la dénomination de Diptères. En effet, leur histoire nalurelle, proprement dite, est à peine avancée d'un pas depuis Réaumur et de Géer, c'est-à-dire depuis plus de soisante ans, et leur distribution metbodique, ainsi que la con. 


\section{(2)}

naissance et la distinction des espèces, est encore extrèmement peu complète, malgré les travaux de Fabricius et de MM. Latreille, Panzer, Fallen, et même de Meigen qui s'en est occupé d'une manière plus spéciale. C'est à remplir cette lacune que s'est consacré M. Robineau Desvoidy dans le travail étendu qu'il a soumis au jugement de l'Académie. Mais pour rendre ce jugement un pev digue de la confiance qu'elle a bien voulu nous accorder, qu'il nous soil permis de nous occuper un moment de ce qui avait été fait sur le même sujet, et des principes qui avaient sirvi de base pour la distribution méthodique des Mouches avant M. Robineau.

Tous les Diptères communément, et, dans le langage vulgaire, réunis sous le nom collectif de Mouches, n'étaient, jusqu'à Fabricius, définis que par l'existence d'une seule paire complète d'ailes, car on regardail déjà les balanciers comme les rudimens de l'autre paire qui existe, à moins que d'avortement, dans tous l's hexapodes. C'est ce que l'on voit très-bien dans les premières éditions du Systema natura jusqu'en ${ }^{1748}$, où le nombre des genres n'est encore que de sept : Oëstre, Asile, Hippobosrque, Taon, Mouche, Cousin et Tipulè. Il fut bientôt porté à treize, divisés en deux sections, toujours d'après la considération du raême appareil.

Geoffroy l'enlomologiste, qui introduisit dans la classification des Insectes la considération nouvelle du nombre des arlicles du tarse, et une analysc plus détaillée de la forme des antennes, établit dans les Diptères ì peu près le mème nombre de genres que Linné. Il en créa cependant six nouveaux; savoir : Stratyomis, ou Mouches armées, dont l'histoire a été si bien faite par Réaumur, Stomoxe, Volucelle, Némotèle, Scatops et Bihio; mais il n'admit pas les trois que Linné venait d'introduire duns la dixième ćdition de son Systema naturee. Ainsi, en les comptant, il n'y avait encore en 1762 , époque de la publication de l'ouvrage classique de Geoffroy, que seize genres dans tout l'ordre des Diptères, et ces genres étaient très-faciles à reconnaitre.

Mais bientût arriva la célèbre innovation de Fabricius, qui, exagerant peut-être l'importance de l'appareil de la bouche, 
qu'il ne connaissait réellement pas, cependant, d'une maničre philosophique, abandonna les erremens suivis par ses prédecesseurs, et distribua tous les Insectes d'après cette seule considération. Les Diptères se trouvèrent convenablement réunis dans la classe des Antliata avec les mêmes subdivisions établies par Linné. Le nombre des genres carıctérisés par les différences dans l'appareil locomoteur de la bouche, et dans la forme des antennes, fut de bonne heure augmenté, d'abord de quatre, puis enfin de sept, et portè par conséquent à vingt-trois. Dans la seconde édition de son Entomologie systématique, publiee en $179^{5}$, le nombre des espèces connues n'était encore que d'environ sept cents.

C'est peu de temps après que commença à paraitre dans la distribution des Animaus, et par conséquent en entomologic, l'établissernent et la dénomination de Familles, que la botanique avait dejà depuis long-temps employécs avec tant de succès. Blumenbach suivait cependant encore le système de Linné, tandis que M. Cuvier combinait déjà cesystème avec celui de Fabricius, qu'il eut même l'avantage d'avair pour collaborateur dans cette partie de son ouvrage.

C'est à MM. Latreille et Duméril qu'est due cette introduction: le premier dans son Précis des caractères des Insectes, publié en 1796; le second dans les tableaux qui sont à la fou du premier volume des Leçons d'Anatomie comparée de M. Cuvier. M. Latreille, dix ans après, développa son système cntomologique dans son Genera Crustaceorum et Insectorum; puis avec de nouveaux perfectionnemens, tírés de ses propres observations et de celles que Fabricius avait données par la publication de son Systema Antliatorum en 1805, dans ses Considérations générales surl'ordre naturel des Crustacés, Arachnides et Insectes, publices en 1810 . Jlen rèsulta pour les Diptères, que les quatorze genres de Linné et de Fabricius furent élevés au rang de familles, sous des dénominations correspondantes; d'où les Tipulaires, les Stratyomydes, les Taoniens, les Rhagionides, les Mydasiens, les Asiliques, les Empides, les Anthraciens, les Bombyliens, les Vésiculeux, les Syrphies, les Conopsaires et les Muscides. Chacune de ces familles fut ensuite subdivisée en genres sur des 


\section{(4)}

caractères plus minutieux, de manière que dans cet ouvrage en 1810, le nombre des genres de Diptères adoptés de Fabricius, de Meigen, et créés par M. Latreille lui-même, fut porté à cent dix-sept pour tout l'urdre, et à vingt-huit pour la famille des Muscides, correspondant à peu près au genre Musca de Linné.

Il n'est pas nécessaire de dire qu'à mesure qu'on eut besoin d'arriver à des caractéristiques de divisions génériques plus nombreuses, on fut obligé de faire entrer quelquefois de nouvelles considérations; mais surtout d'examiner de plus près, avec une loupe plus forte, les organes dont on devait tirer les caractères, en mesuranl surtout la longueur et la grosseur des articles de lantenne.

Jusque-là, les dénominations de familles étant tirées des noms génériques linnéens ou fabriciens, on avait toujours un fil pour se reconnaître; l'on pouvait en rester, pour ainsi dire, au point de la science que l'on voulait, et connaitre le non d'un Insecte suivant l'un ou l'autre de ces auteurs, les seuls qui eussent donné un species complet. Mais uutre le conflit qui résulta de ce que FaLricius et M. Latreille, travaillant dans le même temps, et d'après le même grincipe, qu'il fallait ćtablir des subdivisions dans les genres nombreux en espèces, furent souvent conduits chacun de sun côté à l'établissement des mêmes genres, sous des dénominations différentes, la confusion fut encore augmentée, parce yue l'on crut devoir abandonner les noms de genre de Linné et de Fabricius pour l'établissement et la dénomination des familles, ct les tirer de quelques points de l'organisation. C'est encore l'un de nous, M. Duméril, qui eut le premier cette idée, et qui l'cxécuta à la fin de 1799 , dans les tableaux qui font suite au premier volume des Lecons d'Anatomie comparée de M. Cuvier, ct depuis, avec plus de détails, dans sa $Z$ oologie analytique; en sorle que dans le mème ordre, les familles établies par MM. Latreille et Duméril, ne se correspondent plus, non-seulement dans leurs dénominations, mais encore dans leur composition. Dans la méthode de M. Duméril, l'ordre des Diptères est divisé en cinq familles seulement; tantôt d'après la disposition des appendices buccaux, tantốt d'après la forme des antennes et mème l'babitation; d'où les Sclérostômes, les Aplocèrcs, les Chétoloxes, les 
Astômes ct les Hydromyes. Ainsi, il fut assez difficile de remon ter aux genres de Fabricius, et encore plus à ccux de Linné; et les avantages de la méthode naturelle furent à peu près perdus, ì ce qu'il semble à votre rapporleur. Quant aux genres, M. Dumćril n'en augmenta que fort peu le nombre. Il adopta ceux de M. Latreille et ceux de la seconde édition de l'Entomologic systématique de Fabricius.

Cetle dissidence entre les deux entomologistes qui ont eu pour but l'établissement des familles naturelles parmi les Insectes, devint encore plus frappante quand M. Latreille, dans le volume du Règne animal de M. Cuvier, qu'il a rédigé, eut aussi abandonné les dénominations de familles qu'il avait formées avec les noms de genres de Fabricius, en eut créé d'autres tirés de quelque point del'organisation. On peut dire qu'alors la confusion aurait été à son comble, suivant votre rapporteur, si les auteurs étrangers dans les Species qu'ils on t publiés dans ces derniers temps, ne fussent pas revenus a la première marche de $\mathbf{M}$. Latreille, qu'il serait si important de voir adoptèe d'unc manière définitive.

Sur ces entrefaites, en effet, les entomologistes allemands $\mathrm{n} e$ se bornant pas à des classifications sans spécialités, c'est-à-dir' sans description des espèces, se mirent à reprendre les travaux de Fabricius en leur appliquant les principes de la méthode naturelle, en s'appugant sur une étude approfondie des espèces do leur pays ou d'Europe. C'est, pour ne parler que de l'ordre d'In. sectes dont nous devons nous occuper, ce qu'ont fait Panzer, Wiedman, et surtout Fallen et Meiren. Ce dernier, en effet, dans son Histoire systématique des Insectes à deux ailes d'Europe, en 4 volumes in-80, dont le dernier n'u paru qu'en 1824 , " considérablement augmenté lé nombre des genres de Diplèrs, puisqu'il le porte à près de quatre cents; mais ses familles qui ne sont qu'au nombre de vingt-trois, concordent assez bien avec lcs genres de Fabricius. Malheureusement encore ces familles ne correspondent pas toujours i celles de M. Latrcille, et encore moins à celles de M. Duméril. Toutufois, M. Meigen nous a fait connaitre près de quatre cents espèces de Mouches proprement dites, dont plus de trois cents font partie de son genre Tachina. Il a aussi introduit, comme offrant de bons caractères, la disposition 


\section{(6)}

des nervures des ailes, dont Jurine a tiré un parti si avantageux dans les Hyménoptères. Sa Terminologie, imitée d'illiger, est aussi plus complète que dans aucun entomologiste.

C'est une chose véritablement déplorable pour la science, que ces dissidences entre les entomologistes les plus estimés; et cependant nous aurions besoin en ce moment d'un parfait accord, puisque M. Robineau Desvoidy, dans le travail gu'il a soumis an jugement de l'A cadémie, déclare qu'il ne s'est occupé que du genre Musca de Fabricius; ce sont done à pen près les Muscides de M. Latreille et de M. Lamarck; mais encore faut-il en retrancher les genres Diopsis, Scenopina et Achias? Ce ne sont pas non plus exactement les Muscides de Meigen; car sous les mémes dénominations, les auteurs allemands ne comprennent point absolument les mèmes espèces, et M. Robineau ne donne pas de définition de ses Myodaires. Nous savons cependant maintenant qu'il les definit surtoul d'après le caractère de la larve, qui est complètement apode et même sans mamelons, outre que l'Insecte parfait n'a que deux soies au suçoir.

Nous venons de voir que les organes dont les entomologistes tiraient leurs caractères distinctifs des Muscides, étaient essenticllement les antennes. M. Robineau ayant besuin d'arriver it la connaissance et ì la distinction d'un nombre d'espèces quintuple et sextuple de ce qu'on connaissait en France avant lui, a dû étudier ce grand genre d'une manière bien plus minutieuse. II u'a erpendant nullement observé leur organisation profonde, et plusieurs partics extérieures importantes ont même été négligées.

L'une de celles qu'il a envisagées avec le plus de soin est la tête, et surtout la partie antérieure; il a étudié les différentes régions de la face, qu'il a dù définir avec rigueur autant que cola était possible, et par conséçuent dénommer. Malheureusement ses dénominations ne concordent pas toujours avec celles données par ses prédécesseurs, et entre autres avec celles d'Illiger et de Mcigen, les seuls auteurs qui aient itabli une terminologie des Diptères un peu complète. Un autre défaut de ses dénominations, c'cst qu'elles ne sont plus simples, ce qui était impurtant, puisqu'elles devaient souvent être accompagnées d'ćpithètes carractéristiques, et qu'clles portent ic nom d'os, frui 
convenait pcut-être cncore moins dans cette partic de l'entomologie que dans toute autre.

M. Desvoidy a aussi porté la plus grande attention sur la composition de l'antenne, dont il regarde avec juste raison la soic comme la continuation. La proportion, la forme des trois articles qui composent la partie basilaire, la direction, la longueur, la distinction, la proportion même des articles de la base de la soie, l'état nu, ou plus ou moins villeux de la partie inarticulée, sont éludiés avec un soin bien plus minutieux encore que dans Meigen lui-même ; et c'est en général sur la considération de cet organe que la plupart des genres sont établis.

La consideration des cuillerons ou de ces petits appendices arrondis, squamiformes, que l'on remarque à la racine postérieure de l'aile des Mouches, est presque nouvelle ' et d'une grande utilité dans leur classification proposée par M. Robineau : c'est même là-dessus que sont établies ses premières divisions. Quoique cet organe, qui paraît produire le bourdonnement dalls ces animaux, mais dont l'utilité est encore inaperçuc, s'efface dans la série par des nuances presque insensibles, il a fait l'heureuse remarque que leur développement est assez bien en harmonie avec les principales habitudes des Mouches : ainsi les espèces les plus actives, les plus grosses, les plus colorées, les Mouches de haut vol, si l'on peut employer cette expression, ont des cuillerons considérables, tandis qu'ils sont très-pelits et même rudimentaires ou nuls dans les espèces qui n'abandonnent pas le lieu où elles sont nées, où elles se nonrrissent el se reproduisent.

M. Robineau a aussi aperçu avec la plus grande raison que le système de coloration des Mouches indique assez bien le rapport naturel des espèces entre elles, mais en outre leur séjour babituel; qu'ainsi les espèces qui vivent à l'air libre, aux rayons du soleil, ont des coulcurs bien plus vives que les espèces tinides vivant à l'abri sur des champignons pourris : aussi s'est-il beaucoup servi de ce nouveau caractère daus sa distribution des tribus.

\footnotetext{
'Je dis presque, parce qu'en effet M. Eatreille avait apercu qu'on pourati s'en scrvir, et s'en est servi lui-méme avec ayantage.
} 


\section{( 8 )}

La forme générale du corps des Mouches etant aussi en rapport plus ou moins nécessaire avec la puissance de vol, avec l'habitude de pénétrer dans l'intérieur des corps dont elles se nourrissent, ou de se tenir même dans l'intervalle des feuilles de Graminées aquatiques, n'a pas dù ètre négligée et avec raison.

La terminaison de l'abdomen, dont les derniers anneaux, ordinairement rentrés en tube de lunette, sont quelquefois constamment sorlis et assez solides pour constituer une espèce particulière de tarière, scrvant à la femelle pour déposerses œufs dans le tissu des corps organisés, a pu aussi fournir de bons caractères.

Enfin, les lieux qu'habitent les différentes espèces de Mouches, l'espèce de corps organisés qui leur est pour ainsi deslinée pour nourriture à l'état de larve ou à celui d'insecte parfait, ont aussi guidé $M$. Robincau dans l'ètablissement de ses familles et de ses tribus, et comme ces circonstances sont plus ou moins bien traduites par le système de coloration, par la gran. cleur des cuillerons, la forme du corps, on conçoit que cette considération a pu étre d'une grande ulilité pour les espèces que leur pelitesse permettait difficilement d'observer d'une manic̀re un peu positive, et cu'il était cependant nécessaire de classer.

Telles sont les principales bases de la classification des Mouches proposée par M. Robineau, et dont il nous reste à donner l'exirait avant d'arriver aux conclusions.

M. Robineau élève le genre Musca de Fabricius au rang d'ordre sous le nom de Mrodnans (Myodaria), qu'il définit, comme il a été dit plushaut, d'uprèsla composition de la trompe etla forme de la larve.

Cet ordre est ensuite partagé en dix familles, savoir :

Les Calyptérées (Calypteratce) qui tirent leur nom du grand développement du cuilleron, quoique dans les dernières espèces il diminue d'une manière très-sensible. Elles ont pour caraclère cssentiel la soie distinclement triarticulée. M. Robineau comprend dans cette famille les plus grosses espèces, celles que nous rencontrons journellement dans nos demeures, autour de nos habitations, qui volent et bourdonnent avec le plus de force, et qui sont le plus vivement colorées. Elles affectcnt des tcintes d'un noir-brun, brunes ou métalliques. 


\section{$(g)$}

La considération de la soie antennaire nue, ou plus ou moins velue, sert à partager celte famille en deux sections, dont la prenière est divisée en sept, et la seconde en huit tribus, qui correspondent quelquefois aux divisions génériques de Fabricius et de M. Latreille, et qui , alors, en tirent leur nom.

Chacune de ces tribus est en outre partagée en un nombre da genres qui ne monte pas à moins de deux cent vingt-quatre, d'après des caractères extrêmement minutieux, tirés principalement de la longueur proportionnelle du second et du troisième article de la partie basilaire de l'antenne.

Les Macromydes (Macromyda), au nombre de dix-neuf genres, ont pour type le G. Echinomya de M. Duméril, ou le Musca grossa de Linné. Elles sont remarquables par leur grosseur, par les poils dont elles sont hérissèes, et surtout parce que le second article des antennes est ordinairement plus long que le troisième, au contraire de ce qui a lieu dans les autres Mouches.

Les Cyclémydes (Cyclemyda) sont moins nombreuses, et ne forment que huit genres groupess autour du Musca zonafa de Fabricius. Elles ont le troisième article antennaice cylindriforme, ordinairement plus que double des deux autres, qui sont trèscourts. Leur fuce est le plus souvent bombée. On ignore les mœurs de leurs larves.

Les Bombonydes (Bumbomyda) forment seize genres, dont toutes les espèces paraissent nouvel les, et dont le Musca bi-cincta de Faloricius peut étre le type. Le troisième article des antennes est un peu comprimé sur les côtés. Lc front et la face ne sont plus bombés. Elles se trouvent dans les lieux secs, arides, exposés au soleil.

Les Entomobies (Entomobiak), dont le principal caractère est d'avoir la face oblique, et de vivre, comme l'indique leur nom, aux dépens des autres Insectes, et entre autres des larves de Lépidoptères nocturnes, ont pour type le Musca puparum de Fabricius. M. Robineau en compte plus de deur cents espèces assez vivement colorées, et réparties dans quarante-sept genres, Celui à qui il donne le nom d'A rabette vit aux dépens des Hyminoptèrcs. C'est à turt qu'on a éerit que ces Mouches ne déposent 
qu'un seul ceuf dans chaque larve, car il en a retiré douze individus d'une chenille da Grand-Paon.

Les Ocyptérées (Ocypterata), beaucoup moins nombreuses, répondent au G. Ocyptera de Fabricius. Elles se distinguent principalement par la forme allongée et cylindrique de leur corps, par des teintes fauves sur les côtés de l'abdomen, ct leur habitude de vivre sur les plantes. Elles n'offrent que trois genres.

Les Gastrodées (Gastrodaca), caractérisées principalement par la largeur de lcur ventre, dont on ne-distingue que les premiers segmens, contiennent encore moins d'espèces, et deux seuls genres, dont l'un est le G. Tachina de Fabricius, ayant le Musca rotundata pour type.

Les Lépidomydes ( Lepidomyda), qui terminent cette section, ne renferment qu'une vingtaine d'espèces, presque toutes nouvelles, réparties dans six genres. Par une singularité remalquable, les espèces dn G. Voidia paraissent propres it Paris. Les antennes sont déjà raccourcies, les cuillerons de grandeur moyenne, et le corps sub-arrondi et déprimé.

Les tribus qui composent la seconde section sont :

Les Phasiennes (Phasianc) qui se groupent en dix genres autour du G. Phasia de Fabricius, adopté par Meigen, et qui comprend les Musca subcoleoptrata, crassipennis, etc., etc. La tête est grosse et transverse. Les ailes sont maculées; le corps est déprimé, et l'abdomen bémisphérique. Elles reaferment les Mouches nobles de Gcoffroy.

Les Pherbellées (Pherbellax), composées de six genres nouveaux, dont l'un a pour type le Musca eompressa de Fabriciurs, sont assez rapprochées des Ocyptérées; mais les autennes sont plus longues; le troisième article est ordinairement cylindrique et beaucoup p.lus long que le second; la face est aplatie, et la soie au moins tomenteuse. C'est sur un genre de cette tribu, le G. Phorophylla, que M. Desvoidy a admis deux paires de palpes inférieurs.

Les Tomenteuses (Tomentosa) qui paraissent ne contenir que des espèces nouvelles, divisées cn cinq genres, ont pour caractères les antennes plus courtes, verticales; la soie toujours 


\section{( II )}

tomenteuse; le corps cylindrique, déprimé, avec des teintes mélangées de noir et de gris. Les larves sont ignorées.

Les Macropodées (Macropodea), dont le nom indique le principal caractère, sont aussi formées d'espèces pour la plupart nouvelles, au nombre de trente-six, réparties dans quatorze genres. Leur face est comme écrasće; leurs ailes sont trigones et epaisses. Elles se trouvent sur les Ombellifères des collines calcaires.

Les Théramydes (Theramyda), moins nombrcuses en genres, puisqu'il n'y en a que huit, renferment cependant plus d'espèces, parce que sous le nom générique de Myophore sont placées les nombreuses espèces voisines de nos Mussa carnaria, vivipara, lardaria, si communes dans les champs. Elles se dislinguent principalement par des antennes un peu raccourcies, lont le second article est comme gibbeux, et par leur corps it teintes grises en lignes ou en plaques. La plupart paraissent etrc vivipares.

Les Muscides (Muscida) forment la tribu la plus nombrense en genres et en espèces. En effet, $M$. Robineau n'en caractérise pas moins de deux cents espèces réparties en quarante-quatre genres. Le Musca meridiana forme son G. Stygia. Le Musca alteralibis est le type de son G. Pollenia, qui en contient trentequatre autres. Le Musca vonitoria constitue le G. Calliphora. Dans celui qu'il nomme Chrysomya se trouvent la brillante Mouche César, et beaucoup d'autres espèces que les auteurs avaient jusqu'ici regardées, peut-être avec raison, comme de simples variétés.

Son G. Biomye contient unc Mouche qui fatigue cruellement les gros quadrupèdes, et qu'il nomme à cause de cela $B$. stimulans. Enlin la Mouche, si commune dans nos habitations qu'clle en a reçu le nom de $M$. domestique, constilue avec neuf autres cspèces le G. Musca. Cette tribu se distingue de la précédente, principalement parce que le deuxième article antennaire est toujours sillonnë, et que le corps n'est ni oblong, ni gris. L'anus des mâles n'est point non plus replié en un tube solide.

La tribu des Aricines (Aricina), beaucoup moins nombreuse en espèces et en genres que la précédente, a déjà ses cuillerons 
sensiblement diminués. Elle a pour type le Musca testacea de Fabricius. Comme elle, toutes les espèces qui la composent offrent des teintes fauves ou testacées; l'abdomen est ordinatrement ponctué ainsi que les ailes; le corps est cylindriforme, quelquefois diprimé. Elles vivent souvent sur les végétaux attaqués de carie.

Les Gagatées (Gagatea) font encore mieux le passage vers la famille suivante par leurs mours, par la diminution des cuillerons, et meme de leur grosseur en général. Les espèces sont ordinairement noires, d'où les noms de Melanophora, de Nigria, d'Afrella et mème de Melania, qui devra être changé, parce qu'il est déjà employé dans une autre partie de la Zoologie. Le corps est cylindrique, la face presque supprimée par le grand développement des régions optiques. Quelquefois le second article des antennes est plus long que le troisième.

La seconde famille des Myodaires porte le nom de Mésomydes (Mesomrda), à cause de leur taille moyenne, et du moindre développement des cuillerons. C'est une suite directe des deux dernières tribus des Calyptérées, en ce que la grosseur diminue beaucoup, et que le système de coloration est moins vif, plus étiolé. Du reste, nous ne voyons pas de caractères bien tranchés pour sa séparation. Aussi la première subdivision en trois section ${ }^{\mathrm{S}}$ porte-t-elle sur trois degrés de dévcloppement du cuilleron, et les autres sur la nudité ou sur le degré de villosité de la soie. It en résulte cing tribus.

Les Limoselles (Limosella), évidemment très-voisincs des Gagatées, sonı cependant en général plus allongées. Leurs teintes sont plus grises, plus cendrées, et la soie antennaire est ordinairement villeuse. Elles vivent, comme l'indique leur nom, sur le bord de l'eau. Presque toutes les espèces de cette tribu, au nombre de plus de soixante, réparties dans quatorze genres, sont nouvelles, et se groupent autour du Musca punctata de Gmelin.

Les Éleutherées (Eleutherata) ont encore les cuillerons assez larges; la soie antennaire est ordinairement nue. Da reste, celle tribu pourrait sans inconvénient être réunie à la précédente, d'au tant plus qu'elle ne renferme qu'un petit nombre d'espèces, cinq pour quatrc genres.

La tribu des Chorellées (Chorellea), ani tire son nom de la 
Mouche danseuse, M. Chorca de Fabricius, à cause de ses mouvemens dans les airs, renferme un plus grand nombre d'espèces en général assez petites, de couleur cendrée ou grise. Les cuillerons sont de moyenne taille : la soie est plus villeuse, et l'abdomen des mâles est atténuć et comme vide. Elle renferme vingtquatre genres et plus de quatre-vingts espèces, presque toutes nouvelles, parmi lesquelles se trouve l'Anthomya pluvialis de M. La treille.

Les Hylémydes ( Hylemyda) commencent la seconde section de cette famille, dans laquelle les cuillerons sont très-petits. C'est cependant encore le même aspect, le même système de coloration el d'ailes. Le corps est un peu plus cylindrique : le ventre des mâles n'est pas attéuué; elles vivent surtout dans les bois.

Enfin les Stigmatatées (Stigmatatea) ont les cuillerons plus petits, toujours la même forme, les mêmes teintes, le mème système d'ailes. Mais la région frontale est plus déveluppée, et ordinairement colorée en rouge. Cette tribu contient une soixantaine d'espèces, réparties en vingt-un genres.

La troisième famille renferme toutes les espèces de Mouches en général assez petites, qui vivent dans les matières animales ou végćtales en putréfaction, ce qu'indiquent la petitesse de leurs cuillerons, leur coloration pâle et le peu de consistance de leur corps. C'est de cette dernière particularité qu'a èté tiré le nom de Malicosômes (Malacosoma). C'est peut-être dans cette famille que M. Robineau a fait le plus de découvertes en espèces. Il la divise en cinq tribus.

Les Pégomydes (Pegomyda), ainsi nommées de leurs larves mineuses de feuilles, ont une très-grande analogie avec la dernière section des Cborellées, dont elles diffèrent spécialement par la forme quadrilatère du front et la petitesse des cuillerons. Elles en ont encore davantage avecla tribu suivante par la coloration de la région frontale et celle des antennes à leur base. Ce sont cependant des mœurs toutes différentes. Aussi le ventre des femelles est-il toujours pourvu d'une petite tarière. Toutes les larves mineuses dont Réaumur et de Géer ont fait l'histoirc avec tant d'intérêt appartiennent à cette tribu, qui contient huit espèces disposées en six genres. 
Les Scatophagines (Scatophagince), dont le nom indique l'habitude principale de vivre dans le fumier, et en général sur les matières organisées en putréfaction, ont pour type le Musca scybalaria de Linné et de Fabricius, si commun aux environs de nos villes. Cette tribu, qui ne diffère essentiellement de la précédente que parce que les espèces qui la composent déposent leurs ceufs sur des débris organisés, et que les antennes sont un peu plus longues, a du reste les mêmes teintes, la même forme de corps et d'ailes. Elle est diviséc en buit genres, contenant trenteune espèces, dont plusieurs sont nouvelles. Nous signalerons cornme une découverte intéressante de l'auteur, celle qui, malgré l'ursenic dont on les a imprégnées, détruit les pièces desséchées de nos collections anitomiques. Nous noterons l'observation déjà faite dans d'autres parties de la Zoologie, que la Mouche jaunevelue, qui vit sur nos excrémens, est beaucoup plus nombreuse et surtout plus grosse et plus velue autour des villes que dans les bois, et qu'ainsi elle a aussi éprouvé l'influence de la socièté de l'homme.

La tribu des Mycétomydes (Mycetomydo), qui tire également son nom de sun habitude de vivre dans les champignons, est encore très-rapprochée des deux précédentes par l'aspect général. Mais la consistance du corps est encore plus molle : la soie antennaire est toujours plus ou moins villeuse : le ventre da mâle se replie constamment en dessous. Les antennes, distantes à la base, sont composées de trois articles distincts, dont le dernier n'est jamais sphérique : enfin les ailes sont souvent liturées. Parmi les dix-neuf espèces que M. Robineau définit dans les huit genres de cette tribu, toutes à peu près sont nouvelles. La plus grosse et la plus remarquable est celle qui vit dans la truffe, et dont Réaumur n'avait connu que la larve.

Les Térhénides (Terhenida), dont la dénomination indique Ja mollesse, et qui constituent la quatrième tribu, ont encore l'abdomen recourbé en dessous. Aussi ne diffërent-elles des $\mathbf{M y -}$ cétomydes, que parce que leur corps est encore plus resserré. Leur couleur est jaune de biscuit, et leurs yeus sont purpurescens. Elles ne se trouvent que dans les lieux ombragés, sur les feuilles des arbres et des plantes aquatiques. Ce sont de petites espèces de 


\section{( 15$)$}

Mouches assez nombreuses, jusqu'alors complètement négligécs, que M. Robineau répartit dans quinze genres.

Les Malacomydes (Malacomyda), qui ont pour caractères distinctifs d'avoir les antennes courles, avec le troisième article lenticulaire ou sphérique, le corselet brunâtre et le corps rougeâtre, sans que l'abdomen soit recourbé en dessous, quoi que assez nombreuses, puisque M. Robineau en porte le nombre à plus d'une douzaine, réparties dans cinq genres, sont toutes nouvelles, et se trouvent aussi dans les lieux ombragés et humides, quelquefois meme dans nos appartemens. La mollesse de leur constitution les rend extrênement difficiles à conserver daos les collections.

La quatrième famille porte le nom d'Aciphorées (Aciphorea), parce que le ventre de la femelle a ses derniers anneaux solides, non rétractiles, et qui, produisant l'effet de la tarière des Tenthrèdes, sert à introduire les œufs sous l'épiderme des plantes, où leur présence fait naître des galles. Sans ce caractère, cette famille serait artificielle; car elle comprend des espèces de Mouches qui ont beaucoup de ressemblance avec les trois dernières tribus précédentes. Les ailes sont plus souvent bigarrées de fascies et de points noirs. Ce sont des Insectes essentiellement phytophages, à l'état de larves comme à l'état adulte. Chaque espèce est souvent fixée sur une espèce particulière de plante; mais non pas toujours : ce qui, suivant l'observation de M. Robineau, a causć heaucoup de confusion dans les travaux des entomologistes. Il s'est efforcè d'établir solidement la distinction des espèces nombreuses de cette familie dans laquelle il n'a pu instituer de tribus, et qu'il répartit de suite en vingt-quatre genres, surtout d'après la considération du péristôme.

La cinquième famille ne renferme aussi qu'une seule tribu. Ellc est désignée par la dénomination de Palomydes (Palomyda), parce que toutes les espèces qui la composent, vivent sur les plantes herbacées des marais. Ce sont toujours de petites Mouches à corps mou, sans cuillerons. Le corps plus ou moins oblong, les ailes étroites, quelquefois mouchetées, les couleurs flavescentes annoncent ce groupe qu'il est assez difficile de définir comme famille, autrement que par un ensemble de caractères, qui chacun 
se retrouve dans une autre section. Cependant les genres qu'elle contient sont assez distincts, quoique nombreux; les entomologistes avaient déjà établi les G. Tetanocera, Loxocera, Sepedon et Dyctia. M. Robineau en établit vingt-sept autres, comprenant au moins soixante espèces.

La sixième famille, cclle des Napéellées (Napeellece), est beaucoup plus aisée à caractériser par la forme du front et de la face larges et développćs, le péristơme carré, les antennes distantes, horizontales, dont le troisième article tend à s'arrondir, la longueur des pates et les teintes d'un noir mat. A leurs deux états, elles vivent dans les endroits humides et ombragés, sur les substances animales et végétales en décomposition. Cette famille renferme deux tribus.

Celle des Napéelles (Napeella) proprement dites offre le troisième article des antennes cylindrique, et le corps d'un noir luisant, tandis que celui-là est sphérique ou lenticulaire, et celui-ci d'un noir mat dans celle des Putrellidées (Putrellidea). D'ailleurs, l'insecte parfait et sa larve, dans la première, se trouverit sur les plantes aquatiques, au lieu que ceux de la seconde vivent de préférence dans les débris animaux. Parmi les cinquante et quelques espèces de Napẻellées, partagées en onze genres par M. Robinea:i, l'on n'avait encore bien défini que celle qui forme le G. Ochtera de M. Latreille. Les Putrellidées, au nombre de soixante environ, réparties en douze genres, comprennent le Musca cellarum des auteurs, et une foule de petites Mouches qui vivent sur les excrémens.

La septième famille porte le nom de Phylomydes (Phytomyda), parce que les espèces qui la cornposent se trouvent sur les plantes, sans s'y donner de grands mouvemens. Aussi sont-elles assez souvent sans cuillerons. Leur corps est mou, allongé, quelquefois mème filiforme, coloré de teintes métalliques; ce qui les rapproche évidemment des Ophyres parmi les Calyptérées. Il s'en trouve néanmoins quelques-unes diont le système de coloration est plus jấle et plus étiolé.

Les différences assez nombreuses que ces Phytomydes présentent, ont conduit M. Robineau à former quatre petites tribus.

Les Hẹliadées (Heliadea), ainsi nommécs parce qu'elles se 


\section{( 17 )}

trouvent plus au soleil que les autres, ce qu'indique le poli de leur corps, orné de teintes brillantes noires ou dorées, ont le Musca aurata de $\mathbf{F a b r i c i u s ~ p o u r ~ t y p e . ~}$

Les Myodines (Myodina) ne diffèrent de la tribu prćcédente, que par la plus grande longueur du troisième arlicle antennaire, et par la soie ordinairement nue. C'est décidément cette tribu qui comprend la mouche de l'olivier, dont M. Robineau fait le G. Elaïmya.

Les Thélidomyes (Thelidomya), dont le corps allongć, trèsfiliforme, rappelle celui des Ichneumons, est porté sur de longues pates, et terminé par une espèce de tarière sur les femelles. Le G. Calobate de M. Latreille en fait partie, ainsi que les Mouches tipulaires de Fabricius, réunies sons le nom générique de $\mathrm{Cli}-$ donies.

Les Hydrellees ( Hydrellea), dont le nom n'est pas tout-ì -fait en rapport avec les habitudes, puisque la plupart vivent sur les fleurs, ont une forme de corps qui les rapproche des Thélidomyes et se groupent autour du G. Micropeza établi sur le Musca cynipsad de Fabricius. M. Robineau en signale unf cinquantaine d'espèces, réparties dans dix genres peu distincts.

La dénomination de Micromydes (Micromyda) imposéc par M. Robineau à la huitième famille, indique qu'elle renferme en général les plus petites espèces de Mouches. Elles le sont en effet telloment, que nos épingles à Insectes les plus fines, peuvent a peine suffire à les piquer sans les détruire entièrement. Il la définit d'après la briéveté des antennes, la petitesse du corps ordinairement noir ou flavescent, et parce que le ventre de la femeile est terminé par quelques anneaux formant taricre. Toutes les larves sont granivores, et les femelles déposent leurs culs dans l'ovaire des fleurs.

Malgré la petitesse de ces espèces, M. Robincau en a observé un assez grand nombre pour être obligé de les partager en six tribus.

Les Anthidulées (Anthidulea), dont le principal caractère est d'avoir la région stemmatique très-développéc, les ailes claires et le bouton des balanciers arrondi, renferment une 


\section{( 38$)$}

soixantaine d'espèces réparties dans seize genres. Le Musca sallatrix de Linné en est le type.

Les Pherbonydes (Pherbornyda), qui ne forment qu'un genre contenant, il est vrai, seize espèces, ont beaucoup de rapport avec les Anthidulées, mais leurs ailes ont cinq à six nervures droites, et le bouton des balanciers est allongé. Leur système d'ailes est en efet assı z particulier.

Les Anthidulinées (Anthidulinea), dont le nom est peut-être un peu trop semblable à celui de la première tribu, n'en diffèrent en effet que parce que la région stemmatique n'est pas développée, et que leur teinte générale est plus flavescente; clles vivent aussi davantage dans les lieus humides. M. Robin rau en caractérise une quarantaine d'espèces réparties en douze genres.

Les Herbellidéés (Herbellidea), dont le nom indique l'habitude de vivre parmi les herbes, et cependant sur les fleurs, ont le corps arrondi, assez brillant, les ailes élargies, souvent trigones, les cuillerons un peu développées, et la soie antennaire toujours nue. M. Robineau en compte environ trente espèces disIribuéés dans une douzaine de genres.

Les Floridulées (Floridulea) ont les ailes moins élargies, le rorps plus allongé, moins consistant, sans cuillerons. Quoique M. Robineau regarde lui-méme son travail sur ce groupe de petiles Moucbes comme encore fort incomplet, il n'en decrit pas moins une quarantaine d'espèces, dont il forme seize genres. Il pense que chaque espèce de plante en nourrit une espèce particulière, ce qui sans doute est un peu exagéré.

Les Ptéromydes (Pteromyda), dernière tribu de cette famille, ont les ailes peu larges, mais plus longues que l'abdomen, ce yui fournit un caractère particulier. Elles ont, en outre, le corps : fflé, en général noirâtre, et le dernier article antennaire cylindrique, ce qui les rapproche un peu de la tribu des Gagatées. Elles ne renferment que neuf espèces pour quatre gẹnres.

La neuvième famille, établie par M. Robineau , porte le nom de Muciphorées (Muciphorea); elle a plus de rapports avec les Malacosómes qu'avec aucune uutre famille, à cause de ses teintes flavescentes plus ou moins étiolées, et des espèces qui la compoecul ct qui se nourrissent de produits, soit animaux, soit végétaux, 


\section{( 19$)$}

en décomposition; mais elle en diffère par la forme des antennes. Ces Mouches sont, en outre, benucoup plus petites; le nom de Micromydes leur convenant tout aussi bien qu'à la famille pricédente. Elle comprend quatre tribus.

Les Dorinées (Dorinea), dont la plupart des caractères rappellent les Palomydes, mais qui s'en distinguent parce que la soie antennaire est plumeuse sur le dos sculement, et que le troisième article est lenticulaire. Elles renferment treize espèces distribuées en cinq genres, et comprenant le Tephrytis florum de Fabricius.

Les Mongomydes (Mungomyda), que li forme cylindrique d" troisième article des antennes, ainsi que la villositć de la soie sur ses deur faces, distinguent des Dorinées, sont aussi plus molles, plus flavescentes; elles vivent dans les liquides végétaux en décomposition. M. Robineau n'en compte encore qu'une ving* taine d'espèces partagées en six genres; mais il suppose qu'on en découvrira beaucoup d'autres; celle qui vil sur les pommes pourries est la plus grosse.

Les Gibbomydes (Gibbomyda) sont aisément distinguéespar la gibbosité de leur corselet, particularité dont leu nom a été tiré ; mais elles le sont également par la forme presque carrée de la face, par les cils frontaux fortement redressés en arrière, par les palpes sensiblement ciliés en bas, et par les ailes spinosules i la base de leur bord antérieur. Elles vivent, du reste, comme les autres Muciphorées, sur les matières organisées en dissolution : l'une dans le fromage (c'est le Musca putris casei de Linné), unc: autre aux dépens de nos collections entomologiques. On peut grouper toutes les Gybbomydes, dont le nombre est d'une vingtaine environ, autour du G. Phora de M. Latreille. M. Robineau en propose huit.

Les Mycénides (Mycenida), dont la découverte est due lout cntière à M. Desvoidy, ne contiennent que quatre ou cinq cspèces réparties en deux genres. Elles sont caractérisées d'une manière exclusive par la disposition de la soie antennaire, qui remonte sur le troisième article et qui s'implante prestrue à son sommet, ce qui les rapproche des Dolichopes. Elles on le purt 
(20)

d'une Mouche avec des teintes noires, et elles vivent dans lés champignons.

Enfin, la dixième famille est celle des Céphialémydes (Cephalemyda), qui ont la têle grosse, le dernier article antennaire styliforme et la bouche très-petite. Le type est le G. Pipunculus de $M$. Latreille, donı M. Robineau caractérise dix espèces, qui ont le corps cylindrique et noir, et qu'on prend sur les fleurs. On ignore totalement les mours des larves, et divers caractères portent à croire que cette section de Diptères n'appartient pas à l'ordre des Myodaires.

Telle est l'analyse exacte, quoique trop brève malgré sa longueur, du grand travail que M. Robineau a soumis au jugement de l'Académie, travail rédigé presque entièrement, et accompagné de cinq cadres ou tableaux contenant toutes les espèces de Diptèrcs de la famille des Mouches observées et recucillies par lui dans une petite étendue du département de l'Yonne.

Le nombre tolal de ces espèces est d'ì peu près dix-huit cents, dont plus de quatorze cents sont nouvclles, ou du moins nouvellement défnics, et parmi lesquelles dix-sept à dix huit genres seulement sont exotiques.

Vos Commissaires ont examiné avec attention l'ourrage de M. Robineau, sans cependant avoir pu le faire sur tous les genres, et a plus forte raison sur toutes les espèces, qui, étant.dessécheces et pour la plupart extrêmement petites, ne pouvaient être analysées.

Le nombre des espèces nouvelles leur a réellement paru extrêmement considèrable. Il se pourrait cependant que $M$. Desvoidy ne s'étant pas encore fait une idée un peu positive de l'espèce, quı n'est bien confirmée que lorsqu'clle est caractérisée par des différences appréciables dans l'appareil générateur, ait considéré de simples variétés de circonstances, c'est-ä-dire de grosscur, de villosité et de vivacité dans les couleurs, comme formant des espèces distinctes.

Quoi qu'il en soit, an considérant toutes ces espèces et en les rapprocluant sous différens points de vae, M. Robineau a ćtí conduit nécessairement à les grouper autour des espèces principales, ce qui a constitué ses fomilles ct ses tribus, qui nous sent- 


\section{( $2 \mathrm{~T})$}

blent en général assez naturelles, mais quelquefois peu nettement caractérisćes.

La considération de la proportion des arlicles basilaires de l'antenne, ainsi que des articles distincts de la soie, la nudité ou la villosité plus ou moins considérable de sa partie non articulée, ont servi à l'établissement des genres, qui nous semblent évidemment trop nombreus, d'autant plus qu'ils ne nous paraissent que rarement confirmés par des différences dans la structure des ailes et dans celles de la trompe, parties malheureusement un peu trop négligées dans le travail de M. Robineau. En étudiant, par exemple, un certain nombre de genres qui constituent la première famille, celle des Calyptérées, nous nous sommes convaincus qu'ils ne reposent, le plus souvent, que sur de très-légères différences dans la proportion du second et du troisicme article antennaire. Quelquefois même le genre n'a pas le caractère de sa tribu.

C'est une heureuse idée sans doute que d'avoir fait marcher de front les différettices de classification des espèces avec celles de mœurs et d'habitudes, et d'avoir ainsi formé des familles, suivant l'espèce de nourriture, à l'élat de larve ou à l'état parfait. Mais il faut craindre d'aller trop loin, et que la distinction des genres et même des espèces ne vînt ì reposer seulement sur la différence des plantes ou du séjour, et non plus sur celles de l'organisalion.

En général, toute la partic systématique nous a paru pécher par une trop grande multiplicité de coupes de premier, de sccond, et même de troisième degré. Le nombre des genres, par exemple, est tel que, l'un portant l'autre, ils ne contiennent pas trois espèces. On conçoit que $M$. Robineau ait pu avoir besoin de cet échafaudage pour arriver à la destination d'espèces aussi nombreuscs et aussi rapprochées ; mais il aurait dû peut-être en faire disparaitre une partie.

Du reste, les noms de famille, de tribus, et même ceux de genres, sont bien formés, courts et euphoniques. Il en est peu qui doivent étre rejetés, parce qu'ils sont déjà employés dans d'autres parties de la Zoologie. Les moins bons sont souvent ceux qui sont tirés de noms d'hommés plus ou moins cólébres dans 
les sciences naturelles, et surtout en entomologie, parce qu'ils ne sont pas toujours brefs ct faciles à prononcer. D'ailleurs, ils offrent quekquefois le petit inconvénient d'allier le nom d'un homme distingué à une épithète désagréable.

Vos Commissaires ne regardent cependant pas le travail de M. Robineau, tel qu'il le leur a remis, comme terminé.

$1^{\circ}$. Parce qu'il n'a peut-t̂tre pas suffisamment circonscrit son sujet en le définissant nettement, ce qu'il ne pouvait faire qu'en donnant un tableau préliminaire des Diptères.

$2^{\circ}$. Parce qu'il n'a pas fuit précéder son travail d'une terminologie un peu étendue, ce qui élait d'une rigoureuse nécessité, quoique difficile. Il se serait alors rendu compte des deux paires de palpes inférieurs, qu'il admet dans les genres Phorophylle et Phyto. En effet, il aurait vu que tous les Diptères ont ces memes parties, mais seulement moins distinetes, et qu'elles constituent les lèvres de la trompe, l'apparence plus grande des quatre palpes étant sans doute due aux extrénités prolongées de chaquc lèvre. Il aurait également vu que la considération des nervures des ailes, qu'il a négligées, et dont Meigen, au contraire, s'est servi avec beaucoup d'avantage, pourrait confirmer plusieurs de ses grandes divisions. Peut-ètre aussi aurait-il été conduit à employer la considération de la trompe, qui, bien analysée, nous parait devoir fournir de bons caractères, quoique d'un difficile emploi.

$3^{\circ}$. Parce qu'il n'a pas établi de synonymie avec les auteurs les plus récens, et qui se sont spécialement occupés du même sujet, comme Fallen et surtout Meigen, lacune difficile sans doute, et peut-étre peu importante en apparence, mais que nous l'invitons fortement à remplir, d'abord par esprit de justice, ensuite pour ne pas embarrasser encore la science de noms différens imposés aux mèmes espèces, aux mềmes divisions. En voyant en effet que Meigen, par suite des travaux de Panzer, de Fallen, de Wiedeman et dessiens, décrit près de trois cents espèces d'Allemagne, qui ne sont pas dans le Systema Antliatorum de Fabricius, il est impossible de croire que plusieurs de celles de M. Robineau ne formeront pas un double emploi.

$4^{\circ}$. Enfin, parce que M. Robineau est encore assez loin d'avoir 


\section{(23)}

visité ct étudic toutes les collections de Paris, ce qu'il serait très-important qu'il continuât (car il a déjà commencé à le faire), afin que son travail largement basé sur l'observation complète et positive, faite sur le vivant, de nos espèces de France, puisse ĉtre t́tendu par une analogie bien conduite à toutes les espèces recueillies dans les différentes parties du monde, et servît ensuile aux considérations philosophiques de distribution géographique, et à la détermination définitive des espèces. Nous pouvons mème assurer que M. Robineau, qui a dù nécessairement penser à ce travail, s'en occupe avec activité, et que déjà le nombre des es pèces de Mouches, en y comprenant celies observées dans les collections de Paris, monte h plus de trois mille.

Vos Commissaires pensent aussi que dans la rédaction finale de son ouvrage, M. Robineau fera bien de proportionner son style au sujet, et de n'ambitionner dans un pareil travail que la clarté et la simplicité des descriptions, et de ne donner qu'une analyse rigoureuse des faits pour les généralités qui leur ont paru quelquefois un peu prétentieuses, défaut peu important, qui tient trop évidemment à l'âge de l'auteur pour ne pas étre excusé.

Malgré ces légères critiques, qui sont même plutôt des avis pour le rendre plus complet, nous n'en regardons pas moins l'ouvrage de $M$. Robincau comme d'une très-grande valeur intrinsèque, par cela seul qu'il renferme l'observation el la distinction d'un nombre d'espèces de Mouches quatre à cing fois plus considérable que celui que l'on connaissait dans les auteurs les plus récens, et ensuite comme indiquant une nature et une direction d'esprit, une persévérance et une patience d'observation malheureusement trop rares nijourd'hui en Zoologie, ou il semble bien plus aisé de s'élever à des considérations générales avant même de connaître un petit nombre de spécialités. L'heureuse alliance que M. Robineau a pu faire des connaissances botaniques et entomologiques, c'est-à-dire, des plantes et des insectes qui vivent ì leurs dépens, a aussi donné à son travail quelque chose de neuf, qu'il se propose d'appliquer aux autres parties de l'entomologie qui en sont susceptibles.

En conséquence, vos Commissaires vous pruposent d'insérer le travail de M. Robineau dans le recueil des Savans étrangers, dont 


\section{( 24 )}

ils le regardent comme très-digne. Ils vous proposent mème d'en faciliter et d'en accélérer la publication par tous les moyens qui sont à votre disposition. Sans cela, il est à craindre que, ne trouvant pas de libraire qui veuille en faire les frais, l'auteur nevoie cinq ou six annèes de recherches assidues perdues pour sa réputálion, et qu'ainsi rebuté par un coup d'essai aussi malheureux, il ne continue pas ses travaux entomologiques, ce qui nous semble devoir être une perte réelle pour la science, comme pour la gloire de la France, dont la zoologie est encore si peu avancée.

LATREILLE.

C. DUMÉRIL.

D. DE BLAINVILLE, Rapporteur.

Certifé conforme à l'original,

Le Secrétaire perpétuel Conseiller-d'Étal, Commandeur de l'Urdre royal de la Légion-d'Honneur,

Le Baron CUVIER.

IMPRIMERIE DE J. TASTU, RUE DE VAUGIRARD, N. 36 . 